\title{
Evaluierung der Umsetzung von HORIZON 2020, EUREKA, COSME, EEN und ERA in Österreich
}

\section{Endbericht}

AIT Austrian Institute of Technology (Projektleitung):

Michael Dinges, Peter Biegelbauer, Robert Kalcik, Anna Wang, Matthias Weber

Joanneum Research:

Michael Ploder, Wolfgang Polt, Jürgen Streicher, Maximilian Unger

KMU Forschung Austria:

Iris Fischl, Peter Kaufmann

ZSI - Zentrum für Soziale Innovation:

Helmut Gassler, Stefanie Konzett-Smoliner, Klaus Schuch

Wien, Juli 2018

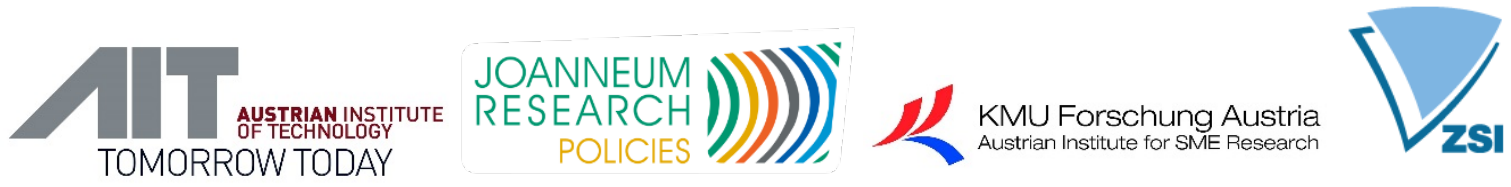



AIT Austrian Institute of Technology

Center for Innovation Systems \& Policy

\author{
Evaluierung der Umsetzung von \\ HORIZON 2020, EUREKA, \\ COSME, EEN und ERA \\ in Österreich
}

Endbericht

Giefinggasse 4, 1210 Wien, Austria

Juli 2018 


\section{Inhalt}

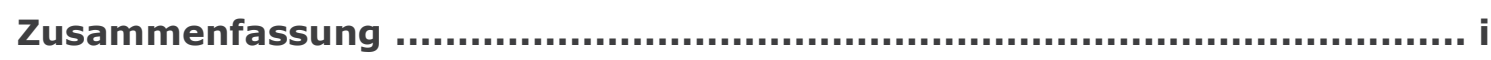

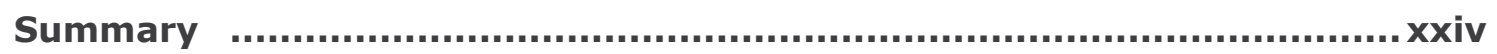

1 Zielsetzung und Methodik der Evaluierung........................................ 1

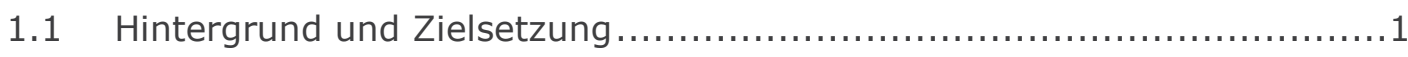

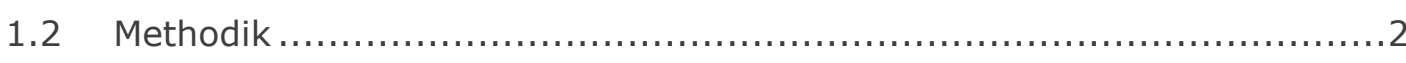

2 Das Leistungsspektrum von FFG-EIP ......................................... 7

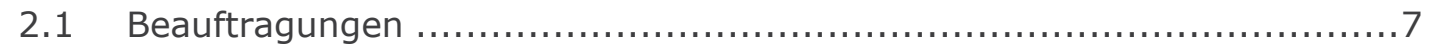

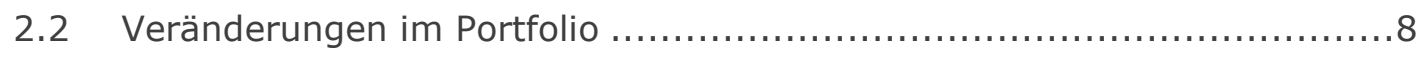

3 Beratung und Betreuung - Fokus Wissenschaft .......................... 14

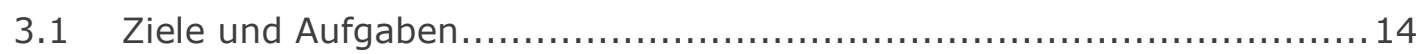

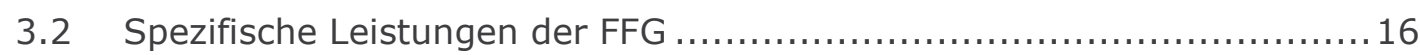

3.3 Ergebnisse der empirischen Erhebungen .............................. 19

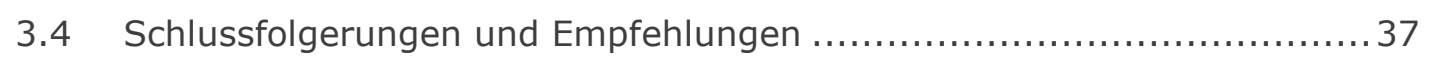

4 Beratung und Betreuung - Fokus Wirtschaft................................ 43

4.1 Ziele und Aufgaben.................................................. 43

4.2 Leistungen der FFG...................................................... 47

4.3 Spezifische Leistungen: EUREKA, COSME, EEN .......................... 50

4.4 Ergebnisse der empirischen Erhebungen .............................. 55

4.5 Ergebnisse zu EUREKA, COSME und EEN ................................6 66

4.6 Schlussfolgerungen und Empfehlungen ................................. 76

5 Governance und Effizienz der EIP-Beauftragung........................... 82

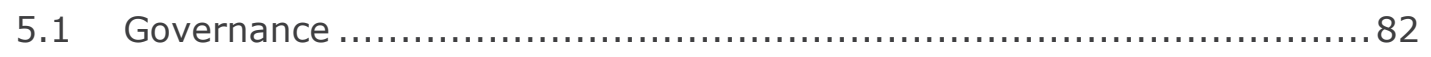

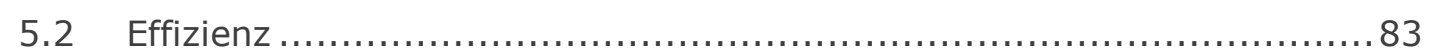

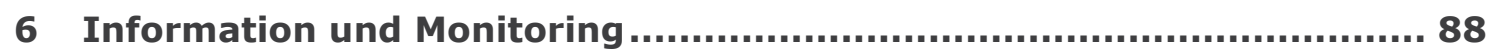

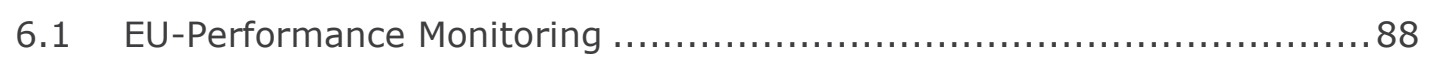

6.2 Ergebnisse der empirischen Erhebungen .............................. 93

6.3 Schlussfolgerungen \& Empfehlungen.................................. 107

7 Koordination im Europäischen Forschungsraum: ERA-

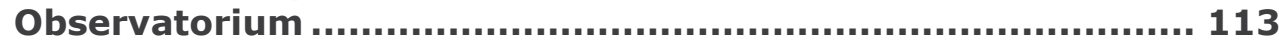

7.1 Ziele und Aufgaben................................................ 113

7.2 Informationen und Austauschmöglichkeiten über EU-Politiken........... 114

7.3 Bedarfsgerechte Beratung und Betreuung für H2O2O und multilaterale Initiativen ............................................... 117

7.4 Unterstützung für den Strukturwandel im Politikbereich ................ 130

7.5 Schlussfolgerungen und Empfehlungen .............................. 134

8 Ausblick und Szenarien ............................................................. 140 
8.1 Positionierung der österreichischen FTI-Politik in FP9 als neuer Rahmen für die nationalen Unterstützungsstrukturen ................... 140

8.2 Erwartungen der österreichischen FTI-Community ..................... 142

8.3 Szenarien zur österreichischen Positionierung in FP9 und ihrer

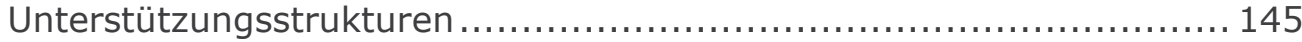

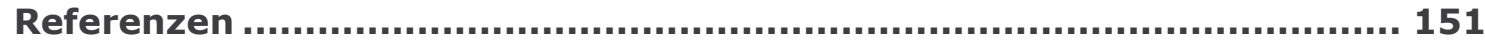

Annex I: Zielerreichung im Rahmen der WFA .................................. 153

Annex II: Weiterentwicklung der WFA ............................................. 162

Annex III: Zugriffsstatistiken und alternative Tools EU-PM................. 172

Annex IV: TeilnehmerInnen an ExpertInneninterviews und

Fokusgruppen ....................................................................... 183

Annex V: Mitglieder des internationalen ExpertInnenbeirates............... 187 


\section{Abbildungsverzeichnis}

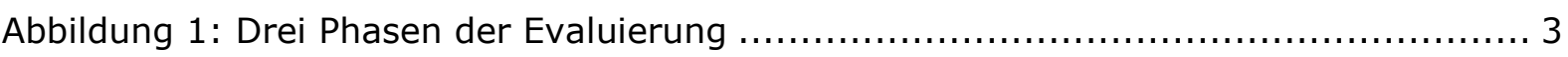

Abbildung 2: Zielsetzungen der $\mathrm{H} 2020$ Beauftragung .................................... 8

Abbildung 3: Persönliche Beratungen zu Rahmenprogrammen und MULLAT ................12

Abbildung 4: Persönliche Beratungen zu Rahmenprogrammen und MULLAT ................13

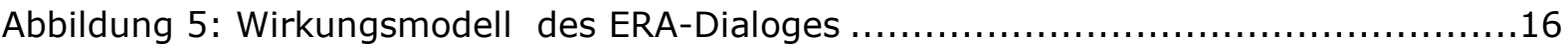

Abbildung 6: Anzahl First und Second Level Beratungen ................................17

Abbildung 7: TeilnehmerInnen der FFG Akademie nach Organisationstyp .................18

Abbildung 8: Zielgruppen der FFG-Akademie nach Veranstaltungsinhalten ................18

Abbildung 9: Erfahrungshintergrund der erreichten Wissenschaftsakteure ................20

Abbildung 10: Verwendete Unterstützungseinrichtungen nach Erfahrungshintergrund ...21

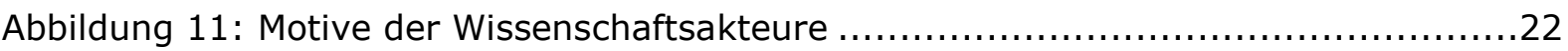

Abbildung 12: In Anspruch genommene Informationsleistungen von FFG-EIP.............23

Abbildung 13: Bewertung der öffentlichen Informationsleistungen (Wissenschaft) ........24

Abbildung 14: Bewertung der persönlichen Beratungsleistungen durch FFG-EIP ..........25

Abbildung 15: Die Nutzung von Weiterbildungsleistungen der FFG-Akademie ...............27

Abbildung 16: Bewertung der Trainings durch FFG-EIP .................................... 28

Abbildung 17: Gesamtbewertung der Leistungen von FFG-EIP nach Schulnoten (in \%)..29

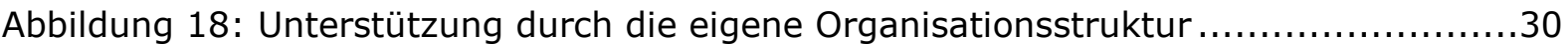

Abbildung 19: Das Leistungsangebot der FFG-EIP nach Kundensegment (Wirtschaft) ....46

Abbildung 20: Logik der Wirkungskette von FFG-EIP (Wirtschaft) .......................46

Abbildung 21: Aktivitäten der FFG-EIP im Fokus "Wirtschaft" im Zeitablauf ................47

Abbildung 22: Praktischer Erfahrungshintergrund der Unternehmen .....................55

Abbildung 23: Allgemeiner Erfahrungshintergrund nach Unternehmensgröße ..............56

Abbildung 24: In Anspruch genommene Unterstützungseinrichtungen .....................5

Abbildung 25: Motive für Inanspruchnahme von FFG-EIP Leistungen..................... 58

Abbildung 26: Bekanntheitsgrad und Nutzung von öffentlichen Informationsleistungen .59

Abbildung 27: Wirkung der Beratungsleistungen............................................ 60

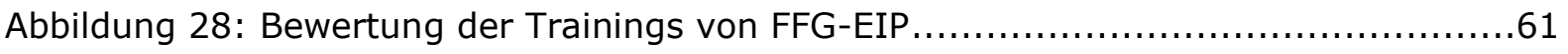

Abbildung 29: Gesamthafte Bewertung der FFG-EIP Leistungen für Unternehmen........65

Abbildung 30: Gesamthafte Bewertung der FFG-EIP Leistungen für Unternehmen........66

Abbildung 31: Bewertung der EEN-Leistungen und Tools durch Intermediäre ..............75

Abbildung 32: Budgetverteilung auf thematische vs. nicht-thematische Aktivitäten .......85

Abbildung 33: Vergleich EIP Aufwand mit Beteiligungsstruktur Österreichs in H2020.....86

Abbildung 34: Aktivitäten EU-PM sowie Gewichtung, Stand November 2016 ..............90

Abbildung 35: Bekanntheit Leistungen EU-PM (Wissenschaft) ............................ 95

Abbildung 36: Bewertung der Leistungen EU-PM, Schulnoten (Wissenschaft) ..............96

Abbildung 37: Bekanntheit Leistungen EU-PM (Unternehmen) .......................... 96

Abbildung 38: Bewertung der Leistungen EU-PM, Schulnoten (Unternehmen) .............97

Abbildung 39: Bekanntheit \& Nutzung Leistungen EU-PM (Intermediäre) ..................98

Abbildung 40: Die Bedeutung des Europa Forum Forschung (Wissenschaftsakteure) ...114

Abbildung 41: Die Bedeutung des Europa Forum Forschung für Intermediäre............115

Abbildung 42: Die Bedeutung des ERA Portal Austrias ................................ 117

Abbildung 43: Zufriedenheit mit MULLAT Beratungsleistungen (Wissenschaft) ............125 
Abbildung 44: Zufriedenheit mit MULLAT Beratungsleistungen (Wirtschaft)

Abbildung 45: Angemessenheit der nationalen Mittel für MULLAT Beteiligungen

Abbildung 46: Zugriffsstatistiken Online-Dienste EU-PM, monatlich

Abbildung 47: Darstellung VINNOVA H2020 Visualization Tool, Säulen

Abbildung 48: Darstellung VINNOVA H2020 Visualization Tool, Calls .174

Abbildung 49: Darstellung VINNOVA H2020 Visualization Tool, Projekte

Abbildung 50: Darstellung VINNOVA H2020 Visualization Tool, Einzelprojekt....

Abbildung 51: Darstellung Dashboard EU-Participant Portal, Österreich

Abbildung 52: Darstellung öffentliches Portal EU-PM, Kennzahlenvergleich AT - DE .... 178

Abbildung 53: EU-PM, Kennzahlenvergleich Top 30 am Beispiel Beteiligungen ....

Abbildung 54: EU-PM, Übersicht AT-Beteiligung nach Programmen

180

Abbildung 55: EU-PM, Vertragsnehmer auf Programmebene am Beispiel ERC-Grants .. 181

Abbildung 56: EU-PM, Beteiligungen nach Bundesland am Beispiel Oberösterreich 


\section{Abkürzungsverzeichnis}

\begin{tabular}{|c|c|}
\hline Abkürzung & Definition \\
\hline ACR & Austrian Cooperative Research \\
\hline AWS & Austria Wirtschaftsservice Gesellschaft \\
\hline BMASGK & $\begin{array}{l}\text { Bundesministerium Arbeit, Soziales, Gesundheit und Konsumenten- } \\
\text { schutz }\end{array}$ \\
\hline BMBWF & Bundesministerium für Bildung, Wissenschaft und Forschung \\
\hline BMDW & Bundesministerium für Digitalisierung und Wirtschaftsstandort \\
\hline BMI & Bundesministerium für Inneres \\
\hline BMNT & Bundesministerium für Nachhaltigkeit und Tourismus \\
\hline BMVIT & Bundesministerium für Verkehr, Innovation und Technologie \\
\hline COSME & $\begin{array}{l}\text { Programm für die Wettbewerbsfähigkeit von Unternehmen und für } \\
\text { KMU }\end{array}$ \\
\hline CPPP & Contractual public-private partnership \\
\hline CSA & Coordination and Support Action \\
\hline $\mathrm{C} 3$ & Core Customer Concept der FFG-EIP \\
\hline EARTO & European Association of Research and Technology Organisations \\
\hline EEN & Enterprise Europe Network \\
\hline EFF & Europa Forum Forschung \\
\hline EFRE & Europäischer Fonds für regionale Entwicklung \\
\hline EIC & European Innovation Council \\
\hline EIMC & Enhancing the Innovation Management Capacity of SMEs \\
\hline EIP & Bereich „Europäische und Internationale Programme" der FFG \\
\hline EIT & European Institute of Technology \\
\hline EJP Cofund & European Joint Programme Cofund \\
\hline EK & Europäische Kommission \\
\hline ERA & European Research Area \\
\hline ERAC & European Research Area Committee \\
\hline ERC & European Research Council \\
\hline ESIF & European Structural and Investment Funds \\
\hline EU-PM & EU-Performance Monitoring \\
\hline F\&E & Forschung und Entwicklung \\
\hline
\end{tabular}




\begin{tabular}{|c|c|}
\hline FFG & Österreichische Forschungsförderungsgesellschaft \\
\hline FoS & Forschungsservicestelle \\
\hline FP7 & $\begin{array}{l}\text { 7. EU-Rahmenprogramm für Forschung, Technologische Entwicklung } \\
\text { und Demonstration }\end{array}$ \\
\hline FWF & Fonds zur Förderung der wissenschaftlichen Forschung \\
\hline FTI & Forschung, Technologie und Innovation \\
\hline GPC & $\begin{array}{l}\text { High Level Group for Joint Programming } \\
\text { (Groupe de haut niveau pour la Programmation Conjointe) }\end{array}$ \\
\hline H2020 & $\begin{array}{l}\text { Horizon 2020, 8. EU-Rahmenprogramm für Forschung und Innova- } \\
\text { tion }\end{array}$ \\
\hline JPI & Joint Programming Initiative \\
\hline$J R$ & Joanneum Research \\
\hline JTI & Joint Technology Initiative \\
\hline KAM & Key Account Management \\
\hline KIC & Knowledge and Innovation Communities \\
\hline KLIEN & Klima- und Energiefonds \\
\hline KMU & Kleine und mittlere Unternehmen \\
\hline NCP & National Contact Point \\
\hline OECD & Organisation for Economic Cooperation and Development \\
\hline PPP & Public-private partnership \\
\hline P2P & Public-public partnership \\
\hline RFTE & Rat für Forschung und Technologieentwicklung \\
\hline SFG & Steirische Wirtschaftsförderung \\
\hline SFIC & Strategic Forum for International S\&T Cooperation \\
\hline TAFTIE & The European Network of Innovation Agencies \\
\hline Uniko & Österreichische Universitätenkonferenz \\
\hline WFA & Wirkungsorientierte Folgenabschätzung \\
\hline WKÖ & Wirtschaftskammer Österreich \\
\hline ÖAW & Österreichische Akademie der Wissenschaften \\
\hline
\end{tabular}





\section{Zusammenfassung}

\section{Hintergrund, Zielsetzungen und Methodik der Evaluierung}

Im Jahr 2013 wurde die FFG, und dort konkret der Bereich für Europäische und Internationale Programme (FFG-EIP), von der Republik Österreich ${ }^{1}$ und der Wirtschaftskammer Österreich mit der Unterstützung der Umsetzung von Horizon 2020 (H2020), dem europäischen Rahmenprogramm für Forschung und Innovation und der European Research Area (ERA) für den Zeitraum 2014-2020 beauftragt. Die Beauftragung soll eine optimale Beteiligung österreichischer FTI-Akteure in H2020 und in multilateralen FTI-Initiativen (MULLATs) erreichen. FFG-EIP baut in Hinblick auf die Unterstützung der österreichischen FTI-Akteure auf den Kompetenzen und gesammelten Erfahrungen vorheriger Beauftragungen auf. Darüber hinaus ist FFG-EIP die offiziell nominierte Nationale Kontaktstelle (NCP) der europäischen Kommission für H2020 und alle multilateralen Initiativen und Programme, die in Verbindung mit H2020 und ERA stehen. Im Jahr 2014 wurde die FFG überdies mit dem Monitoring der österreichischen Beteiligung in H2020 und ERA betraut. Darüber hinaus ist die FFG mit PartnerInnen auch für Unterstützungsleistungen für EUREKA, COSME und das Enterprise Europe Network (EEN) verantwortlich.

Um den übergeordneten Zielsetzungen der Beauftragung Rechnung zu tragen, verfolgt FFG-EIP vier zentrale Zielsetzungen, die den ergebnisorientierten Handlungsrahmen als nationale Kontaktstelle für H2020 Rechnung trägt:

1. Ziel 1: Unterstützung der erfolgreichen Beteiligung durch Information und Beratung

2. Ziel 2: Systemorientierte Unterstützung österreichischer FTI-Organisationen

3. Ziel 3: Kompetenter Partner für europäische FTI-Programme und ERA-Entwicklungen

4. Ziel 4: Analyse und FTI-Politikunterstützung

Vor diesem Hintergrund, ist es das Ziel dieser Evaluierung eine systematische Gesamtbetrachtung der Umsetzung von $\mathrm{H} 2020$ und ERA in Österreich sowie von EUREKA, COSME und EEN vorzunehmen. Die drei Schwerpunktbereiche der Evaluierung sind:

1. Beauftragung der FFG mit der Beratung und Betreuung von H2020 und ERA (EIP-Beauftragung)

2. Beauftragung der FFG mit dem EU-Performance Monitoring (EU-PM-Beauftragung)

3. Das ERA-Observatorium Austria, das einen übergeordneten Rahmen für die Integration der einzelnen Aufgabenstellungen im Bereich der Governance der EU-FTI-Politik in Österreich auf Seiten des BMBWF bildet.

\footnotetext{
${ }^{1}$ Zum Beauftragungszeitpunkt 2013 vertreten durch das Bundesministerium für Wirtschaft, Familie und Jugend; das Bundesministerium für Verkehr, Innovation und Technologie; das Bundesministerium für Land-und Forstwirtschaft, Umwelt und Wasserwirtschaft; das Bundesministerium für Gesundheit sowie das Bundesministerium für Wissenschaft und Forschung als federführendes Ressort.
} 
Darüber hinaus werden im Rahmen der Evaluierung ergänzend die Beauftragungen zu EUREKA, COSME und dem EEN analysiert.

Den vielfältigen Einzelfragestellungen der Evaluierung liegen folgende Meta-Fragestellungen zugrunde:

1. Generelle Würdigung der Umsetzung: Sind die Ausrichtung und Umsetzung der vorhandenen Formate und Unterstützungsleistungen - unter dem gegebenen budgetären Rahmen - grundsätzlich geeignet bzw. entsprechend implementiert, um die erwünschten Wirkungen der Maßnahmen in Hinblick auf den Output so effizient und effektiv wie möglich zu erzielen?

2. Würdigung spezifischer Leistungen: Wo sind - angesichts von beobachtbaren und zu erwartenden Entwicklungen im FTI-Bereich (inklusive FTI-Politik) - die Formate und Unterstützungsleistungen adäquat und wo sind Nachbesserungen anzusetzen?

3. Handlungsbedarf und Handlungsoptionen: Wo gibt es weiße Flecken oder wo sind neue und bessere Ansätze und Instrumente zu schaffen? Nach welchen Prinzipien sind diese im Wesentlichen zu gestalten?

Das Evaluierungsdesign fußte auf einer Triangulation sich ergänzender Methoden und Perspektiven: Dieser Ansatz kombiniert unterschiedliche quantitative und qualitative Methoden so, dass Befunde von mindestens zwei Quellen bestätigt werden. Hinzu kam ein internationaler Beirat aus renommierten europäischen ExpertInnen, der das Evaluierungsteam bei der Reflexion der Ergebnisse, dem Ziehen von entsprechenden Schlussfolgerungen und der Ableitung von Empfehlungen für die Anpassung und Weiterentwicklung des österreichischen Angebotsportfolios unterstützte².

Im Mittelpunkt der Erhebungsphase stand die Gewinnung robuster Daten mithilfe von Desk-Research, ExpertInneninterviews und einer Online-Befragung.

Darauf aufbauend erfolgte eine Validierung und Reflexion der Zwischenresultate gemeinsam mit den AuftraggeberInnen. Davon ausgehend erfolgte eine vertiefte Analyse in Form von Fokusgruppeninterviews, ergänzenden ExpertInneninterviews und einer DelphiBefragung.

Die Ergebnisse dieser Phase bildeten wiederum die Basis für die Entwicklung von Empfehlungen unter Einbindung internationaler Sichtweisen durch eine Fokusgruppe in Brüssel und partizipativer Workshops mit dem internationalen Beirat der Evaluierung sowie der Gruppe der AuftraggeberInnen.

Die Schlussfolgerungen und Empfehlungen der Evaluierung beruhen auf einer Triangulation der Ergebnisse und Erkenntnisse der eingesetzten Methoden, um spezifische Stärken und Schwächen jeder einzelnen Methode zu kompensieren, was zur Validität und Reliabilität der Empfehlungen beiträgt.

\footnotetext{
2 Die Liste der Mitglieder des internationalen Beirats befindet sich in Annex V.
} 


\section{Zentrale Beobachtungen und Empfehlungen}

Die nachfolgenden Empfehlungen der Evaluierung orientieren sich an den drei Schwerpunktbereichen der Evaluierung und damit den verschiedenen Beauftragungen der FFG: Beratung und Betreuung, EU Performance Monitoring, ERA Observatorium. In Bezug auf den Schwerpunktbereich Beratung und Betreuung wird außerdem unterschieden zwischen Empfehlungen, die sich spezifisch auf die Zielgruppen Wissenschaft und Wirtschaft beziehen und solchen, die einen zielgruppenübergreifenden Charakter haben. Die Effizienz und Governance dieser speziellen Beauftragung wird ebenfalls gesondert behandelt.

\section{Beratung und Betreuung - Fokus Wissenschaft}

Das Leistungsspektrum von FFG-EIP für den Bereich Wissenschaft beinhaltet sowohl spezifische Informations- und Beratungsleistungen für AntragstellerInnen in $\mathrm{H} 2020$ als auch Betreuungsinstrumente für Zielgruppen auf der Führungsebene von Forschungsorganisationen. Zu letzteren zählen insbesondere die Vizerektorate für Forschung, aber auch die Forschungsservicestellen an den Universitäten.

Die Relevanz der Leistung der FFG-EIP in den Bereichen 1) Bewusstseinsbildung \& Information, 2) Programm- und Projektberatung sowie 3) strategische Beratung und ERA-Orientierungswissen in Bezug auf den Bedarf der FFG-KundInnen ist generell hoch. Dies trifft insbesondere auf die persönlichen Beratungsleistungen und die Angebote zur Weiterbildung im Rahmen der FFG-Akademie zu.

Das individuelle Dienstleistungsspektrum von FFG-EIP ist insgesamt auch effektiv, trägt zur Steigerung der Antrags-Kompetenz der ForscherInnen bei und erhöht die Erfolgswahrscheinlichkeit in der Antragstellung. Es existieren jedoch Potenziale zur Erhöhung der Effektivität, die durch zielgruppengerechte Anpassungen im Leistungsportfolio erzielt werden können.

\section{Empfehlung: Zielgruppengerechte Ansprache von ForscherInnen}

Um den Zugang zu den Dienstleistungen von FFG-EIP zu erhöhen und die Effektivität der angebotenen Dienstleistungen zu steigern, sollte eine zielgruppengerechte Ansprache von erfahrenen und weniger erfahrenen ForscherInnen erfolgen. Schwerpunkte des Beratungsangebotes sollten besser aufgezeigt und deren potentielle Nutzen für die unterschiedlichen Zielgruppen sollte klar dargelegt werden. Dies könnte durch die Darstellung von Leistungsmodulen/-paketen für spezifische Gruppen geschehen, in denen entsprechende Leistungen zielgruppengerecht und übersichtlich gebündelt werden.

Eine mit der Beauftragung 2014 neu eingeführte Leistung der FFG-EIP zielt darauf ab, die Selbstbefähigung der Forschungsorganisationen in Hinblick auf den Europäischen Forschungsraum und H2020 zu unterstützen. Diesbezüglich sind deutliche Fortschritte erzielt worden, die von den Universitäten bereitgestellten Kapazitäten an den jeweiligen Forschungsservicestellen sind jedoch sehr heterogen. Gleichzeitig steigt die Bedeutung von europäischen FTI-Netzwerken und strategischen Partnerschaften in den europäischen Rahmenprogrammen, und es besteht sowohl für den außeruniversitären als auch für den 
universitären Bereich ein Bedarf, verstärkt strategisch auf internationaler Ebene zu agieren. Dies legt eine entsprechende Anpassung der Unterstützungsleistungen der FFG-EIP nahe.

\section{Empfehlung: Spezifische Leistungen für Servicestellen entwickeln}

Hintergrundwissen zu den Arbeitsprogrammen von H2020 sollten zeitnah, kurz und konkret an Servicestellen weitergegeben werden. Das inkludiert die oft informellen Diskussionen im Vorfeld, Einschätzungen was in einem Call wirklich gefragt sein wird, und andere Hintergrundinfos. Da diese Informationen oft begrenzt gültig sind (z.B. Arbeitsdokumente), sollte die FFG eine mit den Delegierten abgestimmte (informelle) Policy entwickeln, welche die Informationsweitergabe regelt.

Call-Inhalte und Work-Programmes sollten so aufbereitet werden, dass diese besser und schneller an die FTI-Community kommuniziert werden können.

Informationen zu Entwicklungen rund um FP9 (wie z.B. Ergebnisse aus informellen Meetings, aktuelle Diskussionen, worauf sollte man in Zukunft achten) sollten schneller verfügbar gemacht werden.

Daten und Informationen, Reports, etc. sollen nicht nur aus dem Link/Bericht selbst bestehen, die FFG sollte auch die wichtigsten Punkte kurz hervorheben.

Neben den unmittelbaren Unterstützungsleistungen kommt der Weiterentwicklung der 'Selbstermächtigung' (Empowerment) der Forschungsorganisationen eine Schlüsselrolle zu. Hierfür sind die Forschungsservicestellen und Vizerektorate der Universitäten die zentralen Ankerpunkte. Über das Instrument der ERA-Dialoge konnte die FFG diesbezüglich bereits einen sinnvollen ersten Schritt setzen, um das Bewusstsein über die Bedeutung von europäischen Agenden in den Organisationen zu stärken. Weiterführende Strategiebildungsprozesse müssen vornehmlich von den Forschungsorganisationen selbst verantwortet werden und befinden sich bei der Mehrzahl der Organisationen noch in einer frühen Entwicklungsphase. Ohne eine Erweiterung der organisationsinternen Kapazitäten erscheint das Potenzial der Forschungseinrichtungen, eine aktive Rolle im Europäischen Forschungsraum zu übernehmen, beschränkt. Die FFG verfügt über eine gute Basis an Beratungsleistungen um Forschungsservicestellen und Vizerektorate anzusprechen, es besteht jedoch Bedarf, die Professionalisierung der Servicestellen und die strategische Selbstermächtigung weiter voranzutreiben.

\section{Empfehlung: Professionalisierung der Servicestellen und strategisches Em- powerment in Hinblick auf ERA weiter forcieren}

Um die Professionalisierung der Servicestellen und das strategische Empowerment weiter zu stärken, sollten die ERA-Dialoge weitergeführt, der Kontakt zu den Forschungsservicestellen systematisiert und zugleich die Unterstützungsleistungen gezielt auf die Bedarfe der jeweiligen Universitäten abgestimmt werden.

In den ERA-Dialogen sollten Aktivitäten, die auch einen Niederschlag auf der Ebene der Fakultäten und Institute finden, weiter vorangetrieben werden. Diese sollten weiterhin den Interessen und Herausforderungen der jeweiligen Universität entsprechen und umsetzbar und verbindlich sein. 
Zwischen den Forschungsservicestellen der Universitäten sollte ein regelmäßiger Erfahrungsaustausch unter Einbeziehung von Service-Personal des außeruniversitären Forschungssektors institutionalisiert werden, der auch zur Stärkung der Selbstorganisation dient. Als Vorbild könnte der Bundesarbeitskreis der EU-ReferentInnen (BAK) in Deutschland dienen.

\section{Beratung und Betreuung - Fokus Wirtschaft}

Die Evaluierung zeigt, dass das Leistungsportfolio im Bereich Beratung und Betreuung der FFG-EIP von Akteuren aus dem Bereich der Wirtschaft überwiegend positiv bis sehr positiv bewertet wird. Durch das EIP-Leistungsangebot wird das Informationsniveau für das ,Ökosystem' H2020-Unterstützung allgemein gehoben sowie auch spezifischere Beratungsbedarfe der Unternehmen erfüllt.

Aufgrund der vorliegenden Befunde fallen die Empfehlungen dementsprechend knapp und im Sinne einer Fortsetzung der entsprechenden Leistungen aus. Optimierungspotential wird im Bereich des,Erwartungsmanagements', d.h. der klareren Vermittlung was die angebotenen Leistungen sind und was sie nicht sind, gesehen.

\section{Empfehlung: Verbesserung des Erwartungsmanagements}

Grundsätzlich ist aufgrund der positiven Erfahrungen eine Strategie des "weiter wie bisher" angebracht. Das zielgruppenspezifische Angebot sollte jedoch noch transparenter dargestellt werden. Dies betrifft insbesondere ein realistisches ,Erwartungsmanagement', das zum einen die Möglichkeiten und Grenzen (bzw. die damit verbundenen Kosten) der Teilnahme an den europäischen FTI-Programmen aufzeigt und zum anderen die Unterstützungsleistungen von FFG-EIP bei der Antragsstellung und Projektabwicklung beinhaltet.

Eine derartige Strategie ist kompatibel mit dem expliziten "Nicht-Ziel" des EIP, die Zahl der Antrag stellenden Unternehmen zu maximieren (um den Preis, ungeeignete Unternehmen bzw. Unternehmen mit ungeeigneten Projekten zur Teilnahme zu "drängen").

Ein besonderes Element im Beratungs- und Betreuungsangebot der FFG-EIP im Bereich Wirtschaft stellt das C3-Konzept (Core Customer Concept) dar, das speziell auf die avanciertesten Nutzer abgestimmt ist. Es wird von den begünstigten Unternehmen als sehr gut eingeschätzt, aber es stellt sich die Frage, wie die Erfahrungen dieser herausragenden Gruppe von Unternehmen auch für andere nutzbar gemacht werden könnten.

\section{Empfehlung: Wirkungspotenzial der C3 Beratung verbessern}

Da das C3 Instrument auf eine relativ kleine Zielgruppe abgestimmt ist, bleibt der positive Impact des Angebots auf die unmittelbare Zielgruppe beschränkt. Eine verstärkte Multiplikatorwirkung und Skalierung der Wirkung erscheint hier notwendig. Diesbezüglich sind folgende Anpassungen denkbar:

Verstärkter Mitteleinsatz für C3, sodass insgesamt mehr Unternehmen in C3 betreut und beraten werden können, 
Einbeziehung der C3 Unternehmen in digitale "Peer Learning" Gruppen (im Sinne der Nutzung der Erfahrung und Expertise der C3 Unternehmen), in denen die österreichischen Projektpartner der C3-Unternehmen einbezogen werden, wobei diese von ExpertInnen der FFG-EIP moderiert werden,

Verstärkte Kooperation der C3 Unternehmen mit Intermediären (wie z.B. der WKO).

\section{EUREKA - Initiative für anwendungsnahe Forschung und Entwicklung in Europa}

Die Zusammenführung der Aktivitäten des EUREKA-Büros unter dem Dach von FFG-EIP kann als gelungen bezeichnet werden. Die Verteilung der Verantwortlichkeiten und Aufgaben auf unterschiedliche Personen im Team und die damit einhergehende, teamübergreifende Vernetzung von Beratungsmöglichkeiten und -aktivitäten sind besonders hervorzuheben.

Das steigende Interesse an entsprechenden Beteiligungen führt mitunter dazu, dass EUREKA, mit Abzügen auch Eurostars, als Alternative zu anderen Förderangeboten fungiert, was die Frage nach der strategischen Positionierung von EUREKA bzw. Eurostars (u.a. im Hinblick auf die Komplementarität mit den Angeboten von H2020) aufwirft.

Aufgrund der unterschiedlichen Ausgestaltung und Dotierung von EUREKA in den Mitgliedsländern spielt das im EUREKA-Büro zusammenlaufende Know-how, die Einbettung in die FFG und die gesammelte Erfahrung eine wesentliche Rolle für die effektive und effiziente Beratung und Projektbetreuung. Die Koordinierung und Synchronisierung zwischen EUREKAs Mitgliedsländern bleibt jedoch EUREKAs größte Herausforderung.

\section{Empfehlung: Nationale Positionierung EUREKAs sinnvoll - Eurostars stärken}

Die Positionierung EUREKAS im europäischen Kontext ist abhängig von Struktur und Ausgestaltung der europäischen Förderlandschaft sowie den unterschiedlichen Netzwerk- und Kooperationsstrukturen. Vor diesem Hintergrund ist EUREKA auf nationaler Ebene als niederschwellige Förderalternative mit guten Erfolgsaussichten im Vergleich zum Rahmenprogramm H2020 und anderen Formen transnationaler FTI-Partnerschaften sinnvoll positioniert.

Insbesondere die aktive Positionierung von Eurostars als Alternative zum stark überzeichneten KMU-Instrument des Rahmenprogramms sollte weiter forciert werden.

Das Programmmanagement von EUREKA wird als schlank in den Strukturen, effizient und transparent wahrgenommen. Der themenoffene, bottom-up-Ansatz von EUREKA spiegelt sich gut im FFG Basisprogramm wider, das eng mit dem EUREKA Büro zusammenarbeitet. Kommunikation im Team und Synergien mit EIP werden gut genutzt. Dementsprechend empfiehlt die Evaluierung auch eine Beibehaltung des jetzigen Modus operandi.

Empfehlung: Effizientes Programmmanagement und schlanke Strukturen beibehalten 
Die Integration des EUREKA-Büros im FFG-EIP ermöglicht Synergien im Bereich der Beratung und Förderung kooperativer anwendungsnaher Forschung und Entwicklung zu nutzen. Die nationale Programmabwicklung erfolgt in effizienter Weise.

Vor diesem Hintergrund soll die Integration des EUREKA-Büros im FFG-EIP weiter beibehalten werden.

\section{COSME - Programm für die Wettbewerbsfähigkeit von Unternehmen und für KMU}

Die FFG ist vom BMDW damit beauftragt, COSME zu bewerben und österreichische Organisationen bei Fragestellungen rund um das Programm zu unterstützen. Das sowohl thematisch als auch inhaltlich breite Spektrum an Maßnahmen innerhalb von COSME Zugang zu Finanzierungsmitteln, Zugang zu Märkten, Förderung der unternehmerischen Initiative und Kultur sowie Verbesserung der Rahmenbedingungen für die Wettbewerbsfähigkeit - ist auf das Generalziel der "Stärkung der Wettbewerbsfähigkeit" ausgerichtet. Das Programm ist damit in hohem Ausmaß auch für das ganze Spektrum der eigentlichen Zielgruppe, die Gesamtheit europäischer KMU, relevant. Dieses Charakteristikum von COSME führt allerdings auch dazu, dass wenig gezielte Lenkungseffekte (i.S. der Verfolgung spezifischer Politikziele oder der Adressierung weniger entwickelter Märkte) erwartet werden können.

Darüber hinaus ist die generelle Bekanntheit des Programms eher gering. Die Außenwahrnehmung (der Möglichkeiten) im Rahmen von COSME scheint mehrheitlich auf die Standbeine Finanzierungsinstrumente sowie das EEN (siehe unten) zu fokussieren; auch die KMU-bezogenen Policy-Berichte werden breiter wahrgenommen. Diese selektive Wahrnehmung bzw. partielle Mangel an Bekanntheit von Elementen des Programms ist aufgrund des Programmcharakters als Politikentwicklungs- und Politikunterstützungsinstrument, der indirekten Wirkungsweise (Intermediäre als den KMU vorgeschaltete Zielgruppe) sowie der hohen Streuung bzw. Breite von Themen und Maßnahmen durchaus nachvollziehbar. Ein Handlungsbedarf, die Bekanntheit von COSME durch gezielte Maßnahmen seitens der FFG-EIP zu steigern, wird vom Evaluierungsteam nicht gesehen. Das Schnittstellenmanagement in Bezug auf die einzelnen Maßnahmen in COSME dürfte, mit gut abgestimmten externen und internen Schnittstellen, weitgehend effizient erfolgen.

\section{Empfehlung: Weiterführung eines proaktiven Programm- und Schnittstellenma- nagements im FFG-EIP}

In COSME gibt es mit Blick auf internationale Aktivitäten von Unternehmen zahlreiche Anknüpfungspunkte in der Betreuung durch die FFG - nicht zuletzt durch die tendenzielle Wiedereingliederung von Innovationsaspekten in thematische/sektorale Calls im Rahmen von COSME, die auch durch die Pilotierung einzelner Maßnahmen (mit potentiellem Innovationsbezug) unterstrichen wird.

Vor diesem Hintergrund kann das Schnittstellenmanagement (zeitnahe Antizipation und Bewerbung bzw. Verteilung von Calls zu den Multiplikatoren und Intermediären etc.) als wichtige und richtige Verortung bei der FFG bewertet werden; der ständige Einbezug 
(pro-aktive Ansprache) der MultiplikatorInnen erscheint - nicht zuletzt aufgrund der nachvollziehbaren selektiven Wahrnehmung und dem partiellen Mangel an Bekanntheit des Programms mit all seinen Möglichkeiten - unablässig.

Allerdings weist das Datenmonitoring der Teilnahmen und der Programmergebnisse Schwächen auf, die aber nicht der FFG zur Last gelegt werden können. Diesbezüglich ist primär die Europäische Kommission gefragt, ihren COSME Data Hub weiterzuentwickeln.

\section{Empfehlung: Verbesserung des Datenmonitorings durch die Europäische Kom-} mission

Hinsichtlich der Transparenz und des Datenmonitorings der Teilnahmen und Programmergebnisse besteht zunächst vor allem auf Seite der Europäischen Kommission eindeutig Verbesserungspotenzial. Der COSME Data Hub ist ein erster Schritt in Richtung besseres Monitoring und Übersichtlichkeit, dennoch fehlt bislang ein strukturiertes Notifizierungssystem für Ausschreibungen, was die Recherchearbeiten der FFG zumindest Großteils ersetzen und die Effizienz wie auch die Effektivität des Programmmanagements durch die FFG erhöhen würde.

\section{EEN - Enterprise Europe Network}

Die Relevanz des EEN, das als Maßnahme kontinuierlich im Rahmen von COSME ausgeschrieben bzw. umgesetzt wird, ist insbesondere durch den breiteren, umfassenderen Zugang (im Unterschied zu einem Projektfokus) sowie auf die üblicherweise auch langfristigere Begleitung von Unternehmen gegeben. Zudem ist der Fokus der Leistungen auf die Unterstützung internationaler Kooperations- und Technologietransferaktivitäten der Unternehmen und dabei das breite Spektrum von Kooperationsaktivitäten (FTI-Kooperationen sowie reine Technologiepartnerschaften, Lizensierungen, Vertriebsvereinbarungen etc.) von hoher Bedeutung für die Zielgruppe, insbesondere für KMU.

\section{Empfehlung: Beibehaltung der Integration der EEN-Leistungen im FFG-EIP}

Das EEN weist eine hohe Bekanntheit unter den Stakeholdern und Intermediären bzw. Förderagenturen auf und wird seiner Rolle als regional verankertes Netzwerk mit ebensolchen Knotenpunkten/Anlaufstellen gerecht. Das EEN hat mit seinen Leistungen/Instrumenten zumeist einen hohen Stellenwert im jeweiligen Beratungsportfolio. Zudem scheinen die EEN-Leistungen/Instrumente organisatorisch gut in die Portfolios integriert bzw. mit weiteren relevanten Maßnahmen (z.B. Beratung zu H2020 und anderen Unterstützungsmöglichkeiten bzw. Förderungen) verknüpft und abgestimmt. Häufig wird mittels EEN-Leistungen im Vorfeld und fast immer ergänzend zu H2020 gearbeitet.

\section{Beratung und Betreuung - übergreifende Empfehlungen}

Über die für die Zielgruppen Wissenschaft und Wirtschaft formulierten Empfehlungen hinaus gibt es mehrere Ansatzpunkte für Verbesserungen, die beide Gruppen gleichermaßen betreffen. Sie weisen gleichzeitig über den Verantwortungsbereich von FFG-EIP hinaus und betreffen die gesamte FFG. 
Während die Website von FFG-EIP ein breites Spektrum an Informationen zu Programmen und Aktivitäten auf europäischer und internationaler Ebene anbietet, wird die Aufmachung, der Überblick und die Identifikation von spezifischen Informationen und Ansprechpersonen vielfach als verbesserungswürdig empfunden.

\section{Empfehlung: Neugestaltung des Internet-Auftritts}

Eine Neugestaltung des Internet-Auftritts von FFG-EIP erscheint notwendig, um Informationen besser an die angesprochenen Zielgruppen vermitteln zu können. Die Homepage von FFG-EIP bietet derzeit eine Fülle an Informationen und guten Inhalten, ist jedoch unübersichtlich und wenig benutzerfreundlich gestaltet. So sind die Suchfunktion, das Auffinden von Programminformationen zu H2020 sowie von Kontaktpersonen schwierig. Unklar ist auch, an welche Stellen/Personen sich man in Hinblick auf Beratungen, NCPs, FFG Akademie und deren Anmeldungsprozesse wenden kann. Der derzeitige Internet-Auftritt von FFG-EIP und von EU-PM legt eine grundlegende Neuausrichtung der FFG-Website insgesamt nahe, in deren Rahmen folgende Aspekte berücksichtigt werden sollten:

\section{Einfacher, intuitiver, zielgruppenspezifischer Zugang:}

- Differenzierung nach Relevanz der Information

- Differenzierung zwischen Basisinformationen (Programminfo) und detaillierten Fragen (z.B. in Bezug auf Antragstellung und zugehörige Services der FFG)

- Bessere Platzierung der Suchfunktion der FFG

\section{Verbesserung der Navigationsstruktur:}

- Sofort-Einstieg zu europäischen Förderprogrammen und zugehörigen FFG Service-Angeboten

- Einführung der kontextabhängigen Benutzerführung sowie eine bessere Darstellung der angebotenen Services: Leistungen wie die "Partnersuche", die im FFG-Portfolio keine oder nur eine untergeordnete Rolle spielen werden derzeit als zentrale Leistungen hervorgehoben, Services wie die verschiedenen Beratungsinstrumente und EUPM sind versteckt.

- Bessere Identifikation zentraler Ansprechpartner: Identifikation und Erreichbarkeit von NCPs und FFG-ExpertInnen sind unklar. Schwerpunkte der Beratungsexpertise ebenso. Beispielsweise könnte der/die richtige AnsprechpartnerIn für eine Leistung/ein Programm jeweils auf der Seite aufgelistet werden.

Wiederholt wird im Rahmen der Evaluierung darauf verwiesen, dass die Antragstellenden in erster Linie ein für ihren Bedarf passendes Förderprogramm suchen, und dementsprechend die getrennten Verantwortlichkeiten an der FFG für nationale und europäische Programme ein Hemmnis für eine nahtlose Beratung darstellen. Dementsprechend sollte ein besonderes Augenmerk auf die Vernetzung und den raschen Informationsfluss zwischen den jeweils für nationale und europäische Programme verantwortlichen Bereichen der FFG gelegt werden. 


\section{Empfehlung: Bessere Vernetzung europäischer und nationaler Programme}

Innerhalb der FFG sollte mit entsprechenden Maßnahmen darauf abgezielt werden, eine optimale "Vernetzung" europäischer und nationaler Programme im Beratungsservice herzustellen. Dies ist vor dem Hintergrund zu sehen, dass viele (vor allem kleinere) Unternehmen nicht zwischen den einzelnen Abteilungen der FFG differenzieren und die Trennung zwischen den Leistungsangeboten des EIP und den diversen Angeboten im Rahmen der nationalen Förderprogramme nicht wahrnehmen.

Angesichts der mittlerweile sehr geringen Erfolgsquote und der häufigen Ablehnung auch von Projekten, die prinzipiell als sehr gut (bzw. förderwürdig) beurteilt wurden, ist eine mögliche Überlegung, entsprechende Anschlussunterstützung bzw. -beratung (zum Beispiel in Richtung „Umwandlung" der Projektidee für ein nationales Förderprogramm) zur Verfügung zu stellen.

\section{Beratung und Betreuung - Governance und Effizienz der EIP-Beauftragung}

Die Beauftragung des EIP mit Informations-, Beratungs-, Betreuungs- und Vernetzungsleistungen erfolgt gesondert und ist nicht Teil des Rahmenvertrags der FFG mit den EigentümervertreterInnen des Bundes (BMVIT und BMDW). Trotz des damit verbundenen administrativen Mehraufwands aufgrund des separaten finanziellen Berichtswesens weist diese besondere Konstruktion eine Reihe von Vorteilen auf, aufgrund derer diese spezielle Form der Beauftragung als sinnvoll anzusehen ist.

\section{Empfehlung: Separate Beauftragung des Bereichs Europäische und Internationale Programme beibehalten}

Um den mit der Beratung und Betreuung der europäischen FTI-Instrumente einhergehenden Zielen und Bedürfnissen gerecht zu werden, erscheint aus heutiger Sicht die separate Beauftragung des Bereichs „Europäische und Internationale Programme" der FFG trotz eines administrativen Mehraufwandes sinnvoll. Hierdurch kann die effektive Einbeziehung mehrerer Ministerien sowie der gesetzlichen Interessensvertretung der österreichischen Wirtschaft in den Steuerungsstrukturen von FFG-EIP gewährleistet werden.

Mit der separaten Beauftragung gehen regelmäßige Berichtspflichten der FFG-EIP einher, die im Zuge einer flexibleren Vertragsgestaltung in Zukunft noch an Bedeutung gewinnen könnten.

\section{Empfehlung: Beibehaltung der regelmäßigen Berichtspflichten}

Angesichts der Größe und Dimension der Beauftragung ist die regelmäßige Berichtslegungspflicht und das damit verbundene laufende Monitoring und Anpassung der Aktivitäten sinnvoll und notwendig. Sollte der zukünftige Vertrag mit FFG-EIP flexibler gestaltet werden, werden diese Berichtspflichten noch mehr an Bedeutung gewinnen. Wir empfehlen deshalb das Beibehalten des derzeitigen Berichtswesens unter Berücksichtigung der Anpassung und Weiterentwicklung der WFA-Indikatoren.

Im Hinblick auf das finanzielle und inhaltliche Controlling im Rahmen der EIP-Beauftragung, mit zahlreichen, teils überlappenden Aktivitätstypen wurde bislang weitgehend eine thematische Kostenzuordnung vorgenommen. Um Intransparenz zu vermeiden und 
eine adäquate Einschätzung des Aufwands insbesondere für neue Beratungsinstrumente (ERA-Dialog, C3) zu ermöglichen, wird für die Zukunft die Einführung eines funktional strukturierten Monitoringsystems empfohlen.

\section{Empfehlung: Verbesserung der EIP-Kostenaufzeichnung}

Die Budgetallokation der EIP-Beauftragung ist angesichts der Beteiligungsstruktur Österreichs in H2020 angemessen. Darüber hinaus ist die Effizienz der Abwicklung gegeben. Um jedoch die Unschärfen der Kostenzuordnung zu beheben und den Mitteleinsatz der verschiedenen Beratungs- und Betreuungsinstrumente besser abschätzen zu können, empfiehlt das Evaluierungsteam die Umstellung der EIP Kostenaufzeichnungsstruktur von thematischen Kostenträgern auf funktionsbezogene Kostenträger. Dies wäre vor allem im Hinblick auf die Bewertung der neuen Betreuungsinstrumente ERA-Dialog und C3 relevant, aber auch um die Kosten von anderen Instrumenten wie Berichte und Analysen oder FFGAkademie nachzuvollziehen. Nachdem es in der Praxis häufig zu einer engen Verzahnung zwischen unterschiedlichen funktionsbezogenen Leistungen kommt (z.B. unterschiedlichen Arten von Beratungen), ist dabei auf eine ausgewogene Balance zwischen Differenziertheit und Umsetzbarkeit zu achten.

\section{EU Performance Monitoring}

Die Evaluierung hat ergeben, dass grundsätzlich ein hohes Maß an Zufriedenheit der NutzerInnen mit dem Informationsangebot und der Funktionalität der Produktpalette des EU Performance Monitoring besteht, und das über alle Zielgruppen hinweg. Auch die Abgrenzung der Zielgruppen und die Bereitstellung zielgruppenspezifischer Angebote werden als sinnvoll und gelungen erachtet.

\section{Empfehlung: Kein Handlungsbedarf bei Zielgruppenabgrenzung}

Die Zufriedenheit der NutzerInnen beider Zielgruppen ist hoch und die entsprechende Abgrenzung der Zielgruppen wird als funktional eingeschätzt. In Bezug auf Letzteres wird jedenfalls aktuell kein Handlungsbedarf geortet. Insbesondere wird die Einrichtung einer eigenen Zielgruppe für regionale Kontaktstellen als nicht erforderlich erachtet. Der Dialog mit den regionalen Kontaktstellen kann jedoch weiter verstärkt werden.

Kritische Anmerkungen betreffen u.a. die Funktionalität der Produkte, insbesondere des Webauftritts und des öffentlichen Online-Portals sowie die Qualität und Aktualität der zur Verfügung gestellten Daten. Dies betrifft etwa die Verbesserung der Auffindbarkeit der EU-PM-Leistungen über die FFG-Homepage sowie die Vermittlung des Leistungspotentials (und der -grenzen) des öffentlichen Portals. Im Vergleich zu ähnlichen Portalen - insbesondere jenem von VINNOVA und dem H2020-Participant Portal der Kommission - weist das EU-PM-Portal eine Reihe von Spezifika auf (z.B. Fokus auf zentrale Kennzahlenund Performancevergleiche auf Programm- und Institutionenebene; Vergleiche auf Ebene der Bundesländer), die eine Weiterführung als eigenständiges EU-PM-Portals auch für die Zukunft nahelegen. 


\section{Empfehlung: Weiterführung des öffentlichen Online-Portals}

Die Evaluierung spricht sich für eine Aufrechterhaltung des öffentlichen Online-Portals aus. Gleichzeitig sollten Verbesserungen in der Profilierung des Portals im Vergleich zu anderen Portalen erfolgen. Für Auswertungen auf Projektebene könnte zudem bspw. direkt auf das VINNOVA-Tool verwiesen werden.

Ein Vermittlungsproblem besteht aus Sicht des Evaluierungsteams auch hinsichtlich der verwendeten Datengrundlagen zur Analyse der Performance innerhalb des Rahmenprogramms, deren Begründung und Aussagekraft. Wenngleich gerade auf Ebene der Programmdelegierten viele Möglichkeiten der individuellen und - im Vergleich zu EU-PM-Daten - häufigeren Informationsgewinnung verfügbar sind, besteht der Vorteil des im Rahmen der Beauftragung festgelegten Fokus auf eCORDA als Datengrundlage in der regelmäßigen Vergleichbarkeit und der Qualitätskontrolle der Daten.

\section{Empfehlung: Usability des Online-Portals stärken}

Adaptierungen beim öffentlichen Portal sind bei der Vermittlung der Datengrundlagen des Portals und dessen Leistungsfähigkeit vorzunehmen. Dies umfasst die Adaption des

Portal-Manuals, das zwar Informationen zur Berechnung der einzelnen Kennzahlen, nicht aber zur grundlegenden Datenstruktur, zur Fristigkeit und Grenzen der Analysierbarkeit enthält. Auch in den Berichtsprodukten, insbesondere den standardisierten Cockpitberichten und Quickinfos, sollen Hinweise zur Datengrundlage und -qualität integriert werden.

In Bezug auf die Integration zusätzlicher Daten zu den transnationalen FTI-Partnerschaften ist EU-PM selber von der Bereitstellung entsprechender Quellen auf EU-Ebene abhängig, gemäß der Prämisse, hier - analog zur Verwendung von eCORDA als zentrale Quelle - auf bereits erhobene und validierte Daten zurückgreifen zu wollen. Aktuell ist die Datenbereitstellung zu transnationalen FTI-Partnerschaften auch auf EU-Ebene noch äußerst lückenhaft, wenngleich hier in jüngerer Vergangenheit positive Entwicklungen zu beobachten sind, die zu einer weiteren Verbesserung der Datenlage beitragen sollten.

\section{Empfehlung: Beibehaltung der laufenden Integration zusätzlicher Datenbe- stände}

Das EU-PM kommt seinem Auftrag zur Prüfung der Integration weiterer Datenquellen nach. Entsprechende Entwicklungen sollen auf EU-Ebene weiter von EU-PM aktiv begleitet werden. Der Lenkungsausschuss soll weiter über neue Datenbestände und Analysemöglichkeiten informiert werden.

Aufgrund der nun zur Verfügung stehenden Vielfalt an Analysemöglichkeiten und dem damit verbundenen Aufwand in der verknüpften Auswertung wurden solche bis dato nur auf Ebene von großen Einzelinstitutionen wie forschungsstarken Universitäten und Unternehmen vorgenommen.

\section{Empfehlung: Kommunikation über Auswertungsmöglichkeiten und -grenzen stärken}

Aus Sicht des Evaluierungsteams kommt EU-PM seinem Auftrag des Vorantreibens der Datenverknüpfung zwischen nationalen und EU-Förderdaten nach. Die Herausforderung 
für die Akteure, insbesondere der Zielgruppe 1, besteht in Zukunft darin, vor dem Hintergrund der vielfältigen Auswertungsmöglichkeiten entsprechend konzise Anfragen zu formulieren (Beispiel: „Reüssieren FördernehmerInnen im nationalen Programm xy überdurchschnittlich in EU-Programmen?"). Gleichzeitig ist von Seiten des EU-PM Teams an der Kommunikation über die bestehenden Möglichkeiten zu arbeiten, bspw. im Rahmen von Workshops mit den Delegierten.

Das vertrauliche Online-Portal wird im Verhältnis zur Möglichkeit der direkten Anfrage bei EU-PM sehr gut angenommen. In Zukunft sollte das Augenmerk verstärkt auf die Entwicklung individualisierter Angebote gelegt werden, die eine bedarfsgerechte Auswahl von Verknüpfungsmöglichkeiten einzelner Programme und Akteure vor dem Hintergrund der Themen-/Programmverantwortlichkeiten der jeweiligen NutzerInnen ermöglichen. Der Dialog mit den NutzerInnen ist hierfür zu stärken. Insgesamt erfolgt die Leistungsbereitstellung aus Sicht des Evaluierungsteams in effizienter Weise.

\section{Empfehlung: Dialogformate zur Spezifizierung von EU-PM Dienstleistungen ent- wickeln}

Das Nutzungspotenzial des vertraulichen Online-Portals wird durch die Selbstermächtigung der Zielgruppe 1 bereits weitgehend ausgeschöpft.

Gleichzeitig gilt es entsprechende Formate des Dialogs zwischen AuftraggeberInnen, NutzerInnen und EU-PM zu entwickeln, um vor dem Hintergrund zunehmender Analysemöglichkeiten durch die Integration neuer Datenquellen a) über neue Services und Möglichkeiten zu informieren und b) darauf aufbauend entsprechend konzise und tatsächlich verfolgbare Analyseaufträge zu erarbeiten. Ein Vorschlag wäre, im Rahmen des TicketSystems einmal jährlich eine Bedarfserhebung unter allen NutzerInnen durchzuführen.

Insgesamt gibt es aufgrund der grundsätzlich positiven Beurteilung des EU-PM und des angehäuften Know-hows bereits zum jetzigen Zeitpunkt eine Reihe von Argumenten für eine Fortführung des EU-PM. Angesichts der Flut an unterschiedlichen Datenquellen besteht für das EU-PM die Möglichkeit eine "gate keeper"-Funktion in der Informationsvermittlung zu übernehmen, durch die das EU-PM zugleich eine zentrale Rolle als „Informations-Broker" im Rahmen des ERA-Observatoriums wahrnehmen kann. Die über EUPM bereitgestellten Daten bilden einen zentralen Referenzrahmen für die nationale PolicyDebatte. Sie finden Verwendung in offiziellen Dokumenten und Berichten (wie bspw. den Forschungs- und Technologiebericht der Bundesregierung) sowie Strategiebildungsprozessen auf Bundesebene.

\section{Empfehlung: EU-PM fortführen}

Das Evaluierungsteam spricht sich für eine Fortführung des EU-PM aus. Diesem kommt eine zentrale Position als "Informations-Broker" für die nationale Policy-Debatte in Bezug auf ERA zu.

Gleichzeitig gilt es den Mehrwert qualitätsgeprüfter Auswertungen und der damit verbundenen weiteren Analyse- und Verknüpfungsmöglichkeiten mit nationalen Daten als politikunterstützenden Services besser zu vermitteln und dadurch die "Erzählhoheit" des EU-PM weiter zu stärken. 


\section{ERA-Observatorium}

Im ERA Observatorium sind eine Reihe von Aktivitäten zusammengefasst, die den Informationsaustausch und die bessere Abstimmung zwischen jenen Akteuren ermöglichen sollen, die eine wesentliche Rolle bei der Formulierung und Vermittlung österreichischer Positionen in Bezug auf die europäische FTI-Politik spielen. Grundsätzlich werden die Aktivitäten im Rahmen des ERA-Observatoriums mehrheitlich positiv beurteilt.

Die Arbeit der EU-Koordinationsabteilung wird dabei fast durchgehend positiv und integrativ wahrgenommen und orientiert sich stark an einem gemeinsamen Erfahrungsaustausch. Die vielfältigen Kommunikationsangebote werden von den beteiligten Fachressorts geschätzt.

Derzeit scheinen jedoch Versuche einer stärkeren Koordination für ressortübergreifende Maßnahmen und eine stärkere Verbindung von nationalen und internationalen Programmen vorerst auch im Rahmen des ERA Observatoriums an ihre Grenzen gestoßen zu sein. Dass ressortübergreifende Koordination in Zukunft vermehrt an Bedeutung gewinnen könnte, zeigt sich bereits heute an der hohen Bedeutung der strategischen Partnerschaftsinstrumente, in der FTI-Akteure aktuell keinen national koordinierten Zugang ausmachen können. Eine informelle Abstimmung im Sinne von Information (Europa Forum Forschung) und gegenseitigem Austausch (Delegierten Roundtable) wurde gut angenommen, während eine weitergehende Abstimmung (z.B. ERA Policy Forum) bislang keine hinreichende Unterstützung fand.

Vor dem gegebenen institutionellen Hintergrund werden seitens der Evaluierung lediglich Anpassungen beim Europa Forum Forschung nahegelegt.

\section{Empfehlung: Dialog im Europa Forum Forschung stärken}

Die Funktion Information/Kommunikation zu relevanten EU-Politiken wird im Wesentlichen gut abgedeckt. Das diesbezüglich wichtigste Instrument, das Europa Forum Forschung, sollte neben seiner angestammten Informationsfunktion zukünftig verstärkt Dialogelemente beinhalten. Interaktive und kreative Zugänge, beispielsweise aus dem Bereich der Gruppenmoderation, könnten hierbei stärker zum Einsatz kommen.

Darüber hinaus stellt die funktionale Einbeziehung anderer Akteure, beispielsweise anderer FTI-Ministerien, in die Gestaltung des Forums eine Option dar, welche den Kooperationsgedanken im Politikfeld stärken könnte.

Das ERA Portal Austria wird gut angenommen. Lediglich eine weitere Verbesserung der Übersichtlichkeit der Website, insbesondere der Suchfunktion erscheint notwendig.

Auch die Beratung und Betreuung ministerieller Ressorts für H2020 funktioniert im Großen und Ganzen gut. Der EIP-Lenkungsausschuss wird in Bezug auf seine Funktionsweise positiv beurteilt. Die thematischen Begleitgruppen der Programmdelegierten sind allerdings sehr unterschiedlich aufgesetzt, was darauf verweist, dass die Funktion der Aufnahme und Weitergabe von Informationen durch Delegierte unterschiedlich interpretiert wird. 


\section{Empfehlung: Beitrag zur Orientierung der Aufgabenstellung von Delegierten}

Wir empfehlen eine stärkere Orientierung der unterschiedlichen Aufgabenstellungen von Delegierten über die definierten Aufgaben und über die formale Vertretung österreichischer Interessen in Brüssel hinaus. Ein Element könnte in diesem Zusammenhang beispielsweise die Kommunikation mit der wissenschaftlichen Community im Rahmen der thematischen ExpertInnengruppen der Delegierten sein.

Der Delegierten-Roundtable ist ein sinnvolles Instrument und wird hervorragend bewertet.

In Bezug auf die Funktion der Beratung und Betreuung ministerieller Ressorts könnte auch das gegenwärtig diskutierte Verbindungsbüro in Brüssel Unterstützungsarbeit leisten. Dabei ist das Potenzial dieses Büros auch im Zusammenhang mit den allgemeinen Zielen der österreichischen EU-FTI-Politik im Hinblick auf die angestrebte Positionierung des Landes im europäischen Forschungs- und Innovationsraum zu sehen. Zum jetzigen Zeitpunkt ist noch keine einheitliche Position in Bezug auf die Einrichtung eines solchen Büros erkennbar. Sie kann sich auch erst auf der Grundlage einer klareren Weichenstellung hinsichtlich der zukünftigen Rolle, die Österreich bei der Gestaltung der europäischen FTI-Politik spielen möchte, herauskristallisieren.

\section{Empfehlung: Zielsetzungen eines EU-Verbindungsbüros mit FTI-Community klä- ren}

Vor der Einrichtung eines Verbindungsbüros sind strategische Weichenstellungen hinsichtlich Zielen, Aufgaben und damit einhergehend Aufbau, Finanzierung und Positionierung einer derartigen Organisationseinheit vorzunehmen. Vor dem Hintergrund der bisher polarisierten Debatte, die sich unter anderem in verschiedenen Fokusgruppen und der Delphi Befragung derart geäußert hat, dass sich die Mehrheit der österreichischen Akteure für ein derartiges Verbindungsbüro und eine Minderheit deutlich dagegen ausgesprochen hat, wird eine weiterführende Diskussion mit den wichtigsten Akteursgruppen empfohlen.

Die Funktion der strategischen Beratung der Politik wird im Moment vom ERA Council Forum wahrgenommen, das der unmittelbaren Beratung des Wissenschaftsministers in Angelegenheiten der EU-FTI-Politik dienen soll. Vor dem Hintergrund der im Regierungsprogramm der Bundesregierung angedachten Neuorganisation und Konzentration der die FTI-Politik beratenden Organe, wird die Beibehaltung einer starken europäischen Komponente empfohlen. Die Erfahrungen des ERA Council Forums zeigen auch den Mehrwert hochrangiger ausländischer Mitglieder eines solchen Gremiums, die mit ihrer Außenperspektive zusätzliche Blickwinkel und Erfahrungen einbringen können. Wenn diese mit einem guten Verständnis der Problemstellungen und der Machbarkeit im nationalen Kontext verknüpft werden kann, kann ein solches Gremium für die FTI-Politik von hohem Nutzen sein. Angesichts der wachsenden Bedeutung der FTI-Politik als Querschnittsmaterie sollte nicht zuletzt auch vor dem Hintergrund internationaler Erfahrungen die politikfeldübergreifende Verankerung eines zukünftigen Beratungsorgans ins Auge gefasst werden. 


\section{Empfehlung: Europäische Dimension in politisch beratenden Gremien berück- sichtigen}

Im Zuge des Neuaufsetzens der FTI-politischen Ratslandschaft in Österreich wird empfohlen, eine starke europäische Dimension in den Aufgaben und bei der Besetzung des neu zu konstituierenden Gremiums vorzusehen.

Um dem nicht zuletzt von der europäischen Ebene verstärkten Querschnittscharakter der FTI-Politik Rechnung zu tragen, sollte ein zukünftiges Gremium sich auch explizit an direkt und indirekt mit FTI-Agenden betraute Ressorts wenden können.

Um der Balance zwischen vertrauensvoller Beratung auf höchster politischer Ebene und der Notwendigkeit von Transparenz und Offenheit Rechnung zu tragen, wird außerdem eine regelmäßige Information über die Aktivitäten eines zukünftigen Beratungsgremiums empfohlen.

Daneben gibt es eine Reihe von Formaten, die dazu beitragen sollten, einen Prozess des

Strukturwandels im FTI-Politikbereich zu unterstützen. Den meisten dieser Instrumente ist gemein, dass sie im Normalfall keine aktive Koordination betreiben, sondern bei gegenseitiger Information verbleiben.

Zwei Instrumente, mit denen versucht wurde, über diese Vorgehensweise hinauszugehen, existieren hingegen nicht mehr. In Bezug auf die Arbeitsgruppe MULLAT zu transnationalen FTI-Partnerschaften konnte zwischen BMBWF und BMVIT kein Einvernehmen über die Zielsetzung und Zugangsweise der Arbeitsgruppe hergestellt werden. Das ERA Policy Forum Austria scheiterte am Versuch, alle österreichischen Ministerien mit FTIKompetenzen zu einer aktiven Koordination im Hinblick auf eine Reihe von als wesentlich wahrgenommenen Themenbereichen zu bewegen.

Tatsächlich ist vor diesem Hintergrund in Österreich eine nationale strategische Abstimmung im Hinblick auf die EU-FTI-Politik kaum vorhanden. Dies zeigt sich gerade bei den transnationalen FTI-Partnerschaften, wo die fehlende nationale Beteiligungsstrategie zu einer größeren Anzahl von Beteiligungen geführt hat, die in ihren Budgets häufig unterkritisch blieben.

Der im Regierungsprogramm festgehaltene neue Prozess für eine FTI-Strategie der Bundesregierung über 2020 hinaus bietet eine Chance für eine Überarbeitung der Koordinationsanstrengungen in der österreichischen EU-FTI-Politik.

\section{Empfehlung: ERA-Formate bündeln und aktivere Koordination zwischen den Bundesministerien stärken}

Die vielfältigen Formate zum Informationsaustausch über potenziell strukturverändernde Entwicklungen hin zum Europäischen Forschungsraum werden in unterschiedlicher Intensität wahrgenommen. Eine Vereinfachung oder Bündelung der verschiedenen Formate wird empfohlen (z.B. ERA-Reporting Board und ERA-Roundtable).

Zugleich erscheint angesichts neuer Instrumente auf EU-Ebene eine aktivere Koordination zwischen verschiedenen Politikfeldern geboten. Vor dem Hintergrund der bisherigen Erfahrungen wird empfohlen, einen vorbereitenden Prozess zu starten, um diese Form 


\section{Ausblick und Szenarien}

\section{Positionierung der österreichischen FTI-Politik in Bezug auf FP9}

Hinweise für die weitere Ausgestaltung der Rolle und Aufgaben der FFG-EIP können sich nicht auf die Korrektur bestehender Defizite bzw. Verbesserungsvorschläge vor dem Hintergrund der aktuellen Aufgaben und Herausforderungen beschränken, sondern müssen auch die sich verändernden Rahmenbedingungen berücksichtigen. Zu diesen Veränderungen zählt insbesondere auch das 9. Rahmenprogramm für Forschung und Innovation der EU-Kommission. Es stellt einen zentralen Bezugspunkt sowohl für die Arbeit der FFG-EIP als auch für die Governance wichtiger Dimensionen österreichischer FTI-Politik dar. Die bisherigen Vorarbeiten weisen auf eine Reihe von möglichen Neuerungen im nächsten Rahmenprogramm hin. Hierzu zählen insbesondere:

- Die Einrichtung eines Europäischen Innovationsrats (EIC), der zur Stärkung der Innovationsdynamik in Europa durch die Unterstützung insbesondere von Start-ups und Scale-ups mit hohem Wachstumspotenzial beitragen soll;

- Eine zunehmende Bedeutung des missionsorientierten Ansatzes in den thematisch orientierten Säulen des Rahmenprogramms, für dessen Umsetzung größere Initiativen (z.B. im Sinne von strategischen Partnerschaften) ebenso erforderlich sein werden wie ein explizites Alignment mit sektoralen Politikfeldern;

- Die bessere Abstimmung von Rahmenprogramm und Europäischen Strukturund Investitionsfonds (ESIF), um den wachsenden Disparitäten in der europäischen Forschungs- und Innovationslandschaft entgegen zu wirken.

Darüber hinaus ist mit Veränderungen des thematischen Portfolios der Forschungs- und Innovationsförderung auf europäischer Ebene zu rechnen, wobei beispielsweise im Falle der Verteidigungsforschung noch offen ist, ob diese innerhalb oder außerhalb des Rahmenprogramms verankert werden wird.

Zugleich muss betont werden, dass zum gegenwärtigen Zeitpunkt noch viele Unsicherheiten bezüglich der Ausgestaltung des nächsten Rahmenprogramms bestehen, die sich erst sukzessive in den nächsten Monaten verringern werden.

Die zukünftige Rolle und die damit in Zusammenhang stehenden Aufgaben der FFG-EIP hängen aber nicht nur vom Rahmenprogramm ab, sondern auch von der angestrebten

Positionierung Österreichs in Bezug auf die europäische FTI-Politik ab. Die Reformulierung der angestrebten Positionierung und Rolle Österreichs auf europäischer Ebene wird der zukünftigen FTI-Strategie obliegen, deren Entwicklung für das Jahr 2019 erwartet wird und für die derzeit Grundlagen erarbeitet werden (u.a. durch die aktuelle OECD Innovation Policy Review). Grundsätzlich sind dabei unterschiedliche Rollenverständnisse möglich, die von einer weitgehenden Anpassung an auf europäischer Ebene definierte Prioritäten und Strategien bis hin zum Anspruch einer proaktiven (Mit-)Gestaltung der europäischen FTI-Politik reichen. Innovationsführer wie Schweden oder Finnland, mit denen 
sich Österreich häufig vergleicht, stellen dabei den Anspruch an sich selbst, auch die europäische Politik zu prägen; ein Anspruch, für dessen erfolgreiche Einlösung es durchaus Belege gibt.

Die Entwicklungen auf beiden genannten Ebenen - Veränderungen im europäischen Rahmenprogramm und angestrebte nationale Positionierung in Bezug auf die Gestaltung der europäischen Forschungs- und Innovationspolitik - haben naturgemäß auch Konsequenzen für die Weiterentwicklung der österreichischen EU FTI-Politik und die mittelfristige Positionierung der FFG-EIP, die sowohl die nationalen FTI-Akteure als auch die nationale FTI-Politik bei ihren Aufgaben an der Schnittstelle zu Europa unterstützen soll.

\section{Szenarien zur österreichischen Positionierung in FP9 und ihrer Unterstützungsstrukturen}

Die Diskussion der Erwartungshaltungen der österreichischen FTI-Community hat gezeigt, dass Veränderungen sowohl in der Governance auf nationaler und an der Schnittstelle zur europäischen Politikebene erforderlich sein werden, als auch in den Unterstützungsleistungen für die FTI-Community. Das Ausmaß dieser Veränderungen ist von der angestrebten Positionierung Österreichs in Bezug auf die europäische FTI-Politik abhängig. Die drei nachfolgend skizzierten Szenarien spiegeln Handlungsoptionen und die jeweiligen Konsequenzen auf nationaler Ebene wider, die diese für die Unterstützungsstrukturen hätten. Sie sind als Denkanstöße für die weitere Diskussion in Österreich zu verstehen, nicht zuletzt auch im Hinblick auf die Entwicklung einer neuen FTI-Strategie.

\section{Szenario 'Enhancement in Continuity': Inkrementelle Weiterentwicklung}

Der Leitgedanke dieses Szenarios besteht darin, dass der gut etablierte und in weiten Teilen auch gut funktionierende Status Quo weiterentwickelt wird und im Zuge dessen Anpassungen der FTI-Politik an die Erfordernisse neuer Instrumente und Initiativen auf europäischer Ebene vorgenommen werden, ohne dass wesentliche Veränderungen in Österreich, z.B. im Bereich der Governance, vorgenommen würden. Dementsprechend bleiben Elemente der nationalen Strategiebildung und Prioritätensetzung (z.B. im Hinblick auf die österreichische Beteiligung an strategischen Partnerschaften) vergleichsweise schwach ausgeprägt, bzw. auf einzelne Ministerien beschränkt. Österreichische Organisationen beteiligen sich weiterhin - je nach individueller Interessenslage und Möglichkeit an einer Vielzahl von Initiativen, sehen sich dabei aber mit der Gefahr einer unterkritischen Ressourcenausstattung konfrontiert und können daher nur eine Nebenrolle in den größer dimensionierten europäischen Initiativen spielen. Eine explizite Fokussierung entlang etwaiger nationaler Schwerpunkte unterbleibt daher.

Im Einklang mit diesem Bild verbleibt die Positionierung Österreichs in EU-Initiativen weitgehend bei den FTI-Organisationen selbst und im Aufgabenbereich der Fachministerien, die diese Positionierung nach jeweils ressortspezifischen Prioritätensetzungen vornehmen.

Aufgrund des Verzichts auf eine umfassende nationale Positionierungs- und Profilierungsstrategie in Bezug auf die europäische Ebene kann auch keine herausstechende proaktive 
Rolle bei der weiteren strategischen Ausgestaltung des Rahmenprogramms gespielt werden.

Grundsätzlich wird in diesem Szenario die unterstützende Rolle von EU-Koordinationsabteilung und FFG-EIP beibehalten und ggf. punktuell gestärkt. Konkret manifestiert sich dies im Aufgreifen der - in weiten Bereichen inkrementellen - Empfehlungen der vorliegenden Evaluierung, darüber hinaus aber auch in einer Reihe zusätzlicher Charakteristika:

- Die Teilnahme der FTI-Akteure am europäischen Rahmenprogramm und weiteren Partnerschaften entwickelt sich entlang der vorherrschenden österreichischen Besonderheiten, d.h. den bestehenden strukturellen (z.B. zentrale FTI-Akteure) und thematischen (z.B. existierende ,Stärken') Profilen, weiter.

- Die FFG-EIP intensiviert ihre Anstrengungen, um strategische Potenziale auf FTIAkteursebene zu erkennen und die FTI-Community diesbezüglich anzusprechen. Dies erfolgt im Rahmen der von den einzelnen Ministerien vorgegebenen Leitlinien, d.h. die Verantwortlichkeit für die Entwicklung der inhaltlichen Strategien verbleibt bei den einzelnen Ministerien. Diese unterstützen im Rahmen ihrer thematischen Verantwortlichkeiten europäische Aktivitäten durch das Bereitstellen zusätzlicher finanzieller Mittel und den Einsatz weiterer Koordinierungsinstrumente (z.B. über ihre Eigentümerfunktionen).

- Informationen zu Verschränkungsmöglichkeiten von nationalen und europäischen Programmen (z.B. Stärken, Schwächen und Komplementaritäten Österreichs in Bezug auf europäische Initiativen) werden seitens der FFG-EIP aufgezeigt, aber es werden nicht aktiv Partnerschaften initiiert oder (an)gesteuert.

- Die Zusammenarbeit zwischen den Ressorts in Bezug auf europäische Initiativen bleibt weiterhin in den Rahmen der jeweiligen Verantwortlichkeiten eingebettet. Aufgrund dieser Abgrenzungen bleibt die Koordination innerhalb der für FTI-Agenden zuständigen Ressorts schwach ausgeprägt, ebenso wie jene zwischen FTIund sektoralen Politikfeldern.

- Die EU-Koordinationsabteilung ist weiterhin als zentraler Knoten und Schnittstelle zwischen EU FTI-Politik, FTI-Ministerien und FTI-Community positioniert. Die Aufgaben der EU-Koordinationsabteilung konzentrieren sich dabei auf Informationsvermittlung und Aktivierung der FTI-Community.

- Angesichts der verteilten Verantwortlichkeiten und begrenzten Politikkoordination kommt einem FTI-politischen Beratungsgremien in Bezug auf EU FTI-Agenden die Rolle zu, Empfehlungen hinsichtlich wichtiger übergreifender Politikmaßnahmen zu erarbeiten, die für die nachhaltige Stärkung der Performance des österreichischen Forschungs- und Innovationssystems erforderlich wären.

- Im Einklang mit diesen Stoßrichtungen wäre die Einrichtung eines Verbindungsbüros in Brüssel zwar denkbar, aber nicht zwingend erforderlich.

\section{Szenario 'Smart and Proactive Alignment': FTI-Politik als Impulsgeberin im europäischen Mehrebenensystem}

In diesem Szenario strebt die österreichische Politik einen Ausbau ihrer Rolle hin zum (pro)aktiven Mitgestalter europäischer FTI-Politik an, um die sich daraus ergebenden 
Chancen der EU FTI-Politik gezielter und effektiver für Österreich zu nutzen (,smart alignment').

Um sich besser als integraler Bestandteil des Mehr-Ebenen Innovationsökosystems in Europa positionieren zu können, strebt Österreich im Rahmen seiner neuen FTI-Strategie eine Reihe von Veränderungen im nationalen System an, über die eine intensivere und einflussreichere Mitwirkung an der Ausgestaltung und in der Folge an der Nutzung der europäischen FTI-Politik möglich wird. Die Festlegung eines solchen Konzepts als Leitlinie für eine kohärente österreichische Position zur EU FTI-Politik wäre ein zentraler Bestandteil einer neuen österreichischen FTI-Strategie.

Dies umfasst neben der weiteren Exzellenzorientierung der Universitäten und der Stärkung der unternehmerischen Kultur insbesondere auch die Entwicklung einer europäischen Beteiligungsstrategie, inkl. nationaler Prioritäten, um in ausgewählten Feldern eine gewichtige und prägende Rolle spielen zu können. Hiermit einher geht eine enge Verschränkung nationaler und europäischer/internationaler Programme, die sich beispielsweise in einer selektiven Beteiligung an strategischen Initiativen der EU oder in einer verbesserten inhaltlichen Komplementarität/Abstimmung zwischen nationalen und europäischen Förderausschreibungen manifestieren könnte.

Die aktive Beteiligung an der Gestaltung europäischer FTI-Instrumente und -Inhalte erfolgt in abgestimmter Form zwischen Ministerien, FFG-EIP und FTI-Community, orchestriert durch die nationale Politik.

Mit anderen Worten, dieses Szenario geht von einer ausgeprägten politischen Leadership-Rolle bei der Stärkung der europäischen Rolle Österreichs im Einklang mit der nationalen FTI-Strategie aus.

Um diese federführende Rolle der Politik bei der strategischen Positionierung Österreichs auf europäischer Ebene ebenso wie im Hinblick auf die Konzeption nationaler Programme und Politiken (,smart and proactive alignment') realisieren zu können, bedarf es der Unterstützung durch eine starke FFG mit ihren nationalen und europäischen Aktivitäten, aber auch der Weiterentwicklung der interministeriellen Abstimmungsmechanismen. Im Hinblick darauf sollten die Unterstützungsstrukturen in Österreich entlang folgender Stoßrichtungen weiterentwickelt werden:

- Interministerielle Abstimmungen dienen nicht nur der Abgrenzung von Verantwortlichkeiten (negative Koordination), sondern ermöglichen Komplementaritäten und Synergien im Sinne der in einer neuen FTI-Strategie festgelegten europäischen Positionierung (positive Koordination). Nationale Strategiebildungsprozesse zu europäischen Leitthemen werden auf politischer Ebene und/oder höchster Beamtenebene gestützt ("ERA Policy Forum 2.0") und tragen so zu einer klaren europäischen Positionierung bei und werden angesichts der wachsenden Dringlichkeit solcher Positionierungen als notwendig und realistisch angesehen.

- Der EU-Koordinationsabteilung kommt in diesem Prozess eine Schlüsselrolle zu, die durch eine entsprechend sichtbare Verankerung im Politiksystem unterstrichen wird (z.B. durch Zuschreibung besonderer Kompetenzen, oder eine Verankerung im Bundeskanzleramt). 
- Ergänzend zur Stärkung des politischen Alignment kommt einer höheren Autonomie und Gestaltungsfreiheit der FFG (vgl. die entsprechenden Empfehlungen der FFG-AWS Evaluierung und das "VINNOVA Modell") eine große Bedeutung zu. Diese Form der höheren Autonomie würde dazu dienen, die Teilnahme an ERA-Initiativen vor dem Hintergrund der nationalen Strategie und komplementär zu österreichischen Besonderheiten auszurichten, z.B. durch aktives Anstoßen und Unterstützen von nationalen Partnerschaften innerhalb der strategischen Rahmenvorgaben und Prioritäten der Politik.

- Dementsprechend wäre auch der Kapazitäts- und Kompetenzaufbau auf Seiten der FFG-EIP zu forcieren, um Schnittstellen nach Brüssel zu intensivieren und Interaktionen mit der österreichischen FTI-Community, aber auch mit Partnern in Europa, zu stärken, um auf diese Weise die Positionierung der nationalen Prioritäten auch auf der europäischen Ebene zu unterstützen.

- Im Einklang mit diesen Überlegungen wäre innerhalb der FFG und in Zusammenarbeit mit den Fachministerien eine engere Verzahnung nationaler und europäischer Programme anzustreben, um bei der Beratung von FTI-Akteuren einen nahtlosen Übergang zwischen Programmen auf unterschiedlichen Ebenen zu ermöglichen.

- Zur Flankierung dieses proaktiven Szenarios kommt der starken Verankerung des EU FTI-Themas in den Agenden eines im Regierungsprogramm vorgesehenen neu zu gestaltenden hochrangigen Beratungsgremiums zu FTI-politischen Fragen eine wichtige Rolle zu. Dadurch soll die effektive Einbettung des österreichischen Forschungs- und Innovationssystems in den europäischen Kontext und die Mitgestaltung eben dieses europäischen Kontexts durch kompetente ExpertInnen mit unabhängiger Binnen- und Außenperspektive unterstützt werden.

- Im Rahmen dieses Szenarios würde auch ein nationales, d.h. von Politik und/oder FFG getragenes, Verbindungsbüro in Brüssel eine sinnvolle Ergänzung bilden.

\section{Szenario 'Distributed Empowerment': Stärkung und Vernetzung der FTI- Community}

In diesem Szenario erfolgt die Ausweitung der Mitgestaltung und Beteiligung an der europäischen FTI-Politik vor allem über die Stärkung und bessere Vernetzung der FTI-Akteure selbst, die seitens der österreichischen Politik bei diesem Prozess der Selbstermächtigung (Empowerment) unterstützt werden. Dies ist insofern ein plausibler Zugang als die Beteiligung an den größeren Initiativen (z.B. strategische Partnerschaften) mit teils geringeren Förderquoten nur dann erfolgreich sein kann, wenn sie sehr eng an die strategischen Interessen der FTI-durchführenden Organisationen geknüpft sind.

Dieses Szenario setzt wiederum eine eigenständige Entwicklungsstrategie der Organisationen voraus, sowie die Fähigkeit zur Selbstorganisation in schlagkräftige nationale Netzwerke. Es benötigt darüber hinaus aber auch entsprechende Ressourcen und Kapazitäten, deren Mobilisierung durch die FTI-Politik unterstützt wird (z.B. durch finanzielle Anreize, Vernetzungsmaßnahmen, Informationsaustausch, etc.). Die Erfahrungen anderer 
europäischer Länder (z.B. Finnland, Niederlande, etc.) bei der Mobilisierung ihrer FTICommunities für die Mitgestaltung der EU FTI-Politik liefern hierfür Anstöße, die aber auch zeigen, dass es bei diesem Selbstermächtigungsprozess sowohl Gewinner als auch Verlierer geben kann.

Auf politischer Ebene erfordert dieses Szenario keine ex ante Festlegung auf prioritäre Themen, um die herum eine Mobilisierung der FTI-Community angestrebt wird, da sich diese aus dem Engagement und der effektiven Vernetzung der FTI-Akteure heraus ergeben. Dies ist auch die zentrale Herausforderung dieses Szenarios, nämlich durch entsprechende selektiv wirkende Anreize zu gewährleisten, dass sich in der tendenziell dispersen FTI-Community Österreichs derartige Schwerpunkte herausbilden können, die dann auch auf EU-Ebene wirkmächtig werden. Bisherige Erfahrungen haben gezeigt, dass es entweder Leitakteure geben muss, die die Mobilisierung von Netzwerken vorantreiben (z.B. einzelne starke Unis, COMET-Zentren, Forschungseinrichtungen oder Unternehmen) und/oder eine gestaltende Rolle der Politik, ggf. verankert in den Fachministerien und deren Aktivitäten (z.B. Silicon Austria Labs), um die Entstehung hinreichend großer Strukturen in Österreich zu ermöglichen.

Ergänzend zu den bereits heute existierenden Unterstützungsleistungen und -strukturen ergibt sich in diesem Szenario die Notwendigkeit, die eigenverantwortliche Strategiefähigkeit der FTI-Akteure und ihre Fähigkeit zur nationalen und europäischen Vernetzung und kollektiven Strategiebildung mit den Mitteln der Politik und der FFG-EIP zu unterstützen. Dies legt Stoßrichtungen wie die folgenden nahe:

- Damit die österreichische FTI-Community auf europäischer Ebene eine aktive Gestaltungs- und Beteiligungsrolle einnehmen kann, ist der Kapazitätsaufbau nationaler FTI-Netzwerke voranzutreiben, was instrumentell über stärkere (finanzielle) Anreize aber auch durch gezielte Informations-, Vernetzungs- und Strategieaktivitäten umgesetzt werden könnte. Angesichts der Fokussierung auf eine Stärkung der FTI-Community und ihrer autonomen Vernetzung ist die interministerielle Koordination in diesem Szenario von weniger zentraler Bedeutung.

- Die EU Koordinationsabteilung fungiert dabei als zentraler Verbindungsknoten zwischen nationaler FTI-Community, Fachministerien und (politischen) EUGremien und flankiert den von der FTI-Community getragenen Ansatz zur Mitgestaltung und Mitwirkung an europäischen Initiativen. Eine angemessen dotierte Ausstattung der EU Koordinationsabteilung oder auch der Fachministerien mit Mitteln zur wettbewerblichen (Ko-)Finanzierung derartiger, von der FTI-Community getragenen Aktivitäten würde es erlauben, diesen Mobilisierungsprozess voranzutreiben.

- Mit den so zur Verfügung stehenden Mitteln würde die FFG eigenverantwortlich entsprechende Programme und Aktivitäten umsetzen können. Im Leistungsangebot der FFG-EIP verschiebt sich der Schwerpunkt hin zur Unterstützung von FTI-Akteuren bei der Beteiligung an größeren Initiativen wie den strategischen Partnerschaften, um beispielsweise die Zusammenarbeit zwischen nationalen FTI-Netzwerken mit europäischen Initiativen zu ermöglichen. 
- Dies wird andere und ggf. zusätzliche Kompetenzen und Kapazitäten auf Seiten der FFG-EIP erfordern. Darüber hinaus würde die FFG-EIP über ein sehr hohes $\mathrm{Ma}$ an strategischer Autonomie verfügen müssen, um in Abstimmung mit der FTI-Community Unterstützungsleistungen zu entwickeln und bereitzustellen. Dabei wäre die FFG-EIP nur innerhalb eines vergleichsweise allgemein gehaltenen Rahmens an inhaltlich-thematische Vorgaben seitens der Fachministerien gebunden.

- Ähnlich wie im zweiten Szenario ist auch hier eine enge Verschränkung der nationalen Programme mit den europäischen Initiativen sinnvoll, hier allerdings ausgerichtet auf die von der FTI-Community forcierten Bereiche. Ein solcher Ansatz würde es nahelegen, auch innerhalb der FFG den Austausch zwischen den verschiedenen national und europäisch ausgerichteten Bereichen weiter zu intensivieren.

- Aufgrund der vergleichsweise schwach ausgeprägten Führungsfunktion seitens der Politik könnte sich ein hochrangiges Beratungsgremium in diesem Szenario stärker an die FTI-Community richten, um für diese eine übergreifende Orientierungsrolle zu übernehmen.

- Auch in diesem Modell wäre ein Verbindungsbüro in Brüssel eine wichtige sinnvolle Ergänzung. In diesem Szenario würde es aber im Wesentlichen von der FTI-Community selbst getragen. 


\section{Summary}

\section{Background, objectives and methodology of the evaluation}

In 2013, the FFG (Austrian Research Promotion Agency), more specifically, the Department for European and International Programmes (FFG-EIP), was commissioned by the Republic of Austria and the Austrian Chamber of Commerce to support the implementation of HORIZON 2020 and ERA for the period 2014-2020. The primary aim of this measure is to foster the participation of Austrian RTI (research, technology and innovation) participants in the European framework programme for research and innovation, Horizon 2020 (H2020) and in multilateral initiatives (MULLATS). With respect to supporting Austrian RTI participants, the EIP builds on the skills and collective experience of previous assignments. The FFG-EIP is also the officially nominated national contact point (NCP) of the European Commission for the framework programme and all initiatives and programmes in connection with $\mathrm{H} 2020$, referred to as the transnational or multinational initiatives of the European Research Area (MULLATs). In 2014, the FFG was entrusted by this organisation with monitoring Austria's involvement in HORIZON 2020 and ERA. With other partners, the FFG is also responsible for providing support services for EUREKA, COSME and the Enterprise Europe Network (EEN).

In order to fulfil the overarching objectives of the assignment, the FFG-EIP is pursuing four key objectives, which take account of the results-oriented action framework as national contact point for $\mathrm{H} 2020$ :

1. Objective 1: Support successful participation by means of information and advice

2. Objective 2: System-orientated support of Austrian RTI organisations

3. Objective 3: Expert partner for European RTI programmes and ERA developments

4. Objective 4: Analysis and RTI policy support

Keeping these goals in mind, the aim of this evaluation is to undertake a systematic general overview of the implementation of $\mathrm{H} 2020$ and ERA, as well as EUREKA, COSME and EEN in Austria. The evaluation focuses on three key areas:

1. Commissioning the FFG with providing advisory and support services for HORIZON 2020 and ERA (EIP assignment)

2. Commissioning the FFG with EU performance monitoring (EU-PM assignment)

3. The ERA Observatory Austria, which forms an overarching framework for the integration of the individual tasks in the field of governance for EU-RTI policy in Austria on the part of the BMBWF (Federal Ministry of Education, Science and Research).

The evaluation also includes an analysis of the assignments for EUREKA, COSME and EEN. 
The following meta questions form the basis of the various individual questions in the evaluation:

4. General evaluation of the implementation: Are the orientation and implementation of available formats and support services - within the allocated budget suitable and appropriately implemented in order to achieve the desired effects of measures regarding output as efficiently and effectively as possible?

5. Evaluation of specific services: With respect to observable and anticipated developments in the RTI domain (including RTI policy), where are support service formats adequate and where should improvements be made?

6. Action required and options for action: Where are any white spots encountered or where should new and better approaches and tools be established? According to which principles should these essentially be designed?

The evaluation design was based on the triangulation of complementary methods: This approach combines various quantitative and qualitative methods to confirm findings from at least two sources. An international advisory board of renowned experts was also involved to support the evaluation team in its reflection on findings, drawing relevant conclusions and deriving recommendations for modifying and enhancing the Austrian solution portfolio.

Central to the survey phase was the collection of robust data using desk research, expert interviews and an online questionnaire.

Based on this, interim results were validated and reflected together with the clients. Subsequent in-depth analysis followed in the form of focus group interviews, additional expert interviews and a Delphi survey.

The results of this phase in turn formed the basis for developing recommendations, which took international perspectives into account through a focus group in Brussels and participatory workshops with the international advisory board and the evaluation's clients.

The conclusions and recommendations of the evaluation are based on the triangulation of the results and findings from the methods used, in order to compensate for the specific strengths and weaknesses of each individual method, thus contributing to the validity and reliability of recommendations.

\section{Key observations and recommendations}

The following recommendations from the evaluation are orientated to the three focus areas of the evaluation and thus the various FFG assignments: Consultancy and support, EU performance monitoring, ERA Observatory. With reference to the advice and support focus area, a distinction is also made between recommendations that relate specifically to the target groups of science and businesses and those that span all target groups. The efficiency and governance of this specific assignment is also discussed separately. 


\section{Advisory and support for the scientific community}

The range of FFG-EIP services for science includes specific information and support services for proposers in $\mathrm{H} 2020$ as well as support tools for target groups at management level in research organisations. The latter particularly includes the Vice Rectorates for research, but also research service centres in universities.

The relevance of the FFG-EIP service in the areas of 1) raising awareness \& information, 2) programme and project consultancy and 3) strategic consultancy and ERA orientation knowledge with regard to FFG customer requirements is generally high. This is particularly true for personal consultancy services and services for continuing education as part of the FFG Academy.

The individual FFG-EIP range of services has positive overall effects because it contributes to enhancing researchers' expertise in drafting proposals and increases the success rates of applications. However, there is potential to increase effectiveness, which may be achieved by making target group-orientated modifications in the service portfolio.

\section{Recommendation: Target group-orientated address to researchers}

To increase access to FFG-EIP services and to enhance the effectiveness of offered services, there should be a target group-orientated approach for addressing experienced and less experienced researchers. The presentation of the core areas of the consultancy service should be improved and the consultancy's potential benefits for the various target groups should be pinpointed. This could be achieved by presenting service modules/packages for specific groups, in which relevant services are clearly packaged to suit the different target groups.

A service recently introduced by the FFG-EIP as part of the 2014 assignment aims to support the self-empowerment of research organisations with regard to the European Research Area and H2020. Significant progress has been achieved in this respect. However, the capacities provided by universities to the respective research service points are very heterogeneous. At the same time, the significance of European RTI networks and strategic partnerships in European framework programmes is increasing and greater strategic action is required at international level, both within and beyond the university sector. This suggests a corresponding amendment to FFG-EIP support services.

\section{Recommendation: Develop specific services for service points}

Background knowledge on $\mathrm{H} 2020$ work programmes should be communicated to service points promptly, concisely and precisely. This includes the, frequently informal, discussions held in advance, assessments of what is really being sought in a call and other background information. As this information is often valid only for a limited period (e.g. working papers), the FFG should develop an (informal) policy agreed with delegates which sets out how information should be disclosed.

Call content and work programmes should be drafted so that they can be better and more quickly communicated to the RTI community. 
Information on developments regarding FP9 (such as conclusions of informal meetings, current discussions, where attention should be focused in future) should be made available more quickly.

Data and information, reports, etc. should not only consist of the link/report itself; the FFG should also briefly highlight the key points.

As well as direct support services to the scientific community, a key FFG-EIP goal is to enhance the empowerment of research organisations. The target groups of empowerment support are the research services and Vice-Rectorates of universities. Using the ERA Dialogue instrument, the FFG has already taken a meaningful first step in this regard to raise awareness of the significance of European agendas in organisations. Research organisations themselves should be primarily responsible for further strategy development processes, and in this regard most organisations are still in the early stages of development. Without expanding capacity within organisations, the potential of research organisations to play an active part in the European Research Area appears to be somewhat limited. The FFG has a sound base of consultancy services to reach out to research service points and Vice Rectorates. However, service point professionalisation and strategic empowerment still needs to be accelerated.

\section{Recommendation: Continued acceleration of professionalisation of service points and strategic empowerment with regard to the ERA}

To reinforce the professionalisation of service points and strategic empowerment, ERA dialogues should be pursued to systematise contact with research service points and, at the same time, the support services targeted to the requirements of the respective universities should be co-ordinated.

Within ERA dialogues, activities that impact at faculty and institute level should also continue to be promoted. These should also be in line with the interests and challenges of the respective university and should be both feasible and mandatory.

Regular knowledge sharing between university research points involving service personnel from outside the university research sector should be institutionalised, which will also serve to strengthen self-management. The Federal Working Group for EU officers (BAK) in Germany should serve as an example in this respect.

\section{Advisory and support for the business community}

\section{FFG-EIP}

The evaluation shows that the business sector rates the FFG-EIP service portfolio for the consultancy and support of participants largely as positive to very positive. The EIP service portfolio has generally increased the level of information for the H2020 "ecosystem" and also fulfils the more specific consultancy requirements of organisations.

Therefore, the recommendations are based on the available findings and largely point to the continuation of relevant services. Optimisation potential is evident in the area of 
"expectations management", i.e. better clarification of what the services provided are/are not.

\section{Recommendation: Improve expectations management}

Based on the positive experiences, a strategy of "business as usual" is generally appropriate. However, the target group-specific offers should be made more transparent. This particularly involves realistic "expectations management" which, on the one hand, indicates the opportunities and limitations (or the associated costs) of participating in European RTI programmes and, on the other hand, includes FFG-EIP support services for project application and project management.

This type of strategy is compatible with the explicit "non-objective" of EIP, to maximise the number of organisations submitting applications (not "pressing" unsuitable organisations or organisations with unsuitable projects to participate).

The $\mathbf{C} \mathbf{3}$ concept (core customer concept) is a specific element of the FFG-EIP consultancy and support service for the business sector, which is selectively used on the most advanced customers. Beneficiary organisations' evaluation of this service is very good. However, the question is how to make the experience of these beneficiaries useful to other organisations.

\section{Recommendation: Improve the impact potential of C3 support}

As C3 support is targeted at a relatively small group, the positive impact of the service on the direct target group remains limited. A greater multiplier effect and greater scalability of impact is necessary. To this end, the following modifications may be possible:

Greater allocation of resources for C3, enabling C3 support and consultancy for more organisations.

Involvement of C3 organisations in digital peer learning groups (for the purpose of using the experience and expertise of $\mathrm{C} 3$ organisations), involving Austrian project partners of C3 organisations, with these groups being moderated by FFG-EIP experts.

More cooperation between $\mathrm{C} 3$ beneficiaries and intermediaries (such as the Austrian Chamber of Commerce (WKO)).

\section{EUREKA - Initiative for application-orientated research and development in Europe}

The integration of the activities of the EUREKA Bureau under the umbrella of FFG-EIP can be described as a success. The allocation of responsibilities and duties to various people in the team and thus the cross-team interlinking of consultancy services and activities is worth mentioning.

The growing interest in EUREKA funding opportunities means that, EUREKA, and also Eurostars with allowances, functions as an alternative to other funding programmes. This 
raises questions concerning the strategic positioning of EUREKA and Eurostars, particularly with regard to complementarity with funding opportunities from the European framework programme.

Based on the different structure and funding of EUREKA in member states, the collective expertise within the EUREKA Bureau, its integration into the FFG and the collective experience is key for the effective and efficient consultancy and project support. However, coordination and synchronisation between EUREKA's member states remains EUREKA's biggest challenge.

\section{Recommendation: National positioning of EUREKA reasonable boost Eurostars}

EUREKA's positioning in a European context depends on the structure and organisation of the European funding landscape and the various network and cooperation structures. Against this backdrop, EUREKA is reasonably positioned at national level as an easily accessible funding alternative with good success rates compared with the $\mathrm{H} 2020$ framework programme and other forms of transnational RTI partnerships.

The active positioning of Eurostars as an alternative to the framework programme's heavily over-subscribed SME instrument should be accelerated.

EUREKA programme management is perceived as lean in structure, efficient and transparent. EUREKA's open-theme, bottom-up approach is well reflected in the FFG General Programme (FFG Basisprogramm) department, which cooperates closely with the EUREKA Bureau. Communication in the team and synergies with EIP are used well. The evaluation thus also recommends continuation of the current modus operandi.

\section{Recommendation: Maintain efficient programme management and lean struc-} tures

The integration of the EUREKA Bureau into FFG-EIP enables the use of synergies in consultancy and support for collaborative applied research and development. National programme management is efficient.

Against this backdrop, the EUREKA Bureau should remain integrated in FFG-EIP..

\section{COSME - Programme for the competitiveness of organisations and SMEs}

The FFG is commissioned by the BMDW to promote COSME and support Austrian organisations with questions about the programme. Both thematically and content-wise, the broad range of measures within COSME - access to funding, access to markets, support for business initiatives and culture, and improvement of framework conditions for competitive capacity - is orientated to the general objective of "increasing competitive ability".i It is thus, to a large extent, relevant for the broad spectrum of its actual target group, all European SMEs. However, this feature of COSME also means that few specific steering attempts (in the sense of pursuing specific political objectives or addressing less-developed markets) can be expected.

Furthermore, general awareness of the programme is rather low. External perception (of opportunities) within the context of COSME appears to focus mostly on the pillars of financial instruments and the EEN (see below); SME-related policy reports are more 
widely known. This selective perception or partial lack of awareness of elements of the programme is completely understandable due to the nature of the programme as a policy development and policy support tool, its indirect mode of action (intermediaries as the primary target group) and the extensive spread and range of themes and measures. A need for action to raise awareness of COSME by means of specific measures on the part of the FFG-EIP was not identified by the evaluation team. Interface management with regard to the individual measures in COSME are largely efficient with well-coordinated internal and external interfaces.

Recommendation: Continuation of proactive programme and interface management in the FFG-EIP

With a view to organisations' international activities there are a number of points of reference within COSME in the support by the FFG - not least by means of the reintegration of innovation aspects into thematic/sectoral calls as part of COSME, which are also highlighted by the piloting of individual measures (with potential regard to innovation). In this light, the evaluation concludes that interface management (timely anticipation and advertisement or circulation of calls to multipliers and intermediaries, etc.) needs to be and is appropriately located at the FFG. However, the constant involvement (proactively approaching) of multipliers is imperative - not least because of the verifiable selective perception and partial lack of awareness of the programme and all its possibilities.

However, data monitoring of participations and programme outcomes shows weaknesses, which are not the FFG's fault. In this regard, the European Commission is primarily responsible to enhance its COSME data hub.

\section{Recommendation: Improve data monitoring by the European Commission}

Regarding the transparency and data monitoring of participations and programme outcomes, there is clearly potential for improvement, especially on the part of the European Commission. The COSME data hub is a first step towards better monitoring and transparency. However, so far there has been no structured notification system for calls for proposals, which could replace a large part of the FFG's research efforts and increase the effectiveness and efficiency of the FFG's programme management.

\section{EEN - Enterprise Europe Network}

EEN and its calls are implemented as a measure within COSME. The EEN's relevance is mostly, a result of its broader, more comprehensive access (as opposed to a project focus) and also, the generally longer term support of organisations. The focus of services on supporting international cooperation and technology transfer activities of organisations and thus the wide range of cooperation activities (R\&D\&I cooperations, pure technology partnerships, licensing, distribution agreements, etc.) is also highly relevant for the target group, especially for SMEs.

\section{Recommendation: Continue with the integration of EEN services into FFG-EIP}

The EEN is well known amongst stakeholders, intermediaries, and funding agencies and fulfils its role as a regionally anchored network with likewise embedded nodes/points of 
contact. With its services/tools, the EEN is a highly significant component of FFG's support services. EEN services/tools also appear to be well integrated in portfolios from an organisational point of view and are well-linked and coordinated with other relevant measures (e.g. support for HORIZON 2020 and other support/funding opportunities). EEN services are frequently used in advance and complementarily to HORIZON 2020.

\section{Consultancy and support - overarching recommendations}

Beyond the target group-specific recommendations for the scientific and businesses communities, there are a number of potential improvements applicable to both. At the same time, they transcend the range of responsibilities of the FFG-EIP and concern the entire FFG.

Whilst the FFG-EIP website provides a wide range of information on programmes and activities at European and international level, the presentation, structure and identification of specific information and contact persons could be improved.

\section{Recommendation: Redesign the website}

Redesigning the FFG-EIP website is necessary to enable better communication of information to the target groups. At present, the FFG-EIP homepage provides a wealth of information and good content. However, its layout is confusing and not especially user-friendly. The search function and finding programme information on $\mathrm{H} 2020$ and contact persons are difficult. It is also unclear to whom reference should be made regarding advisory and support services, NCPs, FFG Academy and what its registration processes are. The current FFG-EIP and EU-PM websites suggest that a fundamental redesign of the FFG website is necessary. In doing this, consideration should be given to the following:

1. Easier, more intuitive, more target group-specific access:

- Differentiation according to relevance of information

- Differentiation between basic information (programme information) and detailed questions (e.g. regarding applications and associated FFG services)

- Better positioning of the FFG search function

2. Improving the navigation structure:

- Immediate access to European funding programmes and associated FFG services offered

- Implementation of context-dependent user prompting and better presentation of services offered: Services such as "Partner search" that play little or no part in the FFG portfolio are currently highlighted as key services, whereas services, such as the various consultancy tools and EU-PM, are hidden. Better identification of key contact persons: Identification and how to reach NCPs and FFG experts are unclear. The same applies to the main points of consultancy expertise. For example, the appropriate contact person for a service/programme could be listed on the page for that service/programme. 
The evaluation repeatedly states that, from the point of view of applicants who are primarily searching for a suitable funding programme for their proposal, having separate responsibilities at the FFG for national and European programmes gets in the way of finding the advice they need. Particular attention should be paid to cross-linking and the rapidflow of information between the FFG departments responsible for national and European programmes.

\section{Recommendation: Better cross-linking of European and national programmes}

Within the FFG, appropriate measures should aim to establish and optimise "cross-linking" of European and national programmes within the consultancy service. This should be seen against the backdrop that many (especially smaller) organisations make no distinction between the individual departments of the FFG and do not recognise the division of services offered by the EIP and the various services offered as part of the national funding programmes.

Meanwhile, in view of the very low success rates and frequent rejection of projects that have been assessed as very good (or above threshold), consideration should potentially be given to establishing relevant connection support or consultancy (for example towards "transforming" project ideas for submission to a national funding programme).

\section{Governance and efficiency of the EIP contract}

Commissioning the EIP with information, consultancy, support and networking services is done separately and is not part of the framework agreement of the FFG with the federal government proprietors (BMVIT and BMDW). Despite the additional associated administrative work and expense due to separate financial reporting, this particular design features a range of benefits, such that this specific form of commissioning can be considered to be reasonable.

\section{Recommendation: Continue separate commissioning of the European and Inter- national Programme Department (FFG-EIP)}

To fulfil the objectives and requirements concomitant with consultancy and support for the European RTI instruments, from the current perspective, separate commissioning of the FFG "European and International Programme" department appears to be appropriate, despite the additional administrative costs. Doing this is a means of ensuring the effective involvement of various ministries and the Austrian Economic Chamber in the FFG-EIP governance structures.

Separate commissioning entails regular reporting obligations for the FFG-EIP, which may become even more important should future contract arrangements become more flexible.

\section{Recommendation: Continue regular reporting obligations}

In view of the size and scale of the contract, the regular reporting obligation and the associated ongoing monitoring and adaptation of activities are both prudent and necessary. If the future contract with the FFG-EIP is to be more flexible, these reporting obligations 
will become even more important. We therefore recommend that current reporting obligations be continued, taking into account the suggestions for the modification and enhancement of WFA indicators.

With regard to financial reporting as part of EIP commissioning, a thematic allocation of costs and staff hours, with many, partly overlapping, types of activities, has largely been applied so far. To enhance transparency and enable adequate estimation of costs of various activities, particularly new services (ERA Dialog, C3), we recommend the introduction of a functionally structured monitoring system for the future.

\section{Recommendation: Improve EIP cost recording}

In view of Austria's participation structure in H2020, the FFG-EIP budget allocation for various activities is reasonable. Implementation is also efficient. However, to eliminate any uncertainties regarding cost allocation and to enable better estimation of the budget allocated to specific support services, the evaluation team recommends that the EIP cost recording structure be changed from thematic to functional. This would be particularly relevant as regards the evaluation of the new ERA Dialog and C3 activities and also understanding the costs of other services, such as reports, analyses and the FFG Academy. Since various functional services are often closely interlinked in practice (e.g. various types of support), care should be taken to ensure a balance is struck between sophistication and feasibility of implementation.

\section{EU performance monitoring}

In general, the evaluation has revealed a high level of user satisfaction across all target groups regarding the range of EU performance monitoring information and products. Demarcation of the target groups and the provision of target group-specific services are also appropriate and successful.

\section{Recommendation: No need for action regarding target group differentiation}

Satisfaction amongst users in both target groups is high and the corresponding demarcation of target groups is functional. In any case, there is no need for any action with regard to the latter. In particular, it is deemed unnecessary to establish a separate target group for regional contact points. However, further dialogue with regional contact points could be strengthened.

Critical comments particularly concern product functionality, especially the website and public online portals, as well as the quality and relevance of data provided. This involves making it easier to find EU-PM services via the FFG homepage and in better communicating the functionalities (and limitations) of the public portal. Compared with similar portals, particularly VINNOVA and the Commission's H2020 participant portal, the EU-PM features a range of specific characteristics (e.g. focus on essential key performance indicator and performance comparisons at programme and institution level; comparisons at federal state level). On this basis, continuation of an independent EU-PM portal also appears to be expedient for the future. 


\section{Recommendation: Continuation of the public online portal}

The evaluation recommends the continuation of the public online portal. At the same time, the portal's profiling should be improved, as compared with other portals. For example, for project level analyses, EU-PM could redirect to the VINNOVA tool.

The evaluation team also sees a communication problem regarding the database used to analyse performance in the framework programme, its justification and significance. Although at the level of programme delegates, various options are available for individual and - compared with EU-PM data - more frequent information gathering, EU-PM's advantage of using eCORDA as a basis, as stipulated in its contract, is the comparability and data quality control.

\section{Recommendation: Improve usability of the online portal}

Adaptations should be made to the public portal regarding the communication of the portal's database and its performance. This includes adapting the portal manual, which includes information on the calculation of individual key performance indicators, but not the underlying data structure, maturity and limits of analysability. Reporting products, particularly standardised cockpit reports and quick information, should also include references to the underlying data and data quality.

Regarding the integration of additional data on transnational R\&I partnerships, the EU-PM itself relies on the provision of appropriate sources at EU level, in accordance with the assumption, here - comparable with the use of eCORDA as a central source - of wanting to rely on data that has already been collected and validated. At present, the data provision on transnational R\&I partnerships at EU level remains extremely limited, although positive developments in the recent past have been observed, which should contribute to further improving the data situation.

\section{Recommendation: Ongoing integration of additional databases}

The EU-PM complies with its mandate to review the integration of other data sources. Corresponding developments on EU level should continue to be actively supported by the EU-PM. The steering committee should also be informed of new databases and analysis options.

With regard to the range of analysis options now available and the associated expense of interlinked analysis, linking European and national funding data has so far only been done for R\&I-intensive individual institutions, such as research-orientated universities and large enterprises.

\section{Recommendation: Improve communication regarding analysis options and limi- tations}

The evaluation team finds that the EU-PM complies with its mandate to promote the integration of national and EU funding data. The challenge in future for the parties involved, particularly target group 1 , will be to formulate concise queries in light of the possibility for complex analysis (for example: "Will funding recipients in national programme $x y$ have above-average success in EU programmes?"). At the same time, the EU-PM team 
needs to work on better communicating the existing options, for example in workshops with delegates.

The confidential online portal is very well received compared to the possibility for direct enquiries to the EU-PM. However, a key consideration in future will undoubtedly be greater individualisation with regard to the demand-driven selection of combinations of individual programmes and participants depending on subject/programme responsibilities of respective users. New formats should be designed for this to enable interaction with users regarding increasingly individualised services. Overall, the evaluation team considers that services are provided efficiently.

\section{Recommendation: Develop dialogue formats for specifying EU-PM services}

The potential for further empowerment of target group 1, i.e., these users can obtain information themselves via the confidential online portal, is already largely exhausted.

At the same time, it is important to develop appropriate formats for the dialogue between clients, users and the EU-PM to a) provide information on new services and opportunities and b) accordingly develop concise and feasible analysis assignments in light of growing analysis options resulting from the integration of new data sources. One recommendation would be to conduct a needs assessment of all requesters once a year through the ticket system.

On the basis of the generally positive assessment of the EU-PM and the expertise accumulated so far, there are a number of arguments for continuation of the EU-PM in principle. Given in particular the flood of various data sources, there is potential here for the EU-PM to have a "gatekeeper" function in the communication of information, through which the EU-PM can simultaneously fulfill its central role as "information broker" as part of the ERA Observatory. The data provided via the EU-PM represents the central reference framework for the national policy debate and is used in official documents and reports (for example, the Federal government's research and technology report) and strategy development processes at federal level (compared with data provided elsewhere, e.g., via programme committees).

\section{Recommendation: Continue the EU-PM}

The evaluation team recommends the continuation of the EU-PM. It holds a key position as "information broker" for the national policy debate regarding ERA.

At the same time, there should be better communication of the added value of qualitycontrolled analyses and other associated analysis and connection opportunities with national data as policy-supporting services, thus further strengthening the "narrative power" of the EU-PM.

\section{ERA Observatory}

The ERA Observatory combines a range of activities, which should enable the exchange of information and better coordination between actors who play a key part in formulating and communicating Austrian positions regarding European RTI policy. In general, the activities within the ERA Observatory are perceived as positive. 
The work of the EU coordination department is almost unanimously perceived as positive and inclusive and strongly orientated to a joint exchange of experience. The relevant sectoral ministries appreciate the diverse range of formats for communication and exchange.

At present, attempts to strengthen coordination for cross-sectoral measures and strengthen connections between national and international programmes within the ERA Observatory appear to have reached their limits. The fact that cross-sectoral coordination could become even more important in future is already being made apparent by the great importance of strategic partnership initiatives, where RTI policymakers, and the wider RTI community, currently cannot developed a nationally coordinated approach. Informal coordination, as in information exchange (Europa Forum Forschung) and exchange of experiences (Delegates Round Table) has been well accepted, whereas there has so far been insufficient support for more intensive coordination (e.g. ERA Policy Forum).

Against this institutional backdrop, the evaluation only suggests modifications regarding the Europa Forum Forschung.

\section{Recommendation: Improve dialogue in the Europa Forum Forschung}

The ERA Observatory fulfils its mandate to provide information/communication on relevant EU policies. The key tool in this respect, the Europa Forum Forschung, should in future include stronger dialogue elements in addition to its established information function. Greater use could be made of interactive and creative approaches, for example methods from group facilitation.

The functional involvement of other participants, for example, other RTI ministries, in organising the forum is also another option that could strengthen policy cooperation and coordination.

The ERA Portal Austria is well regarded. Only further improvements to the website's structure, particularly the search function, appear to be necessary.

Generally speaking, advisory and support for $\mathbf{H 2 0 2 0}$ for ministries is also working well. The EIP steering committee's mode of operation is perceived positively. However, the thematic expert groups of programme delegates are positioned and work very differently, which indicates that delegates interpret their role as information receiver and conveyers very differently.

\section{Recommendation: Contribution to the guidance of delegates' assignments}

We recommend stronger orientation of delegates' various assignments beyond the tasks specified and beyond the formal representation Austrian interests in Brussels. One element in this connection, could be, for example, communication with the scientific community as part of delegates' thematic expert groups.

The delegates' round table is a useful tool and assessed as excellent.

With reference to the function of advice and support for ministerial departments, the liaison office in Brussels currently being discussed could also be a useful supporting structure. In the process, this office could potentially be seen as associated with the general objectives of the Austrian EU-RTI policy regarding the country's positioning in the Euro- 
pean Research and Innovation Area. There is currently still no consistent position regarding the implementation of such an office. This may only become apparent on the basis of a clear course of action regarding the future role that Austria would like to play in shaping European RTI policy.

\section{Recommendation: Clarify objectives of an EU liaison office with the RTI commu- nity}

Before establishing a liaison office, strategic courses of action must be set regarding objectives, tasks and the associated structure, financing and positioning of this type of organisation. Against the backdrop of the debate which has so far been polarised, and which has been voiced in places such as various focus groups and in the Delphi questionnaire, that the majority of Austrian participants are in favour of such a liaison office, with only a minority being against, further discussion with key groups of actors is recommended.

The role of strategic advice for policy is currently exercised by the ERA Council Forum, which advices the Minister for Science and Research directly on matters pertaining to EURTI policy. Against the backdrop of the envisaged reorganisation and reduction of the bodies advising on RTI policy in the federal government programme, retention of a strong European component is recommended. ERA Council Forum experience also shows the added value of senior international membership of such a body, whose outside perspective may bring additional viewpoints and experiences. If this can be combined with a good understanding of the problems and feasibility in the national context, such a body could be very beneficial for RTI policy. Given the increasing significance of RTI policy as an interdisciplinary issue, and against the backdrop of international role models, consideration should also be given to incorporating a future consultancy body across policy fields.

\section{Recommendation: Consider the European dimension in boards advising on pol-} icy

In the course of the restructuring of the RTI policy advisory landscape in Austria, we recommend provision be made for a strong European dimension in tasks and with regard to membership of the to be constituted board.

To take into account, not only on European level, the increased cross-sectoral nature of RTI policy, a future board should also be able to refer explicitly to ministries directly and indirectly tasked with RTI obligations.

To take account of the balance between confidential advice at the highest political level and the need for transparency and openness, regular provision of information regarding the activities of a future advisory board is also recommended.

There is also a range of formats that should assist with supporting a structural transformation process in the RTI policy area. What most of these tools have in common is that they do not usually engage in any active coordination, but rather remain as instruments for sharing information. 
Two tools, with which an attempt was made to depart from this procedure, no longer exist. In the case of the MULLAT working group on transnational RTI partnerships, no agreement could be reached between BMBWF and BMVIT regarding the working group's objectives and approach. The ERA Policy Forum Austria failed in an attempt to motivate all Austrian ministries with RTI responsibilities to actively coordinate regarding a range of subject areas seen as significant.

In fact, there is scarcely any national strategic coordination in Austria regarding EU-RTI policy. This is especially evident with regard to transnational RTI partnerships, where the lack of a national investment strategy has resulted in a great number of participants often remaining subcritical in their budgets.

The new process foreseen in the government programme for an RTI strategy for the government beyond 2020 provides an opportunity for the review of coordination efforts in Austrian EU-RTI policy.

\section{Recommendation: Pool ERA formats and strengthen active coordination be- tween federal ministries}

The various formats for the exchange of information about potentially structure-changing developments contributing to the European Research Area are known to varying degrees. We recommend simplifying or grouping the various formats (e.g. ERA Reporting Board and ERA Round Table).

At the same time, given new instruments at EU level, more active coordination between various policy fields is more necessary than ever. In light of past experiences, we recommend the initiation of a preparatory process for the long-term incorporation of this form of coordination into Austrian governance and RTI strategy.

\section{Outlook and scenarios}

\section{Positioning of Austrian RTI policy with regard to FP9}

Suggestions for the further design of the roles and tasks of the FFG-EIP should not be limited to rectifying existing deficiencies or improvement proposals against the backdrop of current tasks and challenges, but should rather take account of the changing framework conditions. These changes also particularly include the EU Commission's 9th Framework Programme for Research and Innovation. It is a key point of reference, both for the work of the FFG-EIP and the governance of key dimensions of Austrian RTI policy. Previous preliminary work points to a range of possible new developments in the next framework programme. These particularly include

- Setting up a European Innovation Council (EIC), which should contribute towards strengthening innovative drive in Europe by supporting start-ups and scale-ups in particular with a high growth potential;

- Increasing importance of a mission-orientated approach in the thematic pillars of the framework programme, whose support will also require larger scale initiatives (e.g. in terms of strategic partnerships), as well as explicit alignment with sectoral policy fields; 
- Better coordination of the framework programme and European Structure and Investment Fund (ESIF) to counter increasing disparities in the European research and innovation landscape.

Changes to the thematic portfolio of research and innovation funding at European level should also be anticipated, where, for example, if defence research is still unresolved, whether or not this should be incorporated within the framework programme.

At the same time, it must be stressed that many uncertainties remain regarding the design of the next framework programme, which will only reduce successively in the coming months.

However, the future role and thus the resulting FFG-EIP tasks do not only depend on the framework programme; they also depend on Austria's intended positioning regarding European RTI policy. The reformulation of Austria's intended positioning and role at European level will be dependent upon the future RTI strategy, whose development for 2019 is anticipated and for which the basis is currently being established (by means which include the current OECD innovation policy review). In principle, there is potential for various ideas on roles, ranging from extensive adjustment to priorities and strategies defined at European level to the aspiration of a proactive (co-)development of European RTI policy. Innovation leaders, such as Sweden and Finland, with which Austria often compares itself, also aspire to shape European policy - with some degree of success in achieving this being well documented.

Developments at both levels mentioned - changes in the European framework programme and intended national positioning regarding shaping European research and innovation policy - naturally also have consequences for the further development of Austrian EU RTI policy and the medium-term positioning of the FFG-EIP, which should support both national RTI participants as well as national policy in their responsibilities as the interface to Europe.

\section{Scenarios on Austrian positioning in FP9 and its supporting structures}

Discussion of the expectations of the Austrian RTI community has shown that changes will be necessary in governance at national level and also at the level of the interface with European policy, as well as in the support services for the RTI community. The extent of these changes depends on Austria's intended positioning regarding European RTI policy. The three scenarios outlined below reflect options for action at national level and the respective consequences that these would have for the support structures. They should be seen as "food for thought" for further discussion in Austria, not least, also with regard to the development of a new RTI strategy.

\section{"Enhancement in continuity" scenario: Incremental change}

The central idea of this scenario is that the well-established and also the largely wellfunctioning status quo is maintained and enhanced, in the course of which RTI policy is modified to the requirements of new tools and initiatives at European level, but without 
significant changes having to be made in Austria, e.g. in the area of governance. Consequently, elements of national strategy planning and prioritisation (e.g. with regard to Austrian participation in strategic partnerships) is negligible

or limited to individual ministries. Furthermore, Austrian organisations participate in a range of initiatives, depending on individual interests and opportunities, but face the risk of sub-critical resources and can thus only play a secondary role in large-scale European initiatives. There may thus be no explicit focus alongside possible national priority areas.

In accordance with this picture, Austria's positioning in EU initiatives remains largely with the RTI organisations themselves and within the remit of line ministries, which adopt this positioning in accordance with the respective ministry-specific set of priorities.

Due to a comprehensive national positioning and profiling strategy with reference to the European level being waived, no prominent proactive role can be played in the further strategic design of the framework programme.

In principle, the supporting role of the EU coordination department and FFG-EIP is retained in this scenario and may be selectively reinforced. This is specifically manifested in taking up the - in many areas, incremental - recommendations of this evaluation, but also in a range of additional characteristics:

- The participation of RTI actors in the European framework programme and other partnerships progresses alongside the prevailing Austrian characteristics, i.e. the existing structural (e.g. central RTI actors) and thematic (e.g. existing "strengths") profiles.

- The FFG-EIP is intensifying its efforts to recognise strategic potential at RTI actor level and to address the RTI community in this regard. This is done as part of the guidelines specified by individual ministries, i.e. the responsibility for developing thematic strategies remains with the individual ministries. In the context of their thematic responsibilities, these support European activities by providing additional financial resources and by using other coordination tools (e.g. by means of their ownership functions).

- Information on options for linking national and European programmes (e.g. Austria's strengths, weaknesses and complementarities with regard to European initiatives) is provided by the FFG-EIP. However, partnerships are not actively initiated or controlled.

- Cooperation between ministerial departments regarding European initiatives continues to remain embedded in the frameworks for the respective responsibilities. Because of these boundaries, coordination among the ministries responsible for RTI agendas remains weak, as does that between RTI and sectoral policy areas.

- The EU coordination department is also positioned as a central node and interface between EU RTI policy, RTI ministries and the RTI community. The responsibilities of the EU coordination department thus focus on communicating information and mobilisation of the RTI community. 
- Given the divided responsibilities and limited policy coordination, the role of an RTI policy advisory board regarding EU-RTI agendas is to develop recommendations regarding key overarching policy measures necessary for the sustainable strong performance of the Austrian research and innovation system.

- In accordance with these directions of impact, it would be plausible, though not absolutely necessary, to establish a liaison office in Brussels.

\section{"Smart and proactive alignment" scenario: RTI policy as a catalyst in the European multilevel system}

In this scenario, Austrian policy aims to develop its role to that of (pro)active contributor to European RTI policy, to make more targeted and effective use of the opportunities resulting from this for Austria ("smart alignment").

To be able to better position itself as an integral element of the multilevel innovation ecosystem in Europe, as part of its new RTI strategy, Austria pursues a range of changes in the national system, which make it possible to achieve more intensive and more influential cooperation in the design and subsequently in the use of European RTI policy. Specifying such a concept as a guideline for a consistent Austrian position on EU RTI policy would be a central element of a new Austrian RTI strategy.

As well as the continued focus on excellence in universities and strengthening of entrepreneurial culture, this would also include the development of a European participation strategy in particular, including national priorities, to be able to play a substantial and formative role in selected areas. This is accompanied by the close interrelation of national and European/international programmes, which could manifest, for example, in selective involvement in EU strategic initiatives or in improved thematic complementarity/coordination between national and European funding opportunities.

Active participation in the design of European RTI tools and content is co-ordinated between ministries, FFG-EIP and the RTI community and orchestrated by national policy.

In other words, this scenario emanates from a strong policy leadership role with the strengthening of Austria's European role in line with national RTI strategy.

To realise this leading role in policy with Austria's strategic positioning at European level and with regard to the design of national programmes and policies ("smart and proactive alignment"), support is required by means of a strong FFG with its national and European activities, as well as the enhancement of interministerial coordination mechanisms. In this regard, the support structures in Austria should be enhanced along the following lines:

- Not only does interministerial coordination serve to delimit responsibilities (negative coordination), it also enables complementarities and synergies in terms of European positioning (positive coordination) as specified in a new RTI strategy. National strategy planning processes on key European topics are supported at policy level and/or at the highest official level ("ERA Policy Forum $\left.2.0^{\prime \prime}\right)$ and thus contribute to a clear European positioning. They are also seen as necessary and realistic, given the increasing urgency of these positionings. 
- The EU coordination department has a key role in this process, which is emphasised by a corresponding visible integration in the policy system (e.g. by the attribution of specific skills or integration in the BKA).

- As well as stronger political alignment, the FFG's greater autonomy and freedom to design strategies (see the corresponding recommendations of the FFGAWS evaluation and the "VINNOVA model") is of major importance. This form of greater autonomy would also serve to align participation in ERA initiatives against the backdrop of national strategy and to complement Austrian characteristics, e.g. through the active initiation and support of national partnerships within the overall strategic guidelines and policy priorities.

- Enhancement of the FFG-EIP's capacity and skills should also be accelerated accordingly, to strengthen interfaces to Brussels, interactions with the Austrian RTI community and interfaces with partners in Europe, and thus to also support the positioning of national priorities at European level.

- In accordance with these considerations, efforts should be made within the FFG and in cooperation with line ministries to achieve closer interlinking of national and European programmes to enable a seamless transition between programmes at various levels when advising RTI actors.

- To support this proactive scenario, there is an important role to be played by the strong integration of EU RTI issues in the agendas of a newly designed high-level advisory board on RTI policy issues, as provided for in the government programme. Thus support should be provided for the effective integration of the Austrian research and innovation system in the European context and active participation in shaping this European context through professional experts with independent internal and external perspectives.

- As part of this scenario, a national liaison office - i.e. supported by policy and/or FFG - in Brussels would also be a useful addition.

\section{"Distributed empowerment" scenario: Strengthening and cross-linking the RTI community}

This scenario sees the extension of participation and involvement in European RTI policy, particularly by means of strengthening and improved cross-linking of RTI actors themselves, supported by the Austrian policy of empowerment regarding this process. This approach is promising because participation in larger initiatives (e.g. strategic partnerships), which sometimes have lower funding rates, can only be successful if they are very closely linked to the strategic interests of organisations implementing RTI.

This scenario in turn assumes that organisations have an independent development strategy and that there is an ability to self-organise in strong national networks. However, it also requires appropriate resource and capacity, whose mobilisation is supported by RTI policy (e.g. by means of financial incentives, networking activities, information sharing, etc.). The experiences of other European countries (e.g. Finland, the Netherlands, etc.) in mobilising their RTI communities to participate in EU RTI policy therefore provide the impetus. However, they also show that there can be losers as well as winners in this empowerment process. 
At policy level, this scenario does not require any ex ante commitment to priority issues, around which efforts will be made to mobilise the RTI community, as these will result from the commitment and effective networking of RTI actors. This is also the key challenge of this scenario, namely to use appropriate and selective incentives to ensure that, in Austria's, generally speaking, scattered RTI community, such focuses can emerge, which are then also influential at EU level. Past experience has shown that there must be either leading players, who promote the mobilisation of networks (e.g. individual strong unis, COMET centres, research institutions or companies), and/or an enablingrole of policy, possibly incorporated in line ministries and their activities (e.g. Silicon Austria Labs), to sufficiently enable the development of large structures in Austria.

In addition to the support services and structures that currently exist, the need arises in this scenario to support the autonomous strategic abilities of RTI actors and their ability for national and European networking and collective strategy planning with government and FFG-EIP resources. This suggests the following directions of impact:

- To ensure that the Austrian RTI community can assume an active role in shaping and participation at European level, capacity development of national RTI networks should be promoted. This could be implemented instrumentally by means of (financial) incentives as well as targeted information, networking and strategy activities. Given the focus on strengthening the RTI community and its autonomous networking, interministerial coordination in this scenario is less significant.

- The EU coordination department functions here as a central connecting node between the national RTI community, line ministries and (policy) EU boards and supports the RTI community's approach to participation and cooperation in European initiatives. Providing the EU coordination department or line ministries with appropriate resources for competitive (co-)financing of those joint activities by the RTI community would permit this mobilisation process to be promoted.

- With the available resources, the FFG would be able to implement the relevant programmes and activities autonomously. In the FFG-EIP's service portfolio, the focus shifts to supporting RTI actors with participation in bigger initiatives, such as strategic partnerships, to enable, for example, cooperation between national RTI networks with European initiatives.

- This will require other and possibly additional skills and capacities on the part of the FFG-EIP. The FFG-EIP would also need to have a very high degree of strategic autonomy to develop and provide support services in coordination with the RTI community. It would only be linked within a comparatively general framework of thematic orientations on the part of line ministries.

- As in the second scenario, close interconnection of national programmes with European initiatives also makes sense here, aimed at those areas being expedited by the RTI community. This approach would also suggest further intensification of the discussion within the FFG between the various national and European programme areas. 
- Because of the relatively weak directing role played by policy, a high-level advisory board could make a stronger appeal to the RTI community in this scenario to assume an overarching guiding function.

- A liaison office in Brussels would also be an important and useful addition in this model. However, in this scenario, the RTI community would essentially perform this role. 


\section{Zielsetzung und Methodik der Evaluierung}

\subsection{Hintergrund und Zielsetzung}

Im Jahr 2013 wurde die FFG, und dort konkret der Bereich für Europäische und Internationale Programme (FFG-EIP), von Seiten der österreichischen Bundesregierung sowie der Wirtschaftskammer Österreich mit der Unterstützung der Umsetzung von H2020 und ERA beauftragt. Im Jahr 2014 wurde die FFG überdies mit dem Monitoring der österreichischen Beteiligung in H2020 und ERA betraut. Darüber hinaus ist die FFG mit Partnern auch für Unterstützungsleistungen für EUREKA, COSME und den Enterprise Europe Network (EEN) verantwortlich.

Vor diesem Hintergrund, ist es das Ziel dieser Evaluierung, eine systematische Gesamtbetrachtung der Umsetzung von H2020 und ERA in Österreich sowie von EUREKA, COSME und EEN vorzunehmen. Die drei Schwerpunktbereiche der Evaluierung sind:

1. Beauftragung der FFG mit der Beratung und Betreuung von H2020 und ERA (EIPBeauftragung);

2. Beauftragung der FFG mit dem EU-Performance Monitoring (EU-PM-Beauftragung);

3. Das ERA-Observatorium Austria, das einen übergeordneten Rahmen für die Integration der einzelnen Aufgabenstellungen im Bereich der Governance der EUFTI-Politik in Österreich auf Seiten des BMBWF bildet.

Darüber hinaus werden im Rahmen der Evaluierung ergänzend die Beauftragungen zu EUREKA, COSME und dem EEN analysiert.

Ohne die Komplexität jedes einzelnen Schwerpunktbereiches plus Zusatzbereiche zu stark vereinfachen zu wollen, liegen der Evaluierung folgende Meta-Fragestellungen zugrunde:

- Generelle Würdigung der Umsetzung: Sind die Ausrichtung und Umsetzung der vorhandenen Formate und Unterstützungsleistungen - unter dem gegebenen budgetären Rahmen - grundsätzlich geeignet bzw. entsprechend implementiert, um die erwünschten Wirkungen der Maßnahmen in Hinblick auf den Output so effizient und effektiv wie möglich zu erzielen?

- Würdigung spezifischer Leistungen: Wo sind - angesichts von beobachtbaren und zu erwartenden Entwicklungen im FTI-Bereich (inklusive FTI-Politik) - die Formate und Unterstützungsleistungen adäquat und wo sind Nachbesserungen anzusetzen?

- Handlungsbedarf und Handlungsoptionen: Wo ist weißen Flecken zu begegnen oder wo sind neue und bessere Ansätze und Instrumente zu schaffen? Nach welchen Prinzipien sind diese im Wesentlichen zu gestalten? 


\subsection{Methodik}

Das Evaluierungsdesign besteht aus drei großen Phasen basierend auf einer Triangulation sich ergänzender Methoden. Der mixed-method Ansatz kombiniert unterschiedliche quantitative und qualitative Methoden der empirischen Wirtschafts- und Sozialforschung so, dass Befunde von mindestens zwei Quellen bestätigt werden. Hinzu kommt ein internationaler Beirat aus renommierten europäischen ExpertInnen, der das Evaluierungsteam bei der Reflexion der Ergebnisse, dem Ziehen von entsprechenden Schlussfolgerungen und der Ableitung von Empfehlungen für die Anpassung und Weiterentwicklung des österreichischen Angebotportfolios unterstützte.

Im Mittelpunkt von Phase 1, der Erhebungsphase, stand die empirische Erhebung zur Gewinnung robuster Daten mithilfe von Desk-Research, Interviews und einer Online-Befragung im Vordergrund. Sie wurde mit dem Zwischenbericht abgeschlossen.

Aufbauend auf den Erkenntnissen der ersten Phase der Evaluierung, erfolgte in Phase 2 eine Validierung und Reflexion der gewonnenen Erkenntnisse. Der Reflexionsworkshop am 14. Dezember 2017 stellte den Auftakt der Phase 2 dar, die methodisch von der Umsetzung von Fokusgruppeninterviews, ergänzenden ExpertInneninterviews und einer Delphi-Befragung geprägt war.

In Phase 3 der Evaluierung wurde die Entwicklung von Empfehlungen in den Mittelpunkt der Aktivitäten gerückt. Insbesondere über die Einbindung internationaler Sichtweisen durch eine Fokusgruppe in Brüssel und partizipative Workshops mit dem internationalen Beirat der Evaluierung sowie der Gruppe der AuftraggeberInnen wurden umsetzbare, handlungsrelevante Empfehlungen entwickelt.

Die Schlussfolgerungen und Empfehlungen der Evaluierung beruhen auf einer Triangulation der Ergebnisse und Erkenntnisse der eingesetzten Methoden, um spezifische Stärken und Schwächen jeder einzelnen Methode zu kompensieren. Die aus dem mixed-method Zugang resultierenden Erkenntnisse stützen sich deshalb jeweils auf verschiedene Erhebungsmethoden und tragen zur Validität und Reliabilität der Empfehlungen bei. 

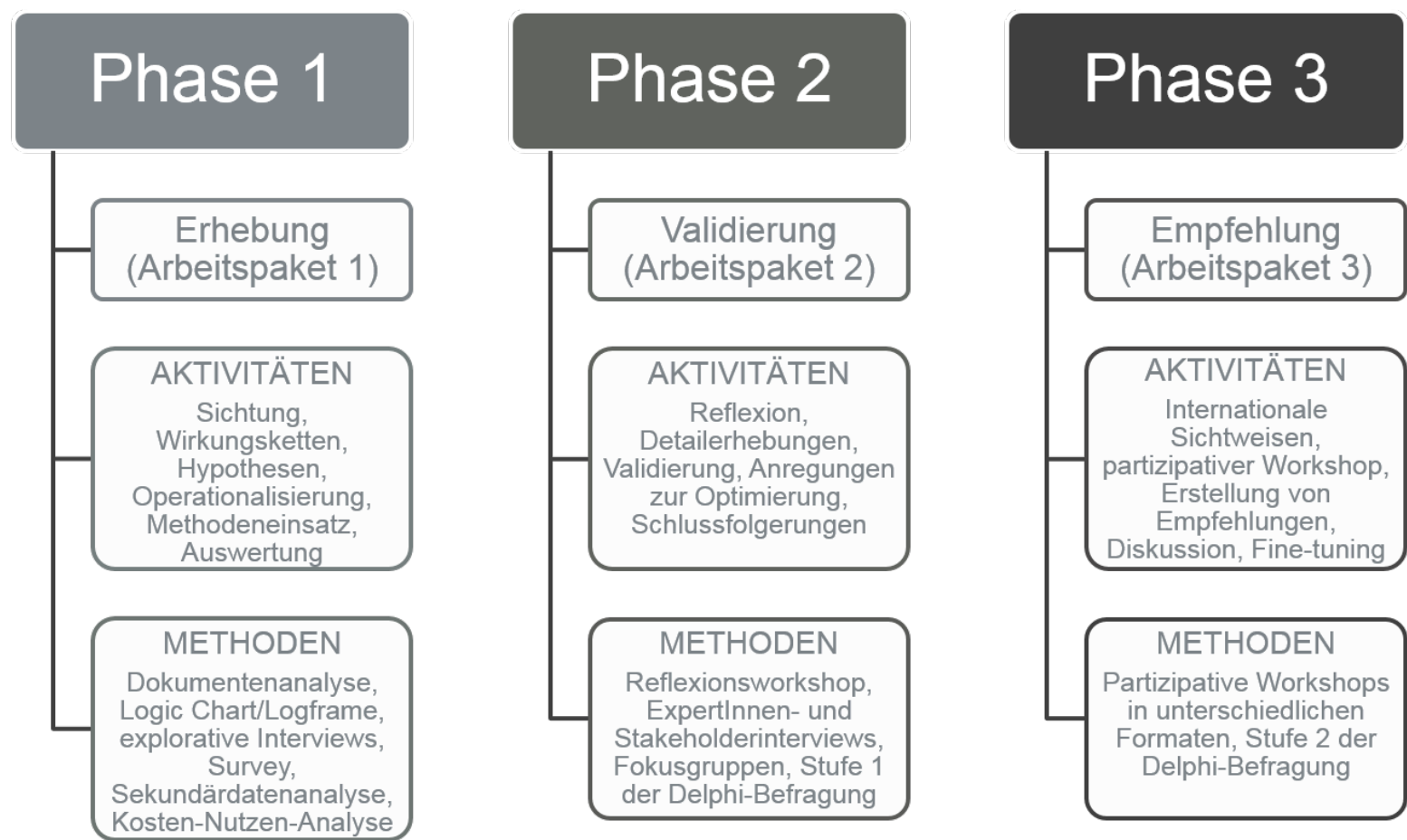

Quelle: Eigene Darstellung

\section{Desk Research}

Als Basis der Evaluierung und zur Ergänzung der Daten und Informationen der Erhebungen wurde eine Dokumentenanalyse durchgeführt. Diese geben ein Bild der konzeptionellen Grundlagen und der Umsetzung von H2020, EUREKA, COSME, EEN und ERA im Kontext der Arbeit der FFG-EIP. Herangezogene Dokumente und Daten inkludierten von den AuftraggeberInnen und FFG-EIP zur Verfügung gestellte Strategiedokumente, interne Analysen und Berichte sowie Monitoring Daten, Protokolle, Abrechnungen, etc.

\section{ExpertInneninterviews}

Im Verlauf der Evaluierung wurden mehr als 50 Interviews mit 45 VertreterInnen der Ministerien, Delegierten und ExpertInnen in H2020, WKO, FFG, Intermediären (SFG, Business Upper Austria, ecoplus, aws, etc.) sowie Unternehmen und Forschungsservicestellen der Universitäten zu den Themenbereichen der Evaluierung durchgeführt (siehe Annex IV: TeilnehmerInnen an ExpertInneninterviews und Fokusgruppen für Liste der befragten ExpertInnen). Die Gespräche waren einerseits explorativ und dienten als Einstieg in die Evaluierung, wo die Ergebnisse des Desk Research sowie erste daraus folgende Hypothesen zu den Themen der Evaluation erörtert und getestet wurden. Andererseits wurden in späteren Phasen ergänzende ExpertInneninterviews geführt, um vertiefende Einschätzungen zu Bedarfslagen der Stakeholder und zur Wirksamkeit der bestehenden Maßnahmen zu ermitteln. 


\section{Online-Befragung}

Als wesentlicher Eckpunkt der Evaluierung wurden drei Online-Befragungen durchgeführt und nach Organisationstypen (KMU, Großunternehmen, Universitäten, außeruniversitäre Forschungseinrichtungen) und Erfahrungshintergrund in europäischen FTI-Programmen (erfahren, unerfahren) ausgewertet. Dabei wurde zwischen drei AdressatInnenkreise unterschieden:

1. Forschende in der Wissenschaft: Dabei handelt es sich um Forschungstreibende innerhalb von Hochschulen, Forschungsinstitutionen, die bereits EIP Beratungsleistungen in Anspruch genommen haben sowie solche, die H2020 Projekte eingereicht haben. Der Fokus lag auf den Beratungsleistungen des EIP in Bezug auf H2020 sowie bi- und multilaterale Kooperationen und die Bekanntheit und Nutzung unterschiedlicher Stakeholder-Formate des ERA-Observatoriums.

2. Forschende in Unternehmen: Dabei handelt es sich um Forschungstreibende innerhalb von Unternehmen, die bereits EIP Beratungsleistungen in Anspruch genommen haben sowie solche die H2020 Projekte eingereicht haben. Der Fokus lag auf den Beratungsleistungen des EIP in Bezug auf H2020 sowie bi- und multilaterale Kooperationen und die Bekanntheit und Nutzung unterschiedlicher Stakeholder-Formate des ERA-Observatoriums.

3. Intermediäre: Dabei handelt es sich um VertreterInnen nationaler und regionaler Agenturen und Fördereinrichtungen sowie WKÖ national und regional. Der Fokus der Befragung lag auf der Kooperation mit EIP, der Governance und nationalen Vernetzung zu H2O20 und bi- und multilateralen Kooperationen (z.B. EUREKA, COSME); Leistungen des EU-PM, Adäquanz der Struktur des ERA-Observatoriums.

Das Sampling erfolgte in Kooperation mit der FFG auf Basis der INNOMAN-Datenbank, die alle KundInnenkontakte von FFG-EIP beinhaltet. Die Online-Befragung wurde im Zeitraum 31.10.2017 - 24.11.2017 durchgeführt und mit einem Support Desk (schriftlich, telefonisch) und Reminder-Emails begleitet.

\section{Tabelle 1: Rücklauf der Online-Survey}

\begin{tabular}{|l|l|l|l|l|l|}
\hline & $\begin{array}{l}\text { Sample } \\
\text { (Personen) }\end{array}$ & $\begin{array}{l}\text { Ausgeschiedene } \\
\text { (Personen) }\end{array}$ & $\begin{array}{l}\text { Bereinigtes } \\
\text { Sample }\end{array}$ & $\begin{array}{l}\text { Gewertete } \\
\text { Fragebögen }\end{array}$ & Rücklauf \\
\hline $\begin{array}{l}\text { Hochschulen / } \\
\text { Forschungseinrichtungen }\end{array}$ & 1188 & 56 & 1132 & 325 & $29 \%$ \\
\hline Unternehmen & 915 & 52 & 863 & 209 & $24 \%$ \\
\hline Intermediäre & 80 & 12 & 68 & 32 & $47 \%$ \\
\hline
\end{tabular}

Quelle: Online-Befragung, Joanneum Research.

3 Nicht zustellbar / keine Teilnahme möglich / in Organisation akkordierte einmalige Beantwortung 
Der Rücklauf liegt bei durchschnittlich 28\%: Bei den Hochschulen und Forschungseinrichtungen sind es $29 \%$, $24 \%$ bei Unternehmen und $47 \%$ bei Intermediären (siehe Tabelle $1)$.

Im Bericht werden die Ergebnisse der Online-Befragungen an relevanten Stellen abgebildet. Diese Abbildungen beinhalten auch Hinweise auf die Anzahl der RespondentInnen der jeweiligen Frage $(n=\ldots)$.

\section{Fokusgruppen}

In Phasen 2 und 3 wurden insgesamt sieben partizipative Fokusgruppen mit dem ERA Council Forum, ERA-KorrespondentInnen/Vize-RektorInnen der Universitäten, VertreterInnen der Forschungsservicestellen der Universitäten, außeruniversitären Forschungseinrichtungen, Unternehmen, regionalen Agenturen und Fördereinrichtungen sowie eine Fokusgruppe in Brüssel mit internationalen ExpertInnen durchgeführt. Die TeilnehmerInnen der Fokusgruppen waren zielgruppenspezifisch ausgewählte ExpertInnen, die unter der Moderation erfahrener ProjektmitarbeiterInnen zentrale Fragestellungen der Evaluierung erörtert haben. Diese waren in erster Linie zukunftsorientiert ausgerichtet um Anregungen und Weiterentwicklungspotential zu diskutieren, dienten aber auch als vertiefendes Erhebungsinstrument. Die insgesamt sieben Fokusgruppen fanden im Zeitraum Januar 2018 - April 2018 statt (siehe Annex IV: TeilnehmerInnen an ExpertInneninterviews und Fokusgruppen für Liste der Fokusgruppen TeilnehmerInnen).

\section{Delphi-Befragung}

Eine zweistufige Delphi-Befragung ist ein Survey mit dem Ziel ein konvergentes Meinungsbild durch Einschätzungen und Bewertungen ausgewiesener ExpertInnen zu erlangen. Die zweistufige Delphi-Befragung diente der Validierung der empirischen Ergebnisse und als weitere Grundlage zur Weiterentwicklung der Argumentationslinien und Empfehlungen. Hier wurde ein breites und gleichzeitig höchstrelevantes Spektrum an ExpertInnen befragt um deren Erfahrungswissen abzuholen. 190 ausgewählte ExpertInnen des österreichischen FTI-Systems wurden in der ersten Runde der Befragung adressiert, die sich in fünf Gruppen gliedern lassen: i) Vize-RektorInnen/ERA KorrespondentInnen der Universitäten, ii) Management der außeruniversitären Forschungsorganisationen, iii) Management in H2020 besonders aktiver Unternehmen, iv) Politikakteure, d.h. Delegierte und ExpertInnen in H2020, und VertreterInnen der Ministerien sowie v) VertreterInnen nationaler/regionaler Agenturen und Fördereinrichtungen.

Es wurde auf eine ausgewogene Zusammensetzung der ExpertInnen der verschiedenen Kategorien geachtet, um unterschiedliche Sichtweisen adäquat einzuholen.

Mithilfe offener und geschlossener Fragen zu vorläufigen Thesen und Schlussfolgerungen wurden die ExpertInnen zu ihren Einschätzungen über ausgewählte Erkenntnisse der Evaluierung befragt. Die Antworten der ersten Runde wurden im Anschluss verdichtet und in einer zweiten Runde einer erneuten Bewertung unterzogen. Durch dieses Verdichten zwischen erster und zweiter Befragungsrunde konnte sowohl eine inhaltliche Nachjustierung der Thesen und Schlussfolgerungen als auch eine Validierung derselben erzielt werden. In der zweiten Befragungsrunde wurden nur diejenigen ExpertInnen kontaktiert, 
die ihre Einschätzung bereits in der ersten Runde abgegeben haben. In der ersten Runde der Befragung haben 69 ExpertInnen Stellung genommen, das entspricht einem Rücklauf von über 36\%. Von diesen 69 haben 39 an der zweiten Runde der Befragung teilgenommen.

\section{Workshops}

Zusätzlich wurde die Evaluierung durch mehrere Reflexionsworkshops unter Einsatz von partizipativen Methoden begleitet, die dazu dienten, die Stoßrichtung des Projekts laufend zu optimieren und aus den Evaluierungsergebnissen valide Schlussfolgerungen zu ziehen.

Der Zwischenbericht wurde in einem ersten Reflexionsworkshop mit AuftraggeberInnen, FFG sowie internationalem Beirat reflektiert. Während im ersten Teil des Workshops die Zwischenergebnisse den AuftraggeberInnen, dem Beirat und VertreterInnen der FFG-EIP vorgestellt und diskutiert wurden, wurde im zweiten Teil des Workshops - nun ohne Einbeziehung der FFG-EIP - mit dem Beirat und der Gruppe der AuftraggeberInnen in einem World Café mögliche Schwerpunkte der Phasen 2 und 3 der Evaluierung diskutiert. In den Phasen 2 und 3 fanden ein Reflexionsworkshop mit dem internationalen Beirat sowie ein weiterer Reflexionsworkshop mit der Gruppe der AuftraggeberInnen und der FFG statt, um die analytischen Befunde der Evaluierung und vorläufige Empfehlungen zu diskutieren und weiterzuentwickeln. Der vorläufige Endbericht samt Empfehlungen wurde in einem abschließenden Ergebnisworkshop mit den AuftraggeberInnen reflektiert.

\section{Interaktion mit der FFG-EIP}

In Abstimmung mit dem federführend auftraggebenden Ressort BMBWF wurden MitarbeiterInnen der FFG-EIP zu ausgewählten Zeitpunkten der Evaluierung in das Projekt mit einbezogen. Dies war insbesondere zu Beginn des Projekts erforderlich, um den Zugang zu zentralen Dokumenten und spezifischen Sachinformationen zu gewährleisten, die als Grundlage für die Evaluierung herangezogen wurden. Außerdem wurden gezielte Anfragen an die FFG-EIP spezifiziert, um detaillierte quantitative Daten über die verschiedenen Tätigkeiten der FFG-EIP zu erhalten.

Die Einbeziehung von VertreterInnen der FFG-EIP in ausgewählte Projektworkshops diente zum einen der Überprüfung der vom Evaluierungsteam erhobenen empirischen Beobachtungen; zum anderen sollte dadurch die Möglichkeit geboten werden, über den Dialog mit den AuftraggeberInnen und dem Evaluierungsteam gezielt formativ zur Evaluierung beizutragen, und zwar insbesondere im Hinblick auf zukünftige Herausforderungen und Aufgaben, die sich in Bezug auf die Unterstützungsstrukturen für künftige europäische Programme stellen werden.

Der FFG-EIP wurde außerdem Gelegenheit geboten, zum Zwischen- und vorläufigen Endbericht Stellung zu beziehen und Sachinformationen zu überprüfen. Wertende Aussagen der FFG fanden nicht Eingang in die Evaluierung; ebenso blieb die Formulierung der Empfehlungen davon unberührt. 


\section{Das Leistungsspektrum von FFG-EIP}

In diesem Kapitel wird das Dienstleistungsspektrum der FFG, das im Rahmen der Beauftragungen „FFG-EIP" und "EUREKA, COSME und EEN", den österreichischen FTI-Akteuren zur Verfügung gestellt wird, analysiert und bewertet. Im ersten Abschnitt werden jene Leistungen von FFG-EIP kurz vorgestellt, die allen FTI-Akteuren in Österreich offenstehen und grundlegende Änderungen im Leistungsspektrum von FFG-EIP spezifiziert.

In den zwei darauffolgenden Abschnitten werden diese Leistungen sowie das spezifische auf das Beratungs- und Dienstleistungsspektrum für die Kernzielgruppen aus den Bereichen "Wissenschaft" und "Wirtschaft" in Hinblick auf ihre Relevanz und Effektivität untersucht. Dabei wird im Bereich „Wirtschaft" auch auf die gesonderten Beauftragungen zu „EUREKA, COSME und EEN" eingegangen.

\subsection{Beauftragungen}

Der Bereich „Europäische und Internationale Programme" der FFG (FFG-EIP) wurde vom Bund, vertreten durch mehrere Ministerien, und der WKO für den Zeitraum 2014-2020 mit der Leistung von Informations-, Beratungs- und Vernetzungsservices beauftragt, um eine optimale Beteiligung österreichischer FTI-Akteure in $\mathrm{H} 2020$, und in multilateralen FTI-Initiativen (MULLATs) zu erreichen. FFG-EIP baut in Hinblick auf die Unterstützung der österreichischen FTI-Akteure auf den Kompetenzen und gesammelten Erfahrungen vorheriger Beauftragungen auf. Darüber hinaus ist FFG-EIP die offiziell nominierte Nationale Kontaktstelle (NCP) der Europäischen Kommission für das Rahmenprogramm und alle Initiativen und Programme in Verbindung mit H2020, die sogenannten transnationalen oder multilateralen Initiativen des Europäischen Forschungsraums (MULLATs).

Die Beauftragung von FFG-EIP verfolgt für die AuftraggeberInnen BMBWF, BMDW, BMVIT, BMASGK und WKO vier übergeordnete Ziele, die Teil der nationalen FTI-Strategie Österreichs sind ${ }^{4}$ :

1. Halten des bisherigen Niveaus der Beteiligung der österreichischen Forschungsorganisationen und ihren Forschenden an H2020 und am Europäischen Forschungs- und Innovationsraum: Die ausgezahlten Rückflüsse sollen weiterhin über dem Wert der österreichischen Eigenmittelzahlungen zum EU-Haushalt liegen;

2. Stärkung der österreichischen Forschung und Innovation durch Internationalisierung;

3. Abstimmung der Information und Beratung über nationale FFG-Programme, europäische und multilaterale Förderprogramme;

4. Stärkung des österreichweiten Interessensaustausches im Bereich Forschung und Innovation im EU-Kontext.

4 Siehe: Bundesministerium für Wissenschaft und Forschung (2013), Antrag auf Einvernehmensherstellung: FFG Unterstützung österreichischer FTI-Akteure im Europäischen Forschungs- und Innovationsraum 2014 - 2020, Wien. 
Um den übergeordneten Zielsetzungen der Beauftragung Rechnung zu tragen, verfolgt FFG-EIP vier zentrale Zielsetzungen, die den ergebnisorientierten Handlungsrahmen als nationale Kontaktstelle für H2020 Rechnung trägt (siehe Abbildung 2).

\section{Abbildung 2: Zielsetzungen der H2020 Beauftragung}

Die FFG ist Kompetenzzentrum für europäische Forschungs- und Innovationsprogramme und unterstützt die österreichische

Wissenschaft und Wirtschaft in ihrer Beteiligung und Verankerung im Europäischen Forschungs- und Innovationsraum. Als Nationale

Kontaktstelle bietet die FFG ein integriertes Beratungs- und Betreuungsangebot für ihre Kunden zur bestmöglichen Nutzung europäischer FTl-Entwicklungen und -Programme.

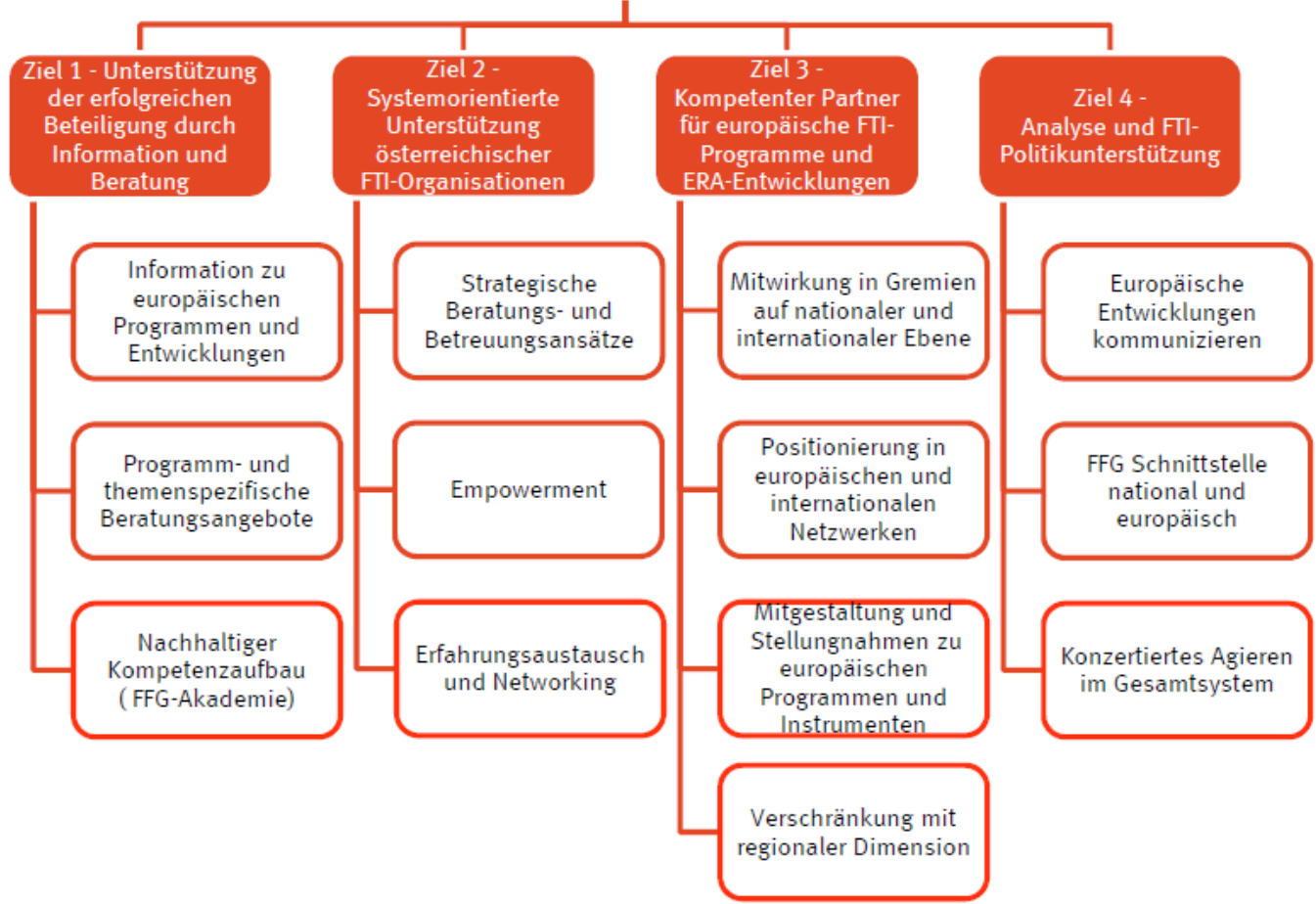

Quelle: FFG-Anbot: Unterstützung österreichischer FTI-Akteure im Europäischen Forschungs- und Innovationsraum, 10. Juni 2013, finale Version.

Während Ziel 1 und Ziel 2 insbesondere die öffentlichen und privaten österreichische FTI-Akteure betreffen, verdeutlichen Ziel 3 und Ziel 4 insbesondere die Kooperation der FFG mit "Politik-Akteuren", die die nationalen Ressorts, nationale und europäische Intermediäre und Agenturen (z.B. regionale Wirtschaftsförderungsagenturen) sowie die Europäische Kommission umfassen.

\subsection{Veränderungen im Portfolio}

Die gegenständliche FFG-EIP Beauftragung zur Unterstützung österreichischer FTI-Akteure im Europäischen Forschungs- und Innovationsraum 2014-2020 legt im Gegensatz zu früheren Beauftragungen, ein stärkeres Augenmerk auf eine systemorientierte Unterstützung und eine Selbstbefähigung der Akteure. Damit reagierte man auch auf die Ergebnisse der Evaluation von 2010, die das Resümee zog, "dass die Dienstleistungen 
von FFG-EIP die Universitäten und Forschungseinrichtungen davon abhalten, die notwendigen internen Kapazitäten für das Forschungsmanagement aufzubauen". ${ }^{5}$

Informations-, Beratungs- und Trainingsleistungen zu H2020, multilateralen Programmen und ERA-Entwicklungen (Ziel 1), zählen zwar nach wie vor zu den Kernaufgaben der Beauftragung, jedoch sollen in der Beauftragung vor allem ${ }^{6}$ :

- Wissenschafts- und Wirtschaftsorganisationen in ihren Strategie- und Positionierungsprozessen so unterstützt werden, dass sie in der Lage sind, europäische Programme und ERA-Entwicklungen mit maximaler Hebelwirkung für eigene Zielsetzungen einsetzen zu können.

- Das Kooperationsprofil mit den Unterstützungsstrukturen (insbesondere) an Universitäten so ausgerichtet werden, dass mittelfristig Erstanfragen und Projektabwicklungsfragen verstärkt organisationsintern abgewickelt werden können.

Weitere Anforderungen an Veränderungen des Leistungsportfolios betrafen:

- Eine stärkere Betreuung von Unternehmen insgesamt und KMU aufgrund der stärkeren Innovationsorientierung von $\mathrm{H} 2020$.

- Die Aufnahme von ERA-Initiativen und Programmen ins Leistungsportfolio von FFG-EIP und die Unterstützung der Governance-Strukturen von MULLATs.

- Den Ausbau der strategischen Intelligenz im Sinne von Zusammenschau und Überblick bieten.

Zur Umsetzung der Beratungs- und Betreuungsleistungen für ihre KundInnen hat FFGEIP eine Toolbox entwickelt, die die zentralen Dienstleistungen von EIP beschreibt. Die in der Toolbox spezifizierten Leistungen sprechen alle Forschenden an Unternehmen, Universitäten, Fachhochschulen und außeruniversitären Forschungsorganisationen in Österreich an. Die zum Einsatz kommenden Instrumente lassen sich in drei Funktionsgebiete gliedern:

1. Bewusstseinsbildung und Informationsvermittlung zur Förderung der Bekanntheit und Reputation europäischer und multilateraler Programme,

2. Programm- und Projektberatung entlang aller Projektphasen und

3. Strategische Beratung und ERA-Orientierungswissen zur Unterstützung der optimalen Nutzung vorhandener Förderprogramme.

Untenstehende Tabelle gibt einen kurzen Überblick über wesentliche Eckpfeiler des Dienstleistungsspektrums der EIP-Beauftragung, die allen österreichischen FTI-Akteuren zur Verfügung stehen ${ }^{7}$.

5 Technopolis (2010)

6 Siehe: FFG (2013), FFG-Anbot: Unterstützung österreichischer FTI-Akteure im Europäischen Forschungs- und Innovationsraum, 10. Juni 2013, finale Version, Wien.

7 Die FFG-Toolbox mit allen Tools inkl. EUREKA, COSME und EEN befindet sich in Annex 1 des Zwischenberichts hinzugefügt. 
Tabelle 2: Das Basis-Dienstleistungsspektrum von FFG-EIP für alle FTI-Akteure

\begin{tabular}{|c|c|}
\hline Aufgabenbereich & Instrumente \\
\hline $\begin{array}{l}\text { Bewusstseinsbildung } \\
\text { und Informationsver- } \\
\text { mittlung }\end{array}$ & $\begin{array}{l}\text { 1. FFG-EIP Website: Bietet allgemeine Informationen über die Ge- } \\
\text { samtstruktur des europäischen und multilateralen Programmportfolio } \\
\text { sowie Förderangebote für alle Zielgruppen. Zusätzlich bieten spezifi- } \\
\text { sche Seiten gezielte Unterstützung bei der Implementierung von } \\
\text { H2020 Projekten (z.B.: rechtliche und finanzielle Aspekte). } \\
\text { 2. eNewsletter: Bieten eine Zusammenschau über nationale und eu- } \\
\text { ropäische Nachrichten } \\
\text { 3. "Awareness" und Informationsveranstaltungen: Bieten Infor- } \\
\text { mationen zum H2020 Programmportfolio und der zugehörigen „Pro- } \\
\text { grammmechanik". Das sind vor allem Veranstaltungen zum Start von } \\
\text { Ausschreibungsrunden, Roadshows, Kooperationsbörsen, etc. }\end{array}$ \\
\hline $\begin{array}{l}\text { Programm- und } \\
\text { Projektberatung }\end{array}$ & $\begin{array}{l}\text { 4. Persönliche Beratung \& Proposal Checks für Wissenschaft und } \\
\text { Wirtschaft wird während aller Projektphasen angeboten. An Universi- } \\
\text { täten und Organisationen mit eigenen „Unterstützungsstrukturen" } \\
\text { werden ForscherInnen und MultiplikatorInnen im Rahmen des Second- } \\
\text { level Supports bei programmspezifischen Fragen beraten. Multiplikato- } \\
\text { rInnen/Intermediäre werden durch den Einsatz unterschiedlicher In- } \\
\text { strumente aus der Toolbox befähigt, den First-level Support sukzes- } \\
\text { sive eigenständig wahrzunehmen. } \\
\text { Im Zeitraum } 2014 \text { - } 2016 \text { wurden insgesamt } 18.318 \text { persönliche Be- } \\
\text { ratungen und } 630 \text { Proposalchecks (Angabe aus LB 2016) von der FFG } \\
\text { durchgeführt. } \\
\text { 5. FFG Akademie: Im Rahmen der FFG Akademie werden Trai- } \\
\text { ningsveranstaltungen und Webinare des EIP zur Informationsver- } \\
\text { mittlung und Wissensaufbau gebündelt. Der besondere Schwerpunkt } \\
\text { liegt auf Projektentwicklung, Antragstellung sowie dem Management } \\
\text { von H2020 Projekten. Diese werden ergänzt durch das Format EU- } \\
\text { Networking zur besseren Vernetzung der österreichischen FTI-Akteure } \\
\text { mit EU-ExpertInnen. Diskussionsrunden im Rahmen des EU-Networ- } \\
\text { kings beschäftigen sich mit aktuellen Entwicklungen der europäischen } \\
\text { Forschungspolitik und Forschungsförderung. } \\
\text { Im Zeitraum } 2014 \text { - } 2016 \text { wurden insgesamt } 66 \text { Trainings im Rahmen } \\
\text { der FFG Akademie durchgeführt. Insgesamt } 2149 \text { Personen nahmen } \\
\text { das Trainingsangebot in Anspruch und investierten je nach Trainings- } \\
\text { dauer 1-2 Arbeitstage. } \\
\text { Im Zeitraum } 2014 \text { - September } 2017 \text { wurden insgesamt } 45 \text { Webinare } \\
\text { von rund } 3.600 \text { Personen verfolgt. Die durchschnittliche Bewertung } \\
\text { (nach Schulnoten) liegt bei 1,68. }\end{array}$ \\
\hline $\begin{array}{l}\text { Strategische Beratung } \\
\text { und ERA Orientierungs- } \\
\text { wissen }\end{array}$ & $\begin{array}{l}\text { 6. ERA-Themendossiers/Analytische Programmberichte: Diese } \\
\text { analysieren die österreichische Beteiligung in bestimmten Themen }\end{array}$ \\
\hline
\end{tabular}

${ }^{8}$ Die Liste der Webinare wurde separat von der FFG zur Verfügung gestellt. 


\begin{tabular}{|l|l|}
\hline Aufgabenbereich & Instrumente \\
\hline $\begin{array}{l}\text { (H2020 und MULLATs) und zeigen wichtige Entwicklungen und Hand- } \\
\text { lungsfelder auf. Quantitative Daten liefert das EU-Performance-Moni- } \\
\text { toring. Die Hauptzielgruppe sind Delegierte und andere Policymaker, } \\
\text { werden aber auf entsprechend angepasstem Detailierungslevel auch } \\
\text { öffentlich zur Verfügung gestellt. Speziell die Themendossiers zu ERC } \\
\text { und MSCA, Positionierung der Wissenschaft, Social Sciences and Hu- } \\
\text { manities (SSH) und Health sprechen auch Wissenschaftsakteure an. } \\
\text { Bis September 2017 wurden 7 Themendossiers in Abstimmung mit } \\
\text { themenverantwortlichen Ressorts und der WKO erstellt }{ }^{9} \text {. Analytische } \\
\text { Programmberichte werden von EU-PM 1x pro Jahr zur Verfügung ge- } \\
\text { stellt. Der Zeitpunkt der Publikation ist abhängig vom jeweiligen Pro- } \\
\text { gramm. } \\
\text { 7. Policy Briefs: In_brief sind anlassbezogene Policy Briefs über ak- } \\
\text { tuelle Entwicklungen mit strategischer und/oder operativer Relevanz } \\
\text { für die österreichische FTI-Community. } \\
\text { Bis September 2017 wurden 2 Policy Briefs erstellt }{ }^{10} . \\
\text { 8. Analysen: Analysen von Evaluierungsergebnissen sowie Netzwerk- } \\
\text { und Beteiligungsanalysen (ex ante und ex post) gewinnen an Bedeu- } \\
\text { tung. Netzwerk- und Beteiligungsanalysen werden unter Einbeziehung } \\
\text { der AuftraggeberInnen, des EU-Performance-Monitorings und den } \\
\text { "themenverantwortlichen AkteurInnen" in den Ressorts durchgeführt. }\end{array}$ \\
\hline
\end{tabular}

Quelle: Eigene Darstellung basierend auf FFG-EIP Toolbox

Gesamthaft betrachtet stellen persönliche Beratungen nach wie vor das Kerngeschäft von FFG-EIP dar.

In der Datenbank INNOman von FFG-EIP waren mit Stichtag 31.12.2016 rund 30.300 natürliche Personen und rund 13.600 Organisationseinheiten aus Österreich erfasst, die als InteressentInnen für die Beteiligung an den Rahmenprogrammen und multilaterale Initiativen gelten. 31\% dieser InteressentInnen stammen von Österreichs Universitäten, 11\% von anderen Forschungsorganisationen. Auf den Unternehmensbereich entfallen insgesamt 39\% der InteressentInnen. 19\% der InteressentInnen stammen aus anderen Organisationen (z.B. Nationale Verwaltung, Städte und Gemeinden, Intermediäre). Seit dem Jahr 2014 wurden jedes Jahr rund 530 Personen neu im INNOman erfasst, die eine Beratung zu den Rahmenprogrammen oder MULLAT erhalten haben.

Untenstehende Abbildung 3 gibt einen Überblick über die langfristige Entwicklung der persönlichen Beratungen durch FFG-EIP. In den Jahren 2014/2015/2016 wurde jede Person pro Jahr durchschnittlich 3,4 Mal zu den Rahmenprogrammen und MULLAT persönlich beraten, Organisationen rund 6 Mal. Auffallend ist, dass die Anzahl der Beratungen im Jahr 2009, rund 2 Jahre nach Start des 7. Rahmenprogramms (Laufzeit 2007 - 2013) ihren absoluten Höhepunkt erreichte. Demgegenüber war die Anzahl der Beratungen zu

9 Siehe die ERA Themendossiers der FFG

10 Ebenda. 
H2020 im ersten Jahr des Programmstarts (2014) am höchsten und ist seither rückläufig. Die sinkende Anzahl der persönlichen Beratungen seit Start von H2020 spiegelt einerseits die Strategieänderung im Rahmen der Neubeauftragung von FFG-EIP für den Bereich Wissenschaft wider, andererseits zeigt die Erfahrung von FFG-EIP, dass gerade in der Anfangsphase von neuen Rahmenprogrammen der Beratungsbedarf besonders hoch ist.

\section{Abbildung 3: Persönliche Beratungen zu Rahmenprogrammen und MULLAT}

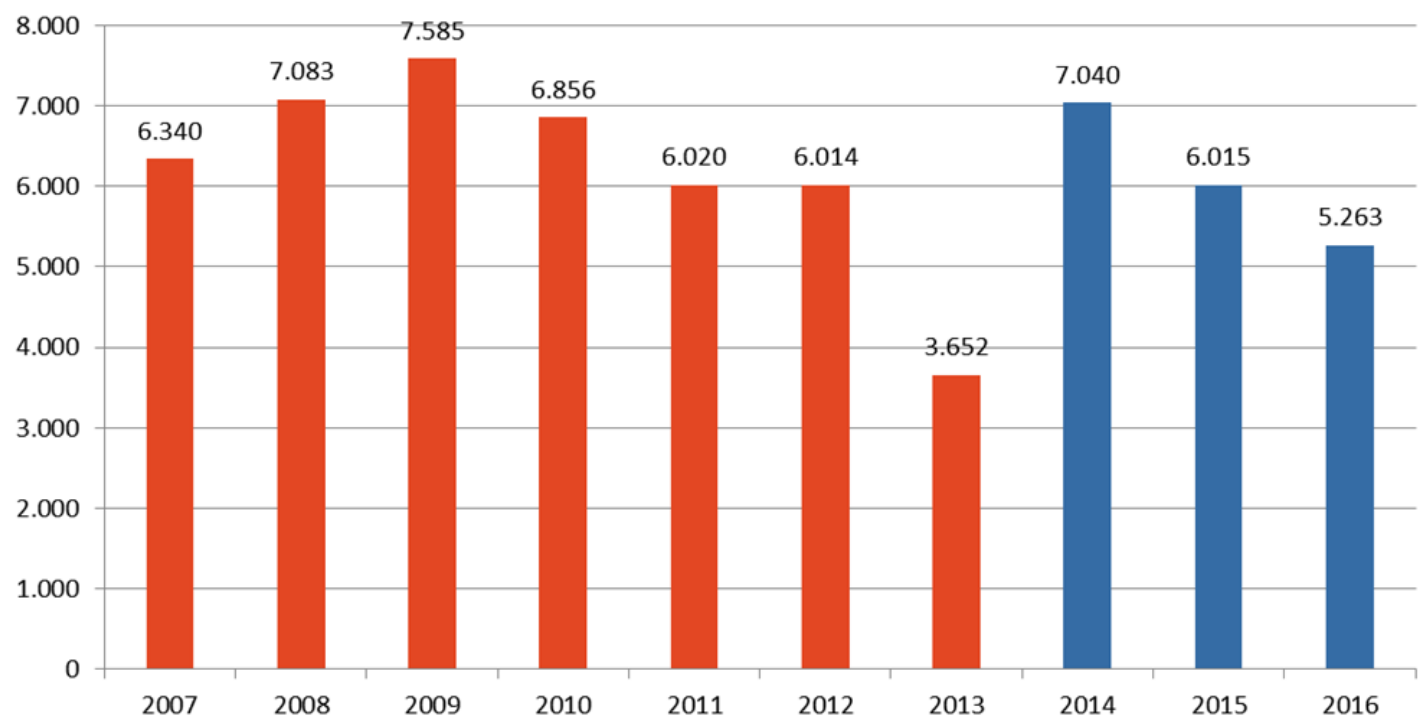

Quelle: FFG: Zusammenfassende Statistiken aus Leistungsbericht und Quartals-Monitoring

Gestützt wird diese These durch den sinkenden Anteil an Beratungen für Universitäten und Forschungseinrichtungen, während der Anteil der Beratungen für kleine und mittlere Unternehmen (KMU) und sonstige Einrichtungen gestiegen ist (siehe Abbildung 4).

Als wesentliches Effektivitätskriterium für die Beratungsleistungen der FFG wird als Indikator eine überdurchschnittliche Bewilligungsquote jener Projekte, die von EIP beraten wurden (Allgemeine Beratung, Detailberatung, Proposal-Check) in der Beauftragung angeführt. Als Vergleichswert wird die durchschnittliche Bewilligungsquote EU-weit herangezogen. Ungeachtet potentieller struktureller Gründe (z.B., dass sich tendenziell erfahrenere ProjektwerberInnen von der FFG beraten lassen) lässt die höhere Bewilligungsquote indirekte Rückschlüsse auf die positive Wirkung der Beratungsleistungen zu, zumal der positive Effekt der Beratungen auch von Ergebnissen der Surveys (vgl. Abb. 13 und 14) und Interviews unterstrichen wird. 


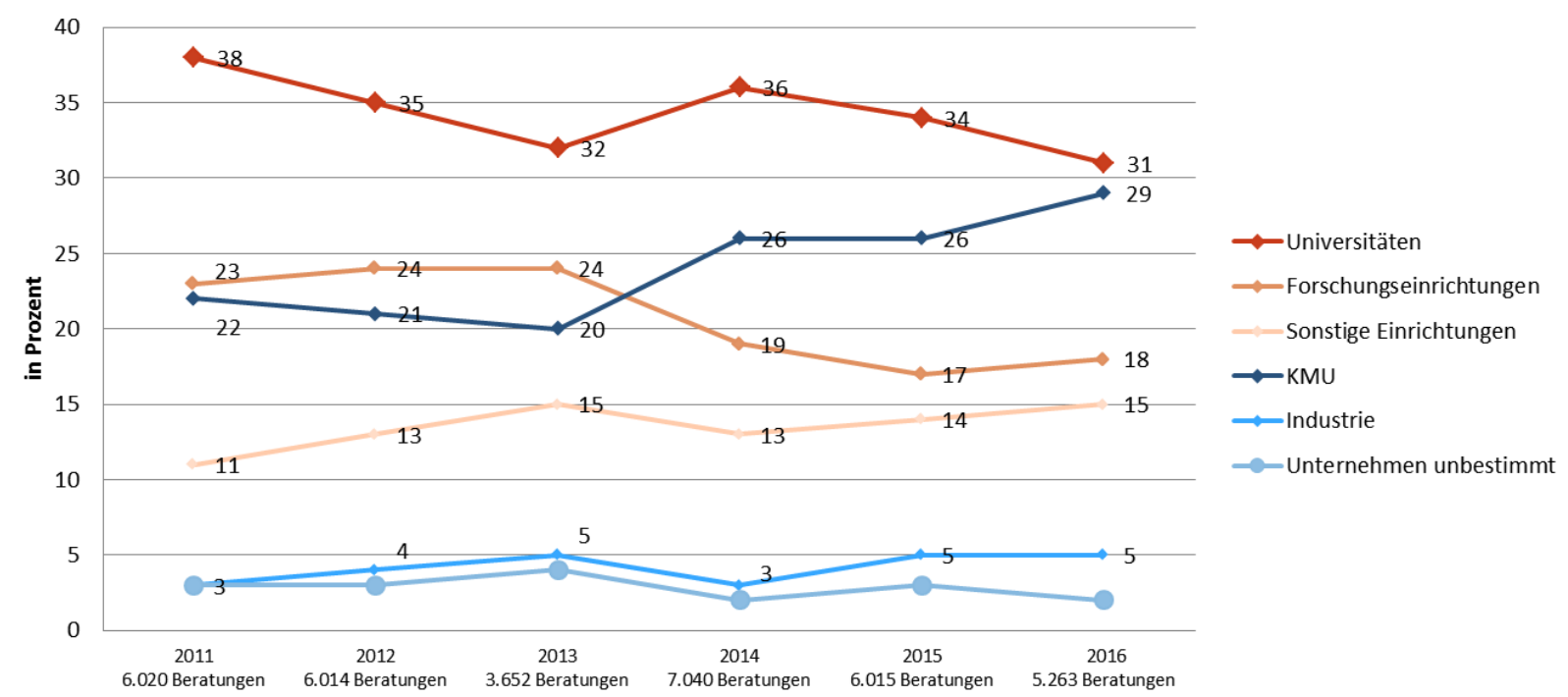

Quelle: FFG: Zusammenfassende Statistiken aus Leistungsbericht und Quartals-Monitoring

Über die oben genannten Leistungen hinausgehend, betreffen wesentliche Neuausrichtungen des Leistungsspektrums von FFG-EIP insbesondere die Instrumente zur strategischen Beratung und ERA-Orientierungswissen für Universitäten und Forschungsorganisationen (ERA-Dialog und Strategiegespräche) sowie zur Unterstützung von Unternehmen mit hohem FTI-Potenzial und Engagement - das Core Customer Concept (C3). Auf ERA-Dialoge und Strategiegespräche wird in Abschnitt 2.2, auf das Core Customer Concept wird in Abschnitt 2.3 eingegangen.

Zudem wurden für die österreichische FTI-Community und Policy-Akteure neue Berichtsformate entwickelt, die in unterschiedlicher Intensität und Frequenz Informationen zu aktuellen Entwicklungen in Hinblick auf ERA und zur österreichischen Beteiligung mit strategischer und/oder operativer Relevanz bereitstellen. Die Erstellung der Berichte erfolgte dabei in enger Kooperation mit dem EU-Performance Monitoring. 


\section{Beratung und Betreuung - Fokus Wissenschaft}

In diesem Abschnitt werden die spezifischen Ziele und Aufgaben von FFG-EIP sowie das Leistungsangebot für die österreichischen FTI-Akteure im Bereich „Wissenschaft" dargestellt, analysiert und bewertet. Die Bewertung der Leistungen von FFG-EIP für Wissenschaftsakteure stützt sich dabei auf folgende empirische Erhebungen:

1. Die Online Befragung der universitären und außeruniversitären FTI-Akteure, die Leistungen von FFG-EIP in Anspruch genommen haben. Diese liefert, differenziert nach dem Erfahrungshintergrund der FTI-Akteure, ein Bild über die Bekanntheit und das Nutzungsverhalten von Leistungen der FFG, sowie die Zufriedenheit mit denselben.

2. Interviews mit VertreterInnen von FFG-EIP und VertreterInnen von Forschungsservicestellen, denen als MultiplikatorInnen im Sinne eines Empowerments der Forschungseinrichtungen eine besondere Bedeutung zukommt.

3. Drei Fokusgruppen Interviews mit a) VertreterInnen von Außeruniversitären Forschungseinrichtungen, b) VertreterInnen von Forschungsservicestellen, und c) den ERA-KorrespondentInnen der Universitäten.

4. Eine Delphi-Befragung, die Einschätzungen über die zukünftig notwendige strategische Orientierung der FFG und die Bedarfslagen der Wissenschaftsakteure vor dem Hintergrund sich ändernder Rahmenbedingungen liefert.

Im Folgenden werden zuerst die spezifischen Ziele und Aufgaben von FFG-EIP im Leistungsbereich Wissenschaft dargestellt. Die Ergebnisse der empirischen Erhebungen werden dann in Themenblöcken zusammengefasst und geben Auskunft über die Funktionstüchtigkeit des FFG-Dienstleistungsspektrums.

Die Darstellung der Ergebnisse differenziert dabei im Wesentlichen zwischen FFG-EIP Leistungen, die insbesondere der Unterstützung von ForscherInnen dienen und Leistungen, die vor allem die Selbstermächtigung auf der Organisationsebene stärken sollen.

\subsection{Ziele und Aufgaben}

Die primäre Aufgabe von FFG-EIP im Leistungsbereich Wissenschaft ist es, über ein spezifisches Angebot an systemisch orientierten Serviceleistungen zur erfolgreichen Beteiligung österreichischer Akteure in H2020 und strategischen Partnerschaften beizutragen.

Wesentliche operative Zielsetzungen von FFG-EIP für den Leistungsbereich Wissenschaft ${ }^{11}$ sprechen dabei zwei Ebenen an:

- Erstens soll die Unterstützung von Personen zur erfolgreichen Beteiligung in $\mathrm{H} 2020$ und anderen multilateralen Initiativen durch Information und Beratung beitragen. Die Bereitstellung von Informations- und Beratungsleistungen der

11 Die Zielgruppe Wissenschaft umfasst It. FFG Zwischenbilanz zur Programmevaluierung konkret: 1. Hochschulen, Fachhochschulen, Außeruniversitäre Forschungseinrichtungen, Privatuniversitäten. 
gesamten FFG-EIP Toolbox wie Trainings, Proposal-Checks, Newsletters und Informationsveranstaltungen ist dafür auch in der laufenden Beauftragung eine Kernaufgabe des EIP.

- Zweitens soll FFG-EIP Beiträge zur Selbstbefähigung der Forschungsorganisationen im europäischen Kontext über die Implementierung eines systemorientierten Beratungsansatzes leisten. FFG-EIP setzt dabei sowohl auf Maßnahmen zum „Empowerment" der organisationsinternen Servicestrukturen, als auch auf eine spezifische Ansprache des Forschungsmanagements (z.B. Vizerektorate für Forschung).

Die zweite Beratungsebene stellt eine zentrale Veränderung in den Zielen und Aufgaben der laufenden EIP-Beauftragung dar: Universitäten und Forschungseinrichtungen sollen im Aufbau und der Weiterentwicklung ihrer strategischen Kompetenz und autonomen Selbststeuerung zur Beteiligung in europäischen und multilateralen FTI-Kooperationen gezielte strategische Betreuung durch das EIP erhalten.

FFG-EIP will daher Forschungsservicestellen (FoS) in der Übernahme des First-Level Supports für ForscherInnen unterstützen. Damit geht eine Fokussierung der FFG auf den Second-Level Support einher:

- Laut FFG beinhaltet der First Level Support allgemeine Anfragen zu H2020 (Erstanfragen, Erstberatungen) sowie wiederkehrende generische Fragestellungen zur Projektabwicklung, Budgetplanung, Managementstrukturen, und Personalkostenabrechnung in $\mathrm{H} 2020$.

- Der Second Level Support beinhaltet die Beantwortung von Detailfragestellungen bzgl. der Einordnung der Projektidee, die Durchführung von Proposal Checks, rechtliche und finanzielle Spezialfragen, spezifische Fragestellungen zum European Research Council sowie zum Vertragsmanagement.

Als Unterstützung für die verstärkte Übernahme des First-Level Supports setzt die FFG auf die Umsetzung folgender Instrumente zur Unterstützung der Forschungsservicestellen:

- Bereitstellung und laufende Aktualisierung von Basisinformationen und häufig angefragten Detailinformationen über geeignete Informationstools (Website, Newsletter, FAQ, etc.);

- Bereitstellung von Hintergrundinformationen (FTI-Entwicklungen, Draft Arbeitsprogramme etc.) in einem geschlossenen online-Bereich;

- Trainings im Rahmen der FFG-Akademie zu komplexen, häufig nachgefragten Themenbereichen fokussieren auf Fragen der Universitäten und großer Forschungseinrichtungen (z. B. Recht und Finanzen, EU-Projektmanagement)

- Persönliche Beratung durch die FFG-ExpertInnen;

- Erfahrungsaustausch in der Runde der ARGE Forschungsförderung ${ }^{12}$, in der sich die MitarbeiterInnen der Forschungsservices österreichischer öffentlicher Universitäten zusammengeschlossen haben, um den Themenkomplex Wissenschaft und Forschung professionell unterstützen zu können.

\footnotetext{
12 Link zur Website der ARGE Forschungsförderung
} 
- Dialogformate für spezifische Gruppen (ProjektmanagerInnen, KoordinatorInnen etc.) oder ad-hoc Webinare bei aktuellen Anlässen.

Simultan wurde ein neues Betreuungsinstrument zur Unterstützung der Profilbildung und Positionierung der österreichischen Wissenschaft im Europäischen Forschungsund Innovationsraum entwickelt: Der ERA-Dialog ist ein regelmäßiger Austausch mit der Führungsebene der Universität/Forschungseinrichtung (Vize-Rektorate bzw. Management, Forschungsservicestellen) zur systematischen Auseinandersetzung mit Entwicklungen und Beteiligungsmöglichkeiten in ERA. Die Führungsebene soll durch den kontinuierlichen Prozess auf das EU-Thema sensibilisiert werden und es in weiterer Folge stärker in der Strategie der Organisation verankern. Zusätzlich zum ERA-Dialog, findet ein Erfahrungsaustausch zwischen den VizerektorInnen und dem BMBWF im Rahmen der Gruppe der ERA-KorrespondentInnen der Universitäten statt (Siehe Kap. 7.4.3).

\section{Abbildung 5: Wirkungsmodell des ERA-Dialoges}

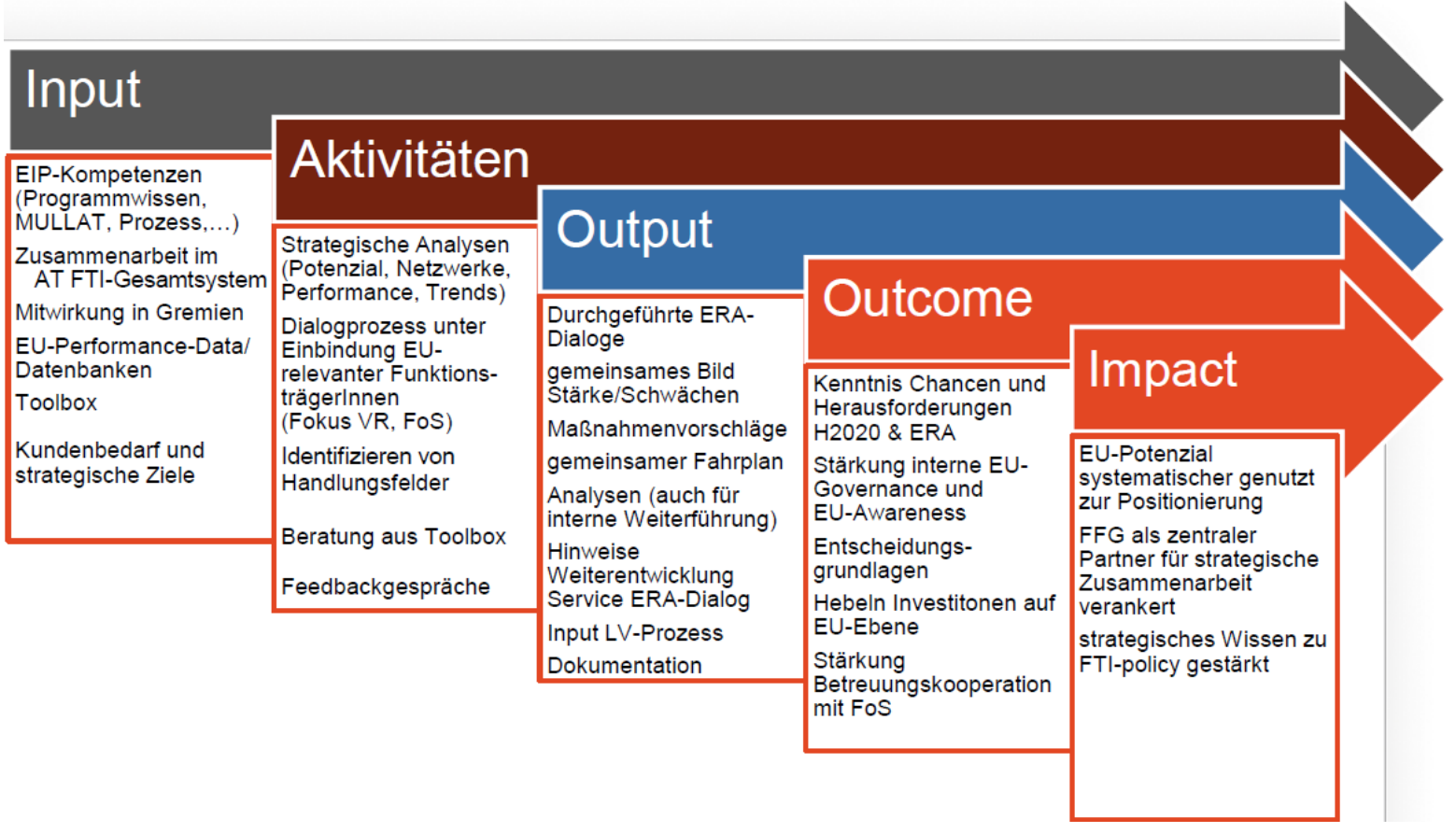

Quelle: FFG (2017)

Auswertungen und Potenzialanalysen zeigen mögliche Handlungsfelder und Optionen auf, um die Weiterentwicklung der Internationalisierungsstrategien der Universität zu unterstützen. Abbildung 5 bildet das Ziel- und Leistungsverständnis des ERA-Dialoges inkl. erwarteter Wirkungen ab.

\subsection{Spezifische Leistungen der FFG}

Programm- und Projektberatung entlang aller Projektphasen, Maßnahmen zur Bewusstseinsbildung und Informationsvermittlung zur Förderung der Bekanntheit und Reputation europäischer und multilateraler Programme, sowie strategische Beratung und ERA-Orientierungswissen zur Unterstützung der optimalen Nutzung vorhandener Förderprogramme umfassen das Leistungsspektrum für die österreichischen Wissenschaftsakteure. Dabei geht es auch darum, EU FTI-Entwicklungen möglichst früh in den 
Dialog mit Entscheidungs- und LeistungsträgerInnen der Universitäten einzubringen und damit ihre Positionierungsbemühungen zu unterstützen.

\section{Abbildung 6: Anzahl First und Second Level Beratungen}

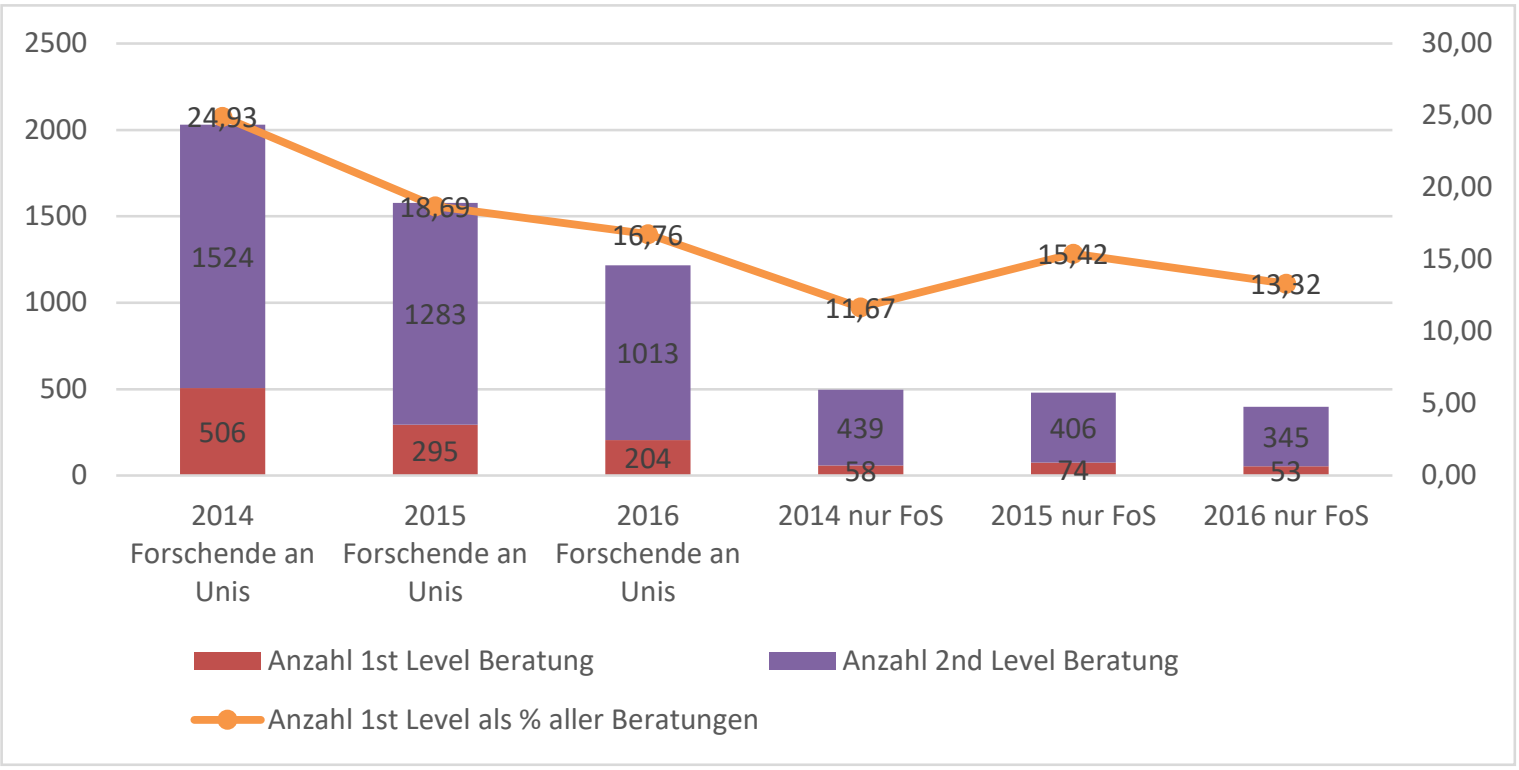

Quelle: FFG (2017)

Der Fokus der persönlichen Beratung der Forschenden in der Wissenschaft der FFG liegt auf der 2nd-Level Beratung zu programmspezifischen Fragen wie Einordnung der Projektidee zum passenden Programm, ERC, rechtlich/finanzielle Spezialfragen, etc. Die Fokussierung auf den 2nd Level Support ist im Sinne der Ziele der Beauftragung: Die Selbstermächtigung der Forschungsorganisationen und die Unterstützung beim Aufbau und Weiterentwicklung strategischer Kompetenzen.

Abbildung 6 zeigt den Verlauf der Beratungen für Universitäten für den Zeitraum 20142016 differenziert nach Forschenden und Forschungsservicestellen (FoS). Auf die FoS entfielen insgesamt 28,5\% aller Beratungen der Universitäten. Bei gesamt rückläufigen Beratungszahlen im Universitätsbereich, ist vor allem bei der Beratung der Forschenden an Universitäten eine deutliche Reduktion der Anzahl der 1st-Level Beratungen im Verlauf der Beauftragung feststellen.

In Hinblick auf die Antragsberatung (Einstiegscheck bis Proposalcheck) liegt der Fokus im Wissenschaftsbereich auf Anträgen von österreichischen KoordinatorInnen. Inhaltlich deckte die FFG das gesamte Themenspektrum von H2020 ab. Gegebenenfalls unterstützt EIP auch die Konsortialbildung durch die Vermittlung von ProjektpartnerInnen (organisational und personell). 


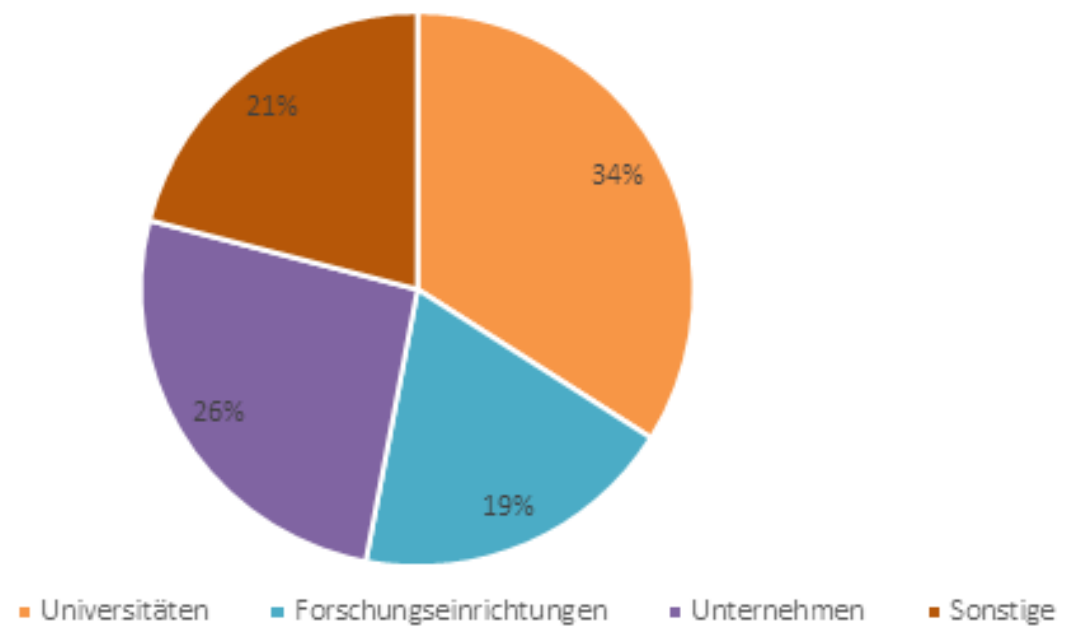

Quelle: FFG - Hintergrunddokument FFG Akademie (2017)

Im Rahmen der FFG Akademie werden Trainingsveranstaltungen und Webinare des EIP zur Informationsvermittlung und Wissensaufbau gebündelt. Von den insgesamt mehr als 4.000 TeilnehmerInnen von 2014-2016, stammen mehr als die Hälfte von Universitäten und Forschungseinrichtungen. Laut FFG arbeiten rund $12 \%$ aller TeilnehmerInnen an den Veranstaltungen der FFG-Akademie für die Forschungsserviceeinrichtungen an Österreichs Universitäten und außeruniversitären Forschungseinrichtungen.

Das Gros der Trainings fokussierte auf den Bereich Antragstellung (46). 6 Trainings fokussierten auf den Bereich Projektmanagement, 3 auf den Bereich Orientierung und 2 auf Verwertung. Für die "FoS" wurden insgesamt 9 Spezialtrainings zur H2020- Kompetenzerweiterung (Stichwort: „First level support", „Empowerment”) entwickelt, darüber hinaus steht das gesamte Veranstaltungsangebot für diese Zielgruppe offen.

\section{Abbildung 8: Zielgruppen der FFG-Akademie nach Veranstaltungsinhalten}

\begin{tabular}{|c|c|c|c|c|}
\hline \multicolumn{5}{|l|}{ ZIELGRUPPEN } \\
\hline \multicolumn{5}{|c|}{$\begin{array}{l}\text { MULTIPLIKATORINNEN: Mitarbeiterlnnen von Forschungsservices und regionalen Agenturen sowie } \\
\text { KonsulentInnen }\end{array}$} \\
\hline ORIENTIERUNG & $\begin{array}{l}\text { PROJEKT- } \\
\text { ENTWICKLUNG UND } \\
\text { ANTRAGSTELLUNG }\end{array}$ & $\begin{array}{l}\text { PROJEKT- } \\
\text { MANAGEMENT }\end{array}$ & VERWERTUNG & EU-NETWORKING \\
\hline $\begin{array}{l}\text { Für alle, die } \\
\text { Orientierung zu } \\
\text { und Überblick } \\
\text { über europäische } \\
\text { FTl- } \\
\text { Förderprogramme } \\
\text { wünschen. }\end{array}$ & $\begin{array}{l}\text { Für alle, die ein } \\
\text { Projekt in Horizon } \\
2020 \text { einreichen } \\
\text { möchten bzw. bei } \\
\text { der Einreichung } \\
\text { mitarbeiten. }\end{array}$ & $\begin{array}{l}\text { Für alle, die ein } \\
\text { in Horizon } 2020 \\
\text { gefördertes } \\
\text { Projekt effizient } \\
\text { abwickeln } \\
\text { wollen. }\end{array}$ & $\begin{array}{l}\text { Für Kleine und Mittlere } \\
\text { Unternehmen, die } \\
\text { einen Überblick sowie } \\
\text { Detailwissen zu } \\
\text { Verwertungs- } \\
\text { möglichkeiten im } \\
\text { europäischen Kontext } \\
\text { erhalten möchten. }\end{array}$ & $\begin{array}{l}\text { Für alle, die mit „EU- } \\
\text { ExpertInnen“ ins } \\
\text { Gespräch kommen } \\
\text { möchten, um ihr } \\
\text { Netzwerk zu } \\
\text { erweitern. }\end{array}$ \\
\hline
\end{tabular}

Quelle: FFG - Hintergrunddokument FFG Akademie (2017) 
Als neues Instrument zur strategischen Beratung und zur Bereitstellung von ERA-Orientierungswissen unterstützt der ERA-Dialog Universitäten, Fachhochschulen und Forschungseinrichtungen mit eigenen Supportstrukturen bei der strategischen Positionierung und Profilbildung im Europäischen Forschungsraum.

Die Betreuung soll die Führungsebene (Vize-Rektorate, Management), Fakultäts- und Institutsleitung sowie die Forschungsservicestellen mit einem regelmäßigen, dialogorientierten Beratungsansatz ansprechen. Die Inhalte sollen dabei über die reine H2020 Antragsberatung hinausgehen und die komplexe EU FTI-Förderlandschaft in die Strategie der Organisationen übersetzen. Dabei unterstützt das FFG-EIP vor allem mit strategischen Analysen zu Stärken und Schwächen und entwickelt Maßnahmenvorschläge. Durch den ERA-Dialog soll auch die interne ERA-Awareness und Governance gestärkt und das strategische Wissen auf Führungsebene verankert werden.

Derzeit laufen 12 ERA-Dialoge mit Universitäten und 2 ERA-Dialoge mit außeruniversitären Forschungsorganisationen. Ende 2017 kamen eine weitere Universität sowie eine weitere Forschungseinrichtung hinzu.

\subsection{Ergebnisse der empirischen Erhebungen}

\subsubsection{Persönliche Unterstützungsleistungen}

\section{Erfahrungshintergrund der FFG-KundInnen}

Die Ergebnisse der Online Befragung der FFG KundInnen, die Beratungsleistungen in Anspruch genommen haben zeigen, dass 75\% der erreichten Wissenschaftsakteure "erfahren" im Umgang mit europäischen Förderprogrammen sind und zumindest an der Einreichung von erfolgreichen Projektanträgen mitgearbeitet haben (Summe aus Koordination und Mitarbeit). Von diesen sind wiederum knapp zwei Drittel als KoordinatorInnen für ihre Organisation oder das Gesamtprojekt tätig. 25\% der erreichten Akteure verfügen über keine oder wenig Erfahrung in der Einreichung und Durchführung von europäischen Forschungs- und Innovationsprojekten.

Unterschiede im Erfahrungshintergrund existieren zwischen den Organisationstypen. Während sich im Universitätsbereich rund 37\% der erreichten FFG KundInnen als wenig erfahren charakterisieren, liegt der Anteil unerfahrener FFG KundInnen im Bereich der außeruniversitären Forschungseinrichtungen bei lediglich $10 \%$.

Die Befragung und die Interviews zeigen, dass die FFG für ForscherInnen aus dem Wissenschaftsbereich klar die zentrale Unterstützungseinrichtung für Fragestellungen zu europäischen FTI-Initiativen darstellt. Mit einem Nutzungsgrad von insgesamt knapp $83 \%$ ist die FFG bedeutender als KollegInnen mit Erfahrungen im EU-Bereich (73\%) sowie organisationsinterne Servicestellen, die 56\% der RespondentInnen in Anspruch nehmen. Auf die Aufgabenteilung zwischen organisationsinternen Servicestellen und der FFG wird dabei in Abschnitt 3.3.2 eingegangen. 


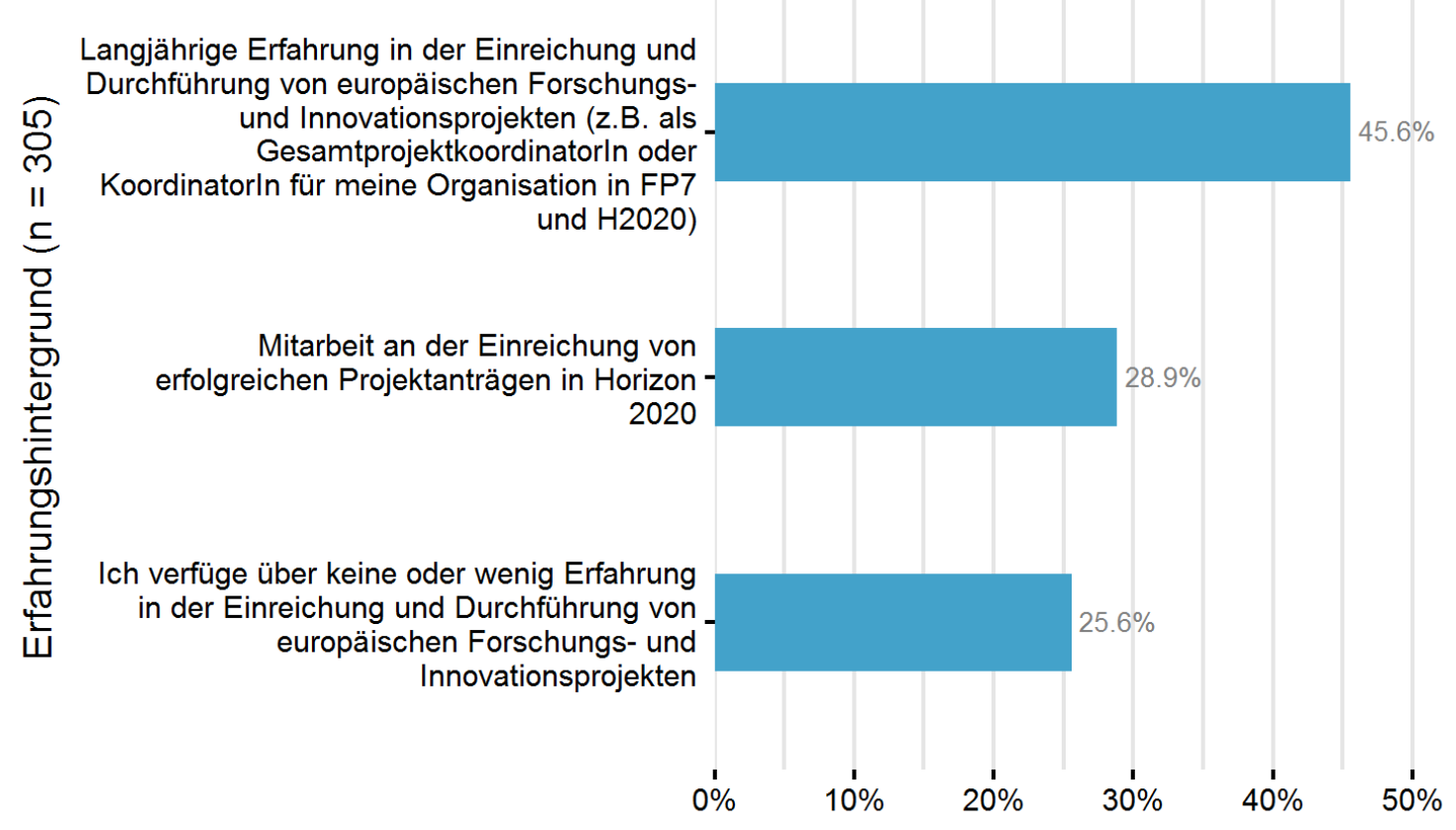

Quelle: Online Befragung, Joanneum Research.

Eine differenzierte Auswertung zwischen erfahrenen und nicht erfahrenen Antragstellenden (siehe Abbildung unten) zeigt, dass "erfahrene Akteure" Leistungen der FFG in einem größeren Ausmaß in Anspruch nehmen als "weniger erfahrene Akteure".

Dies mag auf den ersten Blick überraschen, weil gerade die weniger erfahrenen AntragstellerInnen den größten Bedarf an Unterstützungsleistungen haben, jedoch sind die erfahrenen AntragstellerInnen besser über das Angebot informiert und wissen unter Umständen besser, welche Art der Unterstützung sie benötigen.

Eine differenzierte Auswertung nach Organisationstyp zeigt, dass ForscherInnen an Universitäten mit 63\% Leistungen organisationsinterner Servicestellen häufiger in Anspruch nehmen als ForscherInnen an außeruniversitären Forschungseinrichtungen.

Eine sehr untergeordnete Rolle im Wissenschaftsbereich nehmen regionale Beratungsstellen und private DienstleisterInnen ein. Die FFG ist somit der zentrale Partner der österreichischen Forschungsakteure für Unterstützung zu H2020. 


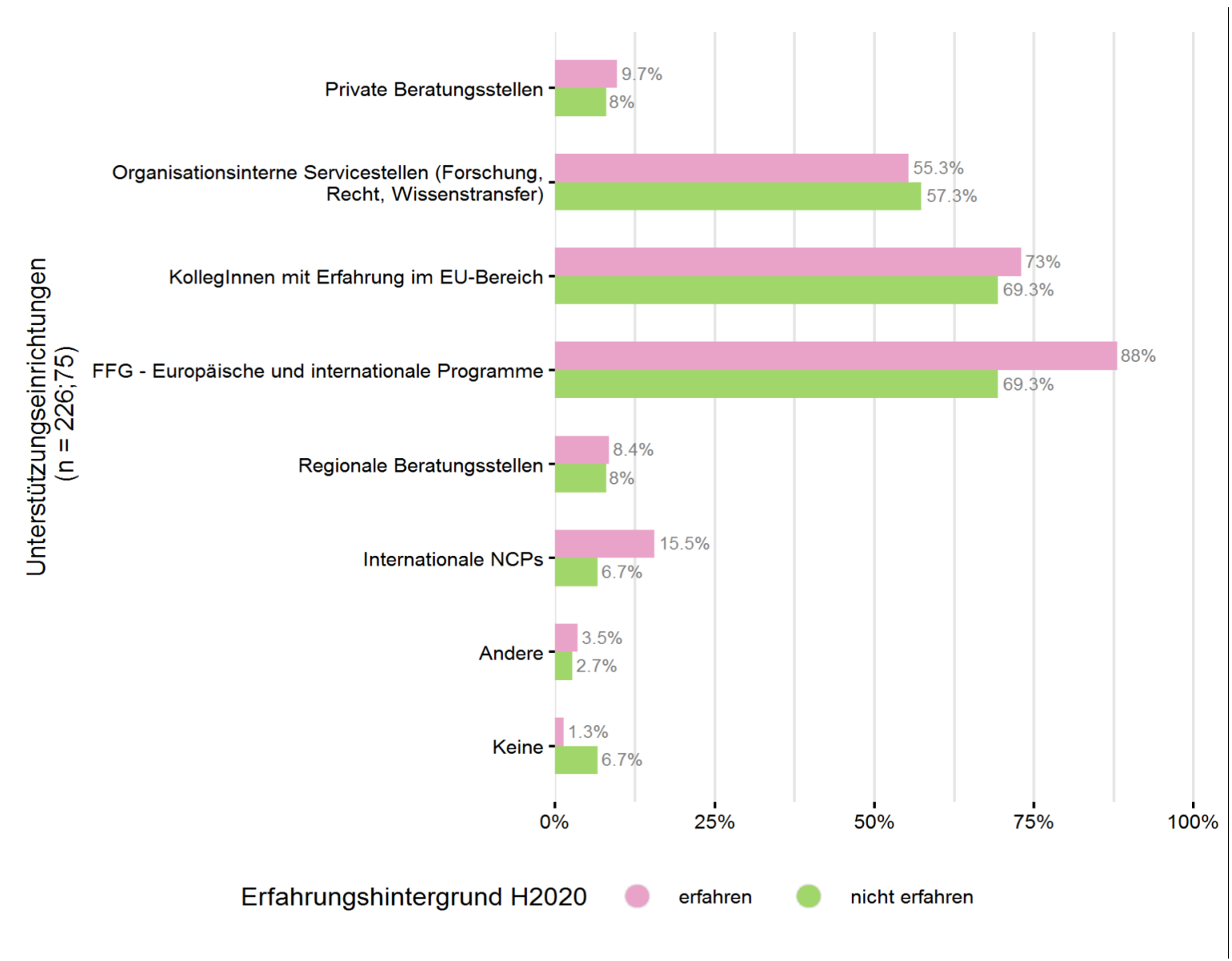

Quelle: Online Befragung, Joanneum Research.

\section{Motive für Inanspruchnahme von FFG-EIP Leistungen}

Die stärksten Bedarfslagen zur Inanspruchnahme von FFG-EIP Leistungen der erreichten Wissenschaftsakteure sind:

- Spezifische Fragen zu Arbeitsprogrammen und Ausschreibungen (57\%),

- Fragen im Rahmen der Erstellung eines Projektantrages (48\%),

- Informationen zu Trends und Entwicklungen in H2020 (46\%),

- Informationen über spezielle Instrumente von H2020 (35\%),

- Allgemeine Informationen zu Formalerfordernissen (33\%).

Eine untergeordnete Bedeutung haben demgegenüber:

- Die Unterstützung bei der Partnersuche (4\%-7\%),

- Fragestellungen zu Schutz- und Eigentumsrechten und Verwertungsstrategien $(9 \%)$,

- Interesse an der Beteiligung von multilateralen Initiativen (11\%). 


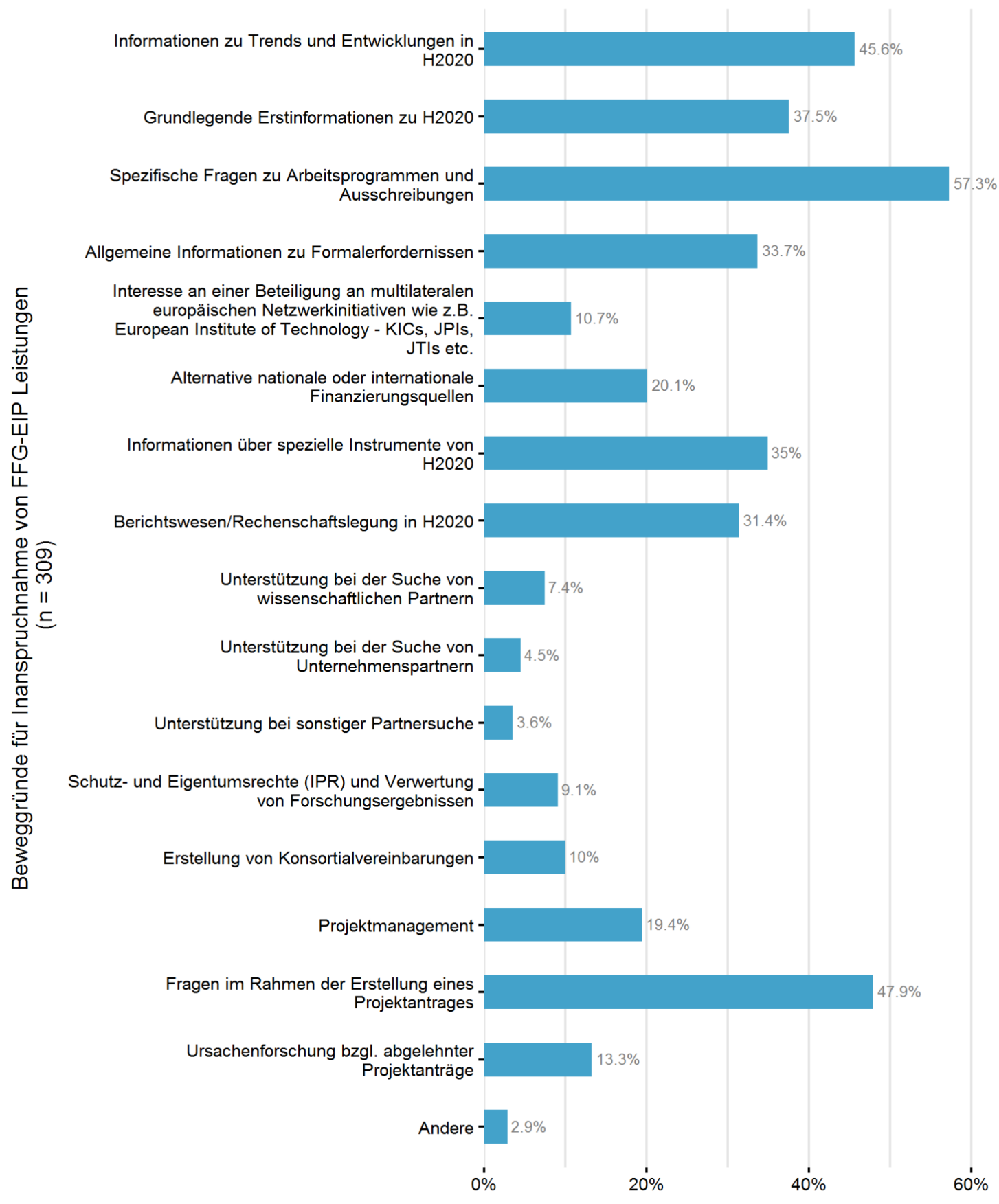

Quelle: Online Befragung, Joanneum Research.

\section{Nutzung, Qualität und Wirkung der Leistungen}

Korrespondierend zu den Motiven, nehmen die befragten Wissenschaftsakteure insbesondere folgende öffentliche Informationsleistungen von FFG-EIP in Anspruch:

- Informationen zu europäischen Forschungsrahmenprogramm (spezifische Ausschreibungen, inhaltliche Schwerpunktsetzungen) (93\%),

- Überblick über Informationen und Förderangebote zum europäischen Programmportfolio $(91 \%)$, 
- Informationen zur Beantragung und Abwicklung von H2020 Projekten (rechtliche, finanzielle und administrative Aspekte) (85\%),

- Informationen zu Instrumenten und Themen von H2020 (82\%).

Darüber hinaus nahmen 61\% der RespondentInnen an Awareness- und Informationsveranstaltungen der FFG teil. Die Website zum europäischen Programmportfolio wurde von $57 \%$ der erreichten Akteure genutzt. Die ERA-Themendossiers (31\%) sowie Informationen zu europäischen multilateralen Initiativen (44\%) erzielten die geringsten Nutzungsquoten der öffentlichen Informationsleistungen. Aufgrund der spezifischen Ausrichtung und Zielgruppe der FTI-Partnerschaftsinstrumente ist die Nutzung dieser Informationsleistung jedoch als hoch zu bewerten.

\section{Abbildung 12: In Anspruch genommene Informationsleistungen von FFG-EIP}

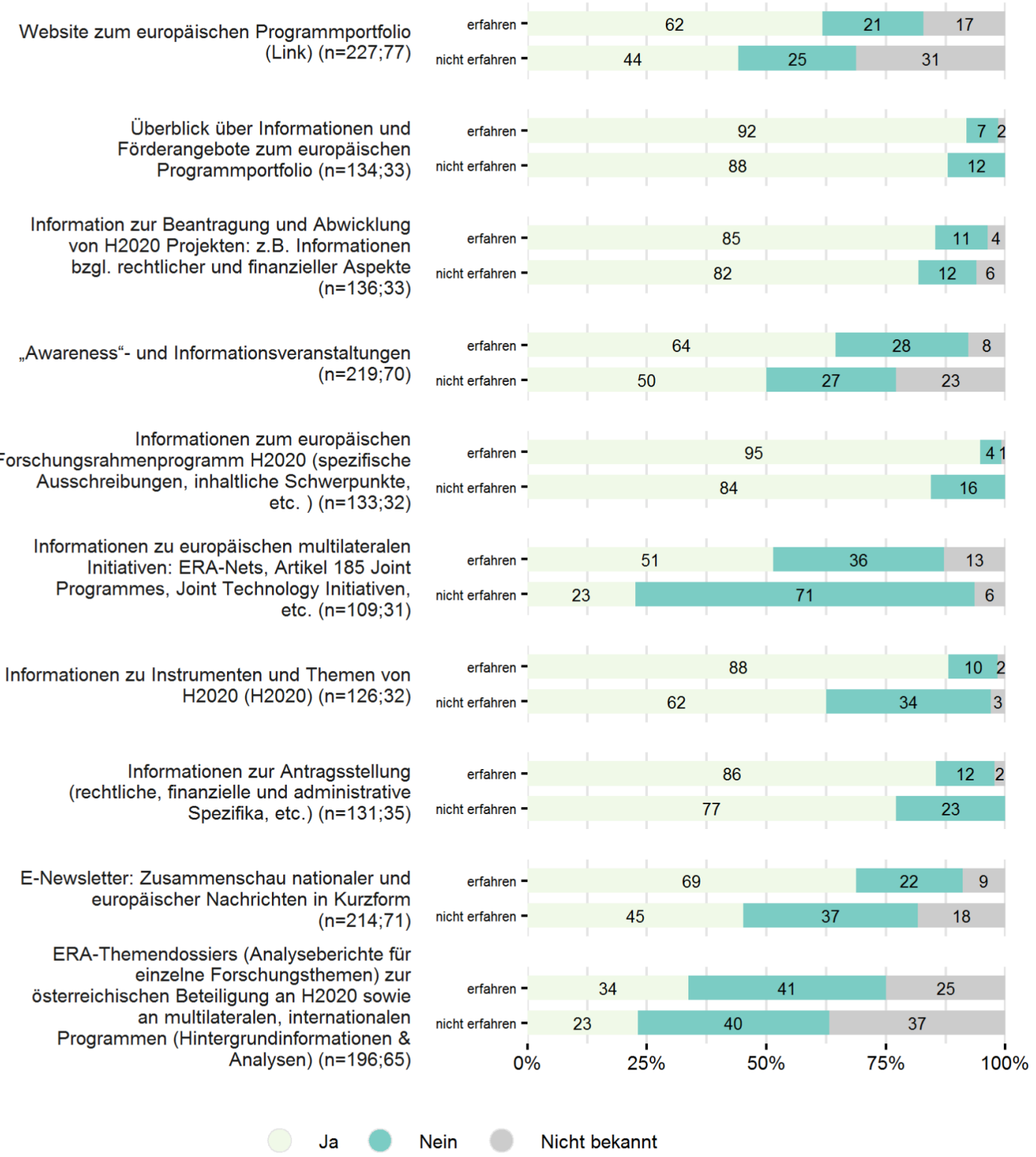

Quelle: Online Befragung, Joanneum Research. 
Zwischen außeruniversitären Forschungseinrichtungen und Universitäten existieren keine Unterschiede in der Nutzung von Informationsleistungen. Erfahrene Akteure greifen jedoch deutlich häufiger auf Dienstleistungen wie die Website zum europäischen Programmportfolio, Informationen zu Instrumenten und Themen von H2020 sowie auf Awareness- und Informationsveranstaltungen zu (siehe Abbildung oben).

Das Angebot an zur Verfügung gestellten Informationsleistungen wird von RespondentInnen im Wissenschaftsbereich überwiegend gut bewertet. $91 \%$ stimmen zumindest eher zu, dass sie sich aufgrund der Informationen insgesamt gut über die Möglichkeiten zur Einreichung an EU-Programmen informiert fühlen. 85\% stimmen zumindest eher zu, dass das Angebot an Informationsdienstleistungen insgesamt klar und übersichtlich strukturiert ist. Zwischen erfahrenen/nicht-erfahrenen Akteuren existieren nur geringfügige Unterschiede in der Bewertung, und zwischen außeruniversitären Forschungseinrichtungen und Universitäten existieren de facto keine Unterschiede.

\section{Abbildung 13: Bewertung der öffentlichen Informationsleistungen (Wissenschaft)}

Ich wurde auf Fördermöglichkeiten aufmerksam gemacht, die mir anderweitig nicht bekannt wären. (n=195:51)

Sie haben es mir ermöglicht, zeitgerecht strategische Planungen für die Einreichung in EU-Programmen vorzunehmen. $(n=196 ; 47)$

Ich erhielt Informationen über das Angebot an persönlichen Unterstützungsleistungen und Trainings der FFG in Bezug auf EU-Programme $(n=193 ; 48)$

Ich fühle mich aufgrund der Informationen insgesamt gut informiert über die Möglichkeiten zur Einreichung in EU-Programmen. $(n=193 ; 50)$

Das Angebot an Informationsdienstleistungen der FFG H2020 und andere EU-Programme betreffend ist insgesamt klar und übersichtlich strukturiert. $(n=194 ; 50)$
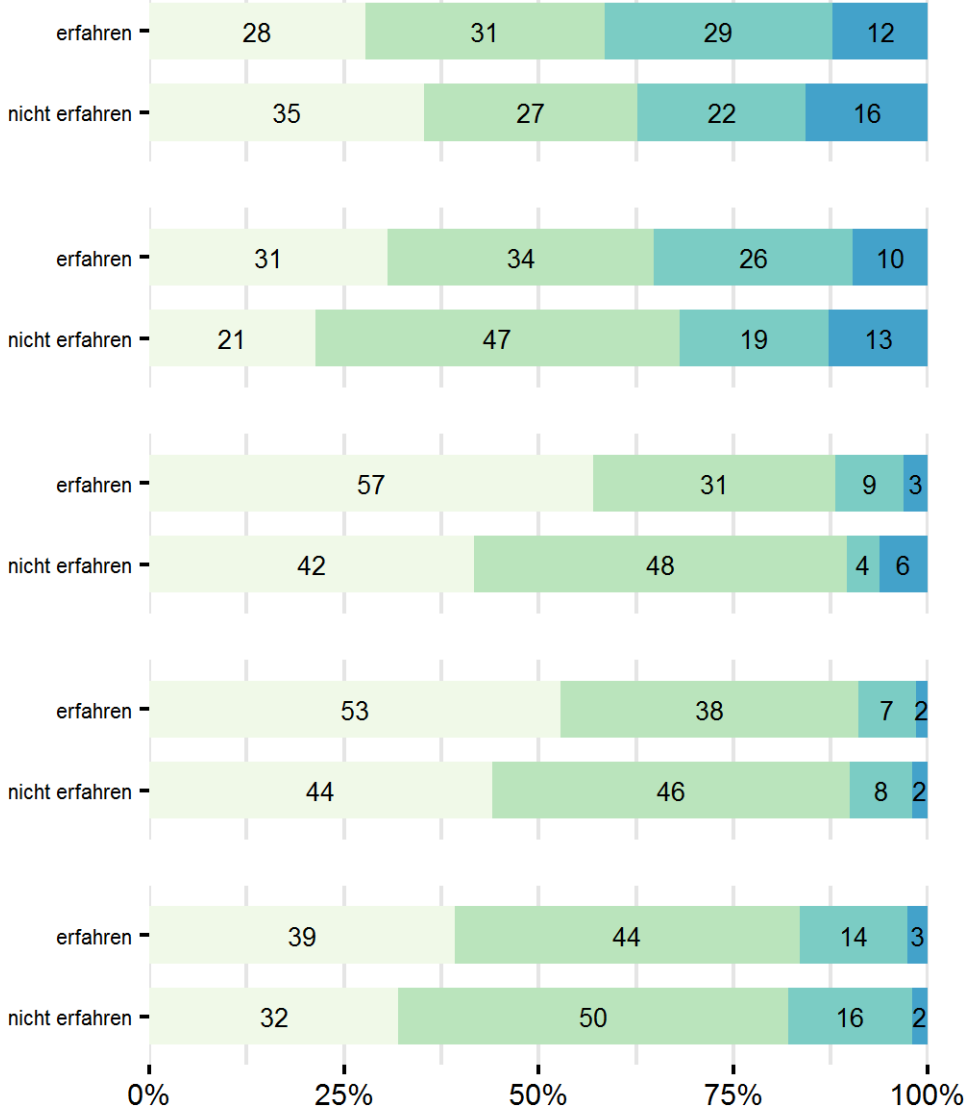

Stimme zu

Stimme eher zu

Stimme eher nicht zu

Stimme nicht zu

Quelle: Online Befragung, Joanneum Research. 
Vor dem Hintergrund der grundsätzlich sehr positiven Bewertung der Informationsleistungen wurden im qualitativen Teil der Befragung insbesondere die Unübersichtlichkeit des gesamten Leistungsportfolios und der Homepage von FFG-EIP kritisiert, die auch lediglich von $62 \%$ der erfahrenen und $44 \%$ der nicht erfahrenen KundInnen in Anspruch genommen wird. Diese Erkenntnis deckt sich weitgehend mit den Ergebnissen der Interviews mit den Servicestellen, die Aufmachung, Überblick und Möglichkeiten zur Identifikation von spezifischen Informationen und Ansprechpersonen als verbesserungswürdig empfinden.

Über das öffentliche Informationsangebot hinausgehend, haben rund 2/3 der RespondentInnen persönliche Beratungsleistungen in Form von Beratungsgesprächen, Einstiegschecks, und Unterstützung hinsichtlich der Formalerfordernisse im Rahmen der Antragstellung von FFG-EIP in Anspruch genommen.

\section{Abbildung 14: Bewertung der persönlichen Beratungsleistungen durch FFG-EIP}

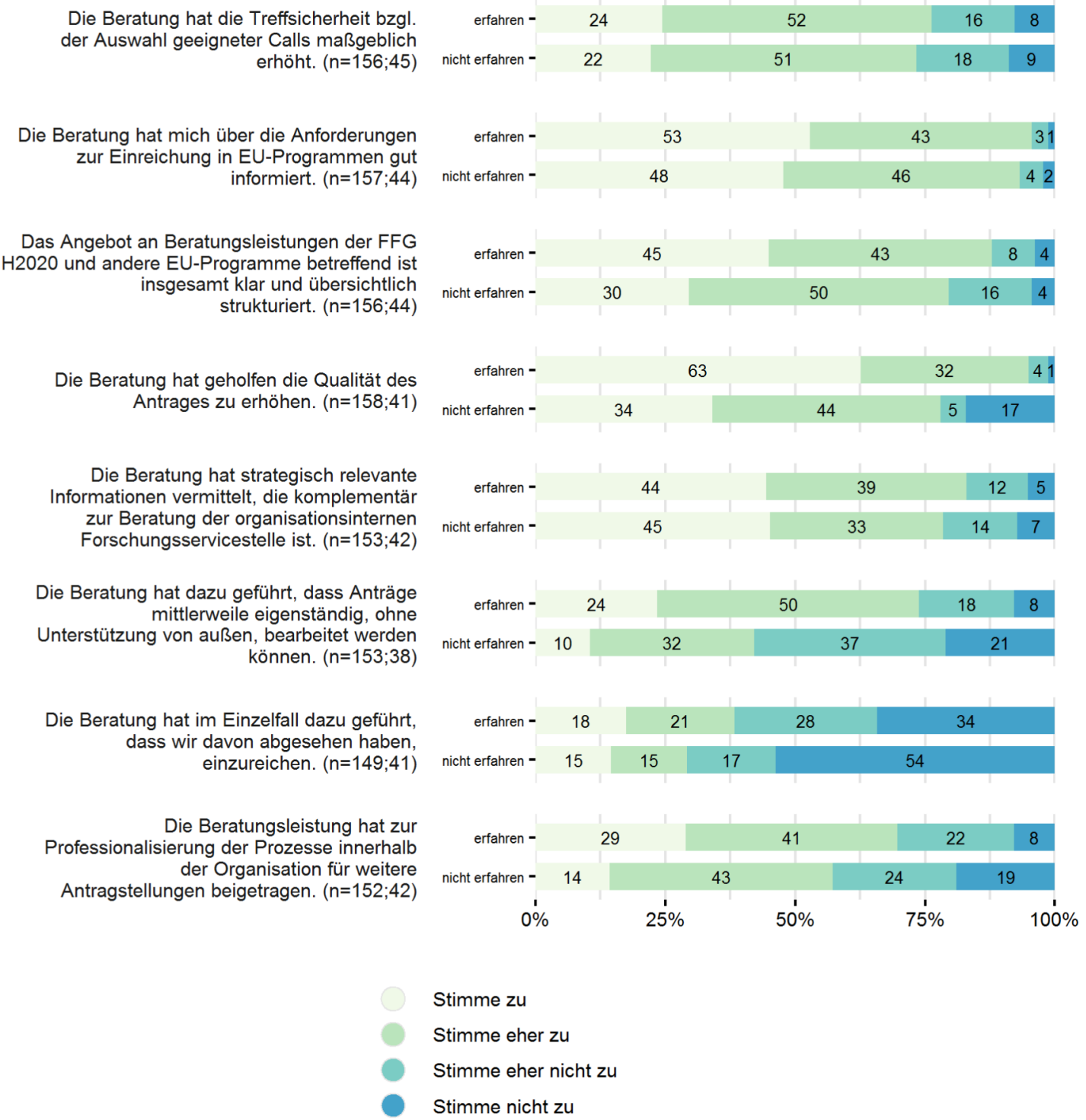

Quelle: Online Befragung, Joanneum Research. 
Die Unterstützung in der Partnersuche und die Vermittlung von Projektpartnern spielt hingegen da facto keine Rolle für die befragten FTI-Akteure. Wie obenstehende Abbildung zeigt, zeitigen auch die persönlichen Beratungsleistungen von FFG-EIP positive Wirkungen und informieren gut über 1) Anforderungen zur Einreichung in EU-Programmen, 2) leisten einen Beitrag zur Erhöhung der Qualität des Antrages und 3) zur Vermittlung von strategisch relevanten Informationen, die komplementär zur Beratung der organisationsinternen Forschungsservicestellen sind. Auffällig sind hier die Unterschiede in den Einschätzungen von erfahrenen und nicht erfahrenen AntragstellerInnen. Es ist anzunehmen, dass Erfahrene auch bereits länger KundInnen der FFG sind, und sich durch langjährige Beratung bereits interne Kompetenzen aufbauen konnten, z.B. Anträge selbst bearbeiten, Professionalisierung der internen Prozesse, etc. Dies sind auch die Kategorien mit größeren Unterschieden zwischen erfahrenen und nicht erfahrenen AntragstellerInnen. Außerdem können erfahrene Antragstellende tendenziell besser einschätzen, was sie von der FFG erwarten können und wo die Grenzen von deren Beratungsangebot liegen.

Ca. $60 \%$ der erreichten Wissenschaftsakteure haben auch an Weiterbildungsleistungen von FFG-EIP im Rahmen der FFG-Akademie teilgenommen, die aus Trainings und Webinaren besteht. In Hinblick auf die Nutzung der wesentlichsten Trainingsangebote zeigt sich, dass erfahrene KundInnen Webinare, Trainingsveranstaltungen und Basisinformationen im Rahmen der H2020 Kompaktveranstaltungen deutlich stärker in Anspruch nehmen als nicht erfahrene KundInnen. 


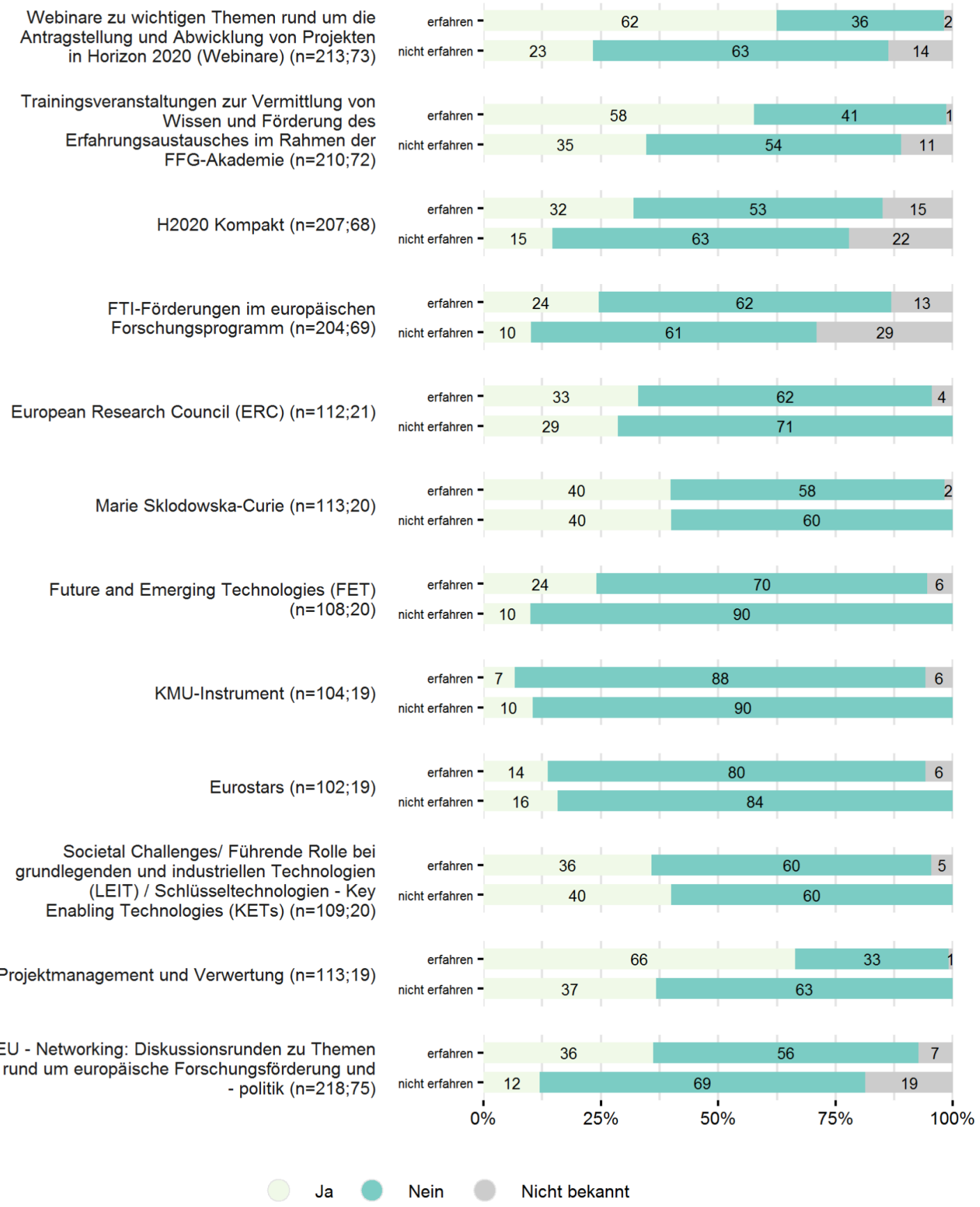

Quelle: Online Befragung, Joanneum Research.

Auch die Bewertung der Trainingsveranstaltungen zeigt ein positives Bild in Hinblick auf die Qualität und Wirkung dieser Unterstützungsleistungen. Zudem gibt es keine signifikanten Unterschiede zwischen erfahrenen und nicht erfahrenen RespondentInnen sowie zwischen Universitäten und außeruniversitären Einrichtungen. 94\% der RespondentInnen bestätigen einen positiven Beitrag zur Professionalisierung der Projektplanung und die Vermittlung der benötigten Qualifikationen für eine erfolgreiche Teilnahme am Rahmenprogramm. 98\% der RespondentInnen sehen die angebotenen Webinare als wertvolle ergänzende Information zur Antragstellung bzw. zum Projektmanagement. 


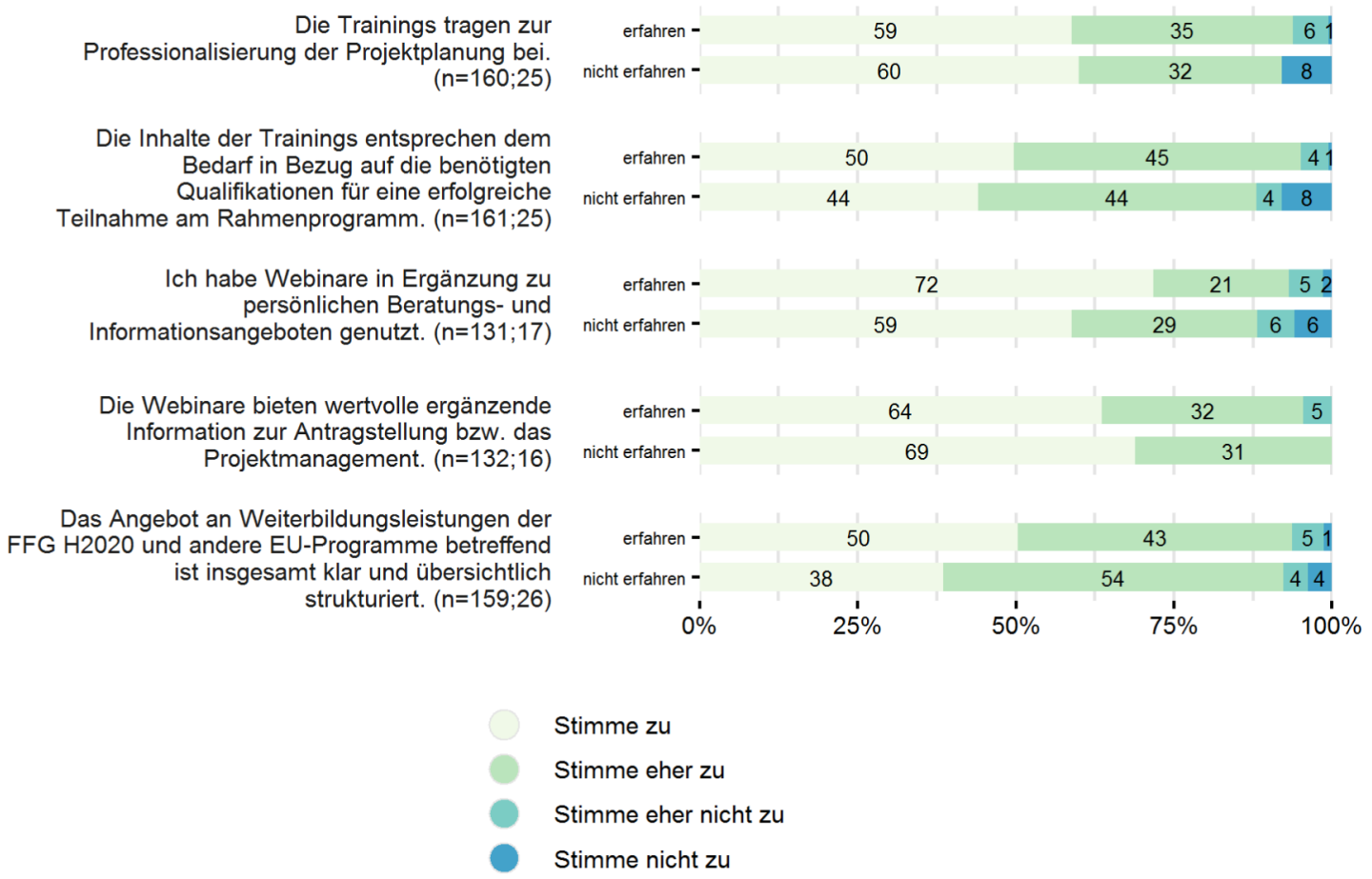

Quelle: Online Befragung, Joanneum Research.

\section{Gesamthafte Bewertung durch die KundInnen}

Insgesamt bewerten 34\% der erreichten Wissenschaftsakteure das Beratungs- und Leistungsangebot von FFG-EIP nach Schulnoten als Sehr gut, $42 \%$ als Gut, $9 \%$ als Befriedigend, $2 \%$ als Genügend und $1 \%$ als Nicht Genügend. $12 \%$ der RespondentInnen haben zu dieser Frage keine Angabe gemacht.

Kritische Anmerkungen in den offenen Textfeldern betrafen insbesondere die Betreuungstiefe und -qualität, wie folgende Aussagen zeigen:

- „Auf konkrete Probleme wird nicht eingegangen, die Themen werden oft allgemein behandelt";

- "Keine klaren Auskünfte bei konkreten Abrechnungsrückfragen";

- "Die individuellen Beratungsleistungen aus vorigen EU Programmen, z.B. FP7 haben mehr gebracht als die ERA Dialoge und strategischen Beratungen. Die Rolle von externen Organisationen in einer verordneten Beraterrolle ist fragwürdig.";

- "Noch speziellere und gezieltere Informationen und Schulungen zu technischen Antragsteilen wie Impact, IPR, und Datenmanagement.".

Auch die rechtzeitige Weitergabe von Informationen und Erreichbarkeit der BeraterInnen, die auch auf der Homepage nicht einfach eruierbar ist, scheint nicht immer gegeben. 


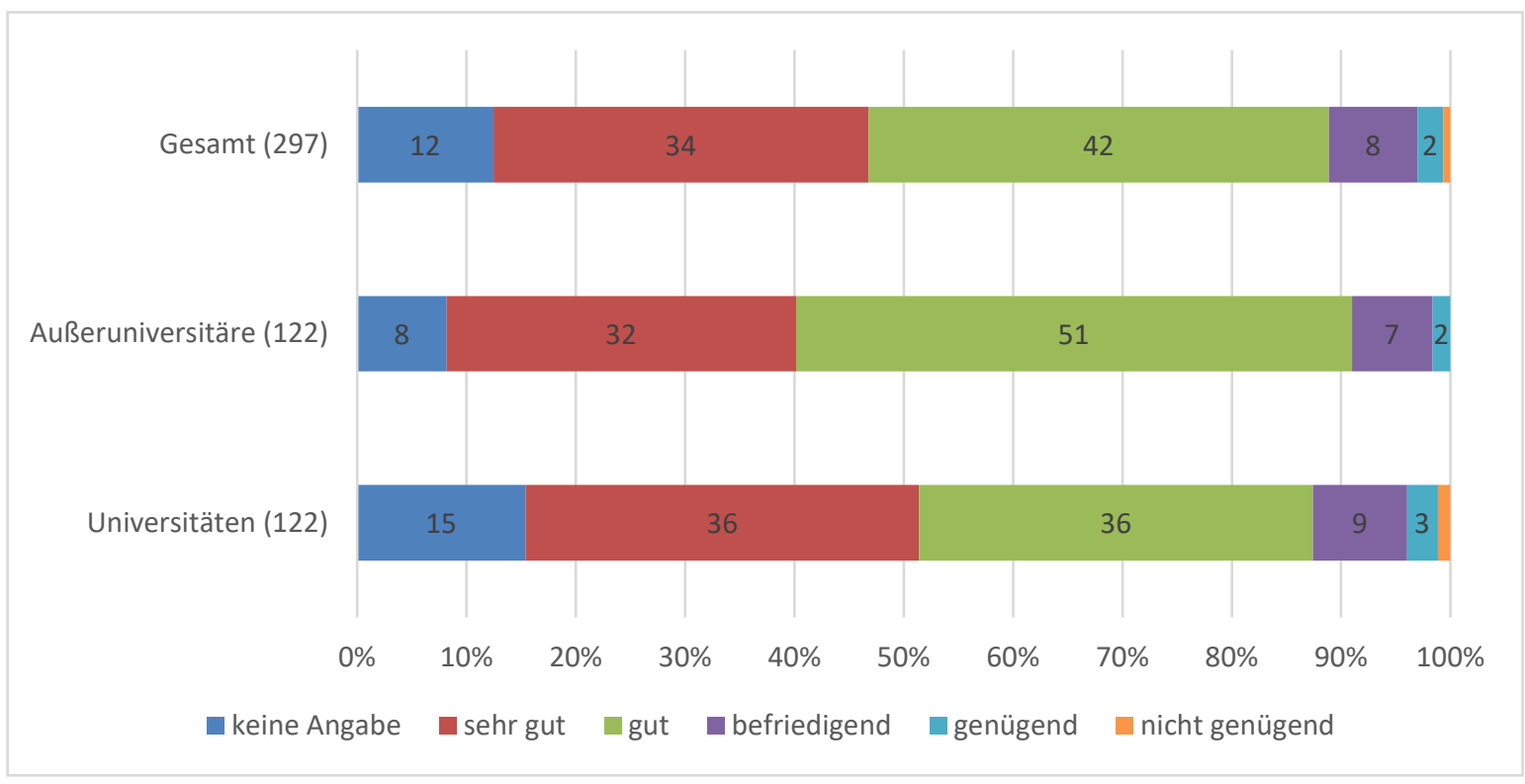

Quelle: Online Befragung, Joanneum Research.

\subsubsection{Unterstützung der Forschungsorganisationen}

Das allgemeine Informations- und Beratungsangebot von FFG-EIP, ergänzt um das strategische Beratungsangebot des ERA-Dialoges bildet auch den Rahmen für die Unterstützung der Universitäten und außeruniversitäre Forschungseinrichtungen auf Organisationsebene. Vor dem Hintergrund des Wirkungsziels, die Selbstbefähigung der Akteure zu stärken, werden in diesem Abschnitt folgende Bereiche analysiert:

- Unterstützungsleistung für ERA und H2020 in den Organisationen,

- Aufgabenverteilung zwischen FFG-EIP und den Organisationsstrukturen,

- die Wirkung des strategischen Beratungsinstruments der ERA-Dialoge.

\section{Die Unterstützungsleistung für ERA und H2020 in den Organisationen}

In Hinblick auf die Unterstützungsleistung ihrer eigenen Organisation sind 58\% der RespondentInnen der Ansicht, dass die Hinzuziehung der hausinternen Forschungsservices an ihrer Organisation die Regel ist. Rund die Hälfte der erreichten TeilnehmerInnen ist der Ansicht:

- dass ihre Organisation professionelle Unterstützung in der Prozessbegleitung von FTI-Projekten bietet,

- über Trends und Entwicklungen im europäischen Forschungsraum informiert und

- aktiv auf die Beratungsleistungen der FFG hinweist.

Lediglich ein Drittel der RespondentInnen ist der Ansicht, dass ihre Organisation regelmäBig Aus- und Weiterbildungsveranstaltungen im Kontext von H2020 anbietet.

Die Befragung zeigt, dass die Unterstützung durch die eigene Organisationsstruktur im universitären Bereich stärker ausgeprägt ist als im Bereich der außeruniversitären For- 
schung, in der das Engagement in ERA/H2020 auch eher den einzelnen Forschenden obliegt. Dies ist insofern beachtlich, als dass der außeruniversitäre Bereich gemessen an der Größe des Sektors eine höhere Antragsintensität und Beteiligung an den RP aufweist.

\section{Abbildung 18: Unterstützung durch die eigene Organisationsstruktur}

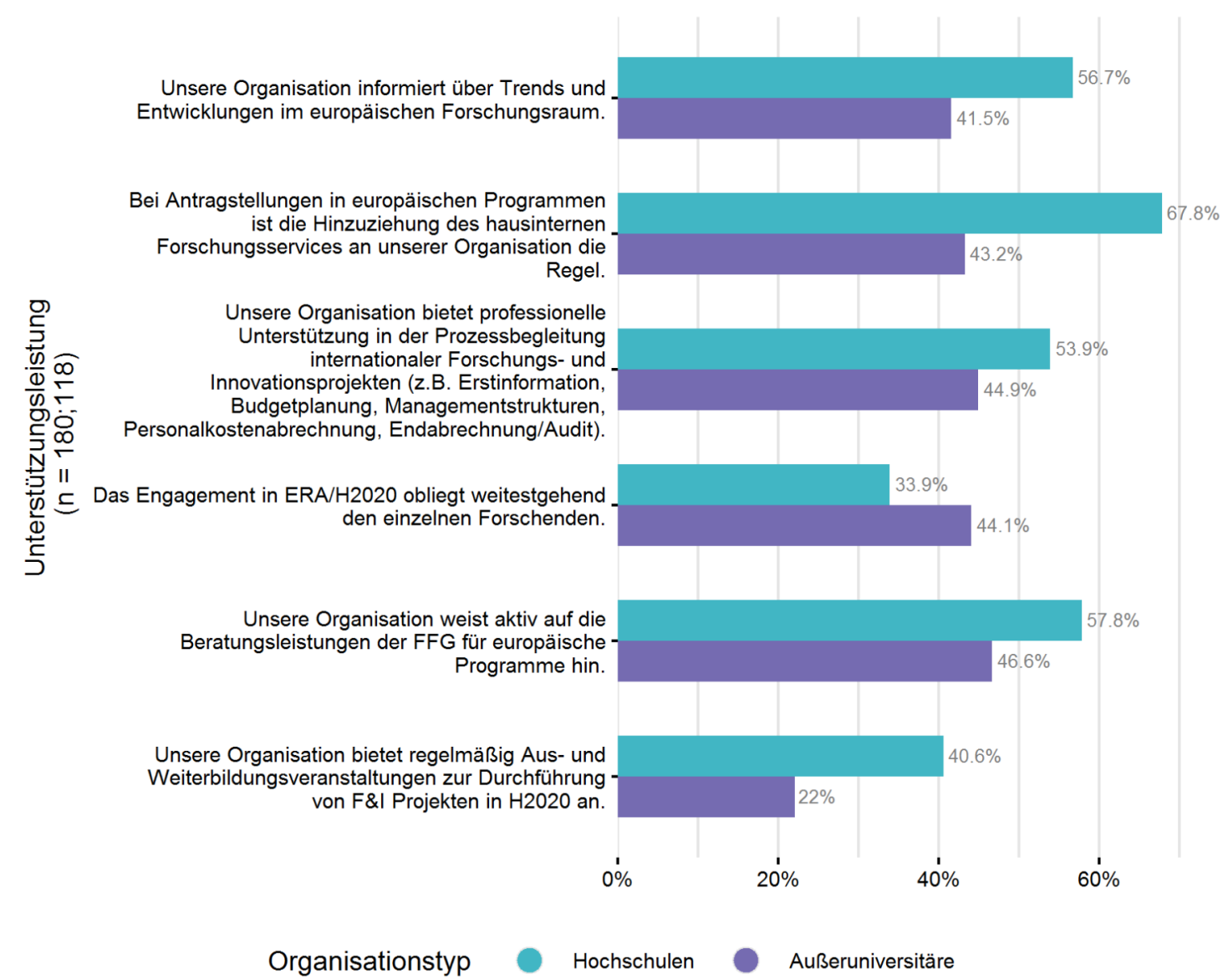

Quelle: Online Befragung, Joanneum Research

Im außeruniversitären Forschungsbereich sind die Unterstützungsstrukturen für das Antragswesen öfter auf Ebene der einzelnen Organisationseinheiten angesiedelt, sodass ein direkter Vergleich nur bedingt möglich scheint. Außerdem handelt es sich bei den außeruniversitären Einrichtungen um eine sehr heterogene Gruppe von Organisationen, die auch eine große Anzahl kleiner Einrichtungen umfasst, die häufig gar keine hausinternen Forschungsservices besitzen und daher stärker von der Unterstützung durch die FFG-EIP abhängig sind als die im Vergleich deutlich größeren Universitäten.

In Ergänzung zur Online-Befragung der FFG-KundInnen zeigen die Ergebnisse der Interviews mit den FoS der Universitäten sowie die Ergebnisse der Fokusgruppeninterviews mit den VizerektorInnen und den Forschungsservicestellen, dass die personelle Ausstattung und somit die Unterstützungskapazität und das Leistungsspektrum der Servicestellen sehr unterschiedlich sind:

- Für den Bereich der Projektberatung werden zwischen 2 und 6 VZÄ je Universität aufgewandt, wobei diese in der Regel nicht nur zu H2020 beraten, sondern auch 
nationale Förderprogramme berücksichtigen. Für europäische Programme und Initiativen werden zwischen 1-4 VZÄ aufgewandt.

- Zusätzlich zur inhaltlichen Projektberatung bieten die Forschungsservicestellen Expertise in den Bereichen Vertragserstellung/Recht, IPR und Technologietransfer, sowie Abrechnungen und Audits.

- Außerdem gibt es Beispiele, in denen für großvolumige und langfristige Fördermaßnahmen (z.B. für eine Beteiligung an den Initiativen des European Institute of Technology) gezielte Support-Aktivitäten aufgebaut wurden, um über das übliche Projektmanagement hinaus die Umsetzung dieser Maßnahmen zu unterstützen.

Ungeachtet der unterschiedlichen Kapazitäten, die nicht mit der Größe der Universität korrespondieren, berichten insbesondere die FoS von einem Kapazitäts- und Wissensaufbau, der in Hinblick auf H2020 und ERA in den letzten Jahren erfolgt sei. Einige Universitäten sahen sich hier bereits in FP7 als gut aufgestellt, gleichzeitig wird jedoch ein Wachsen der Aufgaben bei begrenzten Ressourcen konstatiert.

Das Dienstleistungsspektrum der FoS ist meist generisch, und weist eine geringe inhaltliche Spezialisierung in Hinblick auf thematische Schwerpunkte und Instrumente in $\mathrm{H} 2020$ auf. Die Servicestellen versuchen auf die Bedürfnisse aller ForscherInnen einzugehen und bieten insbesondere folgende Unterstützungsleistungen an:

- Allgemeine Informationen zu Drittmittelprojekten an der Universität (interne Regelungen, Personalkosten, Overheads etc.),

- Zuordnung der Projektidee zu geeigneten Förderprogrammen,

- Konkrete Hilfestellungen in Bezug auf Antragstellung, Kostenberechnung und Verbesserung der Projektanträge,

- Beratung zu verschiedenen nationalen, europäischen und internationalen Programmen.

Um auch in inhaltlicher Sicht das gesamte nationale und internationale Programmportfolio abzudecken, wird von den Servicestellen ein enger Kontakt mit den ExpertInnen der FFG und den Delegierten als notwendig erachtet, um 1) inhaltlich- thematische Expertise zu erlangen, und 2) frühzeitig auf programmatische Änderungen reagieren zu können.

FFG-EIP stellt daher für inhaltliche, thematisch orientierte Beratung von FTI-Akteuren meist die Hauptinformationsquelle der Universitäten und Forschungsorganisationen dar.

Neben der FFG greifen die FoS der Universitäten aber auch auf andere Informationsquellen zurück. Wesentliche Informationsträger, die durchwegs in Ergänzung zu FFG-EIP wahrgenommen werden, sind:

- Die KoWi - Kooperationsstelle EU der Wissenschaftsorganisationen der Deutschen Forschungsgemeinschaft (DFG),

- Die Nationalen Kontaktstellen in Deutschland (Projektträger),

- Das Netzwerk des Forschungsmanagements in Europa (EAMA),

- Direkte Kontakte zu Policy-Officers in der Europäischen Kommission,

- EURAXESS und IDEALIST.

An den einzelnen Organisationen wird die Aufgabenteilung mit FFG-EIP letztlich sehr unterschiedlich umgesetzt. Generell wird FFG-EIP als wichtiger thematischer Partner der FoS und als zentrale Beratungsstelle für die Servicestellen gesehen. FFG-EIP hätte mit 
seinem Personalstand die Möglichkeit sehr spezifisches thematisches/fachliches Knowhow aufzubauen, während die Servicestellen durch ihre oftmals geringe Größe eher Allrounder sein müssen.

So fokussiert bspw. eine Servicestelle speziell auf den Europäischen Forschungsrat (ERC) und die Marie-Skodlowska-Curie (MSCA) Instrumente, während Anfragen bzgl. aller anderen Programme recht schnell an FFG-EIP weitergereicht werden. Eine andere Forschungsservicestelle sieht sich selbst als erste Anlaufstelle für alle Programme und schickt AntragstellerInnen bei Bedarf (speziell für den Proposalcheck) an FFG-EIP weiter bzw. fragt bei FFG-EIP an. Eine dritte Forschungsservicestelle betreut vornehmlich KoordinatorInnen, während im Falle von Beteiligungen als ProjektpartnerIn die entsprechenden Anfragen an FFG-EIP weitergereicht werden. Wiederum eine andere Servicestelle leistet über den ganzen Projektzyklus Hilfestellungen, die auch das Mitschreiben an Anträgen beinhaltet und spricht auch einzelne ForscherInnen/Departments bei geeigneten Calls direkt an.

Der persönliche Austausch, die Qualität der Beratungsleistungen und die inhaltliche Expertise von FFG-EIP werden von Servicestellen und VizerektorInnen überwiegend sehr positiv wahrgenommen. Das Bemühen der FFG-EIP aktiv an Forschungsstellen heranzutreten, scheint aus Sicht der FoS vorhanden zu sein.

Durch die langjährige Zusammenarbeit mit den FoS wurden eine Vertrauensbasis und gute Kooperationsverhältnisse aufgebaut, und auch im europäischen Vergleich werden die FFG Services von den FoS gut bewertet.

Zudem werden mit den Forschungsorganisationen auch spezifische Veranstaltungen und Trainings in Kooperation mit der FFG durchgeführt, die dafür auch in die Bundesländer kommt. Auch die Aufgabenteilung zwischen FFG und den FoS wird als prinzipiell gut bewertet. Besonders hervorgehoben wurde dabei der Proposalcheck.

Teilweise kritisiert wurde, dass Trends zu zukünftigen Entwicklungen/Themen in H2020 sowie die Informationsweitergabe von nationalen Delegierten sehr verhalten weitergegeben werden, andererseits aber auch eine Informationsüberflutung stattfinden würde. Diese Aussagen könnten Bedarf nach einer besser kuratierten und zielgruppenspezifischeren Informationsweitergabe über neu zu entwickelnde Kanäle aufzeigen.

Zu Beginn von H2020 wurde zudem die Trennung zwischen First- und Second-Level

Beratung aus Sicht der FoS stark von der FFG beworben, jedoch ohne klare Abgrenzung der Zuständigkeitsbereiche und genauer Definition. Dies hat in den FoS zuerst Angst ausgelöst: "Was kommt da auf uns zu?" „Um diesen Aufgaben gerecht zu werden, müssten wir das Personal um das 2- bis 3-fache aufstocken?". Im Verlauf von H2020 ist der erwartete Schock jedoch ausgeblieben und die FoS sind mit der Qualität und Geschwindigkeit der Leistungen von FFG-EIP zufrieden. Dies lag vor allem daran, dass die FFG eine flexible Auslegung und Handhabung der Differenzierung zwischen First und Second Level Support verfolgte, um auf die unterschiedlichen Bedürfnisse der Organisationen einzugehen.

Vor dem Hintergrund einer allgemeinen Zufriedenheit mit den Dienstleistungen der FFG sehen die befragten Einrichtungen aber durchaus Bedarf an einer Weiterentwicklung des Dienstleistungsportfolios der FFG in Hinblick auf 
- die Bereitstellung von Hintergrundwissen zu den Arbeitsprogrammen von H2020,

- die Aufbereitung von Call-Inhalten die eine zielgruppenorientierte Ansprache an den Einrichtungen ermöglicht, und

- die Weitergabe zu strategischen Entwicklungen im Instrumentenportfolio und zukünftigen Rahmenprogrammen.

Neben der verstärkten Analyse von Trends in H2020 und der Reflexion über die Bedeutung dieser Entwicklungen, erachten die meisten der befragten FoS Basis-Leistungen wie den „Proposal-Check" und die gemeinsame Konzipierung von Trainings und Veranstaltungen als essenziell.

Konstatiert wurde auch, dass sich die FFG Informationsleistungen eher an die sehr informierte/erfahrene Community zu richten scheine, und FFG-EIP sehr spezifisches Wissen anbiete, bei dem man vorher wissen müsse, was man möchte und an wen man sich wendet. Vor diesem Hintergrund, wurden folgende Verbesserungspotenziale angeführt:

- Klärung der generellen Ausrichtung des First- und Second Level Supports;

- Neuausrichtung der Homepage und ein besserer Zugang zu zentralen Informationen (z.B. Factsheets über Formalitäten, Trainingsunterlagen etc.);

- Bessere Zielgruppenorientierung der Services betreffend erfahrener und weniger erfahrener Communities und KundInnen;

- Bessere Spezifizierung der Leistungen der FFG, z.B. in Leistungsmodulen/-paketen für spezifische Gruppen;

- Höhere Geschwindigkeit in Bezug auf die Weitergabe von Informationen;

- Verbesserung der strategischen Positionierung der FFG, als FTI-politischer Akteur, der Lobbying für die Interessen der österreichischen Position betreibt.

\section{Die Rolle von FFG-EIP zur strategischen Unterstützung der Universitäten} und Forschungsorganisationen in ERA

Universitäten und Forschungsorganisationen sollen von der FFG auch darin unterstützt werden, aktiv ihre Internationalisierungsstrategien und strategische Beteiligungen in den Rahmenprogrammen, dem European Institute of Technology und strategischen (multilateralen) FTI-Partnerschaften (JTIs, ERA-NETs etc.) voranzutreiben.

Neben den Unterstützungsleistungen der FFG stellt insbesondere das österreichweit angewandte Instrument der Ziel- und Leistungsvereinbarungen für Universitäten einen Ansatzpunkt zur Profilierung österreichischer Universitäten im Europäischen Forschungsraum dar, während im außeruniversitären Bereich teilweise individuelle Zielsetzungen auf Ebene der Organisationen zur Geltung kommen.

Als Einstiegspunkt in die Analyse wurde daher gefragt, inwiefern die im Rahmen der Leistungsvereinbarungen vereinbarten Zielsetzungen, das Engagement in ERA und H2020 betreffend, innerhalb der Universität kommuniziert werden, und eine Richtschnur für die Planung eigener Aktivitäten darstellen. Die Befragung der ForscherInnen aus dem Universitätsbereich zeigt, dass 
- $\quad 57 \%$ der Ansicht sind, dass die im Rahmen der Ziel- und Leistungsvereinbarungen vereinbarten Zielsetzungen, das Engagement in ERA und H2020 betreffend, innerhalb der Universität aktiv kommuniziert werden, und

- $50 \%$ der Ansicht sind, dass diese Vereinbarungen auch eine Richtschnur für die Planung der eigenen Aktivitäten sind.

Lediglich $17 \%$ sehen jedoch eine Vereinbarung von Zielen auf Ebene der Organisationseinheit (Fakultät/Institut etc.) in Hinblick auf das Engagement in ERA und H2O20 als gegeben an.

Ergänzend zur Einschätzung der ForscherInnen, zeigt die Delphi-Befragung von FTI-ExpertInnen, dass die Mehrheit dem Steuerungsinstrument der Ziel- und Leistungsvereinbarungen neutral gegenübersteht (56\%). Ein gleich großer Anteil von $22 \%$ der erreichten ExpertInnen sieht in den Ziel- und Leistungsvereinbarungen ein geeignetes bzw. nicht geeignetes Instrument, um auf nationaler Ebene die Beteiligung von Universitäten und Forschungsorganisationen in ERA zu stärken.

Jene ExpertInnen, die Ziel- und Leistungsvereinbarungen kritisch gegenüberstehen, betonen die Überfrachtung der Ziel- und Leistungsvereinbarungen, die Begrenztheit bürokratischer Verordnungen als Steuerungsinstrument und die Bedeutung von Anreizsystemen, die noch zu entwickeln wären. Aber auch ExpertInnen, die den Ziel- und Leistungsvereinbarungen für die Profilbildung in ERA positiv gegenüberstehen, betonen die Bedeutung der von der Universität selbst zu wählenden strategischen Ausrichtung der Universität, die in den Vereinbarungen Berücksichtigung finden müsste.

Für das verantwortliche Bundesministerium für Bildung, Wissenschaft und Forschung, ermöglichte das System der Ziel- und Leistungsvereinbarungen in einen koordinierten Dialog zur Positionierung der Hochschulen im Europäischen Forschungsraum zu treten. Ähnlich wie bei den Kapazitäten der Forschungsservicestellen zeigt sich jedoch, dass die strategische Schwerpunktsetzung in Hinblick auf die Beteiligung am europäischen Forschungsraum nicht einheitlich ist.

Insbesondere Volluniversitäten und medizinische Universitäten sehen keine Möglichkeit, strategische Kapazitäten für die Beteiligung an europäischen Programmen aufzubauen. Möglichkeiten zur strategischen Schwerpunktsetzung wurden hier auch vor dem Hintergrund von Förderwahrscheinlichkeiten von 2\% bis 5\% (z.B. HEALTH / Klinischer Bereich) als nicht zielführend erachtet.

Demgegenüber sehen einige spezialisierte Universitäten (insbesondere technische Universitäten) die Entwicklung von internationalen FTI-Strategien und den Aufbau von Support Stellen gerade deshalb als wichtig an, um einen Netzwerk-Aufbau betreiben zu können, der es ihnen erlaubt im Vorfeld von Calls und der Entwicklung von neuen Instrumenten frühzeitig in die richtigen Konsortien und Anträge zu investieren und so die Förderwahrscheinlichkeit bedeutend zu erhöhen.

Auch die Universitäten im Kunst- und Kulturbereich sind in Begriff Unterstützungskapazitäten aufzubauen. Dies geschieht vor allem vor dem Hintergrund, dass die FFG in diesem Bereich kaum in der Lage zu sein scheint (zu geringe Kapazitäten), den speziellen Bedarf der Kunstuniversitäten zu decken und es nur punktuelle Verbindungslinien zur 
FFG gibt. Dies liegt zum einen daran, dass es für die Kunstuniversitäten tendenziell weniger relevante Programmschienen in $\mathrm{H} 2020$ gibt als für breiter aufgestellte Universitäten. Zu den für Universitäten besonders relevanten Programmen der Säule 1 werden die Kunstunis ebenso wie alle anderen Zielgruppen beraten und betreut.

Insgesamt erscheinen die Internationalisierungsstrategien der Universitäten für die inhaltliche Ausrichtung der Servicestellen der Universitäten bisher kaum handlungsrelevant, da ein Großteil der internationalen Forschungsanträge nach wie vor "bottom-up" erfolgt.

Die befragten Akteure konstatieren, dass die Bedeutung strategischer Netzwerke und Kooperationen auf Organisationsebene steige, und vor diesem Hintergrund beispielsweise Universitäten in England, den Niederlanden und Belgien vermehrt in die Professionalisierung der Servicestrukturen für Netzwerkbildung und strategische Antragsentwicklung investieren, die tatsächliche Reaktion darauf, scheint jedoch abhängig vom Grad der Organisationswerdung der Universität, die es in unterschiedlicher Art und Weise erlaubt, TopDown und Bottom-Up Prozesse in einer gemeinsamen Vorgehensweise zu verbinden.

Dass die Bedeutung aktiver Beteiligungsstrategien vermutlich weiter steigen wird, legen auch die Ergebnisse der Delphi-Befragung nahe. 70\% der befragten ExpertInnen vertreten hier die Ansicht, dass die österreichischen Universitäten und auch die außeruniversitären Forschungseinrichtungen ihre Anstrengungen forcieren müssen und verstärkt aktive Beteiligungsstrategien entwickeln sollen. Hervorgehoben werden die Bedeutung von Netzwerken und Kooperationen, die eine Strategieentwicklung auf organisatorischer Ebene erfordert und die Erarbeitung von Alleinstellungsmerkmalen ermögliche. Eine gute Kenntnis über eigene Stärken und innerorganisatorische Konsultationsprozesse werden dafür ebenso als notwendig erachtet, wie eine österreichische Gesamtstrategie zur Forschung an Universitäten und außeruniversitären Forschungsorganisationen. Kritisch gesehen wird jedoch auch, dass eine erhöhte Antragstätigkeit auch mit geringeren Erfolgswahrscheinlichkeiten und höheren Kosten verbunden sein könnten. Auf universitärer Ebene wurde auch die Befürchtung geäußert, dass eine stärkere Industrieorientierung zu Lasten der Grundlagenforschung führen könnte. Ebenso könnte die unabhängige Rolle der ForscherInnen durch die Universität beeinflusst werden. Für den außeruniversitären Sektor wird die Rolle als Bindeglied zur Wirtschaft in Partnerschaften betont. Strategien werden als geeignetes Instrument gesehen um "zufällige" Beteiligungen zu vermeiden, wobei hier einige ExpertInnen bereits eine stärkere Profilbildung als im Universitätsbereich verorten.

\section{Erkenntnisse zu den ERA-Dialogen ${ }^{13}$}

Vor dem Hintergrund der derzeit verfügbaren Kapazitäten der Universitäten und Forschungseinrichtungen versucht die FFG in Ergänzung zum oben diskutierten Informa-

13 Die Dokumentationen der Feedback-Gespräche wurden dem Evaluierungsteam von der FFG in anonymisierter Form zur Verfügung gestellt. 
tions-, Beratungs-und Trainingsangebot die Profilbildung und Positionierung der österreichischen Wissenschaft im Europäischen Forschungsraum über den ERA-Dialog zu unterstützen.

Sowohl die Feedback Gespräche der FFG als auch die Interviews und Fokusgruppen zeichnen ein überwiegend positives Bild von Qualität und Relevanz des ERA-Dialoges.

Die Feedback Gespräche der ERA-Dialoge zeigen, dass die teilnehmenden Organisationen, die Zusammenarbeit mit der FFG als konstruktiv und fruchtbar empfanden, was insbesondere am interaktiven Format und dem ermöglichten Dialog zwischen der FFG und dem Management der Einrichtungen lag.

Zu Beginn der ERA-Dialoge als teilweise unklar empfunden wurden dessen Zielsetzungen und Relevanz für die beteiligten Akteure (Vizerektorate für Forschung und Forschungsservicestellen), wie folgende Aussagen zeigen:

- "Es sei schwer zu beantworten, ob die Erwartungen erfüllt wurden, denn das Ziel hat sich erst in gemeinsamer Arbeit konkretisiert und entwickelt";

- "Zunächst hatten wir andere Vorstellungen vom ERA-Dialog, er erschien zunächst wenig operativ, jedoch im Laufe der Zusammenarbeit ... war überrascht";

- „Die Relevanz der Gespräche war auch nicht unmittelbar bewertbar, aber es gibt eine "Umweg-Rentabilität" (z.B. Zusammenarbeit zu Themenforschungsstrategien)".

Der Nutzen der ERA-Dialoge lag zusammenfassend aus Sicht der teilnehmenden Universitäten vor allem in der Außensicht auf die eigene Organisation und der Zeit für gemeinsame Reflexion und Supervision. So wurden insbesondere von den VizerektorInnen für Forschung die ERA-Dialoge als sehr nützlich empfunden, da diese es ermöglichten, auf strategischer Ebene zu diskutieren um im Anschluss daran bei Bedarf in die Tiefe gehen zu können.

Die in den ERA-Dialogen vermittelten Informationen stellten vor dem Hintergrund begrenzter Kapazitäten auch eine Zeitersparnis für die Management-Ebene der Universität dar, da sie "konzentrierte und für das Management aufbereitete, gut rezipierbare und verständliche Information" vermittelt.

Die FFG wurde im Rahmen des ERA-Dialoges von den VizerektorInnen auch als unabhängiger Partner wahrgenommen, mit guter Kenntnis der Rahmenbedingungen und der gesamten FTI-Landschaft (nicht nur Programme), ohne "Geltungsdrang" und Eigeninteresse zum Forschungsthema. Dem gegenüber wiesen die Servicestellen der Universitäten auf ein Vertrauensproblem zwischen Universitäten und FFG hinsichtlich der Rolle als Beratungsstelle der Universitäten als auch der Ministerien hin. Befürchtet wurde insbesondere, dass die FFG im Zuge ihrer Beratungstätigkeit für Universitäten Daten über diese sammelt, die in der Folge für die Unterstützung des Ministeriums genutzt werden könnten. Durch eine unreflektierte Weitergabe von Datenmaterial könnten Universitäten beschädigt werden und das Vertrauensverhältnis zwischen FFG und den Universitäten könne sich nicht weiterentwickeln.

Auf Ebene der Organisationsleitung bewirkten die Gespräche jedenfalls eine Erhöhung der Awareness für europäischen Programmen und Initiativen. Die VizerektorInnen berichteten beispielsweise von einer besseren Einschätzung der Relevanz und Bedeutung 
einzelner europäischer Instrumente, einer differenzierteren Bewertung von Beteiligungsbzw. Erfolgskennzahlen, oder einer besseren Einschätzung der Bedeutung von Gremien (Beratungsgremien, Steuerungsgremien etc.) in den Governance-Strukturen der strategischen Partnerschaftsprogramme. In einzelnen Fällen wurden auf Basis der strategischen Informationen des ERA-Dialoges auch organisatorische Umsetzungsmaßnahmen gesetzt. So berichtete eine Universität, dass auf Basis des ERA-Dialoges in zwei ausgesuchten Bereichen gezielt in Personen investiert wurde, um die Beteiligungen auf EU-Ebene in diesen Bereichen zu forcieren.

Die Bedeutung das EU-Thema (strategisch) auch auf Ebene der Fakultäten und Institute zu verankern und die interne Kommunikation diesbezüglich zu stärken wurde erkannt, gleichzeitig jedoch auch als existierende Lücke wahrgenommen. Diese Lücke bestätigen auch die Einschätzungen der Servicestellen und die Ergebnisse der Online Befragung. So wurde bspw. im Bereich der Volluniversitäten die Möglichkeit eine EU-Beteiligungsstrategie auf Organisationsebene herzustellen aufgrund des Fehlens einer "gemeinsamen Identität" und einer „zu großen Heterogenität der Fakultäten" als schwierig erachtet.

Die Servicestellen konstatierten zwar einen eher geringen Nutzen des ERA-Dialoges für ihre konkrete Arbeit, strichen jedoch eine erhöhte Aufmerksamkeit der Vizerektorate für ihre eigene Arbeit sowie die Reflexion zu Stärken und Schwächen der Einrichtungen hervor.

Die Erhebungen zeigten auch möglichen Änderungsbedarf in Hinblick auf Ausrichtung und Umsetzung der ERA-Dialoge auf. Von den Universitätsleitungen und den Forschungsservicestellen wurde angemerkt, dass ein stärkeres Augenmerk (an den Universitäten) auf die Verbindung zwischen der strategischen und der operativen Ebene zu legen sei. Angeregt wurde daher die Konzeption thematischer und interdisziplinärer Workshops zu Querschnittsthemen mit z.B. mehreren Instituten oder Fakultäten. In mehreren Feedback-Gesprächen wurde auch zum Ausdruck gebracht, dass es nun wichtig wäre die derzeitige Dynamik aufrecht zu halten und Verbindlichkeit zu schaffen. Dies könne einerseits durch eine kürzere Frequenz der Meetings, andererseits durch die Definition eines klaren Maßnahmenkatalogs auf Seiten der FFG und der Universitäten mit kurz-, mittel- und langfristigen Zielen erreicht werden.

\subsection{Schlussfolgerungen und Empfehlungen}

Das Leistungsspektrum von FFG-EIP für den Bereich Wissenschaft beinhaltet sowohl spezifische Informations- und Beratungsleistungen für Antragsteller in H2020 als auch Betreuungsinstrumente für "Enabler" oder „Policy-orientierte" Zielgruppen in den Forschungsorganisationen. Zu letzteren zählen insbesondere die Forschungsservicestellen und die Vizerektorate für Forschung an den Universitäten.

\section{Relevanz der Leistungen von FFG-EIP}

Die KundInnen der FFG aus dem Wissenschaftsbereich konsumieren insbesondere Leistungen der FFG, um Fragen zu Arbeitsprogrammen und Calls, Trends und Entwicklungen zu H2020 sowie Fragen bei der Erstellung eines Projektantrages zu beantworten. Das 
entsprechende Leistungsportfolio der FFG in den Bereichen 1) Bewusstseinsbildung \& Information, 2) Programm- und Projektberatung sowie 3) strategische Beratung und ERAOrientierungswissen trifft den Bedarf der FFG-KundInnen sehr gut.

Die Bedarfslagen der Forschungsakteure und die Position der FFG als der zentrale Partner für Unterstützung zu H2020 spiegeln die hohe Relevanz der FFG-Aktivitäten wider. Dies trifft insbesondere auf die persönlichen Beratungsleistungen und die Angebote zur Weiterbildung im Rahmen der FFG-Akademie zu, die von den befragten KundInnen überwiegend sehr positiv bewertet werden.

\section{Effektivität der Leistungen für KundInnen}

Das individuelle Dienstleistungsspektrum von FFG-EIP ist insgesamt effektiv, trägt zur Steigerung der Antrags-Kompetenz der ForscherInnen bei und erhöht die Erfolgswahrscheinlichkeit in der Antragstellung. Es existieren jedoch Potenziale zur Erhöhung der Effektivität, die durch Anpassungen im Leistungsportfolio erzielt werden können.

Die Instrumente der EIP-Toolbox sind so gestaltet, dass sie allen KundInnen, unabhängig vom Erfahrungshintergrund mit den Rahmenprogrammen, der institutionellen Zugehörigkeit und der Funktion innerhalb ihrer Organisation offenstehen. Dies garantiert auch den Zugang zu neuen Zielgruppen, die erstmals mit dem Rahmenprogramm in Kontakt kommen.

Die empirischen Erhebungen haben gezeigt, dass erfahrene KundInnen das volle Dienstleistungsspektrum der FFG-Services nutzen. Das verfügbare Informationsangebot und die Beratungsleistungen der FFG tragen dazu bei, dass sich die KundInnen gut informiert über Einreichmöglichkeiten in EU-Programmen fühlen und ihre Antragskompetenz steigern konnten.

Weniger erfahrene KundInnen nehmen insbesondere die FFG-Services auf der Website, Trainings und Webinare deutlich seltener in Anspruch als erfahrene KundInnen. Qualitative Rückmeldungen aus Interviews und Fokusgruppen zeigen, dass insbesondere Potenziale zur verbesserten Darstellung und Strukturierung des Informations- und Beratungsangebotes existieren.

\section{Empfehlung: Zielgruppengerechte Ansprache von ForscherInnen}

Um den Zugang zu den Dienstleistungen von FFG-EIP zu erhöhen und die Effektivität der angebotenen Dienstleistungen zu steigern, sollte eine zielgruppengerechte Ansprache von erfahrenen und weniger erfahrenen ForscherInnen erfolgen. Schwerpunkte des Beratungsangebotes sollten besser aufgezeigt und deren potentielle Nutzen für die unterschiedlichen Zielgruppen sollte klar dargelegt werden. Dies könnte durch Leistungsmodule/-pakete für spezifische Gruppen geschehen, in denen entsprechende Leistungen zielgruppengerecht und übersichtlich gebündelt werden. Dadurch könnten auch neue Stakeholdergruppen, die in Zukunft an Bedeutung im Rahmenprogramm gewinnen könnten (z.B. zivilgesellschaftliche Organisationen), stärker in den Fokus der Aufmerksamkeit gerückt werden. 


\section{Empfehlung: Neugestaltung des Internet-Auftritts}

Eine Neugestaltung des Internet-Auftritts von FFG-EIP erscheint notwendig, um Informationen besser an die angesprochenen Zielgruppen vermitteln zu können. Die Homepage von FFG-EIP bietet derzeit eine Fülle an Informationen und guten Inhalten, ist jedoch unübersichtlich und wenig benutzerfreundlich gestaltet. So sind die Suchfunktion, das Auffinden von Programminformationen zu H2020 sowie von Kontaktpersonen schwierig. Unklar ist auch, an welche Stellen/Personen sich man in Hinblick auf Beratungen, NCPs, FFG Akademie und deren Anmeldungsprozesse wenden kann. Der derzeitige Internet-Auftritt von FFG-EIP und von EU-PM legt eine grundlegende Neuausrichtung der FFG-Website insgesamt nahe, in deren Rahmen folgende Aspekte berücksichtigt werden sollten:

\section{Einfacher, intuitiver, zielgruppenspezifischer Zugang:}

- Differenzierung nach Relevanz der Information

- Differenzierung zwischen Basisinformationen (Programminfo) und detaillierten Fragen (z.B. in Bezug auf Antragstellung und zugehörige Services der FFG)

- Bessere Platzierung der Suchfunktion der FFG

2. Verbesserung der Navigationsstruktur:

- Sofort-Einstieg zu europäischen Förderprogrammen und zugehörigen FFG Service-Angeboten

- Einführung der kontextabhängigen Benutzerführung sowie eine bessere Darstellung der angebotenen Services: Leistungen wie die "Partnersuche", die im FFG-Portfolio keine oder nur eine untergeordnete Rolle spielen werden derzeit als zentrale Leistungen hervorgehoben, Services wie die verschiedenen Beratungsinstrumente und EU$\mathrm{PM}$ sind versteckt.

- Bessere Identifikation zentraler AnsprechpartnerInnen: Identifikation und Erreichbarkeit von NCPs und FFG-ExpertInnen sind unklar. Schwerpunkte der Beratungsexpertise ebenso. Beispielsweise könnte der/die richtige AnsprechpartnerIn für eine Leistung/ein Programm jeweils auf der Seite aufgelistet werden.

\section{Effektivität und Wirkung der Informations- und Beratungsdienstleistungen}

Die Selbstbefähigung der Forschungsorganisationen in Hinblick auf den Europäischen Forschungsraum und H2020 zu unterstützen ist eine neue Aufgabe für FFG-EIP. Die im Rahmen der Evaluierung durchgeführten Analysen haben gezeigt, dass der Erfahrungshintergrund der ForscherInnen und die Leistungsfähigkeit der Organisationsstrukturen sehr unterschiedlich sind. Die Mehrheit der erreichten FFG-KundInnen (58\%) ist der Ansicht, dass die Hinzuziehung der hausinternen Forschungsservices an ihrer Organisation mittlerweile die Regel ist. Die Kapazitäten der Universitäten sind jedoch sehr heterogen in Hinblick auf 1) die Kapazitäten zur Unterstützung von ForscherInnen für H2O20 und Partnership-Programme, 2) den Status der Organisationsentwicklung und zugehörige Strategiebildungsprozesse sowie 3) inhaltlich/thematische Unterstützungsmöglichkeiten. Gleichzeitig steigt die Bedeutung von europäischen FTI-Netzwerken und strategischen Partnerschaften in den europäischen Rahmenprogrammen und es besteht sowohl für den außeruniversitären Bereich als auch für universitären Bedarf, verstärkt internationale Be- 
teiligungsstrategien zu entwickeln. Als beratende Unterstützerin wird FFG-EIP überwiegend als unabhängige Partnerin wahrgenommen, die insbesondere thematische Kompetenzen und Wissen zu spezifischen Instrumenten von H2020 und Partnership-Programmen zur Verfügung stellt.

Die Zielsetzung, zur Selbstbefähigung der Forschungsorganisationen über Stärkung des "First Level Supports" an Universitäten und die Begleitung von Strategieprozessen beizutragen ist daher relevant, jedoch mit Risiken behaftet. Zum einen benötigt die FFG Hintergrundwissen zum Leistungsspektrum und zur Spezialisierung der Forschungsorganisationen, um effektiv zur Beratung der Organisationen beitragen zu können. Zum anderen besteht die Gefahr, dass grundlegende Beratungsleistungen weiterhin an die FFG ausgelagert werden, obwohl ein Kapazitätsaufbau und eine strategische Fokussierung der Wissenschaftsorganisationen notwendig sind.

\section{Empfehlung: Spezifische Leistungen für Servicestellen entwickeln}

Hintergrundwissen zu den Arbeitsprogrammen von H2020 sollten zeitnah, kurz und konkret an Servicestellen weitergegeben werden. Das inkludiert die oft informellen Diskussionen im Vorfeld, Einschätzungen was in einem Call wirklich gefragt sein wird, und andere Hintergrundinformationen. Da diese Informationen oft begrenzt gültig sind (z.B. Arbeitsdokumente), sollte die FFG eine mit den Delegierten abgestimmte (informelle) Policy entwickeln, welche die Informationsweitergabe regelt.

Call-Inhalte und Work-Programmes sollten so aufbereitet werden, dass diese besser und schneller an die FTI-Community kommuniziert werden können.

Informationen zu Entwicklungen rund um FP9 (wie z.B. Ergebnisse aus informellen Meetings, aktuelle Diskussionen, worauf sollte man in Zukunft achten) sollten schneller verfügbar gemacht werden.

Daten und Informationen, Reports, etc. sollen nicht nur aus dem Link/Bericht selbst bestehen, die FFG sollte auch die wichtigsten Punkte kurz hervorheben.

Die Forschungsservicestellen und Vizerektorate der Universitäten sind zentrale Ankerpunkte, über die das Empowerment der Forschungsorganisationen weiterentwickelt werden kann. Die Analysen haben gezeigt, dass die FFG prinzipiell über die notwendige inhaltliche Kompetenz und Unabhängigkeit verfügt, um Servicestellen und Vizerektorate effektiv in strategischen Belangen zu beraten. Teilweise existiert diesbezüglich jedoch ein Vertrauensproblem, da die FFG sowohl für die Universitäten, als auch für das verantwortliche Ministerium Beratungsfunktionen wahrnimmt. Einerseits ist das Wissen, das der FFG über die Universitäten zur Verfügung steht, potenziell von hoher Relevanz für die Zielund Leistungsvereinbarungen als dem wesentlichen Instrument zur strategischen Steuerung der Universitäten. Andererseits bilden die Leistungen von FFG-EIP und insbesondere die ERA-Dialoge ein wichtiges Element zur Unterstützung der Universitäten im Rahmen eines offenen und vertrauensvollen Austauschs, das von den Organisationen in unterschiedlichem Ausmaß wahrgenommen werden kann. Dieser potenzielle Zielkonflikt ist inhärenter Teil der Beauftragung, dem in der Praxis dadurch begegnet werden kann, dass die Weitergabe von Erfahrungen aus den verschiedenen Formen des Austauschs mit den Universitäten nur in aggregierter und gefilterter Form erfolgt. 
In Hinblick auf die Leistungen von FFG-EIP existiert, abgesehen vom thematischen Fokus der FFG Beratung, keine strikte Differenzierung zwischen dem First-Level Support, der an den Organisationen selbst wahrgenommen werden soll, und dem Second Level Support der FFG. Dies erscheint angesichts der unterschiedlichen Bedarfslagen der einzelnen Unis und Forschungsservicestellen plausibel.

Für die Forschungsservicestellen existiert über die elektronische FoS-Plattform und die Teilnahme der FFG an Treffen der ARGE Forschungsförderung hinaus kein spezifisches Dienstleistungsangebot zur Stärkung der Beratungskapazitäten der Organisationen. Hinzu kommt, dass die Fähigkeit der Universitäten eine strategische Positionierung im Europäischen Forschungsraum zu betreiben, und aktive Beteiligungsstrategien zu verfolgen, sehr unterschiedlich ausgeprägt ist. Bis auf einige Ausnahmen erscheinen die personellen Unterstützungskapazitäten keinesfalls ausreichend, um eine aktive Beteiligung auf Organisationsebene, z.B. über die Beteiligung an strategischen Partnerschaften, zu forcieren. Über das Instrument der ERA-Dialoge konnte die FFG diesbezüglich jedoch einen sinnvollen ersten Schritt setzen, um das Bewusstsein über die Bedeutung von Europäischen Agenden in den Organisationen zu stärken.

Die ERA-Dialoge stellen daher einen ersten Einstiegspunkt dar, um das Engagement der Organisationen bei den vielfältigen Möglichkeiten, die die europäische Ebene bietet, auf eine neue Stufe zu heben. Strategiebildungsprozesse müssen vornehmlich von den Organisationen selbst verantwortet werden und befinden sich Großteils noch in einer frühen Phase der Entwicklung. Ohne eine Erweiterung der organisationsinternen Kapazitäten, erscheint das Potenzial der Forschungseinrichtungen eine aktive Rolle im Europäischen Forschungsraum zu übernehmen beschränkt.

Die FFG verfügt über eine gute Basis an Beratungsleistungen um Forschungsservicestellen und Vizerektorate anzusprechen, es besteht jedoch Bedarf die Professionalisierung der Servicestellen und das strategische Empowerment weiter zu forcieren.

\section{Empfehlung: Professionalisierung der Servicestellen und strategisches Em- powerment in Hinblick auf ERA weiter forcieren}

Um die Professionalisierung der Servicestellen und das strategische Empowerment weiter zu stärken, sollten die ERA-Dialoge weitergeführt, der Kontakt zu den Forschungsservicestellen systematisiert und zugleich die Unterstützungsleistungen gezielt auf die Bedarfe der jeweiligen Universitäten abgestimmt werden.

In den ERA-Dialogen sollten Aktivitäten, die auch einen Niederschlag auf der Ebene der Fakultäten und Institute finden, weiter vorangetrieben werden. Diese sollten weiterhin den Interessen und Herausforderungen der jeweiligen Universität entsprechen und umsetzbar und verbindlich sein.

Zwischen den Forschungsservicestellen der Universitäten sollte ein regelmäßiger Erfahrungsaustausch unter Einbeziehung von Service-Personal des außeruniversitären Forschungssektors institutionalisiert werden, ${ }^{14}$ der auch zur Stärkung der Selbstorganisation

14 Ein solches Format wurde im 7. Rahmenprogramm seitens der FFG angeboten, aber angesichts der Heterogenität der Interessen der Forschungsservicestellen wieder eingestellt. Angesichts der 

land dienen. ${ }^{15}$

steigenden Anforderungen an europäische Beteiligungen sollte die damalige Entscheidung überprüft werden.

15 Der Bundesarbeitskreis der EU-ReferentInnen (BAK) in Deutschland besteht aus bis zu 2 Mitgliedern je Bundesland und verfolgt die Ziele i) die Beratungsstrukturen zu stärken und ii) die Arbeit der EU-ReferentInnen zu professionalisieren. Über das BAK-Netzwerk werden Informationen und Erfahrungen ausgetauscht, Probleme diskutiert und hochschulspezifische Lösungsansätze erarbeitet. Die Mitglieder des Bundesarbeitskreises treffen sich zweimal jährlich bei BAK-Sitzungen, arbeiten in themenspezifischen Arbeitsgemeinschaften (z.B. AG Recht, AG Projektmanagement) und sind im Alltag eng vernetzt. In regelmäßigen Jour Fixes geben sie ihre Kenntnisse an Stakeholder wie Hochschulleitungen, Verwaltung und WissenschaftlerInnen weiter. Darüber hinaus existiert auch ein EU-Mentoring Programm, welches darauf abzielt das vorhandene Fachund Praxiswissen im kollegialen Austausch zwischen neuen und erfahrenen EU-ReferentInnen weiter zu entwickeln. 


\section{Beratung und Betreuung - Fokus Wirtschaft}

Im Folgenden werden die spezifischen Ziele für die Kundengruppe "Wirtschaft" dargestellt, wobei zu beachten ist, dass zusätzlich zu den Kernzielen für die Wirtschaft auch noch generische Dimensionen wie internationale Kooperation, regionale Kooperation, multilaterale Programme, Stärkung der Gleichstellung und Schnittstellenmanagement bestehen, die ebenfalls die Kundengruppe Wirtschaft (mit) betreffen.

\subsection{Ziele und Aufgaben}

\section{Zielgruppenspezifische Ziele „Wirtschaft"}

Durch einschlägige Maßnahmen und Betreuungsangebot sollen die Potenziale des Europäischen Forschungsraums für die österreichische "Wirtschaft" (Unternehmen und MultiplikatorInnen wie Branchenverbände oder Cluster) bestmöglich genutzt werden. Dabei müssen die jeweiligen Herausforderungen, die sich bei der "Nutzung" der Potentiale des europäischen Forschungsraums ergeben, je nach Unternehmenstyp unterschiedlich berücksichtigt werden.

Kleine- und mittlere Unternehmen (KMU) sehen sich dabei naturgemäß anderen Herausforderungen gegenüber als große Unternehmen. Beispielsweise sind die Voraussetzungen für KMU in Bezug auf eine nachhaltige, strategische Planung ihrer Forschungs- und Entwicklungs- (F\&E) Aktivitäten, die Umsetzung und Verwertung ihrer F\&E- bzw. Innovationsergebnisse, ihre verfügbaren Ressourcen und ihr Zugang zu (Risiko-)Kapital auf den Kapitalmärkten ganz anders gelagert als bei großen Unternehmen. Gleichzeitig finden sich in vielen KMU auch Informationsdefizite in Bezug auf administrative und rechtliche Rahmenbedingungen, die es durch maßgeschneiderte Angebote abzubauen gilt.

\section{Ziele zur intersektoralen Kooperation (Wissenschaft - Wirtschaft)}

Darüber hinaus verfolgt FFG-EIP auch Ziele, die über den Fokus einer Zielgruppe hinausgehen. Österreichische Unternehmen sollen für die Kooperation Wissenschaft-Wirtschaft auch auf europäischer Ebene aktiviert werden. Den Mehrwert intersektoraler Kooperation auf europäischer Ebene zu vermitteln, und die Unterstützung der Akteure in konkreten Kooperationsbeziehungen im Rahmen institutioneller Netzwerke auf europäischer Ebene (wie z.B. PPPs, Europäische Branchenverbände) zählen zu den Hauptaufgaben der FFGEIP.

\section{Ziele zur regionalen Kooperation}

Österreichischen Unternehmen soll Unterstützung bei der optimalen Kombination von Fördermaßnahmen aus $\mathrm{H} 2020$ und anderen innovationsrelevanten Fördermöglichkeiten im Europäischer Fonds für regionale Entwicklung (EFRE) geboten werden. Dabei sollen die Potenziale regionaler KooperationspartnerInnen genutzt werden. Schwerpunkt hierbei ist die Umsetzung der „intelligenten Spezialisierungsstrategien” (Smart Specialisation). 


\section{Ziele zur internationalen Kooperation}

Die Stärkung der internationalen Positionierung österreichischer FTI-Akteure auch im globalen bzw. außereuropäischen Kontext unter optimaler Nutzung und Abstimmung zwischen europäischen und nationalen Förderprogrammen wird explizit angestrebt, wobei sich FFG-EIP als die zentrale Partnerin für ihre österreichischen Kunden in Bezug auf internationale F\&E-Aktivitäten in europäischen und globalen Netzwerken versteht. Notwendig hierfür ist die Forcierung eines pro-aktiven Kontaktmanagements mit PartnerInnen in strategisch definierten Zielländern und der Ausbau einschlägiger Kooperationen mit Agenturen und Organisationen aus dem europäischen Ausland und im TAFTIE Netzwerk aller europäischen Innovationsagenturen (plus Israel).

Neben diesen hier kurz dargestellten Zielen definiert FFG-EIP auch explizite Nichtziele zur klaren $\mathrm{Ab}$ - und Eingrenzung ihrer Aktivitäten. Diese Nichtziele sind:

- Unternehmen nach einem fixen Schema unspezifisch und ohne Rücksicht auf ihre jeweiligen Bedürfnisse, Herausforderungen und auch Möglichkeiten zu betreuen.

- Unternehmen, die nicht über die notwenigen Voraussetzungen verfügen in die Teilnahme an europäischen und internationalen Programmen zu drängen (in diesem Fall kann aber die Vermittlung zu passenden nationalen Fördermöglichkeiten stattfinden).

\section{Untergliederung/Typisierung der Zielgruppe Wirtschaft}

Die FFG anerkennt explizit die große Heterogenität der Zielgruppe Wirtschaft bzw. Unternehmen im speziellen. Auf Basis der F\&E-Erhebung der Statistik Austria wird derzeit von insgesamt ca. 3.000 F\&E-aktiven Unternehmen ausgegangen, wovon der überwiegende Anteil Klein- bzw. Kleinstunternehmen sind. Von diesen ca. 3.000 Unternehmen waren bzw. sind ca. die Hälfte (1.500 Unternehmen) bereits mit der FFG-EIP in Kontakt. Durch die Erweiterungen (z.B. größere Innovationsorientierung, neues KMU-Instrument) in H2020 (gegenüber FP7) wird von Seiten der FFG-EIP nun ein derzeitiges Potential von ca. 5.000 Unternehmen geschätzt (wobei dies die Zahl jener Unternehmen ist, die in den letzten fünf Jahren bei der FFG um eine Förderung in einem nationalen Programm angesucht hat ${ }^{16}$ ). Die FFG geht davon aus, dass von diesem rechnerischen Potential ca. $40 \%$ tatsächlich das EIP für Beratungsangebote etc. nutzen werden (ca. 2.000 Unternehmen).

Aufgrund von programmatischen Neuerungen in H2020 gegenüber FP7 ergeben sich zusätzlich neue Zielgruppen, die wiederum spezifische Anforderungen und Bedürfnisse aufweisen, auf die von Seiten der FFG-EIP instrumentell zu reagieren war. Darunter sind beispielweise die verstärkte Innovationsorientierung (Demonstration / Replikation, Berücksichtigung vermehrt auch von Dienstleistungen und Ausweitung des Innovationsbegriffs über rein technologische Innovationen hinaus), und die Elemente der Risikofinanzierung (Bedarf an einschlägigen Beratungen auch in Richtung Markteinführung, Skalierung etc.).

16 Der Innovationsscheck wird dabei jedoch nicht berücksichtig, da dieser zu niedrigschwellig angelegt ist und daher nicht als „Proxy” für die Affinität zu H2020 angewendet werden kann. 
Aufgrund dieser ausgeprägten Heterogenität segmentiert FFG-EIP die Zielgruppe "Unternehmen" zusätzlich noch nach deren Kenntnisstand bzw. Erfahrungsschatz in Bezug auf europäische FTI-Programme und unterscheidet demnach folgende drei Gruppen:

- "Potentials" (ca. 2.415 Unternehmen bzw. 69\% aller KundInnen auf die 25\% des Betreuungsaufwands entfallen).

- "Experienced" (ca. 1.050 Unternehmen bzw. 30\% aller KundInnen auf die ca. $60 \%$ des Betreuungsaufwands entfallen.

- „Higly Experienced Core Customer" (ca. 35 Unternehmen bzw. 1\% aller KundInnen auf die $15 \%$ des Betreuungsaufwands entfallen).

Diesen KundInnengruppen werden jeweils auf ihre spezifischen Bedürfnisse zugeschnittene Leistungsangebote zugeordnet (siehe Abbildung). Gleichzeitig ist es explizites Ziel, dass die Unternehmen auch in eine jeweils "höhere" KundInnengruppe (z.B. von Weiterentwicklung von „Potentials" zu "Experienced Customers") aufsteigen können. An der Spitze stehen die "Highly Experienced" Unternehmen, die zum einen als sogenannte "Core Customer" ein besonders intensives und individualisiertes Unterstützungs- und Beratungsangebot erhalten, oder zum anderen aufgrund ihrer hohen Erfahrung und europäischen Kompetenz u.U. auch nur rudimentäre Unterstützung von Seiten der FFG-EIP benötigen.

Inputs und Aktivitäten von FFG-EIP sowie deren intendierte Wirkung auf Unternehmen, werden in Abbildung 19 zusammenfassend dargestellt. Die darauf aufbauende Wirkungskette der Interventionen durch FFG-EIP ist in Abbildung 20 erfasst. 


\section{Zielgruppe „Unternehmen“( KMU, Großunternehmen)}

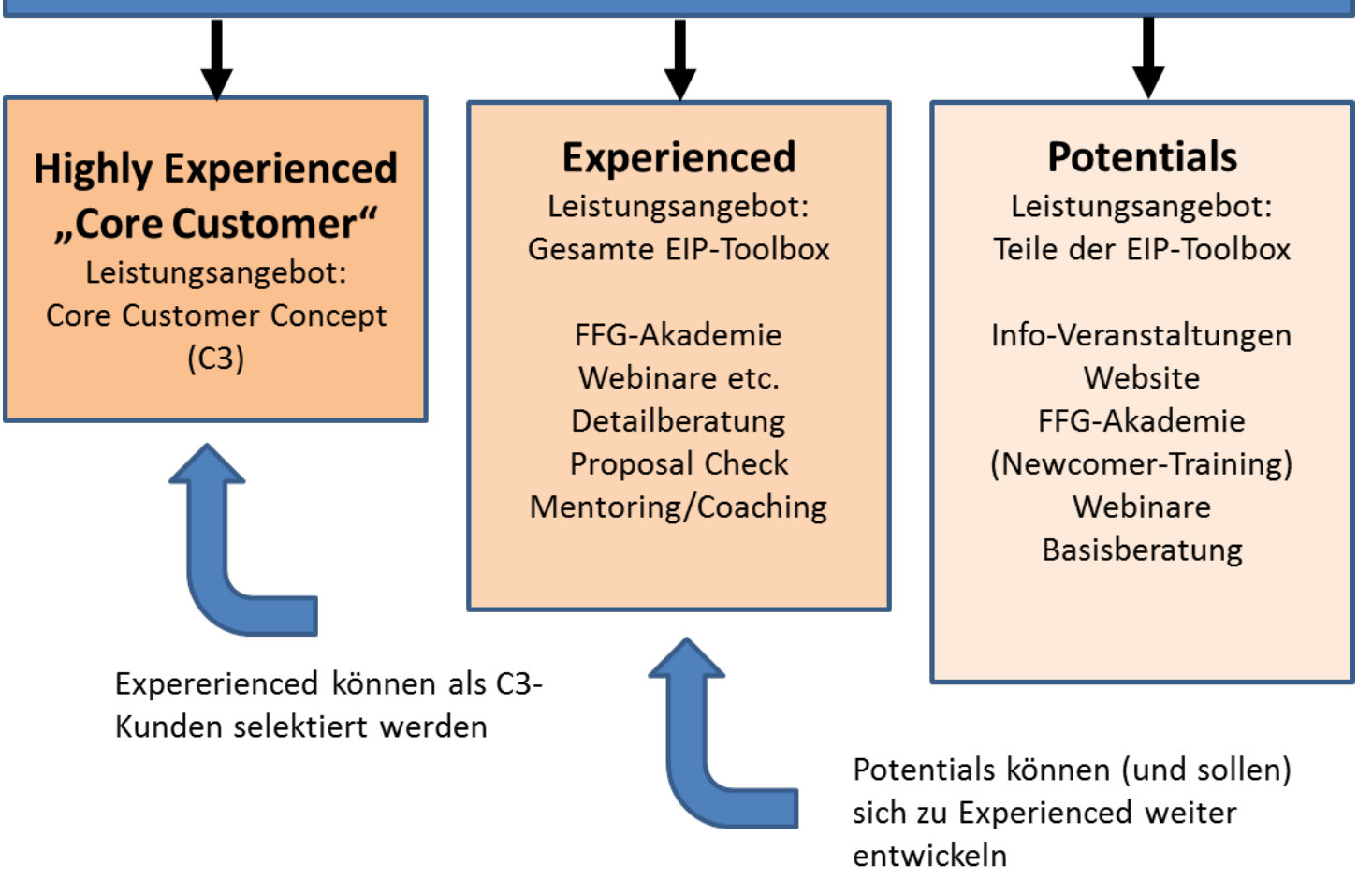

Quelle: FFG, 2014.

\section{Abbildung 20: Logik der Wirkungskette von FFG-EIP (Wirtschaft)}

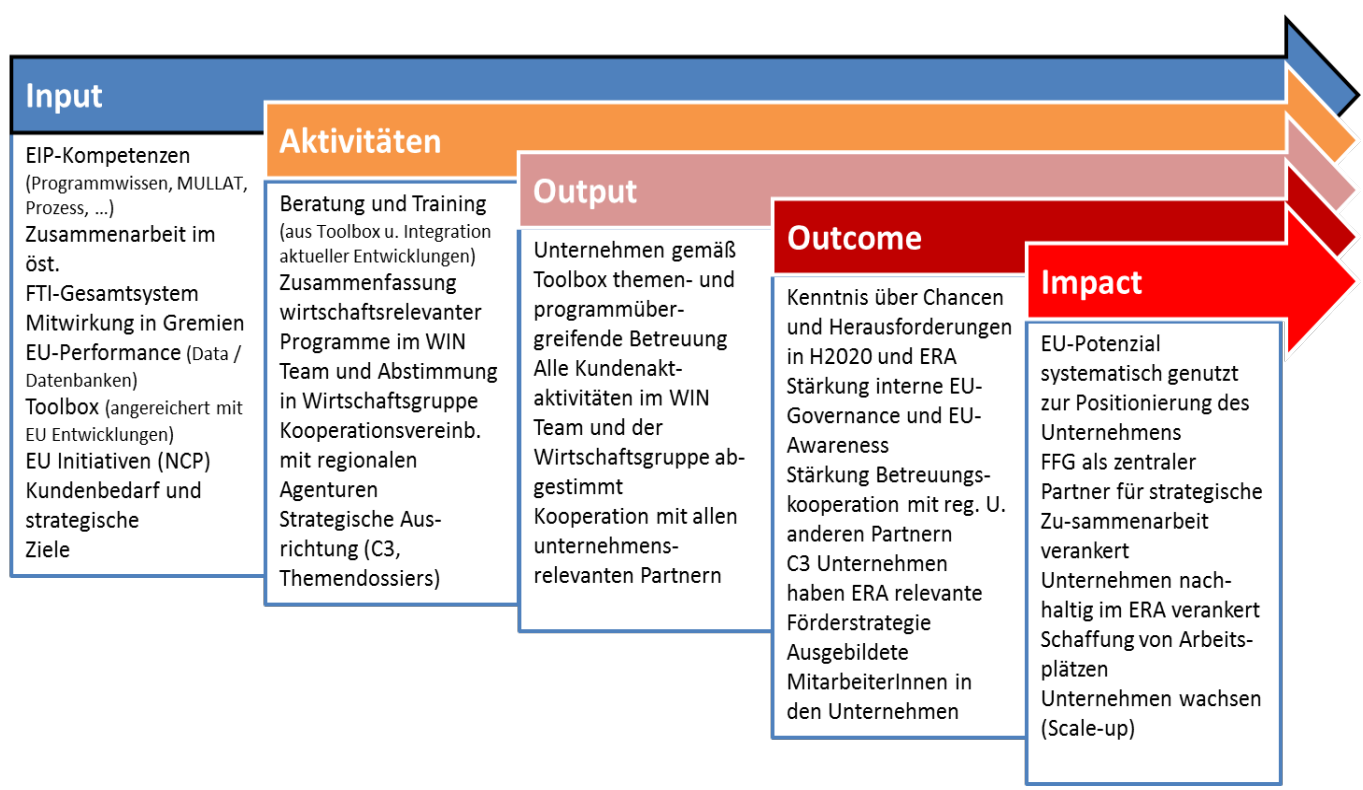

Quelle: FFG, 2014 


\subsection{Leistungen der FFG}

Das Beratungs- und Betreuungsangebot für Unternehmen wird entlang der Instrumente der FFG Toolbox (siehe Abschnitt 2.1) umgesetzt. Die Services adressieren Bewusstseinsbildung, Informationsvermittlung, Programm- und Projektberatung, strategische Beratung, und die spezifischen Programme EUREKA und COSME sowie EEN.

Abbildung 21 stellt die Aktivitätsbündel von FFG-EIP im Rahmen der Beauftragung zusammenfassend dar. Die Unternehmen bzw. MultiplikatorInnen stehen im Zentrum des Betreuungskonzepts und der Betreuungsservices.

\section{Abbildung 21: Aktivitäten der FFG-EIP im Fokus „Wirtschaft” im Zeitablauf}

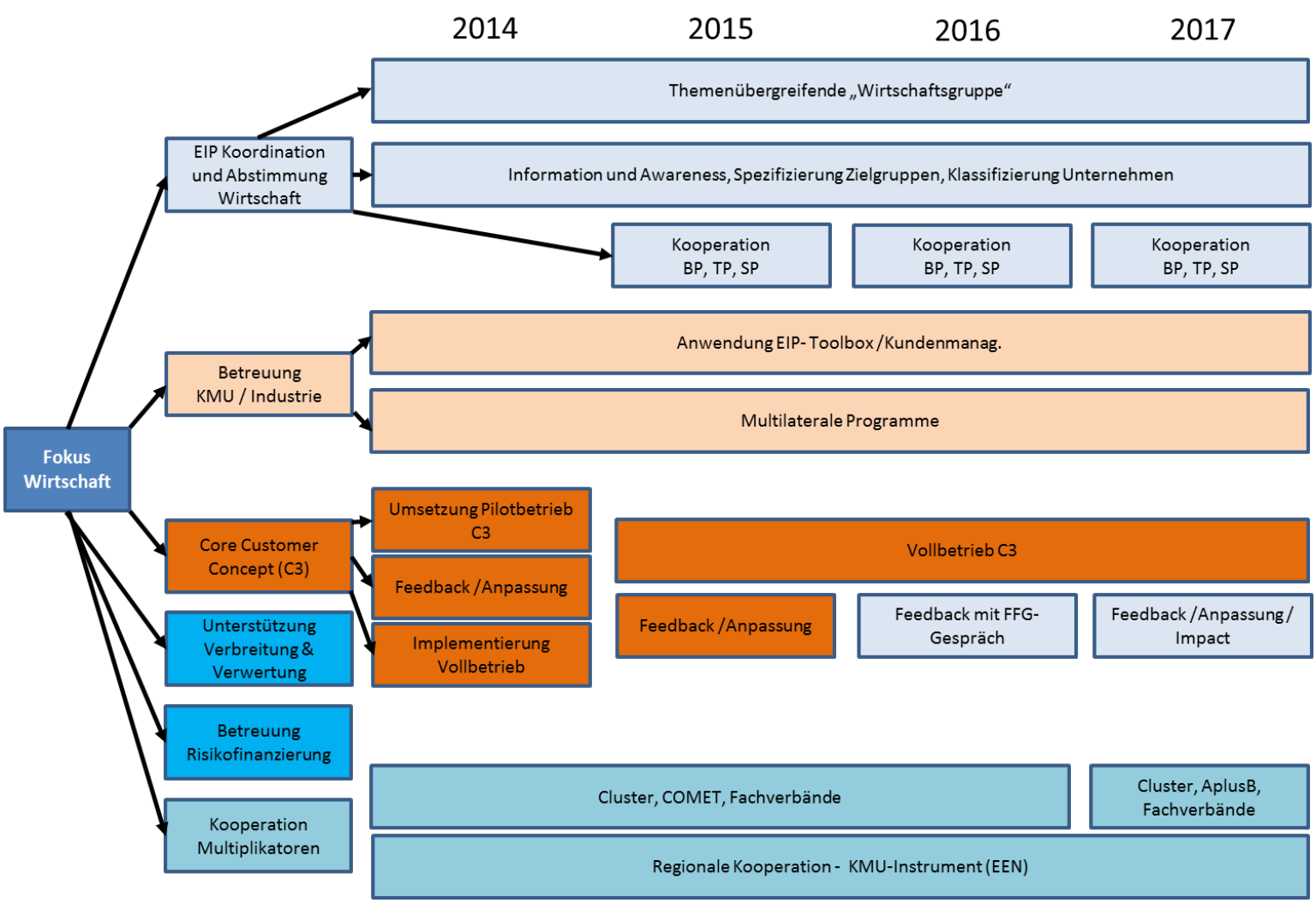

Quelle: FFG-Leistungsberichte 2014ff

\subsubsection{Zusammenarbeit und Schnittstellen bei der Betreuung}

Die Zielgruppe Wirtschaft ist im Bereich FFG-EIP deutlich mit der Innovationsorientierung in den Programmen (vorrangig H2020) verknüpft.

Intern, also im Bereich FFG-EIP, werden die relevanten Aktivitäten innerhalb des sogenannten WIN-Team zusammengefasst. Es repräsentiert personell alle innovationsorientierten Programme und Initiativen mit Relevanz für die Zielgruppe Wirtschaft, um so der Zusammenarbeit zwischen EEN, NCPs sowie regionalen Akteuren in der Wirtschaftsbetreuung gerecht zu werden. Das WIN-Team hat einen engen Konnex zu den thematischen Teams der FFG-EIP. Damit ist auch die Betreuung der Programme EUREKA; COSME bzw. EEN in das WIN-Team integriert, was einen Informationsaustausch und ausreichend Koordinierung der Maßnahmen zur Betreuung der Unternehmen (entlang der 
Wertschöpfungskette) garantieren soll. Relevante Schnittstellen und die Zusammenarbeit mit externen Stakeholdern sind bedarfsorientiert und damit auch in unterschiedlicher Intensität erfolgend definiert:

- Innerhalb der FFG kooperiert FFG-EIP mit anderen Bereichen in Hinblick auf die Entwicklung von Services für KundInnen, die gemeinsame strategische Weiterentwicklung im Rahmen der Thementeams, bis hin zu bereichsübergreifenden Beauftragungen und Projekten.

- Mit regionalen Agenturen und EEN-PartnerInnen erfolgt die Abstimmung anlassbezogen, jedoch sind regelmäßige Meetings zu einzelnen Themenupdates ( $\mathrm{H} 2020$, EEN), gemeinsame Firmenbesuche/Beratungen sowie Veranstaltungen vorgesehen.

- Mit der aws erfolgt die Abstimmung vor allem hinsichtlich der Bereiche KMU-Instrument (IPR) und Finanzierungsinstrumente (COSME und H2020). Vorrangig sind der strategische Informationsaustausch sowie gemeinsame Veranstaltungen und Vermittlung von Unternehmen. Formal ist die aws auch Unterauftragnehmerin für IPR-Leistungen des EEN in der FFG.

- Mit der Wirtschaftskammer erfolgt eine strategische Abstimmung und eine gemeinsame Koordination der österreichischen EEN-Knotenpunkte (siehe dazu Abschnitt 4.4.3). Die Abstimmung erfolgt laufend hinsichtlich EU FTI-relevanter Veranstaltungen und Entwicklungen. Die WKO dient vor allem als Multiplikatorin und Distributorin von Informationen zu den von der WKO vertretenen Unternehmen. Zudem wird im Rahmen großer europäischer Initiativen mit hoher Relevanz für Unternehmen kooperiert (z.B. KIC-Ausschreibungen, EIC, rechtliche Fragestellungen).

- Explizit als weitere wichtige Stakeholder, mit welchen zusammengearbeitet wird, ist die Clusterplattform ${ }^{17}$, das Patentamt und die Industriellenvereinigung angeführt.

Zwei weitere wichtige Elemente, die die Betreuung der Zielgruppe Wirtschaft charakterisieren, sind die Ausrichtung einzelner Betreuungen und Tools auf das neue KMU-Instrument von H2020 sowie die Entwicklung des sogenannten Core Customer Concept (C3).

\subsubsection{Ganzheitliches Servicepaket für KMU aufgrund des neuen KMU- Instruments in $\mathrm{H} 2020$}

In H2020 wird verstärktes Augenmerk auf die Einbeziehung von KMUs in den europäischen FTI-Prozess gelegt. Dies drückt sich nicht zuletzt in der Zielsetzung $20 \%$ aller H2020-Mittel der Säulen 2 und 3 an KMU zu geben sowie im neuen KMU-Instrument aus. Das KMU-Instrument adressiert alle Arten von KMU mit einem hohen Innovations- und Wachstumspotenzial. Es ist ein Querschnittsinstrument, das sowohl im Bereich "Grundlegende und industriellen Technologien" der Säule 2 als auch in der Säule "Gesellschaftliche Herausforderungen" Anwendung findet. Das KMU-Instrument fördert Projekte

17 Siehe auch die Website der Clusterplattform 
nach dem Bottom-up-Prinzip ${ }^{18}$. Nach einer Schätzung der FFG sind lediglich $10 \%$ aller oben genannten rund 5000 Unternehmen für dieses Instrument grundsätzlich geeignet, was bei der Beratung und Betreuung entsprechende Anforderungen stellt. Nicht geeignete Projektideen werden z.B. möglichst früh bezüglich alternativer Fördermöglichkeiten in anderen europäischen, multilateralen oder nationalen Förderprogrammen geprüft. Besonders relevant bei der Beratung und Betreuung zum KMU-Instrument ist eine gute Abstimmung und Verknüpfung der Services mit jenen im Rahmen des EEN sowie mit den NCPs.

\section{Core Customer Concept der FFG-EIP}

Die Ziele des Core Customer Concept bestehen darin, die internationale (EU-)Expertise in speziell ausgewählten Unternehmen weiter zu vertiefen und so einen Beitrag zur Weiterentwicklung ihrer europäischen und internationalen FTI-Strategien zu leisten. Auf Basis unterschiedlicher Kriterien wird aus der Summe der FFG-KundInnen eine Auswahl an potentiellen C3-Unternehmen getroffen. Die Kriterien beziehen sich dabei sowohl "hard facts" (z.B. Höhe und Anzahl der bisherig erhalten FFG-Förderungen) als auch "soft facts" (z.B. Stellung als Frontrunner). Bei der Auswahl geht es dabei nicht unbedingt die "besten" Unternehmen zu identifizieren (im Sinne jener, die am besten die europäischen FTI-Programme "bespielen"), sondern jene, von denen ein hohes Potential in Hinblick auf die Beteiligung in $\mathrm{H} 2020$ zu erwarten sei. Die Liste der C3-Potentials umfasst ca. 300 Unternehmen, von denen von der FFG aber nur ca. 200 tatsächlich als relevant eingestuft werden. Von den restlichen 100 wird angenommen, dass sie keine intensive C3-Betreuung benötigen, weil sie ihre europäische Strategie allein bzw. ohne externe Beratungsund Betreuungsbedürfnisse realisieren können.

Zentraler Kern des C3 Konzepts ist, dass je Unternehmen eine FFG-ExpertIn mit umfassenden Kenntnissen des betreffenden Unternehmens und dessen Umfeld nominiert wird und als alleiniger AnsprechpartnerIn gegenüber dem Unternehmen fungiert.

Das C3-Konzept wird dynamisch implementiert. Innerhalb einer Programmperiode sind jeweils wechselnde Unternehmen unter C3-Betreuung. Angestrebt wird ein jährliches Portfolio von ca. 15-20 C3-betreuten Unternehmen, wobei diese Unternehmen eine Bandbreite von Klein- über Mittel- bis Großunternehmen abdecken sollen. Diese Unternehmen sollen etwa zwei Jahre (wobei natürlich einige Unternehmen je nach Bedarf auch früher "ausscheiden" können) intensiv mit den C3-Instrumenten betreut werden.

Somit sollen bis zu 50 Unternehmen (d.h. ca. ein Viertel der gesamten potentiellen Zielgruppe von 200) im Rahmen der FFG-EIP Beauftragung betreut werden. Damit soll ein Pool an F\&E-intensiven Unternehmen (unterschiedlichster Größenklassen) generiert werden, die überdurchschnittliche Kenntnisse des europäischen FTI-Systems besitzen und mit expliziten Strategien die Programme und Instrumente der europäischen FTI-Politik nutzen (können). Die C3-Instrumente umfassen dabei, neben den üblichen EIP-ToolboxInstrumenten, eine sehr intensive und individuelle Betreuung durch die jeweils den einzelnen Unternehmen zugeordneten EIP-internen ExpertInnen.

\footnotetext{
18 Bis Anfang 2018 als „primarily bottom-up” klassifiziert.
} 


\subsection{Spezifische Leistungen: EUREKA, COSME, EEN}

Neben den Tools zur Bewusstseinsbildung, Informationsvermittlung, Programm- und Projektberatung, strategische Beratung (inkl. „ERA-Orientierungswissen"), die auch zur Beratung zu den spezifischen Programmen EUREKA, COSME und EEN eingesetzt werden (können), sind ebenfalls spezifische Tools für den Einsatz im Rahmen der Beratung zu eben diesen Programmen definiert.

\subsubsection{EUREKA - Initiative für anwendungsnahe Forschung und Entwicklung in Europa}

EUREKA ist eine 1985 gegründete Initiative, die sich als europäisch-internationales Netzwerk für anwendungsnahe Forschung und Entwicklung versteht. Auf der Basis einzelner Programme und Initiativen (v.a. Netzwerkprojekte, Clusterprojekte, EUREKA Eurostars) zielt die Initiative darauf ab, europäische Unternehmen und ihre KooperationspartnerInnen bei grenzüberschreitenden F\&E-Projekten zu unterstützen und somit die Produktivität und Wettbewerbsfähigkeit der europäischen Industrie zu stärken. Derzeit sind 41 Staaten und die Europäische Union EUREKA-Vollmitglieder. Kanada, Chile, Südafrika und Südkorea sind assoziiert.

Die politische Begleitung und administrative Betreuung von EUREKA-Projekten sind dezentral organisiert. Im jährlichen Wechsel übernimmt ein EUREKA-Mitgliedsland den Vorsitz und koordiniert die politische und strategische Weiterentwicklung. Die Gremien werden dabei jeweils vom Vorsitzland geleitet.

Neben seiner dezentralen Struktur ist der themenoffene, bottom-up-Ansatz der Projekt-Calls eine Besonderheit von EUREKA. Interessierte TeilnehmerInnen haben jederzeit die Möglichkeit, Projektanträge zu beliebigen Themen einzureichen. Für ein EUREKA-Projekt wird mindestens ein Unternehmen aus einem anderen EUREKA-Mitgliedsland benötigt. Universitäten und Forschungseinrichtungen können als Subauftragnehmer oder KooperationspartnerInnen in den Projekten teilnehmen.

Das Förderprogramm EUREKA-Eurostars wurde 2008 als eine gemeinsame Initiative von EUREKA und der Europäischen Kommission (EK) gestartet. Gefördert werden marktnahe F\&E-Kooperationen in kleinen, internationalen Konsortien. Als Initiative mehrerer Mitgliedstaaten gemäß Art. 185 Lissabon-Vertrag werden 75\% der Fördermittelmittel national dotiert, die restlichen (maximal) 25\% stammen von der EK („Top-Up"-Finanzierung).

Derzeit nehmen 36 Staaten an Eurostars teil. In den Gesprächen wurde bestätigt, dass die Realisierung von EUREKA Eurostars wesentlich dazu beigetragen hat, dass EUREKA weiter ein relevanter Player auf europäischer Ebene ist. Kritisch wird dabei die Abhängigkeit von der EK gesehen, insbesondere die Bereitschaft, auch künftig einen finanziellen Beitrag zu stellen.

Bei EUREKA Eurostars werden die Projekte durch ein europäisches Evaluationskomitee bewertet, die Vergabe der Mittel erfolgt auf Landesebene durch nationale oder regionale Finanzierungsinstrumente. EUREKA Projekte werden, mit Ausnahme der EUREKA-Cluster, 
durch nationale Fördergeber sowohl evaluiert als auch gefördert. Projekte, die die EUREKA-Kriterien erfüllen und Förderungen erhalten, werden zur Erteilung des EUREKA-Labels vorgeschlagen. Die gesicherte Finanzierung der jeweiligen Projektbeiträge ist notwendig um das Label zu erhalten.

Im Jahr 2014 wurde für EUREKA eine strategische Roadmap erarbeitet, in der u. a. Vorschläge für eine bessere Verankerung im Europäischen Forschungsraum festgelegt und Verbesserungen bezüglich der Komplementarität mit anderen Programmen dargelegt wurden. In Zukunft soll zudem stärker mit außereuropäischen Ländern im Rahmen von EUREKA zusammengearbeitet werden. Einen wichtigen Beitrag liefert das Instrument Globalstars, das darauf abzielt, die internationale Zusammenarbeit von KMU mit PartnerInnen aus Drittstaaten zu verbessern und voranzutreiben. Zudem wurde das Leistungsangebot von EUREKA um das "High-Tech Investment Programme" (Erleichterung des Zugangs zu Risikofinanzierung für die Markteinführungsphase) erweitert.

In Österreich nimmt das EUREKA-Büro im Bereich FFG-EIP die Abwicklung von österreichischen Beteiligungen an EUREKA wahr. EUREKA ist Teil des Rahmenvertrags der FFG mit den Eigentümerressorts. Hauptaufgabe des Büros ist es, als National Project Coordinator (NPC) durch Information, Beratung und Projektbetreuung eine adäquate Beteiligung österreichischer AntragstellerInnen und ProjektnehmerInnen an EUREKA und Eurostars zu ermöglichen bzw. zu unterstützen.

Auf Einzelprojektebene umfasst die Betreuung nicht nur die Phase der Antragstellung, sondern auch die Projektlaufzeit und reicht über das Projektende hinaus. Bezugnehmend auf die Programmebene ergeben sich Aufgaben wie etwa die Mitwirkung bei der Erstellung von Leitfäden oder des Ausführungsvertrags, die Durchführung von Formalprüfung und Fördersynchronisierung. ProjektnehmerInnen werden zum Fortgang der Projektarbeit und dem Stand der Kooperation regelmäßig befragt. Der Input fließt in Early Progress Checks, Final Reports und, etwa 2-4 Jahre nach Abschluss des Projekts, in Market Impact Reports ein. In den Aufgabenbereich des Büros fällt auch die Unterstützung des EUREKA Hohen Repräsentanten (High Level Group Representative) in seinen strategischen Funktionen.

Das EUREKA Büro in FFG-EIP arbeitet in Abstimmung mit dem EUREKA Sekretariat (ESE) in Brüssel, das das gemeinsame Dienstleistungszentrum des EUREKA-Netzwerks bildet und den jeweiligen EUREKA-Vorsitz und die EUREKA-Gremien bei ihrer Arbeit unterstützt, selbst jedoch über kein (Projekt-)Budget verfügt. Zu den Aufgaben von ESE zählen auch die Sammlung und Verteilung von Informationsmaterial über die Projekte, das Unterhalten einer Projektdatenbank, sonstige Öffentlichkeitsarbeit und Betreuung der internationalen Website von EUREKA.

Die Finanzierung von Eurostars Projekten erfolgt im Rahmen einer eigenen FFG Programmlinie, jene von Cluster- und Netzwerk-Projekten speist sich aus den FFG Basisprogrammen. Dabei ist für EUREKA kein fixer Budgetrahmen vorgegeben, auch nicht für die bi- bzw. multilateralen Calls. Die EUREKA inkl. Eurostars-Aktivitäten und -Betreuung erfolgt seit einigen Jahren auf der Basis von jährlichen Arbeitsplänen. Die darin festgelegten Ziele (u.a. Beteiligungsanzahl, Messindikatoren) sowie geplante Vorhaben und Aufgaben werden im Zuge der Erstellung von Jahresberichten mit den erzielten Ergebnissen verglichen und bewertet. 
Der Großteil der TeilnehmerInnen an Eurostars-Projekten und AntragstellerInnen in EUREKA-Projekten (inkl. Cluster) erhielt auch eine Beratung durch das EUREKA-Büro. Laut Jahresbericht wurden 2016 von den MitarbeiterInnen des EUREKA-Büros 155 intensive

Beratungsgespräche (2015: 137) zu EUREKA und Eurostars durchgeführt, hinzu kommt nochmal ungefähr die gleiche Zahl an Kundenkontakten kürzerer Natur (unter 10 Minuten). Daneben wurden Beratungsleistungen zu EUREKA und Eurostars im Rahmen der allgemeinen Beratungen des WIN-Teams erbracht. Wie in den Jahren zuvor nahmen auch 2016 MitarbeiterInnen des EUREKA-Büros an einer Vielzahl an Netzwerkveranstaltungen sowie Konferenzen im In- und Ausland teil.

Während die EUREKA-Projektzahlen aufgrund des Eurostars-Erfolges in den Jahren 2013 sowie 2014 stark rückläufig waren, konnte laut FFG-EIP-Zwischenbilanz zuletzt wieder ein starkes Wachstum verzeichnet werden. So wurden 2015 sowie 2016 rd. $€$ 1,7 Mio. an Förderungen für die EUREKA Netzwerk- und EUREKA Clusterprojekte ausgeschüttet. Für 2017 ist mit einer weiteren Steigerung des Fördervolumens für EUREKA auf ca. $€$ 3,3 Mio. zu rechnen. Mit 24 geförderten Eurostars-Projekten wurde 2016 zudem der bisherige Höchststand seit Programmstart erreicht. Laut Jahresbericht 2016 wird erwartet, dass die Teilnahme an EUREKA-Projekten künftig weiter steigen wird. Bilaterale Ausschreibungen ${ }^{19}$, wie sie zuletzt mit der Türkei und Spanien ausgerufen wurden sowie multilaterale Ausschreibungen im Rahmen von Globalstars (Chile) werden von Seiten der Administration als durchaus erfolgreich und stimulierend angesehen und sollen ausgebaut werden.

\subsubsection{COSME - Programm für die Wettbewerbsfähigkeit von Unternehmen und für KMU}

Das „Programme for the Competitiveness of Enterprises and Small and Medium Sized Enterprises 2014-2020") - ehemals CIP ("Competitiveness Innovation Programme") - ist das zentrale EU-Programm zur Steigerung der Wettbewerbsfähigkeit von Unternehmen, insbesondere KMU. Dieses wurde nach Ende des Vorläuferprogramms CIP um die Innovationsaspekte sowie ICT und Energy Activities im Programm reduziert, die in H2020 eingegliedert wurden. Somit fokussiert COSME noch stärker auf den breiten Themenbereich "Competitiveness". Das Programm wird in vier zentrale Bereiche unterteilt, die zudem auch die Einzelziele des Programms darstellen:20

1. Zugang zu Finanzierungsmitteln ("Accessing Finance"),

2. Zugang zu Märkten ("Opening Markets"),

3. Förderung von unternehmerischer Initiative und -kultur (",Supporting Entrepreneurs"),

4. Verbesserung der Rahmenbedingungen für die Wettbewerbsfähigkeit („Improving Business Conditions").

19 Zentrales Element dieser Ausschreibungen ist die erst vor einigen Jahren eingeführte, flexible Setzung von Deadlines für gemeinsame Projekteinreichungen, bi- oder multilateral. Der Unterschied zu EUREKA Globalstars besteht vor allem in der Teilnahme außereuropäischer PartnerInnen.

20 Vgl. EU-Verordnung 1287/2013 des EU-Parlaments und Rates bzw. aktuell 
COSME ist für die gesamte Laufzeit mit rd. 2,3 Mrd. $€$ ausgestattet, wovon mind. $60 \%$ für den Bereich "Accessing Finance" vorgesehen sind. Maßnahmen in COSME sind kaum direkt für KMU zugänglich. Vielmehr werden zahlreiche Maßnahmen ausgeschrieben (Calls für Proposals, Calls for Tender, Framework Contracts), die Multiplikatoren und Intermediäre in den EU-Mitgliedsstaaten umsetzen sollen, um damit letztlich KMU zu unterstützen. Das Programm adressiert als Politikentwicklungs- und Politikunterstützungsinstrument die primäre Zielgruppe der KMU insgesamt häufig (nur) indirekt, d.h. über Intermediäre, Studienergebnisse, Best-Practice-Austausche, Veranstaltungen und Konferenzen oder Datenaufbereitungen. KMU sind somit eine indirekt adressierte Zielgruppe des Programms.

Die FFG ist vom Bundesministerium für Digitalisierung und Wirtschaftsstandort (BMDW) damit beauftragt, COSME zu bewerben und österreichische Organisationen bei Fragestellungen rund um das Programm zu unterstützen. Die wesentlichen Aufgaben der FFG umfassen folgende Aktivitätsbereiche ${ }^{21}$ :

- Bewusstseinsbildung für diese Programmlinie bei MultiplikatorInnen,

- Information über Ausschreibungen, Beratung und Betreuung von ProjekteinreicherInnen,

- Berichterstattung über die Ergebnisse,

- Nicht öffentlich wirksame Tätigkeiten, wie z.B. die Unterstützung der COSME-Programmdelegierten im Rahmen der Aktivitäten in den Programmkomitees.

Die Hauptaufgabe der FFG im Rahmen der Beauftragung COSME ist die operative Abwicklung diverser Calls for Proposals bzw. Tender. Potentielle österreichische Akteure (Intermediäre bzw. EinreicherInnen) werden dazu identifiziert und informiert. Intermediäre, als potenzielle EinreicherInnen, sind im Programmkontext primär Organisationen (Cluster, Servicestellen, Verbände, Wirtschaftskammern u.ä.), welche die Maßnahmen, die letztlich KMU in unterschiedlicher Art adressieren, umsetzen sollen. Die Informationsarbeit der FFG basiert auf persönlichen Gesprächen oder auf der Ansprache via Telefon und E-mail bzw. über Beiträge bei relevanten Veranstaltungen. Schwerpunkte liegen dabei auf dem "Aufmerksam machen" auf relevante Einreichmöglichkeiten und der Weitergabe von Basisinformationen. In weiterer Folge werden interessierte EinreicherInnen zu inhaltlichen und administrativen Anforderungen bei der Antragstellung unterstützt.

\subsubsection{EEN - Enterprise Europe Network}

Das Enterprise Europe Network ist eine Maßnahme, die (kontinuierlich) im Rahmen des COSME-Programms ausgeschrieben und umgesetzt wird. Es können im Rahmen des Netzwerks u.a. folgende Unterstützungsmaßnahmen ergriffen werden ${ }^{22}$ :

- Bereitstellung von Informations- und Beratungsdiensten,

- Erleichterung von grenzüberschreitenden Partnerschaften,

21 Lt. Leistungsbeschreibungen der Beauftragung

22 Siehe EU-Verordnung Nr. 1287/2013 des EP und des Rates vom 11. Dezember 2013 über ein Programm für die Wettbewerbsfähigkeit von Unternehmen und für kleine und mittlere Unternehmen (COSME) (2014-2020), Art. 10, Abs. 1) 
- Bereitstellung eines Kommunikationskanals zwischen KMU und der Kommission.

Das EEN hat einen Fokus auf (für die jeweiligen Unternehmen maßgeschneiderte) Hilfestellungen bei internationalen Kooperations- und Technologietransferaktivitäten - insbesondere für KMU. Gegenstand der Unterstützung sind dabei Kooperationen unterschiedlicher Art (FTI-Kooperationen sowie reine Technologiepartnerschaften, Lizensierungen, Vertriebsvereinbarungen etc.).

Das EEN-Austria Konsortium besteht aus insgesamt zwölf EEN-PartnerInnen („EENKnoten"), die sowohl regionale Wirtschafts- und FTI-Agenturen als auch Wirtschaftskammern sind. ${ }^{23}$ Koordinatorin des EEN in Österreich ist die WKO. Die FFG zeichnet für die thematische Sub-Koordination der österreichischen EEN-FTI-PartnerInnen verantwortlich und ist zudem selbst ein EEN-Knoten. Beide bieten Unternehmen aus Wien, Niederösterreich und dem Burgenland sämtliche EEN-Serviceleistungen an. In den restlichen Bundesländern sind die jeweiligen EEN-Knoten für ebendiese Serviceleistungen verantwortlich.

Neben ihren allgemeinen Tools zur Beratung und Betreuung der Zielgruppen Wissenschaft und Wirtschaft (siehe Tabelle 2) bedient sich die FFG (wie auch alle anderen elf EEN-Knoten) im Rahmen ihrer EEN-Betreuung folgender Tools zur Information und

\section{Bewusstseinsbildung:}

- EEN-Webseite,

- EEN-Referenzen (Success Stories \& Testimonials),

- Newsletter,

- Awareness und Informationsveranstaltungen,

- Standbetreuung (auf Veranstaltungen),

- Networking auf externen Veranstaltungen,

- Social Media (Twitter, Xing).

Darüber hinaus greift die FFG auf Tools für die operative Arbeit zurück, die den Bereichen Beratung, Kooperationssupport und Innovationssupport zugeordnet werden können.

- Individuelle Beratung zu Internationalisierung, Wissens- und Technologietransfer und F\&E-Projekten,

- Internationale Partnersuche über die Datenbank „Marktplatz" sowie direkte Kontakte,

- Kooperationsbörsen (Stand-Alone sowie im Rahmen von anderen Veranstaltungen oder Messen),

- Key Account Management (KAM): seit 2014 werden jedem KMU, bei einer Förderzusage im KMU-Instrument von $\mathrm{H} 2020$ hinsichtlich einzelner projektbasierter Herausforderungen spezifische Beratungsleistungen angeboten. Diese Beratung wird von den EEN-Knoten bzw. in weiterer Folge von Business Coaches durchgeführt, die in der EEN-Datenbank eingetragen sind.

\footnotetext{
23 Siehe dazu auch die Website des Enterprise Europe Network
} 
- Enhancing the Innovation Management Capacity of SMEs (EIMC): Analyse des Innovationsmanagements (bei einer vergleichsweise geringen Anzahl an ausgewählten Unternehmen) unabhängig von H2020-Teilnahmen.

\subsection{Ergebnisse der empirischen Erhebungen}

Im Folgenden werden die Befunde der Online Befragung in Hinblick auf die Passgenauigkeit und Wirkung der FFG-Services für Unternehmen dargestellt. Darüber hinaus lieferten Interviews und je eine Fokusgruppe mit C3 Unternehmen und regionalen Intermediären sowie Analysen der Feedback-Gespräche von C3 Unternehmen Einschätzungen zur Relevanz und Effektivität des Leistungsspektrums für Unternehmen.

\section{Allgemeiner Erfahrungshintergrund der Unternehmen}

Die Selbsteinschätzung der Unternehmen bezüglich ihres Erfahrungshintergrunds betreffend europäische FTI-Programme zeigt, dass sich knapp zwei Drittel der antwortenden Unternehmen als erfahren einschätzen (vgl. Abbildung 22).

\section{Abbildung 22: Praktischer Erfahrungshintergrund der Unternehmen}

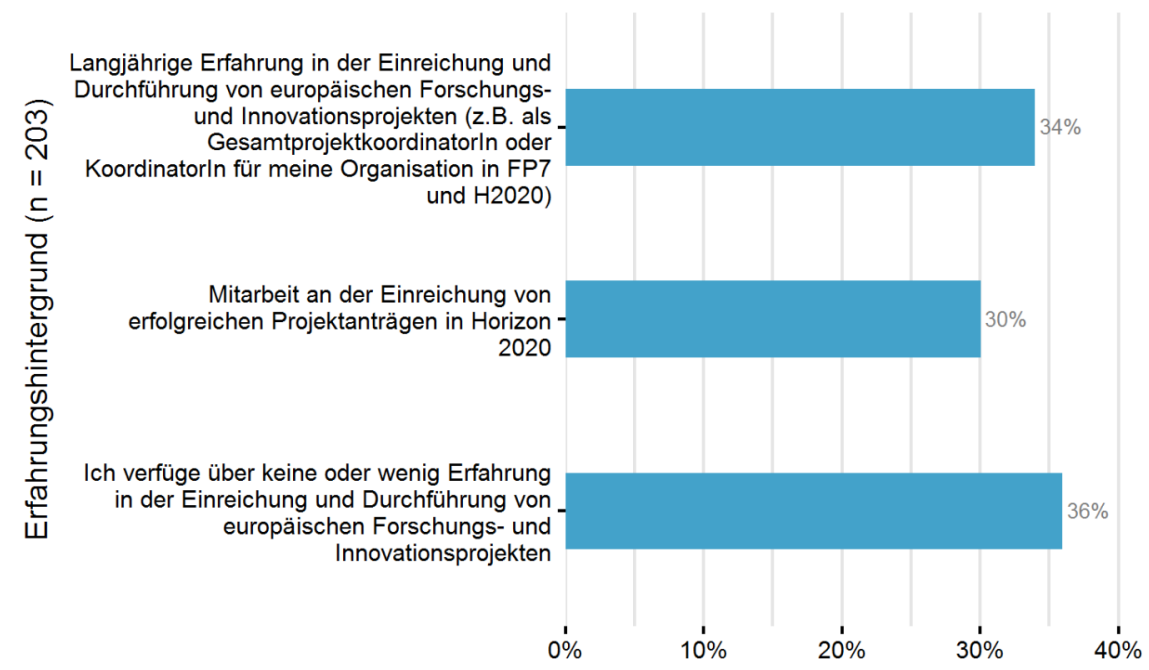

Quelle: Online Befragung, Joanneum Research.

Betrachtet man den praktischen Erfahrungshintergrund der Unternehmen zeigt sich, dass ca. ein Drittel bereits eine langjährige Erfahrung (z.B. als KoordinatorIn) mit den europäischen FTI-Programmen aufweisen, während ein knappes Drittel eine Mitarbeit an der Einreichung von erfolgreichen Projektanträgen vorweisen. Die erfahrenen Unternehmen teilen sich damit annähernd in eine Hälfte mit „intensiver Erfahrung" und eine zweite Hälfte mit „punktueller" Erfahrung.

Bei einer differenzierten Betrachtung des Erfahrungshintergrunds nach kleinen und groBen (bzw. mittleren) Unternehmen ist zu beobachten, dass auch die antwortenden Kleinunternehmen überwiegend (knapp 63\%) Erfahrung mit den europäischen FTI-Programmen aufweisen (Abbildung 23). Dies kann als Hinweis dafür interpretiert werden, dass das spezifische Ziel der FFG-EIP, nämlich speziell die besonderen Herausforderungen von 
kleinen Unternehmen zu adressieren und ebenfalls an die europäische FTI-Politik heran zu führen, erfüllt werden konnte. Darüber hinaus zeigt sich darin auch die beträchtliche Reichweite des Leistungs- und Beratungsangebots der EIP, die durchaus auch die Kleinunternehmen erreicht.

\section{Abbildung 23: Allgemeiner Erfahrungshintergrund nach Unternehmensgröße}
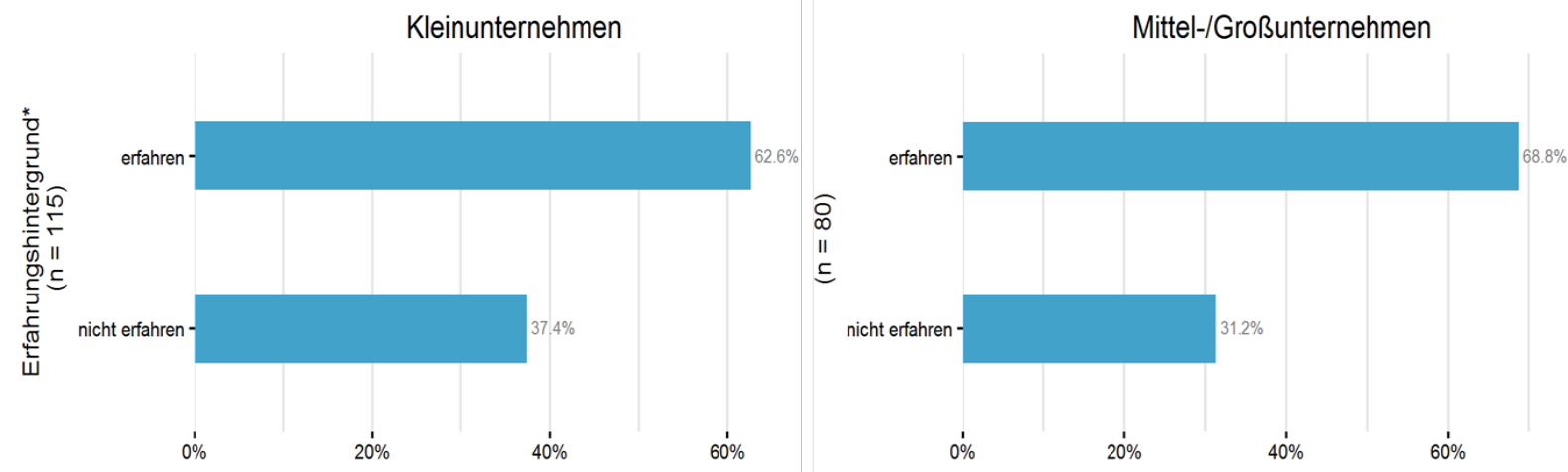

Quelle: Online Befragung, Joanneum Research.

\section{Nutzung von unterschiedlichen Typen von Unterstützungseinrichtungen}

Hinsichtlich der Nutzung verschiedenster Unterstützungseinrichtungen ist mit einer Nennung von $82 \%$ die FFG-EIP eindeutig an erster Stelle, gefolgt von KollegInnen mit einschlägiger europäischer Erfahrung mit 50\%. Alle anderen Typen von Unterstützungseinrichtungen liegen weit dahinter: Regionale Beratungsstellungen und Private Beratungsstellen werden von etwa 20\% der befragten Unternehmen in Anspruch genommen. 


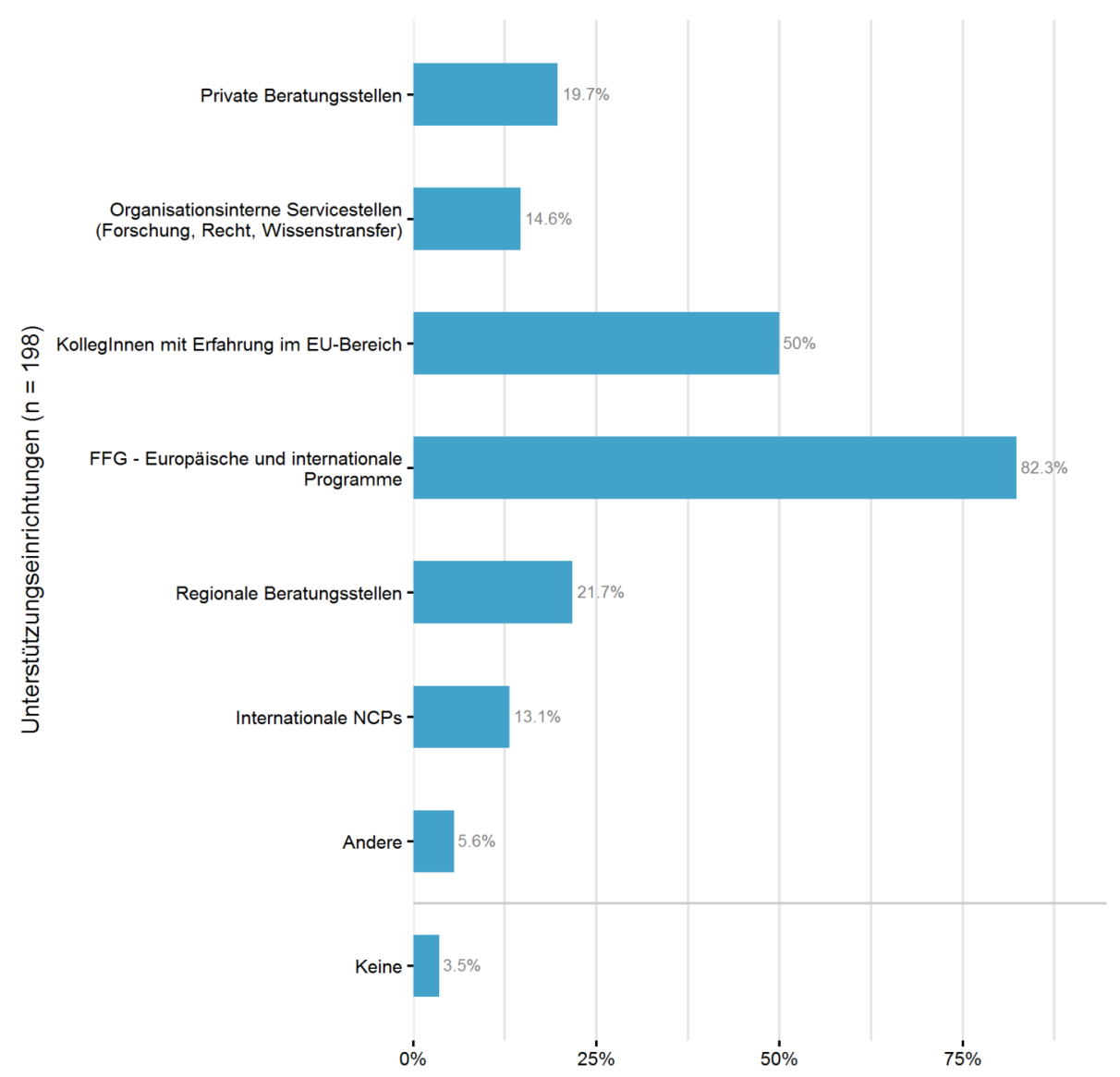

Quelle: Online Befragung, Joanneum Research.

\section{Motive für Inanspruchnahme von FFG-EIP Leistungen}

Die Beweggründe für eine Inanspruchnahme von FFG-EIP-Unterstützungsleistungen sind vielfältig (siehe Abbildung 25).

Hervorstechend ist, dass sich die Angaben für spezifische Information (spezifische Fragen zu Arbeitsprogrammen und Ausschreibungen mit knapp 51\%, Informationen über spezielle Instrumente mit 36\%) als auch allgemeine Informationen (Trends und Entwicklung mit $42 \%$, grundlegende Erstinformation mit 42\%) annähernd die Waage halten (wobei spezifische Bedürfnisse leicht vorne liegen).

Demgegenüber spielen Unterstützungsleistungen bei der Netzwerkfindung bzw. Partnerfindung eine geringe Rolle. Dies wird auch durch einige der qualitativen Interviews bekräftigt, in denen betont wurde, dass die Netzwerkbildung überwiegend "evolutionär" erfolgt, d.h. entlang von bestehenden Kooperationen, die sich im Zeitablauf erweitern. ${ }^{24}$

24 Anzumerken ist, dass sich in einigen qualitativen Rückmeldungen im Zuge des Online-Surveys ein etwas anderes Bild zeichnet, in dem bestimmte Leistungen des EIP (wie z.B. auch die netzwerkorientierte Angebot im Rahmen von „Match Making") durchaus höher gewichtet werden. 


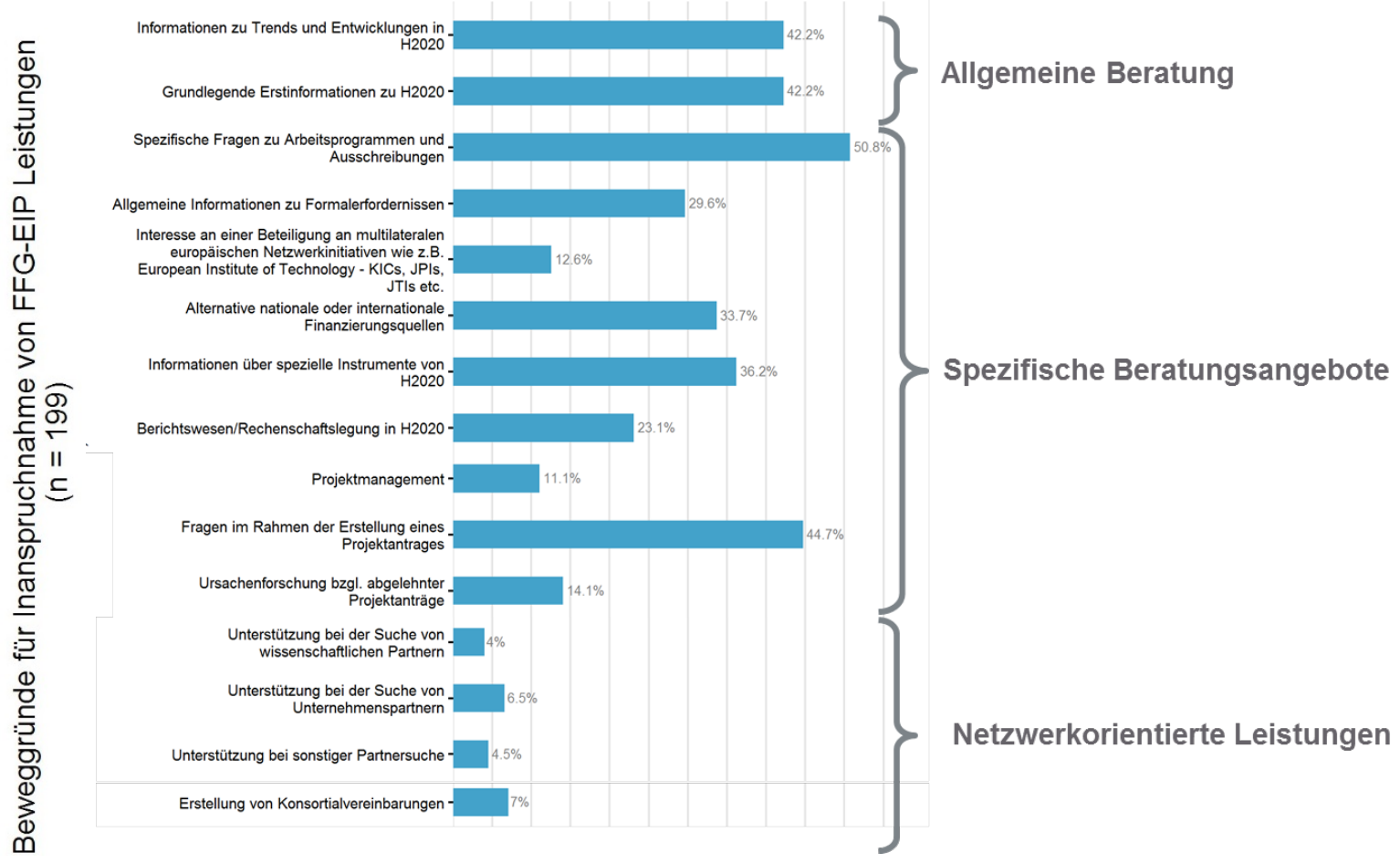

Quelle: Online Befragung, Joanneum Research.

\section{Nutzung, Qualität und Wirkung der Leistungen}

Hinsichtlich der Nutzung der diversen Informationsangebote zeigen sich große Unterschiede, wobei neben der Nutzung auch der Bekanntheitsgrad starke Schwankungen verzeichnet. Hohe Nutzungen finden sich einerseits bei den eher allgemeinen bzw. überblickshaften Informationsangeboten mit Nutzungsgraden von teilweise bis über $90 \%$ und andererseits mit $80 \%$ wiederum bei spezifischen Informationen zu rechtlichen, finanziellen und administrativen Aspekten.

Ähnliches gilt z.B. auch für Beratungsangebote in Bezug auf geistige Eigentumsrechte, deren Bedeutung in einigen qualitativen Anmerkungen betont wurde. 


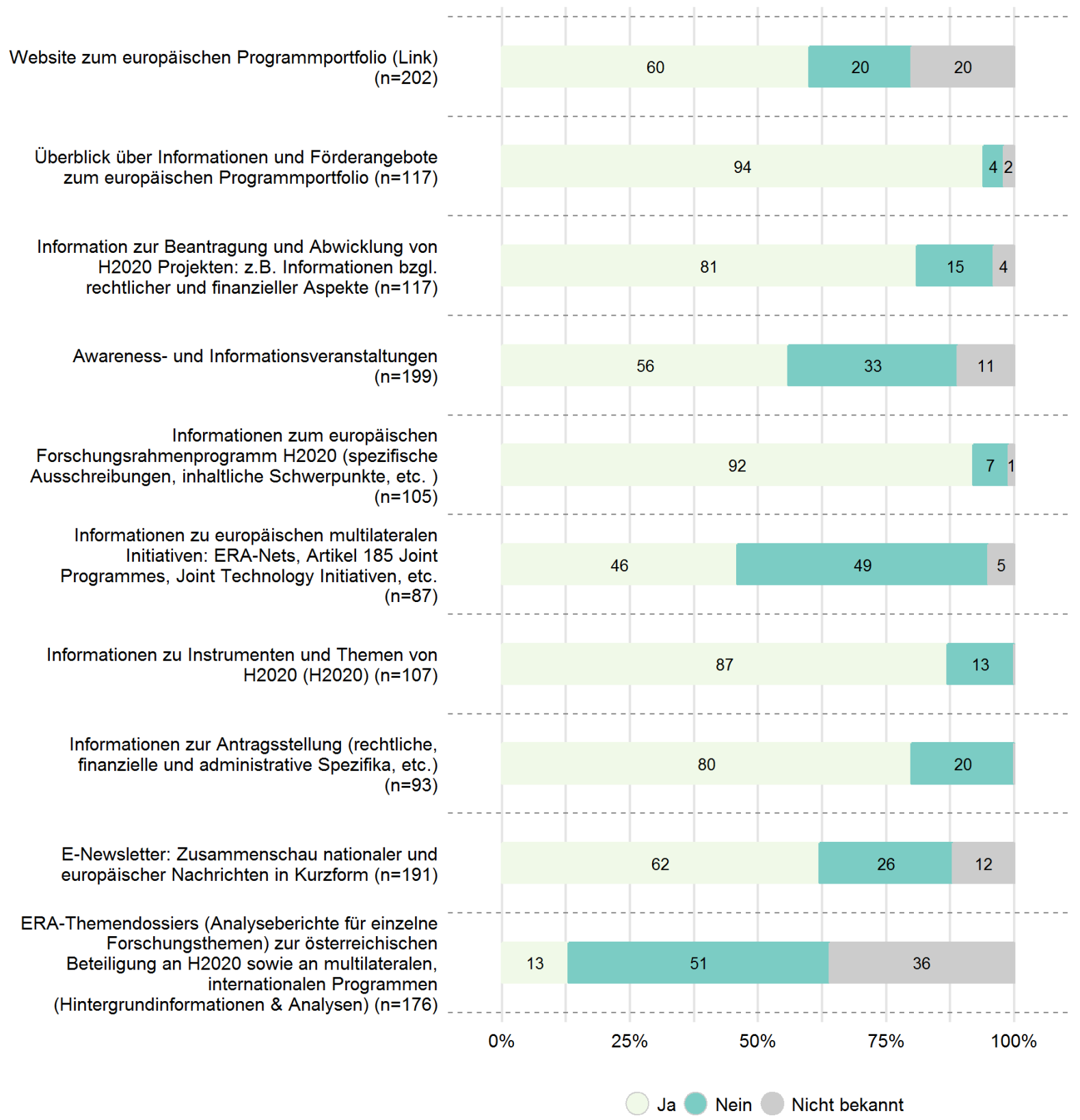

Quelle: Online Befragung, Joanneum Research.

\section{Spezifische Bewertung einzelner Beratungsleistungen}

Rund 2/3 der antwortenden Unternehmen haben eine persönliche Beratungsleistung der FFG in Anspruch genommen. Generell werden die verschiedenen Beratungsleistungen meist gut bis sehr gut bewertet. Überwiegend 70 bis $80 \%$ der Antwortenden stimmen den jeweiligen Aussagen zu (inklusive "stimme eher zu"). Dabei zeigt sich das Muster, dass informationsbezogene Leistungen (z.B. "Anforderungen zur Einreichung in EU-Programmen", "Angebot an Beratungsleistungen", "strategisch relevante Informationen") besonders gut bewertet werden. 
Die Einschätzung inwieweit die Beratungen auch tatsächlich das konkrete Handeln und die Strategieformulierung verändert (z.B. „Anträge mittlerweile eigenständig”, ,abgesehen haben, einzureichen", "Professionalisierung der Beratung") ist ebenfalls positiv, die absolute Zustimmung liegt jedoch hinter den Werten der informationsbezogenen Fragestellungen. Dies ist aber nicht überraschend, da konkrete Informationsvermittlung eindeutig leichter umzusetzen ist als die Übersetzung der im Zuge der letzten Antragsstellung erworbenen Skills in die Fähigkeit eigenständig europäische Förderanträge zu stellen. Dies könnte auch ein trügerisches Sicherheitsgefühl darstellen. Interessant ist auch das Feedback der Unternehmen, dass die Beratung bei etwas über der Hälfte der Unternehmen zu einem Antrag in H2020 führte, was darauf schließen lässt, dass sich verständlicherweise Unternehmen mit einer intensiveren/positiven Erfahrung für die Teilnahme an der Befragung motivieren ließen.

\section{Abbildung 27: Wirkung der Beratungsleistungen}

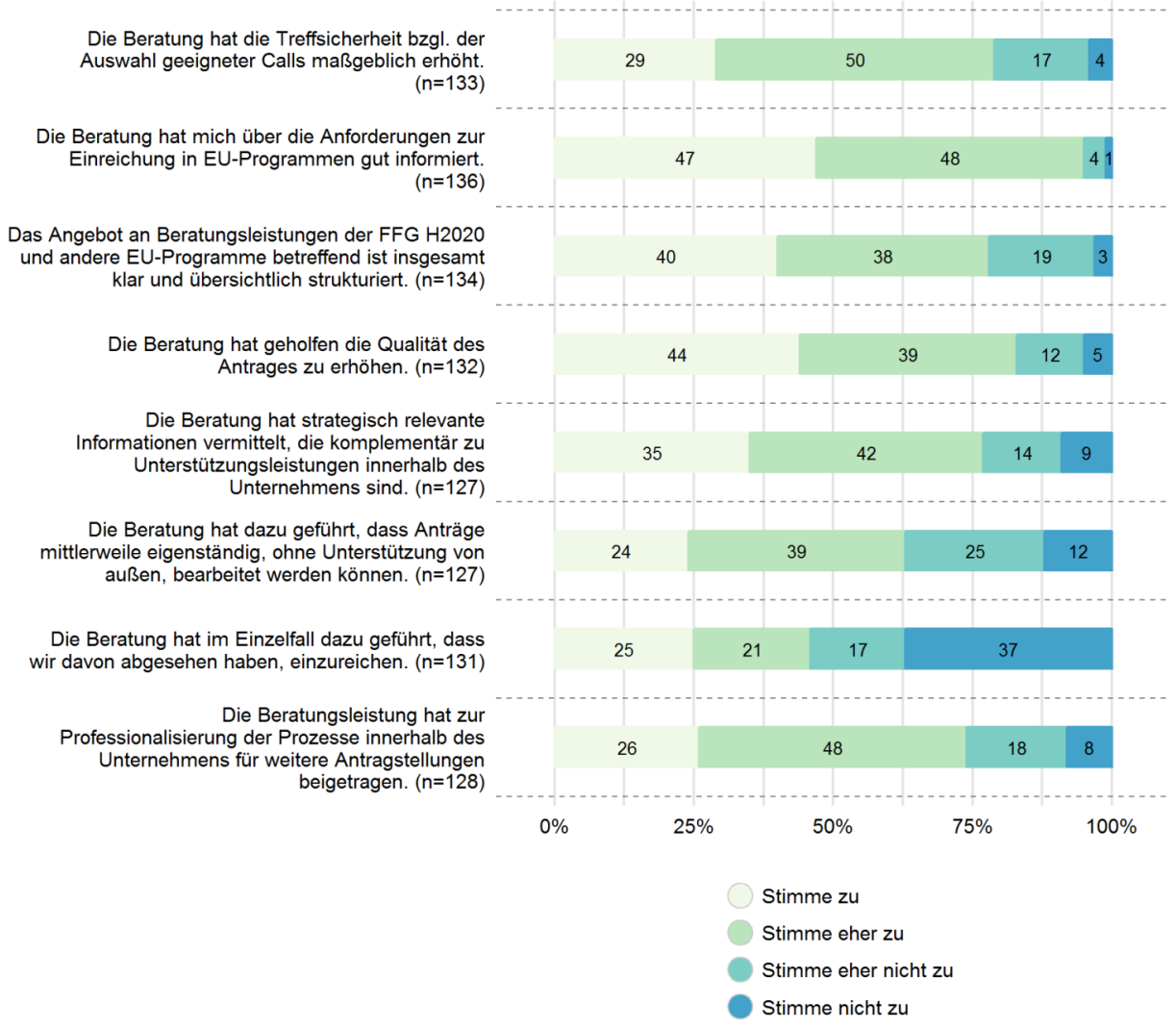

Quelle: Online Befragung, Joanneum Research.

Mehr als 50\% der antwortenden Unternehmen gaben an, an einem Training der FFGAkademie teilgenommen zu haben. Die im Rahmen der Beauftragung neu eingeführten Webinare, die häufig in Ergänzung zu persönlichen Beratungs- und Informationsangeboten genutzt werden, werden von den befragten Unternehmen sehr positiv bewertet. Hoch ist auch die Zustimmung zur Passgenauigkeit der Trainings, die aus Sicht der befragten Unternehmen zur Professionalisierung der Projektplanung beitragen. 


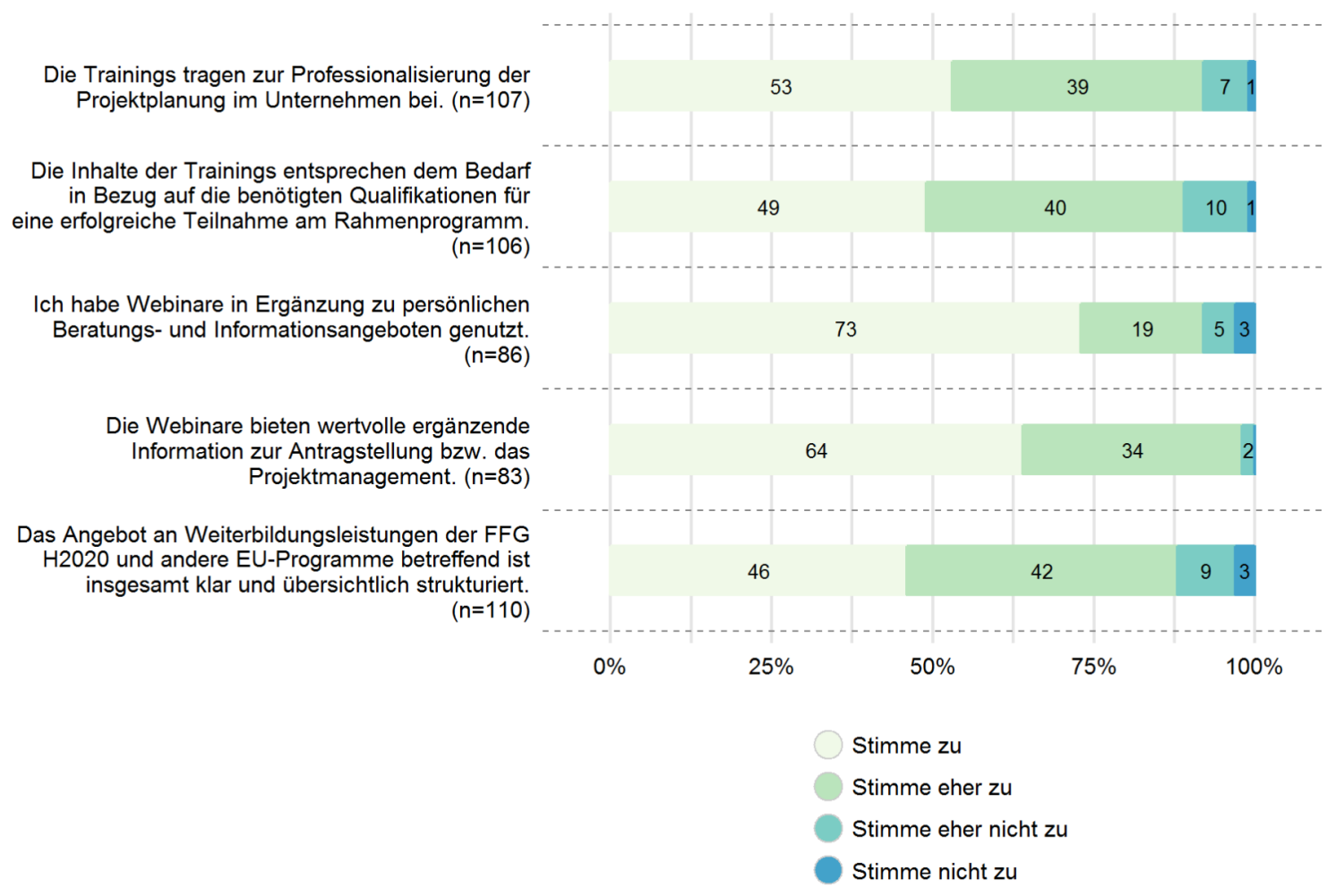

Quelle: Online Befragung, Joanneum Research.

\section{Erkenntnisse zum Core Customer Concept der FFG}

Das Core Customer Concept der FFG bietet einer kleinen, ausgewählten Gruppe an Unternehmen mit hohem FTI-Potenzial und Engagement eine umfangreiche und intensive Betreuung hinsichtlich ihrer optimalen Positionierung im Europäischen Forschungsraum. Dies belegen die Daten der folgenden Tabelle.

Bislang wurden 9 Unternehmen in der Pilotphase 2014, 15 Unternehmen im Jahr 2015 und 15 Unternehmen im Jahr 2016 als "core customer" der FFG betreut. Da einige Unternehmen kontinuierlich über mehrere Jahre betreut wurden, erreichte das C3 Instrument bislang 20 unterschiedliche Unternehmen. Diese C3 Unternehmen bekamen 318 Beratungen (davon 15\% Langberatungen) im Jahr 2015. Im darauffolgenden Jahr waren es 184 Beratungen (davon 40\% Langberatungen). Die Unternehmen besuchten insgesamt 21 FFG Veranstaltungen (zB Workshops, Webinare, Info-Veranstaltungen) im Jahr 2015 und 44 im Jahr 2016. 
Tabelle 3: Betreuung von C3 Unternehmen

\begin{tabular}{|lrr|}
\hline & 2015 & 2016 \\
\hline Anzahl an betreuten C3 Unternehmen & 15 & 15 \\
\hline Beratungen insgesamt & 318 & 184 \\
\hline davon Langberatungen in \% & $15 \%$ & $40 \%$ \\
\hline besuchte EIP Veranstaltungen & 21 & 44 \\
\hline
\end{tabular}

Für die Beratungen sowie deren Vor- und Nachbearbeitungen wendeten die FFG ExpertInnen 30 Personentage im Jahr 2015 und 20 Personentage im Jahr 2016 auf.

Die Auswertung der Feedback-Fragebögen zeigt durchwegs, dass die C3 Unternehmen sehr zufrieden mit den Leistungen der FFG ExpertInnen sind.

Besonders hervorgehoben werden in diesem Zusammenhang die Kompetenz der BeraterInnen, die rasche und direkte Kommunikation sowie die maßgeschneiderte, direkt auf das Unternehmen zugeschnittene Bereitstellung von Informationen und Services.

Die C3 Unternehmen nannten eine Vielzahl verschiedener Erwartungen, die sie im Vorfeld der Durchführung des Core Customer Concepts hatten, die unter den folgenden vier Kategorien subsumiert werden können:

- „Informationen zum Einreichprozess und den Evaluierungskriterien von H2020-Anträgen";

- „Aufbau einer Kommunikationsbasis mit einem/einer FFG ExpertIn der/die direkt mit dem Geschäftsplan des Unternehmens vertraut ist";

- "Erstellung eines für das Unternehmen optimierten Förderplans";

- "Vermittlung von Kontakten (zB zu Coaches, möglichen ProjektpartnerInnen und relevanten Netzwerken".

Die überwiegende Mehrheit der C3 Unternehmen gibt an, dass ihre Erwartungen voll und ganz erfüllt wurden. Weiters wurden die Unternehmen gefragt, inwiefern die Beratungsleistungen des C3 einen Mehrwert für ihr Unternehmen geschaffen haben. Folgende Zusammenstellung gibt einen Überblick über die Antworten der Unternehmen:

- "das gesamte Spektrum der Beteiligungspotentiale wurde systematisch erschlossen in Übereinstimmung mit Unternehmenszielen und -strategien";

- "Integration einer ERA Strategie in den unternehmensinternen Business Plan";

- „zusätzliche Geschäftsfelder wurden interessant gemacht”;

- "bewussteres Planen der Förderstrategie durch kompetente Beratung, besseres Einschätzen der Förderchancen";

- "Die Beratung hat die Qualität der H2020 Anträge maßgeblich verbessert, hat sich aber auch positiv auf das Schreiben nationaler Anträge etc. ausgewirkt";

- "erfolgreich eingeworbene EU Mittel";

- "erfolgreich eingeworbene EU Mittel haben sich positiv auf Unternehmenswert ausgewirkt" und Zugang zu Investitionskapital erleichtert";

- "Unternehmen konnte vom Netzwerk der FFG ExpertInnen profitieren".

Es zeigt sich, dass sich der Erfolg des Core Customer Concepts insbesondere hinsichtlich des Erwerbs von Wissen über den Europäischen Forschungsraum und seinen Funktionsweisen bemerkbar macht.

Eine Vielzahl an Unternehmen gibt an, dass „zusätzliche Geschäftsfelder interessant gemacht wurden", sie nun "den Förderdschungel verstehen können" und die Beratungen 
das Unternehmen „entscheidungsfähig gemacht haben". Ein bewusstes Planen und Aufsetzen einer unternehmensinternen Förderungsstrategie ist somit möglich. Von den Unternehmen werden in diesem Zusammenhang als angenehme "Nebeneffekte" dieser individuellen Betreuung die Ersparnis von Zeit und Kosten gesehen, die durch das eigenständige Einarbeiten in die Logik von H2020 und das Ausarbeiten von Anträgen mit extrem niedrigen Förderchancen entstanden wären.

Als einen weiteren "added value" geben die Unternehmen an, dass sich die Qualität ihrer Forschungsanträge verbessert habe. In diesem Zusammenhang betonen die Unternehmen, dass „die Beratung durch die FFG die Qualität des H2020 Antrags definitiv erhöht hat und zu ihrem Erfolg beigetragen hat"; aber nicht nur die Qualität der H2020 Anträge habe sich verbessert; einige Unternehmen erwähnen, dass sich ihre neu gewonnene Kompetenz im Antragschreiben auch positiv auf andere Anträge (von zB nationalen Programmen) ausgewirkt habe.

Ein weiterer Erfolgsindikator ist natürlich auch die Höhe der erfolgreich eingeworbenen EU Mittel. Diesbezüglich erwähnen die C3 Unternehmen, dass die intensive Betreuung durch FFG ExpertInnen, z.B. auch im Rahmen des Proposal checks maßgeblich zum Erfolg des H2020-Antrags beigetragen habe. Zusätzlich gilt eine EU Förderung als ein Qualitätsmerkmal in InvestorInnenkreisen. Daher gaben einige Unternehmen an, dass sich durch die erfolgreich eingeworbenen EU Mittel der Wert ihres Unternehmens erhöht und Zugang zu Investitionskapital deutlich erleichtert habe. Die folgende Zusammenstellung stellt dar, wie viele Projekte in ERA durch die C3 Unternehmen eingereicht wurden sowie ihren Erfolg.

Tabelle 4: Überblick über C3 Beratungsleistungen

\begin{tabular}{|lr|}
\hline & $2015+2016$ \\
\hline Eingereichte Projekte in ERA der C3 Unternehmen & 141 \\
\hline $\begin{array}{l}\text { Involvierung der EIP ExpertInnen bei diesen Einreichungen } \\
\text { durch Beratung }\end{array}$ & $60 \%$ \\
\hline $\begin{array}{l}\text { Involvierung der EIP ExpertInnen bei diesen Einreichungen } \\
\text { durch Intensivberatung und Proposalcheck }\end{array}$ & $33 \%$ \\
\hline Erfolgreiche Projekte der C3 Unternehmen & 46 \\
\hline $\begin{array}{l}\text { nicht geförderte Projekte der C3 Unternehmen } \\
\text { aber ausgezeichnet als "above threshold" }\end{array}$ & 9 \\
\hline Betreuung durch EIP ExpertInnen bei den erfolgreichen Einreichungen & 34 \\
\hline
\end{tabular}

Insgesamt gab es von Seiten der C3 Unternehmen 141 einschlägige Projekteinreichungen, davon waren 46 erfolgreich. 34 dieser 46 Projekteinreichungen wurden von der FFG begleitet. Zwei Aspekte müssen hinsichtlich dieses Erfolgsindikators beachtet werden: zum einen lassen sich erfolgreiche $\mathrm{H} 2020$ Beteiligungen oftmals erst über einen längeren Zeitraum abschätzen, d.h. die C3 Unternehmen und ihre Aktivitäten sollten über einen längeren Zeitraum analysiert werden. Zum anderen strahlt die C3 Betreuung auf andere Projekteinreichungen aus, daher wäre auch eine Betrachtung der Entwicklung der Inanspruchnahme von nationalen Projekten relevant. 
Abschließend wird von den Unternehmen noch der Aufbau strategischer Partnerschaften als ein wesentlicher Erfolg des Core Customer Concepts gesehen. Die Unternehmen erwähnen in diesem Zusammenhang, dass sie "wertvolle Kontakte zu Partnerorganisationen, Gremien und Netzwerken" erhalten haben und Partnerschaften auch tatsächlich aufbauen konnten.

Gefragt nach dem „Impact” des Core Customer Concepts für ihr Unternehmen gaben jedoch auch einige Unternehmen an, dass die Beratungen zwar "hilfreich" waren, dass jedoch (aufgrund interner oder externer Faktoren) die entsprechenden Strukturen nicht geschaffen werden konnten, sodass besprochene Maßnahmen nicht umgesetzt wurden. Solche kritischen Kommentare waren:

- "Beratung war hilfreich -> aber Umsetzung ist schwierig";

- "besprochenen Maßnahmen mit FFG konnten aufgrund interner und externer Faktoren nicht konsequent umgesetzt werden, C3 Kooperation wurde beendet";

- "der richtige Blickwinkel auf ERA wurde geschaffen, das entsprechende Wissen wurde vorab aber eher nur für nationale Einreichungen verwendet";

- „aktuelle Geschäftsentwicklung lässt EU Fokus momentan nicht zu".

In den durchgeführten Interviews wurde das Core Customer Concept (C3) von den teilnehmenden Unternehmen sehr positiv wahrgenommen und als wertvolle Unterstützung hinsichtlich ihrer FTI-Strategie vis-a-vis Europa bewertet. Bezüglich dieser Strategien finden sich dabei unterschiedliche Ansätze, sowohl was das Antragsverhalten, die Prozesse der Netzwerkfindung als auch die gesuchte bzw. bevorzugte Stellung innerhalb der Konsortien (KoordinatorInnenrolle) betrifft. Hervorgestrichen wurden in den Interviews insbesondere:

- die hohen Kompetenzen der FFG-BetreuerInnen,

- die "kurzen Wege" (direkter Draht zu den FFG-BetreuerInnen),

- die Schulungsangebote für MitarbeiterInnen („MitarbeiterInnen werden im Anlassfahl gezielt hingeschickt") und

- die "selektierte" und "kommentierte" Informationsbereitstellung.

Auch auf die positiven Effekte der Betreuung durch die FFG-EIP für die Strategiefindung wurde hingewiesen. Ebenso geschätzt wird die Hilfestellung bei der operativen Antragstellung. Insgesamt werden von den Unternehmen die aufgrund der Betreuung und Begleitung induzierten Lerneffekte hervorgestrichen und es wurde teilweise auch auf dadurch erfolgte Strategieänderungen hingewiesen. Anzumerken ist, dass von Seiten der Unternehmen die FFG als eine Institution wahrgenommen wird, d.h. eine Differenzierung bezüglich FFG-interner Bereiche wird oft nicht wahrgenommen. Das C3-Konzept wird auch von Seiten der FFG bewusst nicht offensiv nach außen getragen.

\section{Aggregierte Schulnotenbewertung durch die Unternehmen}

Insgesamt bewerten die antwortenden Unternehmen das Leistungsportfolio der FFG in Bezug auf ERA und $\mathrm{H} 2020$ sehr positiv. 76\% bzw. 83\% (bei Nichtberücksichtigung jener Unternehmen, die bei dieser Frage keine Angaben machten) bewerten die Leistungen der FFG mit „Sehr gut" oder "gut". 
Leistungsportfolio der FFG im Bereich ERA und $\mathrm{H} 2020(n=203)$

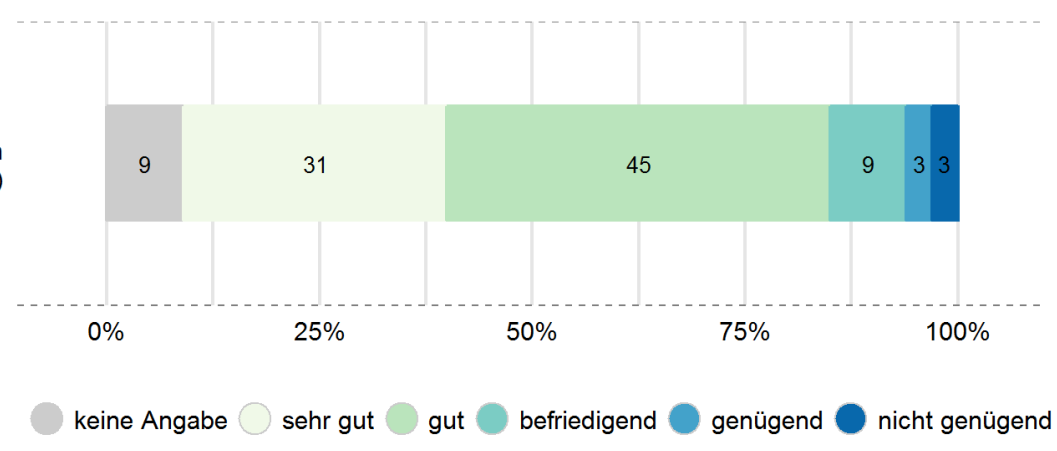

Quelle: Online Befragung, Joanneum Research.

Auch eine spezifische Betrachtung der Bewertung der FFG-EIP Leistungen - differenziert nach der Unternehmensgröße einerseits und dem Erfahrungsgrad der antwortenden Unternehmen andererseits - zeigt durchwegs gute bis sehr gute Bewertungen. Im Detail ergeben sich dabei dann folgende Spezifika (siehe Abbildung 30):

Unterschiede zeigen sich - sowohl bei Kleinunternehmen als auch bei der Gruppe der Mittel- und Großunternehmen - vor allem hinsichtlich des Erfahrungsgrads. Bei nicht erfahrenen Unternehmen ist in beiden Gruppen der Anteil jener Unternehmen, die keine Angabe zur Qualität machen können (bzw. machen wollen) deutlich höher als bei erfahrenen Unternehmen. Dabei ist die Tendenz, keine Bewertung abgeben zu können (wollen), bei Mittel- und Großunternehmen generell etwas stärker ausgeprägt. Insgesamt zeigt sich jedoch in allen Teilgruppen, dass "schlechte" Einschätzungen der EIP-Leistungen nur von einer ganz kleinen Minderheit der Unternehmen abgegeben werden. 

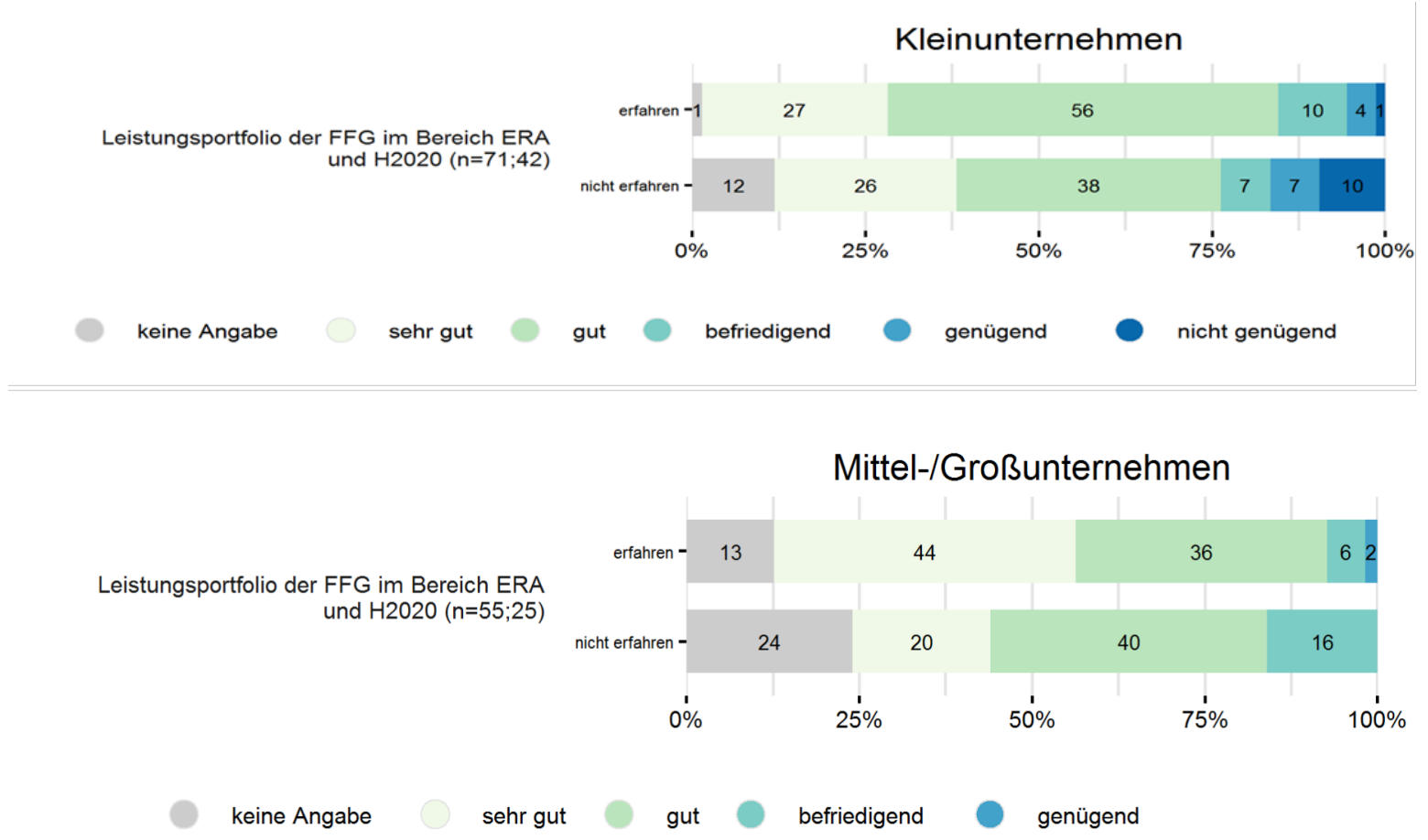

Quelle: Online Befragung, Joanneum Research.

\subsection{Ergebnisse zu EUREKA, COSME und EEN}

\subsubsection{EUREKA - Initiative für anwendungsnahe Forschung und Entwicklung in Europa}

EUREKA bzw. Eurostars wurde unter dem Dach von FFG-EIP in den letzten Jahren als niedrigschwellige Förderalternative mit vergleichsweise hohen Erfolgschancen, neben dem KMU-Instrument und anderen Beteiligungsmöglichkeiten im Rahmen von H2020 und den strategischen Partnerschaften, positioniert. Wie die bisherigen Gespräche zeigen, stellt EUREKA bzw. Eurostars einen wichtigen Teil des Förderangebots dar, nimmt dabei aber keine Sonderstellung ein. Die Eingliederung des EUREKA Büros unter das Dach von FFG-EIP und in das Konzept der ganzheitlichen Betreuung spielte dabei eine wesentliche Rolle. War das EUREKA Büro vor 2013 noch überwiegend als eigenständiges Team tätig, fand It. Meinung einer GesprächspartnerIn mit Beginn der Umsetzung von H2020 ein Paradigmenwechsel - und der (nicht gänzlich abgeschlossene) Versuch einer strategischen Positionierung von EUREKA im Portfolio - statt. In den Freitexten der Online-Befragung wird die Komplementarität von EUREKA zu den europäischen Programmen von einigen Befragten positiv hervorgestrichen. Ein/e Teilnehmer/in kritisiert allerdings eine "stiefmütterliche Behandlung" von EUREKA im Angebotsportfolio von FFG-EIP.

Wie bereits in der FFG-EIP-Zwischenbilanz festgehalten wird heute nicht nur bereichsübergreifend sondern auch stark teamübergreifend gearbeitet. Vor dem Hintergrund der verschiedenen Aktivitäten und Fördermöglichkeiten findet im WIN-Team (in- 
tern sowie mit anderen FFG Einheiten) ein reger Austausch statt. Während sich das EUREKA Büro der FFG-EIP aus personeller Sicht nicht geändert hat, wurden verschiedene Kompetenzen auf mehrere Personen im Team verteilt, auch auf jene, die zuvor nicht für EUREKA tätig waren. EUREKA-relevante Themen wie EEN, Zugang zu Risikofinanzierung und allgemeine Betreuung der Wirtschaft wurden im WIN-Team zuletzt verstärkt konzentriert. Während dadurch die Zusammenarbeit verstärkt und Synergien gewonnen werden konnten, wird die Vernetzung im wachsenden Ausmaß als (zeitliche) Herausforderung wahrgenommen.

Wie in mehreren Gesprächen bestätigt, führt dieser regelmäßige Austausch über betreute Unternehmen aber auch dazu, dass EUREKA, mit Abzügen auch Eurostars, mitunter als „Überlaufgefäß" für andere Angebote fungiert. Der Doktrin folgend, den KundInnen die bestmögliche Lösung zu bieten, werden Unternehmen, die nicht ganz für beispielsweise das H2020-KMU-Instrument in Frage kommen, häufig andere, niederschwellige Alternativen angeboten. Dazu zählt neben den nationalen Basisprogrammen auch EUREKA und Eurostars. Diese Überlauffunktion wird von Seiten der Administration durchaus als positiv gesehen, vor allem vor dem Hintergrund des wachsenden Drucks von Seiten der Europäischen Kommission, KMU zur Beteiligung an F\&E Förderinstrumente zu bewegen. Wenn sich, so die Sichtweise, viele Unternehmen für die attraktiven Angebote unter $\mathrm{H} 2020$ bzw. dem KMU-Instrument ("2 million for 1 single enterprise") bewerben aber letztlich nicht gefördert werden, sei es die Aufgabe von z.B. FFG konstruktiv mit dem teils hohen Interesse umzugehen und für förderwürdige Unternehmen mögliche alternative Angebote zu ermitteln.

Dabei stellte sich in einigen Gesprächen die Frage, ob es z.B. nicht einfacher wäre, ausgewählte Unternehmen gleich auf z.B. Eurostars hinzuweisen, v.a. wenn sich abzeichnet, dass das präferierte Instrument überzeichnet sein würde - eine klare Funktion der Beratung ("sich in die Unternehmen versetzen"). Wie den Gesprächen zu entnehmen war, bieten insbesondere Eurostars-Projekte durch entsprechende Anpassungen die Möglichkeit, die Erfolgswahrscheinlichkeit zu optimieren. Das könnte der einen oder anderen Enttäuschung auf Unternehmensseite mitunter entgegenwirken - und dazu beitragen dem "Seal of Excellence" ("dem Unternehmen gute Möglichkeiten bieten") auf nationaler Ebene eine Funktion zu geben.

Der verstärkte Austausch von EUREKA mit EEN, der im Bereich FFG-EIP zur Entwicklung einer gesamtheitlichen Sichtweise wesentlich beigetragen hat und über nationale wie internationale Workshops forciert wird, wurde im Rahmen verschiedener Gespräche äußerst positiv beurteilt. Während über das FFG-EIP laufend Aktivitäten gesetzt werden um die österreichische Beteiligung an EUREKA Cluster-Projekten zu steigern, etwa über die Organisation von Proposers' Days oder ähnlichen Networking-Aktivitäten, vermissen BeobachterInnen der Szene ein strategisches Interesse österreichischer Unternehmen an einer Kooperation im Rahmen eines Clusters, das über das einfache Lukrieren von Fördermitteln hinausgeht. Dies zeigt sich insbesondere im Vergleich zu anderen Ländern (Frankreich, Niederlande, Finnland), in welchen Priorisierungen im Clusterbereich mit der Industrie vorgenommen und entsprechende Budgets zur Verfügung gestellt werden. Auf Österreich entfällt die „Teilnehmer-Rolle”. Strategische Clusterinitiativen brauchen das Interesse von Unternehmen. In Österreich könnte laut einer InterviewpartnerIn z.B. die Industrie-4.0-Plattform eine solche Multiplikatorfunktion übernehmen. 
EUREKA-Mitgliedsländern steht die Möglichkeit offen, bi- oder multilaterale EUREKA-Calls zu lancieren. Darüber hinausgehende Gestaltungsmöglichkeiten ergeben sich für ein EUREKA-Vorsitzland, indem sich Vorsitzschwerpunkte ebenfalls in Calls umsetzen lassen. Nationale Agenturen, wie im Falle Österreichs die FFG, können diese Calls in weiterer Folge maßgeschneidert umsetzen. So wurde beispielsweise die Globalstars-Initiative auch von FFG-EIP mitinitiiert. Die Governance von EUREKA erfolgt auf nationaler Ebene entsprechend der Bedürfnisse der Ressorts. Während das BMDW primär eine bottom-up orientierte, länderspezifische Politikumsetzung verfolgt (z.B. Danube Region Call, Bi- und Multilaterale Calls), engagiert sich das BMVIT Themen-fokussiert, z.B. über Cluster.

Ein wichtiges Merkmal von EUREKA ist dessen unterschiedliche Ausgestaltung und Dotierung in den Mitgliedsländern. So gibt es in Österreich beispielsweise keine Trennung von Label-Vergabe und Förderentscheidung für EUREKA Projekte. Einreichungen werden im FFG Basisprogramm behandelt, das mit Themenoffenheit und einer bottom-up Orientierung den Ansatz von EUREKA spiegelt. Die enge Zusammenarbeit zwischen dem EUREKA-Büro und den VertreterInnen der Basisprogramme bedingt regelmäßige Abstimmungsprozesse. So werden einerseits auf elektronischem Weg Zeitpläne und Einreichfristen abgestimmt, andererseits finden gemeinsame Monats-Meeting zum bilateralen Austausch statt.

EUREKA Projekte werden nach entsprechender interner Evaluierung vom Beirat für die Basisprogramme für eine Label-Vergabe bzw. die Förderung empfohlen. Nur wenn der, bzw. die anderen internationalen TeilnehmerInnen bei ihrer EUREKA-Heimatinstitution eine Empfehlung erhalten haben, erhält das Projekt ein gemeinsames Label. So kann es manchmal vorkommen, dass ein Förderantrag abgelehnt wird, etwa weil trotz positiver Prüfung beteiligter, ausländischer EUREKA-Büros der Antrag nicht österreichischen Förderregeln entspricht. Oder, dass ein Konsortium einreicht und dann eine PartnerIn ausfällt, weil der nationale Fördergeber die verfügbaren Ressourcen bereits ausgeschöpft hat. Das erklärt auch die unterschiedliche Beteiligung der Länder an EUREKA und, mit Abstrichen, auch an Eurostars. Vor diesem Hintergrund werden österreichische EinreicherInnen dazu angehalten erst dann für ein Projekt auf nationaler Ebene einzureichen, wenn in den Partnerländern Klarheit über Förderentscheidung besteht.

Die jeweiligen NPCs sind bemüht, den Austausch mit beteiligten, ausländischen FördergeberInnen bei Einreichung eines gemeinsamen Projekts rasch aufzunehmen und die Abstimmung bestmöglich zu unterstützen. Wie auch in den Jahresberichten hervorgehoben wird, bleibt die Koordinierung und Synchronisierung jedoch das Hauptproblem von EUREKA, zusätzlich zu schrumpfenden nationalen Budgets in vielen Ländern. Die Vernetzung in EUREKA und die Nutzung des im EUREKA-Büro vereinten Know-hows über die örtlichen Gegebenheiten (verfügbare Mittel für EUREKA) ermöglicht es, die Erfolgswahrscheinlichkeiten in der Zusammenarbeit mit den verschiedenen Ländern gut abschätzen zu können.

Zwischen den nationalen NPCs besteht generell eine gute Kommunikationsbasis und ein reger Austausch. Ausdruck hierfür sind die verstärkt durchgeführten bilateralen und multilateralen Calls. Während die Abstimmung mit den Förderbedingungen der anderen Mitgliedsländer allgemein als herausfordernd angesehen wird, finden sich überraschend viele 
ausländische Einreichungen mit österreichischen Beteiligungen. In einigen Gesprächen wird dies damit erklärt, dass Österreich als verlässliches, stabiles Land angesehen wird, das über ein gut dotiertes Förderwesen und damit verbundene Beratung verfügt.

Die administrativen Plankosten für das Programmmanagement von EUREKA in der FFG-EIP wurden seit 2014 jedes Jahr unterschritten (siehe Tabelle 3) Das schlanke Programmmanagement, das die wesentlichen Grundfunktionen der Abwicklung abdeckt, kombiniert mit der Nutzung der Synergien im EIP (so auch WIN-Team) ist als effizient zu bewerten. Durch das Auflegen von Arbeitsplänen (Plan) und Jahresberichten (IST) wird eine hohe Transparenz der Abwicklung sowie eine angemessene Kontrolle der Mittelverwendung gewährleistet.

Tabelle 5: Administrative Programmkosten EUREKA Programmmanagement FFG-EIP

\begin{tabular}{|l|c|c|c|}
\hline & 2014 & 2015 & 2016 \\
\hline Geplante Programmkosten (in €) & 312.185 & 307.313 & 287.383 \\
\hline Tatsächliche Abrechnung (in €) & 255.174 & 227.659 & 248.548 \\
\hline Abweichung in \% & $-18,0 \%$ & $-26,0 \%$ & $-13,5 \%$ \\
\hline
\end{tabular}

Quelle: Jahresbericht It. Rahmenvertrag, EUREKA inkl. Eurostars-Betreuung im EIP 2014-2016

Der Beitrag von EUREKA Eurostars zur Zielerreichung im Rahmen der WFA wird als insgesamt zufriedenstellen eingeschätzt. Die untere Schwelle des Zielbereichs des Indikators "Teilnahme an 100-120 erfolgreichen Eurostars-Projekten in der Phase von Eurostars-2 (2014-2020)" wurde bereits überschritten: Per Ende März wurden It. EU-PM für 103 Eurostars-2-Projekte ein Vertrag erstellt. Davon wurde lediglich ein Projekt seit 2014 in der Laufzeit storniert. Rund ein Drittel bestehen seit Anfang 2017. Gesetzt den Fall, dass Eurostars-2-Ausschreibungen auch weiterhin im gleichen Rhythmus abgewickelt werden, kann davon ausgegangen werden, dass auch die obere Grenze erreicht bzw. überschritten werden wird. Zu bedenken gilt, dass die Möglichkeit der Projektfinanzierung im engen Verhältnis zu den zur Verfügung stehenden Budgetmitteln steht. Änderungen bei der Mittelausstattung hätten unmittelbare Auswirkungen auf die Projektfinanzierung.

Bei der Interpretation des Indikators "(Erhöhung des Anteils an) innovativer KMU, die mit anderen kooperieren", eine Messgröße im European Innovation Survey (EIS) der sich aus der Europäischen Innovationserhebung (Community Innovation Survey - CIS) ableitet, ist die zeitlichen Verzögerung bei der Bereitstellung der Ergebnisse zu beachten. Der aktuell verfügbare Datenstand ist von 2012/14 und weist einen Anteil von 20,5\% auf - und damit weniger als die angestrebten 22,7\%. Aufgrund des Alters der Daten ist eine entsprechende Bewertung nur bedingt zulässig. Eine bessere Einschätzung ist auf Basis der Ergebnisse der CIS 14/16 zu erwarten, dessen Bereitstellung für Ende 2018 erwartet wird.

Während dieser Indikator die Ansprüche an eine statistische Validität prinzipiell erfüllt, stellt sich an einigen Stellen die Frage nach der Zweckmäßigkeit. So werden bei der Innovationserhebung neben EUREKA Eurostars auch andere Maßnahmen zur Berechnung des Zielwertes herangezogen. Zudem werden in der Innovationserhebung explizit „innovative KMU" adressiert, ein Kriterium, das in EUREKA Eurostars nicht zwangsläufig erfüllt 
sein muss. Abschließend ist festzustellen, dass der KMU-Anteil in den Eurostars-Projekten (2014 bis März 2018) bei $60 \%$ lag.

\subsubsection{COSME - Programm für die Wettbewerbsfähigkeit von Unternehmen und für KMU}

Wie bereits weiter oben genauer ausgeführt, ist die FFG vom BMDW damit beauftragt, COSME zu bewerben und österreichische Organisationen bei Fragestellungen rund um das Programm zu unterstützen. Wie aus den durchgeführten Interviews hervorgeht, sind hauptsächlich die in Tabelle 6 dargestellten Akteure auf nationaler Ebene in den vier

Bereichen des Programms hinsichtlich deren Umsetzung aktiv:

Tabelle 6: Akteure und Aktivitäten in den Programmbereichen von COSME

\begin{tabular}{|c|c|c|}
\hline COSME Bereich & Akteur & Aktivität(en) \\
\hline \multirow[t]{2}{*}{$\begin{array}{l}\text { Zugang zu } \\
\text { Finanzierungsmitteln } \\
\text { („Accessing Finance") }\end{array}$} & aws & $\begin{array}{l}\text { Abwicklung Kreditgarantiefazili- } \\
\text { tät (LGF) und Equity Fazilität für } \\
\text { Wachstum (EFG) } \\
\text { Durch COSME unterstützte aws } \\
\text { Förderungen: } \\
\text { - } \quad \text { aws Garantie für junge Un- } \\
\text { ternehmen } \\
\text { - aws Double Equity } \\
\text { - aws Garantien für Investitio- } \\
\text { - } \text { nen in Österreich } \\
\text { aws Garantien International }\end{array}$ \\
\hline & FFG & $\begin{array}{l}\text { Bekanntmachung bei KMU aus } \\
\text { operativer Ebene, Signposting } \\
\text { von KMU zur AWS; Aktivitäten } \\
\text { bei potentiellen weiteren Finanz- } \\
\text { intermediären auf strategischer } \\
\text { Ebene (z.B. Banken-Roundtable) }\end{array}$ \\
\hline \multirow[t]{2}{*}{$\begin{array}{l}\text { Zugang zu Märkten } \\
\text { („Opening Markets") }\end{array}$} & WKÖ/FFG & $\begin{array}{l}\text { Koordination EEN-AT-Konsor- } \\
\text { tium, EEN-Knoten (i.d.R. WKÖ: } \\
\text { Businessthemen; FFG: FTI-The- } \\
\text { men) }\end{array}$ \\
\hline & $\begin{array}{l}\text { Bundesländer- } \\
\text { akteure }\end{array}$ & EEN-Knoten \\
\hline $\begin{array}{l}\text { Förderung der unternehmeri- } \\
\text { schen Initiative und -kultur } \\
\text { („Supporting Entrepreneurs") }\end{array}$ & WKÖ/WKs/Intermediäre & $\begin{array}{l}\text { z.B. bei div. Entrepreneurship } \\
\text { Calls oder Stimulierung weiterer } \\
\text { ERASMUS Knotenpunkte }\end{array}$ \\
\hline
\end{tabular}




\begin{tabular}{|l|l|l|}
\hline COSME Bereich & Akteur & Aktivität(en) \\
\hline $\begin{array}{l}\text { Verbesserung der Rahmenbedin- } \\
\text { gungen für die Wettbewerbsfä- } \\
\text { higkeit („Improving Business } \\
\text { Conditions") }\end{array}$ & $\begin{array}{l}\text { BMDW } \\
\text { Netzwerk der EU-Botschafter }\end{array}$ & KMU-Politikmaßnahmen \\
\cline { 2 - 3 } & $\begin{array}{l}\text { FFG } \\
\text { Bundesländer- } \\
\text { agenturen } \\
\text { Intermediäre (Verbände, Cluster } \\
\text { etc.) }\end{array}$ & $\begin{array}{l}\text { sektorale und horizontale Calls } \\
\text { (z.B. Cluster, Tourismus, Digita- } \\
\text { lisierung) }\end{array}$ \\
\hline
\end{tabular}

Über $80 \%$ der budgetären Mittel in COSME entfallen auf die Bereiche "Accessing Finance" und "Opening Markets".

Je nach Jahr waren bisher 3\% bis 5\% der Mittel für den Bereich "Supporting Entrepreneurs" und $9 \%$ bis $13 \%$ für den Bereich "Improving Business Conditions" vorgesehen. ${ }^{26}$ Neben den Finanzierungsinstrumenten (Bereich „Accessing Finance"), dem EEN und einigen Rahmenverträgen, bspw. zu jährlichen Berichten über KMU-Entwicklungen, Studien, Best-Practice-Austauschen, Veranstaltungen, Konferenzen u.ä., werden diverse thematische bzw. sektorale Calls veröffentlicht, die in Relation zu den zuvor erwähnten Aktivitäten einen sehr kleinen budgetären Anteil ausmachen. Das Programm ist hier besonders kleinteilig strukturiert und thematisch breit gestreut. Oftmals wird je Call nur ein Projekt EU-weit gefördert. Diese Ausschreibungen sind jedoch auch seitens der FFG in Hinblick auf H2020 relevant, da die Stärkung der Wettbewerbsfähigkeit (adressiert durch COSME) und Innovation (adressiert nun durch H2020) oft zueinander in Verbindung stehen, und einzelne Themen (z.B. Manufacturing, Digitisation etc.) in beiden Programmen - wenn auch aus unterschiedlicher Perspektive - adressiert werden. Die Herauslösung der Innovationsaspekte aus CIP/COSME bei Programmstart dürfte also tendenziell wieder in der Programmumsetzung zurückgenommen werden, eine logische Konsequenz der impliziten Verknüpfung dieser Bereiche. Hier ist die FFG kontinuierlich gefordert, die strategische Positionierung der Zielgruppen zu unterstützen und an der Schnittstelle der beiden Programme entsprechend zu agieren.

Interessant ist die Tatsache, dass in COSME mit einzelnen kleinen thematischen Calls (z.B. Thema Manufacturing) auch pilothafte Calls lanciert werden, die bei hinreichendem Erfolg später auch in H2020 (z.B. Innovation for SMEs), größer und fokussierter, übernommen werden oder auch eine gewisse strukturelle Basis dafür aufbauen sollen, an der dann mit weiteren Calls angesetzt wird. ${ }^{27}$ Auch die Interimsevaluierung von COSME

25 in diesem Programmbereich sind die Calls thematisch am breitesten gestreut innerhalb des ohnehin bereits breiten Portfolios von COSME

26 Siehe dazu auch: Technopolis Group (2017): Interim Evaluation of the COSME Programme

27 Beispiel verknüpfte Calls zum Thema Manufacturing: COSME Call-1: "Access for SMEs to advanced manufacturing support" (Schaffung eines "European Advanced Manufacturing Support

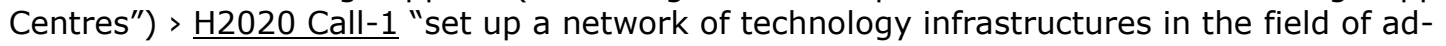
vanced manufacturing for clean production with one-stop-shop access for SMEs" (INNOSUP-03- 
streicht die diesbezügliche Flexibilität des Programms mit weiteren Beispielen hervor: "A key asset of the COSME programme is its flexibility in launching new actions, including pilot actions, in response to emerging needs and changing policy priorities, for example in the area of entrepreneurship and the support to start-ups." ${ }^{28}$ Die FFG arbeitet hier aktiv daran, solche Trends rechtzeitig zu antizipieren und die jeweiligen österreichischen Potenzialträger für solche Ausschreibungen zu identifizieren bzw. zur Teilnahme zu motivieren.

Zentraler Mehrwert der Betreuung des Programms durch die FFG dürfte der Informationsvorsprung durch den Aufbau direkter Kontakte zur Europäischen Kommission und der Teilnahme an internationalen Veranstaltungen sein.

Dies ermöglicht eine zeitgerechtere und adäquatere Information und Beratung der Zielgruppe was einen wichtigen Aspekt darstellt, da die Calls i.d.R. kurzfristig angekündigt werden und darüber hinaus auch oft nur für eine kurze Zeitspanne geöffnet sind. Die FFG arbeitet intensiv an einem ständigen Screening der Community (potentielle EinreicherInnen) sowie an dem Screening, welche Themen/Calls geöffnet werden (kurzfristige Dimension). Die organisatorische Integration der Programmagenden in das WIN-Team ist auch dabei eine notwendige Voraussetzung für ein effizientes Programm- und Schnittstellenmanagement.

Die administrativen Plankosten für das Programmmanagement von COSME in der FFG-EIP wurden seit 2014 jedes Jahr unterschritten, was vor allem auf Unterschreitungen bei den Reisekosten zurückzuführen ist (siehe Tabelle 7). Diese Abrechnungsdaten sind, neben der sinnvollen organisatorischen Integration (WIN-Team etc.), ein wichtiges Indiz für eine relativ effiziente Umsetzung von COSME in der FFG.

Tabelle 7: Administrative Programmkosten COSME Programmmanagement FFG-EIP

\begin{tabular}{|l|r|r|r|}
\hline & 2014 & 2015 & 2016 \\
\hline Geplante Programmkosten (in €) & 99.255 & 99.564 & 122.392 \\
\hline Tatsächliche Abrechnung (in €) & 91.438 & 82.327 & 98.924 \\
\hline Abweichung in \% & $-8 \%$ & $-17 \%$ & $-19 \%$ \\
\hline
\end{tabular}

Quelle: COSME Jahresberichte 2014, 2015, 2016

Eine wesentliche Herausforderung bei der Umsetzung von COSME durch die FFG scheint die Datenlage bezüglich der Rückflüsse zu sein. Insgesamt waren EU-weit für die Implementierung der COSME-Arbeitsprogramme 2014-2016 rd. 823 Mio EUR (2014: 240, 2015: 294, 2016: 289) budgetiert, die durch eine Vielzahl an Einzelaktionen umgesetzt wurden. Hiervon ist die Verteilung von rund $10 \%$ der jeweiligen jährlichen Budgets

2017; Kooperation mit Vertragsnehmer aus COSME Call-1 ist ausdrücklich gewünscht) > $\underline{\mathrm{H} 2020}$ Call-2 "Pan-European advanced manufacturing assistance and training for SMEs" (INNOSUP-062020; Scaling-up des small-scale-Piloten aus CoSME-1) > COSME Call-2 (skills): Curriculum guidelines for key enabling technologies (KET) and advanced manufacturing technologies (AMT)

28 Technopolis Group (2017): Interim Evaluation of the COSME Programme, S. 95 
aus der Datenbank nicht abrufbar, da die Vergabe nicht über offene Ausschreibungen, sondern direkt durch die Europäische Kommission erfolgt. ${ }^{29}$

\section{Seitens der Europäischen Kommission werden wenig Daten zur Verfügung ge-} stellt, und wenn, dann mit starken Verzögerungen und auch nur für Einzelbereiche. Ein Monitoring der sehr breit gestreuten Themen gestaltet sich dementsprechend umständlich und zeitintensiv, und wird nur durch die Kenntnis der FFG von AntragstellerInnen möglich, zu denen im Laufe der Antragstellung persönlicher Kontakt bestand, bzw. andererseits durch direktes Kontaktieren von ReferentInnen innerhalb der Europäischen Kommission, die für bestimmte Themen zuständig sind, in denen eine österreichische Beteiligung vermutet wird. Hier werden also lückenhafte Daten durch aufwendige Kleinarbeit der FFG aufgearbeitet.

Das Monitoring von COSME wurde bisher mit der Einführung des COSME Data Hub ${ }^{30}$ nur im Ansatz verbessert; die relativ schlechte Datenlage besteht nach wie vor in Form von zeitlicher Verzögerung der Übermittlung von Teilnahmedaten, der Unmöglichkeit der Auswertung von Einzelbeteiligungen und der nicht vorhandenen Information zu abgelehnten Beteiligungen. ${ }^{31}$ Auch die Nachvollziehbarkeit der Rückflüsse ist de facto nicht ausreichend gegeben, da nur eine Gesamtzahl seitens der Europäischen Kommission angegeben wird.

Seit Beginn der Laufzeit des Programms mit 2014 erzielten österreichische Organisationen in dem Programm einen Rückfluss von mindestens 13,4 Mio $€ .32$

Laut FFG-EIP-Zwischenbilanz weist COSME keinen hohen Bekanntheitsgrad unter den potentiellen EinreicherInnen auf; aber auch einzelnen MultiplikatorInnen (jene, die die Informationen weiterverbreiten sollen; Ausnahme: FFG und z.T. WKO) dürfte das Programm (mit seinen gesamten Möglichkeiten) eher wenig bekannt sein, wie die Interviewund auch die Befragungsergebnisse zeigen. Eine Ausnahme bilden dabei durchweg die Finanzierungsinstrumente (Bereich: „Accessing Finance") sowie das EEN (Bereich „Opening Markets).

Wie aus den Interviews hervorgeht, wird das Programm - abseits der Finanzierungsinstrumente und dem EEN - von einzelnen Zielgruppen scheinbar aktiver genutzt (z.B. Clustermanagements, Tourismusakteure) und von anderen weniger (z.B. einzelne WKs wobei die Aktivitätslevels offenbar auch organisationsintern variieren). Einzelne Intermediäre nehmen auch bewusst nicht teil, d.h. reagieren nicht auf Calls (Entscheidung der Geschäftsführung in der Organisation, Kosten/Nutzen-Abwägung). In einzelnen Fällen erscheint der Mehrwert einer Teilnahme auch bei Intermediären, die in der Vergangenheit bereits an COSME-Projekten teilgenommen haben, aktuell nicht mehr gegeben. So wird

29 In Form von "ad hoc grants", "direct contracts", „administrative agreements", „membership fees" oder "reimbursement of experts", oder oftmals in Form von "Specific contracts under a framework contract".

30 Siehe dazu das COSME Data Hub mit öffentlich zugänglicher Übersicht über Projekte

31 Siehe dazu auch Technopolis Group (2017): Interim Evaluation oft he COSME Programme (z.B. zusammenfassend S. 97)

32 COSME Jahresbericht über das Jahr 2016 (Stand 10/2017) 
beispielsweise mittlerweile von einer Teilnahme an den Calls zu "Clusters Excellence", abgesehen, da man in Österreich von anderen europäischen KonsortialpartnerInnen (z.B. südeuropäische Cluster) offenbar nicht mehr so viel lernen kann und der Aufwand für Antrag und Durchführung dem Nutzen nicht (mehr) entspricht. Dennoch bietet das Programm nach wie vor - nicht zuletzt aufgrund von wechselnden thematischen/sektoralen Calls - Teilnahmemöglichkeiten für österreichische Intermediäre; gerade in den Bereichen Cluster und Tourismus, in denen bisher eine vergleichsweise gute österreichische Teilnahmeaktivität festgestellt werden konnte, wie einige InterviewpartnerInnen hervorheben.

\subsubsection{EEN - Enterprise Europe Network}

Das EEN-Netzwerk ist in seinem Selbstverständnis ein Servicepartner für Unternehmen, mit einem Fokus auf Internationalisierung, internationaler Kooperation, Forschung und Entwicklung und Innovation.

Der Mehrwert des EEN ist nach Ansicht der meisten InterviewpartnerInnen die dezentrale (regionale) Organisation mit für die Unternehmen maßgeschneiderten Angeboten.

Die Beratung und der Kooperationssupport (Individuelle Beratung, internationale Partnersuche und Kooperationbörsen) stehen im Fokus der FFG-EIP-Beauftragung mit EENLeistungen im Rahmen von COSME. Der Innovationssupport (KAM und EIMC) wird aus Mitteln des H2020-Vertrags finanziert und steht einem vergleichsweise kleinen Teil der Unternehmen zur Verfügung, deren Selektion entweder durch erfolgreiche Antragstellung in H2020 vorgegeben ist (KAM), oder seitens der FFG nach hoch selektiven Kriterien vorgenommen wird (EIMC). Bei der Ausschöpfung der Mittel haben sämtliche KAM-Aktivitäten Vorrang vor EIMC-Aktivitäten.

In Zusammenhang mit dem KMU-Instrument ist vor allem das KAM als EEN-Leistung relevant, das in enger Zusammenarbeit zwischen den EEN-Verantwortlichen in dem FFGEIP und dem NCP für das KMU-Instrument sowie in Abstimmung mit der EIP-internen Wirtschaftsgruppe abgewickelt wird. Mit dem KAM wird ein im KMU-Instrument erfolgreiches Unternehmen hinsichtlich projektspezifischer Herausforderungen von ausgewählten Business Coaches begleitet. Damit kann die Beteiligung eines Unternehmens am KMUInstrument in einen größeren unternehmensrelevanten Kontext gesetzt werden und können zukünftige Potentiale bzw. mögliche Hindernisse dieser Beteiligung zeitnah antizipiert und adressiert werden (mögliche Anschlussförderungen und -finanzierungen, weitere PartnerInnen/Konkurrenten, Märkte etc.).

Das EIMC als weitere EEN-Leistungen steht hingegen nicht in einem notwendigen Zusammenhang mit dem KMU-Instrument. Damit werden KMU adressiert, die ein innovatives Potential und einen Bedarf an einem Aufbau an Strukturen zum Management von Innovationen haben. Die Selektion (einer relativ geringen Anzahl an Unternehmen) erfolgt über die FFG-EIP und dabei spielt auch hier die laufende Zusammenarbeit und Abstimmung von EEN-Verantwortlichen, NCP und Wirtschaftsgruppe eine bedeutende Rolle. Die organisatorische Integration der EEN-Aktivitäten in das WIN-Team ist also auch dementsprechend von hoher Relevanz. 
Im Sinne eines regionalen Netzwerks spielen die einzelnen Knoten (Anlaufstellen in den Bundesländern) eine gewichtige Rolle in der erfolgreichen Umsetzung. Die Rolle der FFG ist hier (da nur für Wien, Niederösterreich und Burgenland zuständig) etwas weniger zentral als beispielsweise bei der Antragsberatung zu H2020, für die vielfach seitens der Bundesländeragenturen an die FFG verwiesen wird. Dennoch - und nicht zuletzt aufgrund der guten organisatorischen Einbettung der EEN-Aktivitäten - erfolgt auch für die Betreuung von Unternehmen aus Wien, Niederösterreich und Burgenland eine sinnvolle, anlassbezogene Abstimmung mit den relevanten Bundesländerakteuren.

\section{Abbildung 31: Bewertung der EEN-Leistungen und Tools durch Intermediäre}

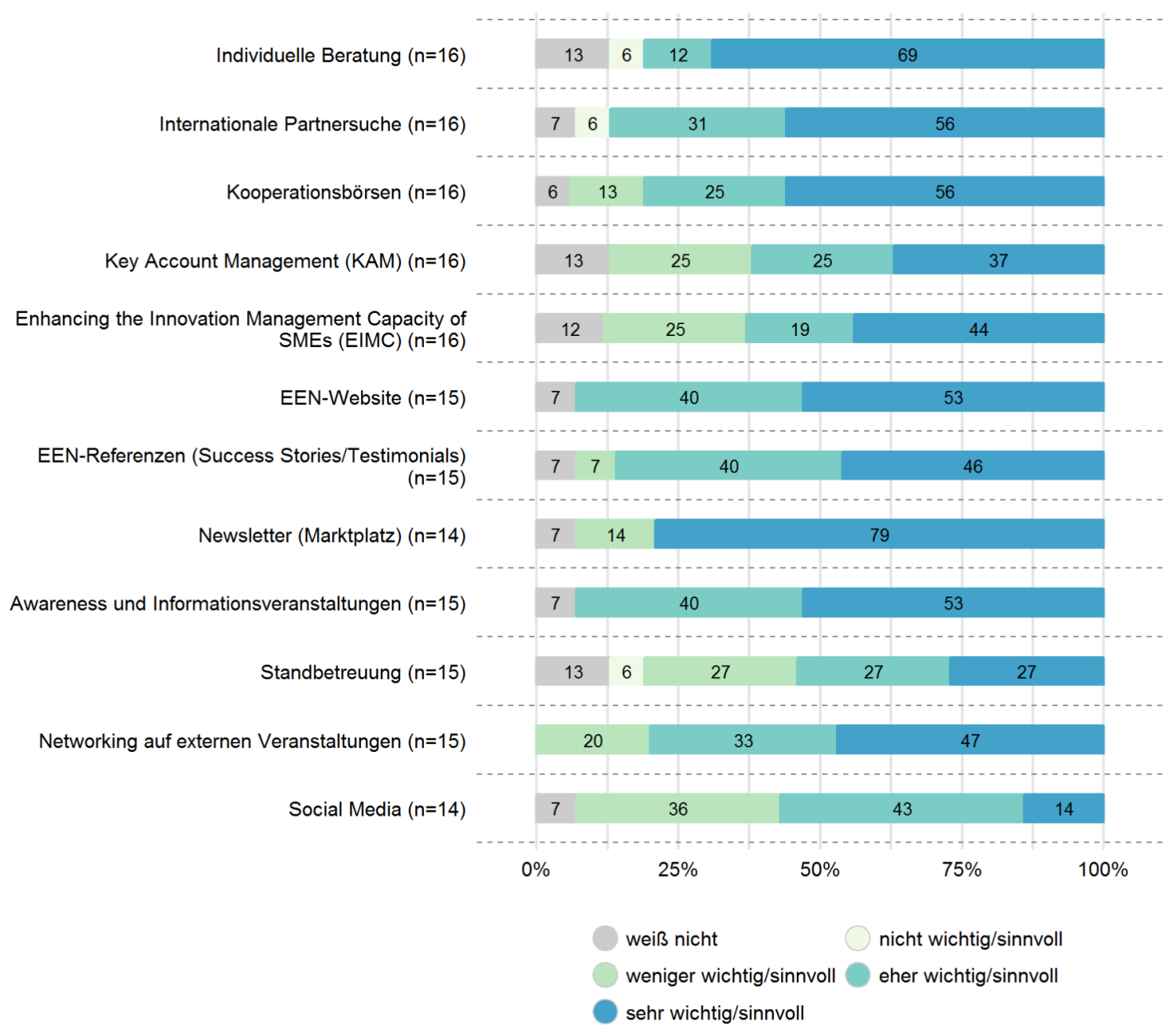

Quelle: Online Befragung, Joanneum Research.

Das EEN wird durchgängig von den umsetzenden Akteuren und auch Stakeholdern als sehr zentral für die Betreuung von Unternehmen bei ihren internationalen Aktivitäten gesehen. Oft wird dabei die Qualität der Tools für entsprechende Leistungen (Instrumente, Datenbank, Datenbasis etc.) hervorgehoben. Auch die Sichtbarkeit (Zitat aus einem Interview: „... das EEN ist als eigenständiges Netzwerk zu sehen") durch eine offenbar gut verankerte Marke, durch professionell kommunizierte "best practise-Beispiele" 
im Sinne von Role Models und das politische Commitment (von allen EU-weiten PartnerInnen im Netzwerk sowie der Kommission) wird durch zahlreiche InterviewpartnerInnen als essentiell eingeschätzt. Die Aussagen einzelner Unternehmen, die an der Unternehmensfokusgruppe teilnahmen, zeigten jedoch, dass Aktivitäten zur Sichtbarkeit möglicherweise auch falsche Erwartungen bei der Zielgruppe erzeugen. Das EEN wird demnach als Förderprogramm/Fördergeber und somit auch als weiterer Player in der Förderlandschaft wahrgenommen, allerdings mit unklaren Möglichkeiten, worüber vereinzelt Unmut herrscht.

Das operative Leistungsportfolio und die einzelnen Tools zur Umsetzung der Beratungen im Rahmen des EEN werden seitens der antwortenden Intermediäre mehrheitlich als wichtig bzw. sehr wichtig/sinnvoll beurteilt (siehe Abbildung 31). Dabei werden gut eingeführte Tools zur konkreten Informationsvermittlung (wie z.B. der Newsletter, individuelle Beratungen, aber auch Partnersuchen und Kooperationsbörsen), im Vergleich zu relativ neuen Tools und Programmlinien (wie z.B. KAM, EIMC und allgemeinen Informationsmöglichkeiten via Standbetreuungen oder Social Media), höher bewertet. Einschränkend muss allerdings dazu angemerkt werden, dass KAM und EIMC nicht allen KMU zur Verfügung stehen und daher diese auch den Intermediären weniger bekannt sein dürften.

Was den EEN-Knoten FFG betrifft, wurden 2015-2016 insgesamt 45 Kooperationsbörsen mit 326 KMU aus Wien, Niederösterreich und Burgenland organisiert. Diese KMU diskutierten in insgesamt 1.804 bilateralen Gesprächen mit ausländischen Firmen und F\&EEinrichtungen mögliche Kooperationen. Im Rahmen von KAM und EIMC wurden insgesamt 37 Firmen in Wien, Niederösterreich und Burgenland betreut.

\subsection{Schlussfolgerungen und Empfehlungen}

FFG-EIP

Das Leistungsportfolio der FFG-EIP wird von den Befragten überwiegend positiv bis sehr positiv bewertet.

Dabei schätzen sich die antwortenden Unternehmen mehrheitlich als bereits erfahren ein; etwa ein Drittel der befragten Unternehmen blickt auf eine langjährige Erfahrung mit europäischen FTI-Programmen (auch in der KoordinatorInnenrolle) zurück.

Als externe Informations- und Beratungsquelle hat die FFG-EIP zum Erstkontakt de facto ein „Alleinstellungsmerkmal" bzw. ein „Unique Selling Proposition" (zweitwichtigste Quelle - mit großem Abstand - für Information und Beratung sind KollegInnen mit einschlägiger "EU-Erfahrung" innerhalb des Unternehmens). Regionale Förderagenturen und private Dienstleister spielen ebenso wie organisationsinterne Serviceeinrichtungen eine untergeordnete Rolle.

Die Nutzung der einzelnen Elemente des Leistungsportfolios zielt dabei vor allem auf (spezifische) Informations- und Beratungsangebote ab. Netzwerkorientierte Leistungen (Unterstützung bei der Partnersuche etc.) spielen demgegenüber eine untergeordnete Rolle. 
Durch das EIP-Leistungsangebot wird das Informationsniveau für das ,Ökosystem' H2020 Unterstützung allgemein gehoben, aber es werden auch die spezifischen Beratungsbedarfe der Unternehmen erfüllt. Insgesamt sind die Unternehmen - und zwar größtenteils unabhängig von der Größe und des Erfahrungshintergrunds - mit den Angeboten bzw. den Leistungen der FFG-EIP sehr zufrieden, d.h. sie bewerten diese in hohem Ausmaß als gut bis sehr gut!

Aufgrund der vorliegenden Befunde hinsichtlich der Erfahrungen der Unternehmen mit dem Leistungsportfolio der FFG-EIP fallen die Empfehlungen dementsprechend knapp im Sinne eines "weiter wie bisher" (und damit verbunden einem hohen Bekanntheitsgrad und Akzeptanz der EIP-Leistungen bei der Zielgruppe) aus. Anpassungen in Richtung einer weiteren Optimierung des Leistungsangebots sollten im Wesentlichen auf eine Verbesserung des Erwartungsmanagements und eine bessere Vernetzung europäischer und nationaler Programme abzielen.

\section{Empfehlung: Verbesserung des Erwartungsmanagements}

Grundsätzlich ist aufgrund der positiven Erfahrungen eine Strategie des "weiter wie bisher" angebracht. Das zielgruppenspezifische Angebot sollte jedoch noch transparenter dargestellt werden. Dies betrifft insbesondere ein realistisches Erwartungsmanagement , das zum einen die Möglichkeiten und Grenzen (bzw. die damit verbundenen Kosten) der Teilnahme an den europäischen FTI-Programmen aufzeigt und zum anderen die Unterstützungsleistungen von FFG-EIP bei der Antragsstellung und Projektabwicklung beinhaltet.

Eine derartige Strategie ist kompatibel mit dem expliziten "Nicht-Ziel" des EIP, die Zahl der Antrag stellenden Unternehmen zu maximieren (um den Preis, ungeeignete Unternehmen bzw. Unternehmen mit ungeeigneten Projekten zur Teilnahme zu "drängen").

\section{Empfehlung: Bessere Vernetzung europäischer und nationaler Programme}

Innerhalb der FFG sollte mit entsprechenden Maßnahmen darauf abgezielt werden, eine optimale "Vernetzung" europäischer und nationaler Programme im Beratungsservice herzustellen. Dies ist vor dem Hintergrund zu sehen, dass viele (vor allem kleinere) Unternehmen nicht zwischen den einzelnen Abteilungen der FFG differenzieren und die Trennung zwischen den Leistungsangeboten des EIP und den diversen Angeboten im Rahmen der nationalen Förderprogramme nicht wahrnehmen.

Angesichts der mittlerweile sehr geringen Erfolgsquote und der häufigen Ablehnung auch von Projekten, die prinzipiell als sehr gut (bzw. förderwürdig) beurteilt wurden, ist eine mögliche Überlegung, entsprechende Anschlussunterstützung bzw. -beratung (zum Beispiel in Richtung "Umwandlung" der Projektidee für ein nationales Förderprogramm) zur Verfügung zu stellen.

Auch das speziell auf die avanciertesten Nutzer abgestimmte Betreuungsangebot der FFG-EIP, nämlich das C3 Konzept (Core Customer Concept) wird von den begünstigten Unternehmen als sehr gut eingeschätzt. Die Interviewergebnisse mit C3 KundInnen sowie die Auswertung der Feedback-Fragebögen der FFG zeigt durchwegs, dass die C3 Unternehmen sehr zufrieden mit den Leistungen der FFG Expertinnen und Experten sind. 
Besonders hervorgehoben werden: die Kompetenz der BeraterInnen, die rasche und direkte Kommunikation sowie die maßgeschneiderte, direkt auf das Unternehmen zugeschnittene Bereitstellung von Informationen und Services.

\section{Empfehlung: Wirkungspotenzial der C3 Beratung verbessern}

Da das C3 Instrument auf eine relativ kleine Zielgruppe abgestimmt ist, bleibt der positive Impact des Angebots auf die unmittelbare Zielgruppe beschränkt. Eine verstärkte Multiplikatorwirkung und Skalierung der Wirkung erscheint hier notwendig. Diesbezüglich sind folgende Anpassungen denkbar:

Verstärkter Mitteleinsatz für C3, sodass insgesamt mehr Unternehmen in C3 betreut und beraten werden können,

Einbeziehung der C3 Unternehmen in digitale „Peer Learning" Gruppen (im Sinne der Nutzung der Erfahrung und Expertise der C3 Unternehmen), in denen die österreichischen ProjektpartnerInnen der C3-Unternehmen einbezogen werden, wobei diese von ExpertInnen der FFG-EIP moderiert werden,

Verstärkte Kooperation der C3 Unternehmen mit Intermediären (wie z.B. der WKO).

\section{EUREKA}

Die Zusammenführung der Aktivitäten des EUREKA-Büros unter dem Dach von FFG-EIP kann als gelungen bezeichnet werden. Die Verteilung der Verantwortlichkeiten und Aufgaben auf unterschiedliche Personen im Team und die damit einhergehende, teamübergreifende Vernetzung von Beratungsmöglichkeiten und -aktivitäten ist besonders hervorzuheben. Vor diesem Hintergrund wurde EUREKA bzw. Eurostars als niedrigschwellige Förderalternative mit vergleichsweise hohen Erfolgschancen neben dem KMU-Instrument und anderen Beteiligungsmöglichkeiten im Rahmen von H2020 und den MULLATs positioniert. Durch die Prüfung von EUREKA-Projekten auf Basis der Vorgaben für die FFG Basisprogramme rückt das (potentielle) Wachstum österreichischer Unternehmen auf Basis von Forschung und Entwicklung in den Vordergrund.

Das steigende Interesse an entsprechenden Beteiligungen führt mitunter dazu, dass EUREKA, mit Abzügen auch Eurostars, als „Überlaufgefäß" für andere Förderangebote fungiert. Während von den GesprächspartnerInnen die Aufgabe von FFG-EIP klar darin gesehen wird, für die KundInnen die bestmögliche Lösung zu finden, d.h. für förderwürdige Unternehmen entsprechende Angebote zu entwickeln, scheint die Frage nach der strategischen Positionierung von EUREKA bzw. Eurostars, u.a. im Hinblick auf die Komplementarität mit den Angeboten des Europäischen Rahmenprogramms, klärungsbedürftig. In diesem Zusammenhang wurde der wachsende Austausch von EUREKA mit EEN als äuBerst positiv beurteilt.

Aufgrund der unterschiedlichen Ausgestaltung und Dotierung von EUREKA in den Mitgliedsländern spielt das im EUREKA-Büro zusammenlaufende Know-how und die gesammelte Erfahrung eine wesentliche Rolle für die Beratung und Projektbetreuung. Die Koordinierung und Synchronisierung zwischen EUREKAs Mitgliedsländern bleibt jedoch EUREKAs größte Herausforderung. 


\section{Empfehlung: Nationale Positionierung EUREKAS sinnvoll - Eurostars stärken}

Die Positionierung EUREKAS im europäischen Kontext ist abhängig von Struktur und Ausgestaltung der europäischen Förderlandschaft sowie den unterschiedlichen Netzwerk- und Kooperationsstrukturen. Vor diesem Hintergrund ist EUREKA auf nationaler Ebene als niederschwellige Förderalternative mit guten Erfolgsaussichten im Vergleich zum Rahmenprogramm H2020 und anderen Formen transnationaler FTI-Partnerschaften sinnvoll positioniert.

Insbesondere die aktive Positionierung von Eurostars als Alternative zum stark überzeichneten KMU-Instrument des Rahmenprogramms sollte weiter forciert werden.

Das Programmmanagement von EUREKA wird als schlank in den Strukturen, effizient und transparent wahrgenommen. Der themenoffene, bottom-up-Ansatz von EUREKA spiegelt sich gut im FFG Basisprogramm wider, das eng mit dem EUREKA Büro zusammenarbeitet. Kommunikation im Team und Synergien mit EIP werden gut genutzt. Hinsichtlich der für EUREKA Eurostars relevanten WFA-Indikatoren ist eine zufriedenstellende Zielerreichung zu attestieren, wobei sich beim CIS-Indikator Fragen der Zweckmäßigkeit stellen. Bei der Erfüllung des Teilnahme-Indikators könnte, in Anlehnung an die Teilnahme im Europäischen Forschungsrahmenprogramm, zwischen der Teilnahme auf der einen und der Leitung solcher Projekte auf der anderen Seite unterschieden werden.

\section{Empfehlung: Effizientes Programmmanagement und schlanke Strukturen beibe-} halten

Die Integration des EUREKA-Büros im FFG-EIP ermöglicht Synergien im Bereich der Beratung und Förderung kooperativer anwendungsnaher Forschung und Entwicklung zu nutzen. Die nationale Programmabwicklung erfolgt in effizienter Weise.

Vor diesem Hintergrund soll die Integration des EUREKA-Büros im FFG-EIP weiter beibehalten werden.

\section{COSME}

Das breite Spektrum an Maßnahmen (thematisch sowie auch hinsichtlich der Instrumente) entspricht dem breiten Generalziel der "Stärkung der Wettbewerbsfähigkeit" und ist damit in hohem Ausmaß auch für die Bandbreite der eigentlichen Zielgruppe, der Gesamtheit europäischer KMU, relevant. Diese Stärke des Programms ist zugleich auch eine gewisse Schwäche, da gerade mit den thematisch offenen Instrumenten, die bis zu 80\% des Budgets von COSME ausmachen, wenig Lenkungseffekte (i.S. der Verfolgung spezifischer Politikziele oder der Adressierung weniger entwickelter Märkte) erwartet werden können.

Die generelle Bekanntheit des Programms ist eher gering. Wahrgenommen werden bei den Intermediären (potentielle Einreicher), aber auch teilweise bei den Multiplikatoren insbesondere die Finanzierungsinstrumente („Access to Finance") sowie das EEN, und teilweise KMU-Policyberichtsleistungen. Der Mehrwert von thematischen/sektoralen Calls scheint einzelnen Intermediären, aber auch einzelnen Multiplikatoren (außerhalb der FFG und WKÖ) öfters nicht eindeutig klar. Auch die Außenwahrnehmung (der Möglichkeiten) 
im Rahmen von COSME scheint mehrheitlich auf die Standbeine Finanzierungsinstrumente sowie das EEN zu fokussieren, was aber wenig über das Ausmaß an Relevanz einzelner Maßnahmen in COSME aussagt. Diese selektive Wahrnehmung bzw. partielle Mangel an Bekanntheit von Elementen des Programms ist aufgrund des Programmcharakters als Politikentwicklungs- und Politikunterstützungsinstrument, der indirekten Wirkungsweise (Intermediäre als den KMU vorgeschaltete Zielgruppe) sowie der hohen Streuung bzw. Breite von Themen und Maßnahmen durchaus nachvollziehbar. Ein Handlungsbedarf, die Bekanntheit von COSME durch gezielte Maßnahmen seitens der FFG-EIP zu steigern, wird vom Evaluierungsteam nicht gesehen. Das Schnittstellenmanagement in Bezug auf die einzelnen Maßnahmen in COSME dürfte, mit gut abgestimmten externen und internen Schnittstellen, weitgehend effizient erfolgen.

\section{Empfehlung: Weiterführung eines proaktiven Programm- und Schnittstellenma-} nagements im FFG-EIP

In COSME gibt es mit Blick auf internationale Aktivitäten von Unternehmen zahlreiche Anknüpfungspunkte in der Betreuung durch die FFG - nicht zuletzt durch die tendenzielle Wiedereingliederung von Innovationsaspekten in thematische/sektorale Calls im Rahmen von COSME, die auch durch die Pilotierung einzelner Maßnahmen (mit potentiellem Innovationsbezug) unterstrichen wird. Vor diesem Hintergrund kann das Schnittstellenmanagement (zeitnahe Antizipation und Bewerbung bzw. Verteilung von Calls zu den Multiplikatoren und Intermediären etc.) als wichtige und richtige Verortung bei der FFG bewertet werden; der ständige Einbezug (pro-aktive Ansprache) der Multiplikatoren erscheint - nicht zuletzt aufgrund der nachvollziehbaren selektiven Wahrnehmung und dem partiellen Mangel an Bekanntheit des Programms mit all seinen Möglichkeiten - unablässig.

\section{Empfehlung: Verbesserung des Datenmonitorings durch die Europäische Kom- mission}

Hinsichtlich der Transparenz und des Datenmonitorings der Teilnahmen und Programmergebnisse besteht zunächst vor allem auf Seite der Europäischen Kommission eindeutig Verbesserungspotenzial. Der COSME Data Hub ist ein erster Schritt in Richtung besseres Monitoring und Übersichtlichkeit, dennoch fehlt bislang ein strukturiertes Notifizierungssystem für Ausschreibungen, was die Recherchearbeiten der FFG zumindest großteils ersetzen und die Effizienz wie auch die Effektivität des Programmmanagements durch die FFG mit Sicherheit noch erhöhen würde.

\section{EEN}

Die Relevanz des EEN, das als Maßnahme kontinuierlich in COSME ausgeschrieben bzw. umgesetzt wird, ist insbesondere durch die breitere, umfassendere Sichtweise (im Unterschied zu einem Projektfokus) sowie auf die üblicherweise auch langfristigere Begleitung von Unternehmen gegeben. Zudem ist der Fokus der Leistungen auf die Unterstützung internationaler Kooperations- und Technologietransferaktivitäten der 
Unternehmen und dabei das breite Spektrum von Kooperationsaktivitäten (FTI-Kooperationen sowie reine Technologiepartnerschaften, Lizensierungen, Vertriebsvereinbarungen etc.) von hoher Relevanz für die Zielgruppe, insbesondere für KMU.

\section{Empfehlung: Beibehaltung der Integration der EEN-Leistungen im FFG-EIP}

Das EEN weist eine hohe Bekanntheit unter den Stakeholdern und Intermediären bzw. Förderagenturen auf und wird seiner Rolle als regional verankertes Netzwerk mit ebensolchen Knotenpunkten/Anlaufstellen gerecht. Das EEN hat mit seinen Leistungen/Instrumenten zumeist einen hohen Stellenwert im jeweiligen Beratungsportfolio. Zudem scheinen die EEN-Leistungen/Instrumente organisatorisch gut in die Portfolios integriert bzw. mit weiteren relevanten Maßnahmen (z.B. Beratung zu H2020 und anderen Unterstützungsmöglichkeiten bzw. Förderungen) verknüpft und abgestimmt. Häufig wird mittels EEN-Leistungen im Vorfeld und fast immer ergänzend zu H2020 gearbeitet.

Ein klares Commitment durch die Europäische Kommission und die nationalen Stakeholder dürfte die Beratungstätigkeiten positiv unterstützen und eine hohe Motivation und Zufriedenheit bewirken. 


\section{Governance und Effizienz der EIP-Beauftragung}

\subsection{Governance}

Die Beauftragung des EIP mit Informations-, Beratungs-, Betreuungs- und Vernetzungsleistungen erfolgt gesondert und ist nicht mit dem Rahmenvertrag der FFG mit den EigentümervertreterInnen des Bundes (BMVIT und BMDW) verbunden. Im Zuge der Interviews hat sich herausgestellt, dass dadurch ein administrativer Mehraufwand verbunden mit dem separaten finanziellem Reporting und Controlling an die AuftraggeberInnen entsteht. Durchschnittlich entfallen auf Monitoring und Berichte 6,5\% des jährlichen Gesamtbudgets. Dieser Kostenträger beinhaltet neben Leistungsmonitoring und Berichterstattung an die Gruppe der AuftraggeberInnen auch Berichte und Analysen für FFG-EIP KundInnen in Wissenschaft und Wirtschaft. Laut FFG liegt der Anteil der der Berichterstattung an die AuftraggeberInnen zugeordnet werden kann, bei rund 5\% des Budgets.

Trotz Unschärfen in der Zuordnung (siehe Kapitel 5.2), kann ein Teil dieser Kosten auf das separate Leistungsmonitoring und Abrechnung an die AuftraggeberInnen zurückgeführt werden und Effizienzsteigerungen könnten möglicherweise durch die Integration der EIP-Beauftragung in den Rahmenvertrag der FFG erzielt werden. Jedoch umfasst die EIP-Beauftragung neben BMDW und BMVIT zusätzliche AuftraggeberInnen, wie BMBWF, WKO und sektorale Ministerien, deren Anforderungen sich von denen des Rahmenvertrags unterscheiden.

\section{Empfehlung: Separate Beauftragung des Bereichs Europäische und Internationale} Programme beibehalten

Um den mit der Beratung und Betreuung der europäischen FTI-Instrumente einhergehenden Ziele und Bedürfnisse gerecht zu werden, erscheint aus heutiger Sicht die separate Beauftragung des Bereichs „Europäische und Internationale Programme" der FFG trotz administrativem Mehraufwand sinnvoll. Hierdurch kann die effektive Einbeziehung mehrerer Ministerien sowie der gesetzlichen Interessensvertretung der österreichischen Wirtschaft in den Steuerungsstrukturen von FFG-EIP gewährleistet werden.

Im Rahmen der Beauftragung gibt FFG-EIP den AuftraggeberInnen jährlich mit einem Arbeitsplan und einem Leistungsbericht über geplante bzw. durchgeführte Aktivitäten einen laufenden Überblick. Im jährlichen Arbeitsplan werden die Schwerpunkte der Arbeit und das Jahresbudget festgelegt und Wirkungsziele und Indikatoren definiert (vgl. Annex I zu WFAs). Der jährliche Leistungsbericht umfasst eine Beschreibung der durchgeführten Aktivitäten und der damit verbundenen Kennzahlen, z.B. Anzahl der Beratungen, Veranstaltungen, etc. Darüber hinaus wird die Zielerreichung der Leistungsindikatoren erhoben und bewertet.

Die jährlichen Arbeitspläne und Leistungsberichte sind umfassend und geben dem Lenkungsausschuss einen guten Überblick über die jeweiligen Tätigkeitsschwerpunkte und Prozesse. Der Leistungsbericht dient den AuftraggeberInnen auch zur Kontrolle in Bezug auf Leistungserfüllung, Zielerreichung und Kosten. Darüber hinaus werden diese für das 
EIP-interne Monitoring und ggf. Anpassung und Weiterentwicklung der Beratungs- und Betreuungsleistungen im Sinne des PDCA-Zyklus (plan-do-check-act) eingesetzt. Im Vergleich zur vorherigen Beauftragung wurde das Berichtswesen in $\mathbf{H 2 0 2 0}$ bereits reduziert: EIP ist nunmehr nicht mehr angehalten Team-spezifische Arbeitspläne und Leistungsberichte vorzulegen. Aus Sicht des Evaluationsteams ist die komprehensive Berichterstattung sinnvoll und sieht hier keine weiteren Möglichkeiten für Kosteneinsparung und Effizienzsteigerung.

\section{Empfehlung: Beibehaltung der regelmäßigen Berichtspflichten}

Angesichts der Größe und Dimension der Beauftragung ist die regelmäßige Berichtslegungspflicht und das damit verbundene laufende Monitoring und Anpassung der Aktivitäten sinnvoll und notwendig. Sollte der zukünftige Vertrag mit FFG-EIP flexibler gestaltet werden, werden diese Berichtspflichten noch mehr an Bedeutung gewinnen. Wir empfehlen deshalb das Beibehalten des derzeitigen Berichtswesens unter Berücksichtigung der Anpassung und Weiterentwicklung der WFA-Indikatoren.

\subsection{Effizienz}

Das Gesamtvolumen der EIP-Beauftragung für die Laufzeit 2014-2020 zur Unterstützung der Umsetzung von H2020 beträgt laut Vertrag maximal 30398000 Euro - das sind rund 4,2 bis 4,4 Millionen pro Jahr. ${ }^{33}$ Die tatsächlich abgerechneten Summen in den Jahren 2014-2016 lagen jeweils unter 4 Millionen Euro und unterschreiten somit deutlich den Referenzkostenplan und die geplanten Budgetwerte ${ }^{34}$ (siehe Tabelle 8)

Tabelle 8: EIP Kostenplan und tatsächliche Kosten 2014-2016 (in Euro)

\begin{tabular}{|l|r|r|r|}
\hline & 2014 & 2015 & 2016 \\
\hline Budgetwert (Plan) & 4101512 & 4064542 & 4275966 \\
\hline Tatsächliche Abrechnung & 3700419 & 3972733 & 3957474 \\
\hline Abweichung & $-10 \%$ & $-2,3 \%$ & $-7,4 \%$ \\
\hline
\end{tabular}

Quelle: FFG-EIP Abrechnungen 2014-2016

Verglichen mit der Beauftragung zum 7. Rahmenprogramm ist das Gesamtvolumen in etwa konstant geblieben: Für den Zeitraum 2007-2012 beliefen sich die angefallenen Kosten im Bereich EIP auf insgesamt rund 20 Millionen Euro, durchschnittlich 3,3 Millionen pro Jahr, im Vergleich dazu knapp 4 Millionen pro Jahr in den Jahren 2015 und 2016. Die Anzahl der VZÄs im Bereich EIP die der Betreuung des Rahmenprogramms zugeord-

33 Gemäß Vertrag zur Beauftragung der FFG-EIP mit der Unterstützung österreichischer FTI-Akteure im Europäischen Forschungs- und Innovationsraum 2014-2020 abgeschlossen zwischen der Republik Österreich und der Österreichischen Forschungsförderungsgesellschaft mbH (FFG)

34 FFG, Abrechnungen des H2020 Beauftragungsvertrages in den Jahren 2014, 2015, 2016 
net werden können, ist über die 2 Rahmenprogrammperioden hinweg annähernd konstant: Sie lagen im Jahr $2012^{35}$ bei 34,97 und im Jahr 2016 bei 33,3736. Das Leistungsspektrum ist währenddessen aber in Richtung einer größeren Fokussierung auf strategische Leistungen erweitert worden (z.B. strategische Beratung und Betreuung der Wissenschafts- und Wirtschaftsorganisationen).

In der Effizienzanalyse der EIP-Beauftragung ist die Anwendung klassischer ökonometrischer Methoden wie die Cost-Benefit-Analysis (CBA) und Cost-Effectiveness-Analysis (CEA) aufgrund der Datenlage, Zielvorgaben und Aufgabenbereiche der Beauftragung nicht möglich. Die Analyse des Mitteleinsatzes im Rahmen dieser Evaluierung beruht auf einer Durchsicht und Bewertung der jährlichen Abrechnungen des EIP, der Beteiligungsstrukturen Österreichs in $\mathrm{H} 2020$ und qualitativen Bewertungen der EIP MitarbeiterInnen und AuftraggeberInnen.

Das EIP verwendet in der Kostenaufzeichnung eine thematische Kostenträgerstruktur, die sich zum Großteil mit den Förderschienen von H2020 deckt - alle "inhaltlichen" Tätigkeiten (inkl. Kundenberatung, Trainings, Analysen, etc.) der EIP-MitarbeiterInnen die sich einem Programm zuordnen lassen, scheinen unter dem jeweiligen Kostenträger auf. Diese Kostenträgerstruktur erschwert die Einschätzung des Aufwands, insbesondere für die neuen Beratungsinstrumente ERA-Dialog und C3 sowie für die FFG-Akademie und Berichte und Analysen zur Bildung der strategischen Intelligenz. „Inhaltliche" Beratungen, Analysen und Berichte im Rahmen von ERA-Dialog, C3, ERA-Themendossier, Policy Briefs, sowie Durchführung von FFG-Akademie Formaten scheinen nicht in den jeweiligen Kostenträgern auf sondern werden den thematischen Kostenträgern zugeordnet (z.B. Im Rahmen des ERA-Dialog wurde zum H2020 Programm Energie beraten - diese Stunden werden auf den thematischen Kostenträger "Energie" gebucht und nicht auf den Kostenträger "ERA-Dialog"). Dieses Problem betrifft auch den Kostenträger "Monitoring und Berichte": Er beinhaltet die Berichterstattung an die AuftraggeberInnen, aber unter anderem findet sich hier teilweise auch der Personaleinsatz für die Erstellung analytischer Berichte wie ERA-Themendossiers - jedoch nicht nach einheitlichem Schema und anfallende Stunden sind wiederum ebenso in den thematischen Kostenträgern wiederzufinden. Eine genaue Einschätzung und Analyse des Mitteleinsatzes nach Funktionsbereichen wie etwa inhaltliche Beratung und Betreuung Forschender, Wissensvermittlung durch Trainings und Workshops im Rahmen der FFG-Akademie, strategische Beratung für die Bereiche Wirtschaft und Wissenschaft, strategische Berichte und Analysen, Unterstützung der Ministerien und Delegierten für H2020, ERA und MULLATs ist somit nicht möglich.

35 Rechnungshof (2014), Nationale Maßnahmen zum 7. Rahmenprogramm für Forschung, technologische Entwicklung und Demonstration

36 FFG (2016), Budget 2016, EIP Organigramm 


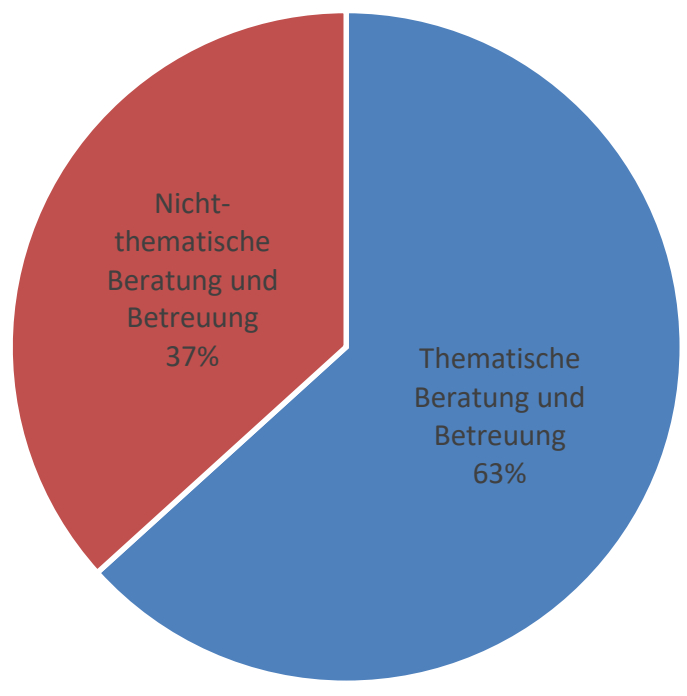

Quelle: Eigene Darstellung, Daten: FFG-EIP Abrechnungen 2014-2016

Durchschnittlich wurde 2014-2016 rund zwei Drittel des EIP-Budgets auf thematische Aktivitäten, inkl. Programm- und Projektberatung, aufgewendet. Ein Drittel lässt sich nicht den Förderprogrammen von H2020 zuordnen - darunter fallen die Kosten für die Bereichsleitung, Beratung und Betreuung zu Recht und Finanzen, Monitoring und Berichte, Koordination und Assistenz der FFG-Akademie, des C3 und ERA-Dialog sowie strategische, unterstützende und beratende Tätigkeiten im Bereich internationale Kooperation und strategische FTI-Partnerschaften.

Es ist zu erwarten, dass in jenen H2020 Programmen, wo Österreich mehr Beteiligungen hat, eine höhere Nachfrage seitens der FTI-Akteure nach EIP Informations-, Beratungsund Betreuungsmaßnahmen besteht. Diese Erwartung wird durch den Vergleich der österreichischen Beteiligungsstruktur mit dem EIP Ressourceneinsatz bestätigt. Die recht hohe Korrelation (Korrelationskoeffizient 0.77) zwischen österreichischen Beteiligungen und EIP Aufwand untermauern diese Vermutung, trotz Schwierigkeiten, die Beratung zu H2020 von anderen Leistungen zu trennen: Der EIP Personal- und Ressourceneinsatz ist deutlich höher in jenen Programmbereichen, wo Österreich mehr Beteiligungen hat und umgekehrt. ${ }^{37}$ In Abbildung 33 wird diese Tendenz vor allem in den H2020 Säulen I und II sehr deutlich ${ }^{38}$.

Der Vergleich der österreichischen Beteiligungen mit dem EIP Aufwand in einigen Programmen von H2020 Säule III ist in diesem Zusammenhang auffällig abweichend. Entgegen den Erwartungen korrelieren Beteiligungen in den Bereichen Energy und Transport

37 Gemessen am durchschnittlichen EIP Mitteleinsatz in den jeweiligen Programmen zwischen 2014-2016, Zahlen aus den EIP Jahresabschlüssen. Datenstand österreichische Beteiligungen in H2020: 30.9.2017

38 Säule I Excellent Science: ERC, FET, MSCA, INFRA. Säule II Industrial Leadership: ICT, NMP, ADVMAT, BIOTECH, ADVMANU, SPACE, RISKFINANCE, SME. Säule III Societal Challenges: HEALTH, FOOD, ENERGY, TPT=TRANSPORT, ENV=ENVIRONMENT, SOCIETY+SWAFS, SECURITY. Einige Programme wurden zusammengefasst um den EIP Kostenträgern zu entsprechen 
nicht mit dem EIP Aufwand. Zurückzuführen ist dies It. FFG im Bereich Transport auf große Initiativen mit vielen ProjektpartnerInnen, womit die Betreuung gesamter Konsortien und weniger einzelner AntragstellerInnen einhergeht. Daher ist die Anzahl der Beratungen trotz gegebener Beratungsintensität niedriger. Trotz gewisser Unschärfe bezüglich der tatsächlichen Intensität der Beratungsleistungen stellen diese Bereiche eine Diskrepanz vom typischen Muster dar.

\section{Abbildung 33: Vergleich EIP Aufwand mit Beteiligungsstruktur Österreichs in H2020}

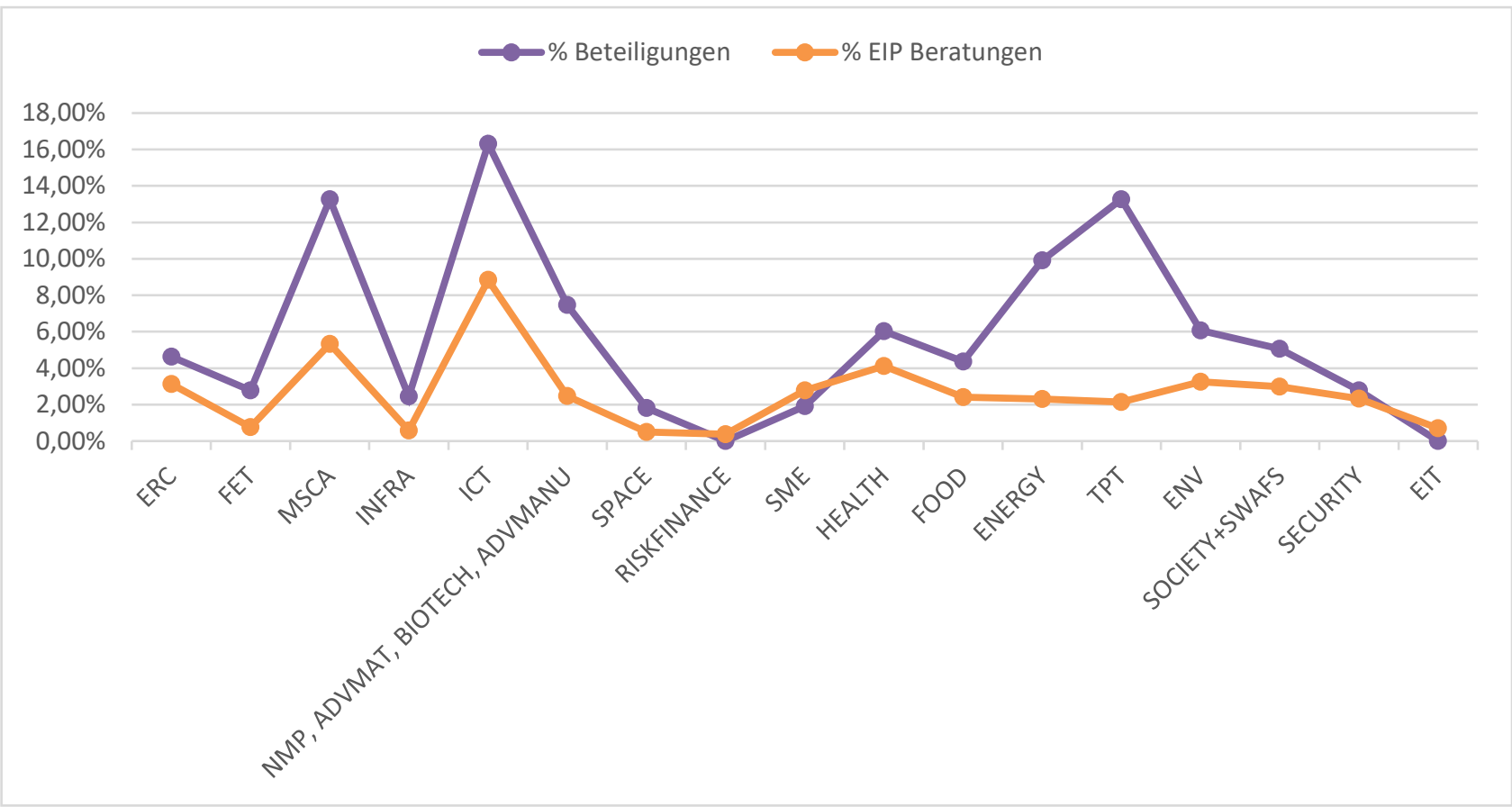

Quelle: eigene Darstellung, Daten: EU-PM Cockpitbericht November 2017, FFG-EIP Abrechnungen

Anhand des Vergleichs der Beteiligungsmuster österreichischer FTI-Akteure an H2020 mit dem FFG-EIP Aufwand lässt sich feststellen, dass die Budgetallokation angemessen ist. Darüber hinaus ist das Gesamtvolumen der Beauftragung nur leicht gestiegen, der Personaleinsatz in etwa konstant verglichen mit der Beauftragung zum 7. Rahmenprogramm während das Aufgaben- und Leistungsspektrum gleichzeitig signifikant erweitert worden ist. Diese Tatsache gekoppelt mit den unterschrittenen Budgets in allen Jahren der aktuellen Beauftragungsperiode weist darauf hin, dass die Leistungen im Rahmen der allozierten Budgets abgewickelt werden konnten.

\section{Empfehlung: Verbesserung der EIP-Kostenaufzeichnung}

Die Budgetallokation der EIP-Beauftragung ist angesichts der Beteiligungsstruktur Österreichs in H2020 angemessen. Darüber hinaus ist die Effizienz der Abwicklung gegeben. Um jedoch die Unschärfen der Kostenzuordnung zu beheben und den Mitteleinsatz der verschiedenen Beratungs- und Betreuungsinstrumente besser abschätzen zu können, empfiehlt das Evaluierungsteam die Umstellung der EIP Kostenaufzeichnungsstruktur von thematischen Kostenträgern auf funktionsbezogene Kostenträger. Dies wäre vor allem im Hinblick auf die Bewertung der neuen Betreuungsinstrumente ERA-Dialog und C3 relevant, aber auch um die Kosten von anderen Instrumenten wie Berichte und Analysen oder FFGAkademie nachzuvollziehen. Nachdem es in der Praxis häufig zu einer engen Verzahnung 
zwischen unterschiedlichen funktionsbezogenen Leistungen kommt (z.B. unterschiedlichen Arten von Beratungen), ist dabei auf eine ausgewogene Balance zwischen Differenziertheit und Umsetzbarkeit zu achten. 


\section{Information und Monitoring}

\subsection{EU-Performance Monitoring}

Beim sog. EU-Performance Monitoring (EU-PM) handelt es sich um eine Projekt-Beauftragung des Bundesministeriums für Bildung, Wissenschaft und Forschung (BMBWF), des Bundesministeriums für Verkehr, Innovation und Technologie (BMVIT), des Bundesministeriums für Digitalisierung und Wirtschaftsstandort (BMDW) sowie des Bundesministeriums für Nachhaltigkeit und Tourismus (BMNT). Die Laufzeit des Projektes ist vom 01. Juli 2014 bis zum 30. Juni 2021 festgelegt. ${ }^{39}$ Die Federführung in der Koordination obliegt gemäß Bundesministeriengesetz (BMG) i.d.g.F dem BMBWF. Zuständig ist dort die Abteilung für EU- und OECD-Forschungspolitik.

\subsubsection{Ziele und Aufgaben}

Zentrale Aufgabe des EU-Performance Monitoring ist die Erfassung, Verarbeitung, Aufbereitung und Kommunikation von Daten über die Beteiligung österreichischer Organisationen - das sind vor allem Unternehmen sowie Forschungs- und Hochschulinstitute - an den EU-Programmen für Forschung und Innovation. ${ }^{40}$

Damit hat EU-PM ab 2014 die Agenden des bis dahin mit dem nationalen PerformanceMonitoring betrauten Konsortiums PROVISO übernommen. "Europäische Programme" umfassen dabei neben Ausschreibungen und Instrumenten des aktuellen Forschungsrahmenprogrammes H2020 auch, nach Maßgabe der Datenverfügbarkeit, andere europaweite F\&E-Programme wie PPPs, ERA-Nets, JTI, JPIs, Artikel 185-Initiativen, EIT-KICs sowie EUREKA. Nach Ermessen des Informationsbedürfnisses von Seiten der AuftraggeberInnen sowie der Datenverfügbarkeit können die Leistungsbereiche auch erweitert werden. So wurde bspw. bereits im ersten Arbeitsprogramm für die Jahre 2014 und 2015 das Leistungsspektrum um die sukzessive Integration von Daten aus den Vorgängerprogrammen von H2020 in die Struktur des EU-PM sowie die anlassbezogene Heranziehung von Daten aus nationalen Förderprogrammen erweitert. ${ }^{41}$ Für das Jahr 2017 wurde zudem die Verschränkung und standardisierte Nutzbarkeit von Daten aus europäischen P2P-F\&E-Netzwerken aus dem, von der FFG geleiteten, H2020 CSA-Projekt ERA-LEARN 2020 als Zielsetzung formuliert.

Durch die Beauftragung des EU-PM an die FFG werden nationale und EU-Beteiligungsdaten unter einem Dach gebündelt, wodurch erstmals eine Zusammenschau europäischer

39 Gem. VERTRAG zur Beauftragung der FFG mit dem Monitoring der österreichischen Performance in den FTI-Aktivitäten der EU 2014 - 2021 abgeschlossen zwischen der Republik Österreich und der Österreichischen Forschungsförderungsgesellschaft mbH (FFG)

40 Siehe hier auch die Website des EU-PM

41 FFG (2016): EU-Performance Monitoring für FTI: Leistungsbericht Juli 2014 bis Dezember 2015, Wien, S.5. 
Förderdaten mit relevanten nationalen Daten möglich ist. Durch die synergetische Nutzung von inhaltlichem und technischem Know-how und der ExpertInnen der FFG soll so "strategische Intelligenz" für FTI-Akteure geboten werden. ${ }^{42}$

Zuständig ist das EU-PM neben der Sammlung und Aufbereitung der von der Kommission und anderen Quellen zur Verfügung gestellten Datenbestände auch für die technische Verschneidung und Integration der Daten in Form einer Datenbank, dem sog. "Data Warehouse".

Damit werden zum einen standardisierte Berichtsprodukte sowie individuelle Sonderauswertungen erstellt, zum anderen werden Daten auch in Form einer Datenbank öffentlich zur Eigenauswertung zur Verfügung gestellt (siehe nachfolgender Abschnitt).

Gemäß Leistungsbeschreibung hat sich das EU-PM für den Beauftragungszeitraum 1.7.2014 - 30.6.2021 die folgenden Ziele in Bezug auf die Datenbereitstellung und Produktpalette gesetzt:43

\section{Datenbereitstellung}

- Sammlung, Aufbereitung, Auswertung und Kommunikation zuverlässiger Daten über die Teilnahme Österreichs in relevanten Förderlinien der EU

- Integration relevanter Informationen aus verfügbaren Datenquellen in den Datengrundstock

\section{Produkte}

- Regelmäßige periodische Auswertungen und ad-hoc Auswertungen auf Anfrage

- Qualitative und quantitative Analysen und Interpretationen über die EU-Performance Österreichs in FTI

- Inhaltliche Zusammenschau von EU-Daten mit relevanten nationalen Förderdaten der FFG

- Regelmäßige Überprüfung der Zielgruppen-Adäquanz

- Bedarfs- und möglichkeitsorientierte Weiterentwicklung

- Betrieb eines Internet-Portals, das seine Inhalte aus einem bei der FFG angesiedelten Data Warehouse bezieht

- Effiziente und effektive Abwicklung unter Nutzung von Synergien zur Beauftragung der FFG zur "Unterstützung österreichischer FTI-Akteure im europäischen Forschungs- und Innovationsraum" (sog. „EIP Beauftragung")

Ziele dieser Aktivitäten sind im Wesentlichen:

- ein kontinuierliches Monitoring der österreichischen Erfolge in den EU-Forschungsprogrammen,

- den Vergleich mit anderen Ländern (internationale Positionierung),

- einen Bundesländer-Vergleich innerhalb Österreichs,

- die Analyse von Stärken und Schwächen in einzelnen Bereichen (z.B. nach Themen, Organisationen und Branchen)

42 Siehe Leistungsbeschreibung des EU-PM, Download 17.05.2018.

43 FFG (2014): Anbot zum EU-Performance Monitoring für FTI. 
- sowie die Unterstützung für strategische und politische Weichenstellungen zu liefern.

Konkret richten sich die Produkte und Aktivitäten des EU-PM an zwei Zielgruppen:

- Zielgruppe 1 / intern: Diese umfasst die beauftragenden Ressorts, Programmdelegierte und NCPs, welche mit einem geschützten Zugriff auf ein Internetportal ausgestattet sind, um auf qualitätsgesicherte Daten (auch personenbezogene!) für die eigene Informationsgewinnung zugreifen zu können. Darüber hinaus werden diese bei individuellen Anfragen prioritär behandelt sowie mit spezifischen Produkten (siehe nächster Abschnitt) adressiert.

- Zielgruppe 2 / extern: Diese umfasst die Öffentlichkeit, Stakeholder und Akteure im FTI-Bereich. Die Zielgruppe 2 erhält Zugang zu den Standardberichtsprodukten sowie zu nichtpersonenbezogenen Datensätzen über das öffentliche Datenportal.

\section{Abbildung 34: Aktivitäten EU-PM sowie Gewichtung, Stand November 2016}

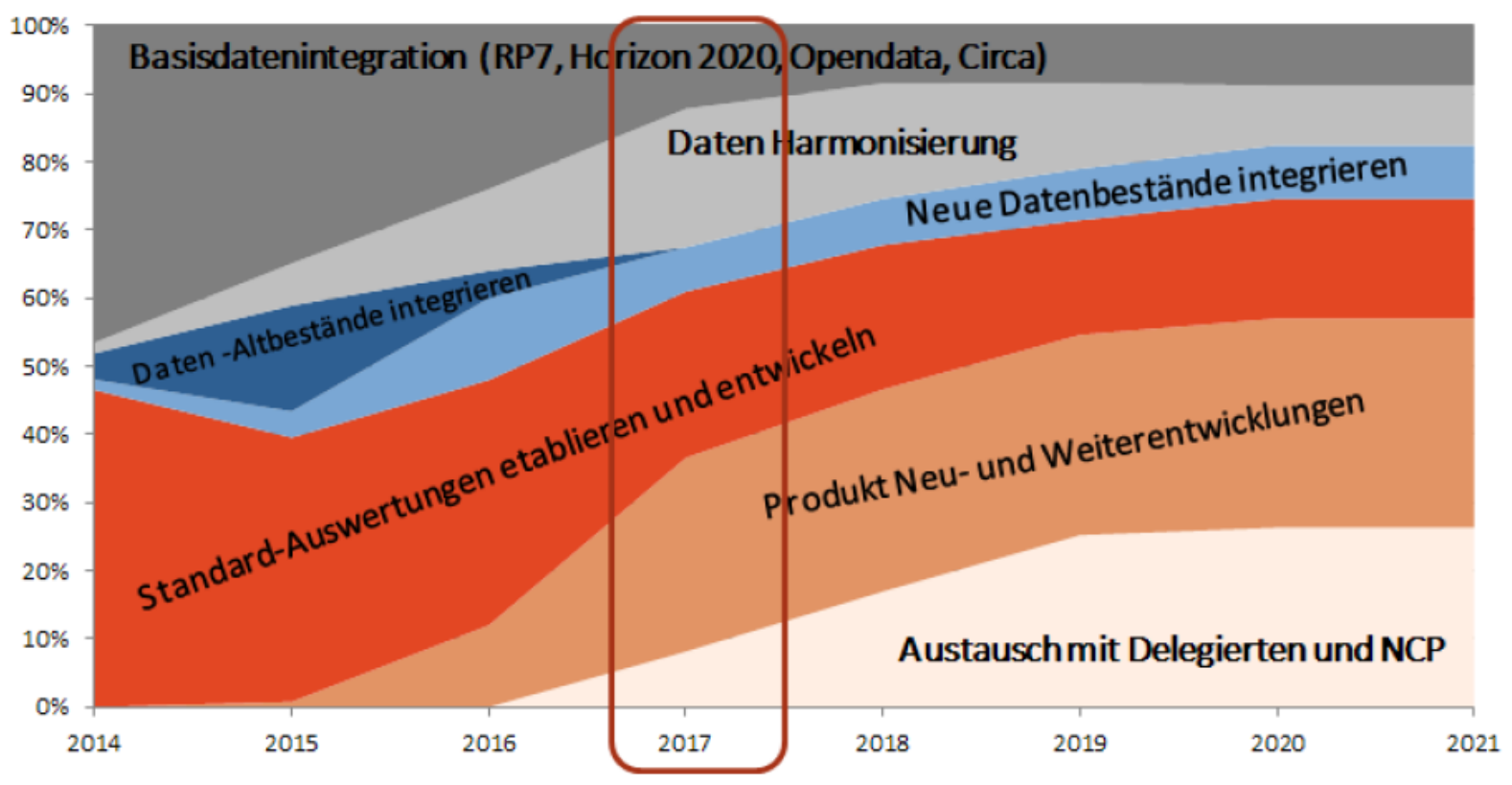

Quelle: EU-PM Leistungsbericht 2016.

Die Abbildung oben stellt die bisherige und prognostizierte Entwicklung des Aktivitätsspektrums auf Basis des EU-PM Arbeitsplanes für das Jahr 2017 (stand November 2016) dar. Hierbei zeigt sich deutlich die Verschiebung der Aktivitätsschwerpunkte von der mehrheitlich abgeschlossenen Basisintegration der Rahmenprogrammdaten und der Entwicklung von Standardauswertungen hin zur Integration neuer Datenbestände (siehe ERA-LEARN 2020) und der Weiterentwicklung von Produkten. Ein stärkerer Fokus als bisher soll auch auf den Austausch mit Programmdelegierten und NCPs gelegt werden. ${ }^{44}$

44 FFG (2016): EU-Performance Monitoring für FTI-Arbeitsplan 2017, S. 3. 
Der Lenkungsausschuss, der sich aus Mitgliedern der auftraggebenden Institutionen des EU-PM zusammensetzt, ist für die Genehmigung der jährlichen Arbeitspläne ${ }^{45}$ sowie der entsprechenden Leistungsberichte zuständig.

In der ersten Leistungsperiode des EU-PM wurde zudem ein sog. Sounding Board mit VertreterInnen der Zielgruppe 2 unterhalten, mit dem Ziel deren spezifische Bedarfe und Wünsche aufnehmen zu können. Aufgrund der großen Heterogenität der Anforderungen, die zum Teil in direktem Widerspruch zu gesetzlichen Regelungen wie dem Datenschutz stehen, wurde auf eine Weiterführung dieses Instrumentes ab der Leistungsperiode 2016 verzichtet ${ }^{46}$.

\subsubsection{Leistungen}

Für seine Produkte hat sich das EU-PM eine Reihe von grundsätzlichen Zielen gesetzt. Diese sollen ${ }^{47}$ :

- über rein deskriptive Beschreibungen hinausgehen und damit aktiv zur Strategieentwicklung und Entscheidungsfindung österreichischer Entscheidungsträger beitragen ("strategische Intelligenz").

- den konkreten Bedürfnissen der Stakeholder entsprechen („maßgeschneidert”).

- laufend den Bedürfnissen entsprechend weiterentwickelt werden.

- über die Analyse der EU-Rahmenprogramme hinausgehen.

- das gesamte verfügbare technische und inhaltliche Know-How der FFG in diesem Bereich nutzbar machen.

Tabelle 9 stellt die Standardprodukte des EU-PM im Überblick dar. Diese umfassen neben verschiedenen Berichtsformaten auch ein öffentliches Online-Portal, über welches individuelle Abfragen zur Beteiligung Österreichs im Rahmenpragramm nach unterschiedlichen Kriterien erstellt werden können. Dieses ist von jenem Internetportal-Zugang für Angehörige der Zielgruppe 1, über das auch vertrauliche und personenbezogene Daten in Form eigener Auswertungen bezogen werden können, zu unterscheiden.

Tabelle 9: Standardprodukte EU-PM

\begin{tabular}{|l|l|}
\hline Produkt & Kurzbeschreibung \\
\hline Überblicksbericht & $\begin{array}{l}\text { Gesamtbericht über die Beteiligung (Programme, The- } \\
\text { men, Bundesländer, Organisationstypen) } \\
-\quad 1 x \text { pro Jahr im Vollumfang } \\
-\quad 2 x \text { pro Jahr als Kurzfassung "Cockpit-Bericht" }\end{array}$ \\
\hline Programmberichte & $\begin{array}{l}\text { Bericht über (Teil-)Programme } \\
\text { (Beteiligungen, Koordinatoren, Rückflüsse, Länderver- } \\
\text { gleiche) }\end{array}$ \\
\hline
\end{tabular}

\footnotetext{
45 Für die erste Periode wurde der Leistungszeitraum ab Beauftragung 2014 bis Dezember 2015 festgelegt.

46 Siehe Leistungsbericht 2016, S. 17

47 Vgl. FFG (2014): Anbot zum EU-Performance Monitoring für FTI, Wien, S. 4.
} 


\begin{tabular}{|c|c|}
\hline Produkt & Kurzbeschreibung \\
\hline & $\begin{array}{l}\text { - 1x pro Jahr, Zeitpunkt der Publikation abhängig } \\
\text { vom jeweiligen Programm }\end{array}$ \\
\hline $\begin{array}{l}\text { Online-Auswerteportal } \\
\text { EU-Performance Monitor }\end{array}$ & $\begin{array}{l}\text { Interaktives, datenbank-basiertes Statistik-Portal mit } \\
\text { verschiedenen Kriterien, Filtermöglichkeiten, etc. }\end{array}$ \\
\hline Endbericht zu FP7 & $\begin{array}{l}\text { Finaler Überblick über das 7. EU-Rahmenprogramm } \\
(2007-2013)\end{array}$ \\
\hline $\begin{array}{l}\text { Endbericht zu H2020 inkl. ERA } \\
\text { Instrumente }\end{array}$ & Finaler Überblick über H2020 \\
\hline
\end{tabular}

Quelle: Siehe EU-PM Leistungsbericht

Darüber hinaus bietet das EU-PM im Rahmen der Beauftragung eine Reihe von Unterstützungsleistungen an Angehörige der Zielgruppe 1. Diese umfassen zum einen die Erstellung sog. Quick-Infos, die als Unterlage für Programmkommittee-Sitzungen einen Überblick über die jeweils aktuelle Call-Performance liefern sollen.

Zudem werden Fokusanalysen auf Anfrage und in enger Abstimmung mit AnfragestellerInnen durchgeführt (nicht nur Zielgruppe 1), die sich spezifischen Schwerpunktbereichen im Detail widmen. FFG-intern werden durch EU-PM auch Auswertungen für die Erstellung der sogenannten Themendossiers ${ }^{48}$ des EIP bereitgestellt. Ziel der Themendossiers ist die Beteiligungsstrukturen österreichischer Akteure im Rahmenprogramm H2020 und damit verbundenen transnationalen FTI-Partnerschaften in ausgewählten Schwerpunktfeldern zu beleuchten und entsprechende Schlussfolgerungen zu Stärken, Schwächen und Handlungsfeldern abzuleiten. Diese sollen dann auch in die Beratungsleistungen der FFG-EIP einfließen.

Zur Unterstützung der Zielgruppe 1 bei der Nutzung der EU-PM Produkte werden zudem Schulungen sowohl für die NCPs als auch über die FFG Akademie angeboten. Die Selbstermächtigung der Akteure in der Informationsgewinnung ist dabei das grundlegende Ziel.

Aktuell (Stand Arbeitsplan 2017) ist das EU-PM auch mit der Berechnung dreier Indikatoren der Wirkungsorientierten Folgenabschätzung (WFA) betraut, nämlich zur Performance von Hochschulen und Außeruniversitären Einrichtungen in H2020, zu den ERC-Grants an Österreich sowie zum Anteil Österreichs an den EU-28 für die ausgezahlten Rückflüsse am EU-Budget für H2020.

Zudem werden im Auftrag des Lenkungsausschusses Themenauswertungen zu spezifischen Bereichen wie Quantentechnologie oder Bioökonomie erstellt, die jedoch nur nach Maßgabe der AuftraggeberInnen im Einzelfall auch öffentlich zugänglich sind.

48 Siehe ERA Themendossiers, Download 24.04.2018 
Im Jahr 2015 war das EU-PM mit der Leitung einer Arbeitsgruppe des ERA-Reporting Boards, bestehend aus externen ExpertInnen, betraut, welche die Entwicklung von Indikatoren zur Innovationslandschaft- und Performance zum Ziel hatte. Ergebnis war das sog. ERA-Dashboard. Dieses soll als Monitoring Instrument der ERA-Roadmap dienen und wird im Rahmen der zweijährig zu erstellenden Fortschrittsberichte veröffentlicht. ${ }^{49}$ Das Dashboard ist seit Juni 2017 online verfügbar. Geplant ist, dass die dort gezeigten Indikatoren alle zwei Jahre mit neuen Werten und mit Kommentaren der jeweiligen Prioritätsverantwortlichen versehen werden. Im Fortschrittsbericht, der alle 2 Jahre erscheinen soll (beginnend mit 2017), sind Teile des Dashboards integriert.

Ein weiterer Aspekt der Beauftragung umfasst die Aufbereitung und Analyse von Datenquellen zu den transnationalen FTI-Partnerschaftsinitiativen voranzutreiben. Entsprechende Aktivitäten sind hier abhängig von der Verfügbarkeit standardisierter Datenquellen auf europäischer Ebene. So ist EU-PM seit 2016 mit der Integration und Auswertung von Daten aus der H2020-Support Action „ERA LEARN 2020 - Strengthening joint programming in Europe", welches von der FFG koordiniert wird, befasst. Im Zug des Projektes sollen Datengrundlagen zu europäischen Public-to-Public-Netzwerken (P2P) und Calls erarbeitet werden, welche durch EU-PM in Bezug auf die österreichische Performance ausgewertet werden sollen. Bislang liegen Daten zu unterschiedlichen Netzwerken (ERANet-Aktivitäten, Artikel 185-Initiativen, Joint Programming Initiativen, European Joint Programming Cofund) und den budgetierten Mitteln für die daraus entstehenden Calls vor. Damit wird dokumentiert, welche Länder durch welche Organisationen an der Umsetzung beteiligt sind. Eine solide Datengrundlage für die Ergebnisse der Calls, also welche Unternehmen, Hochschulen, Forschungseinrichtungen letztlich die Nutznießer dieser Aktivitäten sind, liegt nach wie vor nur rudimentär vor. Eine Diskussion und Einschätzung zum Stand der aktuellen Analysemöglichkeiten erfolgt in den nachfolgenden Abschnitten auf Basis der empirischen Erhebungen.

MitarbeiterInnen des EU-PM nehmen regelmäßig an Veranstaltungen im Rahmen des ERA-Observatoriums, wie dem Europa Forum Forschung, dem ERA-Reporting Board oder dem ERA Roundtable teil, um unter anderem über aktuelle Entwicklungen zur Performance Österreichs in H2020 sowie in spezifischen Themenfeldern zu informieren. Zudem findet ein jährlicher Austausch mit den Programm-Delegierten statt, um Feedback und Anregungen zu den Aktivitäten von EU-PM einzuholen und Weiterentwicklungsmöglichkeiten zu diskutieren.

\subsection{Ergebnisse der empirischen Erhebungen}

Im Folgenden werden die Ergebnisse der empirischen Erhebungen dargestellt. Grundlage hierfür ist zum einen die Online-Erhebung (für eine Beschreibung zu Methode und Ablauf siehe Kapitel 1), in welcher sowohl die Bekanntheit als auch die Bewertung einzelner Produkte bzw. Produktkategorien des EU-PM nach Schulnoten unter Angehörigen der Zielgruppe 2, Hochschulen, Forschungseinrichtungen und Unternehmen sowie intermediärer

49 BMWFW, BMVIT (2017): Österreichischer Forschungs- und Technologiebericht 2017. 
Organisationen (z.B. regionale Förderagenturen) erhoben wurde. Im Bereich intermediärer Organisationen wurde neben der Bekanntheit auch die Verwendung der Produkte für eigene Informations- und Beratungsleistungen abgefragt. Diese Resultate wurden, wie in den vorangegangenen Kapiteln, mit den EU-PM bezogenen Fragestellungen aus den Interviews sowie entsprechenden Resultaten der Fokusgruppen kontextualisiert. Angehörige der Zielgruppe 1 wurden alleinig im Rahmen von Interviews adressiert. Darüber hinaus liefert die Delphi-Erhebung Erkenntnisse zum wahrgenommenen Nutzen des Informationsangebotes zu ERA und H2020 - und damit auch zu den Leistungen des EU-PM in der FTI-Community.

Die Resultate der quantitativen und qualitativen Erhebungen wurden mit Auswertungen der Nutzungsstatistik des öffentlichen sowie des vertraulichen Online-Portals und der EUPM-Website ergänzt, um die Einschätzungen zur Relevanz des EU-PM für die jeweiligen Zielgruppen in Verbindung mit der quantitativen Entwicklung der Nutzung treffen zu können (siehe Tabelle 9). Zusätzlich wurde für das Jahr 2017 eine Auswertung der direkten Anfragen das EU-PM auf Basis des Ticketsystems vorgenommen um Aussagen zu Art und Intensität der Anfragen insbesondere im Vergleich zur Nutzung des vertraulichen OnlinePortals durch die Zielgruppe 1 treffen zu können. Dies erfolgte in enger Abstimmung mit dem EU-PM zur Abschätzung des jeweiligen Aufwandes, da das Ticketsystem alleinig Titel und Typ der AuftraggeberIn der Anfrage liefert.

Ein weiterer Aspekt dieses Kapitels umfasst die vergleichende Analyse zweier, mit dem öffentlichen Portal des EU-PM vergleichbarer, Webdienstleistungen die von VINNOVA bzW. der EU-Kommission betrieben werden. Ein weiterer Abschnitt widmet sich der Diskussion zur Integration weiterer Datenquellen in die Auswertungsmöglichkeiten von EU-PM sowie den Verschneidungsmöglichkeiten mit nationalen Förderdaten.

\section{Bekanntheit, Nutzung und Erfahrungen mit den Produkten - Zielgruppe 2}

Die Bekanntheit der standardisierten Berichtsprodukte sowie der öffentlichen Online-Datenbank für Selbstauswertungen liegt bei Hochschulen und Forschungseinrichtungen deutlich über jener der Unternehmen. So geben $46 \%$ der erreichten Angehörigen von Hochschulen und Forschungseinrichtungen und 35\% der UnternehmensvertreterInnen an die Berichtsprodukte zur Performance Österreichs in H2020 zu kennen (siehe Abbildung 35 und Abbildung 37). Die öffentliche Online-Datenbank ist in beiden Fällen eher von geringerer Bedeutung, 25\% bzw. 17\% der RespondentInnen geben an, diese zu kennen. Sofern bekannt, scheint jedoch die Zufriedenheit der NutzerInnen sowohl mit den Berichtsprodukten als auch der öffentlichen Online-Datenbank hoch zu sein, mit mehrheitlichen Bewertungen von "Gut" bis "Sehr gut" (siehe Abbildung 36 und Abbildung 38). Diese Beobachtungen sind auch stabil in Bezug auf weitere Untergliederung nach Erfahrungsgrad bzw. Institutionentyp. Auch mit ERA und H2020- "unerfahrene" Akteure kennen die standardisierten Berichtsprodukte eher als das öffentliche Online-Portal.

Die Beobachtungen aus der Online-Befragung decken sich mit den Ergebnissen der Interviews, die mit VertreterInnen von Universitäten geführt wurden. Insbesondere die Programm- und Cockpitberichte werden hier als wichtige Informationsquellen und Orientierungspunkte bezeichnet. 
Im Vergleich dazu scheinen für die Interviewten die Möglichkeiten zur Erstellung von Selbstauswertungen über die Online-Datenbank bis dato von untergeordneter Bedeutung bzw. schlichtweg nicht bekannt zu sein (z.B. "Alle Leistungen sind bekannt, mit der Ausnahme der Online Datenbank zur Erstellung eigener Auswertungen; diese wurde eigentlich angekündigt, es war aber nicht klar, dass sie mittlerweile existiert; grundsätzlich wäre diese interessant!").

In zwei der Interviews wurde in diesem Zusammenhang angemerkt, dass die Erstellung individueller Auswertungen für die Hochschulen insbesondere dann interessant wäre, wenn sie auf Einzelinstitutionenebene erfolgen könnte, d.h. wenn sich bspw. eine österreichische Universität in ihrer Performance mit ihren Peers in anderen EU-Ländern vergleichen könnte. Zurzeit seien nach Ansicht der Befragten ja keine Auswertungen auf Institutionenebene bzw. von Institutionen im EU-Vergleich möglich. Als Vorbild für eine derartige Möglichkeit wird das von VINNOVA entwickelte Tool H2020 Visualization genannt, welches z.B. den Vergleich der Top 100-Organisationen im Rahmenprogramm ermöglicht. ${ }^{50}$ Eine vergleichende Darstellung von Logik und Funktionalität des EU-Portals und dem VINNOVA-Tool wird im nachfolgenden Abschnitt zum Vergleich des EU-PM mit anderen Datenportalen unternommen.

Weitere Anmerkungen betrafen die Möglichkeit Daten zu Erfolgs- bzw. Ablehnungsraten in den Programmlinien des Rahmenprogrammes zwischen Einzel-Organisationen vergleichen zu können, um einen Überblick über die Wettbewerbssituation zu gewinnen. Anfragen wie diese waren jedoch einer der Gründe für die bereits erwähnte Einstellung des Sounding Boards, da dieses bis dato nicht mit geltenden Datenschutzbestimmungen in Einklang zu bringen ist bzw. nicht dem aktuellen Auftrag des EU-PM entspricht. ${ }^{51}$

\section{Abbildung 35: Bekanntheit Leistungen EU-PM (Wissenschaft)}

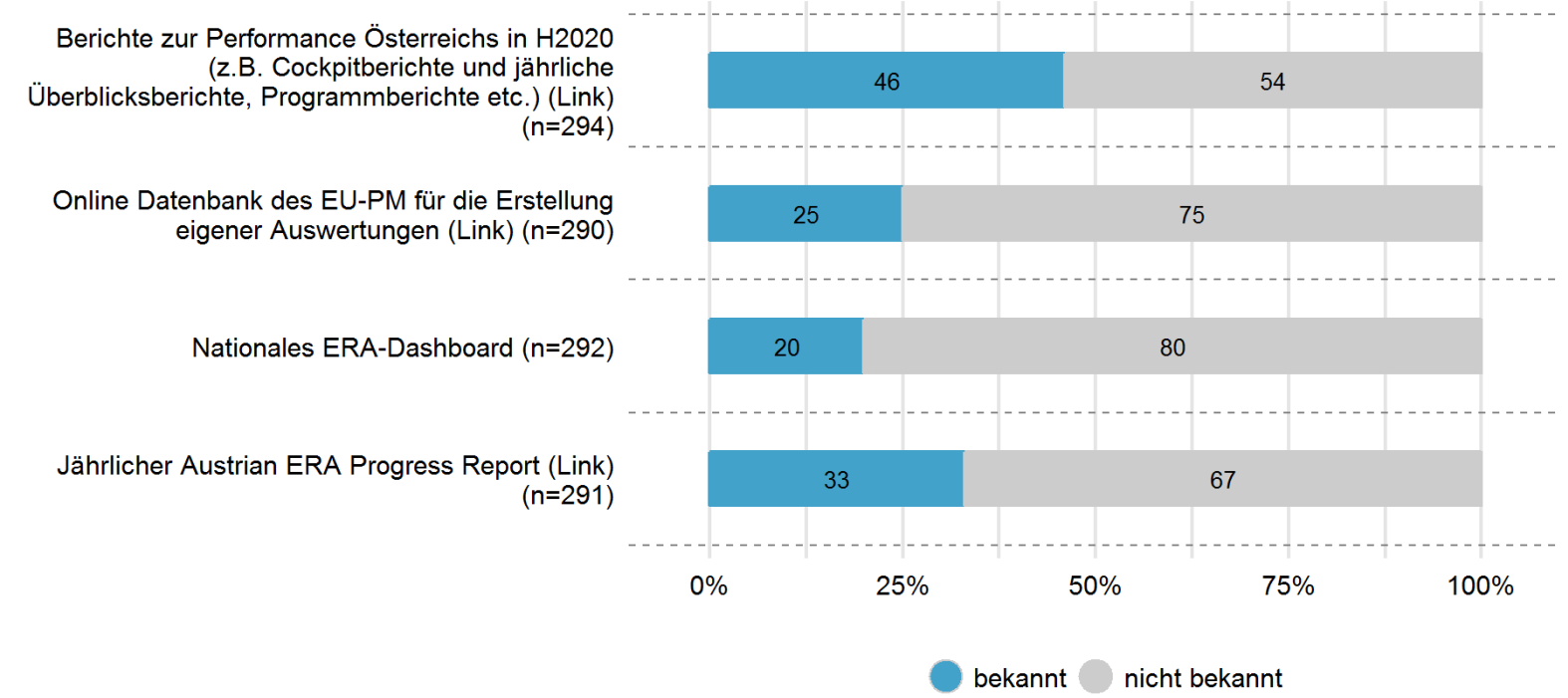

Quelle: Online Befragung, Joanneum Research.

50 Siehe diesbezüglich das VINNOVA H2020 Visualization Tool

51 Diesbezügliche Auswertungen für die eigene Organisation sind jedoch auf Anfrage möglich. 


\section{Abbildung 36: Bewertung der Leistungen EU-PM, Schulnoten (Wissenschaft)}

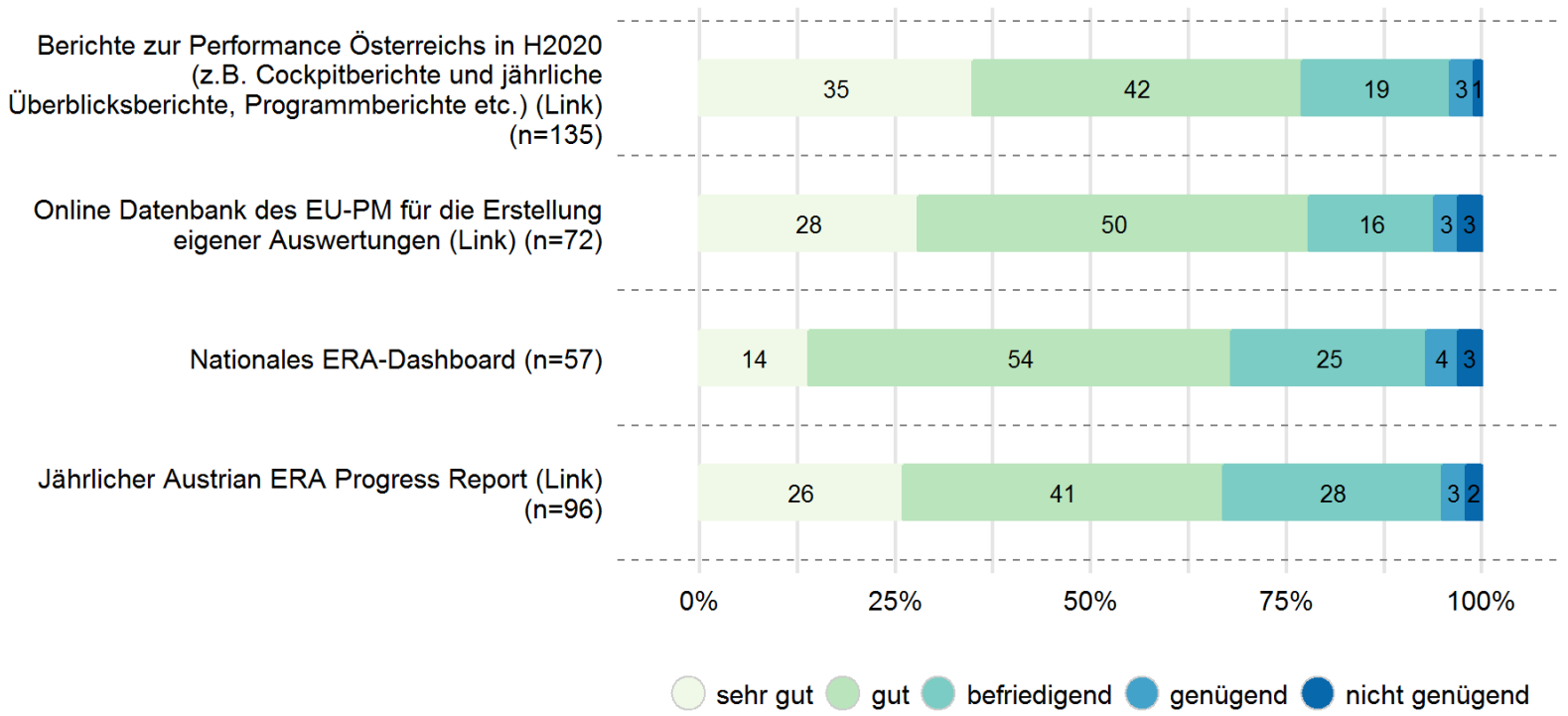

Quelle: Online Befragung, Joanneum Research.

\section{Abbildung 37: Bekanntheit Leistungen EU-PM (Unternehmen)}

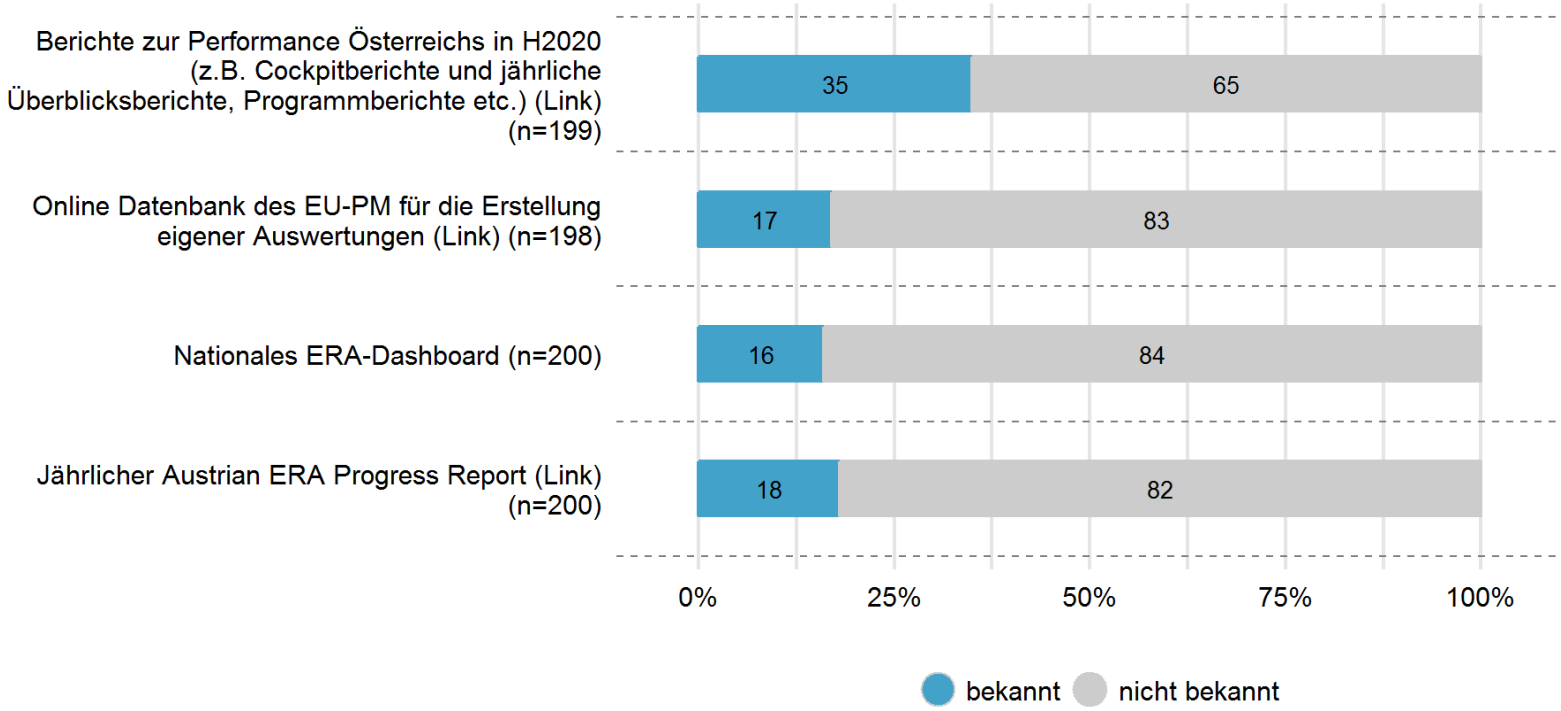

Quelle: Online Befragung, Joanneum Research. 


\section{Abbildung 38: Bewertung der Leistungen EU-PM, Schulnoten (Unternehmen)}

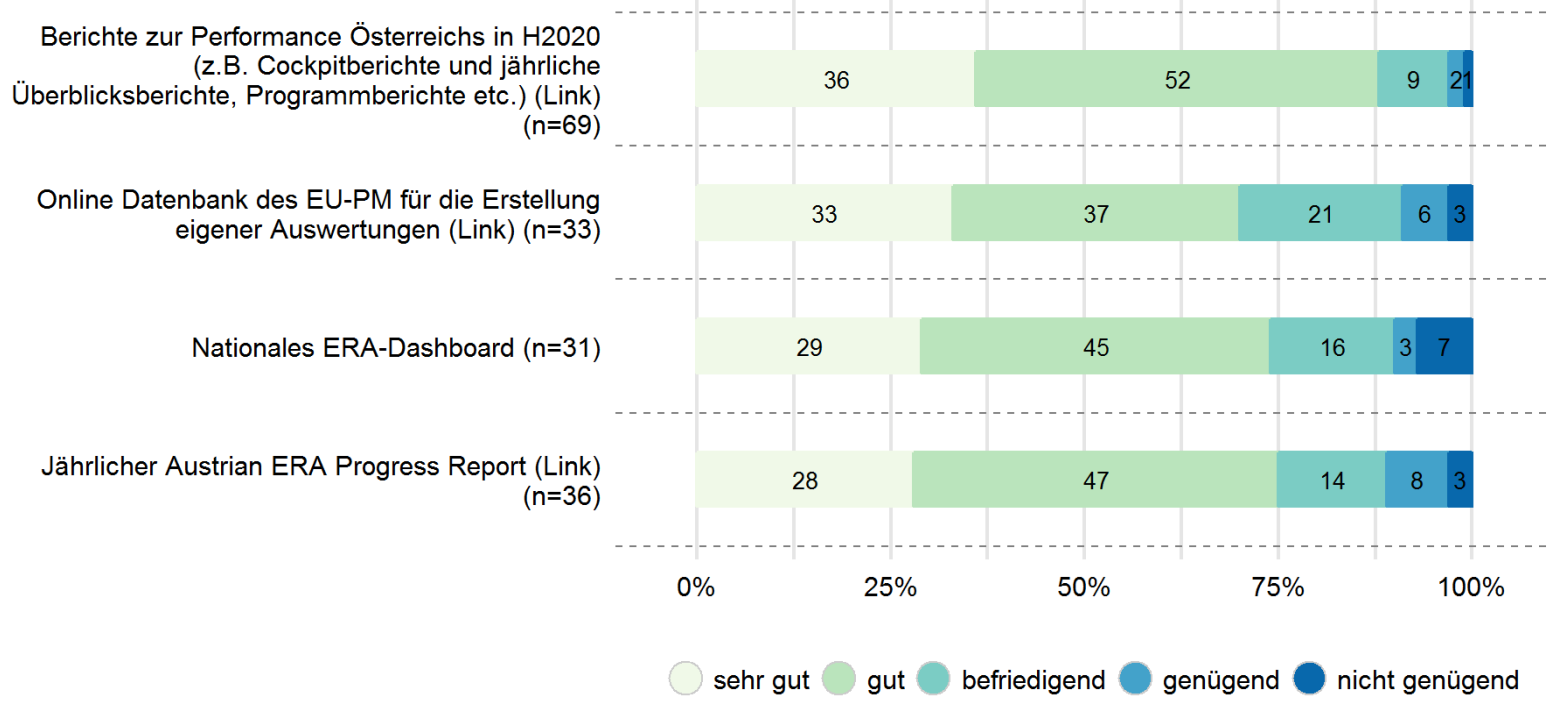

Quelle: Online Befragung, Joanneum Research.

Obwohl ebenfalls der Zielgruppe 2 angehörig, sind die Bekanntheit und die Nutzung des EU-PM Portfolios unter befragten VertreterInnen Intermediärer Organisationen also den regionalen Förder- und Beratungsinstitutionen - deutlich stärker ausgeprägt. Erhoben wurde dabei neben der Bekanntheit der Leistungen, ob diese von den Intermediären wie z.B. regionalen Förderagenturen für die Erbringung eigener Beratungsleistungen oder Informationsaufbereitung an Dritte verwendet werden. So geben $67 \%$ der Befragten dieser Gruppe an, Berichtsprodukte des EU-PM für die eigene Leistungserbringung zu nutzen (siehe Abbildung 39). Die Zufriedenheit mit diesen Produkten, als Grundlage für die eigene Leistungserbringung ist hoch. 92\% der Intermediäre gaben an, dass die von EU-PM bereitgestellten Daten zu Performance-Kennzahlen in Bezug auf H2O2O hilfreich für die eigene Arbeit seien. Immerhin 58\% der befragten Intermediären ist die OnlineDatenbank bekannt, $48 \%$ nutzen sie für die eigene Arbeit.

Insgesamt legt die Zugriffsstatistik des öffentlichen Online-Portals eine wachsende Bekanntheit des öffentlichen Online-Portals nahe, auch wenn insbesondere unter Angehörigen an Hochschulen und Forschungseinrichtungen im Einzelnen noch Informationsdefizite zu bestehen scheinen.

Zwischen 2015 und 2017 ist die Zahl der jährlichen Zugriffe von 1.978 auf 2.459 angestiegen. Gleichzeitig wurde in den Interviews jedoch auch auf die schwierige Auffindbarkeit der EU-PM Website, der öffentlichen Online-Datenbank und die allgemeine Unübersichtlichkeit der FFG-Homepage hingewiesen. Anders als beim öffentlichen Online-Portal sind die Zugriffe auf die EU-PM Website in der Tat im Vergleich zu 2015 rückläufig (von 1.641 auf 1.3262017 ).

Vereinzelt wurden in Interviews mit regionalen Beratungs- und Fördereinrichtungen zudem der Bedarf nach Zugang zu Daten zu abgelehnten Projektanträgen in den Bundesländern geäußert, um entsprechenden Organisationen passgenaue Beratung zukommen 
lassen zu können. So hätten regionale Kontaktstellen früher Zugang zu den Beteiligungsstatistiken gehabt - also auch den Daten zu letztlich abgelehnten Anträgen. Dies deckt sich mit FFG-internen Überlegungen, die im Rahmen eines Interviews geäußert wurden, für die regionalen Kontaktstellen bzw. Intermediäre eine Art "Zielgruppe 1 1/2" zu definieren, die mit regionalspezifischen Auswertemöglichkeiten ausgestattet werden könnte. Die Gewährung erneuter Individualdatenzugänge wäre jedoch auch mit zusätzlichen Lizenzkosten im Rahmen der für das Portal verwendeten IT-Businesslösung verbunden sowie mit Mehraufwand in der Gestaltung der Berechtigungslogik in Bezug auf die Selektion und Abgrenzung der zielgruppenspezifischen Zugriffsmöglichkeiten. Im Rahmen der aktuellen Beauftragung sei vor diesem Hintergrund die bewusste Entscheidung für eine klar abgegrenzte Zielgruppe 1 mit einheitlicher Berechtigungslogik getroffen worden. Diese schließt eine Weitergabe von Individualdaten aus, die über den Kreis der Delegierten und AuftraggeberInnenorganisationen hinausgeht.

\section{Abbildung 39: Bekanntheit \& Nutzung Leistungen EU-PM (Intermediäre)}

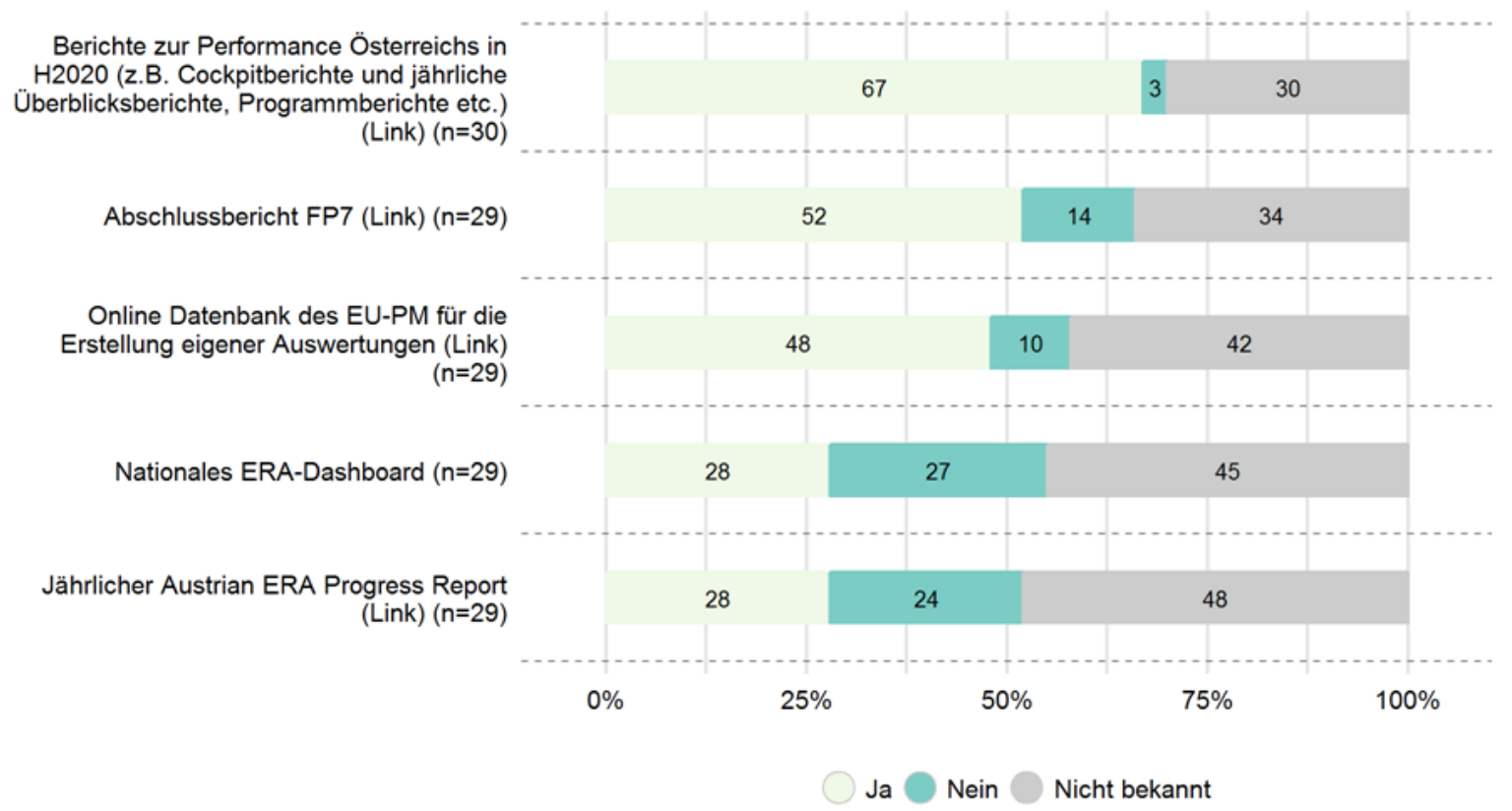

Quelle: Online Befragung, Joanneum Research.

\section{Bekanntheit, Nutzung und Erfahrungen mit den Produkten - Zielgruppe 1}

VertreterInnen der Zielgruppe 1 wurden im Rahmen der Interviews (siehe Kapitel 1 zu Methoden) adressiert. Die Aktivitäten und Produkte des EU-PM umfassten einen Teilbereich des Gesprächsleitfadens. Die Leistungen des EU Performance Monitorings wurden in den Interviews grundsätzlich sehr positiv bewertet. Nach dem Schulnotensystem werden Bewertungen zwischen 1 und 2 vergeben. Besonders geschätzt wird die Möglichkeit, maßgeschneiderte Expertisen von der FFG erhalten zu können, die unmittelbar in Berichte einfließen. Auch die KundInnennähe und das Bemühen der MitarbeiterInnen um Lösungen werden honoriert. 
Die Cockpitberichte zur Performance Österreichs in H2020 sind zwar dem Namen nach nicht immer bekannt, wurden aber nach entsprechendem Hinweis im Gespräch mehrheitlich positiv beurteilt. Die jährlichen Überblicksberichte zur Performance Österreichs in H2020 werden als hilfreiche Überblicksdokumente verwendet und gut bewertet. Die Programmberichte zur Performance Österreichs in bestimmten Programmbereichen von H2020 werden dagegen seltener verwendet, weil stattdessen entweder Spezialauswertungen von der FFG oder von eigenen MitarbeiterInnen angefordert werden.

Das personalisierte Online-Portal zur Erstellung eigener Auswertungen ist großteils bekannt und wird grundsätzlich positiv bewertet. Gleichzeitig wurde im Zuge der Interviews die Frage der (Selbst-)Befähigung der involvierten Akteure diskutiert, dieses entsprechend zu nutzen. Tatsächlich gibt es ein deutliches Auseinanderklaffen zwischen wenigen GesprächspartnerInnen, die das Instrument selber verwenden oder gar im Umgang mit diesem Instrument versiert sind und der Mehrheit der InterviewpartnerInnen, die sich als wenig technikaffin bezeichnet bzw. Zeitgründe für die bislang geringe Auseinandersetzung mit diesem Service angibt und stattdessen im Fall des Falles auf direkte Anfragen beim EU-PM zurückgreift. Die Auswertung der Zugriffe auf das vertrauliche Portal im Jahr 2017 zeigt jedoch, dass deren Anzahl mit 392 (bei 102 registrierten NutzerInnen) fast 4-mal höher war als die Zahl der direkten Anfragen, die VertreterInnen der Ministerien an das EU-PM gerichtet haben (98). ${ }^{52}$ Unter Berücksichtigung der begründeten Annahme, dass eine Reihe von Anfragen aus regelmäßig wiederkehrenden Updates zu bereits erstellten Analyserastern (bspw. Spezialauswertungen zum ERC oder für den aktuellen Universitätsbericht) bestehen, die mit geringem Aufwand verbunden sind, sinkt die Zahl der originären Anfragen sogar noch weiter. Gründe für direkte Anfragen sind dabei oftmals Zeitdruck oder die Komplexität der Fragestellung. Dies deutet darauf hin, dass die direkten Anfragen in Relation zum Eigenzugriff eine deutlich geringere Rolle spielen und spezifische Fragestellungen adressieren. FFG-intern wurden 37 Anfragen an EU-PM gerichtet, die spezifische Analysen zu Drittstaatenkooperationen, Beiträge für die Themendossiers, institutionenspezifische Auswertungen sowie themenspezifische und programmspezifische Fragestellungen umfassen. Auch Vorbereitungen zu Pressekonferenzen sind darunter. Dies ist ein Hinweis für die Verankerung von EU-PM als FFG-interne Informationsquelle.

\section{Vergleich EU-PM mit anderen Datenportalen}

Ein Aspekt, der in den Interviews geäußert wurde, war die Frage nach der prinzipiellen Notwendigkeit einer derart ausgestalteten nationalen Analyseeinheit, vor dem Hintergrund zu Verfügung stehender Datenquellen auf europäischer Ebene und Quellen in anderen Mitgliedstaaten. Hierfür wurde das H2020 Dashboard der EU-Kommission genannt oder auch, z.B. von Seiten einiger Hochschulakteure, das H2020-Visualization

52 Das Ticketsystem des EU-PM unterscheidet nicht direkt zwischen den Zielgruppen. Erfasst werden alle Arten von Anfragen wie sie auch von VertreterInnen der Zielgruppe 2 gestellt werden können. Insgesamt wurden 2017292 Tickets erfasst, wobei die Erstellung der Quickinfos als Standardprodukt herausgerechnet wurde, wodurch sich eine Zahl von 202 Erledigungen ergibt. Als Anfragen durch die Zielgruppe 1 wurden jene durch die Ministerien gezählt. 
Portal der schwedischen Innovationsagentur VINNOVA. Eine durch das Evaluierungsteam vorgenommene, vergleichende Bewertung der unterschiedlichen Herangehensweisen und Auswertemöglichkeiten des öffentlichen EU-PM-Datenportals ${ }^{53}$, des VINNOVA H2020 Visualization Tools ${ }^{54}$ und des öffentlichen Participant Portals der EU Kommission zeigt jedoch, dass hier durchaus unterschiedliche Logiken in Bezug auf Aussagefähigkeit und Darstellungsstrukturen zur Anwendung kommen, die in der Bewertung der Funktionalität zu berücksichtigen sind.

So folgt das VINNOVA-Tool einer stark projekt- und institutionenbezogenen Logik. Mit wenigen Mausklicks kann von der Darstellung der Projekte und Fördermittelausschüttungen auf Ebene der drei Säulen und Programme von H2020 über die einzelnen Calls bis hinunter auf die Einzelprojektebene navigiert werden, wo dann auch die jeweiligen Partnerinstitutionen und deren Anteile am Projekt identifiziert werden können.

Diese Darstellungsform scheint gerade für individuelle Organisationen wie Hochschulen besonders attraktiv zu sein und wird offensichtlich bereits verwendet. Gleichzeitig ist eine vergleichende Analyse wesentlicher Kennzahlen wie eben den Beteiligungs- oder Erfolgsraten zwischen Ländern und Institutionen in diesem Aufbau nicht bzw. nur eingeschränkt möglich (Beteiligungsraten werden auf Länderebene in Bezug auf den Anteil den Fördermitteln nicht an den Projekten dargestellt). Darüber hinaus gibt es keine Möglichkeit für regionalisierte Auswertungen. Auch werden keine Datenqualitätschecks und Bereinigungen wie durch EU-PM vorgenommen (siehe nachfolgender Abschnitt), sondern lediglich über eCORDA bereitgestellte Einzelprojektdaten eingespielt.

Das Participant Portal der EU-Kommission zielt hingegen darauf ab, einen Überblick über die Performance eines Landes über Beteiligungsraten, ausgeschüttete Fördermittel, deren Verteilung nach Säulen/Topics sowie die Top-Performer auf Organisationsund Projektebene zu vermitteln. Ähnlich wie bei der Darstellungsform des VINNOVATools ist jedoch auch hier die Möglichkeit für rasche Direktvergleiche eingeschränkt, beispielsweise was den Vergleich von Erfolgsquoten betrifft.

Demgegenüber liegt der Fokus des öffentlichen Online-Portals von EU-PM auf der komparativen Performanceanalyse wesentlicher Kennzahlen zu Beteiligungen, ausgeschütteten Fördermitteln und insbesondere auch Erfolgsraten auf Ebene von Ländern, Programmen und Organisationstypen. Diese Daten sind für Österreich auch in regionalisierter Form auf Ebene der Bundesländer verfügbar. Auch können die Top-Organisationen in H2020 nach Organisationstyp (bspw. Hochschulen oder Unternehmen) und Land in Bezug auf Kennzahlen wie Beteiligungen, Fördermittel und Koordinationsrollen auf Ebene der einzelnen Programmlinien ausgewiesen werden. Eine Auswertung der Beteiligung von Organisationen auf der Ebene von Einzelprojekten ist hingegen nicht möglich, hier stellt das VINNOVA-Tool eine komplementäre Funktionalität bereit.

53 Siehe öffentliche EU-PM Datenbank; Download 23.04.2018

54 Beim VINNOVA H2020 Visualization Portal handelt es sich um studentisches Projekt in Kooperation mit der KTH- Royal Institute of Technology mit dem Ziel in Visualisierungsinterface zu schaffen. Datengrundlage ist wie bei EU-PM eCORDA. Updates werden durch VINNOVA entsprechend der Fristigkeiten analog zum öffentlichen Portal von EU-PM eingespielt. 
Die in den Interviews geäußerten Anmerkungen zur potenziellen Substituierbarkeit des öffentlichen EU-Portals weisen daher im Vergleich der genannten Instrumente eher auf ein gewisses Informationsdefizit in Bezug auf die Möglichkeiten der jeweiligen Tools hin.

Tatsächlich scheint hier eher eine komplementäre denn substitutive Beziehung zu bestehen, weil jedes Tool einer anderen Nutzungslogik folgt. In Bezug auf das Portal des EU-PM kann hier insbesondere der politikunterstützende Aspekt, durch den Fokus auf Performance-Vergleiche und die Identifikation von Stärken und Schwächen zwischen den einzelnen am Rahmenprogramm teilnehmenden Staaten, hervorgehoben werden. Ein weiterer zentraler Aspekt ist die Performance-Analyse auf Ebene der Bundesländer.

Zwei GesprächspartnerInnen thematisierten auch die Frage - insbesondere vor dem Hintergrund des Kostenaspektes - inwieweit die Erbringung von Serviceleistungen durch das EU-PM überhaupt für eine breitere Öffentlichkeit zugänglich sein sollte, um eine Überfrachtung des EU-PM zu vermeiden. Die Frage der Relevanz des Kostenaspektes für die Leistungserbringung ist vor dem Hintergrund der Einschätzungen zur Kosten-Effizienz der Leistungserbringung zu sehen, wie im nächsten Abschnitt dargestellt.

\section{Datenqualität und Verknüpfung}

Ein weiterer wichtiger Aspekt umfasst den Bereich der Datenqualität und deren Aktualität. So wurde vereinzelt auf Unterschiede in der Zuordnung von Themen durch Programmkomitees mit jener, wie sie EU-PM auf Basis von eCORDA vornimmt, verwiesen, die eine einheitliche Darstellung von Themenbereichen erschwere und laufend spezifische Einzelauswertungen notwendig mache. Weiters attestiert eine InterviewpartnerIn der Zielgruppe 1 ein Aktualitätsproblem, da sich die Auswertungen der FFG immer auf den Status der Angebote nach Abschluss der Ausschreibung beziehen. Nach diesem Zeitpunkt würden sich allerdings immer wieder Verschiebungen ergeben, weil bspw. Projekte neu ausverhandelt werden, einzelne Akteure tatsächlich nicht an einem Projekt teilnehmen können oder neue Akteure auftauchen. Diese Verschiebungen würden sich nicht in den Auswertungen der FFG widerspiegeln. Seitens des EU-PM wird darauf verwiesen, dass die Entscheidung den Angaben aus eCORDA zu folgen - und damit durch die Kommission selbst vorgenommene Zuordnungsveränderungen zu übernehmen - bewusst im Rahmen der Beauftragung getroffen wurde, basierend auf der Überlegung, dass Alternativdarstellungen ebenfalls hohen Erklärungsaufwand produzieren würden. Mit dieser Vorgehensweise könne die Übereinstimmung von Kommissionsangaben und EU-PM-Angaben gewährleistet werden. Weiters leiste EU-PM wichtige Grundlagenarbeit im Bereich der Datenpflege und -integration (wie z.B. die Korrektur von Organisationsnamen oder der NACE ${ }^{55}$ - und NUTS ${ }^{56}$-Zuordnung), die - wie im Folgenden näher erläutert - der Verbesserung der Aufbereitung für die nationale Verwendung diene.

\footnotetext{
55 "Nomenclature statistique des activités économiques dans la Communauté européenne"; Statistische Systematik der Wirtschaftszweige in der Europäischen Gemeinschaft.

56 "Nomenclature des unités territoriales statistiques"; Systematik der Gebietseinheiten für die Statistik.
} 
Gleichzeitig wurde durch das EU-PM jedoch selber auf die Problematik der 14-tägigen Taktung der Datenaktualisierung in einzelnen Programmen im Portal der EU-Kommission verwiesen, die im Vergleich zu den eigenen Produkten naturgemäß dazu führe, dass insbesondere Programmdelegierte mitunter für ihre Bereiche aktuellere bzw. abweichende Informationen zur Verfügung hätten. Durch häufige Änderungen der Datenbank auf Seiten der Kommission (z.B. Veränderungen von Bezeichnungen bei Calls und Topics) sei allerdings der standardmäßige Import eine Herausforderung: die entsprechenden Hintergrundinformationen werden aktuell nur 3x im Jahr zur Verfügung gestellt, woran auch die Fristigkeit der nationalen Datenupdates gekoppelt ist, die dann tatsächlich für offizielle Berichte und Statistiken (z.B. Cockpitberichte etc.) zur Performance in H2020 verwendet werden können. Gleichzeitig seien die zukünftige konkrete Weiterführung dieser Aktualisierungen, ihre genauen Intervalle sowie die Taktung der Bereitstellung der Rohdaten durch die Kommission via eCORDA und via Open Data Portal auf Ebene der Kommission noch nicht abschließend diskutiert, die Umsetzung daher noch nicht endgültig absehbar. Allfällige Schlussfolgerungen sind daher zum jetzigen Zeitpunkt nur eingeschränkt möglich und vor dem Hintergrund entsprechender Entwicklungen im Bereich der Datenbereitstellung auf europäischer Ebene zu ziehen.

Von Seiten der Delegierten wird zudem immer wieder der Wunsch geäußert, die Verknüpfung mit weiteren Datenquellen - wie in der Beauftragung verankert - voranzutreiben, um Aussagen über Relevanz und Interaktion unterschiedlicher Instrumente auf Ebene der Fördernehmer treffen zu können. Dies umfasst zum einen die Einbeziehung von Beteiligungs- und Performancedaten nationaler Akteure in transnationalen europäischen FTI-Partnerschaften - Public-Public- (JPI, ERA-Net-Instrumente, Artikel 185 Initiativen) und Public-Private-Initiativen (JTI, EIT, contractual Public-Private-Partnerships) - sowie nicht durch die EU-Kommission kofinanzierter Partnerschaftsinstrumente wie EUREKA Cluster- und Netzwerkprojekte. ${ }^{57}$

Entsprechende Aktivitäten finden bereits statt, seitens des Teams von EU-PM wird jedoch die Verfügbarkeit entsprechender Datenbestände auf europäischer Ebene als notwendige Bedingung hervorgehoben. Wie bereits dargestellt ist EU-PM am Projekt "ERA-Learn 2020" beteiligt, welches u.a. eine Datenerfassung der P2P-Instrumente beinhaltet. Daten zu aktiven und abgeschlossenen Netzwerkpartnerschaften (bis zurück ins 6. Rahmenprogramm), den jeweiligen Trägerorganisationen, der Laufzeit sowie den jeweiligen Calls und den budgetierten Fördersummen werden hier bereits standardisiert verfügbar gemacht. Eine wesentliche Einschränkung besteht jedoch zurzeit noch in Bezug auf die Erfassung der Förderaktivitäten auf Einzelprojektebene. So verfügt das EU-PM aktuell über entsprechende Daten zu Projekteilnahmen in obig angeführten Instrumenten nur dort, wo diese über die FFG abgewickelt und finanziert werden (bspw. ERA-Nets oder die Art. 185 Initiativen Eurostars und AAL und der JPI Urban Europe) und somit in der nationalen Förderdatenbank der FFG aufscheinen. Hier handelt es sich jedoch nur um Daten zu den

57 Vgl. BMVIT, BMWFW (2017): Österreichischer Forschungs- und Technologiebericht 2017; Lagebericht gem. $\S 8$ (1) FOG über die aus Bundesmitteln geförderte Forschung, Technologie und Innovation in Österreich; Kapitel 5.3 für eine detaillierte Darstellung unterschiedlicher Formen transnationaler F\&E-Kooperationen im Europäischen Forschungsraum. 
national geförderten Akteuren ohne entsprechende Angaben zum Gesamtkonsortium. Ein Bericht über die aktuellen Analyse-Möglichkeiten der zur Verfügung stehenden Daten zu den P2P-Instrumenten wurde dem Lenkungsausschuss im Februar 2018 übermittelt. ${ }^{58}$ Darüber hinaus werden jährlich instrumentenspezifische Berichte an die jeweiligen AuftraggeberIn - u.a. BMVIT, BMBWF, KLIEN - zu Calls und Projekten, der nationalen Kofinanzierung für österreichische PartnerInnen die über die FFG abgewickelt wird, sowie Aspekten des Programmmanagements erstellt. ${ }^{59}$

Weiters lobbyiert EU-PM in seiner Rolle als Mitglied der eCORDA-Usergroup für die Integration von Daten zu europäischen Public-Private-Kooperationen (insb. Joint Technology Initiatives, contractual Public-Private-Partnerships) durch die EU-Kommission in eCORDA. Hier gab es im vergangenen Jahr eine Reihe von Weiterentwicklungen, die Zahl eingepflegter Informationen steige mit jeder neuen Datenlieferung der Kommission, jedoch sehr uneinheitlich über die einzelnen Instrumente hinweg. So sind ProjektteilnehmerInnen und entsprechende Fördersummen an Ausschreibungen der JTI ECSEL in eCORDA bereits erfasst, für andere JTIs wie Clean Sky 2 können dagegen bis dato nur Aussagen über NetzwerkpartnerInnen und/oder die ausgeschütteten Mittel in den Calls getätigt werden, nicht jedoch zu Einzelakteuren auf Projektebene. Contractual Public-Private-Partnerships (CPPP) sowie die FET-Flagship-Initiativen sind aufgrund ihrer Natur als über das Rahmenprogramm direkt ausgeschriebene Aktivitäten ebenfalls in eCORDA erfasst. Auch zu den aktuellen Datenbeständen in eCORDA zu transnationalen FTI-Partnerschaften im Bereich der PPP wurde im September 2017 ein Bericht an den Lenkungsausschuss gelegt. ${ }^{60}$

Die Situation im Bereich der Datenerfassungsmöglichkeiten zu den einzelnen Instrumenten der transnationalen FTI-Partnerschaft EUREKA ist analog zu jener bei den P2Ps. Aktuell können nur Daten zu den national geförderten Akteuren, welche über die FFG Programme finanziert werden, dargestellt werden. Auch hier geht der Bezug zu den internationalen PartnerInnen in den Konsortien aktuell noch verloren. Eurostars wird als P2P-Initiative nach Art. 185 auch im Rahmen von ERA-Learn 2020 erfasst. Seitens EU-PM wurde im Jahr 2017 eine Anfrage an das EUREKA-Sekretariat gestellt, um einen Datenbezug zumindest zu den internationalen PartnerInnen von Projekten mit österreichischer Beteiligung herstellen zu können. Diese war zum Zeitpunkt der Evaluierung jedoch noch unbeantwortet. Eine Herausforderung in diesem Zusammenhang, so die Einschätzung des EUPM-Teams, sei zum einen, dass das EUREKA-Sekretariat auf die Meldungen der EUREKANCPs in den jeweiligen Partnerländern angewiesen ist, die sehr uneinheitlich erfolgen würden und zum anderen die zeitliche Fristigkeit. So wäre selbst bei Verfügbarkeit der entsprechenden internationalen Daten der zeitliche Abgleich mit den nationalen EUREKABeteiligungsdaten über die FFG-Förderdatenbank eine Herausforderung, da i.d.R. ein zeitlicher Abstand zwischen der Erteilung des EUREKA-Labels und dem Aufscheinen in der nationalen Förderdatenbank besteht.

58 Dieser Bericht liegt dem Evaluierungsteam vor.

59 EU-PM (2016): Jahresbericht 2016.

60 Dieser Bericht liegt dem Evaluierungsteam vor. 
Die Beauftragung sieht ebenfalls die Verknüpfung zwischen nationalen Förderdaten der FFG sowie EU-Performance-Daten im Rahmen der Möglichkeiten der zur Verfügung stehenden Datenbestände - eCORDA sowie die nationale Förderdatenbank der FFG - vor. Hier wurden insbesondere im Jahr 2017 seitens des EU-PM Anstrengungen zur Harmonisierung der über eCORDA bereitgestellten Stammdaten zum Rahmenprogramm mit den Stammdaten der FFG-Förderdatenbank vorgenommen. Die Herausforderung in der Verknüpfung der Förderdaten auf Ebene individueller Organisationen liegt dabei laut EUPM-Team in mitunter abweichenden Schreibweisen bzw. fehlenden Zuordnungen auf Basis des Firmenbuchregisters. Letztere seien in den Rahmenprogrammdaten noch sehr lückenhaft. Dieser Abgleich mit dem Firmenbuchregister, das auch die Grundlage der eindeutigen Identifikation von Unternehmen in der nationalen Förderdatenbank darstellt, wird von EU-PM vorgenommen. Dies erlaubt auch die Zuordnung von Unternehmenscharakteristika wie den NACE-Code oder der Unternehmensgröße. Ein Problembereich ist hier nach wie vor der Sektor der Klein- und Kleinstunternehmen, die nicht notwendigerweise über eine Firmenbuchnummer verfügen. Abgleiche erfolgten hier, wie auch für Nicht-Unternehmen (wie Hochschulen, Forschungseinrichtungen, Vereine) über die namentliche Zuordnung. Mittlerweile sei eine kritische Größe harmonisierter Daten bis zurück ins 6. Rahmenprogramm erreicht, die laut EU-PM-Team entscheidend sei um aussagekräftige Auswertungen generieren zu können. Die Harmonisierungen werden seit 2017 mit jeder neuen Datenlieferung vorgenommen. Verknüpfte Auswertungen zur Performance nationaler Akteure auf Organisationsebene wurden bis dato anlassbezogen für forschungsstarke Universitäten und Unternehmen vorgenommen. Für die aktuelle Evaluierung des KIRAS-Programmes konnte bspw. analysiert werden, in welchen europäischen Programmen nationale Akteure, die über KIRAS gefördert wurden, reüssieren. ${ }^{61}$ Selbiges sei beispielsweise prinzipiell auch für im Rahmen von EUREKA-Instrumenten geförderte nationale Akteure möglich. Seitens des EU-PM-Teams wird jedoch betont, dass die klare Eingrenzung der Fragestellung für derartige Auswertungen, aufgrund der hohen Komplexität der möglichen Verknüpfungen, zentral sei, z.B. in Bezug auf Abgrenzung von Akteursgruppen, spezifischen Unternehmen oder Programmen. Man bewege sich hier weg von standardisierten Produkten, deren Erstellung einen immer kleineren Anteil an der Arbeitsleistung des EU-PM einnähme - wie auch die entsprechende Abbildung 34 im vorangegangenen Abschnitt zeigt - hin zu immer individuelleren Services. Hier sei der Dialog mit den NCPs und den AuftraggeberInnen zu verstärken.

61 Engelhardt, S.; Künzel, M.; Ecker, B.; Schnabl, A. (2018): Evaluation des österreichischen Sicherheitsforschungsprogramms KIRAS Bericht 2017; Studie im Auftrag des Bundesministeriums für Verkehr, Innovation und Technologie (BMVIT). 


\section{Analyse der Effizienz des Mitteleinsatzes von EU-PM}

Die Gesamtsumme der EU-PM Beauftragung über die gesamte Laufzeit 2014-2021 beträgt laut Vertrag 3.94 Millionen Euro62. In den Jahren 2014-2016 lag der tatsächlich abgerechnete Betrag unter dem jeweiligen Planwert (siehe Tabelle 10). Das Team des EUPM besteht aus fünf Personen zu insgesamt 3.9 VZÄ. Der Vergleich mit dem Vorgängerprojekt PROVISO weist eine bedeutende Reduktion des Personaleinsatzes auf: Neun MitarbeiterInnen zu insgesamt 6.5 bis 7 VZÄ waren laut Rechnungshofbericht bei PROVISO beschäftigt ${ }^{63}$.

Tabelle 10: Budget und Abrechnungen EU-PM 2014-2016 (in Euro)

\begin{tabular}{|l|r|r|r|}
\hline & 2014 & 2015 & 2016 \\
\hline Budgetwert & 300000 & 484833 & 535646 \\
\hline Tatsächliche Abrechnung & 198844 & 459517 & 461653 \\
\hline
\end{tabular}

Quelle: EU-PM Leistungsberichte 2014-2015, 2016

Es ist auch bei EU-PM nicht möglich, eine klassische quantitative Effizienzanalyse durchzuführen. Die Erkenntnisse zum Mitteleinsatz basieren großteils auf einer Dokumentenanalyse und Interviews mit MitarbeiterInnen des EU-PM.

Das EU-PM verwendet für die Kostenaufzeichnung nur einen Kostenträger - d.h. der genaue Aufwand für die einzelnen Standard- und Spezialprodukte lässt sich nicht bestimmen. Einschätzungen zum tatsächlichen Aufwand unterschiedlicher Formen der Leistungserbringung durch EU-PM können lediglich auf Basis der Gewichtung der einzelnen Aktivitäten wie sie in den jährlichen Leistungsberichten dargestellt werden sowie in Rücksprache mit den MitarbeiterInnen des EU-PM erfolgen. Hier zeigt sich, dass die Entwicklung und Erstellung standardisierter Produkte (Cockpitbericht, Quickinfos) sowie das standardisierte, regelmäßige Einpflegen der eCORDA-Daten in das EU-PM-Datawarehouse im Vergleich zu den ersten beiden Jahren nach der Implementierung von EU-PM mittlerweile effizient und beinahe vollautomatisiert durchgeführt werden können. Dies sei mittlerweile Routine und der Aufwand für die Integration und Befüllung der Daten gut abschätzbar. Rund zehn Tage nach der Bereitstellung der Daten durch die Kommission werden die Eckdaten des neuen Datenstandes bereits durch EU-PM kommuniziert. Dies steht im Einklang mit der in den Leistungsberichten dargestellten Verschiebung des Aufwandes (Abbildung 34) hin zu immer stärker individualisierten Formen der Leistungserbringung. Gleichzeitig lasse sich der erwartete Aufwand für die Abarbeitung von Fokusanalysen und individuellen Anfragen mittlerweile auf Basis der Erfahrungen immer besser planen.

Einzig die Aktivitäten rund um die Erstellung des Indikatorensets zum ERA-Dashboard (die Leitung der damit beauftragten Arbeitsgruppe des ERA-Reporting Boards, laufende

62 Gemäß Vertrag zur Beauftragung der FFG mit dem Monitoring der österreichischen Performance in den FTI-Aktivitäten der EU 2014-2021 abgeschlossen zwischen der Republik Österreich und der Österreichischen Forschungsförderungsgesellschaft mbH (FFG)

63 Rechnungshof (2014), Nationale Maßnahmen zum 7. EU-Rahmenprogramm für Forschung, technologische Entwicklung und Demonstration 
Berechnung der festgelegten Indikatoren, Online-Applikation des ERA-Dashboards im Rahmen des öffentlichen Datenportals ${ }^{64}$, die Erstellung eines entsprechenden Manuals) stellten einen unerwarteten Mehraufwand dar, der jedoch vor dem Hintergrund der Effizienzgewinne in obig genannten Bereichen im Rahmen des Budgets letztlich gut abgedeckt werden konnte.

Als wesentliche Herausforderungen für die Zukunft werden die Aktivitäten rund um das Vorantreiben der harmonisierten Integration von Datenquellen zu EU-FTI-Partnerschaftsinstrumenten in eCORDA sowie die laufende Weiterführung und Nutzbarmachung der Verknüpfung zwischen nationalen und EU-Förderdaten genannt.

Dies erfordere neben der entsprechenden Entwicklung individueller Lösungswege auch einen intensiveren Dialog mit den Stakeholdern insbesondere auf Seiten der AuftraggeberInnen um Erwartungen, Anforderungen und Möglichkeiten zu klären. Ein Beispiel für entsprechende geplante Aktivitäten stellt der für Mai 2018 avisierte Workshop im BMVIT dar, um Fragen rund um die Möglichkeiten der Nutzung verknüpfter Stammdaten aus eCORDA und der FFG-Förderdatenbank zu klären. Darüber hinaus wurden Ende 2017 bzw. zu Beginn 2018 an den Lenkungsausschuss zwei Berichte zu Stand und Möglichkeiten der Datenanalyse im Bereich der transnationalen FTI-Partnerschaften (P2P und PPP) übermittelt. ${ }^{65}$ Entsprechende Aktivitäten, insbesondere die Verknüpfung nationaler und EU-Förderdaten, seien aufgrund der notwendigen Bereinigungen und Abgleiche noch nicht im demselben Ausmaß automatisierbar wie es die alleinige Datenintegration aus eCORDA für sich genommen bereits weitestgehend ist.

Ein weiterer Aspekt der Effizienzanalyse betrifft die Nachvollziehbarkeit und Zweckmäßigkeit des Reportings sowie die Interaktion mit dem/der AuftraggeberIn in unterschiedlicher Form. Aus Sicht des Evaluierungsteams stellen die Arbeits- und Kostenpläne in Verbindung mit den Leistungsberichten einen nachvollziehbaren Überblick über geplante und durchgeführte Aktivitäten sowie Gründe für etwaige Abweichungen dar. Gleichzeitig besteht Verbesserungspotential in Bezug auf die klare Darstellung von Zielhierarchien. Aktuell werden in den jährlichen Arbeitsplänen Zielsetzungen in Listenform dargestellt. Ein Vorschlag wäre, eine tabellarisch gegliederte Darstellung von Kann- und Soll-Zielen (letztere direkt abgeleitet aus Beauftragung), inkl. relevanter Einflussfaktoren für die Zielerreichung (siehe Vorschlag Tabelle 11).

64 Link zur öffentlichen EU-PM Datenbank; Download 24.04.2018.

65 Diese Berichte liegen dem Evaluierungsteam vor. 
Tabelle 11: Modell Zieldarstellung Arbeitsplan

\begin{tabular}{|l|l|}
\hline Kann-Ziel & Faktoren der Zielerreichung \\
\hline $\begin{array}{l}\text { Unabhängige Erarbeitung spezifischer Fo- } \\
\text { kusanalysen durch EU-PM }\end{array}$ & $\begin{array}{l}\text { Zustimmung Lenkungsausschuss } \\
\text { Freie Ressourcen } \\
\text { Ausreichende Abdeckung bei integrierten und } \\
\text { abgeglichenen Datenbeständen }\end{array}$ \\
\hline Soll-Ziel & $\begin{array}{l}\text { In Kooperation mit ERA-LEARN 2020 werden } \\
\text { guswertungen zu P2PNetzwerken zur Verfü- } \\
\text { gung gestellt }\end{array}$ \\
\hline
\end{tabular}

Quelle: Eigene Darstellung, Beispiele siehe EU-PM Arbeitsplan für 2017.

Dies wäre in Hinkunft auch eine wichtige Grundlage, um Einschätzungen zur Adäquanz der Governance-Strukturen zu generieren, indem herausgearbeitet wird, welche Maßnahmen auf welche Interventionen zurückgehen und welche Begleitschritte zur Verfolgung derselben durch den Lenkungsausschuss bzw. im Rahmen der monatlichen Abstimmungstreffen zwischen MitarbeiterInnen des EU-PM und des BMBWF als AuftraggeberInnenvertretung gesetzt wurden. Aktuell sind entsprechende Einschätzungen aus Sicht des Evaluierungsteams kaum möglich, außer solchen, die auf der Darstellung der involvierten Akteure beruhen. Vor dem Hintergrund der zu erwartenden steigenden Komplexität der Nutzungsmöglichkeiten in Verbindung mit der Integration neuer Datenquellen und dem Bedeutungsgewinn individueller Analysen steht nämlich auch die Governance vor neuen Herausforderungen. Diese betreffen die Formulierung von Anforderungen bspw. für Schwerpunktthemen für Analysen (wie sie jetzt bereits vereinzelt erfolgen z.B. Industrie 4.0 oder Bioeconomy), auch im Bereich der Nutzung national/EU-verknüpfter Daten, sowie das Monitoring der Nutzung und des Bedarfs entsprechender Angebote. Eine Idee für letzteres wäre bspw. eine im Rahmen des Ticket-Systems einmal jährlich durchzuführende Bedarfserhebung unter allen AnfragestellerInnen zu 1) deren Zufriedenheit mit der jeweilig erbrachten Leistung, 2) dem Bedarf nach themen-/programm-/akteurs-spezifischen Auswertungen als Grundlage für die Erstellung des Arbeitsplanes und zur Nachvollziehbarkeit durch den Lenkungsausschuss.

\subsection{Schlussfolgerungen \& Empfehlungen}

Im Folgenden werden auf Basis der Resultate aus den empirischen Erhebungen in Verbindung mit dem zur Verfügung gestellten statistischen Material zu den Aktivitäten von EUPM sowie im Abgleich mit entsprechenden Dokumenten wie der Beauftragung und den Jahresberichten zentrale Schlussfolgerungen und Empfehlungen synthetisiert. Die Schlussfolgerungen umfassen Einschätzungen zu Relevanz, Effektivität und Effizienz des EU-PM und seiner Leistungen.

Ein zentraler Aspekt ist die grundsätzliche Zufriedenheit der NutzerInnen mit dem Informationsangebot und der Funktionalität der Produktpalette. Die Ergebnisse der Erhebun- 
gen zeigen, dass die Zufriedenheit der NutzerInnen beider Zielgruppen mit dem Informationsangebot von EU-PM generell hoch ist. Insbesondere Angehörige der Zielgruppe 1 heben die Kundennähe und das Bemühen um individuelle Lösungen seitens der MitarbeiterInnen des EU-PM als besonders positiv hervor. Auch die Abgrenzung der Zielgruppen und die Bereitstellung zielgruppenspezifischer Angebote wird als sinnvoll und gelungen erachtet. Zwar wurde vereinzelt der Wunsch von Seiten regionaler Kontaktstellen nach Zugang zu Individual- und Antragsdaten geäußert, wie es in der Periode vor EU-PM der Fall war. Vor dem Hintergrund der bewussten Entscheidung, diesen im Rahmen der Beauftragung von EU-PM - nicht zuletzt aufgrund der hohen Lizenzkosten - nicht mehr zu gewähren und der hohen Zufriedenheit und Nutzung von Informationsleistungen des EUPM für die eigene Leistungserbringung intermediärer Organisationen, wird hier aus Sicht des Evaluierungsteams jedoch aktuell kein Handlungsbedarf geortet.

\section{Empfehlung: Kein Handlungsbedarf bei Zielgruppenabgrenzung}

Die Zufriedenheit der NutzerInnen beider Zielgruppen ist hoch und die entsprechende Abgrenzung der Zielgruppen wird als funktional eingeschätzt. In Bezug auf Letzteres wird jedenfalls aktuell kein Handlungsbedarf geortet. Insbesondere wird die Einrichtung einer eigenen Zielgruppe für regionale Kontaktstellen als nicht erforderlich erachtet. Der Dialog mit den regionalen Kontaktstellen kann jedoch weiter verstärkt werden.

Kritische Anmerkungen betreffen insbesondere die Funktionalität der Produkte, insbesondere des Webauftritts und des öffentlichen Online-Portals sowie die Qualität und Aktualität der zur Verfügung gestellten Daten. Bei ersterem besteht aus Sicht des Evaluationsteams Handlungsbedarf hinsichtlich einer Verbesserung der Auffindbarkeit der EU-PMLeistungen über die FFG-Homepage sowie in der Vermittlung des Leistungspotentials des öffentlichen Portals, wenngleich Zugriffe auf Letzteres stetig steigen. Ein weiterer Aspekt betrifft die Abgrenzung der Leistungsfähigkeit des öffentlichen EU-PM-Portals zu ähnlichen Produkten von VINNOVA bzw. der H2020-Participant Portal der Kommission. Hier kommt das Evaluierungsteam zu dem Schluss, dass dem EU-PM-Portal - vor dem Hintergrund der unterschiedlichen Stoßrichtungen der Vergleichsprodukte (VINNOVA auf Einzelprojektebene und Akteure, H2020-Participant Portal auf Länderüberblick) - mit seinem Fokus auf zentrale Kennzahlen- und Performancevergleiche auf Programm- und Institutionenebene zwischen den beteiligten Ländern sowie auf Ebene der österreichischen Bundesländer hier jedenfalls eine zentrale Funktion als politikunterstützendes Service, gemäß der Position des EU-PM im ERA-Observatorium, zukommt. Auch erfolgt die entsprechende Umsetzung mittlerweile weitestgehend standardisiert und effizient im Aufwand.

\section{Empfehlung: Weiterführung des öffentlichen Online-Portals}

Die Evaluierung spricht sich für eine Aufrechterhaltung des öffentlichen Online-Portals aus. Gleichzeitig sollten Verbesserungen in der Profilierung des Portals im Vergleich zu 
anderen Portalen erfolgen. Für Auswertungen auf Projektebene könnte bspw. direkt auf das VINNOVA-Tool verwiesen werden. ${ }^{66}$

Ein Vermittlungsproblem besteht aus Sicht des Evaluierungsteams auch hinsichtlich der verwendeten Datengrundlagen zur Analyse der Performance innerhalb des Rahmenprogramms, deren Begründung und Aussagekraft. Wenngleich gerade auf Ebene der Programmdelegierten viele Möglichkeiten der individuellen und - im Vergleich zu EU-PM-Daten - häufigeren Informationsgewinnung verfügbar sind, besteht der Vorteil des im Rahmen der Beauftragung festgelegten Fokus auf eCORDA als Datengrundlage in der regelmäßigen Vergleichbarkeit und der Qualitätskontrolle der Daten.

\section{Empfehlung: Usability des Online-Portals stärken}

Adaptierungen beim öffentlichen Portal sind bei der Vermittlung der Datengrundlagen des Portals und dessen Leistungsfähigkeit vorzunehmen. Dies umfasst die Adaption des

Portal-Manuals, das zwar Informationen zur Berechnung der einzelnen Kennzahlen, nicht aber zur grundlegenden Datenstruktur, zur Fristigkeit und Grenzen der Analysierbarkeit enthält. Auch in den Berichtsprodukten, insbesondere den standardisierten Cockpitberichten und Quickinfos, sollen Hinweise zur Datengrundlage und -qualität integriert werden.

In Bezug auf die Integration zusätzlicher Daten zu transnationalen FTI-Partnerschaften ist EU-PM von der Bereitstellung entsprechender Quellen auf EU-Ebene abhängig. Aktuell ist die Datenbereitstellung zu transnationalen FTI-Partnerschaften auch auf EUEbene noch äußerst lückenhaft, wenngleich hier in jüngerer Vergangenheit positive Entwicklungen zu beobachten sind. So bildet ERA-LEARN 2020 zumindest die Partnernetzwerke in den P2P ab. aber Angaben zur Projektteilnahme, Fördersummen auf Einzelinstitutionenebene etc. sind derzeit jedoch im Verantwortungsbereich der jeweiligen nationalen Förderagenturen der P2P Netzwerke und immer noch lückenhaft. In Bezug auf die PPPs werden - insbesondere auch auf Anregung von EU-PM als Teil der eCORDA-Usergroup - bereits Anstrengungen unternommen entsprechende Daten in eCORDA zu integrieren. Wenngleich die Bestände laufend wachsen, ist die Abdeckung auch hier noch sehr lückenhaft. So können bspw. die Aktivitäten insbesondere solcher JTI bis auf die Projektebene hinunter dargestellt werden, die wie ECSEL durch nationalen Programme kofinanziert werden. Für andere Instrumente können oftmals nur, analog zu den P2P, die Netzwerkstruktur und veranschlagte Budgets dargestellt werden. Für Österreich können in jedem Fall Auswertungen auf Ebene der nationalen ProjektpartnerInnen vorgenommen werden, die über die FFG-Instrumente kofinanziert werden. Dies umfasst bspw. österreichische EUREKA Beteiligungen, die in der FFG-Förderdatenbank aufscheinen.

\section{Empfehlung: Beibehaltung der laufenden Integration zusätzlicher Datenbe- stände}

66 Hierbei ist zu berücksichtigen, dass VINNOVA zwar mit gleicher Frequenz wie EU-PM auf eCORDA Daten zurückgreift, diese aber in der Folge nicht in gleicher Weise bereinigt und standardisiert wie EU-PM. 
Das EU-PM kommt seinem Auftrag zur Prüfung der Integration weiterer Datenquellen nach. Entsprechende Entwicklungen sollen auf EU-Ebene weiter von EU-PM aktiv begleitet werden. Der Lenkungsausschuss soll weiter über neue Datenbestände und Analysemöglichkeiten informiert werden.

Im Jahr 2017 wurde die Verknüpfung von eCORDA mit nationalen Förderdaten auf Basis der FFG-Förderdatenbank vorangetrieben. Dies ist insofern herausfordernd, als mit jeder neuen Datenlieferung Abgleiche zur eindeutigen Identifikation (Firmenbuchnummer, Org.- Namen) vorgenommen werden müssen, da Unterschiede zwischen den beiden Quellen bestehen können. Lücken bestehen noch bei den Kleinst- und Kleinunternehmen, die nicht notwendigerweise über eine Firmenbuchnummer verfügen müssen. Prinzipiell konnte nunmehr eine Verschneidung bis zurück ins 6 . Rahmenprogramm vorgenommen werden. An der Integration neuer Daten wird laufend gearbeitet. Aufgrund der Vielfalt an Analysemöglichkeiten und dem damit verbundenen Aufwand in der verknüpften Auswertung, wurden solche bis dato nur auf Ebene von großen Einzelinstitutionen wie forschungsstarken Universitäten und Unternehmen vorgenommen.

\section{Empfehlung: Kommunikation über Auswertungsmöglichkeiten und -grenzen stärken}

Aus Sicht des Evaluierungsteams kommt EU-PM seinem Auftrag des Vorantreibens der Datenverknüpfung zwischen nationalen und EU-Förderdaten nach. Die Herausforderung für die Akteure, insbesondere der Zielgruppe 1, besteht in Zukunft darin, vor dem Hintergrund der vielfältigen Auswertungsmöglichkeiten entsprechend konzise Anfragen zu formulieren (Beispiel: „Reüssieren FördernehmerInnen im nationalen Programm xy überdurchschnittlich in EU-Programmen?"). Gleichzeitig ist von Seiten des EU-PM Teams an der Kommunikation über die bestehenden Möglichkeiten zu arbeiten, bspw. im Rahmen von Workshops mit den Delegierten.

Das vertrauliche Online-Portal wird im Verhältnis zur Möglichkeit der direkten Anfrage bei EU-PM sehr gut angenommen. So gab es 2017 4-mal mehr Zugriffe auf das vertrauliche Portal als direkte Anfragen durch Ministerien. Die Analyse der Anfragestatistik sowie die Aussagen in den Interviews legen zudem nahe, dass die Zahl direkter Anfragen ihrer Natur entsprechend auch nicht durch Maßnahmen des EU-PM gesteuert werden könne. Zentrale Gründe sind nämlich oftmals Zeitdruck oder der Bedarf nach komplexeren Spezialauswertungen und Analysen. Auch machen Folgeupdates zu bereits erstellten Analyserastern einen nicht unbeträchtlichen Teil der Anfragen aus, die jedoch in Bezug auf den Mehraufwand vernachlässigbar sind. Verbesserungspotential wird - wie aus den Interviews mit NutzerInnen hervorgehend - im Bereich der Funktionalität und Gestaltung des vertraulichen Portals geortet (zusätzliche Schulungen, Manual). Hier ist die enge Abstimmung mit den NutzerInnen erforderlich. Dem Evaluierungsteam stand aufgrund der Vertraulichkeit der Daten nur ein eingeschränkter Blick auf die Funktionalitäten zur Verfügung. Ein wesentlicher Aspekt wird sicherlich in Zukunft auch hier die stärkere Individualisierung sein, in Bezug auf die bedarfsgerechte Auswahl von Verknüpfungsmöglichkeiten einzelner Programme und Akteure vor dem Hintergrund der Themen-/Programmverantwortlichkeiten der jeweiligen NutzerInnen. 
Die Leistungsbereitstellung erfolgt aus Sicht des Evaluierungsteams, sofern auf Basis der verfügbaren Materialien beurteilbar, in effizienter Weise. Die Effizienzgewinne welche durch die weitestgehend erfolgreiche Implementierung automatisierter Verfahren zur Erstellung der Standardprodukte erzielt wurden, erlauben einerseits direkte Anfragen entsprechend akkurat abzuarbeiten. Gleichzeitig wird voraussichtlich in Hinkunft, vor dem Hintergrund der steigenden Möglichkeiten in der Verknüpfung unterschiedlicher Datenquellen, der Bedarf nach individuellen Lösungen weiter zunehmen und es damit auch zu einer entsprechenden Verschiebung der Ressourcen kommen. Damit kommen auch auf die Governance neue Herausforderungen zu. Dies betrifft einerseits die Formulierungen von Anforderungen bspw. für Schwerpunktthemen für spezifische Analysen sowie andererseits das Monitoring der Wahrnehmung entsprechender Angebote. Hier müssten neue Formate für die Information von Möglichkeiten und Bedarfserhebungen der Zielgruppe 1 forciert werden. Dies wären bspw. entsprechende Workshops mit den Delegierten. Ein weiterer Vorschlag wäre, einmal jährlich im Rahmen des Ticket-Systems eine Bedarfserhebung unter allen AnfragestellerInnen durchzuführen. Diese könnte folgende Aspekte beinhalten: 1) Zufriedenheit mit der jeweils erbrachten Leistung, 2) Bedarf nach themen-/programm-/akteurs-spezifischen Auswertungen. Somit könnte eine Grundlage für die Erstellung des Arbeitsplanes entstehen und eine bessere Nachvollziehbarkeit durch den Lenkungsausschuss gewährleistet werden.

Empfehlung: Dialogformate zur Spezifizierung von EU-PM Dienstleistungen entwickeln

Das Nutzungspotenzial des vertraulichen Online-Portals wird durch die Selbstermächtigung der Zielgruppe 1 bereits weitgehend ausgeschöpft.

Gleichzeitig gilt es entsprechende Formate des Dialogs zwischen AuftraggeberInnen, NutzerInnen und EU-PM zu entwickeln, um vor dem Hintergrund zunehmender Analysemöglichkeiten durch die Integration neuer Datenquellen a) über neue Services und Möglichkeiten zu informieren und b) darauf aufbauend entsprechend konzise und tatsächlich verfolgbare Analyseaufträge zu erarbeiten. Ein Vorschlag wäre, im Rahmen des TicketSystems einmal jährlich eine Bedarfserhebung unter allen NutzerInnen durchzuführen.

Insgesamt gibt es aufgrund der grundsätzlich positiven Beurteilung des EU-PM und dem bereits angehäuften Know-How bereits zum jetzigen Zeitpunkt eine Reihe von Argumenten für eine grundsätzliche Fortführung des EU-PM. Denn gerade angesichts der Flut an unterschiedlichen Datenquellen besteht das Potential für das EU-PM hier eine "gate keeper"-Funktion in der Informationsvermittlung zu haben. Diese umfasst insbesondere:

- Plausibilitätschecks, Aufbereitung der eCORDA und sonstiger europäischer Datenquellen;

- die Verknüpfung mit nationalen Datenquellen;

- die Online-Tools und Berichtsprodukte, insbesondere auch die diesbezüglich geleistete Vorarbeit in der Analyse;

- die Funktion als nationale Auskunftstelle in Datenfragen;

- sowie die Erstellung individueller, spezifischer Auswertungen. 
Damit erfüllt das EU-PM aus Sicht des Evaluierungsteams seine zentrale Rolle als „Informations-Broker" im Rahmen des ERA-Observatoriums. Indikatoren dafür sind einerseits der hohe Informationsgehalt, der dem Format Europa Forum Forschung von Seiten der FTI-Community attestiert wird und wo regelmäßig MitarbeiterInnen des EU-PM über aktuelle Entwicklungen berichten. Des Weiteren basieren eine Reihe von Berichtsprodukten wir die Themendossiers des FFG-EIP auf Inputs des EU-PM. Die Auswertungen der Anfragestatistik in Bezug auf die FFG-internen Leistungen verdeutlicht zudem die Rolle des EU-PM als Informationsdienstleister innerhalb der eigenen Organisation. Einzig das ERA Council Forum als ein zentrales Element des ERA-Observatoriums verweist in erster Linie auf europäische Quellen bzw. das Domainwissen seiner Mitglieder für die eigene Arbeit zurückzugreifen.

Vor dem Hintergrund der aktuell noch sehr hohen Komplexität der Datenbereitstellung auf EU-Ebene - wenngleich hier prinzipiell ebenfalls bereits vieles verfügbar ist, jedoch zurzeit in weniger gut aufbereiteter Form - kommt den über EU-PM bereitgestellten Daten die Rolle eines zentralen Referenzrahmens für die nationale Policy-Debatte und die Verwendung in offiziellen Dokumenten und Berichten (wie bspw. den Forschungs- und Technologiebericht der Bundesregierung) sowie Strategiebildungsprozessen auf Bundesebene (im Vergleich z.B. zu über Programmkomitees bereitgestellte Daten) zu.

\section{Empfehlung: EU-PM fortführen}

Das Evaluierungsteam spricht sich für eine Fortführung des EU-PM aus. Diesem kommt eine zentrale Position als "Informations-Broker" für die nationale Policy-Debatte in Bezug auf ERA zu.

Gleichzeitig gilt es, den Mehrwert qualitätsgeprüfter Auswertungen und der damit verbundenen weiteren Analyse- und Verknüpfungsmöglichkeiten mit nationalen Daten als politikunterstützenden Services besser zu vermitteln und dadurch die "Erzählhoheit" des EU-PM weiter zu stärken.

Diese Schlussfolgerungen sind jedoch vor dem Hintergrund der aktuellen Struktur der Datenbereitstellung auf europäischer Ebene zu bewerten. Zukünftige Entwicklungen in Bezug auf Dienstleistungen zur Datenbereitstellung auf EU-Ebene können noch nicht abgeschätzt werden. 


\section{Koordination im Europäischen Forschungsraum: ERA-Observatorium}

\subsection{Ziele und Aufgaben}

Das Bundesministeriengesetz 2017 überträgt die Federführung der Koordination der österreichischen EU-FTI-Politik dem Bundesministerium für Bildung, Wissenschaft und Forschung (BMBWF).

Dies inkludiert vor allem:

1. die Beschäftigung mit den EU-Rahmenprogrammen (Support für H2020) und

2. koordinierende Maßnahmen zur Umsetzung des Europäischen Forschungsraums (Artikel 179 AEUV).

Das von der EU-Koordinationsabteilung des BMBWF betreute ERA-Observatorium dient in diesem Zusammenhang als Dachstruktur für die Integration der einzelnen Aufgabenstellungen im Bereich der Governance der EU-FTI-Politik in Österreich.

Das übergeordnete Ziel der Instrumente des ERA-Observatoriums ist die Unterstützung der eigenverantwortlichen Entscheidungsfindung insbesondere von politiknahen FTI-Akteuren im EU-Kontext. Die konkreten Zielsetzungen des ERA-Observatoriums sind die Bereitstellung:

- von Informationen und Austauschmöglichkeiten über die relevanten EU-Politiken (ERA Portal Austria67, Europa Forum Forschung),

- einer bedarfsgerechten Beratung und Betreuung der verschiedenen ministeriellen Ressorts für H2020 (EIP-Beauftragung, Lenkungsausschuss FFG-EIP, thematische ExpertInnengruppen H2020, Delegierten-Roundtable),

- einer strategischen Beratung der Politik (ERA Council Forum Austria) und von intelligenten Prozessen für den Strukturwandel im Politikbereich (ERA-Policy Forum, ERA-Roundtable, Arbeitsgruppen zu EIT, JPI, MULLAT, ERA KorrespondentInnen der Universitäten), sowie eines

- wirkungsorientierten Monitorings der österreichischen Teilnahme an H2020 und ERA (EU-PM, ERA Reporting Board).

Diese Funktionen werden durch die entsprechenden (zuvor in Klammern angeführten) institutionellen Lösungen adressiert, die im Folgenden vorgestellt und auf der Basis von Fokusgruppen, Online-Survey, Delphi-Befragung sowie 33 Interviews mit EU FTI-ExpertInnen (davon fünf Programmdelegierte und neun ExpertInnen für H2020) von Bundesministerien, Wirtschaftskammer Österreich (WKO) sowie aws, FFG und regionalen Agenturen und der Analyse verschiedener Dokumente reflektiert wurden.

67 Siehe die Website des ERA-Portal Austria 


\subsection{Informationen und Austauschmöglichkeiten über EU- Politiken}

Über das EU-Performance Monitoring (siehe Kapitel 4) und die EIP-Beauftragung (siehe Kapitel 2) stellt das BMBWF den Akteuren der FTI-Politik und der FTI-Community strategisch relevante Informationen zur österreichischen Beteiligung in H2020 und ERA-Initiativen zur Verfügung. Darüber hinausgehend adressiert das BMBWF die österreichische FTICommunity über das Veranstaltungsformat „Europa Forum Forschung" direkt. Über das ERA Portal Austria (siehe hierzu die Website des ERA Portal Austria www.era.gv.at) stellt das BMBWF Informationen zu Entwicklungen im Europäischen Forschungsraum zur Verfügung.

\subsubsection{Europa Forum Forschung}

Das Europa Forum Forschung (EFF) ist ein Informations- und Kommunikationsformat, im Rahmen dessen mehrmals im Jahr die FTI-Community adressiert wird. Ziel ist es, aus Sicht des BMBWF komplexe EU FTI-Themen verständlich aufzubereiten und dazu die Community zu informieren. Bei jeweils ca. 100 TeilnehmerInnen aus Bundesministerien, Agenturen, Universitäten, Interessenvertretungen, außeruniversitären Forschungseinrichtungen und vereinzelt auch Unternehmen werden Informationen über EU FTI-Themen ausgetauscht.

Das Europa Forum Forschung kommt in seiner Funktion als Informationskanal für Neuigkeiten aus der EU-FTI-Politik bei den adressierten Zielgruppen sehr gut an. Es wurde von 55\% der RespondentInnen in intermediären Organisationen und $18 \%$ der ForscherInnen aus dem Wissenschaftsbereich bereits genutzt. Das Forum ist jedoch im Unternehmensbereich kaum bekannt (77\% kennen es nicht, lediglich $7 \%$ haben daran teilgenommen).

\section{Abbildung 40: Die Bedeutung des Europa Forum Forschung (Wissenschaftsakteure)}

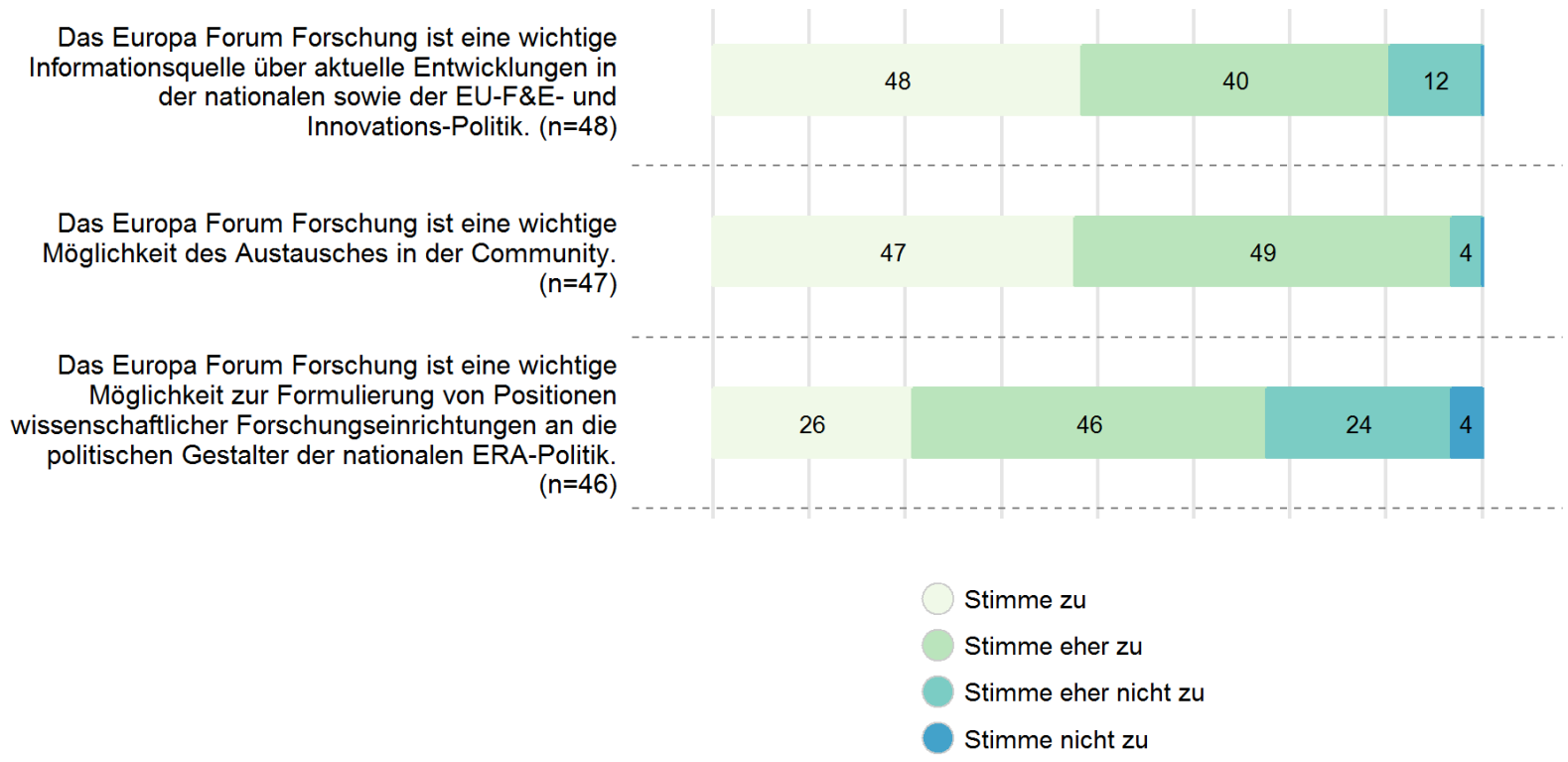

Quelle: Online Befragung, Joanneum Research. 
Sowohl Intermediäre, als auch ForscherInnen im Wissenschaftsbereich sehen das EFF als eine wichtige Informationsquelle über aktuelle Entwicklungen in der nationalen sowie der EU Forschungs- und Innovationspolitik und eine wichtige Möglichkeit des Austausches in der Community.

Während die wissenschaftliche Community im EFF überwiegend die Möglichkeit zur Formulierung von Positionen wissenschaftlicher Forschungseinrichtungen an die politischen Gestalter der nationalen ERA-Politik sieht, schätzt die Mehrheit der befragten Intermediäre diese Rolle für den Unternehmenssektor als wenig bedeutend ein.

\section{Abbildung 41: Die Bedeutung des Europa Forum Forschung für Intermediäre}

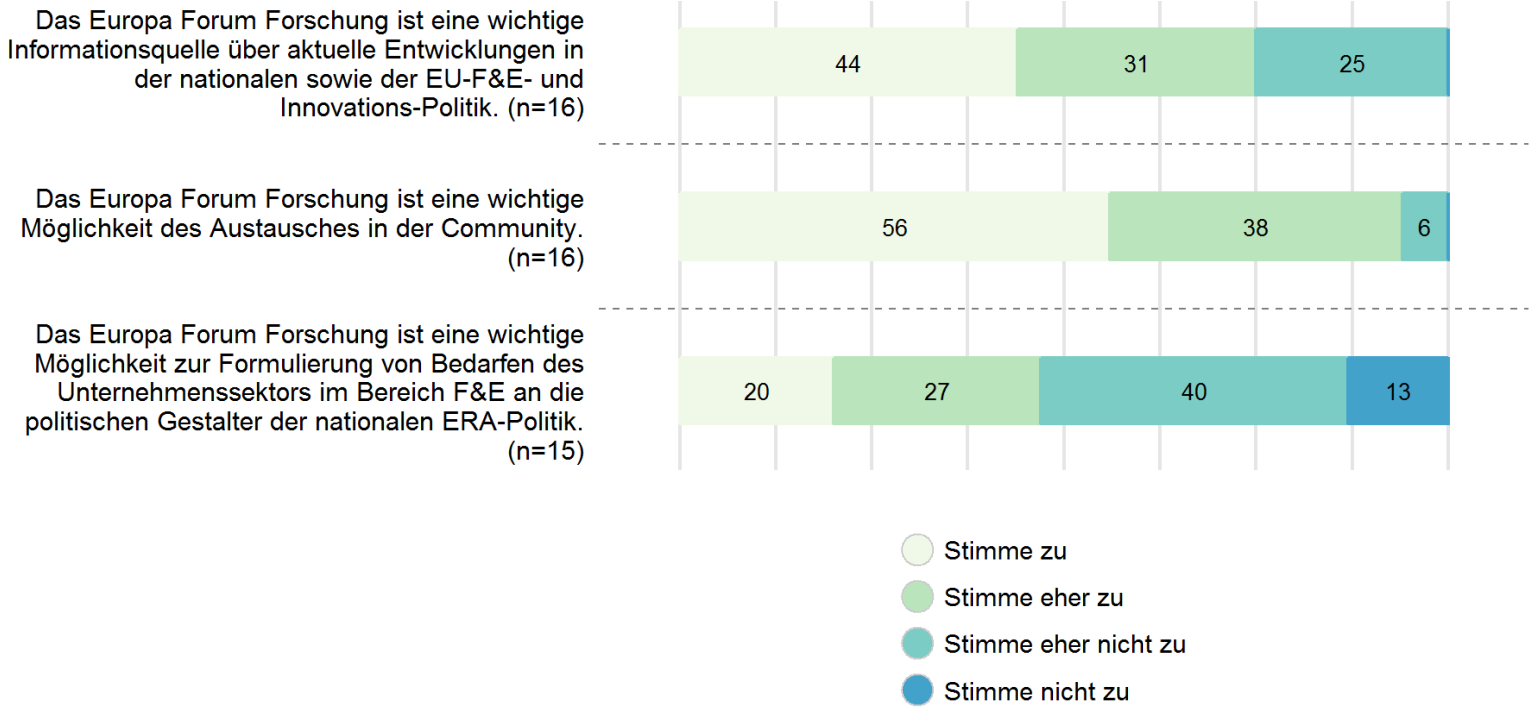

Quelle: Online Befragung, Joanneum Research.

Die im Rahmen der Evaluierung durchgeführten Interviews bestätigen, dass das Forum vor allem für seine Überblicksinformationen und die Möglichkeit des Knüpfens bzw. Auffrischens von Kontakten geschätzt wird. Der Relaunch des Forums mit dem Ziel einer Optimierung des Informationsaustausches (beispielsweise "Bundesländer-Slot") wurde ebenfalls positiv bewertet.

In verschiedenen Sub-Communities wird aber auch ein Bedarf nach einem verstärkten Austausch an Meinungen und Informationen geäußert. So wurde in einigen ExpertInneninterviews und Fokusgruppen die Hoffnung auf einen intensiveren Austausch zwischen den verschiedenen Akteursgruppen und der FTI-Politik im Europa Forum Forschung ausgesprochen. Ein Wunsch nach Verstärkung einer Ownership auch außerhalb des Wissenschaftsressorts wurde im Rahmen der ExpertInneninterviews ebenfalls geäußert.

Diesen Wünschen nach einer Öffnung der Diskussionskultur könnte durch das Einräumen von kommunikativen Freiräumen zum bidirektionalen Austausch als Ergänzung zum vorherrschenden Präsentations-/ Diskussionsmodus entsprochen werden. Dazu würde sich der Einsatz von partizipativen Methoden wie Open Space, Story Telling, Pro Action Café, 
etc. eignen. ${ }^{68}$ Eine weitere Möglichkeit wäre eine Verstärkung der Teilhabe einzelner Akteursgruppen im Agenda Setting und/oder Moderation und/oder GastgeberInnenschaft.

\subsubsection{ERA Portal Austria}

Das ERA Portal Austria stellt eine Informations- und Kommunikationsplattform zum Europäischen Forschungsraum, zu gemeinsamen EU/AT Aktivitäten (z.B. zu den Joint Programming Initiativen der EU, Forschungspartnerschaften u.ä.) und zum österreichischen Forschungssystem dar. Die Zielgruppen und damit UserInnen des Portals sind klar definiert:

- EntscheidungsträgerInnen aus Politik und Verwaltung,

- Personen, die in der Beratung arbeiten, z.B. in FTI-Agenturen, insb. NCPs oder im EEN,

- Strategische Akteure wie z.B. Wissenschaftsmanager, Rektorate, Universitätsservices,

- Politische Verantwortliche aus dem Ausland (v.a. am ERA Beteiligte), der EU-Kommission und ihren Dienststellen etc.

Das ERA-Portal wurde im Jahr 2016 einer umfassenden Evaluierung unterzogen ${ }^{69}$. Die Evaluierung des ERA-Portals charakterisiert das Portal als eine Drehscheibe für den Informationsaustausch zu allen Themen, die den Europäischen Forschungsraum betreffen, und als ein demokratisches Werkzeug der FTI-Politik, das ein breites Feld an FTI-AkteurInnen einbindet. Als Hauptusergruppen identifiziert die Evaluierung Förderagenturen, EU Policy-Maker, VertreterInnen aus Wirtschaft \& Wissenschaft, und EntscheidungsträgerInnen in Ministerien (national und international).

Das bestehende ERA Portal Austria wird in der Evaluierung von seinen UserInnen als gut bekannt, informativ und nützlich beschrieben. Besonders hervorgehoben wird die gute redaktionelle Arbeit und das Sicherstellen der Qualität der Informationen durch ein engagiertes und gut vernetztes BMBWF-Team mit definierten Regeln.

Die Evaluierung des ERA-Portals zeigt auch eine hohe Resonanz des ERA-Portal Austrias auf europäischer Ebene. Das ERA Portal Austria erfüllt für die UserInnen oft auch die Funktion eines nützlichen Kompendiums (bzw. zum Teil eines Archivs), dem als Quelle von ERA-bezogener Information vertraut wird. Sehr positiv wird gesehen, dass wichtige Dokumente direkt zum Download auf dem Portal verfügbar sind (keine veralteten Links).

Zudem stellt die Evaluierung des ERA-Portal Austrias fest, dass auch der Community Bereich um selektierte AkteurInnen zum Wissensaustausch zu verbinden, geschätzt und gut angenommen wird.

\footnotetext{
${ }^{68}$ Es gibt zahlreiche Methoden und Formate, um Debatten in Gruppen stärker partizipativ und bidirektional zu gestalten. Open Space (TeilnehmerInnen bringen eigene Vorschläge ein, die im Sinne einer Agenda durch eine eigene Methode gereiht werden), Story Telling (besonders erfolgreiche Erfahrungen werden durch eine spezifische Methode in Klein- und Großgruppen geteilt und untersucht), oder Pro Action Cafe (in Klein- und Großgruppen werden durch ein Methodenset Themen erarbeitet und Lösungsvorschläge gemacht) sind dafür nur ausgewählte Beispiele.
}

69 Eutema (2016), Evaluierung des ERA Portal Austria, Wien. 
Die in dieser Evaluierung durchgeführte Online-Befragung bestätigt, dass das ERA Portal Austria eine wichtige Informationsquelle über aktuelle Entwicklung in der nationalen sowie der EU Forschungs- und Innovationspolitik ist.

\section{Abbildung 42: Die Bedeutung des ERA Portal Austrias}

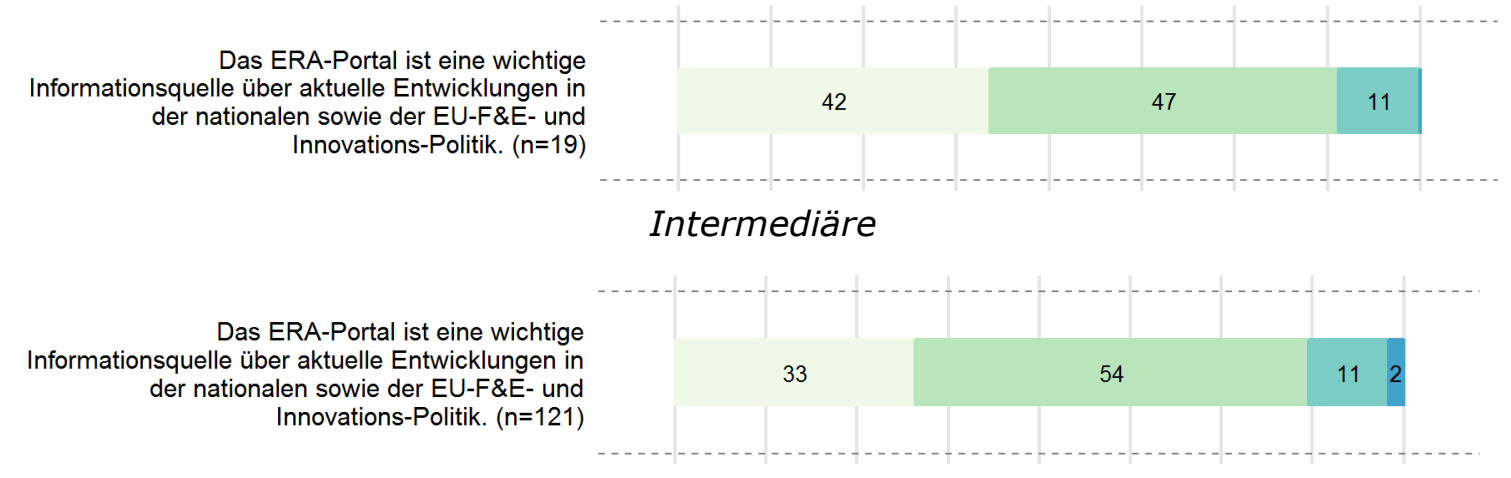

ForscherInnen
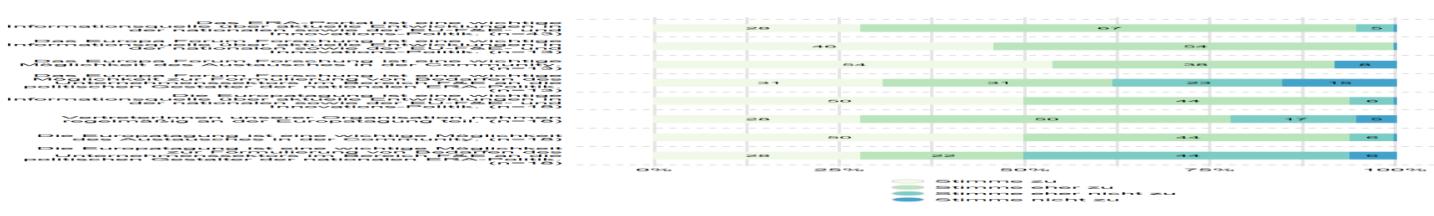

Unternehmen

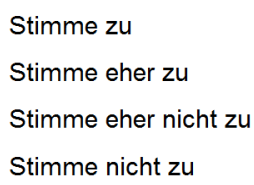

Quelle: Online Befragung, Joanneum Research.

Darüber hinausgehend bestätigen Gespräche mit den FTI-AkteurInnen im Rahmen dieser Evaluierung die Relevanz des ERA Portal Austrias als strategische Informationsquelle, das auch über email-alerts/news über Entwicklungen im europäischen Forschungsraum berichtet. Es wird als umfangreich und aktuell wahrgenommen. Allerdings sollte die Übersichtlichkeit verbessert werden - vor allem mit dem Zweck einer besseren Auffindbarkeit der Inhalte. In diesem Zusammenhang sollte auch eine Überarbeitung der Suchfunktion durchgeführt werden, um die Resultate von Suchanfragen zu verbessern.

\subsection{Bedarfsgerechte Beratung und Betreuung für H2O20 und multilaterale Initiativen}

Zielsetzung der EU-Koordinationsabteilung für die Unterstützung in H2O20 und multilaterale Initiativen ist die strategische und operative Beratung für Forschungscommunity und FTI-Policy Akteure bereitzustellen. Während die FFG-EIP Beauftragung (siehe Kapitel 2 und 3) die Unterstützungsleistungen für forschungsaktive Akteure analysiert, werden in diesem Abschnitt die übergeordneten Unterstützungsstrukturen für 1) österreichische 
Programmdelegierte und EntscheidungsträgerInnen in H2020 sowie 2) die spezifische Unterstützung für multilaterale Initiativen analysiert.

Wesentliche Unterstützungsinstrumente für die österreichischen Programmdelegierten sind: 1) die Zusammenarbeit von FFG-EIP ExpertInnen als NCPs und den nationalen Programmdelegierten, für die jeweils Leitlinien des BMBWF in Hinblick auf ihre Rolle und Aufgaben existieren ${ }^{70}, 2$ ) die thematischen ExpertInnengruppen für die nationalen Delegierten und 3) die Delegierten Roundtables.

\subsubsection{Die Unterstützung für Programmdelegierte}

Der zentrale Ort der Mitgestaltung von H2020 durch die VertreterInnen der Mitgliedstaaten ist, wie auch in früheren Rahmenprogrammen, der Programmausschuss, wobei die wichtigsten Gegenstände der Mitwirkung durch die Mitgliedstaaten in den Rahmenprogrammen die Arbeitsprogramme, Verfahrensfragen und Zeitpläne sind ${ }^{71}$.

Die nationalen Programmdelegierten sind grundsätzlich eigenverantwortlich und es handelt sich bei den Delegierten um Abgesandte der sektoralen Ressorts. Die Auswahl vor dem Hintergrund von Sprachfähigkeit, der Fähigkeit zum Netzwerken, persönlichem Interesse, Wissen um Förderungsmöglichkeiten auf der nationalen Ebene, Flugangst und anderen persönlichen Eigenschaften liegt somit im Aufgabenbereich des jeweiligen sektoralen Resorts.

Dies bedeutet auch, dass der koordinierenden Funktion, die dem BMBWF obliegt, Grenzen gesetzt sind. Seitens des BMBWF wurde versucht, eine Struktur zu bilden, die die eigenverantwortlichen Programmdelegierten über relevante Information und Austauschmöglichkeiten unterstützt und es erlaubt, eine eigene, funktionierende Umgebung aufzubauen. Zu beachten ist dabei auch, dass die Möglichkeiten der Beteiligung und Gestaltungsmöglichkeiten in den relevanten Programmausschüssen beträchtliche Unterschiede zwischen einzelnen Teilbereichen von H2020 zeigen. Wesentliche Herausforderungen für nationale Delegierte sind 1) breitere Agenden der europäischen FTI-Politik, 2) unklar wahrgenommene Zuständigkeiten innerhalb der Europäischen Kommission, sowie 3) die verstärkte Einbindung von sektoralen Generaldirektionen ${ }^{72}$ (z.B. Energy, Connect, Environment, Growth).

\subsubsection{Thematische ExpertInnengruppen und Kooperation mit NCPs}

Um ein optimales Arbeiten in den Programmausschüssen zu ermöglichen und österreichische Interessen zu vertreten, sind eine Reihe von Koordinationsmechanismen notwendig. Neben der persönlichen Kenntnis und der Erfahrung der nationalen Delegierten werden Abstimmungen zwischen Mitgliedsstaaten vorgenommen.

\footnotetext{
70 BMWFW 2013

71 Siehe Pernicka et al 2002 und Technopolis 2016

72 Ibid
} 
In mehreren Themengebieten arbeiten österreichische Delegierte mit Delegierten anderer Länder zusammen und bilden Allianzen. Gute Akteurskenntnisse und Netzwerke zu den wesentlichen nationalen Akteuren in der Wissenschaft als auch in der Wirtschaft, wie auch die Existenz einer nationalen Agenda beeinflussen darüber hinaus die potenziellen Gestaltungsmöglichkeiten der Delegierten ${ }^{73}$.

Die zur Unterstützung der Delegierten gedachten nationalen thematischen ExpertInnengruppen werden sehr unterschiedlich verwendet. Während von manchen Delegierten derartige Gruppen aktiv und regelmäßig verwendet und betreut werden (ähnlich dem in den Niederlanden lange bewährte Sounding Board Modell für EU FTI-Governance ${ }^{74}$ ), werden in anderen Fällen derartige Kontakte unregelmäßig und in informellen Netzwerken bedient.

Als wesentlicher Grund für die Nutzung von ExpertInnengruppen wurde der Austausch mit ExpertInnen angegeben, also auch die Möglichkeit für ExpertInnen, sich zu Themen der EU FTI-Politik zu äußern. Erklärungen für die Nichtnutzung von ExpertInnengruppen waren einerseits "nicht notwendig" bzw. (als Außenzuschreibung) die Organisation der jeweiligen Communities selber, die thematisch geeint und besser strukturiert (oder auch nicht) sein können.

Unklar ist dabei nicht nur, welches Modell in welchem Setting erfolgreicher ist, sondern auch, was Erfolgskriterien für eine thematische ExpertInnengruppe sein könnten. Denkbar wäre etwa eine Tiefenwirkung in die Community, im Sinne des besseren Erkennens von Themen, die nachgefragt werden und so für österreichische Positionen in Brüssel relevant sein würden. Eine Klärung der Zielsetzungen der ExpertInnengruppen über die existierenden schriftlichen Empfehlungen ${ }^{75}$ hinaus erscheint vor diesem Hintergrund sinnvoll.

In Bezug auf die Arbeit zwischen Delegierten und dem National Contact Point FFG ist es mittlerweile grundsätzlich Usus, dass zu Meetings der Programmkomitees die/der Delegierte und eine ExpertIn/NCP fahren. In manchen Fällen wird die Delegierte von ExpertInnen aus anderen Ministerien (Beispiel Sicherheitsforschung, wo neben dem Programmdelegierten häufig auch ExpertInnen aus dem BMI mitfahren) begleitet. Die Arbeitsbeziehungen zu den FFG-NCPs werden von den Delegierten meist als gut bis sehr gut beschrieben.

\subsubsection{Der Delegierten-Roundtable}

Als wesentlicher Unterstützungs- und Koordinationsmechanismus des BMBWF wird der Delegierten Roundtable durchgeführt. Im Rahmen des Delegierten-Roundtable treffen sich durchschnittlich einmal im Quartal die Delegierten zu den H2020 Programmkomitees und tauschen Informationen über ihre Arbeit aus. Der Roundtable findet im Vorfeld des

\footnotetext{
73 Technopolis 2016

74 vgl. Biegelbauer 2004

75 BMWFW 2013
} 
sogenannten Strategischen Programmausschusses von H2020, welche die Delegiertengruppen koordiniert, statt. Bei den TeilnehmerInnen des Roundtable handelt es sich um einen überschaubaren Kreis ohne Beteiligung der FFG-NCPs und anderer ExpertInnen.

Der Delegierten-Roundtable wird als wichtige Informationsleistung durch die EU-Koordinationsabteilung angesehen und wurde von den InterviewpartnerInnen positiv hervorgehoben. Besonders von den Nicht-Forschungsministerien wurde der Austausch mit KollegInnen aus anderen Ministerien sowie der Überblick über die Aktivitäten der Europäischen Kommission und der Initiativen im europäischen Forschungsraum betont.

Zusätzlich zum Delegierten Roundtable gibt es einmal im Jahr ein persönliches Gespräch mit dem Abteilungsleiter der EU-Koordinationsabteilung, bei dem unterschiedliche Themen zu Entwicklungen im Rahmenprogramm und ERA behandelt werden. Aus diesen Gesprächen ist auch schon eine Studie ${ }^{76}$ entstanden. Zudem wird der Arbeitseinsatz für den Politikbereich in diesem Zusammenhang reflektiert und in schriftlicher Art und Weise festgehalten.

Mit dem Delegierten-Roundtable und den persönlichen Gesprächen zwischen der EU-Koordinationsabteilung und den nationalen Delegierten ist es offensichtlich gelungen, ein Instrument zu etablieren, mit dem die Informationsasymmetrie gegenüber der Europäischen Kommission zumindest begrenzt werden kann. Der Austausch über die Strategien auf österreichischer und europäischer Ebene wird dabei als wertvoll empfunden.

\subsubsection{Die Bedeutung von NCPs und nationalen Delegierten für die FTI- Akteure}

Die Arbeit der nationalen Kontaktstelle und der nationalen Delegierten ist für die FTI-Akteure von Bedeutung. Die nationalen FTI-Akteure haben vor allem Interesse an:

- einer frühzeitigen, antizipierenden Informationsvermittlung bzgl. Entwicklungen im Rahmenprogramm und anderen Initiativen,

- einer hohen Komplementarität zwischen nationalen und europäischen Initiativen im FTI-Bereich.

Im Rahmen der Online Befragung wurde diesbezüglich erhoben, inwiefern die Forschungsakteure eine Komplementarität zwischen den europäischen und den nationalen Programmen gegeben sehen, und ob die Schwerpunktsetzung der europäischen FTI-Fördermöglichkeiten den Bedarfslagen der FTI-Akteure entspricht.

Aus Sicht der nationalen Forschungsakteure im Bereich Wissenschaft werden in der durchgeführten Online-Befragung die Komplementarität der thematischen F\&E Fördermöglichkeiten in Österreich von 36\% der befragten Akteure und im Bereich Wirtschaft von $38 \%$ der Akteure zumindest "gut" bewertet.

Die Schwerpunktsetzungen der europäischen FTI-Möglichkeiten werden im Wissenschaftsbereich von $28 \%$ der befragten Akteure und im Wirtschaftsbereich von lediglich $34 \%$ der Akteure als "gut" bewertet.

\footnotetext{
76 Technopolis 2016
} 
In Hinblick auf die Arbeit der NCPs und der nationalen Delegierten fühlen sich $56 \%$ der befragten Wissenschaftsakteure und $75 \%$ der Wirtschaftsakteure zumindest gut bzgl. der Aufnahme von Anregungen durch die NCPs und Delegierten der Ministerien vertreten.

Die befragten Wirtschaftsakteure konstatieren wesentlichen Veränderungsbedarf der Programme in Hinblick auf den Abbau administrativer Hürden (65\%), die Förderung von Innovation (53\%) sowie die thematische Orientierung (39\%). Auch im Wissenschaftsbereich sehen $63 \%$ der befragten Akteure Verbesserungspotenziale im Bereich des Abbaus administrativer Hürden und der thematischen Schwerpunktsetzung (60\%).

\section{Lenkungsausschuss (EIP, EU-PM)}

Als wesentliches Steuerungselement der Aktivitäten von FFG-EIP wurde vom BMBWF ein Lenkungsausschuss für FFG-EIP und EU-PM einberufen. Der Lenkungsausschuss zu FFGEIP bzw. EU-PM dient der Abstimmung der einzelnen den Bereich finanzierenden Organisationen, BMBWF, BMVIT, BMDW, BMNT, BMASGK und WKO (BMASGK und WKO nicht bei EU-PM). Der Lenkungsausschuss des EIP-FFG wurde in mehreren ExpertInneninterviews positiv bewertet. Einige GesprächspartnerInnen führen ihn im Hinblick auf die gute Zusammenarbeit als Good Practice im EU-FTI-Governance Bereich an.

\subsubsection{Unterstützung für transnationale FTI-Partnerschaften}

Transnationale FTI-Partnerschaften zwischen Ländern des europäischen Forschungsraums und internationale Kooperationen nehmen sowohl in $\mathrm{H} 2020$ als auch darüber hinausgehend einen immer größeren Stellenwert ein. In H2020 sind unter Berücksichtigung von Public-Public-Partnerships (JPIs, ERA-NETs, Art. 185) und Public-Private Partnerships (JTIs, cPPPs) bereits $25 \%$ der verfügbaren Mittel in transnationalen FTI-Partnerschaften gebunden.

Während im Zuge der Ausschreibungen innerhalb von H2020 in erster Linie Themen von gesamteuropäischem Interesse adressiert werden sollen, tragen die diversen Formen biund multilateraler Partnerschaften - zumeist bottom-up - durch die jeweiligen Akteure getriebenen Themenfindungs- und Implementierungsprozesse zur Vergemeinschaftung von F\&E-Aktivitäten in Europa bei ${ }^{77}$.

Ziel ist es, über die Bündelung nationaler Mittel im Bereich der F\&E- und Innovationsförderung sowie über die Vernetzung entsprechender Aktivitäten im öffentlichen wie privaten Bereich zur Bildung kritischer und international wettbewerbsfähiger Größen in ausgewählten Schwerpunkten beizutragen ${ }^{78}$. Dem österreichischen Forschungs- und Technologiebericht folgend, ist Österreich in 64 von 108 aktiven Public-Public-Partnership Initiativen engagiert, was einem Anteil von 59\% entspricht. Ein Großteil dieser Beteiligungen entfällt auf Aktivitäten des ERA-Net-Schemas, zudem ist Österreich in 8 von 10 JPIs aktiv.

77 BMWFW, BMVIT. 2017. Österreichischer Forschungs- und Technologiebericht 2017

78 Ebenda 
Die Herausforderungen in Bezug auf die österreichische Beteiligung in den transnationalen FTI-Partnerschaften können wie folgt skizziert werden:

- Auf ministerieller Ebene wurde in mehreren Gesprächen eine grundsätzliche Notwendigkeit der Abstimmung der verschiedenen Ministerien und der FFG in diesem Bereich festgestellt.

- Die FFG steht vor der Herausforderung Unterstützung für die Vielzahl an Instrumenten zu geben, die manchmal lediglich über eine geringe Förderdotierung verfügen. Sie zeichnet auch für die Administration von Antragsverfahren verantwortlich.

- Den österreichischen Forschungs- und Innovationsakteuren sind die Beteiligungsstrategien Österreichs an transnationalen FTI-Partnerschaften oftmals unklar. Zudem wird in einigen Gesprächen vermutet, dass österreichische FTI-Akteure über organisatorische Grenzen hinweg gemeinsame Beteiligungen an Initiativen wie z.B. den KICs des EIT anstreben müssten.

- Für die Zukunft werden von mehreren GesprächspartnerInnen größere Veränderungen auf der europäischen Ebene erwartet. In besonderer Weise bezieht sich das auf die erwartete Missionsorientierung des neuen Rahmenprogramms mit seinen größeren Instrumenten und seiner Stärkung größerer Strukturen.

Vor diesem Hintergrund haben sowohl die FFG als auch die verantwortlichen Ministerien Initiativen gesetzt, um eine bessere Abstimmung zu ermöglichen und die Komplexität für die österreichischen FTI-Akteure zu reduzieren.

\section{Maßnahmen der FFG zur Unterstützung in multilateralen Initiativen}

Mit Beginn von H2020 wurde innerhalb der FFG-EIP die Auseinandersetzung mit den sogenannten multilateralen Initiativen verstärkt. Innerhalb der FFG-EIP wurde ein

MULLAT-Think-Tank eingerichtet, um:

- einen kontinuierlich aktuellen Überblick über die Mittel zu haben, die von H2020 in die transnationalen FTI-Partnerschaften fließen,

- die Zusammenschau über unterschiedliche Instrumente, Fördermechanismen, Förderhöhen etc. zu gewährleisten,

- das Wissen zu diesen Initiativen im EIP zu bündeln,

- die Ressorts in Hinblick auf Entscheidungsprozesse zu Beteiligungen zu unterstützen und

- einen Überblick über die VertreterInnen Österreichs in den diversen Gremien zu erhalten.

Eine der Herausforderungen in Bezug auf die MULLATs besteht im Fehlen eines umfassenden Überblicks über laufende oder in Vorbereitung befindliche Initiativen, die in unterschiedlichen Ressorts verankert und an verschiedene nationale Programme angekoppelt sein können. In der Betreuung der multilateralen Initiativen bedarf es daher der bereichsübergreifenden Zusammenarbeit mit den verantwortlichen Fachministerien, der FFG intern sowie der Kooperation mit dem EU-PM.

Die Ergebnisse aus der Beschäftigung mit den transnationalen FTI-Partnerschaften innerhalb von FFG-EIP wurden zur Unterstützung der ressortübergreifenden Arbeitsgruppe 
MULLAT verwendet, in EU-Networking Meetings eingebracht und haben im Rahmen eines Meet \& Exchange Workshops im Rahmen der NCP-Academy zu einer Diskussion mit der zuständigen Daten-Unit in der EU-Kommission ${ }^{79}$ geführt, in die sich wiederum auch das EU-PM eingebracht hat. Weitere Maßnahmen der FFG sind z.B. EU-Networking Events, Delegierten Austauschrunden sowie die Mitwirkung an der JPI Gruppe Österreich und der EIT Begleitgruppe.

Der Aufbau einer MULLAT-Governance Datenbank, die die Vertretung österreichischer Akteure in allen MULLAT-Gremien abbildet, wird inzwischen als wichtige Diskussionsgrundlage im Rahmen von ERA-Dialogen, C3-Gesprächen sowie Workshops und Strategiegesprächen mit FTI-Organisationen herangezogen, um Beteiligungspotenziale und Handlungsmöglichkeiten zu verorten.

Obwohl die strategische Bedeutung von MULLATs von einer wachsenden Anzahl an FTIAkteuren anerkannt wird, bleibt die systematische Befassung mit den MULLATs die Ausnahme, und damit auch die Nutzung der inzwischen in FFG-EIP aufgebauten Expertise. An einer Universität waren die Auswertungen Ausgangspunkt für einen „MULLAT-Workshop" gemeinsam mit ihren VertreterInnen in Gremien. Damit wurde von der FFG ein Erfahrungsaustausch zum strategischen Nutzen der transnationalen FTI-Partnerschaften ermöglicht.

Funktional deckt der FFG-EIP MULLAT Support \& Service bislang drei wesentliche Bereiche ab:

Erstens versucht die FFG im Rahmen des Supports für FTI-Akteure mit den PartnerInnen der Vernetzungsplattformen ${ }^{80}$ Kontakt zu halten, Strategiegespräche mit ausgewählten Akteuren im österreichischen FTI-System zu führen sowie Informationen zu transnationalen FTI-Partnerschaften im Rahmen der C3 Gespräche und des ERA-Dialogs an die Organisationsleitung zu vermitteln. Dabei hat sich gezeigt, dass eine strategische Orientierung und Herangehensweise in Hinblick auf die transnationalen FTI-Partnerschaften kaum gegeben ist. Screenings von Forschungsschwerpunkten und Beteiligungsstrategien der Organisation sind nicht vorhanden. Um eine erfolgreiche Beteiligung österreichischer Wissenschaftsakteure zu forcieren, wäre eine enge Kooperation der Organisationsleitung mit relevanten Departments und Kooperationen zwischen Akteuren/Organisationen notwendig.

Zweitens bemüht sich die FFG im Hinblick auf neue transnationale FTI-Partnerschaften (wie z.B. FET-Flagship, 5G-PPP81, etc.) frühzeitig auf neue Instrumente aufmerksam zu machen und die Community zu vernetzen. Darüber hinaus hat die FFG einzelne Akteure in der Vorbereitung auf die Beteiligung an den Knowledge und Innovation Communities (KICs) des European Institute of Technology in rechtlicher Hinsicht, und in Hinblick auf die Bedeutung spezifischer Instrumente der KICs beraten.

\footnotetext{
79 DG Research \& Innovation, Unit J5 "H2020 - Information \& Data"

${ }^{80}$ Siehe das Infoblatt der Vernetzungsplattformen für eine Kurzinformation zu den Zielen der Vernetzungsplattformen

${ }^{81} 5$ th Generation communication networks and services
} 
Drittens runden spezifische Veranstaltungen im Rahmen der FFG-Akademie, EU Networking-Veranstaltungen und thematische Events mit ausgewählten Akteuren den Themenbereich MULLAT Support \& Service ab.

Und schließlich unterstützt FFG-EIP im Rahmen des Governance Support insbesondere die FTI-Ressorts (BMBWF, BMDW und BMVIT) in Hinblick auf Auswahl und strategische Beteiligungsmöglichkeiten. Als ExpertInnenorganisation übernimmt die FFG teilweise strategische Vertretungsfunktionen und hat Österreich in der Mutual Learning Exercise zu Alignment ${ }^{82}$ unterstützt. Konkret übernimmt die FFG folgende Arbeiten:

- Teilnahme in der JPI Gruppe Österreich: Diese stellt einen nationalen Austauschkreis in Bezug auf geplante und durchgeführte Aktivitäten zwischen VertreterInnen der JPIs dar.

- Interministerielle AG MULLAT: Zielsetzung war eine bessere Koordination und Prioritätensetzung in Hinblick auf die Beteiligungsstrategie an transnationalen FTIPartnerschaften zu ermöglichen. Dabei hat die FFG versucht, für die Ministerien eine Relevanzeinschätzung von einzelnen transnationalen FTI-Partnerschaften zu erleichtern und ein Self-Assessment Tool entwickelt. Eine stärkere Abstimmung zur Beteiligung an transnationalen FTI-Partnerschaften wurde von einem Ministerium abgelehnt. Die AG MULLAT ist daher derzeit ruhend.

- Mutual Learning Exercise (MLE) on Alignment: Die FFG nahm für Österreich an der MLE on Alignment and Interoperability of Research Programmes teil. Im Ergebnis konnte durch diesen Erfahrungsaustausch zwischen Mitgliedsstaaten eine Vielzahl von Verbesserungsmaßnahmen identifiziert werden, die es nun auf nationaler Ebene weiter zu verfolgen gilt. Darüber hinausgehend nahm die FFG an den Sitzungen der FTI-AG7b teil (siehe dazu weiter unten) und unterstützt die Ministerien in den Arbeitsgruppen von ERAC und GPC.

\section{Der Aufbau von strategischen Unterstützungsleistungen seitens der FFG für die}

Ministerien wird dabei von den GesprächspartnerInnen unterschiedlich, aber tendenziell positiv bewertet. Während diese Dienstleistung von einigen als notwendig im Hinblick auf die Gesamtleistungsentwicklung der FFG und die wachsende Komplexität der EU FTI-Politik bezeichnet wird, sehen andere GesprächspartnerInnen diese Funktion als nicht vorrangig an.

Der dahinter liegende Grund scheint darin zu liegen, dass das Thema der Unterscheidung zwischen Strategiebildung in den Ministerien und operationaler Umsetzung durch die FFG nach Ansicht einiger InterviewpartnerInnen auch 14 Jahre nach der Gründung der FFG noch immer umstritten ist. ${ }^{83}$ Mehrmals wurde angesprochen, dass die FFG keine weiteren Strategiefunktionen übernehmen sollte.

Dies steht im Gegensatz zur ebenfalls vorgefundenen Einschätzung, dass eine derartige Weiterentwicklung seitens der FFG notwendig wäre. Dies wurde einerseits im Hinblick auf

\footnotetext{
82 https://rio.jrc.ec.europa.eu/en/policy-support-facility/mle-alignment-and-interoperability-research-programmes-national-coordination

83 Vergleiche auch Pichler et al 2007, Biegelbauer 2013 und auch die Ergebnisse der FFG/aws-Evaluation
} 
die Unterstützung der Bundesministerien, andererseits aber auch auf die Handlungsfähigkeit im Hinblick auf die Vertretung Österreichs in europäischen Gremien angemerkt. Nach dieser Argumentationslinie findet die Vertretung Österreichs insgesamt in Hinsicht auf eine Lancierung von Themen und Durchsetzung von Interessen zu wenig proaktiv statt und kann auch durch die FFG - unter anderem aus Gründen limitierten strategischen Wissens - zu wenig wahrgenommen werden.

\section{Zufriedenheit mit den Betreuungsstrukturen für multilaterale Beziehungen}

Wie untenstehende Abbildungen aus der Online Befragung zeigen, ist die Zufriedenheit mit den Beratungsleistungen der FFG auch in Hinblick auf die transnationalen FTI-Partnerschaften gegeben. Unklarheiten existieren vor allem in Hinblick auf die Zuständigkeiten anderer nationaler Agenturen und einer Weitervermittlung zu diesen.

\section{Abbildung 43: Zufriedenheit mit MULLAT Beratungsleistungen (Wissenschaft)}

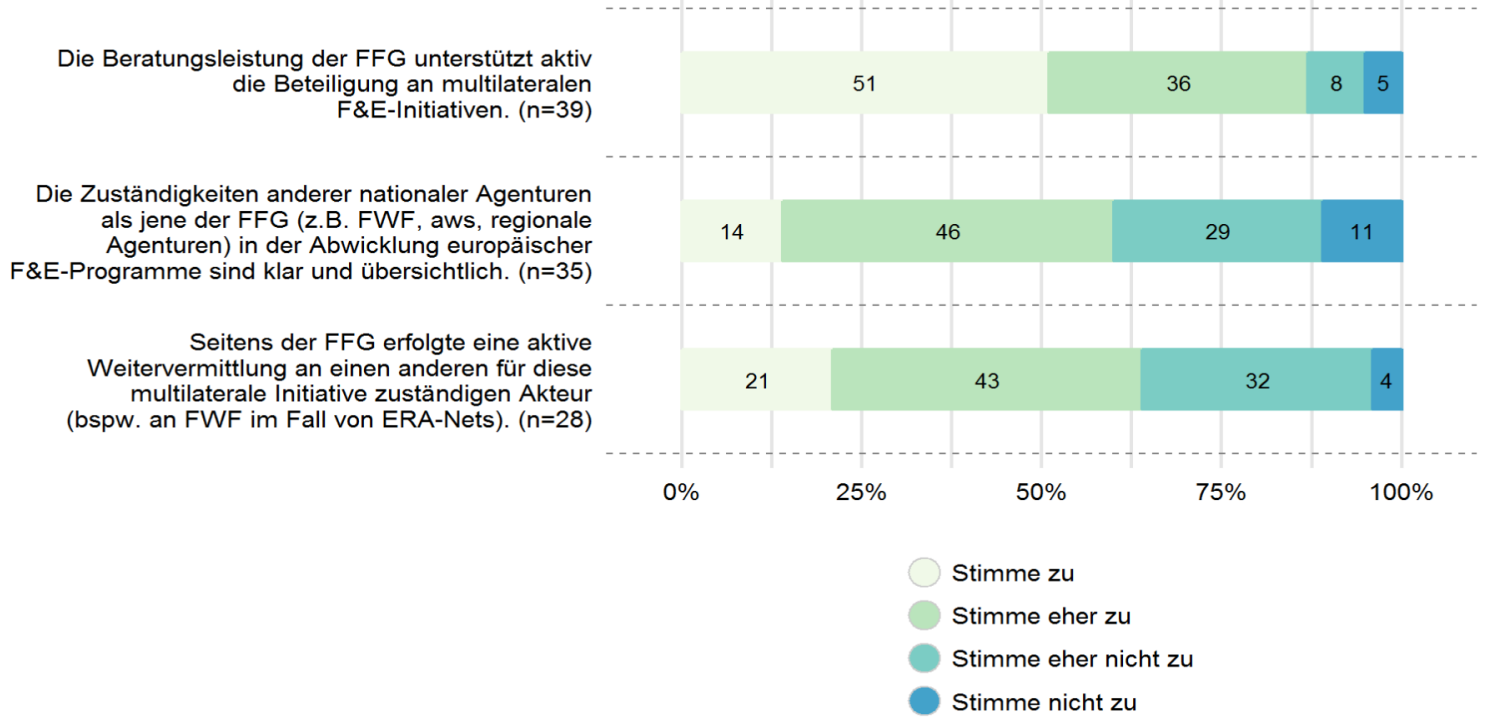

Quelle: Online Befragung, Joanneum Research. 


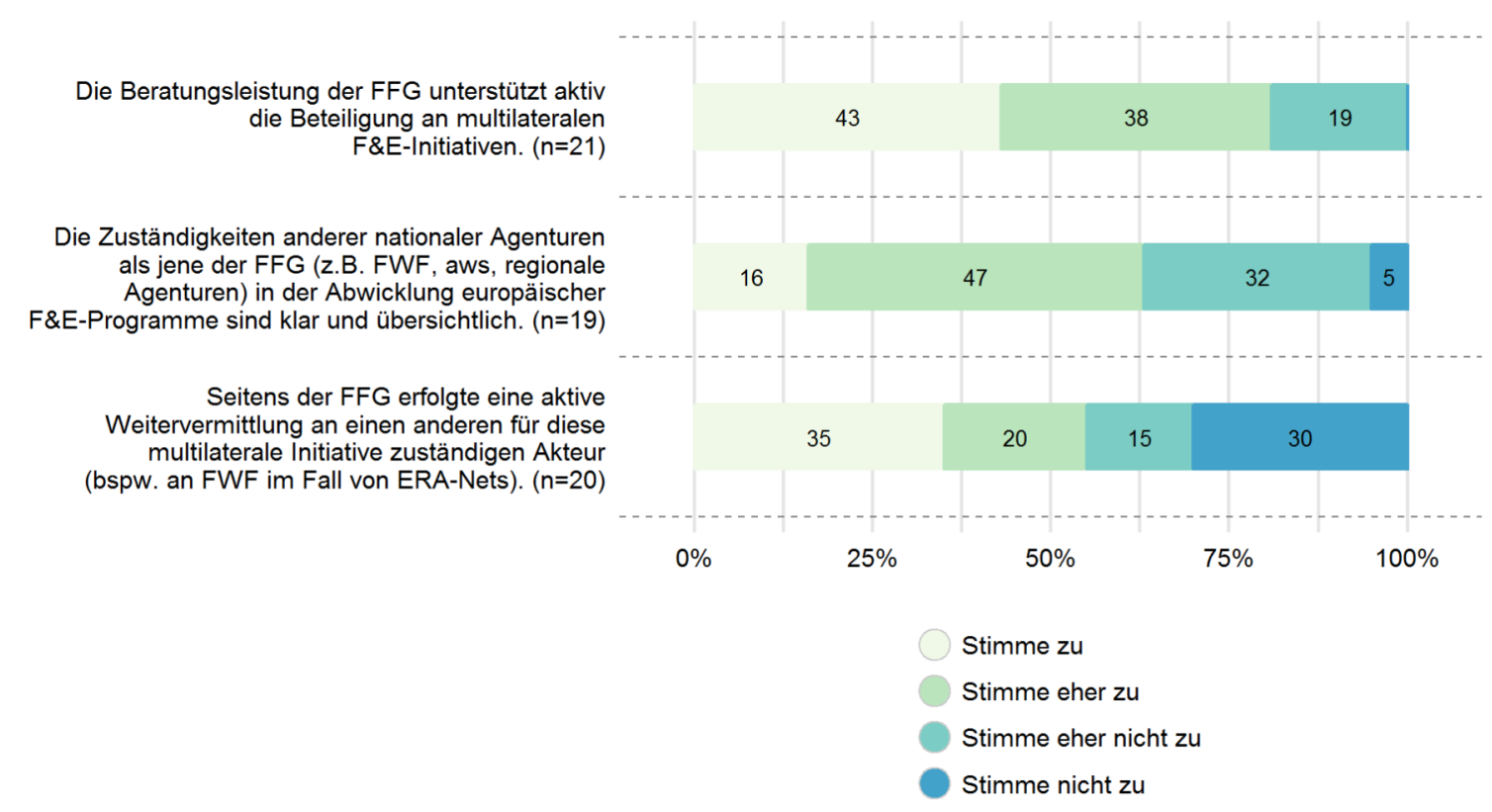

Quelle: Online Befragung, Joanneum Research.

Kritisch wird auch die Angemessenheit der verfügbaren nationalen Mittel für MULLAT gesehen. 24\% der erreichten Wissenschaftsakteure und 14\% der Wirtschaftsakteure bewerten diese nach Schulnoten mit "Nicht Genügend". Weitere $14 \%$ in beiden Gruppen lediglich mit "Genügend". 


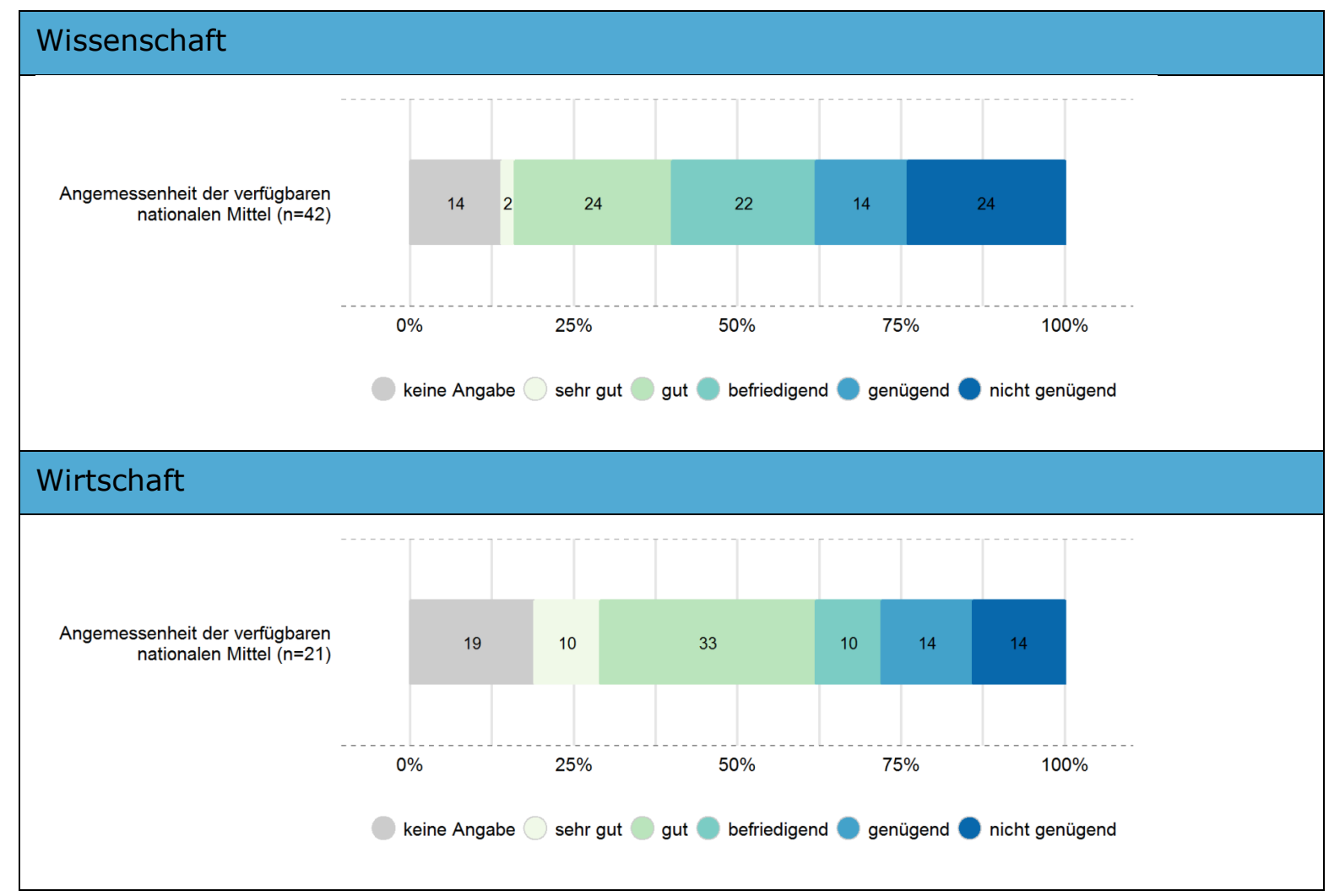

Quelle: Online Befragung, Joanneum Research.

Die Kooperation mit der FFG spielt im EU-FTI-Governance Bereich grundsätzlich eine große Rolle, wobei die Informationsangebote (siehe Kapitel 3) sehr unterschiedlich bewertet wurden. Das Verhältnis zwischen Delegierten und FFG-NCPs wurde in den meisten, wenn auch nicht in allen Fällen, als positiv und vertrauensvoll charakterisiert.

In Hinblick auf die Beteiligung an transnationalen FTI-Partnerschaften versucht FFG-EIP relativ erfolgreich, Komplexität für FTI-Akteure im Gesamtsystem zu reduzieren und auch die Akteure der FTI-Governance zu unterstützen. Die FFG nimmt in diesem Bereich vor allem eine unterstützende Rolle ein, ihr tatsächlicher Handlungsspielraum scheint vor allem im Bereich des operativen Alignments gegeben zu sein.

Die zu erwartende Missionsorientierung des FP9 und die größere Bedeutung von transnationalen FTI-Partnerschaften wird als eine Herausforderung für die Governance des Politikbereichs wahrgenommen. Auch vor diesem Hintergrund wurde das Thema der Koordination der ministeriellen Akteure in der nationalen EU-FTI-Governance in vielen Gesprächen indirekt, in einigen direkt angesprochen. Sowohl Interviews mit verschiedenen Akteursgruppen im FTI-Bereich, als auch Indizien der Online Befragung weisen darauf hin, dass vor dem Hintergrund einer steigenden Bedeutung transnationaler FTI-Partnerschaften im Gesamtsystem, Bedarf an einer österreichweit abgestimmten Beteiligungsstrategie besteht. 


\subsubsection{FTI-Verbindungsbüro in Brüssel}

Die Frage, inwiefern ein österreichisches EU-Verbindungsbüro (Arbeitstitel ARTIH) in Brüssel österreichische FTI-Akteure stärken kann, wurde bereits im Rahmen einer Machbarkeitsstudie zur Etablierung eines österreichischen EU-Verbindungsbüros ${ }^{84}$ eingehend analysiert und bewertet. Ziel der Machbarkeitsstudie war es, eine Entscheidungsgrundlage für die Einrichtung oder Nicht-Einrichtung eines österreichischen FTI-Verbindungsbüros in Brüssel zu schaffen und für den Fall einer Pro-Empfehlung konkrete Empfehlungen für die Finanzierung und Governance zu entwickeln.

Die Machbarkeitsstudie zum EU-Verbindungsbüro zeigt, dass man durch gute Verbindungen zur europäischen Ebene inhaltlich wie zeitlich Informationsvorsprünge gewinnen kann - und dies unabhängig von der konkreten Organisationsform.

Verbindungsbüros leisten aus der Sicht der Studie einen Beitrag, um neue Entwicklungen frühzeitig zu erkennen und die eigene Strategie zu entwickeln und voranzutreiben.

Dadurch sei es leichter möglich, inhaltliche Beiträge frühzeitig und daher mit größerem Gestaltungsspielraum in den europäischen Politik-Gestaltungsprozess einzubringen oder Personen zu platzieren. Die Studie stellt drei Optionen zur Einrichtung eines Verbindungsbüros vor:

- Option 1: Ein vom Bund betriebener ARTIH,

- Option 2: Ein von FTI-Stakeholdern betriebener ARTIH,

- Option 3: Ein agenturbetriebener ARTIH.

Da die mit dem Rahmenprogramm und ERA-Agenden befassten Akteure des Bundes, der Studien folgend keinen Bedarf an einem Verbindungsbüro sehen und viel mehr im Rahmen bestehender Verbindungen "nach Brüssel", vor allem im Rahmen der Programmausschüsse und im Wege der Ständigen Vertretung arbeiten, ist eine Umsetzung als Option 1 aus Sicht der Studie nicht empfehlenswert.

Option 2 wiederum würde aus Sicht der Studie daran scheitern, dass die Gruppe der möglichen österreichischen Stakeholder (hier im engen Wortsinn) derzeit derart inhomogen sei, dass ein funktionierendes Governance-Modell für ein gemeinsam betriebenes Verbindungsbüro unrealistisch erscheint.

Die Studie empfiehlt daher die Umsetzung eines agenturbetriebenen FTI-Verbindungsbüro für die österreichischen FTI-Akteure, angesiedelt beim Bereich Europäische und internationale Programme, EIP in der FFG, und zwar als integraler Teil genau dieser jeweils befristeten Beauftragung und ihrer bewährten Governance. Dem Verbindungsbüro käme dann die Rolle zu, die Interessen der FTI-Akteure zu erkennen, zu filtern und zu bündeln und anderseits Informationen aus Brüssel kommend zu sichten und zielgerecht zu verteilen.

Die zuletzt intensiv diskutierte Option eines FTI-Verbindungsbüros in Brüssel wurde in Fokusgruppen und Delphi Befragung kontrovers debattiert.

84 Technopolis (2017), Machbarkeitsstudie zur Etablierung eines österreichischen EU Verbindungsbüros im FTI-Bereich in Brüssel, Endbericht, Juli 2017, Wien. 
Die Erhebungen in dieser Evaluierung bestätigen, dass die Befürwortung bzw. Ablehnung eines Verbindungsbüros stark von der konkreten Zielsetzung und Aufgabenstellung einer derartigen Einrichtung und dem erwarteten Nutzen für die Stakeholder abhängt:

- Soll über das Verbindungsbüro vornehmlich die Informationsübermittlung nach Österreich gestärkt werden?

- Soll (ggf. in Kooperation mit anderen Nationalstaaten) auch Interessenvertretungsarbeit geleistet werden?

- Wenn ja, sollen primär gesamtstaatliche Interessen oder auch die Interessen der FTI-Communities vertreten werden?

Die Fokusgruppeninterviews haben vor allem gezeigt, dass die intendierten Ziele und möglichen Aufgaben eines EU Verbindungsbüros den potenziellen NutzerInnengruppen nicht bekannt sind. Darüber hinaus bestätigen die Fokusgruppen, dass durchaus unterschiedliche Varianten eines Verbindungsbüros denkbar sind, die von einem gesamtstaatlichen "österreichischen Verbindungsbüro" bis hin zu folgenden Versionen eines "Verbindungsbüros der FTI-Community" reichen:

- Einzelne interessierte FTI-Akteure treten spezifischen europäischen Netzwerken bei: diese Option ist der status quo.

- Die österreichische Wissenschafts-Community kauft sich in eine bestehende Organisation ein

- Die Wissenschaftsakteure (Universitäten, Forschungsorganisationen, ÖAW, etc.) bauen gemeinsam ein Verbindungsbüro auf.

- Die FFG baut ein Verbindungsbüro als nationaler Knoten in Brüssel (derzeit diskutierte Option).

- Die FFG baut in Kooperation mit einem anderen Land ein organisiertes Verbindungsbüro als nationaler Knoten in Brüssel auf.

Letztere könnte in Form eines Einkaufs in eine bestehende Organisation oder des gemeinsamen Aufbaus einer neuen Organisation entstehen. Unabhängig davon könnte sich eine österreichische Einrichtung Infrastrukturen auch mit anderen Büros teilen - ähnlich dem Vorbild Norwegens und der Schweiz.

Die Unklarheit über Ziele und Aufgaben eines EU-Verbindungsbüros spiegelt sich auch in den Ergebnissen der Delphi-Befragung wider. Für die Aussage, dass die Einrichtung eines österreichischen Verbindungsbüros in Brüssel sinnvoll sei, um bessere und zeitnahe Informationen über das Zusammenwirken nationaler und europäischer FTI-Strategien zu erhalten, findet sich knapp keine absolute Mehrheit. 48\% stimmen dieser Aussage zu oder sehr $z u$, aber relativ viele (31\%) lehnen sie eher oder ganz ab. $21 \%$ sind unentschieden.

Am skeptischsten sind die Unternehmen, aber auch bei den Policy-Drivern findet sich keine befürwortende Mehrheit. Es herrscht Sorge vor Parallelstrukturen, einem mangelnden Informationsfluss nach Österreich und einer bürokratisch-politisierten Besetzungspolitik. Dem gegenüber stehen Ansichten, dass der informelle Austausch in Zukunft zunehmen und wichtiger werden würde, weshalb ein solches Büro sinnvoll sei, u.a. auch um österr. Anliegen „nach Brüssel” transportieren zu können. Gleichzeitig wird angemerkt, 
dass es schon einige österreichische Vertretungen in Brüssel gäbe, aber bereits vorhandenes Potenzial zu wenig genutzt werden würde.

Insgesamt zeigt sich, dass die Meinungen zu dieser Aussage polarisiert sind und jedenfalls kein Konsens in Österreich für ein EU Verbindungsbüro in Brüssel besteht. Vor einer Einrichtung eines EU Verbindungsbüros in Brüssel sind jedenfalls Ziele und Aufgaben eines EU Verbindungsbüros mit den potenziellen NutzerInnengruppen zu klären. Dabei ist auch zu berücksichtigen, inwiefern die FFG eine strategische Rolle in Hinblick auf die Vertretung Österreichs einnehmen soll.

\subsection{Unterstützung für den Strukturwandel im Politikbereich}

Ein weiterer Aspekt der Arbeiten der EU-Koordinationsabteilung betrifft die Unterstützung für den Strukturwandel im Politikbereich zur Realisierung des europäischen Forschungsraumes. Wesentliche Elemente dieser Tätigkeiten umfassen 1) das ERA Council Forum Austria, 2) das ERA Policy Forum, 3) den Roundtable zu ERA und die ERA KorrespondentInnen der Universitäten sowie 4) die Arbeitsgruppen zur Internationalisierung, die im Folgenden analysiert werden.

\subsubsection{ERA Council Forum Austria}

Das ERA Council Forum Austria ist ein hochrangiges ExpertInnengremium, das den österreichischen Minister für Bildung, Wissenschaft und Forschung an der Schnittstelle zwischen der europäischen Forschungspolitik und dem nationalen Wissenschafts-, Forschungs- und Innovationssystem berät. Es bietet strategische Orientierung und Beratung im Kontext von "Europa 2020", "Innovationsunion", "EFR-Partnerschaft" sowie "H2020". Nach eigenen Aussagen stehen dabei folgende vier Wirkungsdimensionen im Vordergrund: i) Aufbereitung von entscheidungsrelevanten Informationen über europäische FTIEntwicklungen, ii) Beratung des für die EU-FTI-Politik verantwortlichen Mitgliedes der österreichischen Bundesregierung, iii) Beauftragung von Analysen und Studien zu ausgewählten Fragestellungen über die EU-FTI-Entwicklungen, iv) Bereitstellung von strategischer Orientierung für das verantwortliche Mitglied der österreichischen Bundesregierung.

Das Forum von fünf Mitgliedern wird von der ehemaligen Präsidentin des ERC, Helga Nowotny, geleitet, und tritt zweimal pro Jahr zusammen. Im Rahmen der jährlichen Europatagung hat das ERA Council Forum eine zentrale Rolle in der Diskussion aktueller Entwicklungen der nationalen FTI-Politik in Bezug auf die Ziele des Europäischen Forschungsraumes.

Das ERA Council Forum Austria wird im Politikfeld unterschiedlich bewertet. Einerseits wird das Forum als effektive Schnittstelle zwischen hochrangigen ExpertInnen im Bereich EU-FTI-Politik und Politik gesehen, das durch seinen unabhängigen Außenblick, die langjährige Erfahrung und das hohe Engagement seiner Mitglieder, ihren Zugang zu komplementären Informationskanälen sowie die Schlüssigkeit und Umsetzbarkeit seiner Empfehlungen eine hohe Glaubwürdigkeit besitzt und sowohl das Gehör als auch das Vertrauen des jeweils zuständigen Ministers gefunden hat. Es diente somit als geschütztes Forum für den offenen und vertrauensvollen Austausch über zentrale FTI-politische Thematiken 
mit hoher europäischer Relevanz. Es gibt auch eine Reihe von Hinweisen für wichtige Anstöße des ERA Council Forums für das Agenda-Setting auf ministerieller Ebene und das Aufgreifen von konkreten Vorschlägen bei der Ausgestaltung von Politikmaßnahmen (z.B. in Bezug auf die Internationalisierung des österreichischen Forschungs- und Innovationssystems, die Wettbewerbsfähigkeit des Universitätssystems oder den aktuell laufenden OECD Review of Innovation Policy Austria). Dies zeigt, dass die ursprüngliche Intention des ERA Council Forums in weiten Teilen erreicht werden konnte.

Andererseits wurde in Interviews, Fokusgruppen und den Ergebnissen des Delphi aber auch immer wieder angegeben, dass zu wenig über Funktion und Arbeit des Forums bekannt ist (Zitate sind: "Welche Ziele werden hier verfolgt?", "Wozu ist das da?") bzw. dass die überlappende Zusammensetzung mit anderen Beratungsorganen kritisch gesehen wird („Immer wieder die gleichen ExpertInnen"). Zwar bietet die jährliche Europatagung eine Plattform für den Dialog der FTI-Community mit dem ERA Council Forum, seine Rolle (und damit sein Nutzen) bleibt aber dennoch für viele unklar.

Wenn ein Ziel der österreichischen EU-FTI-Politik Transparenz ist, so sollte im Hinblick auf die von einer im Regierungsprogramm $2017^{85}$ angekündigten Umstrukturierung der FTI-politischen Räte deren Kommunikation und Sichtbarkeit verbessert werden. Allerdings gilt es dabei zwischen der erforderlichen vertraulichen Rolle von Räten an der Schnittstelle zur Politik einerseits, und der Forderung nach Transparenz und Öffentlichkeit andererseits, abzuwägen. Eine Möglichkeit wäre beispielsweise die Verwendung des Europa Forum Forschung für eine regelmäßige Kurzinformation über die letzten Aktivitäten des ERA Council Forum (bzw. dessen Nachfolger).

\subsubsection{ERA Policy Forum Austria}

Das ERA Policy Forum Austria zur Koordination der verschiedenen Bundesministerien mit FTI-Agenden dient der Abstimmung der einzelnen Ministerien auf BeamtInnenebene. Vor dem Hintergrund einer stärkeren Orientierung H2020 an den großen gesellschaftlichen Herausforderungen, versuchte das ERA Policy Forum, Wechselwirkungen zwischen der FTI-Politik und sektoralen Politiken zu antizipieren und Handlungsbedarf für Strukturwandel in Europa/Österreich zu identifizieren. Es sollte einen Beitrag dazu leisten, die FTI-Politik in den Dienst der sektoralen Politiken zu stellen und sektorale Unterstützung für die FTI-Politik zu suchen. Dies wird, besonders vor dem Hintergrund des neuen Rahmenprogramms und seiner Orientierung in Richtung Missionsorientierung, vom Wissenschaftsressort als wichtig erachtet, da ein Unterstützungs-und Koordinationsbedarf entstehen könnte, der durch keine der existierenden Institutionen (Rat für Forschung und Technologieentwicklung bzw. FTI-Taskforce) abgedeckt wird.

Es gab zwei Anläufe eine derartige Koordination von Seiten des Wissenschaftsresorts zu initiieren. Der erste Anlauf bezog sich auf das Thema "Willkommenskultur” im Jahr 2014. Dabei traten Unklarheiten der Zuständigkeiten im Verhältnis zu Sozialministerium und In-

\footnotetext{
85 Siehe das Regierungsprogramm 2017-2022
} 
nenministerium auf. Beim zweiten Anlauf wurde versucht, eine Aktivität zum Thema "demographischer Wandel" zu lancieren; es erwies sich aber als unmöglich, alle entscheidenden Akteure an einen Tisch zu bekommen.

Das ERA Policy Forum ist inklusive der konstituierenden Sitzung im Jahr 2014 insgesamt drei Mal zusammengetroffen. Seit dem dritten Treffen im Mai 2016 ist das Forum nicht mehr zusammengetreten, da - zumindest zu diesem Zeitpunkt - kein Interesse an einer weiterführenden Koordination in Bezug auf EU FTI-Politik vorhanden war.

Die geführten Interviews zum ERA Policy Forum Austria zeigen, dass das Policy Forum vor allem außerhalb der mit FTI-Agenden betrauten Ministerien auf wenig Resonanz gestoßen ist. Ein wesentlicher Faktor dürfte dabei sein, dass zur Ausarbeitung ressortübergreifender Strategien und Maßnahmen nicht nur eine Abstimmung auf Ebene der BeamtInnen notwendig erscheint, sondern auch ein übergreifender politischer Wille zur Verschränkung von Maßnahmen mehrerer Ressorts. Zudem war einigen GesprächspartnerInnen die Abgrenzung zwischen den einzelnen gleichzeitig aktiven Koordinationsformaten unklar (z.B. Namensähnlichkeit zwischen ERA Council Forum und ERA Policy Forum).

\subsubsection{Roundtable zu ERA und ERA-KorrespondentInnen der Universitäten}

Im Rahmen des ERA-Roundtable werden Entwicklungen im europäischen Forschungsraum diskutiert sowie Beratungen zu nationalen Maßnahmen im Kontext der Realisierung des Europäischen Forschungsraums (EFR) in den sechs Prioritäten vorgenommen:

- Effektivere nationale Forschungssysteme (EFR-Priorität 1),

- Länderübergreifende Zusammenarbeit (EFR-Priorität 2),

- Ein offener Arbeitsmarkt für Forscherinnen und Forscher (EFR-Priorität 3),

- Gleichstellungsaspekte (EFR-Priorität 4),

- Wissenstransfer / Open Access (EFR-Priorität 5),

- Internationale Zusammenarbeit (EFR-Priorität 6).

Am ERA-Roundtable nehmen die Akteure teil, die für die nationale Umsetzung der ERAPrioritäten zuständig sind. Dabei handelt es sich um Vertreter des Wissenschaftsressorts. Auch Institutionen, die eine ERA- Partnerschaft mit der Europäischen Kommission haben, sind eingeladen (Uniko, JR und ACR als EARTO- Mitglieder, FWF als Science Europe Mitglied).

Seit dem Jahr 2013 haben 13 Sitzungen des ERA-Roundtable bis einschließlich Juli 2017 stattgefunden. Die Sitzungsprotokolle des ERA-Roundtable zeigen, dass insbesondere die inhaltliche Erstellung der österreichischen ERA-Roadmap ${ }^{86}$, zur Gestaltung des nationalen Reformplans koordinierend im ERA-Roundtable diskutiert und mit erarbeitet wurden. Diese Arbeit schloss insbesondere das BMVIT und die FFG ein. Darüber hinaus wurden in weiterer Folge der Prozess zur Erstellung des ERA-Fortschrittsberichts (ERA-Progress Report Austria ${ }^{87}$ ) im Rahmen des ERA-Roundtable erstellt. Der nationale Fortschrittsbericht zum Monitoring der österreichischen ERA-Roadmap besteht dabei aus zwei Teilen:

86 Siehe hierzu die österreichische ERA-Roadmap

87 Siehe hierzu den ERA-Progress Report Austria 
- Übersicht aus Einzelmaßnahmen in den 6 ERA-Prioritäten

- ERA Dashboard-Indikatoren, die Auskunft über Österreichs Position im europäischen Forschungsraum geben.

Die für das Monitoring der Fortschritte vorgesehenen ERA Dashboard Indikatoren wurden dabei von der Arbeitsgruppe ERA Dashboard erarbeitet und darauf aufbauend auch im ERA-Roundtable diskutiert.

Neben den Arbeiten rund um die ERA-Fortschrittsberichte wurden im Rahmen des ERA Roundtables auch Ergebnisse des nationalen Meinungsbildungsprozesses zu FP9 und der Entwurf eines interministeriell abgestimmten Eckpunktepapiers diskutiert.

Der ERA-Roundtable kann insgesamt als Spiegelformat zum Delegierten-Roundtable angesehen werden. Der ERA Roundtable verfolgt im engeren Sinne die Koordination der nationalen ERA-Prioritäten und zieht dafür auch relevante Akteursgruppen außerhalb der Ministerien heran. Dies fördert zum einen den Austausch zu aktuellem Wissen zu Entwicklungen im Europäischen Forschungsraum zwischen betroffenen Akteuren. Zum anderen werden dem verantwortlichen Wissenschaftsressort Inputs zur Diskussion auf europäischer Ebene und zu Entwicklungen auf Akteursebene zur Verfügung gestellt. Die Bewertung des ERA-Roundtables ist nicht einheitlich; während er grundsätzlich als Forum für den Austausch zwischen VertreterInnen der Ministerien und FTI-Community geschätzt wird, wurden von einigen GesprächspartnerInnen der Umfang, die Aktualität und die zielgruppenspezifische Relevanz der tatsächlich übermittelten Informationen kritisch beurteilt.

Neben dem ERA-Roundtable führt die EU-Koordinationsabteilung auch regelmäßig Arbeitstreffen mit den ERA-KorrespondentInnen der Universitäten durch. Der Kreis der ERA-KorrespondentInnen der Universitäten besteht aus von den Universitäten nominierten VertreterInnen auf Rektoratsebene, meist den VizerektorInnen für Forschung und/oder Internationales. Bei dessen Treffen wird auch Zahlenmaterial zur österreichischen Beteiligung diskutiert und es werden Erfahrungen mit den Rahmenprogrammen ausgetauscht sowie Maßnahmen besprochen, die im Kontext der ERA-Dialoge stehen.

Diese Treffen werden seitens dieser Zielgruppe insgesamt als sinnvoll erachtet und ermöglichen einen direkten Meinungsaustausch auf strategischer Ebene. Sie sind vor allem in Kombination mit dem ERA-Dialog der FFG hilfreich, um den Universitäten Zugang zu Wissen rund um EU-FTI-Programme nahe zu bringen. Als Format erhöhen sie den Wissensstand der EntscheidungsträgerInnen auf Organisationsebene und ermöglichen es, neue Entwicklungen zu antizipieren und in eigene Strategien zu übersetzen.

\subsubsection{Arbeitsgruppen zur Internationalisierung}

Im Jahr 2011 verabschiedete die österreichische Bundesregierung die FTI-Strategie des Bundes 2014-2020. In weiterer Folge wurden zur Umsetzung der Strategie in sieben Bereichen Arbeitsgruppen eingesetzt, wobei sich zwei Arbeitsgruppen im Rahmen von Europa (AG 7b) bzw. über Europa hinaus ( $A G$ 7a) mit dem Thema Internationalisierung beschäftigten. Die AG 7a und 7b der FTI-Strategie sind vielen InterviewpartnerInnen jeweils bekannt. 
Die Tatsache, dass die AG7b mittlerweile eingestellt und stattdessen die Alignment Arbeitsgruppe eingerichtet wurde, ist hingegen einigen GesprächspartnerInnen noch nicht bewusst. Wesentliche Zielsetzung der AG Alignment ist die Aufarbeitung von Empfehlungen aus diversen Arbeitsgruppen zum strategischen und operativen Alignment, wobei vor allem darauf geachtet werden soll(te), in Hinblick auf Verbesserungen "Quick Wins" zu identifizieren und die grundsätzliche Realisierbarkeit einzuschätzen.

Die mittlerweile eingestellte interministerielle Arbeitsgruppe zu den multilateralen Initiativen (MULLAT) ist unter den drei Arbeitsgruppen (EIT, JPIs, MULLAT) interessanterweise am vergleichsweise besten bekannt. Einige GesprächspartnerInnen bedauern, dass die Arbeitsgruppe schon lange nicht mehr zusammengetreten ist.

Die Arbeitsgruppen zum European Institute of Technology (EIT) und JPIs dienen primär der Abstimmung ministerieller Positionen zu diesen europäischen Instrumenten. Die TeilnehmerInnen setzen sich dementsprechend in erster Linie aus Ministerien, FFG, WKO, teils auch IV, FWF und Uniko, und unmittelbar mit EIT und JPIs beauftragten Organisationen zusammen. Die Arbeitsgruppen treffen sich in mehrmonatigen Abständen, im Durchschnitt zweimal pro Jahr.

Grundsätzlich entstand in den Gesprächen über die Arbeitsgruppen zu FTI-AG7b, EIT, JPI und MULLAT der Eindruck, dass von vielen, auch erfahrenen, AkteurInnen im Politikfeld die Initiativen im Detail nicht überblickt werden (was zum Teil auch direkt verbalisiert wird). Die transnationalen FTI-Partnerschaften wurden immer wieder kritisch reflektiert, so auch in mehreren Fokusgruppen (besonders jene mit den Forschungsservicestellen, den außeruniversitären Einrichtungen und den Unternehmen). Wiederholt wird diskutiert, dass die Instrumente immer unübersichtlicher werden, aber auch eine strategische Unklarheit im Hinblick auf die Vorgehensweise österreichischer Ministerien existiert. Insbesondere FTI-Akteure äußerten die grundsätzliche Kritik, dass es in Österreich unklar ist, warum manche Initiativen (beispielsweise im Bereich von JPIs bzw. allgemein transnationale FTI-Partnerschaften) gefördert werden und andere nicht.

Vor dem Hintergrund teilweise sehr kleiner nationaler Beteiligungsbudgets wäre ein Prozess der strategischen Entscheidungsfindung zu den transnationalen FTI-Partnerschaften sinnvoll. In einer Gegenüberstellung der österreichischen Stärkefelder und Interessen sollten Beteiligungen als Grundsatzentscheidungen gesehen werden.

Uneinigkeit herrscht im Politikfeld zum Thema eines möglichen gemeinsamen Fördertopfes, der die Umsetzung einer derartigen Koordination erleichtern würde. Unter anderem stellt sich die Frage, wer für einen derartigen Fördertopf zuständig sein sollte: ein Ministerium, die FFG oder der FWF? In diesem Zusammenhang sind auch die Finanzierungsentscheidungen zu sehen: Wer wäre in letzter Instanz dafür zuständig?

\subsection{Schlussfolgerungen und Empfehlungen}

Die im ERA Observatorium zusammengefassten Aktivitäten, die Informationsaustausch und bessere Abstimmung zwischen den Akteuren ermöglichen sollen, werden mehrheitlich positiv beurteilt. 
Die Arbeit der EU-Koordinationsabteilung wird dabei fast durchgehend positiv und integrativ wahrgenommen und orientiert sich stark an einem gemeinsamen Erfahrungsaustausch. Die vielfältigen Kommunikationsangebote werden von den beteiligten Fachressorts geschätzt.

Derzeit scheinen Versuche einer stärkeren Koordination für ressortübergreifende Maßnahmen und eine stärkere Verbindung von nationalen und internationalen Programmen vorerst auch im Rahmen des ERA Observatoriums an ihre Grenzen gestoßen zu sein. Dass ressortübergreifende Koordination in Zukunft vermehrt an Bedeutung gewinnen könnte, zeigt sich bereits heute an der hohen Bedeutung der strategischen Partnerschaftsinstrumente, in der die insbesondere FTI-Akteure keinen national koordinierten Zugang ausmachen können.

Eine informelle Abstimmung im Sinne von Information (Europa Forum Forschung) und gegenseitigem Austausch (Delegierten Roundtable) wurde gut angenommen, während eine weitergehende Abstimmung (z.B. ERA Policy Forum) keine hinreichende Unterstützung fand.

In der Beurteilung der wesentlichen strategischen Aktivitäten der nationalen Ministerien im EU-FTI-Bereich gibt es einen Konsens, der - vor allem auf der Basis der mit den EUFTI-Governance ExpertInnen durchgeführten Interviews - etwa wie folgt beschrieben werden könnte. Demnach sind die Kernaufgaben der Bundesministerien im EU-FTI-Bereich:

- die Koordination der nationalen Positionen sowie ein Wissensaustausch zu diesen Positionen,

- ein Monitoring der Stärken von Österreich und eine Anpassung von europäischen Programmen an die verstärkten als Teil einer strategischen Arbeit der Delegierten,

- eine Kooperation mit anderen EU-Ländern in der Entwicklung von gemeinsamen Strategien,

- die Einbeziehung und Information von Akteuren sowie

- eine Verteidigung der entsprechenden Bundesmittel gegenüber anderen Politikbereichen auf der nationalen Ebene.

Daraus kann man schließen, dass die fünf Funktionen des ERA-Observatoriums grundsätzlich sinnvoll gewählt sind. Sie werden von den einzelnen Instrumenten allerdings nur teilweise abgedeckt.

\section{Empfehlung: Dialog im Europa Forum Forschung stärken}

Die Funktion Information/Kommunikation zu relevanten EU-Politiken wird im Wesentlichen gut abgedeckt. Das diesbezüglich wichtigste Instrument, das Europa Forum Forschung, sollte neben seiner angestammten Informationsfunktion zukünftig auch Dialogelemente beinhalten. Interaktive und kreative Zugänge, beispielsweise aus dem Bereich der Gruppenmoderation, könnten hierbei stärker zum Einsatz kommen.

Darüber hinaus stellt die funktionale Einbeziehung anderer Akteure, beispielsweise anderer FTI-Ministerien, in die Gestaltung des Forums eine Option dar, welche den Kooperationsgedanken im Politikfeld stärken könnte.

Das ERA Portal Austria wird gut angenommen. Lediglich eine weitere Verbesserung der Übersichtlichkeit der Website, insbesondere der Suchfunktion erscheint notwendig. 
Die Beratung und Betreuung ministerieller Ressorts für H2020 funktioniert im GroBen und Ganzen gut. Der EIP-Lenkungsausschuss wird in Bezug auf seine Funktionsweise positiv beurteilt. Die thematischen Begleitgruppen der Programmdelegierten sind sehr unterschiedlich aufgesetzt, von einem regelmäßig bedienten Netzwerk bis zu einer losen Ansammlung an persönlich bekannten ExpertInnen, deren Netzwerkknoten die Delegierten sind. Einige Delegierte verwenden überhaupt keine Begleitgruppe, was nahelegt, dass die Funktion der Aufnahme und Abgabe von Informationen jeweils auf der nationalen und der europäischen Ebene durch Delegierte unterschiedlich interpretiert wird.

Das jährliche persönliche Gespräch des Abteilungsleiters der EU-Koordinationsabteilung mit den Delegierten dient der gemeinsamen Reflexion der geleisteten Arbeit sowie vorhandener Strukturen auf einer persönlichen Ebene. Dieses wurde von den InterviewpartnerInnen gut beurteilt und erscheint vor dem Hintergrund der unterschiedlichen Situationen der einzelnen Delegierten zweckmäßig.

\section{Empfehlung: Beitrag zur Orientierung der Aufgabenstellung von Delegierten}

Wir empfehlen eine stärkere Orientierung der unterschiedlichen Aufgabenstellungen von Delegierten über die definierten Aufgaben und über die formale Vertretung österreichischer Interessen in Brüssel hinaus. Ein Element könnte in diesem Zusammenhang beispielsweise die Kommunikation mit der wissenschaftlichen Community im Rahmen der thematischen ExpertInnengruppen der Delegierten sein.

Der Delegierten-Roundtable ist ein sinnvolles Instrument und wird hervorragend bewertet.

In Bezug auf die Funktion der Beratung und Betreuung ministerieller Ressorts könnte auch das gegenwärtig diskutierte Verbindungsbüro in Brüssel Unterstützungsarbeit leisten. Dabei ist das Potenzial dieses Büros auch im Zusammenhang mit den allgemeinen Zielen der österreichischen EU-FTI-Politik im Hinblick auf die angestrebte Positionierung des Landes im europäischen Forschungs- und Innovationsraum zu sehen (vgl. Kap. 8.3).

\section{Empfehlung: Zielsetzungen eines EU-Verbindungsbüros mit FTI-Community klären}

Vor der Einrichtung eines Verbindungsbüros sind strategische Weichenstellungen hinsichtlich Zielen, Aufgaben und damit einhergehend Aufbau, Finanzierung und Positionierung einer derartigen Organisationseinheit vorzunehmen. Vor dem Hintergrund der bisher polarisierten Debatte, die sich unter anderem in verschiedenen Fokusgruppen und der Delphi Befragung derart geäußert hat, dass sich die Mehrheit der österreichischen Akteure für ein derartiges Verbindungsbüro und eine Minderheit deutlich dagegen ausgesprochen hat, wird eine weiterführende Diskussion mit den wichtigsten Akteursgruppen empfohlen.

Die Funktion der strategischen Beratung der Politik wird im Moment vom ERA Council Forum wahrgenommen, das der unmittelbaren Beratung des Wissenschafts- und Forschungsministers in Angelegenheiten der EU-FTI-Politik dienen soll. Das Forum wird im Politikfeld unterschiedlich wahrgenommen. In der FTI-Community sind die konkreten Aufgaben und Aktivitäten des Forums wenig bekannt. Auch wurde generell die Zusammen- 
setzung und die personellen Überlappungen zwischen den verschiedenen Räten hinterfragt. Im Hinblick auf die (selbst-)gesteckten Ziele des ERA Council Forums, d.h. vor allem die die vertrauensvolle Beratung der höchsten Ebene der Forschungs- und Wissenschaftspolitik, kann die Arbeit des Forums aus Sicht der unmittelbar beteiligten Akteure als erfolgreich gelten.

Vor dem Hintergrund der im Regierungsprogramm der Bundesregierung angedachten Neuorganisation und Konzentration der die FTI-Politik beratenden Organe, wird einerseits die Beibehaltung einer starken europäischen Komponente empfohlen. Die Erfahrungen des ERA Council Forums zeigen auch den Mehrwert hochrangiger ausländischer Mitglieder eines solchen Gremiums, die mit ihrer Außenperspektive zusätzliche Blickwinkel und Erfahrungen einbringen können. Wenn diese mit einem guten Verständnis der Problemstellungen und der Machbarkeit im nationalen Kontext verknüpft werden kann, kann ein solches Gremium für die FTI-Politik von hohem Nutzen sein. Angesichts der wachsenden Bedeutung der FTI-Politik als Querschnittsmaterie sollte nicht zuletzt auch vor dem Hintergrund internationaler Erfahrungen die politikfeldübergreifende Verankerung eines zukünftigen Beratungsorgans ins Auge gefasst werden.

Darüber hinaus sollte unter Berücksichtigung des Gebotes der Transparenz von Politik unabhängig davon die Kommunikation des Forums bzw. Forum-Nachfolgeorgans und die Kommunikation darüber verbessert werden. Andernfalls hat das fehlende Wissen über das jeweilige Organ das Potenzial andere Elemente von dessen Arbeit im Politikfeld negativ zu beeinträchtigen. Eine Möglichkeit wäre beispielsweise die Verwendung des Europa Forum Forschung für eine regelmäßige Kurzinformation über die letzten Aktivitäten des ERA Council Forum-Nachfolgeorgans.

\section{Empfehlung: Europäische Dimension in politisch beratenden Gremien berück- sichtigen}

Im Zuge des Neuaufsetzens der FTI-politischen Ratslandschaft in Österreich wird empfohlen, eine starke europäische Dimension in den Aufgaben und bei der Besetzung des neu zu konstituierenden Gremiums vorzusehen.

Um dem nicht zuletzt von der europäischen Ebene verstärkten Querschnittscharakter der FTI-Politik Rechnung zu tragen, sollte ein zukünftiges Gremium sich auch explizit an direkt und indirekt mit FTI-Agenden betraute Ressorts wenden können.

Um der Balance zwischen vertrauensvoller Beratung auf höchster politischer Ebene und der Notwendigkeit von Transparenz und Offenheit Rechnung zu tragen, wird außerdem eine regelmäßige Information über die Aktivitäten eines zukünftigen Beratungsgremiums empfohlen.

Die Funktion des wirkungsorientierten Monitorings der österreichischen Teilnahme an H2020 und ERA wird neben der Tätigkeit bzw. der Governance des EU-PM auch durch das ERA Reporting Board wahrgenommen. Der darin angestrebte Dialog zwischen Ministerien und angewandten Forschungsinstituten Österreichs zum Thema Analyse und Datenbereitstellung des EU-FTI-Bereichs wurde durch die Entwicklung eines ERA-Dashboards erfüllt. Seit Ende 2015 finden die Treffen des Instruments nur mehr sporadisch statt und seine Notwendigkeit sollte seitens der EU-Koordinationsabteilung überprüft werden. 
Die Funktion der Unterstützung von Prozessen für den Strukturwandel im Politikbereich ist im Vergleich zu den anderen Funktionen des Observatoriums am wenigsten aktiv. Diese Beobachtung kann auch in einen unmittelbaren Zusammenhang mit dem dafür notwendigen Aufwand bei der Bemühung von einer negativen zu einer positiven Koordination zu gelangen, gestellt werden.

Bei einigen funktionierenden Instrumenten im Bereich dieser Funktion sind die Anforderungen an die Form der Koordination weniger hoch. Dies gilt beispielsweise für die Treffen der ERA-KorrespondentInnen der Universitäten, die eine sinnvolle Kombination mit dem ERA-Dialog der FFG darstellt.

Die Arbeitsgruppen zum EIT und zu den JPIs sind in ihrer Ausrichtung sehr spezialisiert, grundsätzlich sinnvoll und dienen weniger der aktiven Koordination, sondern eher dem, ebenso wichtigen, Informationsaustausch zum jeweiligen europäischen Instrument.

Ähnlich wird auch im ERA-Roundtable allgemein zu den Entwicklungen im europäischen Forschungsraum diskutiert, wobei die diesbezüglichen Treffen oft über keine konkreten thematischen Ausgestaltungen verfügen. Allen zuletzt genannten Instrumenten ist gemein, dass sie im Normalfall keine aktive Koordination betreiben, sondern bei gegenseitiger Information verbleiben.

Zwei Instrumente, mit denen versucht wurde, über diese Vorgehensweise hinauszugehen, existieren hingegen nicht mehr. Die Arbeitsgruppe MULLAT zu transnationalen FTIPartnerschaften scheiterte an der unterschiedlichen Zugangsweise von BMBWF und BMVIT zu den entsprechenden europäischen Instrumenten. Während das damalige BMWFW eine Policy-Perspektive auf die FTI-Partnerschaften einnahm, war die Logik im BMVIT von unmittelbaren thematischen Problemstellungen der beteiligten Abteilungen getrieben. Vor diesem Hintergrund konnte keine Übereinstimmung über die Zielsetzung der Arbeitsgruppe getroffen werden, die dann auch nicht mehr zusammen trat.

Das ERA Policy Forum Austria scheiterte am Versuch, alle österreichischen Ministerien mit FTI-Kompetenzen zu einer aktiven Koordination im Hinblick auf eine Reihe von als wesentlich wahrgenommenen Themenbereichen zu bewegen. Das Instrument beendete seine Arbeit nach nur wenigen Sitzungen, weil die meisten Ministerien nicht mehr vertreten waren.

Tatsächlich ist vor diesem Hintergrund in Österreich eine nationale strategische Abstimmung im Hinblick auf die EU-FTI-Politik kaum vorhanden. Dies zeigt sich gerade bei den transnationalen FTI-Partnerschaften, wo die fehlende nationale Beteiligungsstrategie zu einer größeren Anzahl von Beteiligungen geführt hat, die in ihren Budgets häufig unterkritisch blieben.

Der Grad von Koordinationsanstrengungen in der österreichischen EU-FTI-Politik hängt unmittelbar von den Zielsetzungen dieser Politik ab. Für die Minimierung von Reibungsverlusten bei der Planung und Durchführung der EU-FTI-Politik reicht eine negative Koordination, wie sie im Moment bereits existiert. Für eine aktive Positionierung in der europäischen Diskussion um EU-FTI-Politik und die geschlossene Vertretung nationaler Standpunkte ist je nach dem Grad der Ambition eine jeweils aktivere (positive) Koordination von Nöten. 
Der im Regierungsprogramm festgehaltene neue Prozess für eine FTI-Strategie der Bundesregierung über 2020 hinaus bietet eine Chance für eine Überarbeitung der Koordinationsanstrengungen in der österreichischen EU-FTI-Politik. Um Erfolg zu haben, sollte der Nachfolger des ERA Policy Forums eine klare Mission haben, von der allgemeine Aufgabenstellungen und konkrete Zielsetzungen ableitbar sind. Diese sollte im Rahmen der FTI-Strategie der Bundesregierung formuliert werden. Sollte dies nicht möglich sein, wird das Aufsetzen eines eigenen Diskussionsprozesses zu diesem Thema empfohlen. Für einen derartigen Prozess wäre eine externe Moderation sinnvoll, weil auch das federführende Ministerium nicht gleichzeitig Interessen vertreten und moderieren kann. Die EUKoordinationsabteilung des BMBWF sollte allerdings unbedingt auch eigene Interessen vertreten können - beispielsweise im Sinne eines Motors für eine positive Koordination.

\section{Empfehlung: ERA-Formate bündeln und aktivere Koordination zwischen den Bundesministerien stärken}

Die vielfältigen Formate zum Informationsaustausch über potenziell strukturverändernde Entwicklungen hin zum Europäischen Forschungsraum werden in unterschiedlicher Intensität wahrgenommen. Eine Vereinfachung oder Bündelung der verschiedenen Formate wird empfohlen (z.B. ERA-Reporting Board und ERA-Roundtable).

Zugleich erscheint angesichts neuer Instrumente auf EU-Ebene eine aktivere Koordination zwischen verschiedenen Politikfeldern geboten. Vor dem Hintergrund der bisherigen Erfahrungen wird empfohlen, einen vorbereitenden Prozess zu starten, um diese Form der Koordination nachhaltig in der österreichischen Governance und FTI-Strategie zu verankern. 


\section{Ausblick und Szenarien}

\subsection{Positionierung der österreichischen FTI-Politik in FP9 als neuer Rahmen für die nationalen Unterstützungsstrukturen}

Hinweise für die weitere Ausgestaltung der Rolle und Aufgaben der FFG-EIP können sich nicht auf die Korrektur bestehender Defizite bzw. Verbesserungsvorschläge vor dem Hintergrund der aktuellen Aufgaben und Herausforderungen beschränken, sondern müssen auch die sich verändernden Rahmenbedingungen berücksichtigen. Zu diesen Veränderungen zählt insbesondere auch das 9. Rahmenprogramm für Forschung und Innovation der EU-Kommission (FP9), das von 2021 bis 2027 umgesetzt werden wird und dessen Schwerpunkte und Strukturen zum Zeitpunkt des Verfassens dieser Empfehlungen gerade intensiv diskutiert werden. Es stellt einen zentralen Bezugspunkt sowohl für die Arbeit der FFG-EIP als auch für die Governance wichtiger Dimensionen österreichischer FTI-Politik dar. Derzeit wird seitens der EU-Kommission ein erster Vorschlag für FP9 erarbeitet, und die bisherigen Diskussionen und Vorarbeiten weisen zumindest auf eine Reihe von möglichen Neuerungen hin, die für das nächste Rahmenprogramm zu erwarten sind. Hierzu zählen insbesondere

- Die Einrichtung eines Europäischen Innovationsrats (EIC), der zur Stärkung der Innovationdynamik in Europa durch die Unterstützung insbesondere von Start-ups und Scale-ups mit hohem Wachstumspotenzial beitragen soll;

- Eine zunehmende Bedeutung des missionsorientierten Ansatzes in den thematisch orientierten Säulen des Rahmenprogramms, für dessen Umsetzung größere Initiativen (z.B. im Sinne von strategischen Partnerschaften) ebenso erforderlich sein werden, wie ein explizites Alignment mit sektoralen Politikfeldern;

- Die bessere Abstimmung von Rahmenprogramm und Europäischen Strukturund Investitionsfonds (ESIF), um den wachsenden Disparitäten in der europäischen Forschungs- und Innovationslandschaft entgegen zu wirken.

Darüber hinaus ist mit Veränderungen des thematischen Portfolios der Forschungs- und Innovationsförderung auf europäischer Ebene zu rechnen, wobei beispielsweise im Falle der Verteidigungsforschung noch offen ist, ob diese innerhalb oder außerhalb des Rahmenprogramms verankert werden wird.

Zugleich muss betont werden, dass zum gegenwärtigen Zeitpunkt noch viele Unsicherheiten bezüglich der Ausgestaltung des nächsten Rahmenprogramms bestehen; der entsprechende Vorschlag der Europäischen Kommission wird für Juni 2018 erwartet.

Die zukünftige Rolle und die damit in Zusammenhang stehenden Aufgaben der FFG-EIP hängen aber nicht nur vom Rahmenprogramm ab, sondern auch von der angestrebten Positionierung Österreichs in Bezug auf die europäische FTI-Politik ab. Bislang wurde im Rahmen der bestehenden FTI-Strategie der österreichischen Bundesregierung der Fokus zum einen auf eine weitere Steigerung der österreichischen Beteiligung an europäischen Förderprorammen gelegt und zum anderen auf eine gestaltende Mitwirkung an der Formulierung einer gesamteuropäischen FTI-Politik; dies im Einklang mit der übergeord- 
neten Zielsetzung der Strategie, in die Gruppe der führenden Länder im Bereich Innovation aufzusteigen (Österreichische Bundesregierung 2011). Die Reformulierung der angestrebten Positionierung und Rolle Österreichs auf europäischer Ebene wird der zukünftigen FTI-Strategie obliegen, deren Entwicklung für das Jahr 2019 erwartet wird und für die derzeit Grundlagen erarbeitet werden (z.B. durch die aktuelle OECD Innovation Policy Review).

Grundsätzlich sind dabei - wie sich an den strategischen Positionierungen anderer europäischer Länder zeigt- unterschiedliche Rollenverständnisse möglich, die von einer weitgehenden Anpassung an auf europäischer Ebene definierte Prioritäten und Strategien bis hin zum Anspruch einer proaktiven (Mit-)Gestaltung der europäischen FTI-Politik reichen. Innovationsführer wie Schweden oder Finnland, mit denen sich Österreich häufig vergleicht, stellen dabei den Anspruch an sich selbst, auch die europäische Politik zu prägen; ein Anspruch, für dessen erfolgreiche Einlösung es durchaus Belege gibt. Die Lund-Konferenz der schwedischen Präsidentschaft im Jahr 2009 hat beispielsweise die Ausrichtung und Struktur des gegenwärtigen Rahmenprogramms beeinflusst, und das gezielte Engagement einzelner Länder hat zur Etablierung von strategischen Partnerschaften und multilateralen Kooperationsmechanismen (d.h. ERA-Nets, JPIs, JTIs, etc.) zwischen nationalen FTI- und Politik-Communities beigetragen. ${ }^{88}$

Die Entwicklungen auf beiden genannten Ebenen - Veränderungen im europäischen Rahmenprogramm und angestrebte nationale Positionierung in Bezug auf die Gestaltung der europäischen Forschungs- und Innovationspolitik - haben naturgemäß auch Konsequenzen für die Weiterentwicklung der österreichischen EU FTI-Politik und die mittelfristige Positionierung der FFG-EIP, die sowohl die nationalen FTI-Akteure als auch die nationale FTI-Politik bei ihren Aufgaben an der Schnittstelle zu Europa unterstützen soll.

Dementsprechend lassen sich angesichts der zu erwartenden Veränderungen in der europäischen FTI-Politik (Ebene 1) verschiedene Szenarien für die selbstgesteckten Ambitionen der nationalen FTI-Politik (Ebene 2) und in Konsequenz für die Rolle der EIP (Ebene 3) formulieren. Aus diesen ergeben sich Hinweise für ,bedingte Empfehlungen' zur weiteren Positionierung und Rolle der FFG-EIP und zu Koordinationserfordernissen in Bezug auf die EU FTI-Politik - abhängig von dem Weg, den Österreich im Hinblick auf die europäische FTI-Politik einzuschlagen gedenkt (Kap. 1.3). Zuvor (Kap 8.2) werden aber noch die wesentlichen im Rahmen der Evaluierung erfassten Erwartungen der FTI-Community hinsichtlich des derzeit noch in Diskussion befindlichen 9. Rahmenprogramms für Forschung und Innovation und der zukünftigen Unterstützungsleistungen seitens der FFGEIP zusammengefasst.

88 Diese beiden Beispiele können auch zur Illustrierung der nachfolgend formulierten Szenarien herangezogen werden. Die Lund-Konferenz stellte ein Beispiel par excellence für einen proaktiven von Seiten der FTI-Politik getriebenen Zugang zur Mitgestaltung der EU FTI-Politik dar und entspricht der Logik von Szenario 2, während das gezielte Vorantreiben strategischer Partnerschaften ein Empowerment und eigenverantwortliches Engagement der nationalen FTI-Akteure voraussetzt und damit im Einklang mit Szenario 3 steht. 


\subsection{Erwartungen der österreichischen FTI-Community}

Im Rahmen der Delphi-Befragung lag ein Schwerpunkt auf der Erhebung von Zukunftserwartungen ausgewählter Akteure des österreichischen Forschungs- und Innovationssystems. ${ }^{89}$ Die TeilnehmerInnen am Delphi können dabei als vertraut mit den aktuellen Debatten über das kommende Rahmenprogramm gelten.

Bei der Auswertung wurde der Schwerpunkt auf die Erwartungen an künftige Entwicklungen in der europäischen FTI-Politik gelegt sowie auf die sich daraus ableitenden Anforderungen an FTI-politische Governance und an Unterstützungsleistungen seitens der FFGEIP. Andere, im Rahmen der Delphi-Befragung angesprochenen Instrumentarien zur Stärkung einer europäischen Ausrichtung der Strategien von FTI-Organisationen (z.B. Leistungsvereinbarungen, Anbahnungsfinanzierungen, nationale Kofinanzierungen), die über den Rahmen der vorliegenden Evaluierung hinausgehen würden, werden nicht im Detail diskutiert.

\subsubsection{Europäischer Forschungsraum}

Der Europäische Forschungsraum bildet den umfassenden Bezugsrahmen für die FTI-Politik in Europa. Rund $80 \%$ der RespondentInnen vertraten die Ansicht, dass die österreichischen Universitäten und auch die außeruniversitären Forschungseinrichtungen ihre Anstrengungen forcieren und verstärkt aktive Beteiligungsstrategien entwickeln müssen, um auch in Zukunft im Europäischen Forschungsraum verankert zu bleiben. Hervorgehoben wurde dabei die Beteiligung an Netzwerken und Kooperationen, die eine Strategieentwicklung auf organisatorischer Ebene erfordert. Neben der Identifikation von eigenen Stärken, die über inner-organisatorische Konsultationsprozessen entwickelt werden sollten, gilt auch eine österreichische Gesamtstrategie zur Forschung an Universitäten und Fachhochschulen als bedeutender Faktor. Allerdings wird das Risiko gesehen, dass eine erhöhte Antragstätigkeit auch mit geringeren Erfolgswahrscheinlichkeiten und höheren Kosten verbunden sein könnte.

\section{Multilaterale Partnerschaften}

Eine wichtige Komponente im Europäischen Forschungsraum bilden die multilateralen Partnerschaften. Mehr als die Hälfte (51,5\%) der Antworten spricht sich für eine Konsolidierung der Partnerschaftsinitiativen und -programme aus, wobei H2020 als Richtschnur für eine Vereinheitlichung der Förderrichtlinien in allen transnationalen FTI-Partnerschaften, insbesondere aber auch in regionalen Instrumenten wie Interreg und $\mathrm{EFRE}^{90}$, dienen sollte, um Ineffizienzen und hohe Ressourcenbindung für eine Vielzahl von Programmmodalitäten abzubauen.

89 Die Delphi-Befragung wurde in zwei Runden durchgeführt. Auf Basis der Ergebnisse der ersten Runde wurden die Leitthesen und zugehörigen Schlussfolgerungen adaptiert und der zweiten Befragungsrunde unterworfen. Für weitere Details sei auf Kapitel 1 verwiesen.

90 Europäischer Fonds für regionale Entwicklung (Englisch: European Regional Development Fund ERDF) 
Interessant ist in diesem Zusammenhang die Beobachtung, dass rund zwei Drittel der RespondentInnen $(70,3 \%)$ die Informationsgewinnung zu den Nutzungs-und Beteiligungsmöglichkeiten transnationaler FTI-Partnerschaften als mit großen Aufwand verbunden ansehen. Eines der Hauptprobleme wird dabei in der fehlenden Bündelung des prinzipiell verfügbaren Informationsangebotes, auch auf Seiten der FFG, gesehen. Außerdem wird kritisiert, dass der Diskurs über die Gestaltung von Maßnahmen im Kreis weniger Informierter (Behörden, große Unternehmen, Intermediäre) geführt würde.

Zugleich zeigen sich einige recht klare Erwartungen hinsichtlich der Governance der multilateralen Partnerschaften in Österreich. So wird von den RespondentInnen ein Bedarf an verstärkter strategischer Koordination und an einer gesamthaften Perspektive über die Beteiligung an transnationalen FTI-Partnerschaften geortet, die zwischen den verschiedenen Ressorts, FTI-Intermediären sowie öffentlichen und privaten Stakeholdern, und unter Berücksichtigung der unterschiedlichen Interventionslogiken von PPPs und P2Ps abgestimmt werden sollte.

Knapp mehr als die Hälfte der RespondentInnen konstatiert darüber hinaus eine unterkritische Mittelstreuung über zu viele Programme $(51,1 \%)$ sowie die Notwendigkeit einer strategischen Mittelbündelung im Rahmen eines nationalen Ko-Finanzierungstopfes $(74,0 \%)$. Die Unterstützung für einen solchen Ko-Finanzierungstopf ist speziell unter den Intermediären und den ERA-KorrespondentInnen der Universitäten sehr hoch, während Policy-Akteure, Unternehmen und außeruniversitäre Forschungseinrichtungen eine gröBere Skepsis ausdrücken. Auch wenn zahlreiche Beteiligungen an europäischen Partnerschaftsinitiativen zur Erweiterung nationaler Förderprogramme genutzt werden (z.B. in den Feldern IKT, Sicherheit oder Produktion), findet das Argument hohe Unterstützung, dass viele Chancen für die nationale Forschungscommunity durch die teilweise fehlende oder zersplitterte Mittelallokation in Österreich vergeben würden. Diese Beobachtung wird interessanterweise auch von vielen Policy-Akteuren geteilt, die die aktuelle Streuung der Ko-finanzierungen über viele unterschiedliche Ministerien sowie insgesamt die geringen Dotierungen im Einzelfall ("es braucht Klotzen statt Kleckern") bemängeln. Ein gemeinsamer, mit ausreichenden Mitteln dotierter Topf würde in Zusammenhang mit einer nationalen Beteiligungsstrategie als ein klares Signal zu mehr Effizienz sowie als Orientierung für FTI-Akteure angesehen.

\section{Missionsorientierte Initiativen}

Es ist zu erwarten, dass der missionsorientierte Ansatz in der Forschungs- und Innovationspolitik im Zuge der Umsetzung des nächsten Rahmenprogramms an Bedeutung gewinnen wird.

Vor diesem Hintergrund erfährt die Aussage, dass eine unter den nationalen Stakeholdern abgestimmte Vorgangsweise im Sinne einer "österreichische Missions-Strategie" dringend erforderlich ist, über nahezu alle befragten Gruppen hinweg deutliche Zustimmung $(68,9 \%)$. In den qualitativen Anmerkungen finden sich aber auch kritische Stimmen zu einer solchen Strategie. Die Gründe für diese kritische Haltung liegen vor allem in der Angst vor thematischen Einschränkungen begründet und - damit einhergehend - in einer geringen Bereitschaft, sich auf gemeinsam definierte Schwerpunkte ein- 
zulassen. Andere Kommentare verweisen demgegenüber auf die Möglichkeit, österreichische Interessen und Schwerpunkte verstärkt in die Diskussion über europäische Missionsthemen einzubringen. Zugleich wird von der Mehrzahl der RespondentInnen betont, dass sich die österreichische F\&E-Förderung zwar stärker an den europäischen Missionsthemen ausrichten, zugleich aber auch eigene Akzente beibehalten sollte $(51,7 \%)$.

\section{Strategische Beratungskompetenz der FFG: Vom Informations- zum Bera- tungsbroker}

Angesichts aktueller und neuer Herausforderungen für die österreichische Forschungscommunity durch die Entwicklungen auf europäischer Ebene sollte die FFG ihre Rolle im Bereich des Strategie-Supports stärken. Dieser Aussage stimmen 50,0\% der RespondentInnen zu oder sehr zu, während $27,8 \%$ diese eher oder ganz ablehnen.

Die zustimmenden Personen gaben außerdem an, dass ein verstärkter Strategie-Support durch die FFG bereits aktuell bemerkbar (z.B. durch ERA-Dialoge, Potentialanalysen) und wichtig sei, um die österreichische Forschungscommunity bestmöglich auf geänderte Erfordernisse und neue Herausforderungen im Rahmenprogramm vorzubereiten. Aus den qualitativen Kommentaren lässt sich allerdings auch herauslesen, dass einige Befragte einen Interessenskonflikt dergestalt erkennen, als strategische Beratungsleistungen auch von privaten Anbietern erbracht werden könnten.

Somit kann aus der Delphi-Befragung die Schlussfolgerung gezogen werden, dass ein verstärkter Strategie-Support durch die FFG von der österreichischen Forschungscommunity gewünscht und auch bereits wahrgenommen wird. Der FFG wird auch die notwendige inhaltliche Kompetenz und Unabhängigkeit zugetraut, um als Partnerin der Universitäten und Forschungsorganisationen die Profilentwicklung der Organisationen in beratender Form zu unterstützen $(66,7 \%)$. Allerdings sind Zusatzleistungen wie strategische Beratung nicht aus dem Stand heraus zu bewältigen; es bestehen seitens der FTI-Community Zweifel, ob die FFG-EIP über die erforderliche Ressourcenausstattung für diese weiterreichende Aufgabe verfügt. Nicht zuletzt würde der Ausbau der strategisch orientierten Beratungskompetenz der FFG eine Intensivierung des direkten Austauschs mit den verantwortlichen Stellen der Europäischen Kommission erfordern.

\section{Die Rolle der FFG in Bezug auf eine verstärke Missionsorientierung auf eu- ropäischer Ebene}

Im Falle einer stärkeren Missionsorientierung auf europäischer Ebene müssten nach Meinung von mehr als der Hälfte der RespondentInnen die Unterstützungsaufgaben der FFG für österreichische FTI-Stakeholder verstärkt und vor allem an neue Themensetzungen angepasst werden $(55,2 \%)$, z.B. in Bezug auf Informationsbereitstellung, CommunityManagement und Koordination. Insbesondere Intermediäre erachten eine derartige Stärkung der FFG als notwendig, während Unternehmen und Policy Akteure eine eher kritische Haltung einnehmen. Angesichts der generell hohen Zufriedenheit mit der FFG wird allerdings weniger eine radikale Neuorientierung, sondern eher eine Anpassung an neue Gegebenheiten ( $z$.B. neue Themensetzungen, neue Instrumente, stärkere Innovations- und Diffusionsorientierung, etc.) durch das FP9 eingefordert. 


\section{zungsleistungen}

Der überwiegende Anteil der befragten ExpertInnen (59,2\%) befürwortet die Aussage, dass die FFG in Bezug auf ihre Informations- und Vernetzungsleistungen verstärkt Augenmerk auf die gesamtheitliche Betrachtung der nationalen und europäischen Förderangebote und die bestehenden österreichischen Akteursnetzwerke (Bildung von Clustern, interinstitutionelle Forschungsplattformen) legen sollte. Nur wenige $(7,4 \%)$ stufen diese Herangehensweise negativ ein, während ein Drittel der RespondentInnen unentschieden ist (33,3\%). Dieses Muster zeigt sich auch entlang der Akteurs-Kategorien, wobei die Zustimmung der ERA-KorrespondentInnen der Universitäten am geringsten ausfällt. Die BefürworterInnen dieser Aussage sehen den Hauptvorteil einer gesamtheitlichen Betrachtung von europäischen und nationalen Förderangeboten darin, dass Synergien besser genutzt werden könnten. Außerdem würde die Forschungscommunity bei Informationsund Vernetzungsleistungen durch die FFG - ganz im Sinne eines "one-stop-shops" - einen guten Überblick über nationale und europäische Förderangebote und Akteursnetzwerke (Cluster, Plattformen) erhalten - und damit ein besseres Verständnis des ohnehin komplexen Feldes bekommen. Ein solcher gesamtheitlicher Ansatz hätte nicht nur auf die FFG-EIP Auswirkungen, sondern auf alle Bereiche der FFG und deren Zusammenwirken. Letztlich könne dadurch die strategische Positionierung der österreichischen FTI-AkteurInnen im europäischen bzw. österreichischen Forschungsraum gestärkt werden.

\subsection{Szenarien zur österreichischen Positionierung in FP9 und ihrer Unterstützungsstrukturen}

Die Diskussion der Erwartungshaltungen der österreichischen FTI-Community hat gezeigt, dass Veränderungen sowohl in der Governance auf nationaler und an der Schnittstelle zur europäischen Politikebene erforderlich sein werden, als auch in den Unterstützungsleistungen für die FTI-Community. Das Ausmaß dieser Veränderungen ist von der angestrebten Positionierung Österreichs in Bezug auf die europäische FTI-Politik abhängig. Die drei nachfolgend skizzierten Szenarien spiegeln die Handlungsoptionen auf nationaler Ebene wider und die jeweiligen Konsequenzen, die diese für die Unterstützungsstrukturen hätten. Sie sind zu verstehen als Denkanstöße für die weitere Diskussion in Österreich, nicht zuletzt auch im Hinblick auf die Entwicklung einer neuen FTI-Strategie.

\subsubsection{Szenario 'Enhancement in Continuity': Inkrementelle Weiterentwicklung}

\section{Grundidee}

Der Leitgedanke dieses Szenarios besteht darin, dass der gut etablierte und in weiten Teilen auch gut funktionierende Status Quo weiterentwickelt wird und im Zuge dessen Anpassungen der FTI-Politik an die Erfordernisse neuer Instrumente und Initiativen auf europäischer Ebene vorgenommen werden. Insbesondere bedeutet dies, dass eine extensive Nutzung der vielfältigen Möglichkeiten der EU FTI-Politik weiterhin als im Interesse 
Österreichs liegend anerkannt und verfolgt wird, ohne dass wesentliche Veränderungen in Österreich, z.B. im Bereich der Governance, vorgenommen würden. Dementsprechend bleiben Elemente der nationalen Strategiebildung und Prioritätensetzung (z.B. im Hinblick auf die österreichische Beteiligung an strategischen Partnerschaften) vergleichsweise schwach ausgeprägt, bzw. auf einzelne Ministerien beschränkt. Österreichische Organisationen beteiligen sich weiterhin - je nach individueller Interessenslage und Möglichkeit an einer Vielzahl von Initiativen, sehen sich dabei aber mit der Gefahr einer unterkritischen Ressourcenausstattung konfrontiert und können daher nur eine Nebenrolle in den größer dimensionierten europäischen Initiativen spielen. Eine explizite Fokussierung entlang etwaiger nationaler Schwerpunkte (die im Rahmen einer neuen FTI-Strategie zu reformulieren wären) unterbleibt daher.

Im Einklang mit diesem Bild verbleibt die Positionierung Österreichs in EU-Initiativen weitgehend bei den FTI-Organisationen selbst und im Aufgabenbereich der Fachministerien, die diese Positionierung nach jeweils ressortspezifischen Prioritätensetzungen vornehmen.

Aufgrund des Verzichts auf eine umfassende nationale Positionierungs- und Profilierungsstrategie in Bezug auf die europäische Ebene kann auch keine herausstechende proaktive Rolle bei der weiteren strategischen Ausgestaltung des Rahmenprogramms gespielt werden.

\section{Stoßrichtungen für die Weiterentwicklung der Unterstützungsstrukturen}

Grundsätzlich wird in diesem Szenario die unterstützende Rolle von EU-Koordinationsabteilung und FFG-EIP beibehalten und ggf. punktuell gestärkt. Konkret manifestiert sich dies im Aufgreifen der - in weiten Bereichen inkrementellen - Empfehlungen der vorliegenden Evaluierung, darüber hinaus aber auch in einer Reihe zusätzlicher Charakteristika:

- Die Teilnahme der FTI-Akteure am europäischen Rahmenprogramm und weiteren Partnerschaften entwickelt sich entlang der vorherrschenden österreichischen Besonderheiten, d.h. den bestehenden strukturellen (z.B. zentrale FTI-Akteure) und thematischen (z.B. existierende ,Stärken') Profilen, weiter.

- Die FFG-EIP intensiviert ihre Anstrengungen, um strategische Potenziale auf FTIAkteursebene zu erkennen und die FTI-Community diesbezüglich anzusprechen. Dies erfolgt im Rahmen der von den einzelnen Ministerien vorgegebenen Leitlinien, d.h. die Verantwortlichkeit für die Entwicklung der inhaltlichen Strategien verbleibt bei den einzelnen Ministerien. Diese unterstützen im Rahmen ihrer thematischen Verantwortlichkeiten europäische Aktivitäten durch das Bereitstellen zusätzlicher finanzieller Mittel und den Einsatz weiterer Koordinierungsinstrumente (z.B. über ihre Eigentümerfunktionen).

- Informationen zu Verschränkungsmöglichkeiten von nationalen und europäischen Programmen (z.B. Stärken, Schwächen und Komplementaritäten Österreichs in Bezug auf europäische Initiativen) werden seitens der FFG-EIP aufgezeigt, aber es werden nicht aktiv Partnerschaften initiiert oder (an)gesteuert.

- Die Zusammenarbeit zwischen den Ressorts in Bezug auf europäische Initiativen bleibt weiterhin in den Rahmen der jeweiligen Verantwortlichkeiten eingebettet. 
Aufgrund dieser Abgrenzungen bleibt die Koordination innerhalb der für FTI-Agenden zuständigen Ressorts schwach ausgeprägt, ebenso wie jene zwischen FTIund sektoralen Politikfeldern.

- Die EU-Koordinationsabteilung ist weiterhin als zentraler Knoten und Schnittstelle zwischen EU FTI-Politik, FTI-Ministerien und FTI-Community positioniert. Die Aufgaben der EU-Koordinationsabteilung konzentrieren sich dabei auf Informationsvermittlung und Aktivierung der FTI-Community.

- Angesichts der verteilten Verantwortlichkeiten und begrenzten Politikkoordination kommt einem FTI-politischen Beratungsgremien in Bezug auf EU FTI-Agenden die Rolle zu, Empfehlungen hinsichtlich wichtiger übergreifender Politikmaßnahmen zu erarbeiten, die für die nachhaltige Stärkung der Performance des österreichischen Forschungs- und Innovationssystems erforderlich wären.

- Im Einklang mit diesen Stoßrichtungen wäre die Einrichtung eines Verbindungsbüros in Brüssel zwar denkbar, aber nicht zwingend erforderlich.

\subsubsection{Szenario 'Smart and Proactive Alignment': FTI-Politik als Impulsgeberin im europäischen Mehrebenensystem}

\section{Grundidee}

In diesem Szenario strebt die österreichische Politik einen Ausbau ihrer Rolle hin zum (pro)aktiven Mitgestalter europäischer FTI-Politik an, um die sich daraus ergebenden Chancen der EU FTI-Politik gezielter und effektiver für Österreich zu nutzen (,smart alignment').

Um sich besser als integraler Bestandteil des Mehr-Ebenen Innovationsökosystems in Europa positionieren können, strebt Österreich im Rahmen seiner neuen FTI-Strategie eine Reihe von Veränderungen im nationalen System an, über die eine intensivere und einflussreichere Mitwirkung an der Ausgestaltung und in der Folge an der Nutzung der europäischen FTI-Politik möglich wird. Die Festlegung eines solchen Konzepts als Leitlinie für eine kohärente österreichische Position zur EU FTI-Politik wäre ein zentraler Bestandteil einer neuen österreichischen FTI-Strategie.

Dies umfasst neben der weiteren Exzellenzorientierung der Universitäten und der Stärkung der unternehmerischen Kultur insbesondere auch die Entwicklung einer europäischen Beteiligungsstrategie, inkl. nationaler Prioritäten, um in ausgewählten Feldern eine gewichtige und prägende Rolle spielen zu können. Hiermit einher geht eine enge Verschränkung nationaler und europäischer/internationaler Programme, die sich beispielsweise in einer selektiven Beteiligung an strategischen Initiativen der EU oder in einer verbesserten inhaltlichen Komplementarität/Abstimmung zwischen nationalen und europäischen Förderausschreibungen manifestieren könnte.

Die aktive Beteiligung an der Gestaltung europäischer FTI-Instrumente und -Inhalte erfolgt in abgestimmter Form zwischen Ministerien, FFG-EIP und FTI-Community, orchestriert durch die nationale Politik. 
Mit anderen Worten, dieses Szenario geht von einer ausgeprägten politischen Leadership-Rolle bei der Stärkung der europäischen Rolle Österreichs im Einklang mit der nationalen FTI-Strategie aus.

\section{Stoßrichtungen für die Weiterentwicklung der Unterstützungsstrukturen}

Um diese federführende Rolle der Politik bei der strategischen Positionierung Österreichs auf europäischer Ebene ebenso wie im Hinblick auf die Konzeption nationaler Programme und Politiken (,smart and proactive alignment') realisieren zu können, bedarf es der Unterstützung durch eine starke FFG mit ihren nationalen und europäischen Aktivitäten, aber auch der Weiterentwicklung der interministeriellen Abstimmungsmechanismen. Im Hinblick darauf sollten die Unterstützungsstrukturen in Österreich entlang folgender Stoßrichtungen weiterentwickelt werden.

- Interministerielle Abstimmungen dienen nicht nur der Abgrenzung von Verantwortlichkeiten (negative Koordination), sondern ermöglichen Komplementaritäten und Synergien im Sinne der in einer neuen FTI-Strategie festgelegten europäischen Positionierung (positive Koordination). Nationale Strategiebildungsprozesse zu europäischen Leitthemen werden auf politische Ebene und/oder höchster Beamtenebene gestützt („ERA Policy Forum 2.0") und tragen so zu einer klaren europäischen Positionierung bei und werde angesichts der wachsenden Dringlichkeit solcher Positionierungen als notwendig und realistisch angesehen.

- Der EU-Koordinationsabteilung kommt in diesem Prozess eine Schlüsselrolle $z u$, die durch eine entsprechend sichtbare Verankerung im Politiksystem unterstrichen wird (z.B. durch Zuschreibung besonderer Kompetenzen, oder eine Verankerung im $B K A)$.

- Ergänzend zur Stärkung des politischen Alignment kommt einer höheren Autonomie und Gestaltungsfreiheit der FFG ( $\mathrm{vgl}$. die entsprechenden Empfehlungen der FFG-AWS Evaluierung und das "VINNOVA Modell") eine große Bedeutung zu. Diese Form der höheren Autonomie würde dazu dienen, die Teilnahme an ERA-Initiativen vor dem Hintergrund der nationalen Strategie und komplementär zu österreichischen Besonderheiten auszurichten, z.B. durch aktives Anstoßen und Unterstützen von nationalen Partnerschaften innerhalb der strategischen Rahmenvorgaben und Prioritäten der Politik.

- Dementsprechend wäre auch der Kapazitäts- und Kompetenzaufbau auf Seiten der FFG-EIP zu forcieren, um Schnittstellen nach Brüssel zu intensivieren und Interaktionen mit der österreichischen FTI-Community, aber auch mit PartnerInnen in Europa, zu stärken, um auf diese Weise die Stärkung der nationalen Prioritäten auch auf der europäischen Ebene zu unterstützen.

- Im Einklang mit diesen Überlegungen wäre innerhalb der FFG und in Zusammenarbeit mit den Fachministerien eine engere Verzahnung nationaler und europäischer Programme anzustreben, um bei der Beratung von FTI-Akteuren einen nahtlosen Übergang zwischen Programmen auf unterschiedlichen Ebenen zu ermöglichen.

- Zur Flankierung dieses proaktiven Szenarios kommt der starken Verankerung des EU FTI-Themas in den Agenden eines im Regierungsprogramm vorgesehenen neu zu gestaltenden hochrangigen Beratungsgremiums zu FTI-politischen Fragen 
eine wichtige Rolle zu. Dadurch soll die effektive Einbettung des österreichischen Forschungs- und Innovationssystems in den europäischen Kontext und die Mitgestaltung eben dieses europäischen Kontexts durch kompetente ExpertInnen mit unabhängiger Binnen- und Außenperspektive unterstützt werden.

- Im Rahmen dieses Szenarios würde auch ein nationales d.h. von Politik und/oder FFG getragenes Verbindungsbüro in Brüssel eine sinnvolle Ergänzung bilden.

\subsubsection{Szenario 'Distributed Empowerment': Stärkung und Vernetzung der FTI-Community}

\section{Grundidee}

In diesem Szenario erfolgt die Ausweitung der Mitgestaltung und Beteiligung an der europäischen FTI-Politik vor allem über die Stärkung und bessere Vernetzung der FTI-Akteure selbst, die seitens der österreichischen Politik bei diesem Prozess der Selbstermächtigung (Empowerment) unterstützt werden. Dies ist insofern ein plausibler Zugang als die Beteiligung an den größeren Initiativen (z.B. strategische Partnerschaften) mit teils geringeren Förderquoten nur dann erfolgreich sein kann, wenn sie sehr eng an die strategischen Interessen der FTI-durchführenden Organisationen geknüpft sind.

Dieses Szenario setzt wiederum eine eigenständige Entwicklungsstrategie der Organisationen voraus sowie die Fähigkeit zur Selbstorganisation in schlagkräftige nationale Netzwerke. Die direkte und aktive Mitgestaltung europäischen FTI-Initiativen durch die FTICommunity benötigt darüber hinaus aber auch entsprechende Ressourcen und Kapazitäten, deren Mobilisierung durch die FTI-Politik unterstützt wird (z.B. durch finanzielle Anreize, Vernetzungsmaßnahmen, Informationsaustausch, etc.). Die Erfahrungen anderer europäischer Länder (z.B. Finnland, Niederlande, etc.) bei der Mobilisierung ihrer FTICommunities für die Mitgestaltung der EU FTI-Politik liefern hierfür Anstöße, die aber auch zeigen, dass es bei diesem Selbstermächtigungsprozess sowohl Gewinner als auch Verlierer geben kann.

Auf politischer Ebene erfordert dieses Szenario keine ex ante Festlegung auf prioritäre Themen, um die herum eine Mobilisierung der FTI-Community angestrebt wird, da sich diese aus dem Engagement und der effektiven Vernetzung der FTI-Akteure heraus ergeben. Dies ist auch die zentrale Herausforderung dieses Szenarios, nämlich durch entsprechende selektiv wirkende Anreize zu gewährleisten, dass sich in der tendenziell dispersen FTI-Community Österreichs derartige Schwerpunkte herausbilden können, die dann auch auf EU-Ebene wirkmächtig werden. Bisherige Erfahrungen haben gezeigt, dass es entweder Leitakteure geben muss, die die Mobilisierung von Netzwerken vorantreiben (z.B. einzelne starke Unis, COMET-Zentren, Forschungseinrichtungen oder Unternehmen) und/oder eine gestaltende Rolle der Politik, ggf. verankert in den Fachministerien und deren Aktivitäten (z.B. Silicon Austria Labs), um die Entstehung hinreichend großer Strukturen in Österreich zu ermöglichen. 


\section{Stoßrichtungen für die Weiterentwicklung der Unterstützungsstrukturen}

Ergänzend zu den bereits heute existierenden Unterstützungsleistungen und -strukturen verweist dieses Szenario auf die Notwendigkeit, die eigenverantwortliche Strategiefähigkeit der FTI-Akteure und ihre Fähigkeit zur nationalen und europäischen Vernetzung und kollektiven Strategiebildung mit den Mitteln der Politik und der FFG-EIP zu unterstützen. Dies legt Stoßrichtungen wie die folgenden nahe:

- Damit die österreichische FTI-Community auf europäischer Ebene eine aktive Gestaltungs- und Beteiligungsrolle einnehmen kann, ist der Kapazitätsaufbau nationaler FTI-Netzwerke voranzutreiben, was instrumentell über stärkere (finanzielle) Anreize aber auch durch gezielte Informations-, Vernetzungs- und Strategieaktivitäten umgesetzt werden könnte. Angesichts der Fokussierung auf eine Stärkung der FTI-Community und ihrer autonomen Vernetzung ist die interministerielle Koordination in diesem Szenario von weniger zentraler Bedeutung.

- Die EU Koordinationsabteilung fungiert dabei als zentraler Verbindungsknoten zwischen nationaler FTI-Community, Fachministerien und (politischen) EU-Gremien und flankiert den von der FTI-Community getragenen Ansatz zur Mitgestaltung und Mitwirkung an europäischen Initiativen. Eine angemessen dotierte Ausstattung der EU Koordinationsabteilung oder auch der Fachministerien mit Mitteln zur wettbewerblichen (Ko)finanzierung derartiger, von der FTI-Community getragener Aktivitäten würde es erlauben, diesen Mobilisierungsprozess voranzutreiben.

- Mit den so zur Verfügung gestellten Mitteln würde die FFG eigenverantwortlich Programme und Aktivitäten umsetzen können. Im Leistungsangebot der FFG-EIP verschiebt sich der Schwerpunkt hin zur Unterstützung von FTI-Akteuren bei der Beteiligung an größeren Initiativen wie den strategischen Partnerschaften, um beispielsweise die Zusammenarbeit zwischen nationalen FTI-Netzwerken mit europäischen Initiativen zu ermöglichen.

- Dies wird andere und ggf. zusätzliche Kompetenzen und Kapazitäten auf Seiten der FFG-EIP erfordern. Darüber hinaus würde die FFG-EIP über ein sehr hohes $\mathrm{Maß}$ an strategischer Autonomie verfügen müssen, um in Abstimmung mit der FTI-Community Unterstützungsleistungen zu entwickeln und bereitzustellen. Dabei wäre sie nur innerhalb eines vergleichsweise allgemein gehaltenen Rahmens inhaltlich-thematisch Vorgaben seitens der Fachministerien gebunden.

- Ähnlich wie im zweiten Szenario ist eine enge Verschränkung der nationalen Programme mit den europäischen Initiativen sinnvoll, hier allerdings ausgerichtet auf die von der FTI-Community vorangetriebenen Bereiche. Ein solcher Ansatz würde es nahelegen, auch innerhalb der FFG den Austausch zwischen den verschiedenen national und europäisch ausgerichteten Bereichen weiter zu intensivieren.

- Aufgrund der vergleichsweise schwach ausgeprägten Führungsfunktion seitens der Politik könnte sich ein hochrangiges Beratungsgremium in diesem Szenario stärker an die FTI-Community richten, um für diese eine übergreifende Orientierungsrolle zu übernehmen.

- Auch in diesem Modell wäre ein Verbindungsbüro in Brüssel eine wichtige sinnvolle Ergänzung. In diesem Szenario würde es aber im Wesentlichen von der FTICommunity selbst getragen. 


\section{Referenzen}

Biegelbauer, P. 2013. Wie lernt die Politik - Lernen aus Erfahrung in Politik und Verwaltung, VS Verlag, Wiesbaden, Deutschland

Biegelbauer, P. 2004. „Interessenvermittlung unter den Bedingungen der europäischen Integration: die Erstellung nationaler Positionen zum 5.Forschungsrahmenprogramm der EU in Österreich, den Niederlanden und Schweden", in: Österreichische Zeitschrift für Politikwissenschaft, Vol 33(2), pp. 137-156

Bundesministerium für Wissenschaft und Forschung. 2013. Antrag auf Einvernehmensherstellung: FFG Unterstützung österreichischer FTI-Akteure im Europäischen Forschungs- und Innovationsraum 2014 - 2020.

Bundesministerium für Bildung, Wissenschaft und Forschung. 2018. https://www.bmbwf.gv.at/forschung/europaeisch/rahmenprogramme/eu-performancemonitoring-fuer-fti/

BMWFW. 2013. Empfehlungen für die Tätigkeit von österreichischen Delegierten, Expertinnen und Experten in den programmbegleitenden Ausschüssen von HORIZON 2020.

BMWFW, BMVIT. 2017. Österreichischer Forschungs- und Technologiebericht 2017.

Engelhardt, S.; Künzel, M.; Ecker, B.; Schnabl, A. 2018. Evaluation des österreichischen Sicherheitsforschungsprogramms KIRAS Bericht 2017; Studie im Auftrag des Bundesministerium für Verkehr, Innovation und Technologie (BMVIT).

Enterprise Europe Network Austria. 2017. https://www.enterpriseeuropenetwork.at/

Eutema. 2016. Evaluierung des ERA Portal Austria, Wien.

FFG. 2013. Anbot zur Unterstützung österreichischer FTI-Akteure im Europäischen Forschungs- und Innovationsraum, 10. Juni 2013, finale Version.

FFG. 2014. Anbot zum EU-Performance Monitoring für FTI, Wien.

FFG. 2014. COSME Leistungsbeschreibung (ab 2014).

FFG. 2014. Leistungsbericht 2014 zur Unterstützung österreichischer FTI-Akteure im Europäischen Forschungs- und Innovationsraum 2014-2020.

FFG. 2015. COSME Leistungsbeschreibung 2015.

FFG. 2015. Jahresbericht COSME 2014.

FFG. 2015. Jahresbericht It. Rahmenvertrag - 2014, EUREKA inkl. Eurostars-Betreuung im EIP.

FFG. 2015. Leistungsbericht 2015 zur Unterstützung österreichischer FTI-Akteure im Europäischen Forschungs- und Innovationsraum 2014-2020.

FFG. 2016. COSME Leistungsbeschreibung 2016.

FFG. 2016. EU-Performance Monitoring für FTI, Arbeitsplan 2017, Wien. 
FFG. 2016. EU-Performance Monitoring für FTI: Leistungsbericht Juli 2014 bis Dezember 2015, Wien.

FFG. 2016. Jahresbericht COSME 2015.

FFG. 2016. Jahresbericht It. Rahmenvertrag - 2015, EUREKA inkl. Eurostars-Betreuung im EIP.

FFG. 2016. Jahresbericht It. Rahmenvertrag - 2016, EUREKA inkl. Eurostars-Betreuung im EIP.

FFG. 2016. Leistungsbericht 2016 zur Unterstützung österreichischer FTI-Akteure im Europäischen Forschungs- und Innovationsraum 2014-2020.

FFG. 2016. Organigramm EIP gesamt 2016.

FFG. 2017. EU-Performance Monitoring für FTI: Leistungsbericht 2016, Wien.

FFG. 2017. FFG-Akademie - Betrachtungen zur Wirkung, Internes Hintergrunddokument.

FFG. 2017. Jahresbericht COSME 2016.

FFG. 2017. Zwischenbilanz zur EIP-Programmevaluierung.

Fraunhofer ISI, KMU Forschung Austria. 2017. Evaluierung der Förderungsgesellschaften Austria Wirtschaftsservice (aws) und Forschungsförderungsgesellschaft mbH (FFG), Karlsruhe, Wien.

Österreichische Bundesregierung. 2011. Potenziale ausschöpfen, Dynamik steigern, Zukunft schaffen. Der Weg zum Innovation Leader. Strategie der Bundesregierung für Forschung, Technologie und Innovation, Wien

Pernicka, S., Feigl-Heihs, M., Gerstl, A., Biegelbauer, P. 2002. Wie demokratisch ist die europäischeForschungs- und Technologiepolitik? Der politische Entscheidungsprozess zum fünften Forschungsrahmenprogramm aus österreichischer Perspektive. Baden-Baden

Pichler, R., Stampfer, M., Hofer, R. 2007. Forschung, Geld und Politik. Die staatliche Forschungsförderung in Österreich 1945-2005, Innsbruck

Rechnungshof. 2014. Bericht des Rechnungshofes: Nationale Maßnahmen zum 7. Rahmenprogramm für Forschung, technologische Entwicklung und Demonstration, Wien.

Technopolis. 2010. Evaluation of Austrian Support Structures for FP 7 \& EUREKA and Impact Analysis of EU Research Initiatives on the Austrian Research \& Innovation System.

Technopolis. 2016. Die unterschiedliche Rolle von großen und kleinen Mitgliedstaaten im Kontext von HORIZON 2020.

Technopolis. 2017. Interim Evaluation of the COSME Programme.

Technopolis. 2017. Machbarkeitsstudie zur Etablierung eines österreichischen EU Verbindungsbüros im FTI-Bereich in Brüssel, Endbericht, Juli 2017, Wien.

Vertrag zur Beauftragung der FFG mit dem Monitoring der österreichischen Performance in den FTI-Aktivitäten der EU 2014 - 2021 abgeschlossen zwischen der Republik Österreich und der Österreichischen Forschungsförderungsgesellschaft mbH (FFG) 


\section{Annex I: Zielerreichung im Rahmen der WFA}

Im Rahmen der Beauftragung war auch die Zielerreichung der WFA zu FFG-EIP und H2020 zu überprüfen. Bis dato wurden alle Indikatoren zu diesen WFA für das Jahr 2017 erfasst und im Folgenden dargestellt.

Tabelle 12: Ziele und Zielerreichung der WFA FFG-EIP

\begin{tabular}{|c|c|c|c|}
\hline Ziel & $\begin{array}{l}\text { Ausgangszustand } \\
\text { Zeitpunkt der WFA }\end{array}$ & $\begin{array}{l}\text { Zielzustand } \\
\text { Evaluierungszeit- } \\
\text { punkt }\end{array}$ & Zielerreichung \\
\hline $\begin{array}{l}\text { Optimale Beteili- } \\
\text { gung österreichi- } \\
\text { scher Wissen- } \\
\text { schaftsorganisatio- } \\
\text { nen am H2020 und } \\
\text { den Initiativen zur } \\
\text { Verwirklichung des } \\
\text { Europäischen For- } \\
\text { schungsraums }\end{array}$ & $\begin{array}{l}\text { Europäischer For- } \\
\text { schungsraum (EFR) und } \\
\text { Binnenmarkt für For- } \\
\text { scher/innen ist noch } \\
\text { nicht umgesetzt. H2020 } \\
\text { ist Primärinstrument zur } \\
\text { Annäherung/Umsetzung } \\
\text { der EFR-Ziele: Die gro- } \\
\text { Ben wissenschaftlichen } \\
\text { Einrichtungen müssen } \\
\text { sich auf die veränderten } \\
\text { Fördermechanismen von } \\
\text { H2020 einstellen }\end{array}$ & $\begin{array}{l}\text { Ziel: mehr als die } \\
\text { Hälfte der Univer- } \\
\text { sitäten sollen über } \\
\text { Internationalisie- } \\
\text { rungsstrategien } \\
\text { verfügen. }\end{array}$ & $\begin{array}{l}\text { Erreicht: Die Universität } \\
\text { im Kontext des Europäi- } \\
\text { schen Forschungsraums } \\
\text { ist Teil der Leistungsver- } \\
\text { einbarungen 2016-2018. } \\
\text { In diesem Rahmen wur- } \\
\text { den für } 18 \text { der } 22 \text { öster- } \\
\text { reichischen Universitäten } \\
\text { Vorhaben und zugehörige } \\
\text { Meilensteine sowie Ziele } \\
\text { bzgl. Beteiligung und Po- } \\
\text { sitionierung (z.B. Anzahl } \\
\text { der Einreichungen in } \\
\text { H2020) in ERA definiert. }\end{array}$ \\
\hline $\begin{array}{l}\text { Verstärkte Beteili- } \\
\text { gung der Wirtschaft } \\
\text { entsprechend um- } \\
\text { gesetzter Innovati- } \\
\text { onsorientierung von } \\
\text { H2020; } 20 \% \text { Betei- } \\
\text { ligung der Kleineren } \\
\text { und Mittleren Unter- } \\
\text { nehmen (KMU) }\end{array}$ & $\begin{array}{l}\text { Die Beteiligung der Wirt- } \\
\text { schaft beträgt im } 7 . \text { EU- } \\
\text { Rahmenprogramm der- } \\
\text { zeit in Österreich } 26 \% \\
\text { (7\% große Unterneh- } \\
\text { men, } 19 \% \text { Kleinere und } \\
\text { Mittlere Unternehmen). }\end{array}$ & $\begin{array}{l}\text { Verstärktes Betei- } \\
\text { ligungsinteresse } \\
\text { der Wirtschaft an } \\
\text { H2020; rund } 28 \% \\
\text { Beteiligung Wirt- } \\
\text { schaft gemessen } \\
\text { an der österreichi- } \\
\text { schen Gesamtbe- } \\
\text { teiligung nach Or- } \\
\text { ganisationskate- } \\
\text { gorien bis zum } \\
\text { Jahr } 2017\end{array}$ & $\begin{array}{l}\text { Erreicht: Unternehmen } \\
\text { stellen 38\% der österrei- } \\
\text { chischen Beteiligungen } \\
\text { (Daten Cockpitbericht EU- } \\
\text { PM November 2017). Das } \\
\text { sind } 717 \text { erfolgreiche Un- } \\
\text { ternehmensbeteiligungen } \\
\text { in H2020 }\end{array}$ \\
\hline $\begin{array}{l}\text { Optimale Grundla- } \\
\text { gen für eine strate- } \\
\text { gische Herange- } \\
\text { hensweise und Prio- } \\
\text { ritätensetzung be- } \\
\text { züglich der österrei- } \\
\text { chischen Beteili- } \\
\text { gung }\end{array}$ & $\begin{array}{l}\text { Auf europäischer Ebene } \\
\text { gibt es mittlerweile eine } \\
\text { Vielzahl unterschied- } \\
\text { lichster Initiativen und } \\
\text { Programme im Bereich } \\
\text { Forschung, Technologie } \\
\text { und Innovation (FTI) mit } \\
\text { multilateralem Ansatz } \\
\text { (MULLAT). Die Heraus- } \\
\text { forderung ist: Dieses } \\
\text { vielfältige internationale }\end{array}$ & $\begin{array}{l}\text { Mobilisierung und } \\
\text { Information der } \\
\text { Kund/innen in Be- } \\
\text { zug auf MULLAT z. } \\
\text { B. durch erklä- } \\
\text { rende Darstellun- } \\
\text { gen/von der Pra- } \\
\text { xis abgeleitete } \\
\text { Modelle/Erfolgs- } \\
\text { geschichten auf } \\
\text { der Website, e- }\end{array}$ & $\begin{array}{l}\text { Erreicht: Die Website in- } \\
\text { formiert auf einer eigenen } \\
\text { Seite über die verschiede- } \\
\text { nen Arten der MULLATs. } \\
\text { EIP führte eine Reihe von } \\
\text { Informationsveranstaltun- } \\
\text { gen und Aussendungen zu } \\
\text { MULLATs (z.B. zu BBI JTI, } \\
\text { JTI Shift2Rail, JPI Cli- } \\
\text { mate, ERA-NETs, etc.) } \\
\text { durch. Darüber hinaus in- } \\
\text { formieren ein Bericht über }\end{array}$ \\
\hline
\end{tabular}




\begin{tabular}{|c|c|c|c|}
\hline Ziel & $\begin{array}{l}\text { Ausgangszustand } \\
\text { Zeitpunkt der WFA }\end{array}$ & $\begin{array}{l}\text { Zielzustand } \\
\text { Evaluierungszeit- } \\
\text { punkt }\end{array}$ & Zielerreichung \\
\hline & $\begin{array}{l}\text { Angebot in österreichi- } \\
\text { schen Forschungsorgani- } \\
\text { sationen gezielt zu nut- } \\
\text { zen um Stärkefelder der } \\
\text { jeweiligen Forschungsor- } \\
\text { ganisation (weiter) zu } \\
\text { entwickeln. }\end{array}$ & $\begin{array}{l}\text { Newsletter, spezi- } \\
\text { fische Veranstal- } \\
\text { tungen, Aussen- } \\
\text { dungen. }\end{array}$ & $\begin{array}{l}\text { die Governancestrukturen } \\
\text { und österreichische Betei- } \\
\text { ligung in MULLATs, sowie } \\
\text { eigene MULLAT Kapitel in } \\
\text { den ERA-Themendossiers } \\
\text { und FFG-Themenberich- } \\
\text { ten. }\end{array}$ \\
\hline $\begin{array}{l}\text { Hohe Kund/innen- } \\
\text { zufriedenheit bei } \\
\text { den Zielgruppen be- } \\
\text { züglich der Leistun- } \\
\text { gen der Toolbox; } \\
\text { Nutzen der zentra- } \\
\text { len neuen Bera- } \\
\text { tungsinstrumente } \\
\text { (ERA-Dialog, Be- } \\
\text { treuung Key Player } \\
\text { Wirtschaft) }\end{array}$ & $\begin{array}{l}\text { Kund/innenzufrieden- } \\
\text { heit: Evaluierung der } \\
\text { Services } 2010 \text { des Be- } \\
\text { reichs } 4 \text { der Österreichi- } \\
\text { schen Forschungsförde- } \\
\text { rungsgesellschaft "Euro- } \\
\text { päische und Internatio- } \\
\text { nale Programme "Con- } \\
\text { sulting, advice on future } \\
\text { trends in the Framework } \\
\text { Programmes": } 75 \% \text { wa- } \\
\text { ren damit zufrieden }\end{array}$ & $\begin{array}{l}\text { Verbesserung der } \\
\text { Kund/innenzufrie- } \\
\text { denheit um } 3 \% \\
\text { Punkte bis } 2017\end{array}$ & $\begin{array}{l}\text { Nicht erreicht: Basierend } \\
\text { auf der Survey waren ins- } \\
\text { gesamt } 386 \text { Personen zu- } \\
\text { frieden mit den EIP Leis- } \\
\text { tungen (Note Sehr Gut o- } \\
\text { der Gut). Das sind } 76 \% \\
\text { der Befragten }(\mathrm{N}=508) \text {. }\end{array}$ \\
\hline $\begin{array}{l}\text { European Research } \\
\text { Area Dialoge (ERA- } \\
\text { Dialoge) }\end{array}$ & $\begin{array}{l}\text { Anzahl der ERA-Dialoge } \\
\text { im Jahr 2013: } 2\end{array}$ & $\begin{array}{l}\text { Insg. } 12 \text { laufende } \\
\text { ERA-Dialoge bis } \\
2017\end{array}$ & $\begin{array}{l}\text { Erreicht: Insgesamt } 12 \\
\text { laufende ERA-Dialoge mit } \\
\text { Universitäten und } 2 \text { mit } \\
\text { außeruniversitären For- } \\
\text { schungsorganisationen. } \\
\text { Ende } 2017 \text { kamen zusätz- } \\
\text { lich eine Universität und } \\
\text { eine Forschungseinrich- } \\
\text { tung neu hinzu. } 91\end{array}$ \\
\hline $\begin{array}{l}\text { European Research } \\
\text { Area Dialoge (ERA- } \\
\text { Dialoge) }\end{array}$ & $\begin{array}{l}\text { Rund } 3.000 \text { Beratungen } \\
\text { für den Wissenschafts- } \\
\text { bereich im Jahresdurch- } \\
\text { schnitt zum RP7 }\end{array}$ & $\begin{array}{l}\text { Insg. 30\% weni- } \\
\text { ger First Level Be- } \\
\text { ratungen }\end{array}$ & $\begin{array}{l}\text { Der Anteil der First Level } \\
\text { Beratungen der Forschen- } \\
\text { den ist rückläufig und lag } \\
\text { im Jahr } 2014 \text { bei rund } \\
25 \% \text {, sank } 2015 \text { auf } 19 \% \\
\text { und } 2016 \text { auf } 17 \% \text {. Im } \\
\text { Jahr } 2017 \text { liegt der Anteil } \\
\text { bei } 19 \% \text {. Der Anteil der } \\
\text { First Level Beratungen } \\
\text { der Forschungsservice- } \\
\text { stellen schwankt zwischen } \\
12 \% \text { in } 2014,15 \% \text { im } \\
\text { Jahr } 2015,13 \% \text { im Jahr }\end{array}$ \\
\hline
\end{tabular}

91 FFG (2017), ERA-Dialog: Zusammenfassung Vorbereitung Evaluierung 


\begin{tabular}{|c|c|c|c|}
\hline Ziel & $\begin{array}{l}\text { Ausgangszustand } \\
\text { Zeitpunkt der WFA }\end{array}$ & $\begin{array}{l}\text { Zielzustand } \\
\text { Evaluierungszeit- } \\
\text { punkt }\end{array}$ & Zielerreichung \\
\hline & & & $\begin{array}{l}2016 \text { und } 24 \% \text { im Jahr } \\
2017.92\end{array}$ \\
\hline $\begin{array}{l}\text { Beratung für Klei- } \\
\text { nere und Mittlere } \\
\text { Unternehmen } \\
(\mathrm{KMU})\end{array}$ & $\begin{array}{l}\text { Beratungen von Kleine- } \\
\text { ren und Mittleren Unter- } \\
\text { nehmen im 7. EU-Rah- } \\
\text { menprogramm im Jahr } \\
\text { 2012: } 1.200 \text { (repräsen- } \\
\text { tatives Jahr des 7. Rah- } \\
\text { menprogramms) }\end{array}$ & $\begin{array}{l}\text { Steigerung der } \\
\text { Beratungszahlen } \\
\text { für Kleinere und } \\
\text { Mittlere Unterneh- } \\
\text { men (KMU): um } \\
\text { insg. } 20 \%\end{array}$ & $\begin{array}{l}\text { Erreicht: Beratungszahlen } \\
\text { der Zielgruppe KMU lagen } \\
2014 \text { bei } 1800,2015 \text { bei } \\
1550,2016 \text { bei } 1542^{93} \\
\text { und } 2017 \text { bei } 1630 .{ }^{94} \text { Das } \\
\text { ist eine durchschnittliche } \\
\text { Steigerung der KMU Bera- } \\
\text { tung um } 35 \% .{ }^{95}\end{array}$ \\
\hline $\begin{array}{l}\text { Multilaterale Pro- } \\
\text { gramme/Initiativen } \\
\text { (MULLAT) - Service } \\
\text { und Beratung }\end{array}$ & $\begin{array}{l}\text { Ausgangsbasis 2012: } 10 \\
\text { Langberatungen }\end{array}$ & $\begin{array}{l}\text { bis 2017: } 120 \text { do- } \\
\text { kumentierte Ser- } \\
\text { vice und Bera- } \\
\text { tungsleistungen }\end{array}$ & $\begin{array}{l}\text { Erreicht: Insgesamt } 1243 \\
\text { Beratungen zu MULLATs, } \\
\text { davon } 285 \text { Langberatun- } \\
\text { gen }^{96}\end{array}$ \\
\hline $\begin{array}{l}\text { Multilaterale Pro- } \\
\text { gramme/Initiativen } \\
\text { (MULLAT) - Service } \\
\text { und Beratung }\end{array}$ & $\begin{array}{l}\text { bisher noch keine Analy- } \\
\text { sen und Berichte (neue } \\
\text { Aktivität) }\end{array}$ & $\begin{array}{l}35 \text { Analysen und } \\
\text { Berichte zu den } \\
\text { MULLAT }\end{array}$ & $\begin{array}{l}\text { Kumuliert 2014-2017: } \\
\text { Insgesamt } 44 \text { MULLAT } \\
\text { Analysen/Berichte }^{97}\end{array}$ \\
\hline $\begin{array}{l}\text { Neue Beratungsin- } \\
\text { strumente }\end{array}$ & $\begin{array}{l}\text { es gibt noch keine Feed- } \\
\text { backgespräche (neue } \\
\text { Aktivität) }\end{array}$ & $\begin{array}{l}\text { dokumentierte } \\
\text { Feedbackgesprä- } \\
\text { che; Ergebnis } \\
\text { "gut" nach Schul- } \\
\text { notensystem }\end{array}$ & $\begin{array}{l}\text { Erreicht: Dokumentation } \\
\text { im Rahmen der ERA-Dia- } \\
\text { log Gesprächsprotokolle } \\
\text { (qualitativ); Feedback auf } \\
\text { 10-teiliger Skala ergibt } \\
\text { "gut" nach Schulnoten- } \\
\text { system. C-3 Betreuung: } \\
\text { Insgesamt wurden über } \\
\text { das Jahr } 201720 \text { Unter- } \\
\text { nehmen betreut. Alle bis- } \\
\text { herigen Ergebnisse der } \\
\text { Kundenzufriedenheit } \\
\text { ergaben umgesetzt min- } \\
\text { destens den Wert "gut". } \\
\text { Feedback } 2017 \text { wird in } \\
\text { Q1/2018 eingeholt. } 98\end{array}$ \\
\hline
\end{tabular}

92 FFG (2017), WFA-Ziele EIP Beauftragung - Ergebnisse 2017

93 FFG (2017), Übersicht zu den Zielen/Indikatoren/Ergebnissen aus den LB 2014-2016

94 FFG (2017), WFA-Ziele EIP Beauftragung - Ergebnisse 2017

95 FFG (2017), Übersicht zu den Zielen/Indikatoren/Ergebnissen aus den LB 2014-2016

96 FFG (2017), WFA-Ziele EIP Beauftragung - Ergebnisse 2017

97 FFG 2017), WFA-Ziele EIP Beauftragung - Ergebnisse 2017

98 FFG (2017), WFA-Ziele EIP Beauftragung - Ergebnisse 2017 
Tabelle 13: Ziele und Zielerreichung der WFA H2020

\begin{tabular}{|c|c|c|c|}
\hline Ziel & $\begin{array}{l}\text { Ausgangszustand } \\
\text { Zeitpunkt der WFA }\end{array}$ & $\begin{array}{l}\text { Zielzustand Evaluie- } \\
\text { rungszeitpunkt }\end{array}$ & Zielerreichung \\
\hline $\begin{array}{l}\text { Grundlagen- bzw. Pio- } \\
\text { nierforschung in Ös- } \\
\text { terreich soll durch die } \\
\text { themenoffene, allein } \\
\text { dem } \\
\text { Ekzellenzkritenum } \\
\text { verpflichtete Förde- } \\
\text { rung von im internati- } \\
\text { onalen Wettbewerb } \\
\text { herausragenden } \\
\text { Forscher/innen im } \\
\text { Rahmen des 'Europäi- } \\
\text { schen Forschungsra- } \\
\text { tes vorangetrieben } \\
\text { werden. }\end{array}$ & $\begin{array}{l}\text { Spitzenforschungs- } \\
\text { preise durch den Eu- } \\
\text { ropäischen For- } \\
\text { schungsrat: } 68 \text { Grants } \\
\text { (Status bis } 2011 \text { ) }\end{array}$ & $\begin{array}{l}\text { Spitzenforschungssti- } \\
\text { pendien durch den } \\
\text { Europäischen For- } \\
\text { schungsrat: } 70 \text { Sti- } \\
\text { pendien (Zwischenziel } \\
\text { bis } 2013 \text { ); insgesamt } \\
140 \text { Stipendien bis } \\
2020\end{array}$ & $\begin{array}{l}\text { Zwischenstand Sep- } \\
\text { tember 2017: } 87 \text { be- } \\
\text { willigte ERC Projekte } \\
\text { (Daten: EU-PM Cock- } \\
\text { pitbericht November } \\
2017 \text { ) }\end{array}$ \\
\hline $\begin{array}{l}\text { Das Wachstumspoten- } \\
\text { zial der österreichi- } \\
\text { schen Unternehmen } \\
\text { soll durch Investitio- } \\
\text { nen in grundlegende } \\
\text { und industrielle Tech- } \\
\text { nologien gestärkt } \\
\text { werden. Dies umfasst } \\
\text { die Bereiche IKT, Na- } \\
\text { notechnologie, fortge- } \\
\text { schrittene Werkstoffe, } \\
\text { Biotechnologie, fort- } \\
\text { geschrittene Fertigung } \\
\text { und Verarbeitung so- } \\
\text { wie Raumfahrt. }\end{array}$ & $\begin{array}{l}\text { Neuer Förderansatz, } \\
\text { daher keine Aus- } \\
\text { gangsbasis möglich }\end{array}$ & $\begin{array}{l}\text { Drei Patentanmeldun- } \\
\text { gen pro } 10 \text { Mio. Euro } \\
\text { Förderung bis } 2020\end{array}$ & $\begin{array}{l}\text { Bis } 1 / 09 / 2016 \text { (inclu- } \\
\text { ding grants to named } \\
\text { beneficiaries) erfolg- } \\
\text { ten im gesamten } \\
\text { Spektrum von } \mathrm{H} 2020 \\
\text { insgesamt } 109 \mathrm{~Pa}- \\
\text { tentanmeldungen und } \\
29 \text { bewilligte Patente } \\
\text { über alle EU-MS hin- } \\
\text { weg. }{ }^{99} \text { Zahlen für AT } \\
\text { Patente liegen nicht } \\
\text { vor. Aufgrund der Ge- } \\
\text { samtanzahl der bisher } \\
\text { erfolgten Anmeldun- } \\
\text { gen scheint der Indi- } \\
\text { kator derzeit nicht re- } \\
\text { alistisch. }\end{array}$ \\
\hline $\begin{array}{l}\text { Unter Nutzung des } \\
\text { gesamten Förderzyk- } \\
\text { lus von der Forschung } \\
\text { bis zur Vermarktung } \\
\text { sollen Beiträge zur Lö- } \\
\text { sung gesellschaftli- } \\
\text { cher Herausforderun- } \\
\text { gen geleistet werden, }\end{array}$ & $\begin{array}{l}\text { Neuer Förderansatz, } \\
\text { daher keine Aus- } \\
\text { gangsbasis möglich }\end{array}$ & $\begin{array}{l}\text { Durchschnittlich eine } \\
\text { regulatorische Umset- } \\
\text { zungsmaßnahme zur } \\
\text { Lösung gesellschaftli- } \\
\text { cher Herausforderun- } \\
\text { gen in Österreich pro } \\
10 \text { Mio. Euro Förde- } \\
\text { rung bis } 2020\end{array}$ & $\begin{array}{l}\text { Indikator in dieser } \\
\text { Weise voraussichtlich } \\
\text { nicht vorhanden. }\end{array}$ \\
\hline
\end{tabular}

99 Europäische Kommission (2016), Horizon 2020 Annual Monitoring Report 2015 


\begin{tabular}{|c|c|c|c|}
\hline Ziel & $\begin{array}{l}\text { Ausgangszustand } \\
\text { Zeitpunkt der WFA }\end{array}$ & $\begin{array}{l}\text { Zielzustand Evaluie- } \\
\text { rungszeitpunkt }\end{array}$ & Zielerreichung \\
\hline \multicolumn{4}{|l|}{$\begin{array}{l}\text { die für Österreich pri- } \\
\text { oritär sind. Es handelt } \\
\text { sich dabei um die in } \\
\text { der österreichischen } \\
\text { FTI-Strategie identifi- } \\
\text { zierten Herausforde- } \\
\text { rungen "Klimawan- } \\
\text { del", "Knappe Res- } \\
\text { sourcen" sowie "Le- } \\
\text { bensqualität und de- } \\
\text { mographischer Wan- } \\
\text { del". }\end{array}$} \\
\hline $\begin{array}{l}\text { Die vereinfachten Ver- } \\
\text { fahren und Regeln } \\
\text { von H2020 sollen } \\
\text { dazu beitragen, dass } \\
\text { im Bereich Forschung } \\
\text { die ausgezahlten ös- } \\
\text { terreichischen Rück- } \\
\text { flüsse weiterhin über } \\
\text { dem Wert der öster- } \\
\text { reichischen Eigenmit- } \\
\text { telzahlungen zum EU- } \\
\text { Haushalt liegen }\end{array}$ & $\begin{array}{l}\text { Anteil der österreichi- } \\
\text { schen Eigenmittelzah- } \\
\text { lungen am EU-Budget } \\
2011: 2.24 \% \text { / Anteil } \\
\text { der ausgezahlten ös- } \\
\text { terreichischen Rück- } \\
\text { flüsse am EU-Budget } \\
\text { im Bereich Forschung } \\
\text { im Jahr 2011: } 2.50 \% \\
\text { (das entspricht } 146.5 \\
\text { Mio. Euro) }\end{array}$ & $\begin{array}{l}\text { Anteil der österreichi- } \\
\text { schen Eigenmittelzah- } \\
\text { lungen am EU-Budget } \\
\text { 2020: } 2.40 \% \text { / Anteil } \\
\text { der ausgezahlten ös- } \\
\text { terreichischen Rück- } \\
\text { flüsse am EU-Budget } \\
\text { im Bereich Forschung } \\
\text { im Jahr 2020: } 2.50 \% \\
\text { (das entspricht } 380 \\
\text { Mio. Euro) }\end{array}$ & $\begin{array}{l}\text { Der Anteil der öster- } \\
\text { reichischen Rückflüsse } \\
\text { am EU-Budget im Be- } \\
\text { reich Forschung im } \\
\text { Jahr } 2016 \text { lag bei } \\
2.76 \% \text { (das entspricht } \\
237.83 \text { Mio. Euro). } \\
\text { Kumuliert über den } \\
\text { Zeitraum 2014-2016 } \\
\text { betrachtet liegt der } \\
\text { Anteil der österreichi- } \\
\text { schen Rückflüsse bei } \\
2.69 \% \text { ( } 573.16 \text { Mio. } \\
\text { Euro) }{ }^{100}\end{array}$ \\
\hline $\begin{array}{l}\text { Im Nuklearfor- } \\
\text { schungsbereich soll } \\
\text { die Neuorientierung } \\
\text { der Kernspaltungsfor- } \\
\text { schung in Richtung ei- } \\
\text { ner konsequenten } \\
\text { Konzentration auf Si- } \\
\text { cherheitsfragen fort- } \\
\text { gesetzt werden. Au- } \\
\text { Berdem sollen die } \\
\text { Kosten für ITER kei- } \\
\text { nesfalls den im EU-Fi- } \\
\text { nanzrahmen vorgege- } \\
\text { benen Höchstbetrag } \\
\text { überschreiten }\end{array}$ & $\begin{array}{l}\text { ITER-Budget 2007- } \\
\text { 2011: 1.047 Mrd. } \\
\text { Euro / 2012-2013: } \\
\text { 1.3 Mrd. Euro }\end{array}$ & $\begin{array}{l}\text { ITER-Budget 2014- } \\
\text { 2020: } 2.7 \text { Mrd. Euro } \\
\text { (Preise 2011) }\end{array}$ & $\begin{array}{l}\text { Indikator nicht ver- } \\
\text { fügbar zum Zeitpunkt } \\
\text { der Evaluierung }\end{array}$ \\
\hline
\end{tabular}

100 Daten von EU-PM basierend auf EC http://ec.europa.eu/budget/figures/interactive/index en.cfm 


\section{Komplementäre Beschreibungen zur WFA}

\section{Die erwarteten Wirkungen des Gesamtvorhaben}

Die Beauftragung des Bereichs für Europäische und Internationale Programme der FFG (FFG-EIP) mit der Unterstützung der Umsetzung von H2020 und ERA soll zu einer optimalen Beteiligung österreichischer Wissenschafts- und Wirtschaftsorganisationen im europäischen Rahmenprogramm und in multilateralen FTI-Initiativen (MULLATs) beitragen. Die Beteiligung der Unternehmen, inklusive KMUs, soll entsprechend der stärkeren Innovationsorientierung von $\mathrm{H} 2020$ gestärkt und Universitäten bei der strategischen Positionierung und Profilbildung in ERA unterstützt werden. Darüber hinaus sollte die Beauftragung beitragen eine optimale Grundlage für eine strategische Herangehensweise und Prioritätensetzung bezüglich der österreichischen Beteiligung an den MULLATs zu schaffen. Dies soll mit einer hohen Zufriedenheit der FFG-EIP KundInnen mit der Qualität und Relevanz der Beratungs- und Betreuungsinstrumente einhergehen.

\section{Narrative Gesamtbeurteilung des Erfolgs des Vorhabens}

Die Beauftragung stützt sich auf das in Europa anerkannte Konzept einer gemeinsamen Servicestelle für Wissenschaft, Wirtschaft, Verwaltung und Intermediäre in Forschung, Technologie und Innovation, die mit hoher Kompetenz und Erfahrungen aus bisherigen Beauftragungen sowie ihre eigene starke fachliche Vernetzung die Beteiligung Österreichs begleitet hat. Der Bereich Europäische und Internationale Programme der FFG baut in Hinblick auf die Unterstützung der FTI-Akteure auf den Kompetenzen und gesammelten Erfahrungen vorheriger Beauftragungen auf und bietet ein breites Spektrum an Instrumenten zur Bewusstseinsbildung und Informationsvermittlung, Programm- und Projektberatung und strategischen Beratung und ERA-Orientierungswissen.

Die stärkere Innovationsorientierung von H2020 und die wachsende Anzahl der multilateralen Initiativen ging mit einer entsprechenden Veränderung des Leistungsportfolios der laufenden EIP-Beauftragung einher (siehe auch Zwischenbericht Kapitel 2.1): Wissenschafts- und Wirtschaftsorganisationen sollen in ihren Strategie- und Positionierungsprozessen so unterstützt werden, dass Sie in der Lage sind europäische Programme und ERA-Entwicklungen mit maximaler Hebelwirkung für eigene Zielsetzungen einsetzen zu können. Das Kooperationsprofil mit den Unterstützungsstrukturen (insbesondere) an Universitäten soll so ausgerichtet werden, dass mittelfristig Erstanfragen und Projektabwicklungsfragen verstärkt organisationsintern abgewickelt werden können. Unternehmen und KMU sollen aufgrund der stärkeren Innovationsorientierung von H2020 stärker betreut werden. ERA-Initiativen und Programmen sollen ins Leistungsportfolio von FFG-EIP aufgenommen und die Governance Strukturen von MULLATs sollen unterstützt werden. FFGEIP soll auch den Ausbau der strategischen Intelligenz im Sinne von Zusammenschau und Überblick bieten.

Die Ziele und erwünschten Wirkungen wurden weitgehend erreicht: Die Anzahl der Beratungen für KMUs stieg im Vergleich zu FP7 deutlich, durchschnittlich um 35\%. Die Beteiligung der Wirtschaft ist im Vergleich zum 7. Rahmenprogramm auch deutlich gestiegen, Unternehmen stellten in FP7 26\% der österreichischen Beteiligung dar, in H2O20 sind es bis 2017 38\% (siehe Zwischenbericht Annex I). Durch das neue Betreuungsinstrument für Wissenschaftsorganisationen „ERA-Dialog" scheint vor allem eine direkte Verbindung 
mit den Vize-Rektoraten hergestellt worden zu sein, jedoch kann noch nicht endgültig beantwortet werden ob die Arbeit der FFG dazu beigetragen hat, Strategiebildungsprozesse in Hinblick auf H2020 und ERA zu stärken. Die oft fehlende Verbindung zwischen der strategischen und operativen Ebene der Universitäten selbst (Forschung findet „bottomup" statt) und die damit einhergehende Governance Problematik der Universitäten liegt aber jenseits der Möglichkeiten der FFG. Das Instrument selbst und das interaktive Format wurde überwiegend positiv bewertet, der Nutzen lag aus Sicht der teilnehmenden Universitäten insbesondere in der Außensicht auf die Organisation sowie der Zeit für gemeinsame Reflexion (siehe Zwischenbericht „Erkenntnisse zu den ERA-Dialogen"). Ähnlich ist die Erkenntnis bei Unternehmen: Kunden des C3 weisen auf positive Effekte der Betreuung für die Strategiefindung hin, jedoch kann noch nicht endgültig bewertet werden inwiefern sich diese positiv auf das „Empowerment" ausgewirkt hat (siehe Zwischenbericht „Erkenntnisse zum Core Customer Concept der FFG"). Das Ziel des „Empowerment" der Universitäten wurde nur teilweise erreicht, ist aber auf einem guten Weg: Der Anteil der First Level Beratungen der Forschenden ist rückläufig und lag im Jahr 2014 bei 25\% und sank 2017 auf 19\%. Die Neuausrichtung der Dienstleistungen sei für die Universitäten spürbar gewesen, jedoch muss der interne Support erst aufgestockt werden und in Gesprächen mit Universitäten wurde die persönliche Beratungsleistung der FFG in dieser Übergangsphase als unersetzbar eingeschätzt (siehe Zwischenbericht Kapitel 2.2.3 und Annex I). Auch das Leistungsportfolio insgesamt wird von FTI-Akteuren in Hinblick auf Qualität und Relevanz sehr geschätzt - 76\% der Befragten bewerteten das Angebot mit Schulnote "Sehr gut" oder "gut" (siehe Zwischenbericht Annex I). Verglichen zur vorherigen Beauftragung wurden sehr viele neue Beratungs- und Betreuungsinstrumente (Aufnahme der MULLATs ins Leistungsportfolio, ERA-Dialog, C3, etc.) bei gleichem Personalstand entwickelt. Auch die Budgetallokation des EIP insgesamt scheint in Anbedacht der österreichischen Beteiligungsstruktur in H2020 angemessen zu sein (siehe Zwischenbericht Kapitel 2.4).

\section{Welche Verbesserungspotentiale sind bei der Durchführung der Evaluierung er- sichtlich geworden?}

Die Ergebnisse der Evaluierung weisen auf Potenziale zur verbesserten Darstellung und Strukturierung des Informations- und Beratungsangebotes von FFG-EIP hin, vor allem wurde im Rahmen der Befragung die Unübersichtlichkeit des gesamten Leistungsportfolios und der Homepage kritisiert. Angedacht werden könnte eine Neustrukturierung des Dienstleistungsspektrums entlang spezifischer Bedarfslagen und Vorkenntnissen von Akteursgruppen. In Gesprächen wurde darüber hinaus auf potentielle Verbesserungsmöglichkeiten bezüglich der Geschwindigkeit der Weitergabe von Informationen und der Verbesserung der strategischen Positionierung der FFG hingewiesen (siehe Zwischenbericht Kapitel 2.2.3). Es gibt auch Anzeichen dafür, dass der Einfluss des Angebotes auf strategische Verhaltensänderungen und „Empowerment" noch in einer Entwicklungsphase zu sein scheint. Die Evaluierung zeigt möglichen Änderungsbedarf in Hinblick auf Ausrichtung und Umsetzung einzelner Beratungsinstrumente (ERA-Dialoge und C3) um das strategische "Empowerment" der Universitäten und Unternehmen zu beschleunigen. Die FFG verfügt aber hierfür eine gute Basis an Beratungsleistungen, die Intensität und die Ausrichtung der Leistungen, speziell die für die Forschungsservicestellen, könnten aber geschärft werden (siehe Zwischenbericht Kapitel 2.2.3 und Kapitel 2.3.4). 


\section{Auswirkungen auf Internationalisierung}

Im Zeitraum 2014 bis einschließlich September 2017 haben 445 Organisationen mit KMU-Einstufung, das sind $25 \%$ an allen österr. Beteiligungen, und 343 Unternehmen, die sich nicht als KMU einstufen, das sind 19\% an allen österr. Beteiligungen, an H2020 teilnehmen. Auf die Gesamtlaufzeit von H2020 extrapoliert können ca. 900 KMU Beteiligungen und 700 Beteiligungen von Großunternehmen erwartet werden ${ }^{101}$.

Der Mehrwert der Teilnahme an H2020 für Unternehmen liegt weniger im finanziellen Beitrag der EU, sondern darin - über europäisch finanzierte Projekte mit wissenschaftlichen und UnternehmenspartnerInnen im Ausland zu kooperieren und damit die Vernetzung innerhalb Europas zu intensivieren.

Die Interim-Evaluierung von H2020 zeigt, dass Fördermittel für die Projektteilnehmer hochgradig additional sind und für die Mitgliedsstaaten und die Teilnehmenden Orgnisationen einen Mehrwert schaffen, der über die Förderung nationaler und regionaler Programme hinausgeht (Interim Evaluation H2020, p.214).

Insbesondere in den Bereichen "Industrial Leadership" in dem eine hohe Beteiligung des privaten Sektors vorliegt, hätten $92 \%$ der Projekte gar nicht, oder nur mit bedeutenden Veränderungen durchgeführt werden können. Für 66\% der H2020 Teilnehmenden wäre der internationale Wissenstransfer ohne H2020 geschwächt worden und 72\% hätten negative Auswirkungen auf Kooperationen mit der Industrie und neuen UnternehmenspartnerInnen innerhalb der europäischen Union in Kauf nehmen müssen (Interim Evaluation H2020, p. 223).

\section{Auswirkungen auf die Innovationsfähigkeit}

Die auf EU-Ebene eingesetzten Instrumentarien decken ein breites Spektrum von der Grundlagenforschung bis hin zur Innovationsförderung ab. H2020 legt im Vergleich zum 7. Rahmenprogramm für Forschung und Entwicklung eine höhere Innovations- und Wirkungsorientierung bereits im Programmdesign aufgrund 1) einer Orientierung an großen (gesellschaftlichen) Herausforderungen, 2) Finanzierungsmöglichkeiten vom Labor bis zum Markt und 3) einer integrierten Wirkungsorientierung in Projektanträgen, Reporting und Monitoring (vgl. Interim Evaluation H2020, p. 22).

Die Bedeutung der Rahmenprogramme als Finanzierungsquelle für FuE in Europa steigt, obwohl die nationalstaatliche Finanzierung noch immer deutlich höher ist. Eine Stärkung der Elemente des Wissens- und Erkenntnistransfers hin zur Steigerung der Innovationsfähigkeit ist besonders im Instrumentenportfolio evident, durch die Stärkung umsetzungsorientierter Instrumente in $\mathrm{H} 2020$. Sie spiegelt sich auch in der stärkeren Integration von KMUs in die Säulen II und III von H2020. Nicht zuletzt zeigt sich die Stärkung des Wissens- und Erkenntnistransfers in einem höheren Anteil von Unternehmen an der Gesamtzahl der AntragstellerInnen und ProjektpartnerInnen. Textmining Analysen, die im Rahmen der Interim Evaluierung von H2020 durchgeführt wurden (p.56), zeigen nicht nur in den Säulen II und III sondern auch in den Bereichen der Future und Emerging

\footnotetext{
101 Extrapolation unter der Annahme, dass im 1. Jahr von H2020 die Anzahl der Beteiligungen
} $50 \%$ unter den Folgejahren lag. 
Technologies einen hohen Beitrag zur Stützung der Innovationsfähigkeit von Unternehmen.

Zur stärkeren Innovationsfinanzierung bietet InnovFin über die EIP passende Finanzierungsinstrumente für Forschung und Innovation - für kleine und mittlere Unternehmen (KMU) ebenso wie für Großunternehmen oder Forschungsinstitute. Die InnovFin-Produkte sind für alle förderfähigen Sektoren verfügbar. Sie wurden für H2020 entwickelt und können in den EU-Mitgliedstaaten und in den mit H2020 assoziierten Ländern in Anspruch genommen werden. In Österreich haben bislang 105 KMU bzw. Mid-Caps 120,4 Mio EUR in Anspruch genommen. 


\section{Annex II: Weiterentwicklung der WFA}

Die Erhebung der Zielerreichung im Rahmen der WFA zur EIP-Beauftragung, WFA H2020, WFA Eurostars 2 und WFA COSME sowie die Prüfung der Zweckmäßigkeit der ausgewählten Indikatoren zur Wirkungsmessung sind Teile des Evaluationsauftrages. Im Rahmen der Zwischenberichtslegung wurden die WFA-Indikatoren für das Berichtsjahr 2017 erhoben und im Anschluss daran einer kritischen Würdigung unterzogen. Das Ziel des Reviews ist die Weiterentwicklung des Indikatorensystems im Sinne einer Reduktion und einer Anpassung der einzelnen Indikatoren.

\section{Anpassung der WFA EIP-Indikatoren}

Das Ziel- und Indikatorensystem zwischen WFA EIP und EIP Leistungsindikatoren ist nicht kongruent bzw. verfolgen eine andere Zielsetzung. Die Indikatoren der WFA EIP-Beauftragung sind Indikatoren, die am Anfang einer Beauftragung festgelegt werden und spätestens nach fünf Jahren evaluiert werden (vgl. §11, WFA-Grund-Verordnung). Die EIP Indikatoren stellen Ziele der Aktivitäten und Schwerpunkte der jeweiligen Planungsjahre der Beauftragung dar, die jährlich von FFG-EIP an die AuftraggeberInnen übermittelt werden. Diese setzen die Ziele mit den Maßnahmen der WFA EIP in Bezug, gehen aber über die in der WFA definierten Indikatoren hinaus und dienen auch dem FFG-internen Monitoring der Leistungen. Das Ziel des Reviews ist die Weiterentwicklung der WFA EIPIndikatoren und die damit einhergehende Anpassung der EIP Leistungsindikatoren. 


\begin{tabular}{|c|c|c|c|c|}
\hline $\begin{array}{l}\text { Ziele der WFA EIP-Beauf- } \\
\text { tragung }\end{array}$ & Indikator & Maßnahmen & $\begin{array}{l}\text { Indikator der Maß- } \\
\text { nahme }\end{array}$ & Anpassungsvorschlag \\
\hline \multirow[t]{2}{*}{$\begin{array}{l}\text { 1. Optimale Beteiligung } \\
\text { österreichischer Wissen- } \\
\text { schaftsorganisationen an } \\
\text { H2020 und den Initiativen } \\
\text { zur Verwirklichung des } \\
\text { Europäischen For- } \\
\text { schungsraums }\end{array}$} & \multirow[t]{2}{*}{$\begin{array}{l}\text { Systematische Auseinander- } \\
\text { setzung mit H2020/Europäi- } \\
\text { scher Forschungsraum (Verfol- } \\
\text { gung über die Ergebnisse der } \\
\text { durchgeführten strategischen } \\
\text { Gespräche sog. "ERA-Dia- } \\
\text { loge"). Die großen wissen- } \\
\text { schaftlichen Forschungsein- } \\
\text { richtungen insbes. Universitä- } \\
\text { ten sind hier die Hauptziel- } \\
\text { gruppe. Die Ergebnisse wer- } \\
\text { den im Zuge der Arbeit von } \\
\text { der Österreichischen For- } \\
\text { schungsförderungsgesell- } \\
\text { schaft, Bereich } 4 \text { "Europäische } \\
\text { und Internationale Pro- } \\
\text { gramme" zusammengefasst } \\
\text { und von den AuftraggeberIn- } \\
\text { nen verfolgt. Ziel: mehr als } \\
\text { die Hälfte der Universitäten } \\
\text { sollen über Internationalisie- } \\
\text { rungsstrategien verfügen. }\end{array}$} & ERA-Dialoge & $\begin{array}{l}\text { Insg. } 12 \text { laufende ERA- } \\
\text { Dialoge bis } 2017\end{array}$ & $\begin{array}{l}\text { Das Ziel der Beauftragung und der Maß- } \\
\text { nahme ist die aktive Unterstützung der } \\
\text { Wissenschaftsorganisationen durch FFG- } \\
\text { EIP. Die bereits existierenden (ERA-Dia- } \\
\text { loge) und zukünftigen Maßnahmen und } \\
\text { deren Wirkung (z.B. Feedback-Proto- } \\
\text { kolle) sollten weiterhin in die WFA aufge- } \\
\text { nommen werden. } \\
\text { Über Feedbackgespräche hinaus sollten } \\
\text { folgende Indikatoren aufgenommen wer- } \\
\text { den: } \\
\text { - Zufriedenheit der Zielgruppen mit } \\
\text { den spezifischen Aktivitäten der FFG } \\
\text { - Anzahl der Universitäten, die kon- } \\
\text { krete Umsetzungsmaßnahmen in Ko- } \\
\text { operation mit der FFG in Angriff ge- } \\
\text { nommen haben }\end{array}$ \\
\hline & & $\begin{array}{l}\text { Veränderung } \\
\text { First/Second Level } \\
\text { Support bei ver- } \\
\text { gleichbarer Beteili- } \\
\text { gung }\end{array}$ & $\begin{array}{l}\text { Insg. } 30 \% \text { weniger } \\
\text { First Level Beratungen }\end{array}$ & $\begin{array}{l}\text { Das Empowerment der Wissenschaftsor- } \\
\text { ganisationen soll durch spezifische For- } \\
\text { mate für Vize-Rektorate und For- } \\
\text { schungsservicestellen weiter forciert } \\
\text { werden. Deren Wirkung sollte in die WFA } \\
\text { aufgenommen werden - siehe dazu } \\
\text { oben. }\end{array}$ \\
\hline $\begin{array}{l}\text { 2. Verstärkte Beteiligung } \\
\text { der Wirtschaft entspre- } \\
\text { chend umgesetzter Inno- } \\
\text { vationsorientierung von } \\
\text { H2O20; } 20 \% \text { Beteiligung } \\
\text { der Kleineren und Mittle- } \\
\text { ren Unternehmen (KMU) }\end{array}$ & $\begin{array}{l}\text { Verstärktes Beteiligungsinte- } \\
\text { resse der Wirtschaft an } \\
\text { H2020; rund 28\% Beteiligung } \\
\text { Wirtschaft gemessen an der } \\
\text { österreichischen Gesamtbetei- } \\
\text { ligung nach Organisationska- } \\
\text { tegorien bis zum Jahr } 2017\end{array}$ & KMU Beratungen & $\begin{array}{l}\text { Steigerung der Bera- } \\
\text { tungszahlen für KMU } \\
\text { um insg. } 20 \%\end{array}$ & $\begin{array}{l}\text { Die Anzahl der Beratungen für KMU ist } \\
\text { ein einfach erhebbarer Indikator der das } \\
\text { Interesse der KMU an H2020 aufzeigt. } \\
\text { Der Zielzustand sollte deshalb auf der- } \\
\text { zeitigem Level der Beratungen pro Jahr } \\
\text { gehalten werden. Die Kundenzufrieden- } \\
\text { heit mit den Leistungen sollte wie gehabt } \\
\text { erfasst werden. }\end{array}$ \\
\hline
\end{tabular}




\begin{tabular}{|c|c|c|c|c|}
\hline $\begin{array}{l}\text { Ziele der WFA EIP-Beauf- } \\
\text { tragung }\end{array}$ & Indikator & Maßnahmen & $\begin{array}{l}\text { Indikator der Maß- } \\
\text { nahme }\end{array}$ & Anpassungsvorschlag \\
\hline & & $\begin{array}{l}\text { Unterstützung bei } \\
\text { der Beteiligung der } \\
\text { Wirtschaft (große } \\
\text { Unternehmen) }\end{array}$ & $\begin{array}{l}\text { Steigerung der Wirt- } \\
\text { schaftsbeteiligung auf } \\
450 \text { (jährlich rund 112) } \\
\text { bis zum Evaluierungs- } \\
\text { zeitpunkt } 2017\end{array}$ & $\begin{array}{l}\text { Die Steigerung der Wirtschaftsbeteili- } \\
\text { gung ist ein erhebbarer Indikator, steht } \\
\text { aber in keinem direkten Zusammenhang } \\
\text { mit den Aktivitäten der FFG. Alternativ } \\
\text { könnte der Erfolg der FFG-Beratung an- } \\
\text { hand folgenden Indikators gemessen } \\
\text { werden: } \\
\text { - Halten/Steigerung überdurchschnitt- } \\
\text { lichen Erfolgsrate der Unternehmen } \\
\text { in H2020, die eine FFG Beratung in } \\
\text { Anspruch genommen haben. }\end{array}$ \\
\hline $\begin{array}{l}\text { 3. Optimale Grundlagen } \\
\text { für eine strategische Her- } \\
\text { angehensweise und Prio- } \\
\text { ritätensetzung bezüglich } \\
\text { der österreichischen Be- } \\
\text { teiligung }\end{array}$ & $\begin{array}{l}\text { Mobilisierung und Information } \\
\text { der Kund/innen in Bezug auf } \\
\text { MULLAT z. B. durch erklärende } \\
\text { Darstellungen/von der Praxis } \\
\text { abgeleitete Modelle/Erfolgsge- } \\
\text { schichten auf der Website, e- } \\
\text { Newsletter, spezifische Veran- } \\
\text { staltungen, Aussendungen. }\end{array}$ & $\begin{array}{l}\text { MULLAT - Service } \\
\text { und Beratung, die } \\
\text { zentral gesellschaft- } \\
\text { liche Herausforde- } \\
\text { rungen adressieren, } \\
\text { insbes. ERA-NET, } \\
\text { JPI, EIT/KIC und EIP }\end{array}$ & $\begin{array}{l}\text { Bis 2017: } 120 \text { doku- } \\
\text { mentierte Service- und } \\
\text { Beratungsleistungen }\end{array}$ & $\begin{array}{l}\text { Service- und Beratungsleistungen zu den } \\
\text { FTI-Partnerschaften sind Teil der EIP-Be- } \\
\text { auftragung und gehören somit zu den } \\
\text { zentralen Aktivitäten. Da diese ohnehin } \\
\text { wahrgenommen werden könnte für die } \\
\text { letzten Jahre von H2020 anstatt der An- } \\
\text { zahl der Beratungen folgender Indikator } \\
\text { eingesetzt werden: } \\
\text { FFG-EIP sollte auch über aktuelle Ent- } \\
\text { wicklungen zum kommenden Rahmen- } \\
\text { programm und den damit einhergehen- } \\
\text { den Veränderungen in der FTI-Partner- } \\
\text { schaftslandschaft informieren und bera- } \\
\text { ten. Dies beinhaltet das zur Verfügung } \\
\text { stellen von gut aufbereiteten Informati- } \\
\text { onsgrundlagen in Hinblick auf: } \\
\text { - Strategic Partnerships } \\
\text { - Missionsorientierung } \\
\text { - FP9 }\end{array}$ \\
\hline
\end{tabular}




\begin{tabular}{|c|c|c|c|c|}
\hline $\begin{array}{l}\text { Ziele der WFA EIP-Beauf- } \\
\text { tragung }\end{array}$ & Indikator & Maßnahmen & $\begin{array}{l}\text { Indikator der Maß- } \\
\text { nahme }\end{array}$ & Anpassungsvorschlag \\
\hline & & $\begin{array}{l}\text { Beitrag zur Ausge- } \\
\text { staltung der multila- } \\
\text { teralen Instrumente } \\
\text { im Wirkungskreis } \\
\text { der FFG }\end{array}$ & $\begin{array}{l}35 \text { Analysen und Be- } \\
\text { richte zu den MULLAT }\end{array}$ & $\begin{array}{l}\text { Als Indikator sollte nicht die Anzahl der } \\
\text { Berichte, sondern die Nutzung erstellter } \\
\text { Berichte erfasst werden. Es gibt derzeit } \\
\text { keine Downloadstatistiken der Berichts- } \\
\text { formate, diese sollte aber eingeführt } \\
\text { werden. }\end{array}$ \\
\hline & & $\begin{array}{l}\text { Neue Beratungsin- } \\
\text { strumente: ERA-Dia- } \\
\text { log, Betreuung Key } \\
\text { Player Wirtschaft }\end{array}$ & $\begin{array}{l}\text { dokumentierte Feed- } \\
\text { backgespräche; Ergeb- } \\
\text { nis "gut" nach Schul- } \\
\text { notensystem }\end{array}$ & $\begin{array}{l}\text { Die Kundenzufriedenheit mit den FFG } \\
\text { Leistungen ist ein erhebbarer und nach- } \\
\text { vollziehbarer Indikator zur Messung der } \\
\text { Relevanz und Effektivität der Beratung } \\
\text { und Betreuung. Die dokumentierten } \\
\text { Feedbackgespräche zu den spezifischen } \\
\text { Beratungsinstrumenten für Wissen- } \\
\text { schaftsorganisationen und Unternehmen } \\
\text { sollten deshalb beibehalten werden. }\end{array}$ \\
\hline $\begin{array}{l}\text { 4. Hohe Kund/innenzu- } \\
\text { friedenheit bei den Ziel- } \\
\text { gruppen bezüglich der } \\
\text { Leistungen der Toolbox; } \\
\text { Nutzen der zentralen } \\
\text { neuen Beratungsinstru- } \\
\text { mente (ERA-Dialog, Be- } \\
\text { treuung Key Player Wirt- } \\
\text { schaft) }\end{array}$ & $\begin{array}{l}\text { Verbesserung der Kund/innen- } \\
\text { zufriedenheit um 3\% Punkte } \\
\text { bis 2017; Überprüfung im } \\
\text { Zuge der geplanten externen } \\
\text { Gesamtevaluierung der Beauf- } \\
\text { tragung } 2017\end{array}$ & & & $\begin{array}{l}\text { Die Kundenzufriedenheit mit den FFG } \\
\text { Leistungen ist ein erhebbarer und nach- } \\
\text { vollziehbarer Indikator zur Messung der } \\
\text { Relevanz und Effektivität der Beratung } \\
\text { und Betreuung und sollte deshalb beibe- } \\
\text { halten werden. }\end{array}$ \\
\hline
\end{tabular}




\section{Anpassung der WFA H2020-Indikatoren}

Für das europäische Forschungs- und Innovationsrahmenprogramm H2020 wurden eine Reihe von Key Performance Indikatoren von der Europäischen Kommission festgesetzt ${ }^{102}$. Diese sollen im Rahmen des jährlichen Monitorings oder in der Zwischen- und Endevaluation von H2020 erfasst werden. Aus Gründen der internationalen Vergleichbarkeit und der Ressourcenschonung sollten Indikatoren zur Wirkungsabschätzung von H2020 in Österreich weitgehend auf die Key Performance Indikatoren von H2020 beziehen.

Die Überprüfung der Indikatoren und Anpassungsvorschläge erfolgen vor dem Hintergrund der in der WFA H2020 gesetzten Ziele.

\begin{tabular}{|c|c|c|c|}
\hline Ziel & $\begin{array}{l}\text { Ausgangszustand } \\
\text { Zeitpunkt der WFA }\end{array}$ & $\begin{array}{l}\text { Zielzustand Evaluie- } \\
\text { rungszeitpunkt }\end{array}$ & Überprüfung und Anpassungsvorschlag \\
\hline $\begin{array}{l}\text { Grundlagen- bzw. Pionierforschung in Ös- } \\
\text { terreich soll durch die themenoffene, allein } \\
\text { dem } \\
\text { Exzellenzkriterium verpflichtete Förderung } \\
\text { von im internationalen Wettbewerb heraus- } \\
\text { ragenden } \\
\text { Forscher/innen im Rahmen des 'Europäi- } \\
\text { schen Forschungsrates vorangetrieben } \\
\text { werden. }\end{array}$ & $\begin{array}{l}\text { Spitzenforschungs- } \\
\text { preise durch den } \\
\text { Europäischen For- } \\
\text { schungsrat: } 68 \\
\text { Grants (Status bis } \\
\text { 2011) }\end{array}$ & $\begin{array}{l}\text { Spitzenforschungssti- } \\
\text { pendien durch den } \\
\text { Europäischen For- } \\
\text { schungsrat: } 70 \text { Sti- } \\
\text { pendien (Zwischenziel } \\
\text { bis } 2013 \text { ); insgesamt } \\
140 \text { Stipendien bis } \\
2020\end{array}$ & $\begin{array}{l}\text { Die Anzahl der Spitzenforschungsstipendien durch den } \\
\text { Europäischen Forschungsrat ist ein erhebbares und nach- } \\
\text { vollziehbares Qualitätsmerkmal für themenoffene, exzel- } \\
\text { lente Forschung in Österreich. } \\
\text { Der Indikator kann jährlich erfasst werden und sollte in } \\
\text { dieser Form beibehalten werden. } \\
\text { Die Anzahl der angestrebten Spitzenforschungsstipendien } \\
\text { sollte die Entwicklung des zur Verfügung stehenden För- } \\
\text { dervolumens des Europäischen Forschungsrates berück- } \\
\text { sichtigen. }\end{array}$ \\
\hline $\begin{array}{l}\text { Das Wachstumspotenzial der österreichi- } \\
\text { schen Unternehmen soll durch Investitio- } \\
\text { nen in grundlegende und industrielle Tech- } \\
\text { nologien gestärkt werden. Dies umfasst die } \\
\text { Bereiche IKT, Nanotechnologie, fortge- } \\
\text { schrittene Werkstoffe, Biotechnologie, fort- } \\
\text { geschrittene Fertigung und Verarbeitung } \\
\text { sowie Raumfahrt. }\end{array}$ & $\begin{array}{l}\text { Neuer Förderansatz, } \\
\text { daher keine Aus- } \\
\text { gangsbasis möglich }\end{array}$ & $\begin{array}{l}\text { Drei Patentanmeldun- } \\
\text { gen pro } 10 \text { Mio. Euro } \\
\text { Förderung bis } 2020\end{array}$ & $\begin{array}{l}\text { Bis 1/09/2016 (including grants to named beneficiaries) } \\
\text { erfolgten im gesamten Spektrum von H2020 insgesamt } \\
109 \text { Patentanmeldungen und } 29 \text { bewilligte Patente über } \\
\text { alle EU-MS hinweg. }{ }^{103} \\
\text { Der Indikator ist für den Bereich "Führende Rolle der In- } \\
\text { dustrie" ein Key Performance Indikator von H2020. Die } \\
\text { bisherige Anzahl der gesamten Patentanmeldungen in } \\
\text { H2020 legt Nahe, dass das europäische Ziel von drei Pa- } \\
\text { tentanmeldungen je } 10 \text { Mio Euro nicht erreicht werden } \\
\text { kann. }\end{array}$ \\
\hline
\end{tabular}

102

https://www.ffg.at/sites/default/files/downloads/page/horizon2020indicators.pdf

103 Europäische Kommission (2016), Horizon 2020 Annual Monitoring Report 2015 


\begin{tabular}{|c|c|c|c|}
\hline Ziel & $\begin{array}{l}\text { Ausgangszustand } \\
\text { Zeitpunkt der WFA }\end{array}$ & $\begin{array}{l}\text { Zielzustand Evaluie- } \\
\text { rungszeitpunkt }\end{array}$ & Überprüfung und Anpassungsvorschlag \\
\hline & & & $\begin{array}{l}\text { Die Anzahl der durch H2020 induzierten Patentanmeldun- } \\
\text { gen sollte weiter erfasst werden. Unklar ist jedoch, in } \\
\text { welcher Weise die europäischen Kommission Daten zu } \\
\text { Patentanmeldungen systematisch erfassen wird und Da- } \\
\text { ten auf Ebene der Mitgliedsstaaten zur Verfügung gestellt } \\
\text { werden. } \\
\text { Die Beteiligung österreichischer Unternehmen an den } \\
\text { Ausschreibungen im Bereich "LEIT" könnte als ein Indika- } \\
\text { tor herangezogen werden, der Auskunft über die Leis- } \\
\text { tungsfähigkeit Innovationskapazität öst. Unternehmen im } \\
\text { internationalen Kontext gibt. } \\
\text { Neben der Anzahl österreichischer Beteiligungen könnte } \\
\text { auch der Zugang zu Risikofinanzierung für Unternehmen } \\
\text { mit innovativen und risikoreichen Projekten aus Öster- } \\
\text { reich und das damit verbundene private Investitionsvolu- } \\
\text { men (Hebeleffekt) als Indikator herangezogen werden. } \\
\text { Beide genannten Indikatoren sind Teil der Key Perfor- } \\
\text { mance Indikatoren von H2020. Indikatoren könnten da- } \\
\text { her mit wenig Aufwand erfasst werden. }\end{array}$ \\
\hline $\begin{array}{l}\text { Unter Nutzung des gesamten Förderzyklus } \\
\text { von der Forschung bis zur Vermarktung } \\
\text { sollen Beiträge zur Lösung gesellschaftli- } \\
\text { cher Herausforderungen geleistet werden, } \\
\text { die für Österreich prioritär sind. Es handelt } \\
\text { sich dabei um die in der österreichischen } \\
\text { FTI-Strategie identifizierten Herausforde- } \\
\text { rungen "Klimawandel", "Knappe Ressour- } \\
\text { cen" sowie "Lebensqualität und demogra- } \\
\text { phischer Wandel". }\end{array}$ & $\begin{array}{l}\text { Neuer Förderansatz, } \\
\text { daher keine Aus- } \\
\text { gangsbasis möglich }\end{array}$ & $\begin{array}{l}\text { Durchschnittlich eine } \\
\text { regulatorische Umset- } \\
\text { zungsmaßnahme zur } \\
\text { Lösung gesellschaftli- } \\
\text { cher Herausforderun- } \\
\text { gen in Österreich pro } \\
10 \text { Mio. Euro Förde- } \\
\text { rung bis } 2020\end{array}$ & $\begin{array}{l}\text { Regulatorische Umsetzungsmaßnahmen im Kontext der } \\
\text { Säule „Gesellschaftliche Herausforderungen" werden } \\
\text { nicht erfasst. Aus Sicht des Evaluierungsteams stellen } \\
\text { diese auch keinen geeigneten Indikator dar, um Beiträge } \\
\text { zur Lösung gesellschaftlicher Herausforderung zu mes- } \\
\text { sen. } \\
\text { Der Nutzen für Österreich könnte an folgenden Indikato- } \\
\text { ren gemessen werden: } \\
\text { - Beitrag Österreichs zu europäischen Partnerschafts- } \\
\text { programmen in den genannten Bereichen und deren } \\
\text { Hebelwirkung durch gepoolte Ressourcen } \\
\text { Beteiligung österreichischer Organisationen in der } \\
\text { Säule Societal Challenges }\end{array}$ \\
\hline
\end{tabular}




\begin{tabular}{|c|c|c|c|}
\hline Ziel & $\begin{array}{l}\text { Ausgangszustand } \\
\text { Zeitpunkt der WFA }\end{array}$ & $\begin{array}{l}\text { Zielzustand Evaluie- } \\
\text { rungszeitpunkt }\end{array}$ & Überprüfung und Anpassungsvorschlag \\
\hline $\begin{array}{l}\text { Die vereinfachten Verfahren und Regeln } \\
\text { von H2020 sollen dazu beitragen, dass im } \\
\text { Bereich Forschung die ausgezahlten öster- } \\
\text { reichischen Rückflüsse weiterhin über dem } \\
\text { Wert der österreichischen Eigenmittelzah- } \\
\text { lungen zum EU-Haushalt liegen }\end{array}$ & $\begin{array}{l}\text { Anteil der österrei- } \\
\text { chischen Eigenmit- } \\
\text { telzahlungen am } \\
\text { EU-Budget 2011: } \\
2.24 \% \text { / Anteil der } \\
\text { ausgezahlten öster- } \\
\text { reichischen Rück- } \\
\text { flüsse am EU- } \\
\text { Budget im Bereich } \\
\text { Forschung im Jahr } \\
2011: 2.50 \% \text { (das } \\
\text { entspricht 146.5 } \\
\text { Mio. Euro) }\end{array}$ & $\begin{array}{l}\text { Anteil der österreichi- } \\
\text { schen Eigenmittelzah- } \\
\text { lungen am EU-Budget } \\
\text { 2020: } 2.40 \% \text { / Anteil } \\
\text { der ausgezahlten ös- } \\
\text { terreichischen Rück- } \\
\text { flüsse am EU-Budget } \\
\text { im Bereich Forschung } \\
\text { im Jahr 2020: } 2.50 \% \\
\text { (das entspricht } 380 \\
\text { Mio. Euro) }\end{array}$ & $\begin{array}{l}\text { Die vereinfachten Verfahren und Regeln von H2020 sen- } \\
\text { ken die Kosten für Antragstellung und Projektadministra- } \\
\text { tion bei den Förderempfängern. } \\
\text { Neben dem Verhältnis österreichischer Eigenmittelzahlun- } \\
\text { gen am EU Budget und Rückflüssen aus dem EU-Budget } \\
\text { könnte in Ergänzung auch die Zufriedenheit mit den ver- } \\
\text { einfachten Verfahren und Regeln überprüft werden. Diese } \\
\text { Überprüfung findet jedoch nicht jährlich statt, sondern } \\
\text { kann nur im Rahmen der Zwischen- und Endevaluation } \\
\text { von H2020 festgestellt werden. }\end{array}$ \\
\hline $\begin{array}{l}\text { Im Nuklearforschungsbereich soll die Neu- } \\
\text { orientierung der Kernspaltungsforschung in } \\
\text { Richtung einer konsequenten Konzentra- } \\
\text { tion auf Sicherheitsfragen fortgesetzt wer- } \\
\text { den. Außerdem sollen die Kosten für ITER } \\
\text { keinesfalls den im EU-Finanzrahmen vorge- } \\
\text { gebenen Höchstbetrag überschreiten }\end{array}$ & $\begin{array}{l}\text { ITER-Budget 2007- } \\
\text { 2011: } 1.047 \text { Mrd. } \\
\text { Euro / 2012-2013: } \\
1.3 \text { Mrd. Euro }\end{array}$ & $\begin{array}{l}\text { ITER-Budget 2014- } \\
\text { 2020: } 2.7 \text { Mrd. Euro } \\
\text { (Preise 2011) }\end{array}$ & $\begin{array}{l}\text { Die österreichischen Beiträge zum ITER Budget sollten } \\
\text { weiter erfasst werden. } \\
\text { Erfasst werden sollte auch, welchen Nutzen österreichi- } \\
\text { sche Organisationen aus der Partizipation an ITER ziehen } \\
\text { (z.B. Rückflüsse an österreichische Unternehmen, Anzahl } \\
\text { gemeinsamer Projekte etc.) }\end{array}$ \\
\hline
\end{tabular}




\section{Weiterentwicklung der WFA COSME}

Grundsätzlich sind in der EU-Verordnung zum Programm Wirkungsindikatoren samt diesbezüglichen Zielsetzungen definiert, ebenso in den jährlichen Arbeitsprogrammen zu den einzelnen Maßnahmen.

Insbesondere was den Bereich Finanzierungsinstrumente, die Förderung unternehmerischer Kultur und das EEN betrifft, so zeigt sich ein durchaus starkes Committment seitens der entsprechenden Stakeholder auf nationaler Ebene. Im offiziellen Dokument des BMDW zum EU-Vorschlag des Programms wird darauf hingewiesen, dass eine Problemanalyse sowie die Abschätzung der Auswirkungen dem Impact Assessment der Europäischen Institutionen entnommen werden können. Als inhaltliche Positionierung Österreichs wird folgendes vorgeschlagen: "die Programmziele decken sich, was den Wirkungsbereich betrifft, mit österreichischen wirtschaftspolitischen Zielen. Daher Unterstützung. Insbesondere strebt Österreich die weitere Verbesserung des Zugangs zu Finanzen durch Eigenkapital- und Kreditbürgschaftsfazilitäten für KMU sowie die Förderung unternehmerischer Kultur an."

Es wird weiters ein (erwarteter) Beitrag zu Wirkungszielen und Maßnahmen im Bundesvoranschlag angeführt:

- Stärkung der Wettbewerbsfähigkeit der österreichischen Unternehmen und Verbesserung des unternehmensfreundlichen Umfelds, insbesondere für kleine und mittlere Unternehmen (KMU) und den Tourismus

- Erhöhung der Attraktivität des Wirtschaftsstandortes, insbesondere Forcierung des Wettbewerbs und Förderung des Unternehmergeistes

- Stärkung der österreichischen Außenwirtschaft

Diese Wirkungsziele (auf Ebene der UG 40) sind mit Blick auf einen Beitrag aus COSMEMaßnahmen nicht mit Indikatoren hinterlegt, was - angesichts der hohen Aggregationsebene der Ziele auch unmöglich bzw. nicht zielführend wäre. Von der Definition von Indikatoren zur Messung der Zielerreichung und Wirkung der österreichischen Beteiligungen an COSME im Rahmen der WFA wurde also bisher Abstand genommen, da in Anbetracht der breiten Ziele, zusammen mit einem breiten/heterogenen Portfolio an Maßnahmen und der Tatsache, dass Österreich nur einen sehr kleinen Teil im Gesamtprogramm wahrnimmt, sowie in Anbetracht der relativ geringen nationalen Mitbestimmung der Arbeitsprogramme, nur der Beitrag zu (ausgewählten) Wirkungszielen auf oberster Ebene zu erwarten ist. Vor allem aber auch das ungenügende Monitoring der Europäischen Kommission im Programm, aber auch die breite Streuung an Maßnahmen, an denen österreichische Intermediäre potenziell teilnehmen können, was zudem aber ungenügend nachvollzogen werden kann (Monitoringproblematik), macht eine konkrete und spezifische WFA schwierig. Vor diesem Hintergrund erscheint es nicht zielführend, einzelne Indikatoren für die WFA abzuleiten. Ein Ansatz könnte jedoch eine Orientierung an den im Arbeitsprogramm definierten Indikatoren sein- zumindest für jene Maßnahmen, die über einen längeren Zeitraum durchgeführt werden (v.a. Finanzierungsinstrumente und das EEN) und auch budgetär das Gros der Mittel ausmachen. Dennoch würde dies nur einen Teil Österreichs Beteiligung widerspiegeln und auf einer Output-Ebene (in Form von PerformanzIndikatoren) erfolgen. 
In Anlehnung an das aktuelle Arbeitsprogramm von COSME erscheinen folgende Performance-Indikatoren als mögliche Schlüsselindikatoren für jene Maßnahmen im Programm, an denen Österreich stabile Teilnahmen aufweist:

- Finanzierungsinstrumente:

- Kreditgarantiefazilität: Finanzierungsvolumina (zT. nach Größenklassen) und Anzahl der finanzierten Firmen

- Risikokapitalfazilität: Investiertes Risikokapital und Anzahl unterstützter Firmen

○ Ggf. zusätzlich investierte Summen

- EEN :

- Anzahl Kooperationsverträge

- Anzahl Firmen als Teilnehmer an Events zur Förderung von Kooperationen (Kooperationsbörsen)

- Anzahl beratener Firmen (individuelle Beratung, Firmenbesuche)

- Anzahl KAM

- Anzahl EIMC

\section{Weiterentwicklung WFA - EUREKA / Eurostars}

Der Beitrag von EUREKA Eurostars zur Zielerreichung im Rahmen der WFA wird als insgesamt zufriedenstellen eingeschätzt. Die untere Schwelle des Zielbereichs des Indikators "Teilnahme an 100-120 erfolgreichen Eurostars-Projekten in der Phase von Eurostars-2 (2014-2020)" wurde bereits überschritten: Per Ende März wurden It. EU-PM für 103 Eurostars-2-Projekte ein Vertrag erstellt. Davon wurde lediglich ein Projekt seit 2014 in der Laufzeit storniert. Rund ein Drittel bestehen seit Anfang 2017. Gesetzt den Fall, dass Eurostars-2-Ausschreibungen auch weiterhin im gleichen Rhythmus abgewickelt werden, kann davon ausgegangen werden, dass bis 2020 auch die obere Grenze erreicht bzw. überschritten werden wird. Zu bedenken gilt, dass die Möglichkeit der Projektfinanzierung im engen Verhältnis zu den zur Verfügung stehenden Budgetmitteln steht. Änderungen bei der Mittelausstattung hätten unmittelbare Auswirkungen auf die Projektfinanzierung.

Bei der Interpretation des Indikators „(Erhöhung des Anteils an) innovativer KMU, die mit anderen kooperieren", eine Messgröße im European Innovation Survey (EIS) der sich aus der Europäischen Innovationserhebung (Community Innovation Survey - CIS) ableitet, ist die zeitlichen Verzögerung bei der Bereitstellung der Ergebnisse zu beachten. Der aktuell verfügbare Datenstand ist von 2012/14 und weist einen Anteil von 20,5\% auf - und damit weniger als die angestrebten 22,7\%. Aufgrund des Alters der Daten ist eine entsprechende Bewertung nur bedingt zulässig. Eine bessere Einschätzung ist auf Basis der Ergebnisse der CIS 14/16 zu erwarten, dessen Bereitstellung für Ende 2018 erwartet wird.

Während dieser Indikator die Ansprüche an eine statistische Validität prinzipiell erfüllt, stellt sich an einigen Stellen die Frage nach der Zweckmäßigkeit. So werden bei der Innovationserhebung neben EUREKA Eurostars auch andere Maßnahmen zur Berechnung des Zielwertes herangezogen. Zudem werden in der Innovationserhebung explizit "innovative KMU" adressiert, ein Kriterium, das in EUREKA Eurostars nicht zwangsläufig erfüllt 
sein muss. Abschließend ist festzustellen, dass der KMU-Anteil in den Eurostars-Projekten (2014 bis März 2018) bei $60 \%$ lag.

\begin{tabular}{|c|c|c|c|c|}
\hline \multirow[t]{2}{*}{ Ziele der WFA } & \multirow{2}{*}{$\begin{array}{l}\text { Zielwert 2014- } \\
2020\end{array}$} & \multirow[t]{2}{*}{ Maßnahmen } & \multicolumn{2}{|c|}{ Indikatoren der Maßnahmen } \\
\hline & & & Zielwert 2017 & $\begin{array}{l}\text { Anpassungsvor- } \\
\text { schlag }\end{array}$ \\
\hline $\begin{array}{l}\text { Ziel 1: Stär- } \\
\text { kung der trans- } \\
\text { nationalen In- } \\
\text { novationsko- } \\
\text { operationen von } \\
\text { österreichischen } \\
\text { forschungsin- } \\
\text { tensiven KMU }\end{array}$ & $\begin{array}{l}\text { Messung durch } \\
\text { den EIS-Indikator } \\
\text { "Innovative SMEs } \\
\text { collaborating with } \\
\text { others" = "Innova- } \\
\text { tive KMU die mit } \\
\text { anderen kooperie- } \\
\text { ren" }\end{array}$ & $\begin{array}{l}\text { Förderung österr. } \\
\text { Eurostars-Teil- } \\
\text { nehmer (natio- } \\
\text { nale Abwicklung } \\
\text { in FFG, in Koordi- } \\
\text { nation mit den } \\
\text { zentralen Abwick- } \\
\text { lungsaufgaben im } \\
\text { EUREKA-Sekreta- } \\
\text { riat) }\end{array}$ & $\begin{array}{l}22,7 \% \text { Anteil } \\
\text { an innovati- } \\
\text { ven KMU die } \\
\text { anderen ko- } \\
\text { operieren }\end{array}$ & $\begin{array}{l}\text { Problem der zeitli- } \\
\text { chen Verzögerung } \\
\text { bei der Datenbereit- } \\
\text { stellung; ab CIS } \\
14 / 16 \text { (Ende 2018) } \\
\text { erste Aussagen } \\
\text { möglich } \\
\text { Eurostars-Pro- } \\
\text { grammdokument } \\
\text { spricht von "for- } \\
\text { schungsintensiven } \\
\text { KMU", ein Unter- } \\
\text { schied zu "innovati- } \\
\text { ven KMU", die im } \\
\text { CIS adressiert wer- } \\
\text { den } \\
\text { Anteil von KMU in } \\
\text { EUREKA Eurostars } \\
\text { erhöhen, derzeit } \\
60 \%\end{array}$ \\
\hline $\begin{array}{l}\text { Ziel 1: Stär- } \\
\text { kung der trans- } \\
\text { nationalen In- } \\
\text { novationsko- } \\
\text { operationen von } \\
\text { österreichischen } \\
\text { forschungsin- } \\
\text { tensiven KMU }\end{array}$ & $\begin{array}{l}\text { Teilnahme an 100- } \\
120 \text { erfolgreichen } \\
\text { Eurostars-Projek- } \\
\text { ten in der Phase } \\
\text { von Eurostars-2 } \\
(2014-2020)\end{array}$ & $\begin{array}{l}\text { Förderung österr. } \\
\text { Eurostars-Teil- } \\
\text { nehmer (natio- } \\
\text { nale Abwicklung } \\
\text { in FFG, in Koordi- } \\
\text { nation mit den } \\
\text { zentralen Abwick- } \\
\text { lungsaufgaben im } \\
\text { EUREKA-Sekreta- } \\
\text { riat) }\end{array}$ & $\begin{array}{l}\text { Teilnahme an } \\
100-120 \text { er- } \\
\text { folgreichen } \\
\text { Eurostars- } \\
\text { Projekten }\end{array}$ & $\begin{array}{l}\text { Gezielter Einsatz } \\
\text { der zur Verfügung } \\
\text { gestellten öst. } \\
\text { Budgetmittel zur } \\
\text { Aufrechterhaltung } \\
\text { des eingeschlage- } \\
\text { nen Weges } \\
\text { Ev. Spezifizierung } \\
\text { Teilnahme an vs. } \\
\text { Leitung von Euro- } \\
\text { stars-Projekten }\end{array}$ \\
\hline
\end{tabular}




\section{Annex III: Zugriffsstatistiken und alternative Tools EU-PM}

Tabelle 15: Zugriffsstatistiken Online-Dienste EU-PM

\begin{tabular}{|l|c|c|c|c|}
\hline Öffentliches Portal & & $\begin{array}{c}2015 \\
\text { (ab Juli) }\end{array}$ & 2016 & 2017 \\
\hline Zugriffe insgesamt & & 1978 & 2310 & 2459 \\
\hline Zugriffe/Monat (letzte drei Monate) & & & & 215 \\
\hline Vertrauliches Portal* & $\begin{array}{c}2014 \\
\text { (ab Juli) }\end{array}$ & 2015 & 2016 & 2017 \\
\hline Logins insgesamt & 217 & 365 & 734 & 392 \\
\hline Logins/Monat (letzte drei Monate) & & & & 36 \\
\hline Anzahl der nominierten User & & & & 102 \\
\hline EU-PM Website & 2014 & 2015 & 2016 & 2017 \\
\hline Besuche insgesamt & 466 & 1641 & 2109 & 1326 \\
\hline Zugriffe/Monat (letzte drei Monate) & & & & 128 \\
\hline
\end{tabular}

*ohne Zugriffe durch Team Datenanalyse

Quelle: EU-PM.

Abbildung 46: Zugriffsstatistiken Online-Dienste EU-PM, monatlich

$$
\text { घ vertrauliches Portal } \square \text { öffentliches Portal } \quad \text { Website }
$$

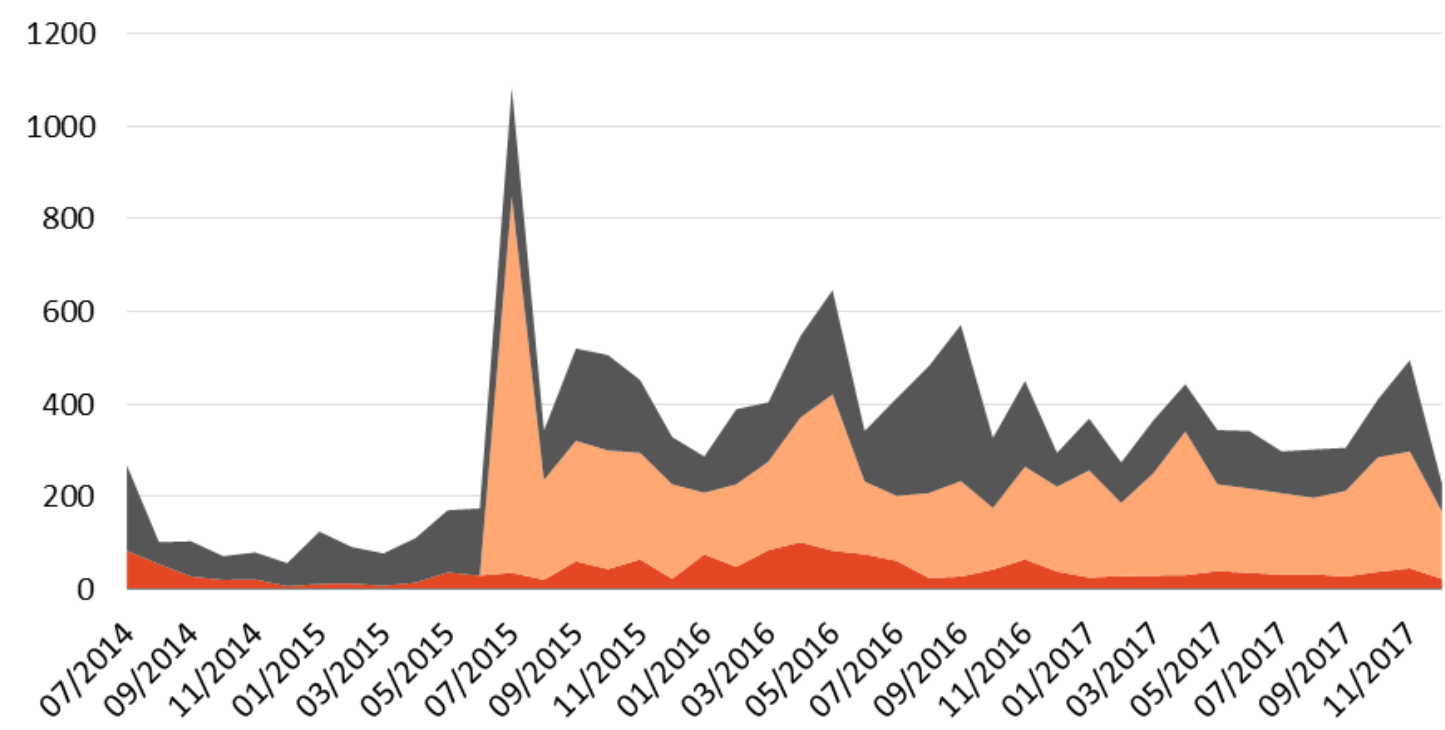

Quelle: EU-PM. 
Abbildung 47: Darstellung VINNOVA H2020 Visualization Tool, Säulen

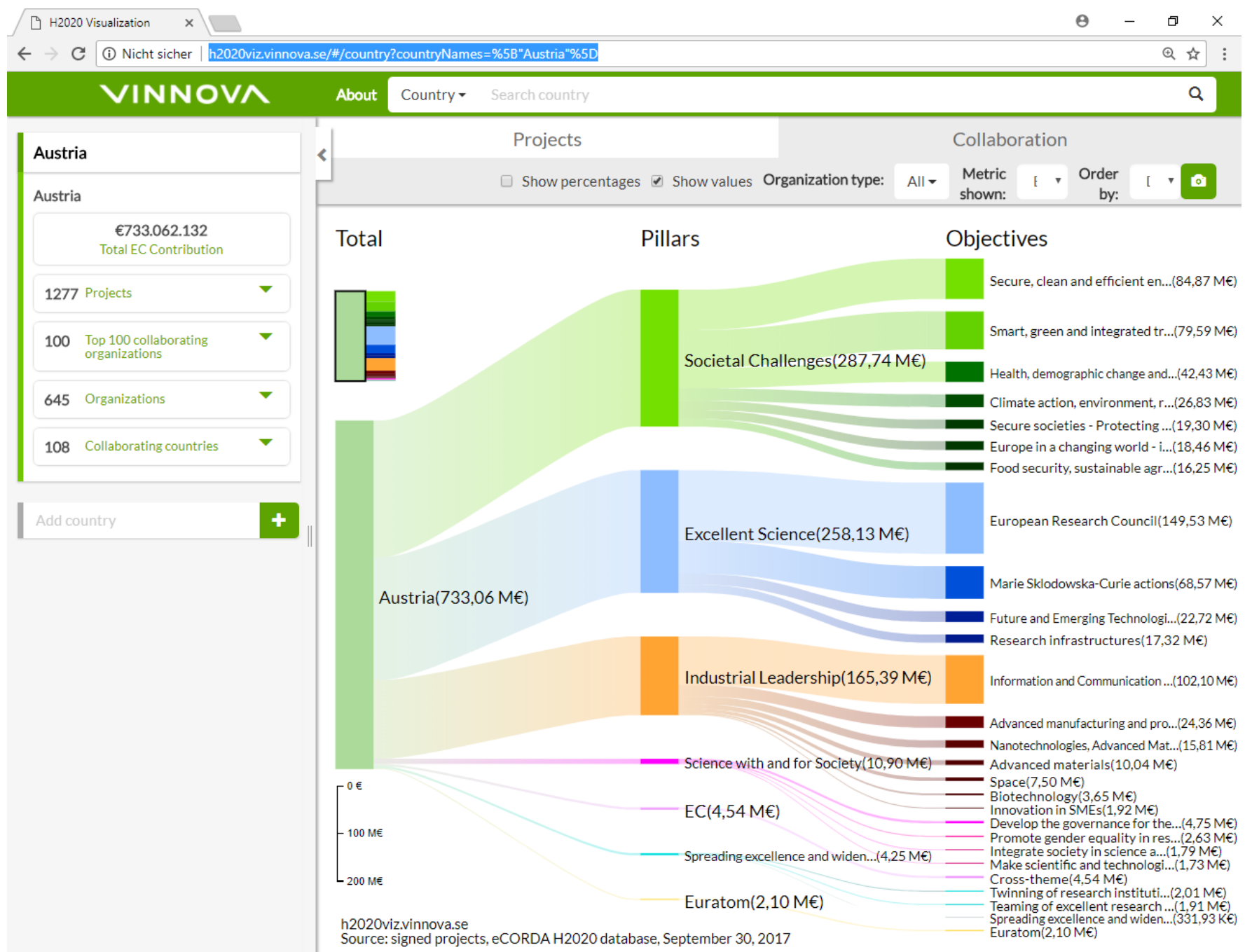

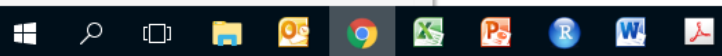

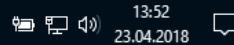

Quelle: http://h2020viz.vinnova.se/\#/; Download 23.04.2018. 
Abbildung 48: Darstellung VINNOVA H2020 Visualization Tool, Calls

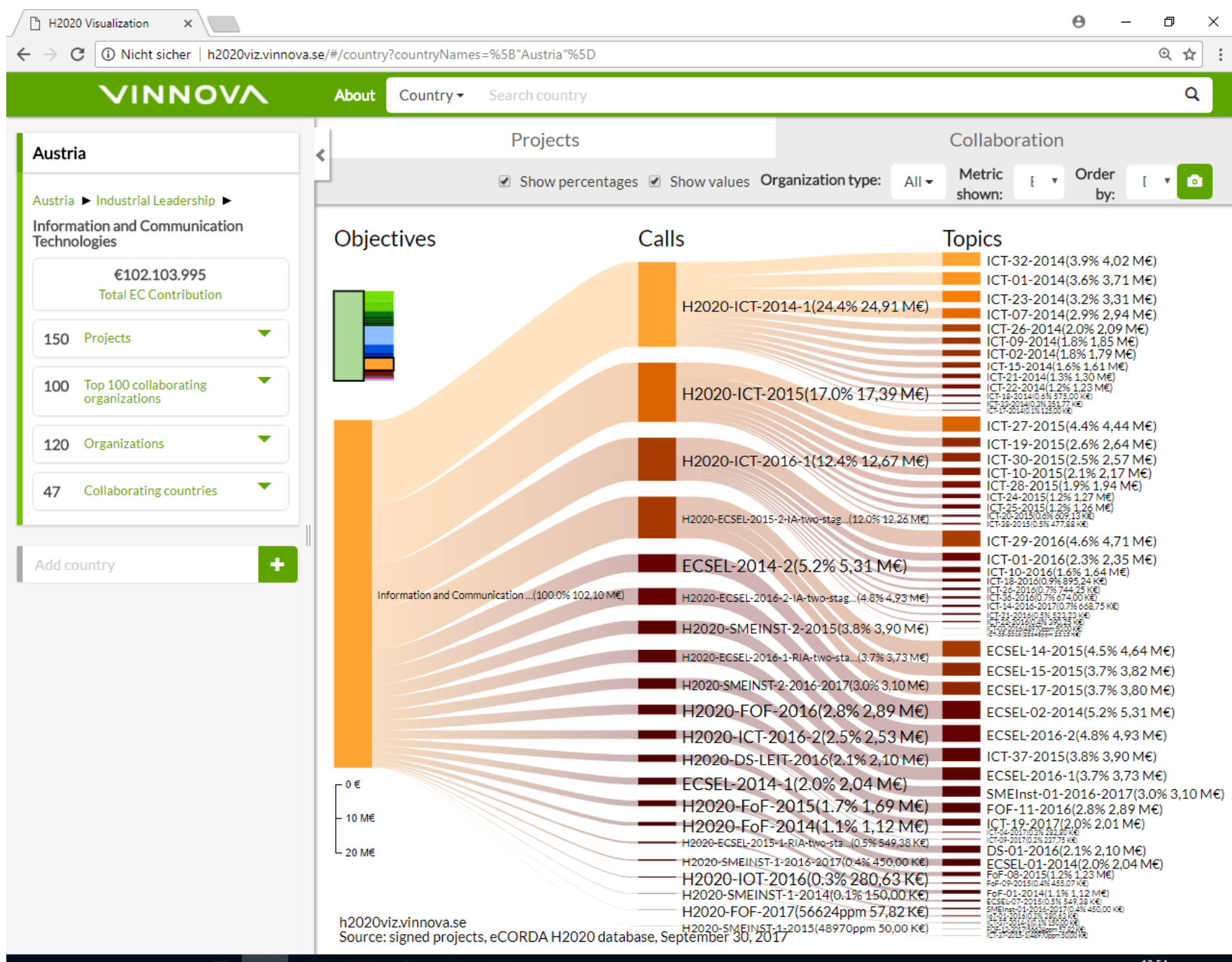

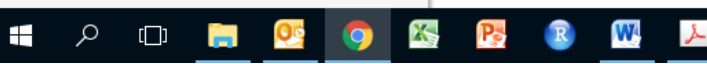

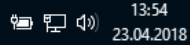

Darstellung am Beispiel Säule II, LEIT-Programm ICT. Quelle: http://h2020viz.vinnova.se/\#/; Download 23.04.2018. 
Abbildung 49: Darstellung VINNOVA H2020 Visualization Tool, Projekte

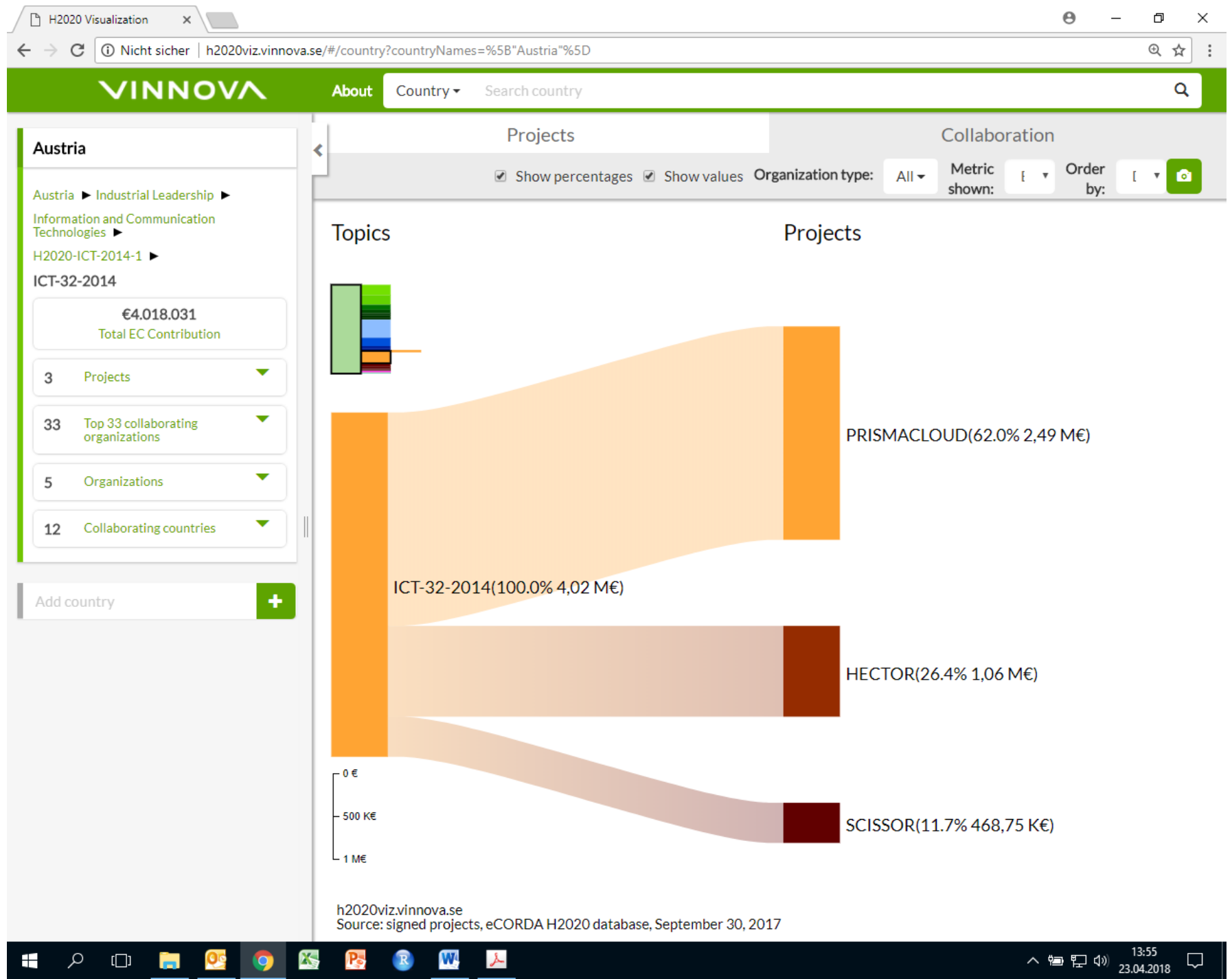

Darstellung am Beispiel LEIT-ICT-32-2014. Quelle: http://h2020viz.vinnova.se/\#/; Download 23.04.2018. 
Abbildung 50: Darstellung VINNOVA H2020 Visualization Tool, Einzelprojekt

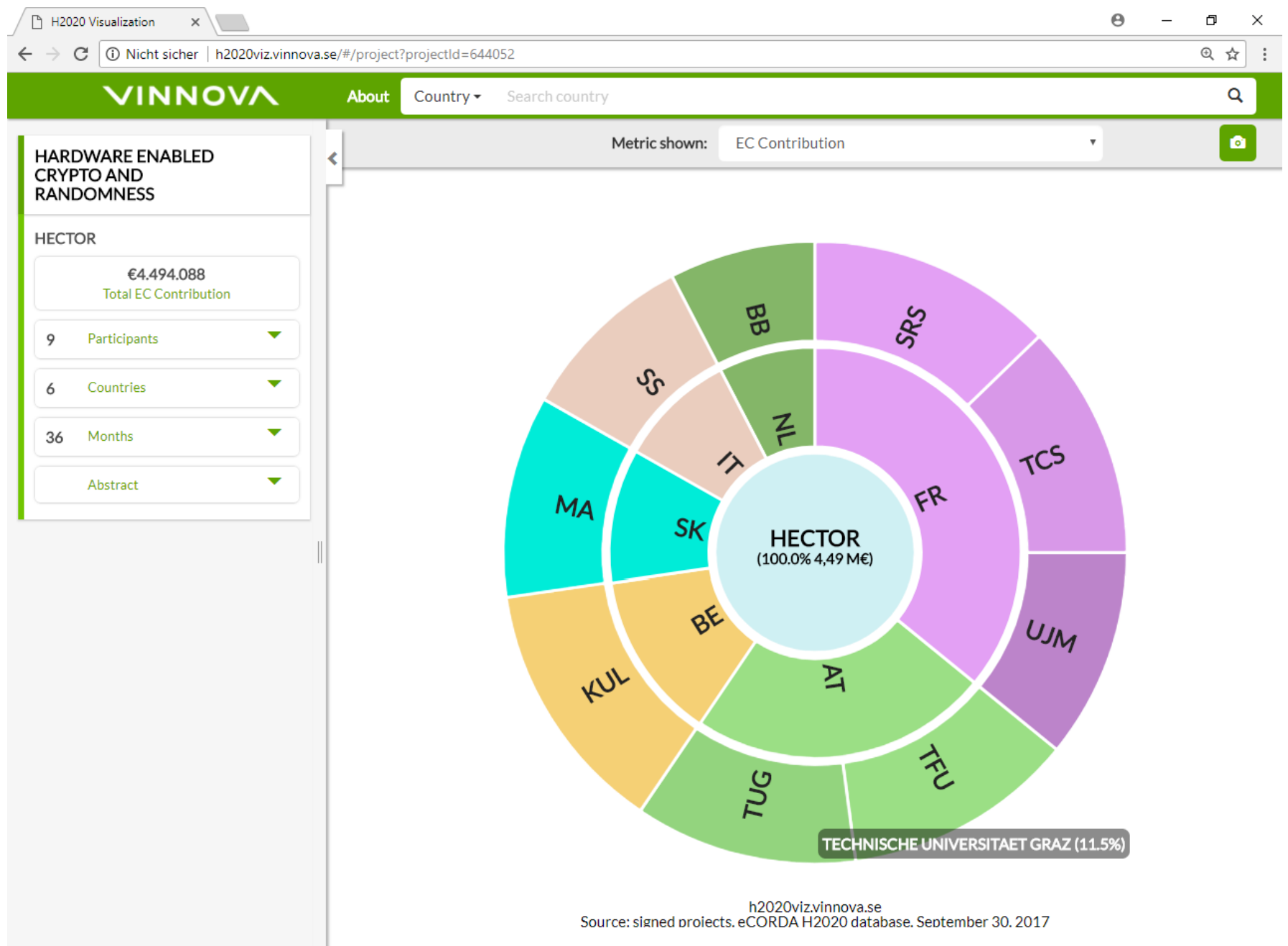

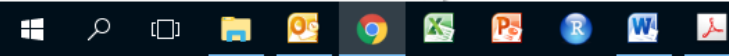

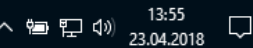

Darstellung am Beispiel LEIT-ICT-32-2014 HECTOR. Quelle: http://h2020viz.vinnova.se/\#/; Download 23.04.2018. 
Abbildung 51: Darstellung Dashboard EU-Participant Portal, Österreich

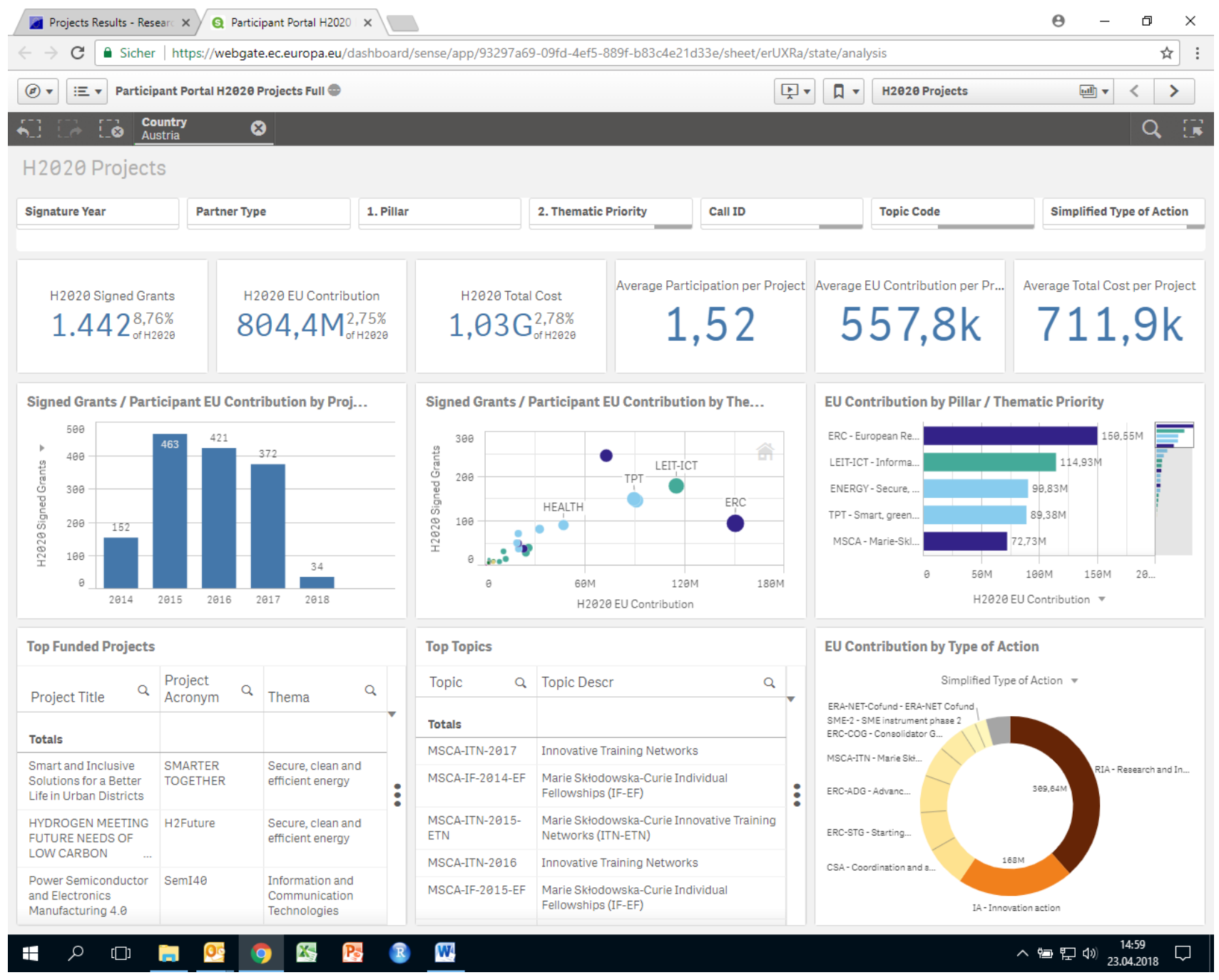

Quelle: https://webgate.ec.europa.eu/dashboard/sense/app/93297a69-09fd-4ef5-889f-b83c4e21d33e/sheet/erUXRa/state/analysis ; Download 23.04.2018. 
Abbildung 52: Darstellung öffentliches Portal EU-PM, Kennzahlenvergleich AT - DE

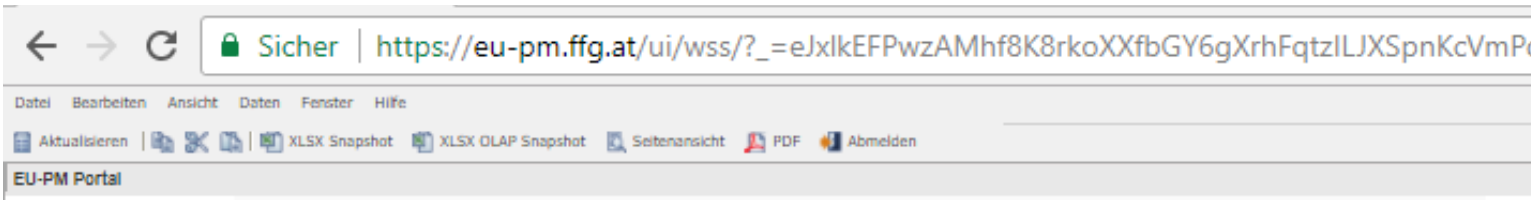

EU.PM Portal

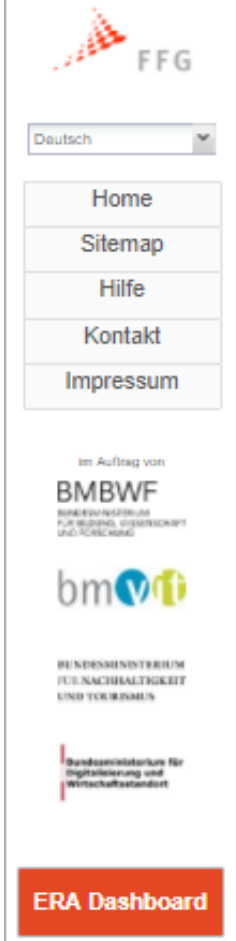

\section{Vergleich zwischen Österreich und Deutschland}

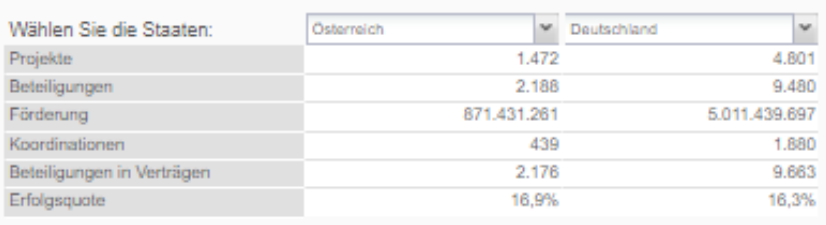

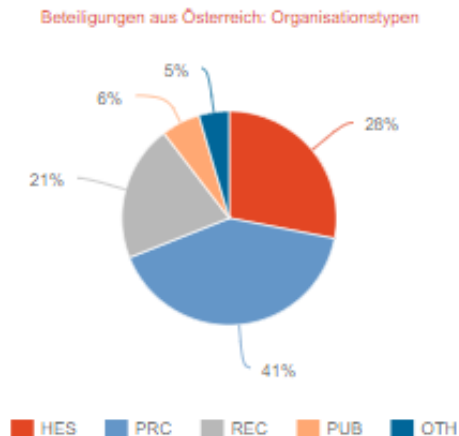

-

Förderung an Osterreich in Mia. Euro für Top Programme
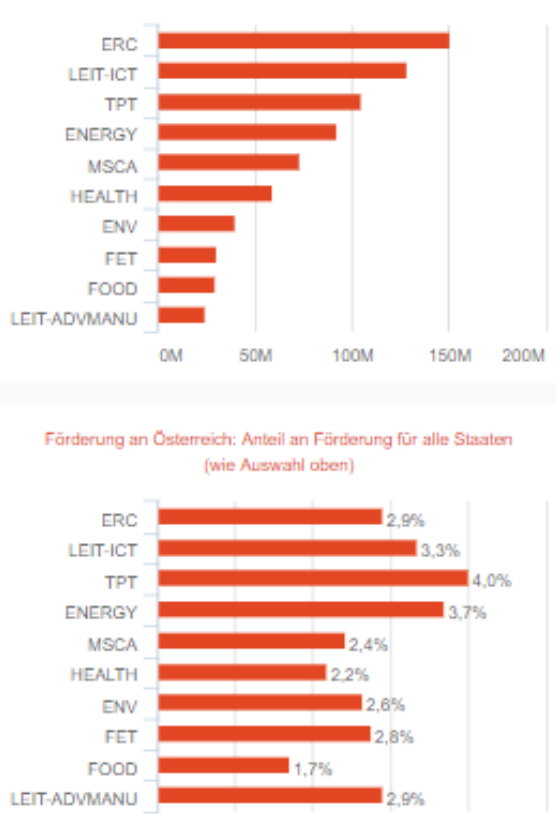

LEIT-ADVMANU

Top 30 Staaten
Beseilgunger aus Deutschland: Organisationstypen

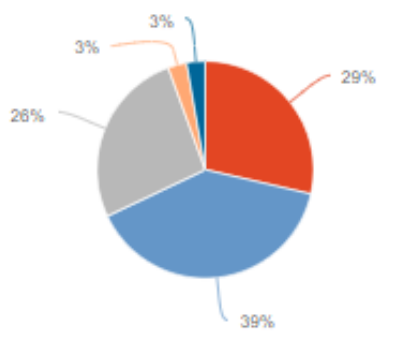

- HES DRC

Förderung an Deutschland in Mir. Euro fûr Tap Programme (wie Auswahl links)

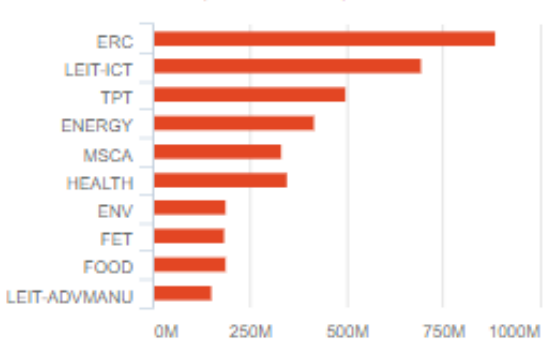

Förderung an Deutschlland: Anteil an Förderung für alle Stasten (wie Auswahl oben)

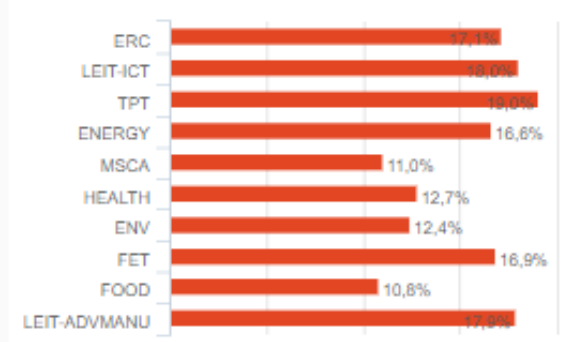

Quelle: https://eupm.ffg.at/ui/login/; Download 24.04.2018 
Abbildung 53: EU-PM, Kennzahlenvergleich Top 30 am Beispiel Beteiligungen

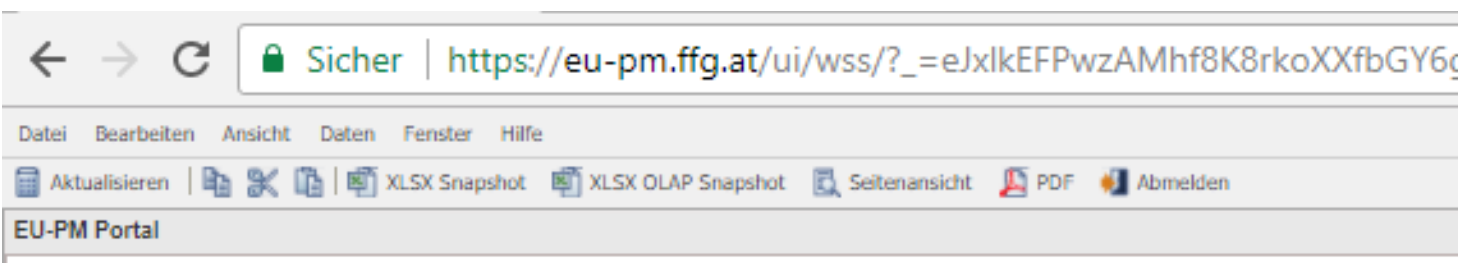

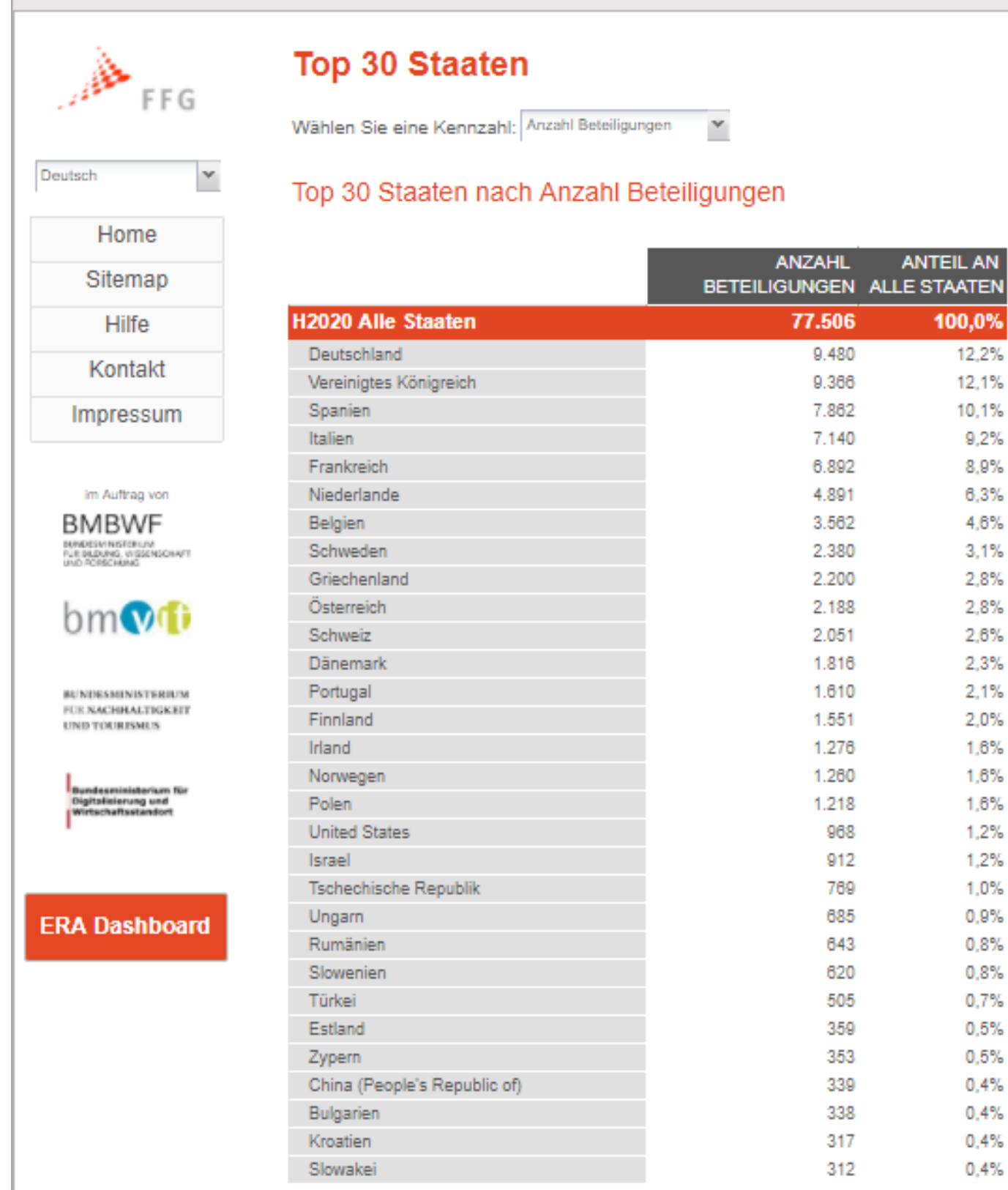

Quelle: https://eupm.ffg.at/ui/login/; Download 24.04.2018 
Abbildung 54: EU-PM, Übersicht AT-Beteiligung nach Programmen

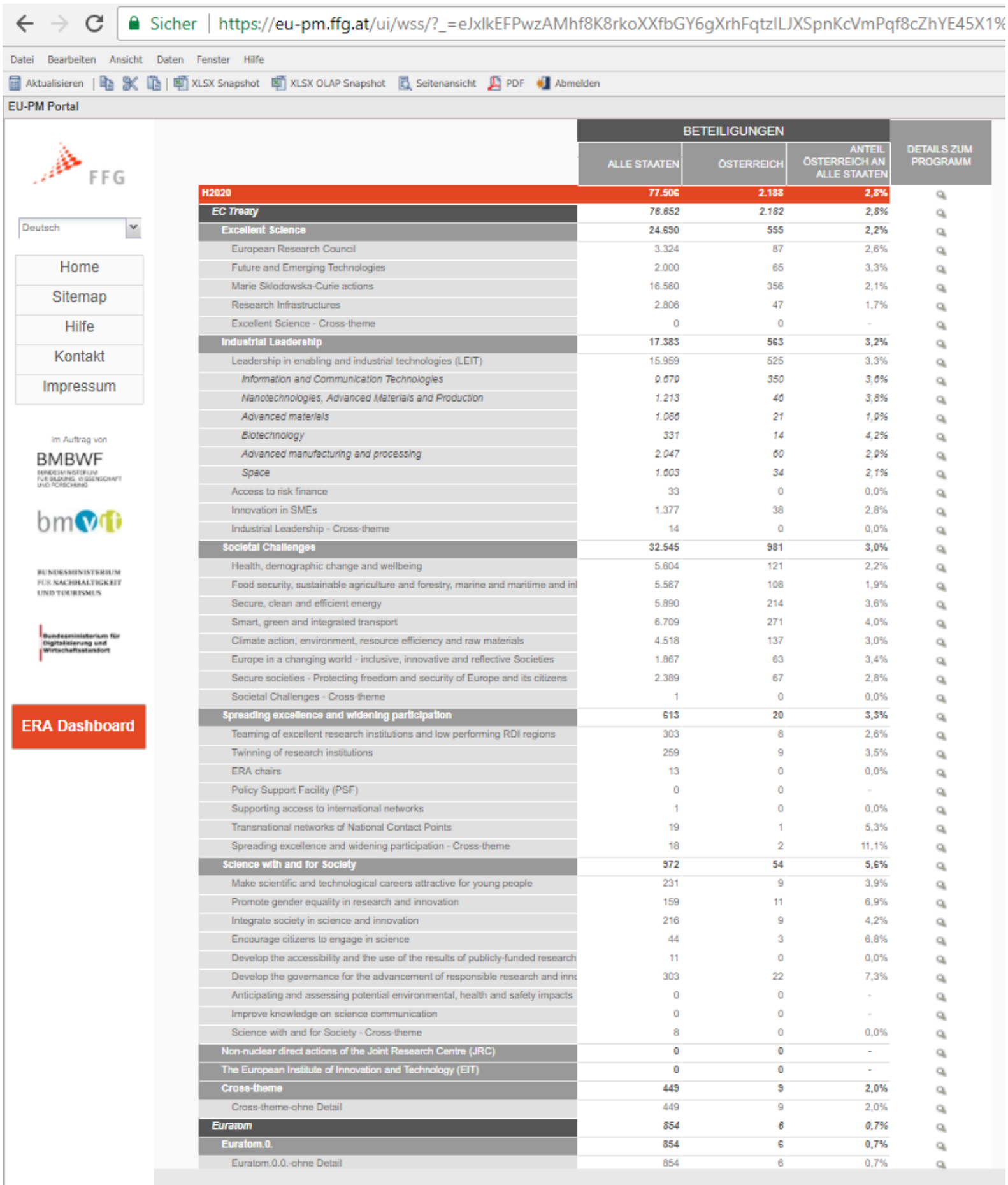

Quelle: https://eupm.ffg.at/ui/login/; Download 24.04.2018 
Abbildung 55: EU-PM, Vertragsnehmer auf Programmebene am Beispiel ERC-Grants

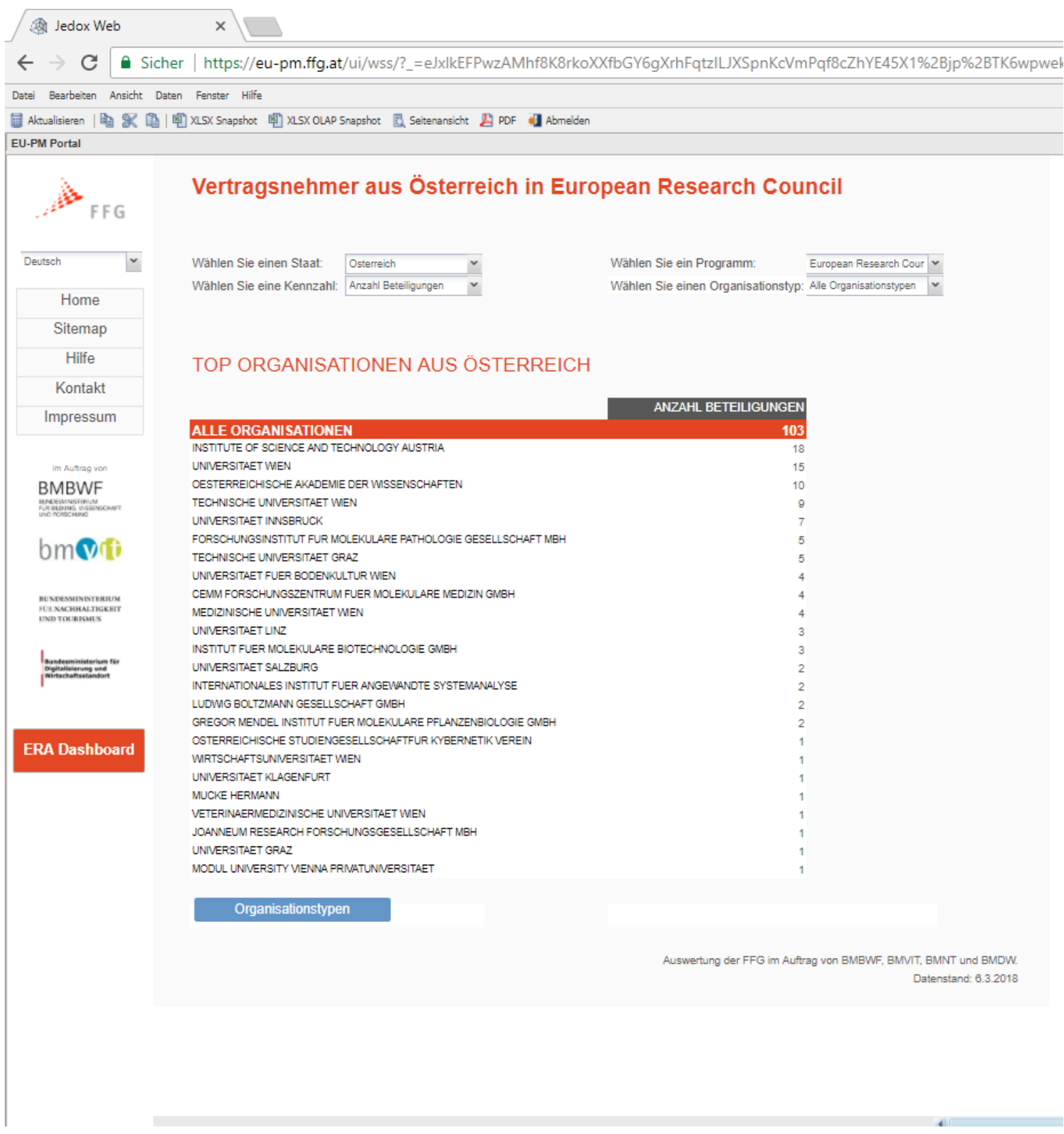

Quelle: https://eupm.ffg.at/ui/login/; Download 24.04.2018 
Abbildung 56: EU-PM, Beteiligungen nach Bundesland am Beispiel Oberösterreich

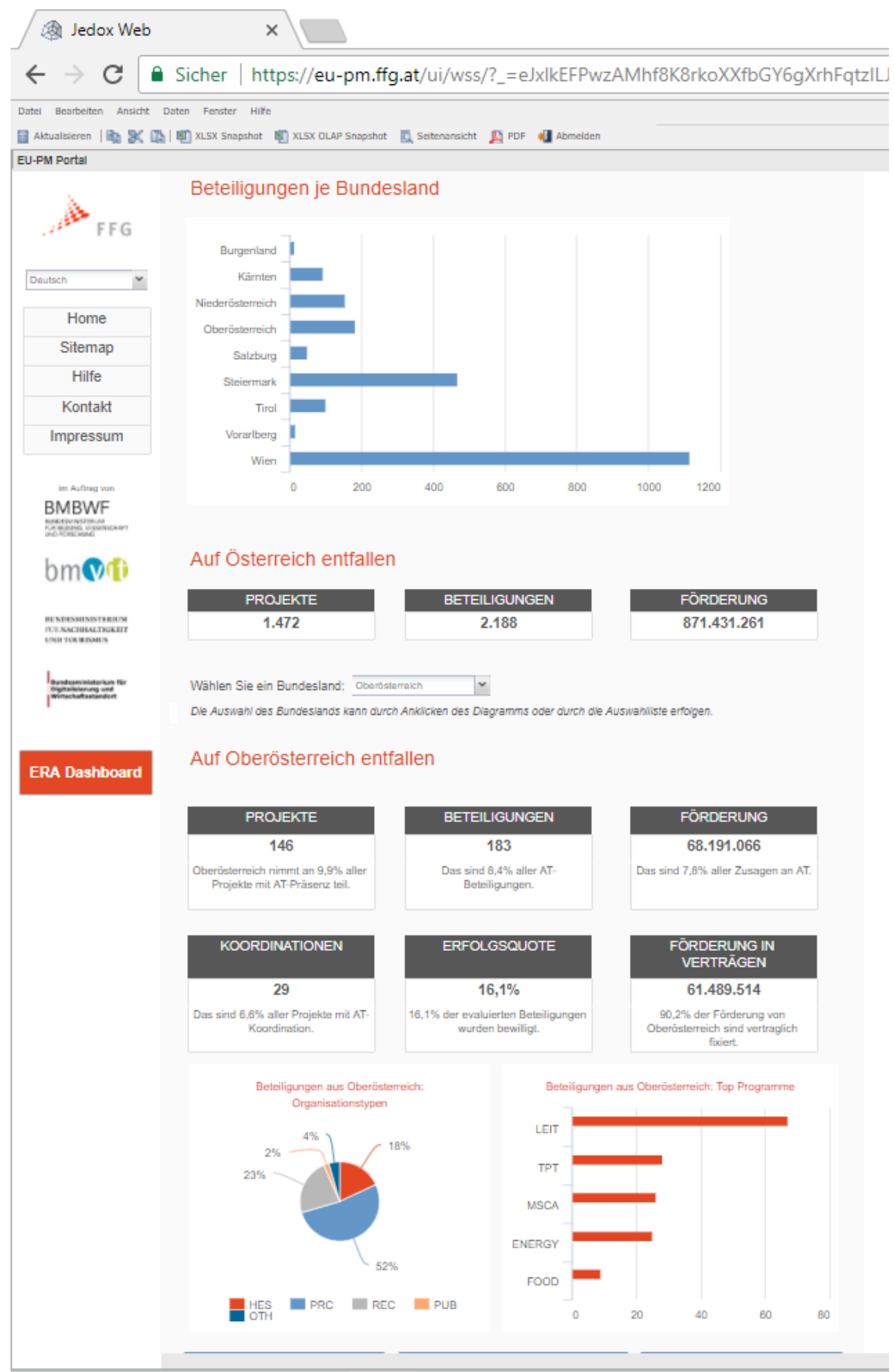

Quelle: https://eupm.ffg.at/ui/login/; Download 24.04.2018 


\section{Annex IV: TeilnehmerInnen an ExpertInneninterviews und Fokusgruppen}

Tabelle 16: ExpertInneninterviews

\begin{tabular}{|c|c|}
\hline Organisation & Name \\
\hline Akademie der bildenden Künste Wien & Mag. Michaela Glanz \\
\hline AWS & Mag. Wassilis Laitsos \\
\hline BMASGK & DI Dr. Eva Claudia Lang \\
\hline BMNT & Dr. Anita Silmbrod \\
\hline BMLV & Mag. Hans Starlinger \\
\hline BMVIT & DI. Brigitte Weiß \\
\hline BMVIT & Dr. Ralph Hammer \\
\hline BMDW & Dr. Stefan Riegler \\
\hline BMDW & Dr. Gabriele Schmid \\
\hline BMDW & Mag. Georg Panholzer \\
\hline BMBWF & Dr. Christian Naczinsky \\
\hline BMBWF & Mag. Martin Schmid \\
\hline BMBWF & Mag. Martina Hartl \\
\hline BMBWF & Mag. Patrizia Jankovic \\
\hline BMBWF & Dipl.-Ing. Ingeborg Schachner-Nedherer \\
\hline Business Upper Austria & Dr. Werner Pamminger \\
\hline Business Upper Austria & DI Dr. Markus Mair \\
\hline Business Upper Austria & Mag. Nicole Döberl \\
\hline ecoplus & Mag. Simone Hagenauer \\
\hline FFG & Mag. Andrea Höglinger \\
\hline FFG & Mag. Birgit Steininger \\
\hline FFG & Mag. Christian Frey \\
\hline FFG & Mag. Markus Bidmon \\
\hline FFG & DI Andrea Kindler \\
\hline FFG & DI Gerald Kern \\
\hline FFG & Mag. Josef Saeckl \\
\hline FFG & DI Thomas Zergoi \\
\hline Innovations- und Technologietransfer Salzburg GmbH & Ing. Mag. Hans-Christian Pfarrkirchner \\
\hline Marinomed & Dr. Andreas Grassauer \\
\hline
\end{tabular}




\begin{tabular}{|l|l|}
\hline Organisation & Name \\
\hline SCL Sensor Technologies & Dr. Ernest Georg Fantner \\
\hline SFG & Dr. Michael Kerschbaumer \\
\hline Standortagentur Tirol & MMag. Klaus Kleewein \\
\hline Technische Universität Graz & Priv.-Doz. Dr.rer.nat. Ursula Diefenbach \\
\hline Technische Universität Wien & DI Siegfried Huemer \\
\hline Ubimet & DI Alexander Arpaci \\
\hline Universität für Bodenkultur Wien & Dipl.Ing. Dr. Elisabeth Denk \\
\hline Universität Innsbruck & Dr. Kurt Habitzel \\
\hline Universität Wien & Mag. Dr. Lukas Zinner \\
\hline Universität Wien & Dr. Helmut Schaschl \\
\hline WISTO Vorarlberg & Mag. Rudolf Grimm \\
\hline WKÖ & MMag. Rudolf Lichtmanegger \\
\hline WKÖ & Mag. Devin Bicer \\
\hline WKÖ Kärnten & Dipl.-Ing. Elisabeth Hauer \\
\hline & Dr. Reinhold Mitterlehner \\
\hline & Mag. Dr. Harald Mahrer \\
\hline
\end{tabular}

Tabelle 17: Fokusgruppen

\begin{tabular}{|l|l|}
\hline Organisation & TeilnehmerInnen \\
\hline ACR & Johann Jäger \\
\hline AIT & Michael Parik \\
\hline Akademie der bildenden Künste Wien & Andrea B. Braidt \\
\hline Austriatech & Florian Kressler \\
\hline BOC Asset Management GmbH & Robert Woitsch \\
\hline Business Upper Austria & Nicole Katharina Döberl \\
\hline Donau-Universität Krems & Viktoria Weber \\
\hline EARTO & Erwan Kermadec \\
\hline ERA Council Forum & Helga Nowotny \\
\hline ERA Council Forum & Hermann Hauser \\
\hline ERA Council Forum & Jana Kolar \\
\hline ERA Council Forum & Jürgen Mlynek \\
\hline ERA Council Forum & Reinhilde Veugelers \\
\hline EUA & Lidia Borrell Damian \\
\hline
\end{tabular}




\begin{tabular}{|c|c|}
\hline Organisation & TeilnehmerInnen \\
\hline FCT, ERAC WG & Eduardo Maldonado \\
\hline Fronius International $\mathrm{GmbH}$ & Jürgen Danzberger \\
\hline Helioz GmbH & Gregor Riss \\
\hline Holzforschung & Angelika Rubick \\
\hline IHS & Bianca Brandl \\
\hline Joanneum Research & Roswitha Katter \\
\hline Kvm-tec electronic $\mathrm{GmbH}$ & Ingrid Pfurtscheller \\
\hline LBG & Jürgen Busch \\
\hline LieberLieber Software GmbH & Rüdiger Maier \\
\hline Medizinische Universität Graz & Tanja Ball \\
\hline Medizinische Universität Graz & Caroline Schober-Trummler \\
\hline Medizinische Universität Innsbruck & Christine Bandtlow \\
\hline Medizinische Universität Wien & Iris Weinbub \\
\hline Medizinische Universität Wien & Michaela Fritz \\
\hline Montanuniversität Leoben & Peter Moser \\
\hline ÖAW & Bedanna Bapuly \\
\hline $\begin{array}{l}\text { Permanent Representation of the Republic of } \\
\text { Slovenia to the EU }\end{array}$ & Sergej Možina \\
\hline Salzburg Research & Veronika Hornung-Prähauser \\
\hline SFG & Robert Hutter \\
\hline Spirit Design Innovation and Brand $\mathrm{GmbH}$ & Georg Wagner \\
\hline Technische Universität Graz & Horst Bischof \\
\hline Technische Universität Graz & Michaela Reininghaus \\
\hline Technische Universität Wien & Johannes Fröhlich \\
\hline Technische Universität Wien & Siegfried Huemer \\
\hline TecSense GmbH & Volker Ribitsch \\
\hline Universität für angewandte Kunst & Barbara Putz-Plecko \\
\hline Universität für Bodenkultur Wien & Elisabeth Denk \\
\hline Universität für Bodenkultur Wien & Josef GlößI \\
\hline $\begin{array}{l}\text { Universität für Musik und darstellende Kunst } \\
\text { Graz }\end{array}$ & Barbara Boisits \\
\hline Universität Innsbruck & Kurt Habitzel \\
\hline Universität Innsbruck & Sabine Schindler \\
\hline Universität Klagenfurt & Friederike Wall \\
\hline
\end{tabular}




\begin{tabular}{|l|l|}
\hline Organisation & TeilnehmerInnen \\
\hline Universität Linz & Alexander Egyed \\
\hline Universität Linz & Isabella Staska \\
\hline Universität Salzburg & Fatima Ferreira-Briza \\
\hline Universität Wien & Lucas Zinner \\
\hline Veterinärmedizinische Universität Wien & Otto Doblhoff-Dier \\
\hline Wirtschaftsagentur Burgenland GesmbH & Michael Sedlak \\
\hline Wirtschaftsagentur Wien & Sylvia Göttinger \\
\hline Wirtschaftsuniversität Wien & Stefan Pichler \\
\hline X-Net Technologies GmbH & Nikolaus Dürk \\
\hline
\end{tabular}




\section{Annex V: Mitglieder des internationalen ExpertInnenbeirates}

Dr. Jean Luc Barras, Abteilungsleiter im Schweizerischen Nationalfonds (SNF) und dort für interdisziplinäre und internationale Zusammenarbeit (inkl. H2020) zuständig

$>$ Prof. ${ }^{\text {in }}$ Dr. ${ }^{\text {in }}$ sc. nat., Dr. ${ }^{\text {in }}$ h. c. sc. Barbara Haering, Mitglied im Europäischen Forschungsrat sowie im ETH-Rat und im Stiftungsrat des Schweizer Nationalfonds

$>$ Dr. Harri Kulmala, CEO des Co-Creation Ökosystems und Innovationsplattform DIMECC (Finnland)

$>$ Dr. Svend Otto Remoe arbeitet im Direktorat des Research Council of Norway und ist dort u.a. als Politikanalyst mit Innovationspolitik betraut

$>$ Prof. ${ }^{\text {in }}$ Dr. ${ }^{\text {in }}$ Sylvia Schwaag-Serger, Direktorin für Internationale Angelegenheiten der schwedischen Innovationsagentur VINNOVA

Unabhängige Unterstützung und Moderation des ExpertInnenbeirates:

$>$ Dr. Dorothea Sturn, ehemalige Geschäftsführerin des Wissenschaftsfonds FWF 
